\title{
TEORIA DE SINGULARIDADES E PROBLEMAS DE BIFURCAÇÃO COM SIMETRIA NOS PARÂMETROS
}

\begin{abstract}
Angela Maria Sitta
Orientadores: Prof. Dr. Ian Stewart (*)

Profa. Dra. Maria Aparecida Soares Ruas

Tese apresentada ao Instituto de Ciências Matemáticas de São Carlos, da Universidade de São Paulo, para obtenção do Título de Doutor em Ciências - Área: Matemática: Geometria e Topologia.
\end{abstract}

(*) Programa Doutorado Sanduíche desenvolvido em conjunto com o ICMSC-USP e Universidade de Warwick - Inglaterra

São Carlos - SP

1993 
Ao meu pai

José Sitta Filho

(in memorian) 


\section{Minha eterna gratidão}

Aos orientadores: Ao Prof. Dr. Ian Stewart, orientador junto à Universidade de Warwick, que com sua precisa orientação abriu todos os caminhos para que este trabalho fosse concretizado. A sua confiança e entusiasmo me motivaram a seguir em frente. $\grave{A}$ Profa. Dra. Maria Aparecida Soares Ruas, orientadora junto ao ICMSC-USP, a quem devo minha formação básica e pacientemente acompanhou cada passo dessa etapa com sugestões e incentivos.

Ao co-orientador junto à Universidade de Warwick, Prof. Dr. Jacques Elie Furter, sempre incansável, pela imensa dedicação e valiosas sugestões.

Ao Prof. Dr. James Montaldi, por ter sugerido a "formulação por caminhos".

Ao Prof. Dr. Washington Luiz Marar, por ter sugerido o Doutorado Sanduíche junto à Universidade de Warwick.

Ao meu orientador acadêmico na primeira fase do doutorado, Prof. Dr. Paulo Ferreira da Silva Porto Junior, pelo incentivo ao Programa Sanduíche.

Ao Prof. Dr. Wilson Maurício Tadini, a quem devo minha vida profissional e formação acadêmica desde os tempos de Mestrado.

Alguns professores merecem espaço nessa página pela colaboração e apoio. São eles: Dr. Brasil Terra Leme, Dr. Carlos Biasi, Dr. Hermínio Cassago Júnior, Dr. José Alberto Cuminato, Dr. Luiz A. da C. Ladeira, Dr. Mark Roberts, Dr. Odelar Leite Linhares e Dr. Sebastião Antonio Izar.

Aos meus procuradores, Hélia Matiko Yano Kodama e Dr. Adalberto Spezamiglio.

Aos amigos: Camilo, Chlöe, Dirce, Gabriela, Greg, Lucia Helena, Miriam, Richard, Sandra e Walter.

Ao IBILCE-UNESP - Campus de São José do Rio Preto. Em particular, aos professores e funcionários do Departamento de Matemática.

Aos professores e funcionários do ICMSC-USP. 
À Universidade de Warwick. Em particular aos professores, funcionários e colegas do Instituto de Matemática.

À Luisa e ao Chico, pela digitação e desenhos.

À $C A P E S$ e ao $C N P q$.

À minha familia, sempre companheira.

$\dot{A}$ Deus, por tudo. 


\begin{abstract}
In this work we apply techniques from singularity theory to multiparameter bifurcation problems with diagonal symmetry on both the state variables and on the bifurcation parameters.

We also present algebraic approaches for the Path Formulation in this symmetric context and describe examples which motivated the point of view which was adopted.
\end{abstract}




\section{RESUMO}

Neste trabalho apresentamos técnicas da teoria de singularidades para problemas de bifurcação multiparamétricos com simetria diagonal nas variáveis-padrão e também nos parâmetros de bifurcação.

Também fazemos uma abordagem algébrica da Formulação por Caminhos neste contexto de simetria e descrevemos aplicações que motivaram o ponto de vista adotado. 


\section{Conteúdo}

1 Teoria geral 1

1.1 Definições gerais e teoremas ...................... 1

1.1.1 Notação e definiçôes . . . . . . . . . . . . . . . . . 1

1.1.2 Germes $\Gamma$-invariantes . . . . . . . . . . . . 2

1.1.3 Germes $\Gamma$-equivariantes . . . . . . . . . . . . . 3

1.2 Definiçốes e resultados devidos a Damon . . . . . . . . . . . 4

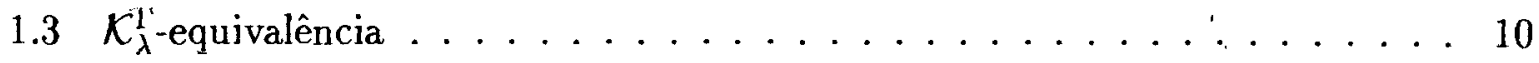

$1.4 \mathcal{K}_{\lambda, u n i}^{\Gamma}(k)$-equivalência $\ldots \ldots \ldots \ldots \ldots \ldots \ldots$

1.5 Espaços tangentes . . . . . . . . . . . . . . . . . . . 12

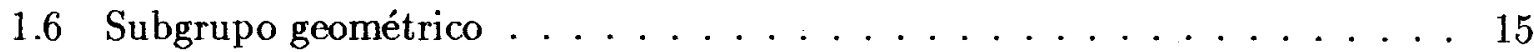

1.7 A Teoria de desdobramento . . . . . . . . . . . . . 17

1.8 Determinação finita . . . . . . . . . . . . . . . 27

1.8 .1 Jatos $\Gamma$-equivariantes . . . . . . . . . . . . 27

1.8.2 A órbita de um $k$-jato $\Gamma$-equivariante . . . . . . . . . 28

1.8.3 Lemas devidos a Damon . . . . . . . . . . . . . . 30

1.8.4 Germes finitamente determinados . . . . . . . . . . . 31

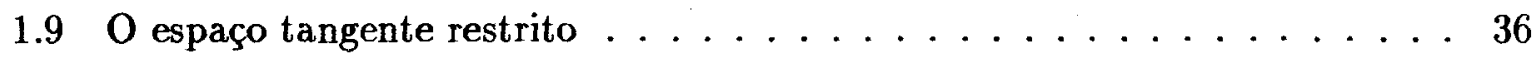

1.10 Problema do reconhecimento . . . . . . . . . . . . . 39

1.10.1 Submódulos intrínsicos e termos de ordem alta . . . . . . . . . . 40 
1.10 .2 Termos de ordem alta $\ldots \ldots \ldots \ldots \ldots \ldots$

$1.10 .3 \mathrm{O}$ subgrupo das $\mathcal{K}_{-}^{\Gamma}$-equivalências unipotentes $\ldots \ldots \ldots \ldots 4$

2 O problema $D_{4} \quad 43$

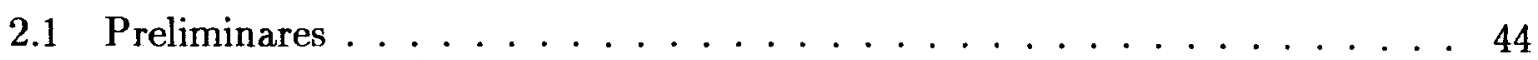

$2.2 \quad K_{\lambda}^{\mathrm{D}_{4} \text {-equivalência } \ldots \ldots \ldots \ldots \ldots \ldots \ldots \ldots \ldots \ldots \ldots \ldots \ldots \ldots \ldots}$

2.3 Espaços tangentes . . . . . . . . . . . . . . 47

2.4 Mudança de coordenadas . . . . . . . . . . . . . . . 49

2.5 Ideais intrínsicos e submódulos . . . . . . . . . . . . . 52

2.6 A forma genérica normal . . . . . . . . . . . . . . 53

2.7 Pré-forma normal . . . . . . . . . . . . . . . 58

2.8 Classificação parcial dos problemas $\mathrm{D}_{4} \ldots \ldots \ldots \ldots \ldots \ldots \ldots$

3 Formulação por caminhos e o problema $D_{4} \quad 71$

$3.1 \Sigma$-teoria para a formulação por caminhos $\ldots \ldots \ldots \ldots$

3.2 Espaços tangentes a um caminho . . . . . . . . . . . . 78

3.3 Formulação por caminhos e o problema $\mathrm{D}_{4} \ldots \ldots \ldots . \ldots 9$

4 Classificação dos problemas $D_{4}$ usando a fomulação por caminhos 108

4.1 Classificação dos problemas $\mathrm{D}_{4}$ com centro organizador $\mathrm{I}$. . . . . . . . . 110

5 Diagramas de bifurcạção $\quad 133$

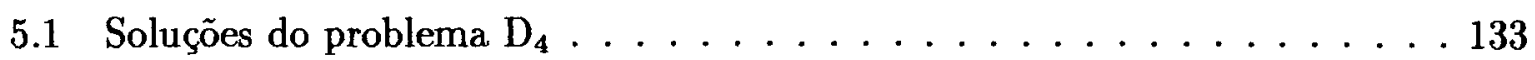

5.2 Estabilidade orbital . . . . . . . . . . . . . 136

5.3 Dados para o problema $\mathrm{D}_{4} \ldots \ldots \ldots \ldots \ldots \ldots$

5.4 Diagramas para a forma normal genérica . . . . . . . . . . . 140

5.5 Diagramas de bifurcação para a forma normal quando $p_{\lambda_{1}}^{o}=0 \ldots 170$ 
6.1 Motivação física . . . . . . . . . . . . . . . . . 224

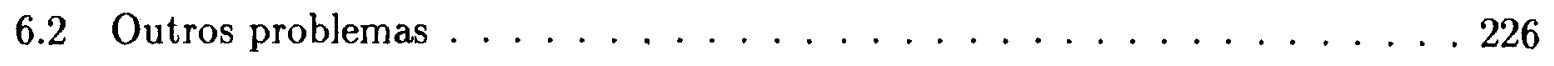

Apêndice $\quad \mathbf{2 2 9}$

$\begin{array}{ll}\text { Bibliografia } & \mathbf{2 3 5}\end{array}$ 


\section{Introdução}

Neste trabalho aplicamos técnicas da teoria de singularidades para o estudo de problemas de bifurcação multiparamétricos com simetria diagonal nas variáveis-padrão e também nos parâmetros de bifurcação.

Em [15] e [16], Golubitsky, Stewart e Schaeffer estudaram problemas de bifurcação com simetria apenas nas variáveis-padrão e com um parâmetro de bifurcação. Em [18], Peters classificou problemas de bifurcação com uma variável padrão e dois parâmetros de bifurcação. Ele também estendeu alguns resultados para os problemas com simetria diagonal tanto nas variáveis-padrão como nos parâmetros de bifurcação. Simultaneamente, em [1], Lavassani escreveu sobre problemas de bifurcação multiparamétricos com simetria apenas nas variáveis-padrão, seguindo [16] e os resultados algébricos de Damon apresentados em [10].

No capítulo 1, adaptamos os resultados sobre desdobramento, a caracterização algébrica da determinação finita e o problema do reconhecimento apresentados em [16], [10], [1] para o contexto onde há simetria diagonal nas variáveis-padrão e também nos multiparâmetros de bifurcação. As técnicas da teoria de singularidades são aplicadas para problemas de bifurcação multiparamétricos $\Gamma$-equivariantes segundo a ação diagonal de um grupo de Lie $\Gamma$ nas variáveis-padrão $x$ e nos multiparâmetros $\lambda$. Observamos que a teoria aqui apresentada também se estende a problemas $\Gamma$-equivariantes onde $\Gamma=\left(\Gamma_{1} \times \mathbf{I}, \mathbf{I} \times \Gamma_{2}\right)$ sendo $\Gamma_{1}$ e $\Gamma_{2}$ grupos de Lie compactos agindo, respectivamente, em $x$ por $g_{1} \cdot x$ e em $\lambda$ por $g_{2} \cdot \lambda$ e $\Gamma$ agindo em $(x, \lambda)$ via a composição das ações acima definidas, ou seja,

$$
\left(g_{1}, g_{2}\right) \cdot(x, \lambda)=\left(\left(g_{1}, \mathbf{1}\right) \cdot\left(\mathbf{1}, g_{2}\right)\right) \cdot(x, \lambda) .
$$

A extensão para o caso onde a ação de $\Gamma$ não é diagonal parece ser tecnicamente possível para os casos mais simples e será objeto de estudo futuro.

No capítulo 2, classificamos através de formas normais os problemas de bifurcação equivariantes com duas variảveis-padrão e dois parâmetros de bifurcação segụndo a ação do grupo diedral $\mathrm{D}_{4}$ nas variáveis e nos parâmetros. Utilizamos os métodos clássicos de determinação do espaço tangente e dos termos de ordem alta para obtenção das formas normais. Os resultados obtidos corrigem a forma genérica normal obtida por Peters em [18] e estendem a classificação para diagramas de codimensão topológica um. Este 
problema foi motivado pelo estudo da envergadura de uma placa quadrada quando um par de forças atua, horizontalmente e verticalmente, sobre a placa.

Consideramos no capítulo 3 o problema da formulação por caminhos dentro de um contexto puramente algébrico e com aplicações para o problema $\mathrm{D}_{4}$-equivariante apresentado no capítulo 2. Esta formulação foi apresentada em [15], onde Golubitsky e Schaeffer relacionaram problemas de bifurcação em uma variável-padrão e sem simetria com um caminho através do desdobramento miniversal de uma cuspóide, no sentido de Teoria das Catástrofes. Até então as técnicas da teoria de singularidades não eram suficientes para tratar dessa caracterização. Por isso, foi introduzido em [15], o conceito de equivalência de contato para problemas com parâmetros distinguidos. Essa idéia básica tem sido estendida e aplicada. Em [4], Montaldi e Mond aplicaram a idéia da $\mathcal{K}_{V}$-equivalência, apresentada por Damon em [11], à teoria de bifurcação equivariante utilizando a formulação por caminhos. Em [23], Bridges e Furter estudaram problemas de bifurcação gradiente e equivariantes através da formulação por caminhos e definiram uma relação de equivalência para caminhos que respeita a equivalência de contato para problemas de bifurcação gradiente. $O$ nosso trabalho apresenta uma abordagem algébrica da formulação de caminhos permitindo classificar as formas normais de uma maneira organizada e precisa. Além disso, minimiza os cálculos que são feitos utilizando-se a os métodos clássicos para obtenção de formas normais como no capítulo 2. Fica ainda em aberto uma caracterização geométrica da $\mathcal{K}_{V}$-equivalência para o nosso caso de simetria diagonal.

No capítulo 4, apresentamos algumas formas normais decorrentes da forma normal genérica dos problemas $\mathrm{D}_{4}$-equivariantes utilizando a formulação por caminhos. Trata-se da aplicação da teoria apresentada no capítulo 3 aos problemas de bifurcação $\mathrm{D}_{4}$-equivariantes introduzidos no capítulo 2.

O capítulo 5 trata da descrição geométrica do comportamento das soluções dos problemas de bifurcação $\mathrm{D}_{4}$-equivariantes através dos diagramas de bifurcação. Estudamos também a estabilidade assintótica dessas soluções. Analisamos em detalhes duas formas normais e seus respectivos desdobramentos.

No capítulo 6 relacionamos alguns problemas em outras áreas, como Mecânica, Física, Química e Teoria de Elasticidade, cuja descrição matemática se reduz a um problema 
$\mathrm{D}_{4}$-equivariante com ação diagonal. Entre esses, o problema da envergadura de uma placa quadrada foj a motivação original da tese e está sendo por nós considerado. 


\section{Capítulo 1}

\section{Teoria geral}

Neste capítulo apresentamos a teoria geral sobre desdobramento, determinação finita e problema do reconhecimento para singularidades multiparamétricas com simetria, ou seja, analisamos os problemas de bifurcação equivariantes onde um grupo de Lie compacto age diagonalmente nas variáveis-padrão e nos multiparâmetros de bifurcação. Os resultados apresentados são baseados no artigo de James Damon [10], na tese de doutoramento de Ali Lari-Lavassami [1] e no livro Singularities and Groups in Bifurcation Theory [16].

\subsection{Definições gerais e teoremas}

\subsubsection{Notação e definiçōes}

Denotamos as coordenadas no espaço padrâo $\mathbb{R}^{n}$ por $x=\left(x_{1} \ldots x_{n}\right)$ e as coordenadas no espaço dos parâmetros $\mathbb{R}^{\ell}$ por $\lambda=\left(\lambda_{1} \ldots \lambda_{\ell}\right)$.

Para multi-índices de números inteiros não negativos $\alpha=\left(\alpha_{1} \ldots \alpha_{n}\right), f_{x_{1}^{a_{1}} \ldots x_{n}^{a_{n}}}$, denota a derivadá parcial $\frac{\partial^{\alpha}}{\partial x} f$ e $\left(D_{x} f\right)$ a Jacobiana de $f$. Também, denotamos o valor na origem $g(0 \ldots 0)$ de qualquer função $g$ reperesentando $f$ ou suas derivadas por $g^{\circ}$.

Para $x \in \mathbb{R}^{n}, \mathcal{E}_{x}$ (ou $\mathcal{E}_{n}$ ) denota o anel usual de germes suaves $f:\left(\mathbb{R}^{n}, 0\right) \rightarrow \mathbb{R}$ e $\mathcal{M}_{x}$ (ou $\mathcal{M}_{n}$ ) seu ideal maximal de germes que se anulam na origem.

Para $y \in \mathbb{R}^{m}, \mathcal{E}_{x, y}\left(\right.$ ou $\left.\mathcal{E}_{n, m}\right)$ denota o $\mathcal{E}_{x^{-}}$módulo dos germes suaves $g:\left(\mathbb{R}^{n}, 0\right) \rightarrow \mathbb{R}^{m}$ e $\mathcal{M}_{x, y}$ (ou $\mathcal{M}_{n, m}$ ) seu submódulo de germes se anulando na origem. Quando $y$ for claro no contexto, denotaremos $\mathcal{E}_{x, y}$ por $\overrightarrow{\mathcal{E}}_{x}$ e $\mathcal{M}_{x, y}$ por $\overrightarrow{\mathcal{M}}_{x}$. 
Seja $G L(n)$ o grupo de todas as transformações lineares invertíveis do espaço $\mathbb{R}^{n}$ nele mesmo e $O(n)$ o seu subgrupo ortogonal n-dimensional.

Seja $\Gamma$ um grupo Lie compacto agindo no $\mathbb{R}^{m}$ e diagonalmente no $\mathbb{R}^{n+\ell}$ via as representações ortogonais

$$
\rho_{n}: \Gamma \rightarrow O(n), \quad \rho_{\ell}: \Gamma \rightarrow O(\ell) \quad \text { e } \quad \rho_{m}: \Gamma \rightarrow O(m)
$$

Denotamos por $\gamma_{n}, \gamma_{\ell}, \gamma_{m}$ as ações no $\mathbb{R}^{n}, \mathbb{R}^{\ell}, \mathbb{R}^{m}$ induzidas por $\rho_{n}, \rho_{\ell}, \rho_{m}$, respectivamente e identificamos $\gamma_{n} \operatorname{com} \rho_{n}(\gamma), \gamma_{\ell} \operatorname{com} \rho_{\ell}(\gamma)$ e $\gamma_{m} \operatorname{com} \rho_{m}(\gamma), \forall \gamma \in \Gamma$.

$\operatorname{Hom}_{\Gamma}\left(\mathbb{R}^{n}\right)$ denota o conjunto de aplicações lineares $\mathbb{R}^{n} \rightarrow \mathbb{R}^{n}$ que comutam com a ação de $\Gamma$ e $\stackrel{\circ}{\mathcal{L}}_{\Gamma}(n)$ a componente conexa $\operatorname{Hom}_{\Gamma}\left(\mathbb{R}^{n}\right) \cap G L(n)$ contendo a aplicação identidade.

A identidade de $G L(n)$ é denotado por $\mathbf{I}_{n}$ e o grupo trivial por 1 .

Sejam $R$ e $S$ anéis, $N$ um $R$-módulo, $M$ um $S$-módulo e $\phi: R \rightarrow S$ um homomorfismo de anel. Um homomorfismo de módulo sobre $\phi$ é por definição uma aplicação $\alpha: N \rightarrow M$ satisfazendo

1. $\alpha\left(n_{1}+n_{2}\right)=\alpha\left(n_{1}\right)+\alpha\left(n_{2}\right), n_{1}, n_{2} \in N$,

2. $\alpha(r n)=\phi(r) \alpha(n), \quad n \in N, \quad r \in R$.

O germe $g:\left(\mathbb{R}^{n}, 0\right) \rightarrow\left(\mathbb{R}^{m}, 0\right)$ induz o homomorfismo de anel, pull-back por $g$,

$$
\begin{aligned}
g^{*}: \mathcal{E}_{m} & \rightarrow \mathcal{E}_{n} \\
f & \mapsto f \circ g .
\end{aligned}
$$

\subsubsection{Germes $\Gamma$-invariantes}

Seja $\mathcal{E}_{(x, \lambda)}^{\Gamma}$ o anel dos germes suaves $\Gamma$-invariantes, isto é,

$$
\mathcal{E}_{(x, \lambda)}^{\Gamma}=\left\{h:\left(\mathbb{R}^{n+\ell} ; 0\right) \rightarrow \mathbb{R} \mid h\left(\gamma_{n} x, \gamma_{\ell} \lambda\right)=h(x, \lambda), \forall \gamma \in \Gamma\right\}
$$

e $\mathcal{M}_{(x, \lambda)}^{\Gamma}$ o seu ideal de germes que se anulam na origem. 
Um resultado de Schwarz [7] diz que existe um conjunto finito de $\Gamma$-polinômios invariantes $\bar{u}_{1}(x, \lambda) \ldots \bar{u}_{r}(x, \lambda)$ tais que todo elemento $h \in \mathcal{E}_{(x, \lambda)}^{\Gamma}$ pode ser escrito como o pull-back por $\bar{u}=\left(\bar{u}_{1} \ldots \bar{u}_{r}\right)$ de uma funçâo de $u=\left(u_{1} \ldots u_{r}\right)$, ou seja,

$$
\mathcal{E}_{(x, \lambda)}^{\Gamma}=\bar{u}^{*} \mathcal{E}_{u}
$$

Analogamente, seja

$$
\mathcal{E}_{\lambda}^{\Gamma}=\left\{\eta:\left(\mathbb{R}^{\ell}, 0\right) \rightarrow \mathbb{R} \mid \eta\left(\gamma_{\ell} \lambda\right)=\eta(\lambda), \forall \gamma \in \Gamma\right\}
$$

e $\mathcal{M}_{\lambda}^{\Gamma}$ seu ideal maximal. Todo elemento $\eta \in \mathcal{E}_{\lambda}^{\Gamma}$ pode ser escrito como uma funçâo de $\bar{v}=\left(\bar{v}_{1} \ldots \bar{v}_{t}\right)$ pulled back por $\left\{\bar{v}_{1} \ldots \bar{v}_{t}\right\}$, polinômios $\Gamma$-invariantes em $\lambda$ dados pelo Teorema de Schwarz, ou seja,

$$
\mathcal{E}_{\lambda}^{\Gamma}=\bar{v}^{*} \mathcal{E}_{v}
$$

\subsubsection{Germes $\Gamma$-equivariantes}

Seja $\overrightarrow{\mathcal{E}}_{(x, \lambda)}^{\Gamma}$ o $\mathcal{E}_{(x, \lambda)}^{\Gamma}$-módulo dos germes suaves $\Gamma$-equivariantes, isto é,

$$
\overrightarrow{\mathcal{E}}_{(x, \lambda)}^{\Gamma}=\left\{f:\left(\mathbb{R}^{n+\ell}, 0\right) \rightarrow \mathbb{R}^{m} \mid f\left(\gamma_{n} x, \gamma_{\ell} \lambda\right)=\gamma_{m} f(x, \lambda), \forall \gamma \in \Gamma\right\}
$$

Pelo Teorema de Finitude de Malgrange-Poénaru [24], $\overrightarrow{\mathcal{E}}_{(x, \lambda)}^{\Gamma}$ é gerado sobre $\mathcal{E}_{(x, \lambda)}^{\Gamma}$ por um conjunto finito de polinômios $\Gamma$-equivariantes $g_{1} \ldots g_{s}$. Consequentemente, usando as coordenadas $\Gamma$-invariantes $u_{i}, 1 \leq i \leq r$, e os polinômios $\Gamma$-equivariantes, $g_{j}, 1 \leq j \leq s$, toda $f \in \overrightarrow{\mathcal{E}}_{(x, \lambda)}^{\Gamma}$ pode ser escrita como

$$
f=\bar{u}^{*}\left(h_{1} g_{1}+\ldots+h_{s} g_{s}\right)
$$

para algum $h_{j} \in \mathcal{E}_{u}$. Usando (1.1) podemos identificar $\overrightarrow{\mathcal{E}}_{(x, \lambda)}^{\Gamma}$ com um submódulo de $\mathcal{E}_{u} \times \ldots \times \mathcal{E}_{u}=\left(\mathcal{E}_{u}\right)^{s}$ de tal forma que cada germe $f \in \overrightarrow{\mathcal{E}}_{(x, \lambda)}^{\Gamma}$ pode ser escrito como $f=\left[h_{1} \ldots h_{s}\right]$ para algum $h_{j} \in \mathcal{E}_{u}$. Note que, em geral, esse submódulo não é a soma direta de cópias de $\mathcal{E}_{u}$ (cf. Capítulo 2).

Seja $\overrightarrow{\mathcal{E}}_{\lambda}^{\Gamma}=\left\{\Lambda:\left(\mathbb{R}^{\ell}, 0\right) \rightarrow \mathbb{R}^{\ell} \mid \Lambda\left(\gamma_{\ell} \lambda\right)=\gamma_{\ell} \Lambda(\lambda), \forall \gamma \in \Gamma\right\} \circ \mathcal{E}_{\lambda}^{\Gamma}$-módulo dos germes suaves $\Gamma$-equivariantes em $\lambda$. 
Analogamente, existe um conjunto finito $p_{1} \ldots p_{\hat{t}}$ de polinômios $\Gamma$-equivariantes em $\lambda$ tais que

$$
\Lambda=\bar{v}^{*}\left(r_{1} p_{1}+\ldots+r_{\hat{t}} p_{\hat{t}}\right)
$$

para algum $r_{k} \in \mathcal{E}_{v}, 1 \leq k \leq \hat{t}$.

Denotamos por $\overrightarrow{\mathcal{M}}_{(x, \lambda)}^{\Gamma}$, e $\overrightarrow{\mathcal{M}}_{\lambda}^{\Gamma}$, respectivamente, os submódulos de $\overrightarrow{\mathcal{E}}_{(x, \lambda)}^{\Gamma}$ e de $\overrightarrow{\mathcal{E}}_{\lambda}^{\Gamma}$, formados por germes que se anulam na origem.

\subsection{Definições e resultados devidos a Damon}

Recordamos algumas definições básicas e alguns resultados devidos a Damon [10] que são necessários para estabelecer os Teoremas de Desdobramento e Determinação Finita.

Sejam $y \in \mathbb{R}^{n}$ e $y^{\prime} \in \mathbb{R}^{m}$. Uma álgebra diferencial (DA-álgebra) consiste de uma $\mathbb{R}$-álgebra $A$ e um homomorfismo de álgebra sobrejetor $\psi: \mathcal{E}_{y} \rightarrow A$. Essas álgebras são anéis locais com ideais maximais $\mathcal{M}_{A}$. Se $\phi: \mathcal{E}_{y^{\prime}} \rightarrow B$ definir outra DA-álgebra, então um homomorfismo de DA-álgebra $\alpha: A \rightarrow B$ será um homomorfismo de álgebra que levanta fornecendo o diagrama comutativo

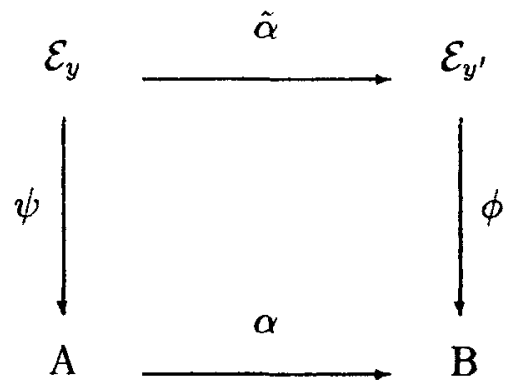

onde $\tilde{\alpha}=g^{*}$ para alguma $g:\left(\mathbb{R}^{m}, 0\right) \rightarrow\left(\mathbb{R}^{n}, 0\right)$.

Exemplo1.2.1. $\mathcal{E}_{(x, \lambda)}^{\Gamma}$ e $\mathcal{E}_{\lambda}^{\Gamma}$ são DA-álgebras.

(i) Defina $\phi:\left(\mathbb{R}^{n+\ell}, 0\right) \rightarrow\left(\mathbb{R}^{r}, 0\right)$ por $\phi(x, \lambda)=\left(\bar{u}_{1}(x, \lambda) \ldots \bar{u}_{r}(x, \lambda)\right)$ onde $\bar{u}=$ $\left(\bar{u}_{1} \ldots \bar{u}_{r}\right)$ é dado pelo Teorema de Schwarz. Isto induz o homomorfismo de álgebra 
sobrejetor

$$
\begin{aligned}
\phi^{*}: \mathcal{E}_{u} & \rightarrow \mathcal{E}_{(x, \lambda)}^{\Gamma} \\
h & \mapsto h \circ \phi .
\end{aligned}
$$

(ii) Analogamente, seja $\left\{\bar{v}_{1} \ldots \bar{v}_{t}\right\}$ o conjunto dos polinômios $\Gamma$-invariantes em $\lambda$. Defina

$$
\begin{aligned}
\psi:\left(\mathbb{R}^{\ell}, 0\right) & \rightarrow\left(\mathbb{R}^{\dagger}, 0\right) \\
\lambda & \mapsto\left(\bar{v}_{1}(\lambda) \ldots \bar{v}_{t}(\lambda)\right) .
\end{aligned}
$$

Isto induz o homomorfismo de álgebra sobrejetor

$$
\begin{aligned}
\psi^{*}: \mathcal{E}_{v} & \rightarrow \mathcal{E}_{\lambda}^{\Gamma} \\
f & \mapsto f \circ \psi .
\end{aligned}
$$

Além disso $\pi_{\ell}:\left(\mathbb{R}^{n+\ell}, 0\right) \rightarrow\left(\mathbb{R}^{\ell}, 0\right)$ induz o homomorfismo de DA-álgebra

$$
\begin{aligned}
\pi_{\ell}^{*}: \mathcal{E}_{\lambda}^{\Gamma} & \rightarrow \mathcal{E}_{(x, \lambda)}^{\Gamma} \\
g & \mapsto g \circ \pi_{\ell}
\end{aligned}
$$

Obviamente, $\pi_{\ell}^{*}(g) \in \mathcal{E}_{(x, \lambda)}^{\Gamma}$ e $\pi_{\ell}^{*}$ é um homomorfismo de álgebra. Resta mostrar que ele levanta para formar o diagrama comutativo

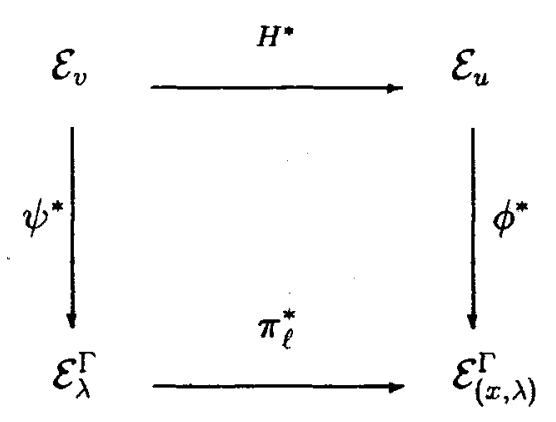

para $H:\left(\mathbb{R}^{r}, 0\right) \rightarrow\left(\mathbb{R}^{t}, 0\right)$ definida da seguinte maneira: para todo $1 \leq j \leq t$, $\bar{v}_{j} \circ \pi_{\ell} \in \mathcal{E}_{(x, \lambda)}^{\Gamma}$ e portanto, existe $h_{j} \in \mathcal{E}_{u}$ tal que

$$
\bar{v}_{j} \circ \pi_{\ell}=\phi^{*}\left(h_{j}\right)=h_{j} \circ \phi
$$

isto é,

$$
\left(\pi_{\ell}^{*} \circ \bar{v}_{j}\right)(x, \lambda)=h_{j}(\bar{u}(x, \lambda)) .
$$

Então, seja $H\left(u_{1} \ldots u_{r}\right)=\left(h_{1}(u) \ldots h_{t}(u)\right) \operatorname{com} u=\left(u_{1} \ldots u_{r}\right)$. 
Consequentemente, para $f \in \mathcal{E}_{v}$,

$$
\begin{aligned}
\left(\left(\phi^{*} \circ H^{*}\right)(f)\right)(x, \lambda) & =(f \circ H \circ \phi)(x, \lambda) \\
& =f\left(h_{1}(\bar{u}(x, \lambda)) \ldots h_{r}(\bar{u}(x, \lambda))\right) \\
& =f\left(\bar{v}_{1}(\lambda) \ldots \bar{v}_{r}(\lambda)\right) \\
& =f\left(\left(\bar{v}_{1} \circ \pi_{\ell}\right)(x, \lambda) \ldots\left(\bar{v}_{r} \circ \pi_{\ell}\right)(x, \lambda)\right) \\
& =\left(f \circ \psi \circ \pi_{\ell}\right)(x, \lambda) \\
& =\left(\left(\pi_{\ell}^{*} \circ \psi^{*}\right)(f)\right)(x, \lambda) .
\end{aligned}
$$

Definição. Sejam $R$ um anel e $\mathcal{I} \subset R$ um ideal. $\mathcal{I}$ será chamado Jacobson se $1+a$ for invertível em $R$ para todo $a \in \mathcal{I}$.

Exemplo 1.2.2. $\mathcal{M}_{(x, \lambda)}^{\Gamma}$ e $\mathcal{M}_{\lambda}^{\Gamma}$ são ideais de Jacobson.

Definição. Seja $(\mathcal{D},<)$ um conjunto finito de índices parcialmente ordenado. Um sistema $d \epsilon$ anéis associado a $\mathcal{D}$ consiste de um conjunto de anéis $\left\{R_{\alpha}: \alpha \in \mathcal{D}\right\}$ junto com os homomorfismos de anéis $\phi_{\alpha \beta}: R_{\alpha} \rightarrow R_{\beta}$ definida por $\alpha \leq \beta$ tais que $\phi_{\beta \gamma} \circ \phi_{\alpha \beta}=\phi_{\alpha \gamma}$, $\alpha \leq \beta \leq \gamma$ e $\phi_{\alpha \alpha}=i d$. Denotamos tal sistema por $\left\{R_{\alpha}\right\}$. Um sistema de ideais $\left\{\mathcal{I}_{\alpha}\right\}$ de $\left\{R_{\alpha}\right\}$ consiste de ideais $\mathcal{I}_{\alpha} \subset R_{\alpha}$ tais que $\phi_{\alpha \beta}\left(\mathcal{I}_{\alpha}\right) \subseteq \mathcal{I}_{\beta}$ para $\alpha \leq \beta$. Dessa forma, $\left\{\left(R_{\alpha}, \mathcal{I}_{\alpha}\right)\right\}$ denota o sistema de anéis e ideais.

Um sistema $\left\{R_{\alpha}\right\}$ para o qual $\mathcal{D}$ satisfaz a propriedade de que cada elemento tem, no máximo, um predecessor imediato é chamado sistema adequadamente ordenado.

Definição. Um sistema de DA-álgebras é um sistema de anéis $\left\{R_{\alpha}\right\}$ para os quais $R_{\alpha}$ é uma DA-álgebra e cada homomorfismo de conexão é um homomorfismo de DA-álgebra.

Exemplo 1.2.3. Segue do Exemplo 1.2.1 que $\left\{\mathcal{E}_{\lambda}^{\Gamma}, \mathcal{E}_{(x, \lambda)}^{\Gamma}\right\}$ é um sistema de DA-álgebras. Exemplo 1.2.4. Sejam

$$
\begin{aligned}
& \mathcal{E}_{(\lambda, \beta)}^{\Gamma}=\left\{g:\left(\mathbb{R}^{\ell+k}, 0\right) \rightarrow \mathbb{R} \mid g\left(\gamma_{\ell} \lambda, \beta\right)=g(\lambda, \beta), \forall \gamma \in \Gamma\right\} \\
& \mathcal{E}_{(x, \lambda, \beta)}^{\Gamma}=\left\{f:\left(\mathbb{R}^{n+\ell+k}, 0\right) \rightarrow \mathbb{R} \mid f\left(\gamma_{n} x, \gamma_{\ell} \lambda, \beta\right)=f(x, \lambda, \beta), \forall \gamma \in \Gamma\right\} .
\end{aligned}
$$

Neste caso $\Gamma$ age trivialmente sobre $\mathbb{R}^{k}$ e assim age ortogonalmente sobre $\mathbb{R}^{n+\ell+k}$. Dessa forma o Teorema de Schwarz se estende a este caso multiparamétrico da seguinte 
maneira: $\mathcal{E}_{(\lambda, \beta)}^{\Gamma}=\bar{v}^{*} \mathcal{E}_{(v, \beta)}$ e $\mathcal{E}_{(x, \lambda, \beta)}^{\Gamma}=\bar{u}^{*} \mathcal{E}_{(u, \beta)}$ onde $\left(\bar{v}_{1} \ldots \bar{v}_{t}\right)$ e $\left(\bar{u}_{1} \ldots \bar{u}_{\tau}\right)$ foram definidos anteriormente na página 3 .

Defina

$$
\begin{aligned}
\Phi:\left(\mathbb{R}^{\ell+k}, 0\right) & \rightarrow\left(\mathbb{R}^{t+k}, 0\right) \\
(\lambda, \beta) & \mapsto\left(\bar{v}_{1}(\lambda) \ldots \bar{v}_{t}(\lambda), \beta\right) .
\end{aligned}
$$

Isto induz o seguinte homomorfismo de álgebra sobrejetor

$$
\begin{aligned}
\Phi^{*}: \mathcal{E}_{(v, \beta)} & \rightarrow \mathcal{E}_{(\lambda, \beta)}^{\Gamma} \\
g & \mapsto g \circ \Phi .
\end{aligned}
$$

Analogamente, defina

$$
\begin{aligned}
\Psi:\left(\mathbb{R}^{n+\ell+k}, 0\right) & \rightarrow\left(\mathbb{R}^{r+k}, 0\right) \\
(x, \lambda, \beta) & \mapsto\left(\bar{u}_{1}(x, \lambda) \ldots \bar{u}_{r}(x, \lambda), \beta\right) .
\end{aligned}
$$

Isto induz o homomorfismo de álgebra sobrejetor

$$
\begin{aligned}
\Psi^{*}: \mathcal{E}_{(u, \beta)} & \rightarrow \mathcal{E}_{(x, \lambda, \beta)}^{\Gamma} \\
f & \mapsto f \circ \psi
\end{aligned}
$$

As projeções

$$
\begin{aligned}
& \mathbb{R}^{n+\ell+k} \stackrel{\pi_{\ell+k}}{\longrightarrow} \mathbb{R}^{\ell+k} \stackrel{\pi_{k}}{\longrightarrow} \mathbb{R}^{k} \\
& (x, \lambda, \beta) \mapsto(\lambda, \beta) \mapsto \beta
\end{aligned}
$$

dão origem ao sistema de DA-álgebras

$$
\mathcal{E}_{\beta} \stackrel{\pi_{k}^{*}}{\longrightarrow} \mathcal{E}_{(\lambda, \beta)}^{\Gamma} \stackrel{\pi_{\ell+k}^{*}}{\longrightarrow} \mathcal{E}_{(x, \lambda, \beta)}^{\Gamma}
$$

pois,

(i) $\quad \pi_{k}^{*}: \mathcal{E}_{\beta} \longrightarrow \mathcal{E}_{(\lambda, \beta)}^{\Gamma}$

$$
h \mapsto h \circ \pi_{k}
$$

$\pi_{k}^{*}(h) \in \mathcal{E}_{(\lambda, \beta)}^{\Gamma}, \pi_{k}^{*}$ é um homomorfismo de álgebra e levanta fornecendo o seguinte diagrama comutativo

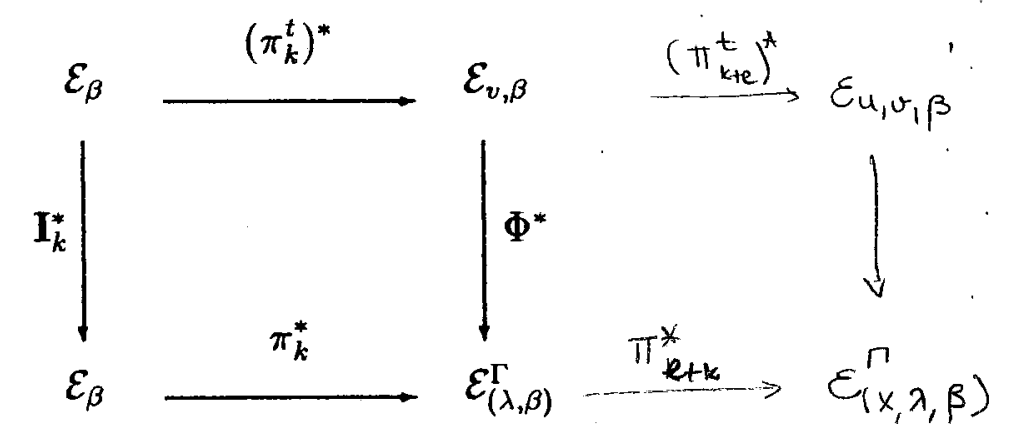


Para isso defina $\pi_{k}^{t}:\left(\mathbb{R}^{t+k}, 0\right) \rightarrow\left(\mathbb{R}^{k}, 0\right)$ como a projeção no $\mathbb{R}^{k}$.

(ii) $\quad \pi_{\ell+k}^{*}: \mathcal{E}_{(\lambda, \beta)}^{\Gamma} \longrightarrow \mathcal{E}_{(x, \lambda, \beta)}^{\Gamma}$

$$
g \mapsto g \circ \pi_{\ell+k}
$$

$\pi_{\ell+k}^{*}(g) \in \mathcal{E}_{(x, \lambda, \beta)}^{\Gamma}, \pi_{\ell+k}^{*}$ é também um homomorfismo de álgebra e levanta dando origem ao seguinte diagrama comutativo

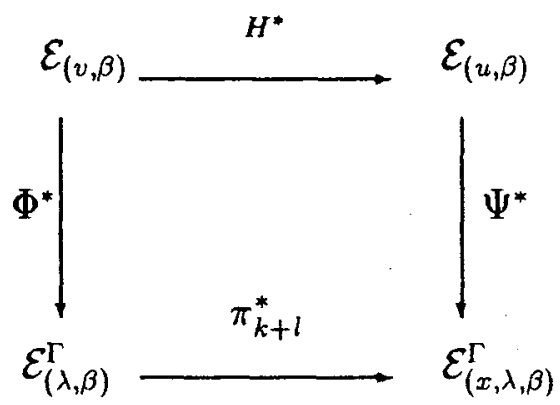

para $H:\left(\mathbb{R}^{r+k}, 0\right) \rightarrow\left(\mathbb{R}^{t+k}, 0\right)$ definida da seguinte maneira: para $1 \leq j \leq t$, $w_{j}:\left(\mathbb{R}^{\ell+k}, 0\right) \rightarrow(\mathbb{R}, 0)$ dada por $w_{j}(\lambda, \beta)=\bar{v}_{j}(\lambda)$ satisfaz $w_{j} \circ \pi_{\ell+k} \in \overrightarrow{\mathcal{E}}_{(x, \lambda, \beta)}^{\Gamma} \quad \mathrm{e}$ assim existe $h_{j} \in \mathcal{E}_{(u, \beta)}$ tal que $\left(w_{j} \circ \pi_{\ell+k}\right)(x, \lambda, \beta)=h_{j}\left(\bar{u}_{1}(x, \lambda) \ldots \bar{u}_{r}(x, \lambda), \beta\right)$. Seja $H\left(u_{1} \ldots u_{r}, \beta\right)=\left(h_{1}(u, \beta) \ldots h_{t}(u, \beta), \beta\right) \operatorname{com} u=\left(u_{1} \ldots u_{r}\right)$. Segue que para $g \in \mathcal{E}_{(v, \beta)}$, $\left(\left(\Psi^{*} \circ H^{*}\right)(g)\right)(x, \lambda, \beta)=(g \circ H \circ \Psi)(x, \lambda, \beta)$

$$
\begin{aligned}
& =g \circ H\left(\bar{u}_{1}(x, \lambda) \ldots \bar{u}_{r}(x, \lambda), \beta\right) \\
& =g\left(h_{1}(\bar{u}(x, \lambda), \beta) \ldots h_{t}(\bar{u}(x, \lambda), \beta), \beta\right) \\
& =g\left(w_{1} \circ \pi_{\ell+k}(x, \lambda, \beta) \ldots w_{t} \circ \pi_{\ell+k}(x, \lambda, \beta), \beta\right) \\
& =g\left(\bar{v}_{1}(\lambda) \ldots \bar{v}_{t}(\lambda), \beta\right) \\
& =\left(g \circ \Phi \circ \pi_{\ell+k}\right)(x, \lambda, \beta) \\
& =\left(\left(\pi_{\ell+k}^{*} \circ \Phi^{*}\right)(g)\right)(x, \lambda, \beta) .
\end{aligned}
$$

Portanto, $\left\{\mathcal{E}_{\beta}, \mathcal{E}_{(\lambda, \beta)}^{\Gamma}, \mathcal{E}_{(x, \lambda, \beta)}^{\Gamma}\right\}$ é um sistema de DA-álgebras.

Para especificar nesse sistema os ideais maximais de Jacobson escrevemos

$$
\left\{\left(\mathcal{E}_{\beta}, \mathcal{M}_{\beta}\right),\left(\mathcal{E}_{(x, \beta)}^{\Gamma}, \mathcal{M}_{(x, \beta)}^{\Gamma}\right),\left(\mathcal{E}_{(x, \lambda, \beta)}^{\Gamma}, \mathcal{M}_{(x, \lambda, \beta)}^{\Gamma}\right)\right\}
$$


e denotamos esse sistema de anéis e jdeajs por $\left\{\left(R_{(x, \lambda, \beta)}, m_{(x, \lambda, \beta)}\right)\right\}$.

Fazendo $\beta=0$, temos o seguinte sistema:

$$
\left\{\left(R_{(x, \lambda)}, m_{(x, \lambda)}\right)\right\}=\left\{\left(\mathcal{E}_{\lambda}^{\Gamma}, \mathcal{M}_{\lambda}^{\mathrm{T}}\right),\left(\mathcal{E}_{(x, \lambda)}^{\mathrm{\Gamma}}, \mathcal{M}_{(x, \lambda)}^{\mathrm{r}}\right)\right\}
$$

Definições. Um $\left\{R_{(\tau, \lambda, \beta)}\right\}$-módulo $M$ consiste de uma soma direta $M_{1} \oplus M_{2} \oplus M_{3}$ tal

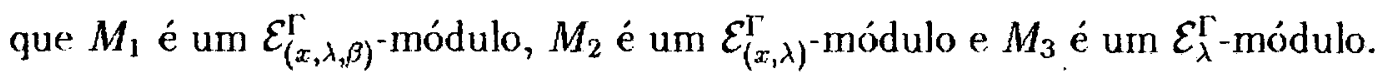

$M$ é dito finitamente gerado se cada $M_{i}$ for um módulo finitamente gerado sobre o anel correspondente, $1 \leq i \leq 3$.

$\operatorname{Um}\left\{R_{(x, \lambda, \beta)}\right\}$-homomorfismo de módulo $\psi: M \rightarrow N$ consiste de uma soma de homomorfismos $\psi_{j i}: M_{j} \rightarrow N_{i}$, para $1 \leq j \leq i \leq 3$, os quais são homomorfismos sobre os homomorfismos de conexão $\pi_{j i}^{*}$.

$N$ é um $\left\{R_{(x, \lambda, \beta)}\right\}$-submódulo de $M$ se $N=N_{1} \oplus N_{2} \oplus N_{3}$, onde $N_{i}$ é um submódulo de $M_{i}$ para todo $1 \leq i \leq 3$. Se $\left\{\mathcal{I}_{(x, \lambda, \beta)}\right\}=\left\{\mathcal{I}_{\beta}, \mathcal{I}_{(\lambda, \beta)}, \mathcal{I}_{(x ; \lambda, \beta)}\right\}$ for um sistema de ideais de $\left\{R_{(x, \lambda, \beta)}\right\}$, definimos

$$
\left\{\mathcal{I}_{(x, \lambda, \beta)}\right\} M=\mathcal{I}_{(x, \lambda, \beta)} \cdot M_{1} \oplus \mathcal{I}_{(\lambda, \beta)} \cdot M_{2} \oplus \mathcal{I}_{\beta} \cdot M_{3}
$$

e é um $\left\{R_{(x, \lambda, \beta)}\right\}$-submódulo de $M$.

Definições semelhantes podem ser feitas para o sistema $\left\{R_{(x, \lambda)}\right\}$, fazendo $\beta=0$.

Note que cada anel em $\left\{R_{(x, \lambda, \beta)}\right\}$ é uma $\mathcal{E}_{\beta}$-álgebra. Dizemos que $\left\{R_{(x, \lambda, \beta)}\right\}$ é um sistema adequadamente ordenado de $\mathrm{DA}$-álgebras sobre $\mathcal{E}_{\beta}$ se cada homomorfismo de conexão for um homomorfismo de $\mathcal{E}_{\beta}$-álgebra e cada anel tem exatamente um predecessor.

Lema 1.2.5. Considere o sistema de DA-álgebras e ideais de Jacobson $\left\{R_{(x, \lambda, \beta)}, \mathcal{J}_{(x, \lambda, \beta)}\right\}$ $=\left\{\left(\mathcal{E}_{\beta}, \mathcal{M}_{\beta}\right),\left(\mathcal{E}_{(\lambda, \beta)}^{\Gamma}, \mathcal{M}_{\beta} \cdot \mathcal{E}_{(x, \beta)}^{\Gamma}\right),\left(\mathcal{E}_{(x, \lambda, \beta)}^{\Gamma}, \mathcal{M}_{\beta} \cdot \mathcal{E}_{(x, \lambda, \beta)}^{\Gamma}\right)\right\}$. Sejam $M$ e $N\left\{R_{(x, \lambda, \beta)}\right\}-$ módulos finitamente gerados. Suponha que $\psi: N \rightarrow M$ é um homomorfismo de módulo sobre $\left\{R_{(x, \lambda, \beta)}\right\}$. Se

$$
\psi(N)+\mathcal{M}_{\beta} \cdot M=M
$$

então

$$
\psi(N)=M \text { e } \psi\left(\left\{\mathcal{J}_{(x, \lambda, \beta)}\right\} N\right)=\left\{\mathcal{J}_{(x, \lambda, \beta)}\right\} M .
$$

Prova. Por ([10], Corolário 6.16, pág. 33), $\left\{R_{(x, \lambda, \beta)}, \mathcal{J}_{(x, \lambda, \beta)}\right\}$ é adequado pois $\left\{R_{(x, \lambda, \beta)}\right\}$ é um sistema adequadamente ordenado de DA-álgebras sobre $\mathcal{E}_{\beta}$. 
O lema segue da definição de sistema adequado de anéis e ideais de Jacobson. ([10], Definiçâo 6.10, pág. 30)

Observação. O Lema 1.2.5 é uma versão generalizada do Teorema de Preparação de Malgrange.

\section{3 $\mathcal{K}_{\lambda}^{\Gamma}$-equivalência}

Seja

$$
\mathbf{M}_{(x, \lambda)}^{\Gamma}=\left\{T:\left(\mathbb{R}^{n+\ell}, 0\right) \rightarrow M_{m}(\mathbb{R}) \mid T\left(\gamma_{n} x, \gamma_{\ell} \lambda\right) \gamma_{m}=\gamma_{m} T(x, \lambda), \forall \gamma \in \Gamma\right\}
$$

o módulo sobre $\mathcal{E}_{(x, \lambda)}^{\Gamma}$ das matrizes que comutam com $\Gamma$.

Precisamos, também, dos seguintes $\mathcal{E}_{(x, \lambda)}^{\Gamma}$-módulos:

$\vec{\Theta}_{(x, \lambda)}^{\Gamma}=\left\{X:\left(\mathbb{R}^{n+\ell}, 0\right) \rightarrow \mathbb{R}^{n} \mid X\left(\gamma_{n} x, \gamma_{\ell} \lambda\right)=\gamma_{n} X(x, \lambda), \forall \gamma \in \Gamma\right\}$,

$\vec{\Theta}_{(x, \lambda)}^{\Gamma, o}=\left\{X \in \vec{\Theta}_{x, \lambda}^{\Gamma} \mid X^{\circ}=0\right\}$ e dos seguintes $\mathcal{E}_{\lambda}^{\Gamma}$-módulos:

$\vec{\Theta}_{\lambda}^{\Gamma}=\left\{\Lambda:\left(\mathbb{R}^{\ell}, 0\right) \rightarrow \mathbb{R}^{\ell} \mid \Lambda\left(\gamma_{\ell} \lambda\right)=\gamma_{\ell} \Lambda(\lambda), \forall \gamma \in \Gamma\right\}$

$\vec{\Theta}_{\lambda}^{\Gamma, o}=\left\{\Lambda \in \vec{\Theta}_{\lambda}^{\Gamma} \mid \Lambda^{o}=0\right\}$.

O grupo de contato $\mathcal{K}_{\lambda}^{\Gamma}$ é definido por

$$
\begin{aligned}
& \mathcal{K}_{\lambda}^{\Gamma}=\left\{(T, X, \Lambda) \in \mathbf{M}_{(x, \lambda)}^{\Gamma} \times \vec{\Theta}_{(x, \lambda)}^{\Gamma, o} \times \vec{\Theta}_{\lambda}^{\Gamma, o} \mid T^{o} \in \stackrel{\circ}{\mathcal{L}}_{\Gamma}(m),\right. \\
& \left.\left(D_{x} X\right)^{o} \in \stackrel{\circ}{\mathcal{L}}_{\Gamma}(n), \quad\left(D_{\lambda} \Lambda\right)^{o} \in \stackrel{\circ}{\mathcal{L}}_{\Gamma}(\ell)\right\} \text {. }
\end{aligned}
$$

Escrevendo $\varphi_{i}=\left(X_{i}, \Lambda_{i}\right), i=1,2$, então a estrutura de grupo de $\mathcal{K}_{\lambda}^{\Gamma}$ é dada pela seguinte multiplicação:

$$
\left(S_{2}, \varphi_{2}\right) \circ\left(S_{1}, \varphi_{1}\right)=\left(S_{2} \cdot\left(S_{1} \circ \varphi_{2}\right), \varphi_{1} \circ \varphi_{2}\right)
$$

onde

$$
S_{2} \cdot\left(S_{1} \circ \varphi_{2}\right)(x, \lambda)=S_{2}(x, \lambda) S_{1}\left(\varphi_{2}(x, \lambda)\right)
$$

e

$$
\varphi_{1} \circ \varphi_{2}(x, \lambda)=\left(X_{1} \circ \varphi_{2}(x, \lambda), \Lambda_{1} \circ \Lambda_{2}(\lambda)\right) .
$$


$\mathcal{K}_{\lambda}^{\Gamma}$ age de uma maneira natural em $f \in \overrightarrow{\mathcal{E}}_{(x, \lambda)}^{\Gamma}$ por

$$
(T, X, \Lambda) \cdot f(x, \lambda)=T(x, \lambda) f(X(x, \lambda), \Lambda(\lambda))
$$

Denotamos a órbita de $\mathrm{f}$ por $\mathcal{K}_{\lambda}^{\Gamma} \cdot f$.

Por definição, dois elementos $f, g \in \overrightarrow{\mathcal{E}}_{(x, \lambda)}^{\Gamma}$ são ditos $\mathcal{K}_{\lambda}^{\Gamma}$-equivalentes se eles pertencerem à mesma $\mathcal{K}_{\lambda}^{\Gamma}$-órbita.

\section{$1.4 \mathcal{K}_{\lambda, u n}^{\Gamma}(k)$-equivalência}

Perturbações dos elementos em $\overrightarrow{\mathcal{E}}_{(x, \lambda)}^{\Gamma}$ são descritas usando o conceito de desdobramento.

$$
\begin{aligned}
& \operatorname{Sejam} \beta=\left(\beta_{1} \ldots \beta_{k}\right) \text { e } \\
& \mathbf{M}_{(x, \lambda, \beta)}^{\Gamma}=\left\{T:\left(\mathbb{R}^{n+\ell+k}, 0\right) \rightarrow M_{m}(\mathbb{R}) \mid T\left(\gamma_{n} x, \gamma_{\ell} \lambda, \beta\right) \gamma_{m}=\gamma_{m} T(x, \lambda, \beta), \forall \gamma \in \Gamma\right\}, \\
& \overrightarrow{\mathcal{E}}_{(x, \lambda, \beta)}^{\Gamma}=\left\{F:\left(\mathbb{R}^{n+\ell+k}, 0\right) \rightarrow \mathbb{R}^{m} \mid F\left(\gamma_{n} x, \gamma_{\ell} \lambda, \beta\right)=\gamma_{m} F(x, \lambda, \beta), \forall \gamma \in \Gamma\right\}, \\
& \vec{\Theta}_{(x, \lambda, \beta)}^{\Gamma, o}=\left\{X:\left(\mathbb{R}^{n+\ell+k}, 0\right) \rightarrow\left(\mathbb{R}^{n}, 0\right) \mid X\left(\gamma_{n}^{\prime} x, \gamma_{\ell} \lambda, \beta\right)=\gamma_{n} X(x, \lambda, \beta), \forall \gamma \in \Gamma\right\}, \\
& \vec{\Theta}_{(\lambda, \beta)}^{\Gamma, o}=\left\{\Lambda:\left(\mathbb{R}^{\ell+k}, 0\right) \rightarrow\left(\mathbb{R}^{\ell}, 0\right) \mid \Lambda\left(\gamma_{\ell} \lambda, \beta\right)=\gamma_{\ell} \Lambda(\lambda, \beta), \forall \gamma \in \Gamma\right\} .
\end{aligned}
$$

Um desdobramento de $f \in \overrightarrow{\mathcal{E}}_{(x, \lambda)}^{\mathrm{r}} \operatorname{com} k$ parâmetros $\beta=\left(\beta_{1} \ldots \beta_{k}\right)$ é um germe de aplicação $F \in \overrightarrow{\mathcal{E}}_{(x, \lambda, \beta)}^{\Gamma}$ tal que $F(x, \lambda, 0)=f(x, \lambda)$.

Denotamos por $\mathcal{K}_{\lambda, u n}^{\Gamma}(k)$ o grupo das $\Gamma$-equivalências para desdobramentos com $k$ parâmetros. É uma extensão de $\mathcal{K}_{\lambda}^{\Gamma}$ da seguinte maneira:

$$
\begin{aligned}
\mathcal{K}_{\lambda, u n}^{\Gamma}(k)= & \left\{(T, X, \Lambda, \Phi) \in \mathbf{M}_{(x, \lambda, \beta)}^{\Gamma} \times \vec{\Theta}_{(x, \lambda, \beta)}^{\Gamma, o} \times \vec{\Theta}_{(\lambda, \beta)}^{\Gamma, o} \times \mathcal{M}_{\beta, \beta} \mid\right. \\
& (T, X, \Lambda) \text { é um desdobramento com } k \text { parâmetros de um elemento de } \mathcal{K}_{\lambda}^{\Gamma} \mathrm{e} \\
& \Phi \text { é um germe de difeomorfismo }\}
\end{aligned}
$$

A ação de $\mathcal{K}_{\lambda, u n}^{\Gamma}(k)$ em $F \in \overrightarrow{\mathcal{E}}_{(\dot{x}, \lambda, \beta)}^{\Gamma}$ é definida por

$$
(T, X, \Lambda, \Phi) \cdot F(x, \lambda, \beta)=T(x, \lambda, \beta) F(X(x, \lambda, \beta), \Lambda(\lambda, \beta), \Phi(\beta)) .
$$

Denotamos a órbita de $F$ por $\mathcal{K}_{\lambda, \text { un }}^{\Gamma}(k) \cdot F$.

Dizemos que $F, G \in \overrightarrow{\mathcal{E}}_{(x, \lambda, \beta)}^{\Gamma}$ são $\mathcal{K}_{\lambda, u n}^{\Gamma}(k)$-equivalentes se eles pertencerem à mesma $\mathcal{K}_{\lambda, \text { un }}^{\Gamma}(k)$-órbita. 
Observações. Para o estudo de desdobramentos, o sistema adequadamente ordenado de DA-álgebras é $\left\{\mathcal{E}_{\beta} . \mathcal{E}_{(\lambda, \beta)}^{\Gamma}, \mathcal{E}_{(x, \lambda, \beta)}^{\Gamma}\right\}$ e para germes em $\overrightarrow{\mathcal{E}}_{(x, \lambda)}^{\Gamma}$ é $\left\{\mathcal{E}_{\lambda}^{\Gamma}, \mathcal{E}_{(x, \lambda)}^{\Gamma}\right\}$.

A definição de $\mathcal{K}_{\lambda, u n}^{\Gamma}(k)$ é suficiente para o que pretendemos. Na teoria geral de Damon (cf. Seç̧âo 1.6), dois grupos de equivalência sâo considerados para desdobramentos. Ambos são subgrupos de $\mathcal{K}_{\lambda, u n}^{\Gamma}(k)$ sendo que o primeiro é dado com $\Phi=\mathbf{I}_{\beta}$ e o segundo ' com $(T, X, \Lambda)$ sendo um desdobramento da identidade de $\mathcal{K}_{\lambda}^{r}$.

\subsection{Espaços tangentes}

O espaço tangente $\dot{a} \mathcal{K}_{\lambda}^{\Gamma}$ no seu elemento identidade é definido por

$$
\mathcal{T}\left(\mathcal{K}_{\lambda}^{\Gamma}\right)=\left\{\left.\frac{d}{d t}\right|_{t=0} \delta_{t} \mid \delta_{t} \in \mathcal{K}_{\lambda}^{\Gamma}, \delta_{0}=\mathbf{1}\right\}
$$

e um cálculo direto mostra que existe uma identificação

$$
\mathcal{T}\left(\mathcal{K}_{\lambda}^{\Gamma}\right) \cong \mathbf{M}_{(x, \lambda)}^{\Gamma} \oplus \vec{\Theta}_{(x, \lambda)}^{\Gamma, o} \oplus \vec{\Theta}_{\lambda}^{\Gamma, o}
$$

Como $\mathbf{M}_{(x, \lambda)}^{\Gamma}$ e $\vec{\Theta}_{(x, \lambda)}^{\Gamma, o}$ são módulos finitamente gerados sobre $\mathcal{E}_{(x, \lambda)}^{\Gamma}$ e $\vec{\Theta}_{\lambda}^{\Gamma, o}$ é um módulo finitamente gerado sobre $\mathcal{E}_{\lambda}^{\Gamma}, \mathcal{T}\left(\mathcal{K}_{\lambda}^{\Gamma}\right)$ é uma soma direta de módulos finitamente gerados sobre o sistema de anéis $\left\{R_{(x, \lambda)}\right\}$.

Note que nessa definiçâo, a origem ficou fixada. Quando a origiem não é fixa, definimos o espaço tangente extendido ([10], pág. 3) e o denotamos por $\mathcal{T}_{e}^{\Gamma}$. Um vetor tangente em $\mathcal{T}_{e}^{\Gamma}$ é visto como o vetor tangente $\left.\frac{d}{d t}\right|_{t=0} E_{t}$ à origem de um desdobramento a um parâmetro $\mathrm{E} \in \mathcal{K}_{\lambda, u n}^{\Gamma}(1)$ da identidade de $\mathcal{K}_{\lambda}^{\Gamma}$. Assim, temos a seguinte identificação

$$
\mathcal{T}_{e}^{\Gamma} \cong \mathbf{M}_{(x, \lambda)}^{\Gamma} \oplus \vec{\Theta}_{(x, \lambda)}^{\Gamma} \oplus \vec{\Theta}_{\lambda}^{\Gamma}
$$

Analogamente, identificamos

$$
\mathcal{T}_{e}\left(\mathcal{K}_{\lambda, u n}^{\Gamma}(k)\right) \cong \mathbf{M}_{(x, \lambda, \beta)}^{\Gamma} \oplus \vec{\Theta}_{(x, \lambda, \beta)}^{\Gamma} \oplus \vec{\Theta}_{(\lambda, \beta)}^{\Gamma} \oplus \mathcal{E}_{\beta, \beta}
$$

Segue que $\mathcal{T}_{e}^{\Gamma}$ é um módulo finitamente gerado sobre o sistema de anéis $\left\{R_{(x, \lambda)}\right\}$ e que $\mathcal{T}_{e}\left(\mathcal{K}_{\lambda, u n}^{\Gamma}(k)\right)$ é um módulo finitamente gerado sobre o sistema de anéis $\left\{R_{(x, \lambda, \beta)}\right\}$. 
Seja $f \in \overrightarrow{\mathcal{E}}_{(x, \lambda)}^{\Gamma}$. A aplicaçâa oŕbita associada com $f$ é uma aplicação

$$
\begin{aligned}
\mathcal{O}_{f}: & \mathcal{K}_{\lambda}^{\Gamma} \rightarrow \overrightarrow{\mathcal{E}}_{(x, \lambda)}^{\Gamma} \\
& (T, X, \Lambda) \mapsto(T, X, \Lambda) \cdot f .
\end{aligned}
$$

Identificando o espaço tangente à $\overrightarrow{\mathcal{E}}_{(x, \lambda)}^{\Gamma}$ com ele mesmo, segue que

$$
\begin{aligned}
& d \mathcal{O}_{f}: \mathcal{T}\left(\mathcal{K}_{\lambda}^{\Gamma}\right) \cong \mathbf{M}_{(x, \lambda)}^{\Gamma} \oplus \vec{\Theta}_{(x, \lambda)}^{\Gamma, o} \oplus \vec{\Theta}_{\lambda}^{\Gamma, o} \rightarrow \overrightarrow{\mathcal{E}}_{(x, \lambda)}^{\Gamma} \\
&(T, X, \Lambda) \mapsto T f+\left(D_{x} f\right) X+\left(D_{\lambda} f\right) \Lambda .
\end{aligned}
$$

Sabemos que $\mathcal{T}\left(\mathcal{K}_{\lambda}^{\Gamma}\right)$ e $\overrightarrow{\mathcal{E}}_{(x, \lambda)}^{\mathrm{r}}$ são módulos finitamente gerados sobre o sistema adequadamente ordenado de DA-álgebras $\left\{R_{(x, \lambda)}\right\}$. Além disso, $d \mathcal{O}_{f}$ é um homomorfismo de módulo sobre esse sistema, pois:

(i) $\mathbf{M}_{(x, \lambda)}^{\Gamma} \oplus \vec{\Theta}_{(x, \lambda)}^{\Gamma, o} \rightarrow \overrightarrow{\mathcal{E}}_{(x, \lambda)}^{\Gamma}$

$$
(T, X) \mapsto T f+\left(D_{x} f\right) X
$$

é um homomorfismo de $\mathcal{E}_{(x, \lambda)}^{\Gamma}$-módulo. Note que $\mathbf{M}_{(x, \lambda)}^{\Gamma} \oplus \vec{\Theta}_{(x, \lambda)}^{\Gamma, o}$ é um $\mathcal{E}_{(x, \lambda)}^{\Gamma}$-módulo e assim trivialmente um módúlo sobre o sistema $\left\{R_{(x, \lambda)}\right\}$.

(ii) $\overrightarrow{\mathcal{E}}_{\lambda}^{\Gamma} \rightarrow \overrightarrow{\mathcal{E}}_{(x, \lambda)}^{\Gamma}$

$$
\Lambda \mapsto\left(D_{\lambda} f\right) \Lambda
$$

é um módulo de homomorfismo sobre $\mathcal{E}_{\lambda}^{\Gamma} \stackrel{\pi_{i}^{*}}{\rightarrow} \mathcal{E}_{(x, \lambda)}^{\Gamma}$.

Uma construção análoga vale para $\mathcal{T}_{e}^{\Gamma}$ e desdobramentos, ou seja, podemos estender $d \mathcal{O}_{f}$ para $\mathcal{T}_{e}^{\Gamma}:$

$$
\begin{aligned}
d \mathcal{O}_{f}: & \mathcal{T}_{e}^{\Gamma} \cong \mathbf{M}_{(x, \lambda)}^{\Gamma} \oplus \vec{\Theta}_{(x, \lambda)}^{\Gamma} \oplus \vec{\Theta}_{\lambda}^{\Gamma} \rightarrow \overrightarrow{\mathcal{E}}_{(x, \lambda)}^{\Gamma} \\
& (T, X, \Lambda) \mapsto T f+\left(D_{x} f\right) X+\left(D_{\lambda} f\right) \Lambda .
\end{aligned}
$$

$\vec{\Theta}_{(x, \lambda)}^{\Gamma}$ é um módulo sobre $\mathcal{E}_{(x, \lambda)}^{\Gamma}, \overrightarrow{\mathcal{E}}_{\lambda}^{\Gamma}$ é um módulo sobre $\mathcal{E}_{\lambda}^{\Gamma}$ e assim $d \mathcal{O}_{f}$ é também um homomorfismo de módulo sobre o sistema $\left\{R_{(x, \lambda)}\right\}$.

Para $F \in \overrightarrow{\mathcal{E}}_{(x, \lambda, \beta)}^{\Gamma}$,

$$
\begin{gathered}
d \mathcal{O}_{F}: \mathcal{T}_{e}\left(\mathcal{K}_{\lambda, u n}^{\Gamma}(k)\right) \simeq \mathbf{M}_{(x, \lambda, \beta)}^{\Gamma} \oplus \vec{\Theta}_{(x, \lambda, \beta)}^{\Gamma} \oplus \vec{\Theta}_{(\lambda, \beta)}^{\Gamma} \oplus \mathcal{E}_{\beta, \beta} \rightarrow \overrightarrow{\mathcal{E}}_{(x, \lambda, \beta)}^{\Gamma} \\
(T, X, \Lambda, \Phi) \mapsto T F+\left(D_{x} F\right) X+\left(D_{\lambda} F\right) \Lambda+\left(D_{\beta} F\right) \Phi
\end{gathered}
$$


$\mathcal{T}_{\epsilon}\left(\mathcal{K}_{\lambda, \text { un }}^{\Gamma}(k)\right)$ e $\overrightarrow{\mathcal{E}}_{(x, \lambda)}^{\Gamma}$ sâo módulos finitamente gerados sobre o sistema $\left\{R_{(x, \lambda, \beta)}\right\}$ e $d \mathcal{O}_{F}$ é um homomorfismo de módulo sobre esse sistema.

Seguindo a teoria geral de ações de grupo em variedades, definimos os espaços tangentes como sendo a imagem da derjvada da aplicação órbita e usando (1.6), (1.7) e (1.8), definimos os espaços tangentes com os quais vamos trabalhar.

Definição. Sejam $f \in \overrightarrow{\mathcal{E}}_{(x, \lambda)}^{\Gamma}$ e $F \in \overrightarrow{\mathcal{E}}_{(x, \lambda, \beta)}^{\Gamma}$.

$$
\begin{gathered}
\mathcal{T}^{\Gamma}(f)=\left\{T f+\left(D_{x} f\right) X+\left(D_{\lambda} f\right) \Lambda \mid T \in \mathbf{M}_{(x, \lambda)}^{\Gamma}, X \in \vec{\Theta}_{(x, \lambda)}^{\Gamma, o}, \Lambda \in \vec{\Theta}_{\lambda}^{\Gamma, o}\right\} . \\
\mathcal{T}_{e}^{\Gamma}(f)=\left\{T f+\left(D_{x} f\right) X+\left(D_{\lambda} f\right) \Lambda \mid T \in \mathbf{M}_{(x, \lambda)}^{\Gamma}, X \in \vec{\Theta}_{(x, \lambda)}^{\Gamma}, \Lambda \in \overrightarrow{\mathcal{E}}_{\lambda}^{\Gamma}\right\} . \\
\mathcal{T}_{e, \text { un }}^{\Gamma}(k)(F)=\left\{T F+\left(D_{x} F\right) X+\left(D_{\lambda} F\right) \Lambda+\left(D_{\beta} F\right) \Phi \mid T \in \mathbf{M}_{(x, \lambda, \beta)}^{\Gamma},\right. \\
\left.X \in \vec{\Theta}_{(x, \lambda, \beta)}^{\Gamma}, \Lambda \in \vec{\Theta}_{(\lambda, \beta)}^{\Gamma}, \Phi \in \mathcal{E}_{\beta, \beta}\right\} .
\end{gathered}
$$

Definindo

$$
\begin{aligned}
& \hat{\mathcal{T}}^{\Gamma}(f)=\left\{T f+\left(D_{x} f\right) X \mid T \in \mathbf{M}_{(x, \lambda)}^{\Gamma}, X \in \vec{\Theta}_{(x, \lambda)}^{\Gamma, o}\right\}, \\
& \hat{\mathcal{T}}_{c}^{\Gamma}(f)=\left\{T f+\left(D_{x} f\right) X \mid T \in \mathbf{M}_{(x, \lambda)}^{\Gamma}, X \in \vec{\Theta}_{(x, \lambda)}^{\Gamma}\right\}, \\
& \hat{\mathcal{T}}_{e, u n}^{\Gamma}(k)(F)=\left\{T F+\left(D_{x} F\right) X \mid T \in \mathbf{M}_{(x, \lambda, \beta)}^{\Gamma}, X \in \vec{\Theta}_{(x, \lambda, \beta)}^{\Gamma}\right\},
\end{aligned}
$$

podemos escrever

$$
\begin{aligned}
& \mathcal{T}_{e}^{\Gamma}(f)=\hat{\mathcal{T}}_{e}^{\Gamma}(f)+\overrightarrow{\mathcal{E}}_{\lambda}^{\Gamma}\left(D_{\lambda} f\right), \\
& \mathcal{T}_{e, u n}^{\Gamma}(k)(F)=\hat{\hat{T}}_{e, u n}^{\Gamma}(k)(F)+\vec{\Theta}_{(\lambda, \beta)}^{\Gamma}\left(D_{\lambda} F\right)+\mathcal{E}_{\beta, \beta}\left(D_{\beta} F\right) .
\end{aligned}
$$

A diferença entre $\hat{T}_{e}^{\Gamma}(f)$ e $\hat{T}^{\Gamma}(f)$ é que $X$ se anula na origem de $\hat{T}^{\Gamma}(f)$. Assim sendo, se $\overrightarrow{\mathcal{E}}_{(x, \lambda)}^{\Gamma}=\overrightarrow{\mathcal{M}}_{(x, \lambda)}^{\Gamma}+\mathbb{R}\left\{Y_{1} \ldots Y_{t}\right\}$ então $\hat{\mathcal{T}}_{e}^{\Gamma}(f)=\hat{\mathcal{T}}^{\Gamma}(f)+\mathbb{R}\left\{\left(D_{x} f\right) Y_{1} \ldots\left(D_{x} f\right) Y_{t}\right\}$.

Os espaços tangentes definidos até agora, tem somente a estrutura de $\mathcal{E}_{\lambda}^{\Gamma}$-módulo.

Definição. O espaço normal estendido é definido por

$$
\mathcal{N}_{e}^{\Gamma}(f)=\frac{\overrightarrow{\mathcal{E}}_{(x, \lambda)}^{\Gamma}}{\mathcal{T}_{e}^{\Gamma}(f)}
$$

e a $\Gamma$-codimensão de um germe $f \in \overrightarrow{\mathcal{E}}_{(x, \lambda)}^{\Gamma}$, denotada $\operatorname{cod}^{\Gamma}(f)$, por

$$
\operatorname{cod}^{\Gamma}(f)=\operatorname{dim}_{\mathbb{R}} \mathcal{N}_{e}^{\Gamma}(f)
$$




\subsection{Subgrupo geométrico}

Nesta seção recordamos a metodologogia geral para teoria de singularidades desenvolvida por Damon em [10] e mostramos como o nosso problema se adapta à ela. Consideremos um subgrupo $\mathcal{G}$ do grupo das equivalências de contato $\mathcal{K}$, agindo em um subespaço linear $\mathcal{F}$ de $\overrightarrow{\mathcal{E}}_{x}$. Assumimos que para cada inteiro positivo $q$, temos um grupo de desdobramentos $\mathcal{G}_{\text {un }}(q)$ (um subgrupo de $\mathcal{K}_{u n}(q)$ ) agindo num subespaço $\mathcal{F}_{u n}(q) \subset \overrightarrow{\mathcal{E}}_{(x, \beta)}$ de desdobramentos com $q$ parâmetros $\beta=\left(\beta_{1} \ldots \beta_{q}\right)$ de germes em $\overrightarrow{\mathcal{E}}_{x}$. Para $q=0$, devemos recuperar $\mathcal{G}$ e $\mathcal{F}$.

Um grupo $\mathcal{G}$ agindo em $\mathcal{F} \subset \overrightarrow{\mathcal{E}}_{x}$ (junto com o correspondente grupo de desdobramento $\mathcal{G}_{\text {un }}(q)$ agindo em $\left.\mathcal{F}_{u n}(q) \subset \overrightarrow{\mathcal{E}}_{(x, \beta)}\right)$ será chamado de subgrupo geométrico de $\mathcal{K}$ se satisfizer as quatro propriedades seguintes:

- naturalidade

- estrutura de espaço tangente

- aplicação exponencial

- condição de filtração

Naturalidade: o grupo e o espaço de desdobramentos são naturais com relação ao pullback.

Podemos considerar elementos em $\mathcal{G}_{\text {un }}(q+1)$ e $\mathcal{F}_{\text {un }}(q+1)$ como desdobramentos de elementos em $\mathcal{G}_{\text {un }}(q)$ e $\mathcal{F}_{\text {un }}(q)$ via o pull-back pela imersão $\mathbb{R}^{q} \hookrightarrow \mathbb{R}^{q+1}$. Dessa forma podemos calcular os espaços estendidos $\mathcal{T}_{e} \mathcal{G}_{u n}(q)$ e $\mathcal{T}_{\varepsilon} \mathcal{F}_{u n}(q)$.

Estrutura do Espaço Tangente: essa propriedade descreve a estrutura algébrica dos espaços tangentes e dos espaços tangentes estendidos e suas respectivas relações.

Existe uma coleção de DA-subálgebras $\left\{R_{x}\right\}$ de $\mathcal{F}$ tal que para cada $\mathbb{R}^{q}$ com coordenadas locais $\beta, \mathcal{T}_{e} \mathcal{G}_{u n}(q)$ e $\mathcal{T}_{e} \mathcal{F}_{u n}(q)$ são $\left\{R_{(x, \beta)}\right\}$-módulos finitamente gerados, com $\mathcal{T} \mathcal{G}_{\text {un }}(q)$ e $\mathcal{T} \mathcal{F}_{\text {un }}(q)\left\{R_{(x, \beta)}\right\}$-submódulos finitamente gerados e, contendo (via naturalidade) $\mathcal{T}_{e} \mathcal{G}$ e $\mathcal{T}_{e} \mathcal{F}$ como $\left\{R_{x}\right\}$-submódulos. Também, para cada $F \in \mathcal{F},\left\{R_{(x, \beta)}\right\}$ se torna 
um sistema de DA-álgebras sobre $\mathcal{E}_{\beta}$ tal que

$$
d \mathcal{O}_{F}: \mathcal{T}_{\epsilon} \mathcal{G}_{u n}(q) \rightarrow \mathcal{T}_{\epsilon} \mathcal{F}_{\text {un }}(q)
$$

é um homomorfismo de $\left\{R_{(x, \beta)}\right\}$-módulos.

Em segundo lugar, requeremos que as aplicaçôes naturais

$$
\frac{\mathcal{T}_{e} \mathcal{G}_{\text {un }}(q)}{\mathcal{M}_{\beta} \mathcal{T}_{e} \mathcal{G}_{\text {un }}(q)} \stackrel{\sim}{\rightarrow} \mathcal{T}_{e} \mathcal{G} \quad \text { e } \quad \frac{\mathcal{T}_{e} \mathcal{F}_{u n}(q)}{\mathcal{M}_{\beta} \mathcal{T}_{e} \mathcal{F}_{u n}(q)} \stackrel{\sim}{\mathcal{T}_{e}} \mathcal{F}
$$

sejam isomorfismos de $\left\{R_{x}\right\}$-módulos. Por último, requeremos que

$$
\mathcal{M}_{x} \mathcal{T}_{e} \mathcal{G} \subset \mathcal{T} \mathcal{G} \quad \text { e } \quad \mathcal{M}_{x} \mathcal{T}_{e} \mathcal{F} \subset \mathcal{T} \mathcal{F}
$$

Esta última condição implica que ambos os espaços tangentes estendidos $\mathcal{T}_{e} \mathcal{G}$ e $\mathcal{T}_{e} \mathcal{F}$ diferem dos espaços tangentes $\mathcal{T} \mathcal{G}$ e $\mathcal{T} \mathcal{F}$ por "campos de vetores constantes".

Aplicação Exponencial: definimos também um grupo $\mathcal{G}_{\text {eq }}(q)$ de equivalências de desdobramentos com $q$-parâmetros considerando os elementos de $\mathcal{G}_{\text {un }}(q)$ que são desdobramentos da identidade em $\mathcal{G}$ junto com um difeomorfismo $\Phi$ definido numa vizinhança da origem do $\mathbb{R}^{q}$ (cf. Seç̧âo 1.4 e [3], [10], [13]). Novamente, podemos considerar "desdobramentos" de elementos de $\mathcal{G}_{\text {eq }}(q)$ como pertencentes a $\mathcal{G}_{\text {eq }}(q+1)$ via o desdobramento a um parâmetro da identidade de $\mathcal{G}_{e q}(q)$. Calculando o espaço tangente estendido à identidade, temos a seguinte relação:

$$
\mathcal{T}_{e} \mathcal{G}_{e q}(q)=\mathcal{T}_{e} \mathcal{G}_{u n}(q) \oplus \mathcal{E}_{\beta}\left\{\frac{\partial}{\partial_{\beta}}\right\}
$$

A restrição da aplicação exponencial para $\mathcal{K}$ induz uma aplicação

$$
\begin{aligned}
\exp : \mathcal{T}_{e} \mathcal{G}_{e q}(q) & \rightarrow \mathcal{G}_{e q}(q+1) \\
\xi & \mapsto \phi_{t}
\end{aligned}
$$

onde $\quad \frac{\partial}{\partial t} \phi_{t}=\xi \cdot \phi_{t}$ e $\phi_{0}=i d$.

Condição de Filtração: $\mathcal{G}_{\text {un }}(q)$ preserva a filtração $\left\{\left\{\mathcal{M}_{x}^{\ell}\right\} \mathcal{F}_{u n}(q)\right\}$ em $\mathcal{F}_{u n}(q)$ e induz uma ação no quociente $\frac{\mathcal{F}_{u n}(q)}{\left\{\mathcal{M}_{x}^{\ell}\right\} \mathcal{F}_{u n}(q)}$ para todo $\ell \geq 0$. 
Note que $\mathcal{G}=\mathcal{K}_{\lambda}^{\Gamma}$ satisfaz as quatro propriedades acima descritas pois tomando $\mathcal{F}=\overrightarrow{\mathcal{E}}_{(x, \lambda)}^{\Gamma}, \mathcal{F}_{u n}(q)=\overrightarrow{\mathcal{E}}_{(x, \lambda, \beta)}^{\Gamma}$ e

$$
\mathcal{G}_{u n}(q)=\left\{(T, X, \Lambda) \in \mathcal{K}_{\lambda, u n}^{\Gamma}(q) \mid \Phi=\mathrm{I}_{\beta}\right\}
$$

temos que: a Naturalidade é óbvia e (1.3), (1.4) fornecem os espaços tangentes estendidos. A propriedade Estrutura de Espaço Tangente segue da. Secção 1.5 e resultados preliminares das seç̧ões anteriores. Para verificar a propriedade Aplicaçâo Exponencial definimos

$$
\mathcal{G}_{\text {eq }}(q)=\left\{(T, X, \Lambda) \in \mathcal{K}_{\lambda, u n}^{\Gamma}(q) \mid(T, X, \Lambda) \text { é um desdobramento da identidade em } \mathcal{K}_{\lambda}^{\Gamma}\right\}
$$

A demonstração das propriedades de $\mathcal{T}_{e} \mathcal{G}_{e q}(q)$ é uma simples extensão da abordagem usada em (1.3). Novamente, omitiremos os detalhes da demonstração por se estender ao nosso contexto. A Condição de Filtração é válida porque ela já existe para a situaçâo não-equivariante e o nosso caso trata de uma ação linear de $\Gamma$.

\subsection{A Teoria de desdobramento}

Sejam $F \in \overrightarrow{\mathcal{E}}_{(x, \lambda, \beta)}^{\Gamma}$ um desdobramento de $f \in \overrightarrow{\mathcal{E}}_{(x, \lambda)}^{\Gamma} \operatorname{com} k$ parâmetros $\beta=\left(\beta_{1} \ldots \beta_{k}\right)$ e $A:\left(\mathbb{R}^{r}, 0\right) \rightarrow\left(\mathbb{R}^{k}, 0\right)$. O pull-back de $F$ sob $A$, denotado por $A^{*} F$, é o desdobramento com $r$ parâmetros de $f$ definido por

$$
\left(A^{*} F\right)(x, \lambda, \alpha)=F(x, \lambda, A(\alpha)), \quad \alpha=\left(\alpha_{1} \ldots \alpha_{r}\right)
$$

Seja $G \in \overrightarrow{\mathcal{E}}_{(x, \lambda, \alpha)}^{\Gamma}$ um desdobramento de $f \in \overrightarrow{\mathcal{E}}_{(x, \lambda)}^{\Gamma} \operatorname{com} r$ parâmetros $\alpha=\left(\alpha_{1} \ldots \alpha_{r}\right)$. Dizemos que $G$ se fatora através de $F$ se existirem $T \in \mathbf{M}_{(x, \lambda, \alpha)}^{\Gamma}, X \in \vec{\Theta}_{(x, \lambda, \alpha)}^{\Gamma}, \quad \Lambda \in \vec{\Theta}_{(\lambda, \alpha)}^{\Gamma}$ e $A:\left(\mathbb{R}^{r}, 0\right) \rightarrow\left(\mathbb{R}^{k}, 0\right)$ satisfazendo $T(x, \lambda, 0)=\mathbf{I}_{m}, X(x, \lambda, 0)=x$ e $\Lambda(\lambda, 0)=\lambda$ tal que

$$
G(x, \lambda, \alpha)=T(x, \lambda, \alpha) F(X(x, \lambda, \alpha), \Lambda(\lambda, \alpha), A(\alpha)) .
$$

$F$ é versal se todo desdobramento $G$ de $f$ se fatora através de $F$. Se $F$ for versal e tiver número mínimo de parâmetros, será chamado de desdobramento miniversal de $f$. Devemos provar que os desdobramentos miniversais de $f$ são únicos a menos de $\mathcal{K}_{\lambda, u n}^{\Gamma}(k)$ equivalência (cf. Teorema 1.7.7). 


\section{Lema 1.7.1. (Lema Algébrico da Teoria de Desdobramento)}

Sejam $f \in \overrightarrow{\mathcal{E}}_{(x, \lambda)}^{\Gamma}$ e $F \in \overrightarrow{\mathcal{E}}_{(x, \lambda, \alpha)}^{\Gamma}$ um desdobramento de $f$ com $k$ parâmetros $\alpha=\left(\alpha_{1} \ldots \alpha_{k}\right)$. As seguintes condiçoes são equivalentes:

1. $\overrightarrow{\mathcal{E}}_{(x, \lambda)}^{\Gamma}=\mathcal{T}_{e}^{\Gamma}(f)+\mathbb{R}\left\{\frac{\partial F}{\partial \alpha_{1}}(., ., 0) \ldots \frac{\partial F}{\partial \alpha_{k}}(., ., 0)\right\}$,

2. $\overrightarrow{\mathcal{E}}_{(x, \lambda, \alpha)}^{\Gamma}=\hat{\mathcal{T}}_{e, u n}^{\Gamma}(k)(F)+\vec{\Theta}_{(\lambda, \alpha)}^{\Gamma}\left(D_{x} F\right)+\mathcal{E}_{\alpha}\left\{\frac{\partial F}{\partial \alpha_{1}} \ldots \frac{\partial F}{\partial \alpha_{k}}\right\}$.

Prova. O fato de que 2. implica 1. é óbvio fazendo $\alpha=0$.

Para mostrar a recíproca usamos o Lema $1.2 .5 \mathrm{com}$

$$
N=\mathcal{T}_{e}\left(\mathcal{K}_{\lambda, u n}^{\Gamma}(k)\right), \quad M=\overrightarrow{\mathcal{E}}_{(x, \lambda, \alpha)}^{\Gamma} \text { e } \psi=d \mathcal{O}_{F} .
$$

Por (1.8), $d \mathcal{O}_{F}$ é um homomorfismo de módulos finitamente gerados sobre o sistema de DA-álgebras $\left\{R_{(x, \lambda, \alpha)}\right\}$. Considere o sistema .

$$
\left\{R_{(x, \lambda, \alpha)}, \mathcal{J}_{(x, \lambda, \alpha)}\right\}=\left\{\left(\mathcal{E}_{\alpha}, \mathcal{M}_{\alpha}\right),\left(\mathcal{E}_{(\lambda, \alpha)}^{\Gamma}, \mathcal{M}_{\alpha} \cdot \mathcal{E}_{(\lambda, \alpha)}^{\Gamma}\right),\left(\mathcal{E}_{(x, \lambda, \alpha)}^{\Gamma}, \mathcal{M}_{\alpha} \cdot \mathcal{E}_{x, \lambda, \alpha}^{\Gamma}\right)\right\}
$$

A condição 1. é equivalente à

$$
d \mathcal{O}_{F}\left(\frac{\mathcal{T}_{e}\left(\mathcal{K}_{\lambda, u n}^{\Gamma}(k)\right)}{\mathcal{M}_{\alpha} \cdot \mathcal{T}_{\varepsilon}\left(\mathcal{K}_{\lambda, u n}^{\Gamma}(k)\right)}\right)=\frac{\overrightarrow{\mathcal{E}}_{(x, \lambda, \alpha)}^{\Gamma}}{\mathcal{M}_{\alpha} \cdot \overrightarrow{\mathcal{E}}_{(x, \lambda, \alpha)}^{\Gamma}}
$$

o qual é equivalente à

$$
d \mathcal{O}_{F}\left(\mathcal{T}_{e}\left(\mathcal{K}_{\lambda, u n}^{\Gamma}(k)\right)\right)+\mathcal{M}_{\alpha} \cdot \overrightarrow{\mathcal{E}}_{(x, \lambda, \alpha)}^{\Gamma}=\overrightarrow{\mathcal{E}}_{(x, \lambda, \alpha)}^{\Gamma} .
$$

Pelo Lema 1.2.5,

$$
d \mathcal{O}_{F}\left(\mathcal{T}_{e}\left(\mathcal{K}_{\lambda, u n}^{\Gamma}(k)\right)\right)=\overrightarrow{\mathcal{E}}_{(x, \lambda, \alpha)}^{\Gamma},
$$

isto é,

$$
\overrightarrow{\mathcal{E}}_{(x, \lambda, \alpha)}^{\Gamma}=\mathcal{T}_{e, u n}^{\Gamma}(k)(F)=\hat{\mathcal{T}}_{e, u n}^{\Gamma}(k)(F)+\vec{\Theta}_{(\lambda, \alpha)}^{\Gamma}\left(D_{\lambda} F\right)+\mathcal{E}_{\alpha}\left\{\frac{\partial F}{\partial \alpha_{1}} \ldots \frac{\partial F}{\partial \alpha_{k}}\right\}
$$

e portanto, 2. se verifica. 


\section{Lema 1.7.2. (Lema da Redução)}

Sejam $f \in \overrightarrow{\mathcal{E}}_{(x, \lambda)}^{\Gamma}$ e $K \in \overrightarrow{\mathcal{E}}_{(x, \lambda, \delta)}^{\Gamma}$ um desdobramento de $f$ com $p$ parâmetros $\delta=$ $\left(\delta_{1} \ldots \delta_{p}\right)$. Seja

$$
\begin{aligned}
L:\left(\mathbb{R}^{n} \times \mathbb{R}^{\ell} \times \mathbb{R}^{p-1}, 0\right) & \rightarrow \mathbb{R}^{m} \\
\left(x, \lambda, \delta_{1} \ldots \delta_{p-1}\right) & \mapsto K\left(x, \lambda, \delta_{1} \ldots \delta_{p-1}, 0\right)
\end{aligned}
$$

um desdobramento com $(p-1)$-parâmetros de $f$. Se existir $Y$, um germe de campo de vetores na origem do $\mathbb{R}^{n} \times \mathbb{R}^{\ell} \times \mathbb{R}^{p}$, da forma

$$
Y(x, \lambda, \delta)=\frac{\partial}{\partial \delta_{p}}+\sum_{j=1}^{n} X_{j}(x, \lambda, \delta) \frac{\partial}{\partial x_{j}}+\sum_{i=1}^{\ell} \Lambda_{i}(\lambda, \delta) \frac{\partial}{\partial \lambda_{i}}+\sum_{s=1}^{p-1} \xi_{s}(\delta) \frac{\partial}{\partial \delta_{s}}
$$

satisfazendo

1. $X \in \overrightarrow{\mathcal{E}}_{(x, \lambda, \delta)}^{\Gamma}$ onde $X=\left(X_{1} \ldots X_{n}\right)$,

2. $\Lambda \in \overrightarrow{\mathcal{E}}_{(\lambda, \delta)}^{\Gamma}$ onde $\Lambda=\left(\Lambda_{1} \ldots \Lambda_{\ell}\right)$

e $T \in \mathbf{M}_{(x, \lambda, \delta)}^{\Gamma}$ tais que

$$
-D_{(x, \lambda, \delta)} K \cdot Y=T K,
$$

então existirá um germe de submersão $C:\left(\mathbb{R}^{p}, 0\right) \rightarrow\left(\mathbb{R}^{p-1}, 0\right)$ tal que $K$ se fatora através de $C^{*} L$

Prova. A idéia é integrar $Y$ para gerar o germe $C$ junto com o germe de difeomorfismo na fonte e, então, integrar o lado direito de (1.9) para criar uma família parametrizada de campos de vetores lineares no $\mathbb{R}^{m}$ necessários para mostrar que $K$ se fatora através de $C^{*} L$.

A condiçâo (1.9) pode ser expressa como

$$
-\frac{\partial K}{\partial \delta_{p}}=\sum_{j=1}^{n} X_{j} \frac{\partial K}{\partial x_{j}}+\sum_{i=1}^{\ell} \Lambda_{i} \frac{\partial K}{\partial \lambda_{i}}+\sum_{s=1}^{p-1} \xi_{s} \frac{\partial K}{\partial \delta_{s}}+T K
$$

para alguma $T \in \mathbf{M}_{(x, \lambda, \delta)}^{\Gamma}$. 
Agora, considere o seguinte sistema de EDOs

$$
\begin{aligned}
\frac{d}{d t} \delta_{p} & =1 \\
\frac{d}{d t} x_{j} & =X_{j}(x, \lambda, \delta), \quad j=1 \ldots n \\
\frac{d}{d t} \lambda_{i} & =\Lambda_{i}(\lambda, \delta), \quad i=1 \ldots \ell \\
\frac{d}{d t} \delta_{s} & =\xi_{s}(\delta), \quad s=1 \ldots p-1
\end{aligned}
$$

Suponha que o fluxo de (1.10) é dado pelo grupo a um parâmetro de germes de difeomorfismos

$$
\begin{aligned}
\Psi_{t}:\left(\mathbb{R}^{n+\ell+p}, 0\right) & \rightarrow \mathbb{R}^{n+\ell+p} \\
(x, \lambda, \delta) & \mapsto\left(\rho_{t}(x, \lambda, \delta), \Lambda_{t}(\lambda, \delta), \Delta_{t}(\delta), t\right)
\end{aligned}
$$

satisfazendo $\Psi_{\delta_{p}}(x, \lambda, \delta)=(x, \lambda, \delta)$.

A equação $\frac{d}{d t} \delta_{p}=1$ implica que as curvas integrais de (1.10) são transversais ao hiperplano $\delta_{p}=0$.

Dado um ponto $P \equiv(x, \lambda, \delta)$ próximo da origem, existe uma curva integral $\gamma_{P}$ de (1.10) tal que $\gamma_{P}\left(\delta_{p}\right)=P$ onde $\delta_{p}$ é a $(n+1+p)$-ésima componente de $P$.

Defina uma submersão $\varphi:\left(\mathbb{R}^{n+\ell+p}, 0\right) \rightarrow \mathbb{R}^{n+\ell+p-1}$ através da projeção de $P \equiv$ $(x, \lambda, \delta)$ ao longo da curva integral $\gamma_{P}$ que passa por ele até o hiperplano $\delta_{p}=0$, ou seja,

$$
\varphi(x, \lambda, \delta)=\left(\pi_{(n+l+p-1)} \circ \Psi_{0}\right)(x, \lambda, \delta)=\left(\rho_{0}(x, \lambda, \delta), \Lambda_{0}(\lambda, \delta), \Delta_{0}(\delta)\right)
$$

Assim, definimos $\varphi(x, \lambda, \delta)=(\bar{\rho}(x, \lambda, \delta), \bar{\Lambda}(\lambda, \delta), \bar{\Delta}(\delta))$ onde $\bar{\rho}(x, \lambda, \delta)=\rho_{0}(x, \lambda, \delta)$, $\bar{\Lambda}(\lambda, \delta)=\Lambda_{0}(\lambda, \delta)$ e $\bar{\Delta}(\delta)=\Delta_{0}(\delta)$.

$\varphi$ é suave por propriedades de EDOs. A restrição de $\varphi$ ao hiperplano $\delta_{p}=0$ é a identidade por definição. Em particular, quando $\delta=0$,

$$
\bar{\rho}(x, \lambda, 0)=x, \quad \bar{\Lambda}(\lambda, 0)=\lambda \text { e } \bar{\Delta}^{o}=0 .
$$


A matriz Jacobiana de $\varphi$ na origem é dada por

$$
\left(\begin{array}{ccccc}
\left(D_{x} \bar{\rho}\right)^{\circ} & \vdots & \star & \vdots & \star \\
\ldots \ldots & & \ldots \ldots & & \ldots \ldots \\
0 & \vdots & \left(D_{\lambda} \bar{\Lambda}\right)^{\circ} & \vdots & \star \\
\cdots \cdots & \ldots \ldots \cdots & \ldots \ldots \\
0 & \vdots & 0 & \vdots & \left(D_{\delta} \bar{\Delta}\right)^{\circ}
\end{array}\right)
$$

Como $\left.\varphi\right|_{\delta_{p=0}}$ é a identidade, rank $(D \varphi)^{\circ}=n+\ell+p-1$. Portanto, $\varphi$ é uma submersão numa vizinhança da origem e segue que

(i) $\operatorname{rank}\left(D_{\delta} \bar{\Delta}\right)^{o}=p-1$ e $\operatorname{assim} \bar{\Delta}:\left(\mathbb{R}^{p}, 0\right) \rightarrow\left(\mathbb{R}^{p-1}, 0\right)$ é uma submersão,

(ii) $\operatorname{rank}\left(D_{\lambda} \bar{\Lambda}\right)^{\circ}=\ell$ e $\operatorname{assim} \bar{\Lambda}_{\delta}:\left(\mathrm{R}^{\ell}, 0\right) \rightarrow \mathbb{R}^{\ell}$ é um difeomorfismo e $\left(D_{\lambda} \bar{\Lambda}\right)^{\circ} \in \mathcal{L}_{\Gamma}(\ell)$.

Além disso, usando o argumento de unicidade de soluções de EDOs, a condição de $\Gamma$ equivariância $\bar{\Lambda}\left(\gamma_{\ell} \lambda, \delta\right)=\gamma_{\ell} \bar{\Lambda}(\lambda, \delta), \forall \gamma \in \Gamma$, é verdadeira.

Mais precisamente, seja $\xi(\lambda, \delta)=\gamma_{\ell}^{t} \bar{\Lambda}\left(\gamma_{\ell} \lambda, \delta\right)$,

$$
\begin{aligned}
\frac{d}{d t} \xi(\lambda, \delta) & =\gamma_{\ell}^{t} \frac{d}{d t} \bar{\Lambda}\left(\gamma_{\ell} \lambda, \delta\right)=\gamma_{\ell}^{t} \Lambda\left(\bar{\Lambda}\left(\gamma_{\ell} \lambda, \delta\right), \bar{\Delta}(\delta)\right) \\
& =\Lambda\left(\gamma_{\ell} \bar{\Lambda}\left(\gamma_{\ell} \lambda, \delta\right), \bar{\Delta}(\delta)\right)=\Lambda(\xi(\lambda, \delta), \bar{\Delta}(\delta))
\end{aligned}
$$

e

$$
\xi(\lambda, 0)=\gamma_{\ell}^{t} \bar{\Lambda}\left(\gamma_{\ell} \lambda, 0\right)=\gamma_{\ell}^{t} \gamma_{\ell} \lambda=\lambda
$$

Consequentemente, por unicidade, $\xi(\lambda, \delta)=\bar{\Lambda}(\lambda, \delta)$ estabelecendo a condição de equivariância requerida.

(iii) Como $\operatorname{rank}\left(D_{x} \bar{\rho}\right)^{\circ}=n, \bar{\rho}_{\lambda, \delta}:\left(\mathbb{R}^{n}, 0\right) \rightarrow \mathbb{R}^{n}$ é um difeomorfismo e $\left(D_{x} \bar{\rho}\right)^{\circ} \in \stackrel{\circ}{\mathcal{L}}_{\Gamma}(n)$.

Usando o mesmo método que em (ii), $\bar{\rho}\left(\gamma_{n} x, \gamma_{\ell} \lambda, \delta\right)=\gamma_{n} \bar{\rho}(x, \lambda, \delta), \forall \gamma \in \Gamma$.

A seguir integramos o campo de vetores $T$ para obter uma família parametrizada de campos de vetores no $\mathbb{R}^{m}$.

Para um dado ponto $\dot{P} \equiv(x, \lambda, \delta)$, seja $\gamma_{P}(t)=(x(t), \lambda(t), \delta(t))$, uma curva integral de (1.10) tal que $\gamma_{P}\left(\delta_{p}\right)=P$.

Considere agora o seguinte sistema linear não autônomo

$$
\begin{aligned}
\frac{d y}{d t} & =-T(x(t), \lambda(t), \delta(t)) y, \text { para } y \in \mathbb{R}^{m} \\
y^{o} & =y_{0} .
\end{aligned}
$$


As soluçooes de $(1.11,1.12)$ dependem de $y_{0}$ e $\gamma_{P}(t)$. Mas, para $P$ próximo da origem, como $\gamma_{p}$ é sempre transversal ao hiperplano $\left\{\delta_{p}=0\right\}$, as soluçôes de $(1.11,1.12)$ realmente dependem somente de $\left(x^{\circ}, \lambda^{\circ}, \delta^{\circ}\right)$ junto com a condição inicial $y_{0}$ quando $t=0$. A soluçâo de $(1.11,1.12)$ é portanto da forma $y(t)=\hat{Y}\left(y_{0}, t ; x^{o}, \lambda^{o}, \delta^{o}\right)$.

Da teoria clássica de EDOs, para $t$ fixado e $\left(x^{\circ}, \lambda^{\circ}, \delta^{\circ}\right)$ próximo da origem, a aplicação $y_{0} \mapsto y(t)$ é um difeomorfismo local ([25], Corolário 9, pág. 58).

Agora,

$$
\begin{aligned}
\frac{d}{d t} K(x(t), \lambda(t), \delta(t)) & =\left(D_{(x, \lambda, \delta)} K\right) \cdot(\dot{x}(t), \dot{y}(t), \dot{\delta}(t))=\left(D_{(x, \lambda, \delta)} K\right) \cdot Y(x(t), y(t), \delta(t)) \\
& =-T(x(t), \lambda(t), \delta(t)) K(x(t), \lambda(t), \delta(t))
\end{aligned}
$$

Como consequência, $K(x(t), \lambda(t), \delta(t))$ satisfaz $(1.11)$, isto é,

$$
K(x(t), \lambda(t), \delta(t))=\hat{Y}\left(K\left(x^{o}, \lambda^{o}, \delta^{o}\right), t ; x^{o}, \lambda^{o}, \delta^{o}\right)
$$

Como todo ponto $P \equiv(x, y, \delta)$ próximo da origem tem uma curva integral de $(1.10)$ passando através dele no tempo $t=\delta_{p}$, deduzimos de (1.13) que

$$
K(x, \lambda, \delta)=\hat{Y}\left(K(\varphi(x, \lambda, \delta), 0), \delta_{p} ; \varphi(x, \lambda, \delta), 0\right)
$$

Seja $\mathrm{E}_{x, \lambda, \delta}(y)=\hat{Y}\left(y, \delta_{p} ; \varphi(x, \lambda, \delta), 0\right)$. $\mathrm{E}_{x, \lambda, \delta}$ é uma família de difeomorfismos locais no $\mathbb{R}^{m}$ e satisfaz

$$
\begin{aligned}
K(x, \lambda, \delta) & \left.=\mathrm{E}_{x, \lambda, \delta} K(\varphi(x, \lambda, \delta), 0)\right) \\
\mathrm{E}_{x, \lambda, 0} & =\mathbf{I}_{m} .
\end{aligned}
$$

Usando a definição de $\varphi$,

$$
\begin{aligned}
K(x, \lambda, \delta) & =\mathrm{E}_{x, \lambda, \delta}(K(\bar{\rho}(x, \lambda, \delta), \bar{\Lambda}(\lambda, \delta), \bar{\Delta}(\delta), 0)) \\
& =\mathrm{E}_{x, \lambda, \delta} \bar{\Delta}^{*} L(\bar{\rho}(x, \lambda, \delta), \bar{\Lambda}(\lambda, \delta), \delta)
\end{aligned}
$$

Definimos o germe de submersão $C$ como $\bar{\Delta}$.

Por um resultado semelhante ao de Mather ([16], Proposição 1.5, pág. 170) podemos também substituir o difeomorfismo não linear $\mathrm{E}_{x, \lambda, \delta}$ por uma matriz $S \in \mathbf{M}_{(x, \lambda, \delta)}^{\Gamma}$, tal que

$$
\begin{aligned}
& K(x, \lambda, \delta)=S(x, \lambda, \delta) \bar{C}^{*} L(\bar{\rho}(x, \lambda, \delta), \bar{\Lambda}(\lambda, \delta), \delta), \\
& S(x, \lambda, 0)=\mathbf{I}_{m} .
\end{aligned}
$$


A simetria da matriz $S$ pode ser obtida da simetria de E ou pode ser obtida calculandose a média sobre $\mathrm{D}_{4}$ diretamente, ou seja, calculando-se a integral de Haar conveniente já que $S$ está próxima da identidade.

Portanto, $K$ se fatora através de $\bar{C}^{*} L$ como desejado.

Observação. Esta prova é baseada na prova do Teorema 2.1 ([16], pág. 238).

\section{Teorema 1.7.3. (O Teorema do Desdobramento)}

Sejam $f \in \overrightarrow{\mathcal{E}}_{(x, \lambda)}^{\Gamma}$ e $F \in \overrightarrow{\mathcal{E}}_{(x, \lambda, \alpha)}^{\Gamma}$ um desdobramento de $f \operatorname{com} k$ parâmetros $\alpha=$ $\left(\alpha_{1} \ldots \alpha_{k}\right)$. As seguintes condições são equivalentes

1. F é versal,

2. $\overrightarrow{\mathcal{E}}_{(x, \lambda)}^{\mathrm{r}}=\mathcal{T}_{e}^{\Gamma}(f)+\mathbb{R}\left\{\frac{\partial F}{\partial \alpha_{1}}(., ., 0) \ldots \frac{\partial F}{\partial \alpha_{k}}(., ., 0)\right\}$.

Prova. A prova é baseada em ([16], §7, pág. 233).

(i) 1. implica 2 .

Seja $p(x, \lambda) \in \overrightarrow{\mathcal{E}}_{(x, \lambda)}^{\Gamma}$ um germe arbitrário e considere o desdobramento a um parâmetro de $f$ dado por

$$
H(x, \lambda, t)=f(x, \lambda)+t p(x, \lambda)
$$

Por hipótese $F$ é versal e dessa forma $H$ se fatora através de $F$, ou seja, existem $T \in \mathbf{M}_{(x, \lambda, t)}^{\Gamma}, X \in \vec{\Theta}_{(x, \lambda, t)}^{\Gamma}, \Lambda \in \vec{\Theta}_{(\lambda, t)}^{\Gamma}$ e $A:(\mathbb{R}, 0) \rightarrow\left(\mathbb{R}^{k}, 0\right)$ satisfazendo $T(x, \lambda, 0)=$ $\mathbf{I}_{m}, X(x, \lambda, 0)=x, \quad \Lambda(\lambda, 0)=\lambda$ e $H(x, \lambda, t)=T(x, \lambda, t) F(X(x, \lambda, t), \Lambda(\lambda, t), A(t))$.

Logo,

$$
\begin{aligned}
\left.\frac{\partial}{\partial t}\right|_{t=0} H(x, \lambda, t), & p(x, \lambda) \\
= & {\left[\frac{\partial T}{\partial t}(x, \lambda, 0) f(x, \lambda)+\left(D_{x} f\right) \frac{\partial X}{\partial t}(x, \lambda, 0)+\left(D_{\lambda} f\right) \frac{\partial \Lambda}{\partial t}(\lambda, 0)\right] } \\
& +\left[\sum_{j=1}^{k} \frac{\partial F}{\partial \alpha_{j}}(x, \lambda, 0) \frac{\partial A_{j}}{\partial t}(0)\right] .
\end{aligned}
$$

Como consequência, $p \in \mathcal{T}_{e}^{\Gamma}(f)+\mathbb{R}\left\{\frac{\partial F}{\partial \alpha_{1}}(., ., 0) \ldots \frac{\partial F}{\partial \alpha_{k}}(., ., 0)\right\}$ e 2 . segue. 
(ii) 2. implica 1 .

Seja $H \in \overrightarrow{\mathcal{E}}_{(x, \lambda, \beta)}^{\Gamma}$ um desdobramento de $f \operatorname{com} r$ parâmetros $\beta=\left(\beta_{3} \ldots \beta_{r}\right)$. Devemos mostrar que $H$ se fatora através de $F$.

Considere a soma de desdobramentos

$$
K(x, \lambda, \alpha, \beta)=F(x, \lambda, \alpha)+H(x, \lambda, \beta)-f(x, \lambda)
$$

Obviamente $K \in \overrightarrow{\mathcal{E}}_{(x, \lambda, \alpha, \beta)}^{\Gamma}$ é um desdobramento com $(k+r)$ parâmetros de $f$.

Afirmamos que existe uma submersão $A:\left(\mathbb{R}^{k+\tau}, 0\right) \rightarrow\left(\mathbb{R}^{k}, 0\right)$ tal que $K$ se fatora através de $A^{*} F$. Concluimos a prova em três etapas.

Etapa 1. Mostramos que a afirmação estabelece 1.

Se for verdadeira, então existirão $T \in \mathbf{M}_{(x, \lambda, \alpha, \beta)}^{\Gamma}, X \in \vec{\Theta}_{(x, \lambda, \alpha, \beta)}^{\Gamma}, \Lambda \in \vec{\Theta}_{(\lambda, \alpha, \beta)}^{\Gamma}$ tais que $T(x, \lambda, 0)=\mathbf{I}_{m}, X(x, \lambda, 0)=x, \Lambda(\lambda, 0)=\lambda \mathrm{e}$

$$
K(x, \lambda, \alpha, \beta)=T(x, \lambda, \alpha, \beta) F(X(x, \lambda, \alpha, \beta), \Lambda(\lambda, \alpha, \beta), A(\alpha, \beta))
$$

Fazendo $\alpha=0$,

$$
H(x, \lambda, \beta)=K(x, \lambda, 0, \beta)=T(x, \lambda, 0, \beta) F(X(x, \lambda, 0, \beta), \Lambda(\lambda, 0, \beta), A(0, \beta)) .
$$

Sejam $S(x, \lambda, \beta)=T(x, \lambda, 0, \beta), \xi(x, \lambda, \beta)=X(x, \lambda, 0, \beta), \tilde{\Lambda}(\lambda, \beta)=\Lambda(\lambda, 0, \beta)$ e $\tilde{A}(\beta)$ $=A(0, \beta)$. Portanto, existem $S \in \mathbf{M}_{(x, \lambda, \beta)}^{\Gamma}, \xi \in \vec{\Theta}_{(x, \lambda, \beta)}^{\Gamma}, \tilde{\Lambda} \in \vec{\Theta}_{(\lambda, \beta)}^{\Gamma}$ e $\tilde{A}:\left(\mathbb{R}^{k}, 0\right) \rightarrow\left(\mathbb{R}^{k}, 0\right)$ satisfazendo $S(x, \lambda, 0)=\mathbf{I}_{m}, \xi(x, \lambda, 0)=x$ e $\hat{\Lambda}(\lambda, 0)=\lambda$ tais que

$$
H=\tilde{A}^{*} F
$$

o que prova que $H$ se fatora através de $F$.

Etapa 2. Mostramos que $A$ existe por indução em $r$.

Seja $L:\left(\mathbb{R}^{n+\ell+k+r-1}, 0\right) \rightarrow \mathbb{R}^{m}$ definida por

$$
L\left(x, \lambda ; \alpha, \beta_{1} \ldots \beta_{r-1}\right)=K\left(x, \lambda, \alpha, \beta_{1} \ldots \beta_{r-1}, 0\right)
$$

Por indução, existe uma submersão $B:\left(\mathbb{R}^{k+r-1}, 0\right) \rightarrow\left(\mathbb{R}^{k}, 0\right)$ tal que $L$ se fatora através de $B^{*} F$. 
É suficiente encontrar uma submersâo $C:\left(\mathbb{R}^{k+r}, 0\right) \rightarrow\left(\mathbb{R}^{k+r-1}, 0\right)$ tal que $K$ se fatora através de $C^{*} L$. O resultado segue pois definindo $A:\left(\mathbb{R}^{k+\tau}, 0\right) \rightarrow\left(\mathbb{R}^{k}, 0\right)$ pela composição de $B$ e $C$, segue que $A$ é uma submersão e que $K$ se fatora através de $A^{*} F$.

Resta provar que existe $C$ satisfazendo a condição desejada.

Etapa 3. Para encontrar $C$, aplicamos o Lema 1.7.2.

Seja $\delta=\left(\delta_{1} \ldots \delta_{k+r}\right)$ onde $\delta_{1}=\alpha_{1} \ldots \delta_{k}=\alpha_{k}, \delta_{k+1}=\beta_{1} \ldots \delta_{k+r}=\beta_{r}$, isto é, $\delta=(\alpha, \beta)$ e $p=k+r$.

Por hipótese,

$$
\overrightarrow{\mathcal{E}}_{(x, \lambda)}^{\Gamma}=\mathcal{T}_{e}^{\Gamma}(f)+\mathbb{R}\left\{\frac{\partial F}{\partial \alpha_{1}}(., ., 0) \ldots \frac{\partial F}{\partial \alpha_{k}}(., ., 0)\right\} .
$$

Pela definição de $K$, podemos substituir $F$ por $K$ na expressão acima e assim, pelo Lema 1.7.1, isto é equivalente à

$$
\overrightarrow{\mathcal{E}}_{(x, \lambda, \delta)}^{\Gamma}=\hat{\mathcal{T}}_{e, u n}^{\Gamma}(p)(K)+\vec{\Theta}_{(\lambda, \delta)}^{\Gamma}\left(D_{\lambda} K^{\prime}\right)+\mathcal{E}_{\alpha}\left\{\frac{\partial K^{\prime}}{\partial \alpha_{1}} \ldots \frac{\partial K}{\partial \alpha_{k}}\right\} .
$$

Além disso, $\frac{\partial K}{\partial \delta_{p}}=\frac{\partial K}{\partial \beta_{\tau}} \in \overrightarrow{\mathcal{E}}_{(x, \lambda, \delta)}^{\Gamma}$ e portanto existem $T \in \mathbf{M}_{(x, \lambda, \delta)}^{\Gamma}, X \in \vec{\Theta}_{(x, \lambda, \delta)}^{\Gamma}$ e $\Lambda \in \vec{\Theta}_{(\lambda, \delta)}^{\Gamma}$ tais que

$$
\begin{aligned}
-\frac{\partial K}{\partial \delta_{p}}(x, \lambda, \delta) & =T(x, \lambda, \delta) K(x, \lambda, \delta)+\sum_{j=1}^{n} X_{j}(x, \lambda, \delta) \frac{\partial K}{\partial x_{j}}(x, \lambda, \delta) \\
& +\sum_{i=1}^{\ell} \Lambda_{i}(\lambda, \delta) \frac{\partial K}{\partial \lambda_{i}}(x, \lambda, \delta)+\sum_{s=1}^{k} \xi_{s}(\delta) \frac{\partial K}{\partial \alpha_{s}}(x, \lambda, \delta) .
\end{aligned}
$$

Assumindo $\xi_{i}^{o}=0, i>k$, podemos escrever

$$
-\frac{\partial K}{\partial \delta_{p}}=\sum_{j=1}^{n} X_{j} \frac{\partial K}{\partial x_{j}}+\sum_{i=1}^{\ell} \Lambda_{i} \frac{\partial K}{\partial \lambda_{i}}+\sum_{s=1}^{p-1} \xi_{s} \frac{\partial K}{\partial \delta_{s}}+T K .
$$

Seja

$$
Y(x, \lambda, \delta)=\frac{\partial}{\partial \delta_{p}}+\sum_{j=1}^{n} X_{j}(x, \lambda, \delta) \frac{\partial}{\partial x_{j}}+\sum_{i=1}^{\ell} \Lambda_{i}(\lambda, \delta) \frac{\partial}{\partial \lambda_{i}}+\sum_{s=1}^{p-1} \xi_{s}(\delta) \frac{\partial}{\partial \delta_{s}} .
$$

Então $-D_{(x, \lambda, \delta)} K \cdot Y=T K$ por $\quad(1.14)$.

Pelo Lema 1.7.2, existe um germe de submersão $C:\left(\mathbb{R}^{p}, 0\right) \rightarrow\left(\mathbb{R}^{p-1}, 0\right)$ tal que $K$ se fatora através de $C^{*} L$. 
Corolário 1.7.4. Seja $g \in \overrightarrow{\mathcal{E}}_{(x, \lambda)}^{\Gamma}$ e seja $W \subset \overrightarrow{\mathcal{E}}_{(x, \lambda)}^{\Gamma}$ um subespaço vetorial tal que

$$
\overrightarrow{\mathcal{E}}_{(x, \lambda)}^{\Gamma}=\mathcal{T}_{e}^{\Gamma}(f) \oplus W
$$

Se $p_{1} \ldots p_{k}$ for uma base para $W$ entâo

$$
G(x, \lambda, \alpha)=g(x, \lambda)+\sum_{j=1}^{k} \alpha_{j} p_{j}(x, \lambda)
$$

será um desdobramento miniversal de $g$.

Prova. Segue diretamente do Teorema 1.7.3.

Teorema 1.7.5. Dois desdobramentos versais de um germe em $\overrightarrow{\mathcal{E}}_{(x, \lambda)}^{\Gamma}$ são equivalentes como desdobramentos se e somente se eles tiverem um mesmo número de parâmetros de desdobramento.

Prova. Como aqui nós não requeremos qualquer condição de equivariância nos parâmetros de desdobramento, a prova é análoga a ([16], Teorema 7.4, pág. 241).

Corolário 1.7.6. Sejam $H$ um desdobramento versal de $g \in \overrightarrow{\mathcal{E}}_{(x, \lambda)}^{\Gamma}$ com parâmetros $\alpha=\left(\alpha_{1} \ldots \alpha_{\ell}\right)$ e $G$ um desdobramento miniversal de $g$ com parâmetros $\beta=\left(\beta_{1} \ldots \beta_{k}\right)$. Seja $\pi: \mathbb{R}^{\ell} \rightarrow \mathbb{R}^{k}$ a projeção canônica. Então $H$ e $\pi^{*} G$ são equivalentes como desdobramentos.

Prova. É imediata usando o Teorema 1.7.5.

Baseados neste corolário, podemos provar o seguinte resultado.

\section{Teorema 1.7.7. (Unicidade do Desdobramento Miniversal)}

Se $f$ e $g \in \overrightarrow{\mathcal{E}}_{(x, \lambda)}^{\Gamma}$ forem dois germes de codimensão finita $\mathcal{K}_{\lambda}^{\Gamma}$-equivalentes e $F$ e $G \in \overrightarrow{\mathcal{E}}_{(x, \lambda, \alpha)}^{\Gamma}$, com $\alpha=\left(\alpha_{1} \ldots \alpha_{k}\right)$, forem dois desdobramentos miniversais de $f$ e $g$, respectivamente, então $F$ e $G$ serão $\mathcal{K}_{\lambda, u n}^{\Gamma}(k)$-equivalentes. Dizemos que $F(G)$ são desdobramentos universais.

Prova. Como $f$ e $g$ são $\mathcal{K}_{\lambda}^{\Gamma}$-equivalentes existe $(S, X, \Lambda) \in \mathcal{K}_{\lambda}^{\Gamma}$ tal que

$$
f(x, \lambda)=S(x, \lambda) g(X(x, \lambda), \Lambda(\lambda)) .
$$

Considere agora o desdobramento $F^{\prime}$ de $f$ dado por

$$
F^{\prime}(x, \lambda, \alpha)=S(x, \lambda) G(X(x, \lambda), \Lambda(\lambda), \alpha) .
$$


Como $G$ é um desdobramento versal de $g$, pelo Teorema 1.7.3,

$$
\overrightarrow{\mathcal{E}}_{(x, \lambda)}^{\Gamma}=\mathcal{T}_{e}^{\Gamma}(g)+\mathbb{R}\left\{\frac{\partial G}{\partial \alpha_{1}}(., ., 0) \ldots \frac{\partial G}{\partial \alpha_{k}}(., ., 0)\right\} .
$$

Considere o seguinte isomorfismo

$$
\begin{aligned}
H: \overrightarrow{\mathcal{E}}_{(x, \lambda)}^{\Gamma} & \rightarrow \overrightarrow{\mathcal{E}}_{(x, \lambda)}^{\Gamma} \\
h & \mapsto H(h)=S(h \circ(X, \Lambda)) .
\end{aligned}
$$

Note que $H(g)=f$ e $H\left(\mathcal{T}_{e}^{\Gamma}(g)\right)=\mathcal{T}_{e}^{\Gamma}(f)$. Além disso, para todo $1 \leq i \leq k$,

$$
\frac{\partial F^{\prime}}{\partial \alpha_{i}}(x, \lambda, 0)=S(x, \lambda) \frac{\partial G}{\partial \alpha_{i}}(X(x, \lambda), \Lambda(\lambda), 0)=H\left(\frac{\partial G}{\partial \alpha_{i}}(x, \lambda, 0)\right)
$$

e assim

$$
\overrightarrow{\mathcal{E}}_{(x, \lambda)}^{\Gamma}=\mathcal{T}_{\epsilon}^{\Gamma}(f)+\mathbb{R}\left\{\frac{\partial F^{\prime}}{\partial \alpha_{1}}(., ., 0) \ldots \frac{\partial F^{\prime}}{\partial \alpha_{k}}(., ., 0)\right\} .
$$

Logo, $F^{\prime}$ é um desdobramento versal de $f$ e, pelo Teorema $1.7 .5, F$ e $F^{\prime}$ são $\mathcal{K}_{\lambda, u n}^{\Gamma}(k)$ equivalentes, ou seja, existe $\left(S^{\prime}, X^{\prime}, \Lambda^{\prime}, A^{\prime}\right) \in \mathcal{K}_{\lambda, u n}^{\Gamma}(k)$ tal que

$$
F(x, \lambda, \alpha)=S^{\prime}(x, \lambda, \alpha) F^{\prime}\left(X^{\prime}(x, \lambda, \alpha), \Lambda^{\prime}(x, \alpha), A^{\prime}(\alpha)\right)
$$

Substituindo $(1.16)$ em (1.15) obtemos $\left(\hat{S}, \tilde{X}, \tilde{\Lambda}, A^{\prime}\right) \in \mathcal{K}_{\lambda, u n}^{\Gamma}(k)$ tal que $F$ é $\mathcal{K}_{\lambda, u n}^{\Gamma}(k)$ equivalente à $G$.

\subsection{Determinação finita}

\subsubsection{Jatos $\Gamma$-equivariantes}

Denotamos por $J_{k}(n+\ell, m)$ o espaço dos $k$-jatos na origem do $\mathbb{R}^{n+\ell}$ dos germes de aplicações suaves do $\mathbb{R}^{n+\ell}$ ao $\mathbb{R}^{m}$. Este espaço é identificado com $\mathrm{P}_{k}$ o qual é o subspaço de $\overrightarrow{\mathcal{E}}_{n+\ell}$ formado por todas as aplicaçóes polinomiais de grau menor ou igual a $k$ e com termo constante nulo.

Para qualquer $f \in \overrightarrow{\mathcal{E}}_{n+\ell}, j^{k} f$ é o polinômio de Taylor de grau $k$ de $f$ calculado em 0 .

Considerando a projeção canônica do espaço de germes ao espaço de jatos

$$
\begin{aligned}
j^{k}: \overrightarrow{\mathcal{E}}_{n+\ell} & \rightarrow J_{k}(n+\ell, m) \\
f & \mapsto j^{k} f
\end{aligned}
$$


definimos a seguinte ação de $\Gamma$ em $J_{k}(n+\ell, m)$ :

$$
\begin{aligned}
\psi_{k}: \Gamma \times J_{k}(n+\ell, m) & \rightarrow J_{k}(n+\ell, m) \\
\left(\gamma, j^{k} f\right) & \mapsto \gamma_{m}^{t} j^{k} f\left(\gamma_{n} \times \gamma_{\ell}\right) .
\end{aligned}
$$

O espaço dos $k$-jatos $\Gamma$-equivariantes, denotado $J_{k}^{\Gamma}(n+\ell, m)$, é dado por

$$
J_{k}^{\Gamma}(n+\ell, m)=\left.J_{k}(n+\ell, m)\right|_{\mathrm{Fix} \psi_{k}} .
$$

Seja $\mathrm{P}_{k}^{\Gamma}$ o subespaço dos polinômios em $\mathrm{P}_{k}$ que são $\Gamma$-equivariantes. Assim, identifica$\operatorname{mos} J_{k}^{\Gamma}(n+\ell, m) \operatorname{com} \mathrm{P}_{k}^{\Gamma}$ e a projeção

$$
\begin{aligned}
j^{k}: \overrightarrow{\mathcal{E}}_{(x, \lambda)}^{\Gamma} & \rightarrow J_{k}^{\Gamma}(n+\ell, m) \\
f & \mapsto j^{k}(f)=j^{k} f
\end{aligned}
$$

é bem definida, ou seja, $j^{k}(f) \in J_{k}^{\Gamma}(n+\ell, m)$. Para provar essa última afirmação, usamos a expansão de Taylor para escrever $f(x, \lambda)=j^{k} f(x, \lambda)+f^{\prime}(x, \lambda)$, onde $f^{\prime}$ é de ordem maior ou igual a $(k+1)$.

Comparando

$$
f\left(\gamma_{n} x, \gamma_{\ell} \lambda\right)=j^{k} f\left(\gamma_{n} x, \gamma_{\ell} \lambda\right)+f^{\prime}\left(\gamma_{n} x, \gamma_{\ell} \lambda\right)
$$

com

$$
\gamma_{m} f(x, \lambda)=\gamma_{m} j^{k} f(x, \lambda)+\gamma_{m} f^{\prime}(x, \lambda),
$$

temos

$$
j^{k} f\left(\gamma_{n} x, \gamma_{\ell} \lambda\right)=\gamma_{m} j^{k} f(x, \lambda), \forall \gamma \in \Gamma
$$

pela unicidade da expansão de Taylor.

\subsubsection{A órbita de um $k$-jato $\Gamma$-equivariante}

Sejam $f \in \overrightarrow{\mathcal{E}}_{(x, \lambda)}^{\Gamma}$ e $F \in \overrightarrow{\mathcal{E}}_{(x, \lambda, \beta)}^{\Gamma} \operatorname{com} \beta=\left(\beta_{1} \ldots \beta_{r}\right) . J^{k} \mathcal{K}_{\lambda}^{\Gamma}$ denota o grupo dos $k$-jatos de elementos de $\mathcal{K}_{\lambda}^{\Gamma}$ e $J^{k} \mathcal{K}_{\lambda, u n}(r)$ o grupo dos $k$-jatos de elementos de $\mathcal{K}_{\lambda, u n}^{\Gamma}(r)$, isto é,

$$
J^{k} \mathcal{K}_{\lambda}^{\Gamma}=\left\{\left(j^{k} T, j^{k} X, j^{k} \Lambda\right) \mid(T, X, \Lambda) \in \mathcal{K}_{\lambda}^{\Gamma}\right\}
$$

e

$$
J^{k} \mathcal{K}_{\lambda, \text { un }}^{\Gamma}(r)=\left\{\left(j^{k} T, j^{k} X, j^{k} \Lambda, j^{k} \Phi\right) \mid(T, X, \Lambda, \Phi) \in \mathcal{K}_{\lambda, u n}^{\Gamma}(r)\right\}
$$


Seja $\mathcal{M}_{n+\ell}^{k+1} \cdot \overrightarrow{\mathcal{E}}_{n+\ell}$ o conjunto de germes de aplicaçâo cujas componentes pertencem a $\mathcal{M}_{n+\ell}^{k+1}$, isto é, são $k$-chatos em $0 \in \mathbb{R}^{n+\ell}$. Assim,

$$
J_{k}(n+\ell, m)=\frac{\overrightarrow{\mathcal{E}}_{n+\ell}}{\mathcal{M}_{n+\ell}^{k+1} \cdot \overrightarrow{\mathcal{E}}_{n+\ell}} .
$$

Seja $\Omega_{(x, \lambda)}^{k+1}=\overrightarrow{\mathcal{E}}_{(x, \lambda)}^{\Gamma} \cap \mathcal{M}_{n+\ell}^{k+1} \cdot \overrightarrow{\mathcal{E}}_{n+\ell}$, isto é, o conjunto das aplicações $\Gamma$-equivariantes cujas componentes sâo $k$-chatas na origem do $\mathbb{R}^{n+\ell}$. Então,

$$
J_{k}^{\Gamma}(n+\ell, m)=\frac{\overrightarrow{\mathcal{E}}_{(x, \lambda)}^{\Gamma}}{\Omega_{(x, \lambda)}^{k+1}} .
$$

A ação de $\mathcal{K}_{\lambda}^{\Gamma}$ em $\overrightarrow{\mathcal{E}}_{(x, \lambda)}^{\Gamma}$ induz uma ação natural no quociente de $J^{k} \mathcal{K}_{\lambda}^{\Gamma}$ por $J_{k}^{\Gamma}(n+\ell, m)$ dada por

$$
\left(j^{k} T, j^{k} X, j^{k} \Lambda\right) \cdot j^{k} f=j^{k}((S, \Lambda, f) \cdot f)
$$

Analogamente, definimos a órbita de $J^{k} \mathcal{K}_{\lambda, \text { un }}^{\Gamma}(r)$ no $J_{k}^{\Gamma}(n+\ell+r, m)$ como sendo o espaço dos $k$ - jatos de desdobramentos com $r$ parâmetros de germes em $\overrightarrow{\mathcal{E}}_{n+\ell}$.

Como sempre denotamos a órbita de $j^{k} f$ sob a ação do $J^{k} \mathcal{K}_{\lambda}^{\Gamma}$ por $J^{k} \mathcal{K}_{\lambda}^{\Gamma} \cdot j^{k} f$ e a órbita de $j^{k} F$ sob a ação de $J^{k} \mathcal{K}_{\lambda, \text { un }}^{\Gamma}(r)$ por $J^{k} \mathcal{K}_{\lambda, \text { un }}^{\Gamma}(r) \cdot j^{k} F$.

Teorema 1.8.2.1. $J^{k} \mathcal{K}_{\lambda}^{\Gamma}$ é um grupo de Lie agindo algebricamente em $J_{k}^{\Gamma}(n+\ell, m)$ e as órbitas sob essa ação sâo subvariedades analíticas de $J_{k}^{\Gamma}(n+\ell, m)$.

Prova. É uma adaptação equivariante da Prova de Mather e é também uma adaptação da prova do Lema 5.6 ([5], pág. 122).

Lema 1.8.2.2. Sejam $f \in \overrightarrow{\mathcal{E}}_{(x, \lambda)}^{\Gamma}$ e $\mathcal{T}_{k}^{\Gamma}(f)$ o espaço tangente à órbita $J^{k} \mathcal{K}_{\lambda}^{\Gamma} \cdot j^{k} f$. Então $\xi \in \mathcal{T}_{k}^{\Gamma}(f)$ se e somente se existir uma decomposição $\xi=T f+\left(D_{x} f\right) X+\left(D_{\lambda} f\right) \Lambda$ módulq $\Omega_{(x, \lambda)}^{k+1}$, onde $T \in \mathbf{M}_{(x, \lambda)}^{\Gamma}, X \in \vec{\Theta}_{(x, \lambda)}^{\Gamma, o}$ e $\Lambda \in \vec{\Theta}_{\lambda}^{\Gamma, o}$.

Prova. Seja $t \mapsto\left(\tilde{T}_{t}, \tilde{X}_{t}, \tilde{\Lambda}_{t}\right)$ a curva em $\mathcal{K}_{\lambda}^{\Gamma}$ passando através do elemento identidade quando $t=0$. Então o vetor tangente a $J^{k} \mathcal{K}_{\lambda}^{\Gamma} \cdot j^{k} f$ é

$$
\xi=\left.\frac{d}{d t}\right|_{t=0} j^{k}\left(\tilde{T}_{t}, \tilde{X}_{t}, \tilde{\Lambda}_{t}\right) \cdot f=j^{k}\left(\left.\frac{d}{d t}\right|_{t=0}\left(\tilde{T}_{t}, \tilde{X}_{t}, \tilde{\Lambda}_{t}\right) \cdot f\right) .
$$


Note que $\left.\frac{d}{d t}\right|_{t=0}\left(\tilde{T}_{t}, \tilde{X}_{t}, \tilde{\Lambda}_{t}\right) \in \mathcal{K}_{\lambda}^{\Gamma}$ e $\tilde{T}_{t}(x, \lambda) \in \stackrel{\circ}{\mathcal{L}_{\Gamma}}(m)$ para $(x, \lambda)$ numa vizinhança pequena da origem, e assim, $\left.\frac{d}{d t}\right|_{t=0} \tilde{T}_{t}(x, \lambda)$ pertence a $\stackrel{\circ}{\mathcal{L}_{\Gamma}}(m)$. Podemos escrever

$$
\tilde{T}_{t}(x, \lambda)=\mathbf{I}_{m}+t T(x, \lambda)+\text { termos de ordem alta em } t .
$$

Consequentemente,

$$
\left.\frac{d}{d t}\right|_{t=0} \tilde{T}_{t}=T \in \mathbf{M}_{(x, \lambda)}^{\Gamma}
$$

Analogamente, como $\tilde{X}_{0}(x, \lambda)=x$ e $\tilde{\Lambda}_{0}(\lambda)=\lambda$, escrevemos

$$
\tilde{X}_{t}(x, \lambda)=x+t X(x, \lambda)+\text { termos de ordem alta em } t
$$

e

$$
\tilde{\Lambda}_{t}(\lambda)=\lambda+t \Lambda(\lambda)+\text { termos de ordem alta em } t
$$

Assim,

$$
\left.\frac{d}{d t}\right|_{t=0} \tilde{X}_{t}=X \in \vec{\Theta}_{(x, \lambda)}^{\Gamma, o} \text { e }\left.\frac{d}{d t}\right|_{t=0} \tilde{\Lambda}_{t}=\Lambda \in \vec{\Theta}_{\lambda}^{\Gamma, o}
$$

Portanto,

$$
\begin{aligned}
\xi & =j^{k}\left(\left.\frac{d}{d t}\right|_{t=0}\left(\tilde{T}_{t}, \tilde{X}_{t}, \tilde{\Lambda}_{t}\right) \cdot f\right) \\
& =j^{k}\left(T f+\left(D_{x} f\right) X+\left(D_{\lambda} f\right) \Lambda\right) \\
& =T f+\left(D_{x} f\right) X+\left(D_{\lambda} f\right) \Lambda \text { módulo } \Omega_{(x, \lambda)}^{k+1} .
\end{aligned}
$$

\subsubsection{Lemas devidos a Damon}

Para estabelecer o Teorema de Determinação Finita recordamos dois lemas devidos a Damon. O lema seguinte é o Lema de Mather para sistemas de DA-álgebras ([10], Lema 7.3, pág. 35) escrito em nosso contexto.

Lema 1.8.3.1. Seja $\eta: N \rightarrow M$ um homomorfismo de $\left\{R_{(x, \lambda, \beta)}\right\}$-módulos finitamente gerados e $M_{0} \subset M$ um $\left\{R_{(x, \lambda, \beta)}\right\}$-submódulo de $\mathbb{R}$-codimensão finita igual a $c$. Então

1. Existe um número $\ell$ que depende de $c$ e da ação do grupo tal que 


$$
M_{0} \subset \eta(N)+\left\{\left(m_{x, \lambda, \beta}\right)^{\ell}\right\} M_{0}
$$

implica

$$
M_{0} \subset \eta(N)
$$

2. Se $\operatorname{dim}_{\mathbb{R}} \frac{M}{\eta(N)}=r<\infty$ então existe um número $\ell^{\prime}$ que depende de $r$ e da ação do grupo tal que

$$
\left\{\left(m_{x, \lambda, \beta}\right)^{\ell^{\prime}}\right\} M \subset \eta(N)
$$

Observação. Um resultado semelhante vale para o sistema de DA-álgebras $\left\{R_{(x, \lambda)}\right\}$.

Agora, suponha que $M$ e $N$ sejam módulos finitamente gerados sobre $\left\{R_{(x, \lambda)}\right\}$ e $\psi: N \rightarrow M$ um homomorfismo de módulo sobre esse sistemas de anéis. Seja $M_{\beta}$ um $\left\{R_{(x, \lambda, \beta)}\right\}$ módulo finitamente gerado pelos geradores de $M$ e $\psi_{1}: N_{\beta} \rightarrow M_{\beta}$ uma extensão de $\psi$, isto é, fazendo $\beta=0, \quad \psi_{1_{\left.\right|_{M}}}=\psi$.

Lema 1.8.3.2. ([10], Corolário 7.5, pág. 36) Na situação acima existe $\ell^{\prime}$ tal que

$$
\left\{\left(m_{x, \lambda}\right)^{\ell^{\prime}}\right\} M \dot{C}^{\prime} \psi(N)
$$

implica

$$
\left\{\left(m_{x, \lambda, \beta}\right)^{\ell^{\prime}}\right\} M_{\beta} \subset \psi_{1}\left(N_{\beta}\right)
$$

\subsubsection{Germes finitamente determinados}

Um germe $f \in \overrightarrow{\mathcal{E}}_{(x, \lambda)}^{\Gamma}$ é $k$ - $\mathcal{K}_{\lambda}^{\Gamma}$-determinado se para todo germe $g \in \overrightarrow{\mathcal{E}}_{(x, \lambda)}^{\Gamma} \operatorname{com} j^{k}(g)=$ $j^{k}(f)$ for $\mathcal{K}_{\lambda}^{\Gamma}$-equivalente a $f$. Um germe é finitamente $\mathcal{K}_{\lambda}^{\Gamma}$-determinado se for $k-\mathcal{K}_{\lambda^{-}}^{\Gamma}$ determinado para algum inteiró $k$.

Obviamente, se $f$ for $k-\mathcal{K}_{\lambda}^{\Gamma}$-determinado então será $\mathcal{K}_{\lambda}^{\Gamma}$-equivalente ao $j^{k}(f)$.

Recorde de (1.2) e (1.6) que

$$
\mathcal{T}\left(\mathcal{K}_{\lambda}^{\Gamma}\right) \simeq \mathbf{M}_{(x, \lambda)}^{\Gamma} \oplus \vec{\Theta}_{(x, \lambda)}^{\Gamma, o} \oplus \vec{\Theta}_{\lambda}^{\Gamma, o} \text { e } d \mathcal{O}_{f}\left(\mathcal{T} \mathcal{K}_{\lambda}^{\Gamma}\right)=\mathcal{T}^{\Gamma}(f)
$$


Considerando a projeção natural $j^{k}: \overrightarrow{\mathcal{E}}_{(x, \lambda)}^{\Gamma} \rightarrow J_{k}^{\Gamma}(n+\ell, m)$, segue que $j^{k}\left(\mathcal{T}^{\Gamma}(f)\right)=\mathcal{T}_{k}^{\Gamma}(f)$, pelo Lema 1.8.2.2 e $\left(\operatorname{Ker} j^{k}\right) \cap \overrightarrow{\mathcal{M}}_{(x, \lambda)}^{\Gamma}=\mathcal{M}_{(x, \lambda)}^{\Gamma} \cdot \Omega_{(x, \lambda)}^{k+1}$.

Consequentemente,

$$
\left(j^{k}\right)^{-1}\left(\mathcal{T}_{k}^{\Gamma}(f)\right)=\mathcal{T}^{\Gamma}(f)+\mathcal{M}_{(x, \lambda)}^{\Gamma} \cdot \Omega_{(x, \lambda)}^{k+1}
$$

\section{Teorema 1.8.4.1. (Teorema de Determinação Finita)}

Dado $f \in \overrightarrow{\mathcal{E}}_{(x, \lambda)}^{\Gamma}$, as seguintes condiçôes são equivalentes:

1. $f$ é finitamente $\mathcal{K}_{\lambda}^{\Gamma}$-determinado,

2. Para algum inteiro $s, \Omega_{(x, \lambda)}^{s} \subset \mathcal{T}_{e}^{\Gamma}(f)$,

3. Para algum inteiro $k, \mathcal{M}_{(x, \lambda)}^{\Gamma} \cdot \Omega_{(x, \lambda)}^{k} \subset \mathcal{T}^{\Gamma}(f)$,

4. $\operatorname{cod}^{\Gamma}(f)$. é finita,

5. $\operatorname{dim}_{\mathbf{R}} \frac{\overrightarrow{\mathcal{M}}_{(x, \lambda)}^{\Gamma}}{\mathcal{T}^{\Gamma}(f)}$ é finita.

Prova. (i) É óbvio que 2. implica 4. pois $\Omega_{(x, \lambda)}^{s}$ tem codimensâo finita em $\overrightarrow{\mathcal{E}}_{(x, \lambda)}^{\Gamma}$.

Para mostrarmos que 4. implica 2., aplicamos o Lema 1.8.3.1 com

$$
\eta=d \mathcal{O}_{f}, N=\mathcal{T}_{\epsilon}^{\Gamma} \text { e } M=\overrightarrow{\mathcal{E}}_{(x, \lambda)}^{\Gamma}
$$

Seja $\left\{R_{(x, \lambda)}\right\}$ o sistema de anéis descrito em (1.2) e de (1.6), $d \mathcal{O}_{f}$ é um homomorfismo de módulos finitamente gerados sobre $\left\{R_{(x, \lambda)}\right\}$.

Usando o Lema 1.8.3.1, $\operatorname{dim}_{\mathbb{R}} \frac{\overrightarrow{\mathcal{E}}_{(x, \lambda)}^{\Gamma}}{\mathcal{T}_{e}^{\Gamma}(f)}<\infty$ implica que existe um $s$ tal que $\Omega_{(x, \lambda)}^{s} \subset \mathcal{T}_{e}^{\Gamma}(f)$

(ii) Analogamente, mostramos que 3. e 5. são equivalentes.

(iii) 2. implica 3 . trivialmente pois

$$
\mathcal{M}_{(x, \lambda)}^{\Gamma} \cdot \Omega_{(x, \lambda)}^{s} \subset \mathcal{M}_{(x, \lambda)}^{\Gamma} \cdot \mathcal{T}_{e}^{\Gamma}(f) \subset \mathcal{T}^{\Gamma}(f)
$$


(iv) Vamos verificar que 3. implica 4. As inclusôes

$$
\mathcal{M}_{(x, \lambda)}^{\Gamma} \cdot \Omega_{(x, \lambda)}^{k} \subset \overrightarrow{\mathcal{M}}_{(x, \lambda)}^{\Gamma} \subset \overrightarrow{\mathcal{E}}_{(x, \lambda)}^{\Gamma}
$$

ocorrem entre espaços de codimensão finita. Portanto, $\mathcal{M}_{(x ; \lambda)}^{\Gamma} \cdot \Omega_{(x, \lambda)}^{k}$ tem codimensão finita em $\overrightarrow{\mathcal{E}}_{(x, \lambda)}^{\Gamma}$.

Além disso,

$$
\mathcal{M}_{(x, \lambda)}^{\Gamma} \cdot \Omega_{(x, \lambda)}^{k} \subset \mathcal{T}^{\Gamma}(f) \subset \mathcal{T}_{e}^{\Gamma}(f)
$$

e $\operatorname{assim} \mathcal{T}_{e}^{\Gamma}(f)$ tem codimensâo finita em $\overrightarrow{\mathcal{E}}_{(x, \lambda)}^{\Gamma}$.

(v) Vamos agora mostrar que 1. e 5. são equivalentes. Primeiro, mostramos que 1. implica 3. e consequentemente 5 .

Suponha que $f$ é $r-\mathcal{K}_{\lambda}^{\Gamma}$-determinado. Com $\ell$ como no Lema 1.8.3.1, seja $b=r+\ell$ e

$$
A=\left\{Z \in J_{b}^{\Gamma}(n+\ell, m) \mid Z^{\circ}=0 \text { e } j^{r}(Z)=j^{r}(f)\right\}
$$

Podemos identificar $A$ com o espaço afim

$$
j^{T}(f)+j^{b}\left(\mathcal{M}_{(x, \lambda)}^{\Gamma} \cdot \Omega_{(x, \lambda)}^{r+1}\right)
$$

e portanto,

$$
T A=j^{b}\left(\mathcal{M}_{(x, \lambda)}^{\mathrm{r}} \cdot \Omega_{(x, \lambda)}^{r+1}\right) .
$$

Por outro lado, como $f$ é $r$ - $\mathcal{K}_{\lambda}^{\Gamma}$-determinado, $A \subset J^{k} \mathcal{K}_{\lambda}^{\Gamma} \cdot j^{k} f$. Logo, $T A \subseteq \mathcal{T}_{k}^{\Gamma}(f)$. Tomando a imagem inversa sob $j^{b}$ e usando (1.17) temos

$$
\mathcal{M}_{(x, \lambda)}^{\Gamma} \cdot \Omega_{(x, \lambda)}^{r+1} \subset \mathcal{T}^{\Gamma}(f)+\mathcal{M}_{(x, \lambda)}^{\Gamma} \cdot \Omega_{(x, \lambda)}^{\ell+r+1}
$$

Para obter 3., aplicamos o Lema 1.8.3.1 com

$$
\eta=d \mathcal{O}_{f}, M=\overrightarrow{\mathcal{M}}_{(x, \lambda)}^{\Gamma}, N=\mathcal{T}\left(\mathcal{K}_{\lambda}^{\Gamma}\right) \text { e } M_{0}=\mathcal{M}_{(x, \lambda)}^{\Gamma} \cdot \Omega_{(x, \lambda)}^{r+1}
$$

Para completar a prova do teorema resta mostrar que 5. implica 1. Seguimos ([10], Teorema 10.2, pág. 42). Para isso, precisamos: 
Proposição 1.8.4.2. Existe um inteiro $r$ tal que se $p \in \Omega_{(x, \lambda)}^{r}$ e $F_{t}=f+t p \operatorname{com} t \in[0,1]$, então dado $t_{1}$, para $t$ suficientemente pequeno, $F_{t_{1}}$ e $F_{\left(t_{1}+t\right)}$ serâo $\mathcal{K}_{\lambda}^{\Gamma}$-equivalentes com os germes de mudança de coordenadas se anulando na origem de $\mathbb{R}^{n+\ell}$. Mais precisamente existirão germes $X \in \overrightarrow{\mathcal{M}}_{(x, \lambda, t)}^{\Gamma}, \Lambda \in \overrightarrow{\mathcal{M}}_{(\lambda, t)}^{\Gamma}$ e $T \in \mathbf{M}_{(x, \lambda, t)}^{\Gamma} \operatorname{com} X(0,0, t)=0$ e $\Lambda(0, t)=0$ tais que

$$
F_{\left(t_{1}+t\right)}=T \cdot F_{t}(X, \Lambda)
$$

Essa proposição conclui a prova de que 5. implica 1. Na verdade, como $X$ e $\Lambda$ se anulam na origem, para cada $t,\left(T_{t}, X_{t}, \Lambda_{t}\right)$ pode ser considerada como um elemento de $\mathcal{K}_{\lambda}^{\Gamma}$. Pela compacidade e conexidade de $[0,1], f=F_{0}$ é $\mathcal{K}_{\lambda}^{\Gamma}$-equivalente à $f+p=F_{1}$. Portanto, $f$ é $(r-1)-\mathcal{K}_{\lambda}^{\Gamma}$-determinado.

Para provar a Proposição 1.8.4.2 precismos dos seguintes Lemas.

Lema 1.8.4.3. Se $\operatorname{dim}_{\mathbf{R}} \frac{\overrightarrow{\mathcal{M}}_{(x, \lambda)}^{\Gamma}}{\mathcal{T}^{\Gamma}(f)}$ for finita, então existirá um inteiro $r$ tal que se $g \equiv f$ módulo $\Omega_{(x, \lambda)}^{r}$ então $\mathcal{M}_{(x, \lambda)}^{\Gamma} \cdot \Omega_{(x, \lambda)}^{r} \subset \mathcal{T}^{\Gamma}(g)$.

Prova. Por 3., existe um inteiro $k$ tal que

$$
\mathcal{M}_{(x, \lambda)}^{\Gamma} \cdot \Omega_{(x, \lambda)}^{k} \subset \mathcal{T}^{\Gamma}(f)
$$

Como $g \equiv f$ módulo $\Omega_{(x, \lambda)}^{r}$, temos que $d \mathcal{O}_{g} \equiv d \mathcal{O}_{f}$ módulo $\Omega_{(x, \lambda)}^{r} \cdot \mathcal{T}_{\epsilon}^{\Gamma}$.

Aplicando ambos os membros a $\mathcal{T}^{\Gamma}\left(\mathcal{K}_{\lambda}^{\Gamma}\right)$ e recordando que $\mathcal{M}_{(x, \lambda)}^{\Gamma} \cdot \mathcal{T}_{\epsilon}^{\Gamma} \subset \mathcal{T}^{\Gamma}\left(\mathcal{K}_{\lambda}^{\Gamma}\right)$, obtemos

$$
\mathcal{T}^{\Gamma}(f) \equiv \mathcal{T}^{\Gamma}(g) \text { módulo } \mathcal{M}_{(x, \lambda)}^{\Gamma} \cdot \Omega_{(x, \lambda)}^{r-1} .
$$

Sejam $\eta=d \mathcal{O}_{g}, N=\mathcal{T}^{\Gamma}\left(\mathcal{K}_{\lambda}^{\Gamma}\right), M=\overrightarrow{\mathcal{M}}_{(x, \lambda)}^{\Gamma}, M_{0}=\mathcal{M}_{(x, \lambda)}^{\Gamma} \cdot \Omega_{(x, \lambda)}^{k} \quad$ and $r=k+\ell+1$, onde $\ell$ como no Lema 1.8.3.1. Por (1.18),

$$
\mathcal{M}_{(x, \lambda)}^{\Gamma} \cdot \Omega_{(x, \lambda)}^{r} \subset \mathcal{T}^{\Gamma}(g)+\mathcal{M}_{(x, \lambda)}^{\Gamma} \cdot \Omega_{(x, \lambda)}^{r-1}=\mathcal{T}^{\Gamma}(g)+\mathcal{M}_{(x, \lambda)}^{\Gamma} \cdot \Omega_{(x, \lambda)}^{k+\ell} .
$$

Pelo Lema 1.8.3.1, $\mathcal{M}_{(x, \lambda)}^{\Gamma} \cdot \Omega_{(x, \lambda)}^{r} \subset \mathcal{T}^{\Gamma}(g)$.

$$
\text { Para } \alpha=\left(\alpha_{1} \ldots \alpha_{s}\right), \text { let } \quad \Omega_{(x, \lambda, \alpha)}^{r}=\mathcal{M}_{(x, \lambda)}^{r} \cdot \overrightarrow{\mathcal{E}}_{(x, \lambda, \alpha)} \cap \overrightarrow{\mathcal{E}}_{(x, \lambda, \alpha)}^{\Gamma} \text { e } \quad \Omega_{(\lambda, \alpha)}^{r}=
$$
$\mathcal{M}_{\lambda}^{r} \cdot \overrightarrow{\mathcal{E}}_{(\lambda, \alpha)} \cap \overrightarrow{\mathcal{E}}_{(\lambda, \alpha)}^{\Gamma}$. Fixando $t_{1}$ na sequência da Proposição 1.8.3.2, veja $F_{t_{1}}$ como um elemento de $\overrightarrow{\mathcal{E}}_{(x, \lambda)}^{\Gamma}$ e $F_{\left(t_{1}+t\right)}$ como seu desdobramento a um parâmetro, isto é, $F_{\left(t_{1}+t\right)} \in \overrightarrow{\mathcal{E}}_{(x, \lambda, t)}^{\Gamma}$. 
Lema 1.8.4.4. Existem germes $\hat{T}_{t} \in \mathbf{M}_{(x, \lambda, t)}^{\Gamma}, \hat{X}_{t} \in \Omega_{(x, \lambda, t)}^{1}$ e $\hat{\Lambda}_{t} \in \Omega_{(\lambda, t)}^{1}$ tais que

$$
\frac{\partial}{\partial t} F_{\left(t_{1}+t\right)}=p=\hat{T}_{t} F_{\left(t_{1}+t\right)}+\left(D_{x} F_{\left(t_{1+t}\right)}\right) \hat{X}_{t}+\left(D_{\lambda} F_{\left(t_{1}+t\right)}\right) \hat{\Lambda}_{t} .
$$

Em outras palavras, $p \in d \mathcal{O}_{F_{\left(t_{1}+t\right)}}\left(\left[\mathcal{T}^{\Gamma}\left(\mathcal{K}_{\lambda}^{\Gamma}\right)\right]_{t}\right)$ onde $\left[\mathcal{T}^{\Gamma}\left(\mathcal{K}_{\lambda}^{t}\right)\right]_{t}$ denota o módulo com os geradores de $\mathcal{T}^{\Gamma}\left(\mathcal{K}_{\lambda}^{\Gamma}\right)$ vistos sobre o sistema de anéis $\left\{\mathcal{E}_{t}, \mathcal{E}_{(\lambda, t)}^{\Gamma}, \mathcal{E}_{(x, \lambda, t)}^{\Gamma}\right\}$ ao invés de $\left\{\mathcal{E}_{\lambda}^{\Gamma}, \mathcal{E}_{(x, \lambda)}^{\Gamma}\right\}$.

Prova. Temos que $F_{\left(t_{1}+t\right)}-F_{t_{1}}=t p \in \mathcal{M}_{t} \cdot \Omega_{(x, \lambda, t)}^{r}$. Por um lado, isso implica que

$$
p=\frac{\partial}{\partial t}\left(F_{\left(t_{1}+t\right)}-F_{t_{1}}\right) \in \Omega_{(x, \lambda, t)}^{r}
$$

Portanto, para mostrar o Lema 1.8.4.4 é suficiente provar que

$$
\Omega_{(x, \lambda, t)}^{r} \subset d \mathcal{O}_{F_{\left(t_{1}+t\right)}}\left(\left[\mathcal{T}^{\Gamma}\left(\mathcal{K}_{\lambda}^{\Gamma}\right)\right]_{t}\right)
$$

Por outro lado, considerando $\left(F_{1} \times \mathbf{I}\right)(x, \lambda, t)=F_{1}(x, \lambda)$ temos

$$
d \mathcal{O}_{F_{\left(t_{1}+t\right)}}=d \mathcal{O}_{\left(F_{1} \times \mathbf{I}\right)} \text { módulo } \Omega_{(x, \lambda, t)}^{r} .
$$

Na notação do Lema 1.8.3.2, sejam $N=\mathcal{T}^{\Gamma}\left(\mathcal{K}_{\lambda}^{\Gamma}\right), \quad M=\overrightarrow{\mathcal{M}}_{(x, \lambda)}^{\Gamma}, \quad M_{t}=\overrightarrow{\mathcal{M}}_{(x, \lambda, t)}^{\Gamma}$ e $N_{t}=\left[\mathcal{T}^{\Gamma}\left(\mathcal{K}_{\lambda}^{\Gamma}\right)\right]_{t}$.

A aplica.̧ão $\psi=d \mathcal{O}_{F_{t_{1}}}: N \rightarrow M$ admite para extensâo $\psi_{1}=d \mathcal{O}_{\left(F_{\left.t_{1} \times \mathbf{I}\right)}\right.}: N_{t} \rightarrow M_{t}$.

Note que $F_{t_{1}}-f=t_{1} p \in \Omega_{(x, \lambda)}^{r}$ e assim pelo Lema 1.8.4.3,

$$
\mathcal{M}_{(x, \lambda)}^{\Gamma} \cdot \Omega_{(x, \lambda)}^{r} \subset d \mathcal{O}_{F_{t_{1}}}\left(\mathcal{T}^{\Gamma}\left(F_{t_{1}}\right)\right) .
$$

Logo, as hipóteses do Lema 1.8.3.2 estão satisfeitas e portanto

$$
\Omega_{(x, \lambda, t)}^{r} \subset \psi_{1}\left(N_{t}\right)=d \mathcal{O}_{F_{\left(t_{1} \times \mathbf{I}\right)}}\left(\left[\mathcal{T}^{\Gamma}\left(\mathcal{K}_{\lambda}^{\Gamma}\right)\right]_{t}\right)
$$

Por $(1.20)$,

$$
\Omega_{(x, \lambda, t)}^{r} \subset d \mathcal{O}_{F_{\left(t_{1}+t\right)}}\left(\left[T^{\Gamma}\left(\mathcal{K}_{\lambda}^{\Gamma}\right)\right]_{t}\right)
$$

Prova da Proposição 1.8.4.2. As hipóteses do Lema da Redução, Lema 1.7.2, são satisfeitas pelo Lema 1.8.4.4 e assim existe uma submersão $C:(\mathbb{R}, 0) \rightarrow\{0\}$ tal que $F_{\left(t_{1}+t\right)}$ se fatora através de $C^{*} F_{t_{1}}=F_{t_{1}}$. Além disso, do fato de que $\hat{X}_{t} \in \Omega_{(x, \lambda, t)}^{1}$ e $\hat{\Lambda}_{t} \in \Omega_{(\lambda, t)}^{1}$, ou seja, que eles se anulam na origem, podemos concluir da prova do Lema da Redução que a mudança de coordenadas $\left(X_{t}, \Lambda_{t}\right)$ preserva a origem. 


\subsection{O espaço tangente restrito}

Dizemos que dois germes $f, g \in \overrightarrow{\mathcal{M}}_{(x, \lambda)}^{\Gamma}$ são fortemente $\mathcal{K}_{\lambda}^{\Gamma}$-equivalentes se eles forem $\mathcal{K}_{\lambda^{-}}^{\Gamma}$ equivalentes com $\Lambda(\lambda)=\lambda$. Portanto, o grupo que induz a $\mathcal{K}_{\lambda}^{\Gamma}$-equivalência forte é

$$
\mathcal{R}\left(\mathcal{K}_{\lambda}^{\Gamma}\right)=\left\{(T, X) \mid\left(T, X, \mathbf{I}_{\ell}\right) \in \mathcal{K}_{\lambda}^{\Gamma}\right\}
$$

O espaço tangente a $\mathcal{R}\left(\mathcal{K}_{\lambda}^{\Gamma}\right)$ no seu elemento identidade é chamado espaço tangente restrito e denotado $\mathcal{R} \mathcal{T}\left(\mathcal{K}_{\lambda}^{\Gamma}\right)$. É definido por

$$
\mathcal{R} \mathcal{T}\left(\mathcal{K}_{\lambda}^{\Gamma}\right)=\left\{\left.\frac{d}{d t}\right|_{t=0} \delta_{t} \mid \delta_{t} \in \mathcal{R}\left(\mathcal{K}_{\lambda}^{\Gamma}\right), \delta_{0}=\mathbf{1}\right\}
$$

e assim existe uma identificação

$$
\mathcal{R} \mathcal{T}\left(\mathcal{K}_{\lambda}^{\Gamma}\right) \simeq \mathbf{M}_{(x, \lambda)}^{\Gamma} \oplus \vec{\Theta}_{(x, \lambda)}^{\Gamma, 0}
$$

A aplicação órbita associada a $f \in \vec{\Theta}_{(x, \lambda)}^{\Gamma, o}$ é

$$
\begin{aligned}
\mathcal{O}_{f}: \mathcal{R}\left(\mathcal{K}_{\lambda}^{\Gamma}\right) & \rightarrow \overrightarrow{\mathcal{E}}_{(x, \lambda)}^{\Gamma} \\
(T, X) & \mapsto(T, X) \cdot f .
\end{aligned}
$$

O espaço tangente a órbita $\mathcal{R}\left(\mathcal{K}_{\lambda}^{\Gamma}\right) \cdot f$ é

$$
\mathcal{R} \mathcal{T}^{\Gamma}(f)=d \mathcal{O}_{f}\left(\mathcal{R T}\left(\mathcal{K}_{\lambda}^{\Gamma}\right)\right)=\left\{T f+\left(D_{x} f\right) X \mid T \in \mathbf{M}_{(x, \lambda)}^{\Gamma}, X \in \vec{\Theta}_{(x, \lambda)}^{\Gamma, o}\right\}
$$

O Teorema seguinte fornece uma condição necesssária para que um germe $p \in \overrightarrow{\mathcal{E}}_{(x, \lambda)}^{\Gamma}$ esteja no conjunto dos termos de ordem alta de $f$, os quais são termos que podem ser removidos da expansão de Taylor de $f$. Ele genelariza o Teorema 1.3 ([16], pág. 168) e parcialmente o Teorema 2.2 ([22]).

Teorema 1.9.1. Dada $f \in \overrightarrow{\mathcal{E}}_{(x, \lambda)}^{\Gamma}$, seja $p$ um germe qualquer em $\overrightarrow{\mathcal{E}}_{(x, \lambda)}^{\Gamma}$.

1. Se $\mathcal{R} \mathcal{T}^{\Gamma}(f+t p)=\mathcal{R} \mathcal{T}^{\Gamma}(f)$ para todo $t \in[0,1]$ entâo $f+t p$ é fortemente $\mathcal{K}_{\lambda^{-}}^{\Gamma}$ equivalente à $f$ para todo $t \in[0,1]$. 
2. Se $\operatorname{cod}^{\Gamma}(f)$ for finita e $\mathcal{T}^{\Gamma}(f+t p)=\mathcal{T}^{\Gamma}(f)$ para todo $t \in[0,1]$ então $f+t p$ será $\mathcal{K}_{\lambda}^{\Gamma}$-equivalente à $f$ para todo $t \in[0,1]$.

3. Se $\operatorname{cod}^{\Gamma}(f)$ for finita $\mathcal{T}_{\epsilon}^{\Gamma}(f+t p)=\mathcal{T}_{e}^{\Gamma}(f)$ para todo $t \in[0,1]$ então $f+t p$ será $\mathcal{K}_{\lambda}^{\Gamma}$-equivalente a $f$ para todo $t \in[0,1]$.

Prova. 1. É semelhante à prova do Teorema 1.3 ([16], pág. 172).

2. Seja $F_{t}(x, \lambda)=F(x, \lambda, t)=f(x, \lambda)+t p(x, \lambda), t \in[0,1]$. Para um dado $t_{1}$ e $t$ suficientemente pequenos afirmamos que existem germes $T_{t} \in \mathbf{M}_{(x, \lambda, t)}^{\Gamma^{*}}, X_{t} \in \Omega_{(x, \lambda, t)}^{1}$ e $\Lambda_{t} \in \Omega_{(\lambda, t)}^{1}$ tais que

$$
\frac{\partial}{\partial t} F_{\left(t_{1}+t\right)}=p=T_{t} F_{\left(t_{1}+t\right)}+\left(D_{x} F_{\left(t_{1}+t\right)}\right) X_{t}+\left(D_{\lambda} F_{\left(t_{1}+t\right)}\right) \Lambda_{t}
$$

Seguindo nossa notação anterior, (1.21) simplesmente significa que $p \in d \mathcal{O}_{F_{\left(t_{1}+t\right)}}\left(\left[\mathcal{T}^{\Gamma}\left(\mathcal{K}_{\lambda}^{\Gamma}\right)\right]_{t}\right)$.

Como na prova do Teorema 1.8.4.2, se (1.21) for verdadeira, então $F_{\left(t_{1}+t\right)}$ e $F_{t_{1}}$ serão $\mathcal{K}_{\lambda}^{\Gamma}$-equivalentes com germes de mudança de coordenadas se anulando na origem de $\mathbb{R}^{n+\ell}$. Então, 2. segue de um argumento de compacidade e conexidade.

Para provar a afirmação, precisamos do seguinte lema.

Lema 1.9.2. Como $f$ tem codimensão finita e $\mathcal{T}^{\Gamma}(f+t p)=\mathcal{T}^{\Gamma}(f)$ para todo $t \in[0,1]$ então

$$
d \mathcal{O}_{F_{\left(t_{1}+t\right)}}\left(\left[\mathcal{T}^{\Gamma}\left(\mathcal{K}_{\lambda}^{\Gamma}\right)\right]_{t}\right)=d \mathcal{O}_{f}\left(\left[\mathcal{T}^{\Gamma}\left(\mathcal{K}_{\lambda}^{\Gamma}\right)\right]_{t}\right)
$$

para todo $t_{1} \in[0,1]$.

Prova. Primeiro observamos que $\left[\overrightarrow{\mathcal{E}}_{(x, \lambda)}^{\Gamma}\right]_{t}=\overrightarrow{\mathcal{E}}_{(x, \lambda, t)}^{\Gamma},\left[\overrightarrow{\mathcal{M}}_{(x, \lambda)}^{\Gamma}\right]_{t}=\overrightarrow{\mathcal{M}}_{(x, \lambda, t)}^{\Gamma}$,

$$
\left[\mathbf{M}_{(x, \lambda)}^{\Gamma}\right]_{t}=\mathbf{M}_{(x, \lambda, t)}^{\Gamma},\left[\overrightarrow{\mathcal{M}}_{\lambda}^{\Gamma}\right]_{t}=\overrightarrow{\mathcal{M}}_{(\lambda, t)}^{\Gamma} \mathbf{e}[\mathbb{R}]_{t}=\mathcal{E}_{t}
$$

Uma combinação dessas igualdades fornece

$$
\left[\mathcal{T}^{\Gamma}\left(\mathcal{K}_{\lambda}^{\Gamma}\right)\right]_{t}=\mathbf{M}_{(x, \lambda, t)}^{\Gamma} \oplus \overrightarrow{\mathcal{M}}_{(x, \lambda, t)}^{\Gamma} \oplus \overrightarrow{\mathcal{M}}_{(\lambda, t)}^{\Gamma}
$$

Para ver, por exemplo, que $\left[\overrightarrow{\mathcal{E}}_{(x, \lambda)}^{\Gamma}\right]_{t}=\overrightarrow{\mathcal{E}}_{(x, \lambda, t)}^{\Gamma}$, considere a inclusão natural

$$
\begin{aligned}
i:\left[\overrightarrow{\mathcal{E}}_{(x, \lambda)}^{\Gamma}\right]_{t} & \rightarrow \overrightarrow{\mathcal{E}}_{(x, \lambda, t)}^{\Gamma} \\
f_{t}(x, \lambda) & \mapsto f(x, \lambda, t) .
\end{aligned}
$$


Queremos provar que $i$ é sobrejetora. Note que $i$ é um homomorfismo de módulo finitamente gerado sobre o sistema de DA-álgebras $\left\{\mathcal{E}_{(x, \lambda, t)}^{\Gamma}, \mathcal{E}_{(\lambda, t)}^{\Gamma}, \mathcal{E}_{t}\right\}$. Por ([10], Corolário 6.16, pág. 33), $\left\{\left(\mathcal{E}_{t}, \mathcal{M}_{t}\right),\left(\mathcal{E}_{(\lambda, t)}^{\Gamma}, \mathcal{M}_{t} \cdot \mathcal{M}_{(\lambda, t)}^{\Gamma}\right),\left(\mathcal{E}_{(x, \lambda, t)}^{\Gamma}, \mathcal{M}_{t} \cdot \mathcal{M}_{(x, \lambda, t)}^{\Gamma}\right)\right\}$ é adequado. Também,

$$
i\left(\left[\overrightarrow{\mathcal{E}}_{(x, \lambda)}^{\Gamma}\right]_{t}\right)+\mathcal{M}_{t} \cdot \overrightarrow{\mathcal{E}}_{(x, \lambda, t)}^{\Gamma}=\overrightarrow{\mathcal{E}}_{(x, \lambda, t)}^{\Gamma}
$$

e assim pelo Lema 1.2.5,

$$
i\left(\left[\overrightarrow{\mathcal{E}}_{(x, \lambda)}^{\Gamma}\right]_{t}\right)=\overrightarrow{\mathcal{E}}_{(x, \lambda, t)}^{\Gamma}
$$

As provas das outras igualdades são semelhantes a essa.

Agora, como $\operatorname{cod}^{\Gamma}(f)$ é finita, para algum inteiro $q$, temos $\left\{p_{i}\right\}_{i=1}^{q} \subset \mathcal{E}_{(x, \lambda)}^{\Gamma}$ tais que

$$
\overrightarrow{\mathcal{E}}_{(x, \lambda)}^{\Gamma}=\mathcal{T}^{\Gamma}(f)+\mathbb{R}\left\{p_{1} \ldots p_{q}\right\}
$$

Calculando []$_{t}$ de ambos os lados de (1.22) e usando as observações preliminares, obtemos

$$
\overrightarrow{\mathcal{E}}_{(x, \lambda, t)}^{\Gamma}=d \mathcal{O}_{f}\left(\left[\mathcal{T}^{\Gamma}\left(\mathcal{K}_{\lambda}^{\Gamma}\right)\right]_{t}\right)+\mathcal{E}_{t}\left\{p_{1} \ldots p_{q}\right\}
$$

Afirmamos que

$$
\overrightarrow{\mathcal{E}}_{(x, \lambda, t)}^{\Gamma}=d \mathcal{O}_{F_{\left(t_{1}+t\right)}}\left(\left[\mathcal{T}^{\Gamma}\left(\mathcal{K}_{\lambda}^{\Gamma}\right)\right]_{t}\right)+\mathcal{E}_{t}\left\{p_{1} \ldots p_{q}\right\}
$$

O Lema segue então de (1.23) e (1.24).

Para provar a afirmação (1.24), definimos

$$
\begin{aligned}
\Psi:\left[\mathcal{T}^{\Gamma}\left(\mathcal{K}_{\lambda}^{\Gamma}\right)\right]_{t} \oplus\left(\mathcal{E}_{t}\right)^{q} & \rightarrow \overrightarrow{\mathcal{E}}_{(x, \lambda, t)}^{\Gamma} \\
\left(T, X, \Lambda, Y_{1} \ldots Y_{q}\right) & \mapsto T F_{\left(t_{1}+t\right)}+\left(D_{x} F_{\left(t_{1}+t\right)}\right) X+\left(D_{\lambda} F_{\left(t_{1}+t\right)}\right) \Lambda+\sum_{i=1}^{q} Y_{i} p_{i} .
\end{aligned}
$$

Note que a imagem de $\Psi$ é igual a expressão do lado direito de (1.24). Assim, é suficient provar que $\Psi$ é sobrejetora. $\Psi$ é um homomorfismo de módulo finitamente gerado sobre sistema de DA-álgebras $\left\{\mathcal{E}_{t}, \mathcal{E}_{(\lambda, t)}^{\Gamma}, \mathcal{E}_{(x, \lambda, t)}^{\Gamma}\right\}$. Pelo conceito de adequado, para mostrar qu $\Psi$ é sobrejetora é suficiente provar que

$$
\mathrm{I}_{m} \Psi \oplus \mathcal{M}_{t} \cdot \overrightarrow{\mathcal{E}}_{(x, \lambda, t)}^{\Gamma}=\overrightarrow{\mathcal{E}}_{(x, \lambda, t)}^{\Gamma}
$$

Como $F_{\left(t_{1}+t\right)}=f+t_{1} p+t p$, a imagem de $\Psi$ pode ser decomposta como

$$
d \mathcal{O}_{\left(f+t_{1} p\right)}\left(\left[\mathcal{T}^{\Gamma}\left(\mathcal{K}_{\lambda}^{\Gamma}\right)\right]_{t}\right)+\mathbb{R}\left\{p_{1} \ldots p_{q}\right\}+\mathcal{M}_{t} \cdot\left\{p_{1} \ldots p_{q}\right\}
$$


Assim, (1.25) pode ser reescrito como

$$
d \mathcal{O}_{\left(f+t_{1} p\right)}\left(\left[\mathcal{T}^{\Gamma}\left(\mathcal{K}_{\lambda}^{\Gamma}\right)\right]_{t}\right)+\mathbb{R}\left\{p_{1} \ldots p_{q}\right\}=\mathcal{M}_{t} \cdot \overrightarrow{\mathcal{E}}_{(x, \lambda, t)}^{\Gamma}
$$

Pelo Teorema de Preparação de Malgrange, isto é equivalente à

$$
d \mathcal{O}_{\left(f+t_{1} p\right)}\left(\left[\mathcal{T}^{\Gamma}\left(\mathcal{K}_{\lambda}^{\Gamma}\right)\right]_{t}\right)+\mathcal{E}_{t}\left\{p_{1} \ldots p_{q}\right\}=\overrightarrow{\mathcal{E}}_{(x, \lambda, t)}^{\Gamma}
$$

Por hipótese,

$$
d \mathcal{O}_{\left(f+t_{1} p\right)}\left(\mathcal{T}^{\Gamma}\left(\mathcal{K}_{\lambda}^{\Gamma}\right)\right)=\mathcal{T}^{\Gamma}\left(f+t_{1} p\right)=\mathcal{T}^{\Gamma}(f)
$$

Por $(1.22)$,

$$
d \mathcal{O}_{\left(f+t_{1} p\right)}\left(\mathcal{T}^{\Gamma}\left(\mathcal{K}_{\lambda}^{\Gamma}\right)\right)+\mathbb{R}\left\{p_{1} \ldots p_{q}\right\}=\overrightarrow{\mathcal{E}}_{(x, \lambda)}^{\Gamma}
$$

Calculando [ $]_{t}$ de ambos os lado, obtemos (1.26) e consequentemente (1.25) é verificado.

Resta provar (1.21).

$$
\frac{\partial}{\partial t} F_{\left(t_{1}+t\right)}=p \in d \mathcal{O}_{f}\left(\left[\mathcal{T}^{\Gamma}\left(\mathcal{K}_{\lambda}\right)\right]_{t}\right)
$$

e assim pelo Lema 1.9.2.,

$$
p \in d \mathcal{O}_{F_{\left(t_{1}+t\right)}}\left(\left[\mathcal{T}^{\Gamma}\left(\mathcal{K}_{\lambda}^{\Gamma}\right)\right]_{t}\right)
$$

Isto conclui a prova de 2 .

3. Usando o mesmo argumento acima, dado qualquer $t_{1}$ podemos mostrar que para $t$ suficientemente pequeno, $F_{\left(t_{1}+t\right)}$ e $F_{t_{1}}$ são $\mathcal{K}_{\lambda}^{\Gamma}$-equivalentes. Embora estejamos trabalhando com o espaço tangente estendido, podemos sempre encontrar germes de mudança de coordenadas que se anulam nas respectivas origens, estabelecendo a $\mathcal{K}_{\lambda}^{\Gamma}$-equivalência requerida. Note que pelo Lema 1.7.2, não temos essa propriedade a menos que a origem seja um ponto singular do campo de vetores que estamos integrando, mas como $f$ tem $\Gamma$-codimensão finita, um argumento padrão da Teoria de Singularidades, análogo ao de ([22], Proposição 2.11) garante que essas equivalências preservam a origem.

\subsection{Problema do reconhecimento}

O problema do reconhecimento trata de estabelecer quando um germe $g \in \overrightarrow{\mathcal{E}}_{(x, \lambda)}^{\Gamma}$ é $\mathcal{K}_{\lambda^{-}}^{\Gamma}$ equivalente a uma forma normal dada. 
Resolver um problema do reconhecimento particular significa caracterizar explicita-

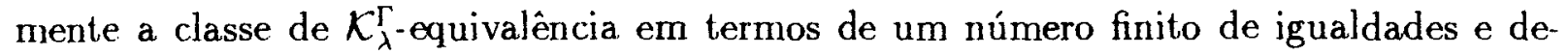
sigualdades polinomiais a serem satisfeitas pelos coeficientes de Taylor dos elementos da classe.

\subsubsection{Submódulos intrínsicos e termos de ordem alta}

Seja $\Phi=(T, X, \Lambda) \in \mathcal{K}_{\lambda}^{\Gamma}$ e considere a aplicação

$$
f \mapsto \Phi(f)=T \cdot f \circ(X, \Lambda)
$$

Definição. Um submódulo $\mathcal{J} \subset \overrightarrow{\mathcal{E}}_{(x, \lambda)}^{\Gamma}$ é intrínsico se $\Phi(f) \in \mathcal{J}$ para toda $f \in \mathcal{J}$ e toda

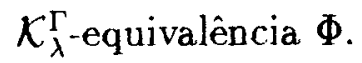

Definição. Seja $V \subset \overrightarrow{\mathcal{E}}_{(x, \lambda)}^{\Gamma}$. A parte intrínsica de $V$ é definida por

IntrV $=\sum\left\{\right.$ submódulos intrínsicos de $\overrightarrow{\mathcal{E}}_{(x, \lambda)}^{\Gamma}$ contidos in $\left.V\right\}$.

Pode também pode ser caracterizado como o major submódulo intrínsico contido em $V$.

Submódulos intrínsicos $\mathcal{J}$ tem a propriedade de que: se $h \in \mathcal{J}$ então $\mathcal{T}^{\Gamma}(h) \subset \mathcal{J}$. (cf. [16], pág. 203).

\subsubsection{Termos de ordem alta}

Seja $f \in \overrightarrow{\mathcal{E}}_{(x, \lambda)}^{\mathrm{r}}$. Dizemos que $p \in \overrightarrow{\mathcal{E}}_{(x, \lambda)}^{\Gamma}$ é de ordem alta com respeito a $f$ se $g+p$ for $\mathcal{K}_{\lambda}^{\Gamma}$-equivalente a $f$ para toda $g$ que for $\mathcal{K}_{\lambda}^{\Gamma}$-equivalente a $f$. Por definição, tais termos não podem pertencer ao conjunto solução do problema de reconhecimento de $f$. Denotamos por $\mathbf{P}(f)$ o conjunto de todos os termos de ordem alta de $f$, ou seja,

$$
\mathbf{P}(f)=\left\{p \in \overrightarrow{\mathcal{E}}_{(x, \lambda)}^{\Gamma} \mid g+p \sim f, \forall g \sim f\right\}
$$

onde $\sim \operatorname{denota} \mathcal{K}_{\lambda}^{\Gamma \text {-equivalência. }}$

Proposição 1.10.2.1. Para cada $f \in \overrightarrow{\mathcal{E}}_{(x, \lambda)}^{\Gamma}$, o conjunto $\mathbf{P}(f)$ é um submódulo intrínsico $\operatorname{de} \overrightarrow{\mathcal{E}}_{(x, \lambda)}^{\mathrm{r}}$.

Prova. ([16], Proposição 7.5, pág. 205). 


\subsubsection{O subgrupo das $\mathcal{K}_{\lambda}^{\Gamma}$-equivalências unipotentes}

Considere a seguinte projeçâo

$$
\begin{aligned}
& \pi: \mathbf{M}_{(x, \lambda)}^{\Gamma} \times \overrightarrow{\mathcal{M}}_{(x, \lambda)}^{\Gamma} \times \overrightarrow{\mathcal{M}}_{\lambda}^{\Gamma} \rightarrow \stackrel{\circ}{\mathcal{L}}_{\Gamma}(m) \times \stackrel{\circ}{\mathcal{L}}_{\Gamma}(n) \times \stackrel{\circ}{\mathcal{L}}_{\Gamma}(\ell) \\
& (T, X, \Lambda) \mapsto\left(T^{\circ},\left(D_{x} X\right)^{\circ},\left(D_{\lambda} \Lambda\right)^{\circ}\right)
\end{aligned}
$$

$\pi$ é um epimorfismo de grupo e seu núcleo, dado por

$$
\mathcal{U}_{\lambda}^{\Gamma}=\left\{(T, X, \Lambda) \in \mathcal{K}_{\lambda}^{\Gamma} \mid T^{o}=\mathbf{I}_{m},\left(D_{x} X\right)^{o}=\mathbf{I}_{n},\left(D_{\lambda} \Lambda\right)^{o}=\mathbf{I}_{\ell}\right\}
$$

é um subgrupo normal de $\mathcal{K}_{\lambda}^{\Gamma}$ formado de difeomorfismos unipotentes e é chamado de subgrupo das $\Gamma$-equivalências unipotentes.

$\mathrm{O}$ espaço tangente à $\mathcal{U}_{\lambda}^{\Gamma}$ no seu elemento identidade é definido por

$$
\mathcal{T}\left(\mathcal{U}_{\lambda}^{\Gamma}\right)=\left\{\left.\frac{d}{d t}\right|_{t=0} \mathcal{U}_{t} \mid \mathcal{U}_{t} \in \mathcal{U}_{\lambda}^{\Gamma} \text { e } \mathcal{U}_{0}=\mathbf{1}\right\}
$$

onde $\mathcal{U}_{t}(x, \lambda)=\left(\tilde{T}_{t}(x, \lambda), \tilde{X}_{t}(x, \lambda), \tilde{\Lambda}_{t}(\lambda)\right)$ satisfaz

$$
\begin{gathered}
\tilde{T}_{t}^{o}=\mathbf{I}_{m}, \tilde{T}_{0}(x, \lambda)=\mathbf{I}_{m} \\
\tilde{X}_{0}(x, \lambda)=x,\left(D_{x} \tilde{X}_{t}\right)^{o}=\mathbf{I}_{n}, \tilde{X}_{t}^{o}=0 \\
\tilde{\Lambda}_{0}(\lambda)=\lambda,\left(D_{\lambda} \tilde{\Lambda}_{t}\right)^{o}=\mathbf{I}_{\ell}, \tilde{\Lambda}_{t}^{o}=0 .
\end{gathered}
$$

Segue que

(i) $\tilde{T}_{t}(x, \lambda)=\mathbf{I}_{m}+t T(x, \lambda)+o(t)$ onde $T(x, \lambda)=\left.\frac{d}{d t}\right|_{t=0} \tilde{T}_{t}(x, \lambda)$,

(ii) $\tilde{X}_{t}(x, \lambda)=x+t X(x, t)+o(t)$ onde $X(x, \lambda)=\left.\frac{d}{d t}\right|_{t=0} \tilde{X}_{t}(x, \lambda)$,

(iii) $\tilde{\Lambda}_{t}(\lambda)=\lambda+t \Lambda(\lambda)+o(t)$ onde $\Lambda(\lambda)=\left.\frac{d}{d t}\right|_{t=0} \tilde{\Lambda}_{t}(\lambda)$.

Usando as condições (1.28), temos que $T \in \mathbf{M}_{(x, \lambda)}^{\Gamma}, T^{\circ}=0, X \in \overrightarrow{\mathcal{M}}_{(x, \lambda)}^{\Gamma},\left(D_{x} X\right)^{\circ}=0$, $\Lambda \in \overrightarrow{\mathcal{M}}_{\lambda}^{\Gamma}$ e $\left(D_{\lambda} \Lambda\right)^{\circ}=\mathbf{0}$.

Portanto,

$$
\mathcal{T}\left(\mathcal{U}_{\lambda}^{\Gamma}\right)=\left\{(T, X, \Lambda) \in \mathcal{K}_{\lambda}^{\Gamma} \mid T^{o}=0,\left(D_{x} X\right)^{\circ}=0,\left(D_{\lambda} \Lambda\right)^{\circ}=0\right\}
$$


Para $f \in \overrightarrow{\mathcal{E}}_{(x, \lambda)}^{\Gamma}$, o $\Gamma$-espaço tangente unipotente a $f$, denotado $\mathcal{T} \mathcal{U}^{\Gamma}(f)$, é definido de um modo análogo ao descrito em (1.5) e portanto é dado por

$$
\begin{array}{r}
\mathcal{T} \mathcal{U}^{\Gamma}(f)=\left\{T f+\left(D_{x} f\right) X+\left(D_{\lambda} f\right) \Lambda \mid(T, X, \Lambda) \in \mathcal{K}_{\lambda}^{\Gamma}\right. \\
\left.\operatorname{com} T^{o}=0, \quad\left(D_{x} X\right)^{o}=0,\left(D_{\lambda} \Lambda\right)^{o}=0\right\}
\end{array}
$$

O Г-espaço tangente restrito unipotente à $f$, denotado $\mathcal{R} \mathcal{T} \mathcal{U}^{\Gamma}(f)$, é dado por

$$
\begin{array}{r}
\mathcal{R} \mathcal{U}^{\Gamma}(f)=\left\{T f+\left(D_{x} f\right) X \mid(T, X) \in \mathbf{M}_{(x, \lambda)}^{\Gamma} \times \overrightarrow{\mathcal{M}}_{(x, \lambda)}^{\Gamma}\right. \\
\left.\operatorname{com} T^{o}=0 \operatorname{e}\left(D_{x} X\right)^{o}=0\right\}
\end{array}
$$

de acordo com as equivalências fortes unipotentes (cf. section 1.9).

Como uma consequência do Teorema 1.17 ([22], pág. 108) temos a seguinte proposição: Proposição 1.10.3.1. Se $f \in \overrightarrow{\mathcal{E}}_{(x, \lambda)}^{\Gamma}$ for de $\Gamma$-codimensâo finita, então

$$
\mathbf{P}(f) \supset \operatorname{Intr} \mathcal{T} \mathcal{U}^{\Gamma}(f)
$$

、 Corolário 1.10.3.2. Seja $p \in \operatorname{Intr} \mathcal{T} \mathcal{U}^{\Gamma}(f)$. Então $f+p$ é $\mathcal{K}_{\lambda}^{\Gamma}$-equivalente a $f$.

Observação. Dada uma forma normal $g$, seja $h \in \overrightarrow{\mathcal{E}}_{(x, \lambda)}^{\Gamma}$ satisfazendo certas condições de não-degenericidade, as quais são definidas por um número finito de desigualdades envolvendo um número finito de derivadas de $h$ na origem. Para provar que $h$ é $\mathcal{K}_{\lambda^{-}}^{\Gamma}$ equivalente a $g$ é suficjente mostrar que

$$
\Phi(h-p)-p^{\prime}=g
$$

onde $\Phi$ é uma $\mathcal{K}_{\lambda}^{\Gamma}$-equivalência, $p, p^{\prime} \in \operatorname{Intr} \mathcal{T} \mathcal{U}^{\Gamma}(g)$ com $p$ sendo termos da expansão de Taylor de $h$ e $p^{\prime}$ termos da expansão de Taylor de $\Phi(h-p)$.

Prova da Observação. De (1.31), segue que

$$
h-p \sim g+p^{\prime}
$$

e pelo Corolário 1.10.3.2, $h-p \sim g$. Usando a definição de $\mathbf{P}(g)$ e a Proposição 1.10.3.1,

$$
h=(h-p)+p \sim g
$$




\section{Capítulo 2}

\section{O problema $D_{4}$}

Um problema de bifurcação $\Gamma$ é um germe $f \in \overrightarrow{\mathcal{E}}_{(x, \lambda)}^{\Gamma}$ tal que $f^{\circ}=0$ e $\left(D_{x} f\right)^{\circ}=0$ com $(x, \lambda)$ denotando as variáveis-padrão e os parâmetros de bifurcação.

Daqui para frente, $\kappa$ denota a reflexão

$$
\begin{aligned}
\kappa: \mathbb{C} & \rightarrow \mathbb{C} \\
z & \mapsto \bar{z}
\end{aligned}
$$

ou, equivalentemente,

$$
\begin{aligned}
\kappa: \mathbb{R}^{2} & \rightarrow \mathbb{R}^{2} \\
(x, y) & \mapsto(x,-y)
\end{aligned}
$$

e $\mu$ denota a ação em $\mathbb{C}$ dada por

$$
\begin{aligned}
\mathbb{C} & \rightarrow \mathbb{C} \\
z & \mapsto\left(e^{i \frac{\pi}{2}} \kappa\right) \cdot z=i \bar{z} .
\end{aligned}
$$

Neste capítulo o nosso objetivo é resolver o problema do reconhecimento para problemas de bifurcação $\mathrm{D}_{4}$ onde o grupo diedral $\mathrm{D}_{4}=<\hat{\kappa}, \hat{\mu}>$ age sobre $(z, \lambda) \in \mathbb{C} \times \mathbb{R}^{2}, \lambda=\left(\lambda_{1}, \lambda_{2}\right)$, por

$$
\begin{aligned}
& \hat{\kappa} \cdot\left(z, \lambda_{1}, \lambda_{2}\right)=\left(\kappa z, \lambda_{1}, \lambda_{2}\right)=\left(\bar{z}, \lambda_{1}, \lambda_{2}\right), \\
& \hat{\mu} \cdot\left(z, \lambda_{1}, \lambda_{2}\right)=\left(\mu z, \kappa\left(\lambda_{1}, \lambda_{2}\right)\right)=\left(i \bar{z}, \lambda_{1},-\lambda_{2}\right) .
\end{aligned}
$$

Esta ação é motivada por um problema em Mecânica. Considere uma placa quadrada, não espessa e não isotrópica e dois pares de forças $F_{1}$ e $F_{2}$ agindo sobre a placa, horizontalmente e verticalmente, como mostra a Fig. 2.1 


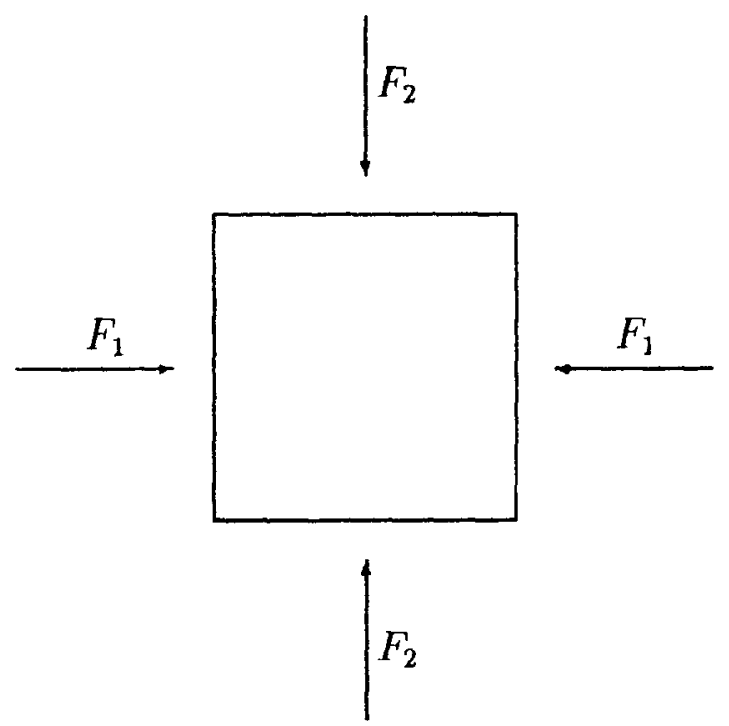

(Fig. 2.1)

Construindo um modelo matemático para essa situação obtemos uma descrição onde duas coordenadas $x_{1}, x_{2}$ representam modos de envergadura diferentes e os dois parâmetros $F_{1}, F_{2}$ correspondem as valores das forças. Para estudar fenômenos, como o da envergadura da placa podemos explorar o fato de que o modelo apresenta a seguinte simetria: a situação física não muda sob as transformaçôes:

$$
\begin{aligned}
& \left(x_{1}, x_{2}, F_{1}, F_{2}\right) \rightarrow\left(x_{1},-x_{2}, F_{1}, F_{2}\right), \\
& \left(x_{1}, x_{2}, F_{1}, F_{2}\right) \rightarrow\left(x_{2}, x_{1}, F_{2}, F_{1}\right) .
\end{aligned}
$$

Essas duas transformaçôes geram uma açầo do grupo $\mathrm{D}_{4}$. Introduzindo novas variáveis

$$
\lambda_{1}=F_{1}+F_{2} \text { e } \lambda_{2}=F_{1}-F_{2}
$$

essa ação aparece como em (2.1).

Alguns resultados preliminares foram estabelecidos por Martin Peters em sua Tese de Doutorado [18].

\subsection{Preliminares}

Usando a mesma notação do capítulo 1, seja

$$
\mathcal{E}_{(z, \lambda)}^{\mathrm{D}_{\mathbf{4}}}=\{h:(\mathbb{C} \times \mathbb{C}, 0) \rightarrow \mathbb{R} \mid h(\hat{\kappa} \cdot(z, \lambda))=h(z, \lambda) \text { e } h(\hat{\mu} \cdot(z, \lambda))=h(z, \lambda)\},
$$




$$
\begin{aligned}
& \overrightarrow{\mathcal{E}}_{(z, \lambda)}^{\mathrm{D}_{4}}=\{f:(\mathbb{C} \times \mathbf{C}, 0) \rightarrow \mathbf{C} \mid f(\hat{\kappa} \cdot(z, \lambda))=\kappa f(z, \lambda) \text { e } f(\hat{\mu} \cdot(z, \lambda))=\mu f(z, \lambda)\}, \\
& \overrightarrow{\mathcal{M}}_{(z, \lambda)}^{\mathrm{D}_{4}}=\left\{f \in \overrightarrow{\mathcal{E}}_{(z, \lambda)}^{\mathrm{D}_{4}} \mid f^{o}=0\right\}, \\
& \mathbf{M}_{(z, \lambda)}^{\mathrm{D}_{4}}=\left\{T:(\mathbb{C} \times \mathbb{C}, 0) \rightarrow G L_{2}(\mathbb{R}) \mid T(\hat{\kappa} \cdot(z, \lambda)) \kappa=\kappa T(z, \lambda)\right. \\
& \mathcal{E}_{\lambda}^{\mathrm{D}_{4}}=\{\eta:(\mathbf{C}, 0) \rightarrow \mathbb{R} \mid \eta(\kappa \lambda)=\eta(\lambda)\}, \\
& \overrightarrow{\mathcal{E}}_{\lambda}^{\mathrm{D}_{4}}=\{\Lambda:(\mathbb{C}, 0) \rightarrow \mathbb{C} \mid \Lambda(\kappa \lambda)=\kappa f(\lambda)\} .
\end{aligned}
$$

Denotamos por $N, \delta, \Delta, u_{4}$ as seguintes expressões:

$$
N=z \bar{z}, \delta=-\frac{1}{2}\left(z^{2}+\bar{z}^{2}\right), \Delta=\delta^{2}, u_{4}=\lambda_{2}^{2}
$$

Seja $\bar{u}=\left(u_{1}, u_{2}, u_{3}, u_{4}, u_{5}\right) \operatorname{com} u_{1}=N, u_{2}=\Delta, u_{3}=\lambda_{1}, u_{4}=\lambda_{2}^{2}$ e $u_{5}=\delta \lambda_{2}$. Note que temos uma única relação: $u_{5}^{2}=u_{2} u_{4}$.

Proposição 2.1.1. 1. $\mathcal{E}_{(z, \lambda)}^{\mathrm{D}_{4}}=\bar{u}^{*} \mathcal{E}_{u}$, isto é, para todo $h \in \mathcal{E}_{(z, \lambda)}^{\mathrm{D}_{4}}$ existe $p \in \mathcal{E}_{u}$ tal que $h(z, \lambda)=p(\bar{u})$.

2. $\overrightarrow{\mathcal{E}}_{(z, \lambda)}^{\mathrm{D}_{4}}$ é gerado por $z, \delta \bar{z}, \lambda_{2} \bar{z}$ como um $\mathcal{E}_{(z, \lambda)}^{\mathrm{D}_{4}}$-módulo.

3. $\mathbf{M}_{(z, \lambda)}^{\mathrm{D}_{4}}$ é o $\mathcal{E}_{(z, \lambda)}^{\mathrm{D}_{4}}$-módulo gerado pelas seguintes aplicações lineares em $\mathbf{C}$ :

$$
\begin{aligned}
S_{1}(z, \lambda) w & =w, & S_{4}(z, \lambda) w & =i \omega \bar{w} \\
S_{2}(z, \lambda) w & =\lambda_{2} \bar{w}, & S_{5}(z, \lambda) w & =-i \lambda_{2} \omega w \\
S_{3}(z, \lambda) w & =\delta \bar{w}, & S_{6}(z, \lambda) w & =-i \delta \omega w
\end{aligned}
$$

onde $\omega=\frac{i}{4}\left(\bar{z}^{2}-z^{2}\right)$.

Prova. Veja ([18], pág. 126-130).

É mais conveniente trabalhar com módulos livres e por isso, daqui para frente, consideraremos $u=\left(u_{1}, u_{2}, u_{3}, u_{4}\right)$ onde como anteriormente $u_{1}=N, u_{2}=\Delta, \quad u_{3}=\lambda_{1} \quad \mathrm{e}$ $u_{4}=\lambda_{2}^{2}$.

Proposição 2.1.2. $\overrightarrow{\mathcal{E}}_{(z, \lambda)}^{\mathrm{D}_{4}}$ é gerado livremente por $z, \delta \vec{z}, \lambda_{2} \bar{z}, \delta \lambda_{2} z$ como um $\mathcal{E}_{u}$-módulo.

Prova. Veja ([18], pág. 131). 
Proposição 2.1.3. $\mathbf{M}_{(z, \lambda)}^{D_{4}}$ é o $\mathcal{E}_{u}$-módulo gerado livremente pelas seguintes aplicaçôes lineares em $\mathbf{C}$ :

$$
\begin{array}{ll}
\hat{S}_{1}(z, \lambda) w=w, & \hat{S}_{5}(z, \lambda) w=i \omega \bar{w} \\
\hat{S}_{2}(z, \lambda) w=\lambda_{2} \bar{w}, & \hat{S}_{6}(z, \lambda) w=-i \lambda_{2} \omega w \\
\hat{S}_{3}(z, \lambda) w=\delta \bar{w}, & \hat{S}_{7}(z, \lambda) w=-i \delta \omega w \\
\hat{S}_{4}(z, \lambda) w=\delta \lambda_{2} w, & \hat{S}_{8}(z, \lambda) w=i \delta \lambda_{2} \omega \bar{w}
\end{array}
$$

Prova. Veja ([18], pág. 133).

Observação. As provas das Proposiçôes 2.1.1. a 2.1.3. podem também ser vistas no Apêndice, fazendo $\alpha=0$.

Proposição 2.1.4. Seja $\bar{v}=\left(\lambda_{1}, u_{4}\right)$.

1. $\mathcal{E}_{\lambda}^{\mathrm{D}_{4}}=\bar{v}^{*} \mathcal{E}_{v}$, isto é, para todo $\eta \in \mathcal{E}_{\lambda}^{\mathrm{D}_{4}}$ existe, $\xi \in \mathcal{E}_{v}$ tal que $\eta(\lambda)=\xi\left(\lambda_{1}, u_{4}\right)$.

2. $\overrightarrow{\mathcal{E}}_{\lambda}^{\mathrm{D}_{4}}$ é gerado livremente por

$$
\left(\begin{array}{l}
1 \\
0
\end{array}\right) \text { e }\left(\begin{array}{l}
0 \\
\lambda_{2}
\end{array}\right)
$$

como um $\mathcal{E}_{\lambda}^{\mathrm{D}_{4}}$-módulo.

Prova. Veja ([18], pág. 131).

\section{$2.2 \mathcal{K}_{\lambda}^{\mathrm{D}_{4}}$-equivalência}

O grupo de contato $\mathcal{K}_{\lambda}^{\mathrm{D}_{4}}$ é

$$
\begin{aligned}
\mathcal{K}_{\lambda}^{\mathrm{D}_{4}}=\left\{(T, X, \Lambda) \in \mathbf{M}_{(z, \lambda)}^{\mathrm{D}_{4}} \times \overrightarrow{\mathcal{M}}_{(z, \lambda)}^{\mathrm{D}_{4}} \times \overrightarrow{\mathcal{M}}_{\lambda}^{\mathrm{D}_{4}} \mid\right. & \left.T^{o},\left(D_{z} X\right)^{o} \text { e }\left(D_{\lambda} \Lambda\right)^{o} \in \stackrel{\circ}{\mathcal{L}}_{\mathrm{D}_{4}}(2)\right\} .
\end{aligned}
$$

Observe que como $n=m, \quad \vec{\Theta}_{(z, \lambda)}^{D_{4, o}}=\overrightarrow{\mathcal{M}}_{(z, \lambda)}^{D_{4}}$.

Dizemos que dois problemas de bifurcação $\mathrm{D}_{4} f, g$ são $\mathcal{K}_{\lambda}^{\mathrm{D}_{4}}$ - equivalentes se existir $(T, X, \Lambda) \in \mathcal{K}_{\lambda}^{\mathrm{D}_{4}}$ tal que $g(z, \lambda)=T(z, \lambda) f(X(z, \lambda), \Lambda(\lambda))$. 


\subsection{Espaços tangentes}

Da Proposiçâo 2.1.2., $f \in \overrightarrow{\mathcal{E}}_{(z, \lambda)}^{\mathrm{D}_{4}}$ pode ser escrita como

$$
f(z, \lambda)=p(u) z+q(u) \delta \bar{z}+r(u) \lambda_{2} \bar{z}+s(u) \delta \lambda_{2} z
$$

onde $u=\left(N, \Delta, \lambda_{1}, u_{4}\right)$ e identificamos $f \operatorname{com}[p, q, r, s]$.

$\mathrm{O} \mathrm{D}_{4^{-}}$espaço tangente estendido à $f \in \overrightarrow{\mathcal{E}}_{(z, \lambda)}^{\mathrm{D}_{4}}$. definido no Capítulo 1 , é

$$
\mathcal{T}_{\varepsilon}^{\mathrm{D}_{4}}(f)=\mathbf{M}_{(z, \lambda)}^{\mathrm{D}_{4}} f+\left(D_{z} f\right) \overrightarrow{\mathcal{E}}_{(z, \lambda)}^{\mathrm{D}_{4}}+\left(D_{\lambda} f\right) \overrightarrow{\mathcal{E}}_{\lambda}^{\mathrm{D}_{4}}
$$

Proposição 2.3.1. $\mathcal{T}_{\epsilon}^{\mathrm{D}_{4}}(f)=\mathcal{E}_{u}<g_{1} \ldots g_{12}>+\mathcal{E}_{\left(\lambda_{1}, u_{4}\right)}<g_{13}, g_{14}>$ onde

$$
\begin{aligned}
& g_{1}=[p, q, r, s] \text {. } \\
& g_{2}=\left[u_{4} r, u_{4} s, p, q\right] \text {. } \\
& g_{3}=[\Delta q, p, \Delta s, r] \text {. } \\
& g_{4}=[N p-\Delta q, p-N q,-N r+\Delta s,-r+N s] \text {. } \\
& g_{5}=[\Delta p-N \Delta q, N p-\Delta q, N \Delta s-\Delta r, \Delta s-N r] \text {. } \\
& g_{6}=\left[-N u_{4} r+\Delta u_{4} s,-u_{4} r+N u_{4} s, N p-\Delta q, p-N q\right] \text {. } \\
& g_{7}=\left[\Delta u_{4} s, u_{4} r, \Delta q, p\right] \text {. } \\
& g_{8}=\left[-\Delta u_{4} r+N \Delta u_{4} s,-N u_{4} r+\Delta u_{4} s, \Delta p-N \Delta q, N p-\Delta q\right] . \\
& g_{9}=\left[2 N p_{N}+4 \Delta p_{\Delta}+p, 2 N q_{N}+4 \Delta q_{\Delta}+3 q,\right. \\
& \left.2 N r_{N}+4 \Delta r_{\Delta}+r, 2 N s_{N}+4 \Delta s_{\Delta}+3 s\right] . \\
& g_{10}=\left[-2 \Delta p_{N}-4 N \Delta p_{\Delta}+\Delta q,-2 \Delta q_{N}-4 N \Delta q_{\Delta}-2 N q+p,\right. \\
& \left.-2 \Delta r_{N}-4 N \Delta r_{\Delta}+\Delta s,-2 \Delta s_{N}-4 N \Delta s_{\Delta}-2 N s+r\right] \text {. } \\
& g_{11}=\left[-2 \Delta u_{4} s_{N}-4 N \Delta u_{4} s_{\Delta}-2 N u_{4} s+u_{4} r,-2 u_{4} r_{N}-4 N u_{4} r_{\Delta}+u_{4} s,\right. \\
& \left.-2 \Delta q_{N}-4 N \Delta q_{\Delta}-2 N q+p,-2 p_{N}-4 N p_{\Delta}+q\right] \text {. } \\
& g_{12}=\left[2 N \Delta u_{4} s_{N}+4 \Delta^{2} u_{4} s_{\Delta}+3 \Delta u_{4} s, 2 N u_{4} r_{N}+4 \Delta u_{4} r_{\Delta}+u_{4} r\right. \text {, } \\
& \left.2 N \Delta q_{N}+4 \Delta^{2} q_{\Delta}+3 \Delta q, 2 N p_{N}+4 \Delta p_{\Delta}+p\right] \text {. } \\
& g_{13}=\left[p_{\lambda_{1}}, q_{\lambda_{1}}, r_{\lambda_{1}}, s_{\lambda_{1}}\right] \text {. } \\
& g_{14}=\left[2 u_{4} p_{u_{4}}, 2 u_{4} q_{u_{4}}, 2 u_{4} r_{u_{4}}+r, 2 u_{4} s_{u_{4}}+s\right] \text {. }
\end{aligned}
$$


Prova. Os geradores $g_{1} \ldots g_{8}$ são obtidos por $g_{i}=\hat{S}_{i}[p, q, r, s], 1 \leq i \leq 8$, onde $\hat{S}_{i}$ são os geradores livres de $\overrightarrow{\mathbf{M}}_{(z, \lambda)}^{\mathrm{D}_{4}}$ dados na Proposiçâo 2.1.3.

Os geradores $g_{9} \ldots g_{12}$ surgem de $\left(D_{z} f\right) X_{j}, 1 \leq j \leq 4$, onde $X_{j}$ sâo geradores livres de $\overrightarrow{\mathcal{E}}_{(z, \lambda)}^{\mathrm{D}_{4}}$ da Proposiçâo $2.1 .2 \mathrm{e}$

$$
g_{13}=\left(D_{\lambda} f\right)\left(\begin{array}{l}
1 \\
0
\end{array}\right), g_{14}=\left(D_{\lambda} f\right)\left(\begin{array}{l}
0 \\
\lambda_{2}
\end{array}\right) .
$$

$\mathrm{O} \mathrm{D}_{4}$-espaço tangente unipotente à $f \in \overrightarrow{\mathcal{E}}_{(z, \lambda)}^{\mathrm{D}_{4}}$, definido no Capítulo 1 , é

$$
\begin{aligned}
\mathcal{T} \mathcal{U}^{\mathrm{D}_{4}}(f)=\left\{T f+\left(D_{z} f\right) X+\left(D_{\lambda} f\right) \Lambda \mid(T, X, \Lambda) \in \mathcal{K}_{\lambda}^{\mathrm{D}_{4}}\right. \\
\left.T^{o}=0,\left(D_{z} X\right)^{\circ}=0,\left(D_{\lambda} \Lambda\right)^{o}=0\right\}
\end{aligned}
$$

\section{Proposição 2.3.2.}

$\mathcal{T} \mathcal{U}^{\mathrm{D}_{4}}(f)=\mathcal{E}_{u}<N g_{1}, \Delta g_{1}, \lambda_{1} g_{1}, u_{4} g_{1}, g_{2}, g_{3}, g_{4}, g_{5}, g_{6}, g_{7}, g_{8}$,

$$
\begin{array}{r}
N g_{9}, \Delta g_{9}, \lambda_{1} g_{9}, u_{4} g_{9}, g_{10}, g_{11}, g_{12}> \\
+\mathcal{E}_{\left(\lambda_{1}, u_{4}\right)}<\lambda_{1}^{2} g_{13}, u_{4} g_{13}, \lambda_{1} g_{14}, u_{4} g_{14}>
\end{array}
$$

com $g_{i}, 1 \leq i \leq 14$, como definidos na Proposição 2.3.1.

Prova. É imediato usando a definição do espaço tangente unipotente à $f$.

Proposição 2.3.3. $\mathcal{T}_{e}^{\mathrm{D}_{4}}(f)=\mathcal{T} \mathcal{U}^{\mathrm{D}_{4}}(f)+\mathbb{R}\left\{g_{1}, g_{9}, g_{13}, \lambda_{1} g_{13}, g_{14}\right\}$.

Prova. Segue das Proposições 2.3.1 e 2.3.2.

$\mathrm{O} \mathrm{D}_{4^{-}}$espaço tangente restrito unipotente $f$, definido no Capítulo 1 , é

$$
\mathcal{R} \mathcal{T} \mathcal{U}^{\mathrm{D}_{4}}(f)=\mathbf{M}_{(z, \lambda)}^{\mathrm{D}_{4}} f+\left(D_{z} f\right) \overrightarrow{\mathcal{M}}_{(z, \lambda)}^{\mathrm{D}_{4}}
$$

Consequentemente,

$$
\begin{array}{r}
\mathcal{R} \mathcal{U}^{\mathrm{D}_{4}}(f)=\mathcal{E}_{u}<N g_{1}, \Delta g_{1}, \lambda_{1} g_{1}, u_{4} g_{1}, g_{2}, g_{3}, g_{4}, g_{5}, g_{6}^{\prime}, g_{7}, g_{8}, \\
N g_{9}, \Delta g_{9}, \lambda_{1} g_{9}, u_{4} g_{9}, g_{10}, g_{11}, g_{12}>
\end{array}
$$

e

$$
\mathcal{T} \mathcal{U}^{\mathrm{D}_{4}}(f)=\mathcal{R} \mathcal{T} \mathcal{U}^{\mathrm{D}_{4}}(f)+\mathcal{E}_{\left(\lambda_{1}, u_{4}\right)}<\lambda_{1}^{2} g_{13}, u_{4} g_{13}, \lambda_{1} g_{14}, u_{4} g_{14}>
$$

A $D_{4}$-codimensão de $f \in \overrightarrow{\mathcal{E}}_{(z, \lambda)}^{\mathrm{D}_{4}}$ é dada por $\operatorname{dim}_{\mathbb{R}} \frac{\overrightarrow{\mathcal{E}}_{(z, \lambda)}^{\mathrm{D}_{4}}}{\mathcal{T}_{e}^{\mathrm{D}_{4}}(f)}$ e denotada $\operatorname{cod}^{\mathrm{D}_{4}}(f)$.

Definimos a $\mathrm{D}_{4}$-codimensão topológica de um problema de bifurcação $\mathrm{D}_{4}$ como sendo sua $\mathrm{D}_{4}$-codimensão menos o número de parâmetros modais. 


\subsection{Mudança de coordenadas}

Seja $e=(T, X, \Lambda)$ uma $\mathcal{K}_{\lambda}^{D_{4}}$-equivalência onde

$$
\begin{aligned}
& X=[a, b, c, d] ; a, b, c, d \in \mathcal{E}_{u} \text { e } a^{o}>0 \\
& \Lambda=\left(\Lambda_{1}, \lambda_{2} \Lambda_{2}\right) ; \Lambda_{1} \in \mathcal{M}_{\lambda}^{\mathrm{D}_{4}},\left(\Lambda_{1}\right)_{\lambda_{1}}^{o}>0 \text { e } \Lambda_{2} \in \mathcal{E}_{\lambda}^{\mathrm{D}_{4}}, \\
& T=\sum_{i=1}^{8} A_{i} \hat{S}_{i} ; A_{i} \in \mathcal{E}_{u} \text { e } A_{1}^{o}>0 .
\end{aligned}
$$

Para simplificar, definimos as seguintes expressões:

$$
\begin{gathered}
\tilde{N}_{1}=a^{2} N-2 a b \Delta+b^{2} \Delta N+c^{2} N u_{4}-2 c d \Delta u_{4}+d^{2} N \Delta u_{4}, \\
\tilde{N}_{2}=-2 a c+2 a d N+2 b c N-2 b d \Delta, \\
D_{1}=a^{2}-2 a b N+b^{2} \Delta+c^{2} u_{4}-2 c d N u_{4}+d^{2} \Delta u_{4}, \\
D_{2}=-2 a c N+2 a d \Delta+2 b c \Delta-2 b d N \Delta, \\
\Delta_{1}=D_{1}^{2}, \quad \tilde{\Delta}_{1}=\Delta \Delta_{1}+u_{4} \Delta_{3}, \\
\Delta_{2}=D_{1} D_{2}, \quad \tilde{\Delta}_{2}=2 \Delta_{2}, \\
\Delta_{3}=D_{2}^{2} .
\end{gathered}
$$

Usando (2.5),

$$
\begin{aligned}
& \tilde{N}=N \circ X=\tilde{N}_{1}+\delta \lambda_{2} \tilde{N}_{2}, \\
& \hat{\Delta}=\Delta \circ X=\tilde{\Delta}_{1}+\delta \lambda_{2} \tilde{\Delta}_{2}, \\
& \tilde{\lambda}_{1}=\lambda_{1} \circ \Lambda=\Lambda_{1}, \\
& \tilde{u}_{4}=u_{4} \circ \Lambda=u_{4} \Lambda_{2}^{2}, \\
& \tilde{\delta}=\delta \circ X=D_{1} \delta+D_{2} \lambda_{2} .
\end{aligned}
$$

Para $p, q, r, s \in \mathcal{E}_{u}$, definimos

$$
\tilde{p}=p\left(\tilde{N}, \tilde{\Delta}, \tilde{\lambda}_{1}, \tilde{u}_{4}\right), \tilde{q}=q\left(\tilde{N}, \tilde{\Delta}, \tilde{\lambda}_{1}, \tilde{u}_{4}\right), \quad \tilde{r}=r\left(\tilde{N}, \tilde{\Delta}, \tilde{\lambda}_{1}, \tilde{u}_{4}\right) \text { e } \tilde{s}=s\left(\tilde{N}, \tilde{\Delta}, \tilde{\lambda}_{1}, \tilde{u}_{4}\right) .
$$

Seja $f \in \overrightarrow{\mathcal{E}}_{(z, \lambda)}^{\mathrm{D}_{\mathbf{4}}}$ dada por $f=[p, q, r, s]$. Novamente para simplificar, fazemos a mudança de coordenadas em duas etapas: na primeira, $(I, X, \Lambda) \cdot f=f^{\prime}$ e na segunda, $(T, I, I) \cdot f^{\prime}=$ $f^{\prime \prime}$, obtendo assim o resultado final. 
Segue que

(i) $f^{\prime}=(l, X, \Lambda) \cdot f=\left[p^{\prime}, q^{\prime}, r^{\prime}, s^{\prime}\right]$ onde

$$
\begin{aligned}
& p^{\prime}=a \hat{p}+D_{1} \Delta b \hat{q}+D_{2} u_{4} c \hat{q}+\Lambda_{2} u_{4} c \hat{r}+D_{1} \Lambda_{2} \Delta u_{4} d \hat{s}+D_{2} \Lambda_{2} u_{4} a \hat{s} \\
& q^{\prime}=b \hat{p}+D_{1} a \hat{q}+D_{2} u_{4} d \hat{q}+\Lambda_{2} u_{4} d \tilde{r}+D_{2} \Lambda_{2} u_{4} b \tilde{s}+D_{1} \Lambda_{2} u_{4} c \tilde{s} \\
& r^{\prime}=c \tilde{p}+D_{1} \Delta d \tilde{q}+D_{2} a \tilde{q}+\Lambda_{2} a \tilde{r}+D_{1} \Lambda_{2} \Delta b \tilde{s}+D_{2} \Lambda_{2} u_{4} c \hat{s} \\
& s^{\prime}=d \tilde{p}+D_{1} c \tilde{q}+D_{2} b \tilde{q}+\Lambda_{2} b \tilde{r}+D_{1} \Lambda_{2} a \tilde{s}+D_{2} \Lambda_{2} u_{4} d \tilde{s}
\end{aligned}
$$

(ii) $f^{\prime \prime}=(T, I, I) \cdot f^{\prime}=\left[p^{\prime \prime}, q^{\prime \prime}, r^{\prime \prime}, s^{\prime \prime}\right] \quad$ onde

$$
\begin{gathered}
p^{\prime \prime}=\left(A_{1}+A_{4} N+A_{5} \Delta\right) p^{\prime}+\left(A_{3}-A_{4}-A_{5} N\right) \Delta q^{\prime}+ \\
\left(A_{2}-A_{6} N-A_{8} \Delta\right) u_{4} r^{\prime} \\
+\left(A_{6}+A_{7}+A_{8} N\right) \Delta u_{4} s^{\prime} \\
q^{\prime \prime}=\left(A_{3}+A_{4}+A_{5} N\right) p^{\prime}+\left(A_{1}-A_{4} N-A_{5} \Delta\right) q^{\prime}+\left(A_{7}-A_{6}-A_{8} N\right) u_{4} r^{\prime} \\
+\left(A_{2}+A_{6} N+A_{8} \Delta\right) u_{4} s^{\prime} \\
r^{\prime \prime}=\left(A_{2}+A_{6} N+A_{8} \Delta\right) p^{\prime}+\left(A_{7}-A_{6}-A_{8} N\right) \Delta q^{\prime}+\left(A_{1}-A_{4} N-A_{5} \Delta\right) r^{\prime} \\
+\left(A_{3}+A_{4}+A_{5} N\right) \Delta s^{\prime} \\
s^{\prime \prime}=\left(A_{6}+A_{7}+A_{8} N\right) p^{\prime}+\left(A_{2}-A_{6} N-A_{8} \Delta\right) q^{\prime}+\left(A_{3}-A_{4}-A_{5} N\right) r^{\prime} \\
+\left(A_{1}+A_{4} N+A_{5} \Delta\right) s^{\prime} .
\end{gathered}
$$

Recorde que $\bar{u}=\left(u, u_{5}\right)$ onde $u=\left(N, \Delta, \lambda_{1}, u_{4}\right)$ e $u_{5}=\delta \lambda_{2}$. Usando a relação $u_{5}^{2}=\Delta u_{4}$ e o Teorema de Taylor com parâmetros ([15], pág. 77), podemos escrever

$$
p(\bar{u})=p_{1}(u)+\delta \lambda_{2} p_{2}(u)
$$

Lema 2.4.1. Sejam $p \in \mathcal{E}_{u}$ e $X, \Lambda$ como em (2.4). Seja $\tilde{p}=p \circ(X, \Lambda)$. Então existem germes $p_{1}, p_{2} \in \mathcal{E}_{u}$ tais que

$$
\tilde{p}(u)=p\left(\tilde{N}_{1}, \tilde{\Delta}_{1}, \tilde{\lambda}_{1}, \tilde{u}_{4}\right)+\delta \lambda_{2} p_{1}(u)+\Delta u_{4} p_{2}(u)
$$

Prova. ([18], pág. 146). 
Seja $f=[p, q, r, s]$. Do Lema 2.4.1, podemos escrever

$$
\tilde{p}(u)=p \circ(X, \Lambda)=\tilde{p}_{1}(u)+\delta \lambda_{2} \tilde{p}_{2}(u)
$$

onde

$$
\hat{p}_{1}(u)=p\left(\hat{N}_{1}, \tilde{\Delta}_{1}, \hat{\lambda}_{1}, \hat{u}_{4}\right)+\Delta u_{4} p_{2}(u) \text { e } \quad \hat{p}_{2}(u)=p_{1}(u)
$$

Analogamente,

$$
\begin{aligned}
& \hat{q}=q \circ(X, \Lambda)=\tilde{q}_{1}+\delta \lambda_{2} \tilde{q}_{2}, \\
& \tilde{r}=r \circ(X, \Lambda)=\tilde{r}_{1}+\delta \lambda_{2} \tilde{r}_{2}, \\
& \hat{s}=s \circ(X, \Lambda)=\tilde{s}_{1}+\delta \lambda_{2} \hat{s}_{2} .
\end{aligned}
$$

Usando essa decomposição, escrevemos as expressôes em (2.7) da seguinte maneira:

$$
\begin{gathered}
f^{\prime}=(I, X, \Lambda) \cdot f=f \circ(X, \Lambda)=\left[p^{\prime}, q^{\prime}, r^{\prime}, s^{\prime}\right] \text { onde } \\
p^{\prime}=a \tilde{p}_{1}+d \Delta u_{4} \tilde{p}_{2}+D_{1} \Delta b \tilde{q}_{1}+D_{2} c u_{4} \tilde{q}_{1}+D_{2} u_{4} \Delta b \tilde{q}_{2} \\
+\Delta u_{4} D_{1} c \tilde{q}_{2}+u_{4} \Lambda_{2} c \tilde{r}_{1}+\Delta u_{4} \Lambda_{2} b \tilde{r}_{2}+u_{4} D_{2} \Lambda_{2} a \tilde{s}_{1} \\
+u_{4} \Delta \Lambda_{2} D_{1} d \tilde{s}_{1}+D_{2} u_{4}^{2} \Delta \Lambda_{2} d \tilde{s}_{2}+\Lambda_{2} \Delta u_{4} D_{1} a \tilde{s}_{2} \\
q^{\prime}=b \hat{p}_{1}+u_{4} c \tilde{p}_{2}+D_{1} a \tilde{q}_{1}+D_{2} u_{4} d \tilde{q}_{1}+D_{2} u_{4} a \tilde{q}_{2}+\Delta u_{4} D_{1} d \tilde{q}_{2} \\
+u_{4} \Lambda_{2} d \tilde{r}_{1}+u_{4} \Lambda_{2} a \tilde{r}_{2}+u_{4} \Lambda_{2} D_{2} b \tilde{s}_{1}+u_{4} \Lambda_{2} D_{1} c \tilde{s}_{1} \\
r^{\prime}=c u_{4} D_{1} b \tilde{s}_{2}+D_{2} u_{4}^{2} \Lambda_{2} c \tilde{s}_{2} \\
+\Delta b \tilde{p}_{2}+D_{2} a \tilde{q}_{1}+D_{1} \Delta d \tilde{q}_{1}+\Delta D_{1} a \tilde{q}_{2}+D_{2} u_{4} \Delta d \tilde{q}_{2} \\
+\Lambda_{2} a \tilde{r}_{1}+u_{4} \Delta \Lambda_{2} d \tilde{r}_{2}+\Lambda_{2} D_{1} \Delta b \tilde{s}_{1}+u_{4} \Lambda_{2} D_{2} c \tilde{s}_{1}+D_{2} \Delta u_{4} \Lambda_{2} b \tilde{s}_{2} \\
s^{\prime}={ }_{2}+a \tilde{p}_{1}+a \tilde{p}_{2}+D_{2} b \tilde{q}_{1}+D_{1} c \tilde{q}_{1}+\Delta D_{1} b \tilde{q}_{2}+D_{2} u_{4} c \tilde{q}_{2}+\Lambda_{2} b \tilde{r}_{1} \\
+u_{4} \Lambda_{2} c \tilde{r}_{2}+\Lambda_{2} D_{1} a \tilde{s}_{1}+u_{4} \Lambda_{2} D_{2} d \tilde{s}_{1}+u_{4} D_{2} \Lambda_{2} a \tilde{s}_{2}+\Lambda_{2} \Delta u_{4} D_{1} d \tilde{s}_{2} .
\end{gathered}
$$




\subsection{Ideais e submódulos intrínsicos}

Recorde que um submódulo intrínsico de $\overrightarrow{\mathcal{E}}_{(z, \lambda)}^{\mathrm{D}_{4}}$ é um submódulo que é invariante sob a ação do grupo das $\mathcal{K}_{\lambda}^{\mathrm{D}_{4}}$-equivalências e um ideal em $\overrightarrow{\mathcal{E}}_{(z, \lambda)}^{\mathrm{D}_{4}}$ é dito intrínsico se for invariante sob a ação do grupo de mudança de coordenadas:

$$
(z, \lambda) \mapsto(X(z, \lambda), \Lambda(\lambda))
$$

onde $X$ e $\Lambda$ satisfazem $(2.4)$.

Recorde ainda que $\left(\overrightarrow{\mathcal{M}}_{(z, \lambda)}^{\mathrm{D}_{4}}\right)^{k}$ é intrínsico para todo $k \geq 1$. Além disso, segue de $(2.6)$ que somas e produtos de ideais intrínsicos $\left\langle\lambda_{1}, u_{4}\right\rangle$ são intrínsicos.

$\mathrm{Na}$ proposição a seguir consideramos a decomposição do Lema 2.4.1.

Proposição 2.5.1. Seja $\mathcal{J}=\left[J_{1}, J_{2}, J_{3}, J_{4}\right]$ um submódulo de codimensão finita de $\overrightarrow{\mathcal{E}}_{(z, \lambda)}^{\mathrm{D}_{4}}$ e $h=[p, q, r, s] \in \mathcal{J} \operatorname{com} p, q, r, s \in \mathcal{E}_{u}$. Se as seguintes condições

1. $p(\tilde{u}) \in J_{1}, q(\tilde{u}) \in J_{2}, r(\tilde{u}) \in J_{3}, s(\tilde{u}) \in J_{4} \operatorname{com} \tilde{u}=\left(\tilde{N}_{1}, \tilde{\Delta}_{1}, \tilde{\lambda}_{1}, \tilde{u}_{4}\right)$ e $p_{1}(u) \in J_{4}$,

2. $<\Delta, u_{4}>\subset J_{1} \subseteq J_{2} \subseteq J_{3} \subseteq J_{4}$,

forem satisfeitas entào $\mathcal{J}$ é um submódulo intrínsico de $\overrightarrow{\mathcal{E}}_{(z, \lambda)}^{\mathrm{D}_{4}}$.

Prova. Seja $e=(T, X, \Lambda)$ uma $\mathcal{K}_{\lambda}^{\mathrm{D}_{4}}$-equivalência.

Primeiro, verificamos que $h^{\prime}=(I, X, \Lambda) \cdot h \in \mathcal{J}$. Seja $h^{\prime}=\left[p^{\prime}, q^{\prime}, r^{\prime}, s^{\prime}\right]$. Usando (2.9), Lema 2.4.1. e as hipóteses, segue que $p^{\prime} \in J_{1}, q^{\prime} \in J_{2}, r^{\prime} \in J_{3}, s^{\prime} \in J_{4}$ e assim $h^{\prime} \in \mathcal{J}$. Seja $h^{\prime \prime}=(T, I, I) \cdot h^{\prime} \operatorname{com} h^{\prime} \in \mathcal{J}$ e $h^{\prime \prime}=\left[p^{\prime \prime}, q^{\prime \prime}, r^{\prime \prime}, s^{\prime \prime}\right]$.

É imediato que $h^{\prime \prime} \in \mathcal{J}$ usando (2.8) e as hipóteses.

Portanto, $\mathcal{J}$ é um submódulo intrínsico de $\overrightarrow{\mathcal{E}}_{(z, \lambda)}^{\mathrm{D}_{4}}$ pois $e \cdot h \in \mathcal{J}$.

Observação 2.5.2. A Proposição 2.5.1. estabelece condições suficientes para um submódulo finito $\mathcal{J}=\left[J_{1}, J_{2}, J_{3}, J_{4}\right]$ de $\overrightarrow{\mathcal{E}}_{(z, \lambda)}^{\mathrm{D}_{4}}$ ser intrínsico, considerando relações particulares entre os ideais $J_{i}, 1 \leq i \leq 4$. A maior dificuldade em trabalhar com ideais e submódulos intrínsicos é determiná-los quando existem simetrias nas variáveis-padrão e nos parâmetros de bifurcação como nesse contexto. A verificação será feita caso-a-caso usando a definição como segue. 
Sejam $e=(T, X, \Lambda)$ uma $\mathcal{K}_{\lambda}^{D_{4}}$-equivalência e $h=[p, q, r, s] \in \mathcal{J}=\left[J_{1}, J_{2}, J_{3}, J_{4}\right]$.

Podemos escrever $h=h_{1}+h_{2}+h_{3}+h_{4} \operatorname{com} h_{1}=[p, 0,0,0], h_{2}=[0, q, 0,0]$, $h_{3}=[0,0, r, 0]$ e $h_{4}=[0,0,0, s]$.

Pelo Lema 2.4.1.,

(i) $h_{1} \circ(X, \Lambda)(u)=\tilde{p}(u)[a, b, c, d]$ com

$$
\tilde{p}(u)=p(\tilde{u})+\delta \lambda_{2} p_{1}(u)+\Delta u_{4} p_{2}(u),
$$

(ii) $h_{2} \circ(X, \Lambda)(u)=\tilde{q}(u)\left[b \Delta D_{1}+c u_{4} D_{2}, a D_{1}+d u_{4} D_{2}, d \Delta D_{1}+a D_{2}, c D_{1}+b D_{2}\right]$,

(iii) $h_{3} \circ(X, \Lambda)(u)=\tilde{r}(u)\left[u_{4} c \Lambda_{2}, u_{4} d \Lambda_{2}, a \Lambda_{2}, b \Lambda_{2}\right]$,

(iv) $h_{4} \circ(X, \Lambda)(u)=\tilde{s}(u)\left[D_{1} \Lambda_{2} \Delta u_{4} d+D_{2} \Lambda_{2} u_{4} a, D_{1} \Lambda_{2} u_{4} c+D_{2} \Lambda_{2} u_{4} b, \quad D_{1} \Lambda_{2} \Delta b+\right.$

$$
\left.D_{2} \Lambda_{2} u_{4} c, D_{1} \Lambda_{2} a+D_{2} \Lambda_{2} u_{4} d\right]
$$

onde decompomos $\tilde{q}(u), \tilde{r}(u), \tilde{s}(u)$ da mesma forma que em (i) e usamos que

$$
\delta \lambda_{2}[p, q, r, s]=\left[\Delta u_{4} s, \varkappa_{4} r, \Delta q, p\right]
$$

Primeiro mostramos que $h^{\prime}=(I, X, \Lambda) \cdot h \in \mathcal{J}$. Para isso, verificamos a relação $h_{i} \circ(X, \Lambda) \in J_{i}, 1 \leq i \leq 4$. Depois disso, usando (2.8) verificamos se $h^{\prime \prime}=(T, I, I) \cdot h^{\prime} \in \mathcal{J}$, para estabelecer que $e \cdot h \in \mathcal{J}$ e assim, que $\mathcal{J}$ é intrínsico.

\subsection{A forma genérica normal}

Teorema 2.6.1. Seja $f=\left[m N+\epsilon_{o} \lambda_{1}, \epsilon_{5}, 1,0\right]$ onde $\epsilon_{o}^{2}=\epsilon_{5}^{2}=1$ e $m \neq \pm 1,0$. Então $f$ tem $\mathrm{D}_{4}$-codimensão 1 e todo problema de bifurcação $\mathrm{D}_{4} h=[p, q, r, s]$ que satisfizer $p^{o}=0, p_{N}^{o} \neq 0, p_{N}^{o} \neq \pm q^{o}, p_{\lambda_{1}}^{o} \neq 0$ e $\quad r^{o} \neq 0$ será $\mathcal{K}_{\lambda}^{D_{4}}$-equivalente à $f$ com os coeficientes $\epsilon_{o}, \epsilon_{5}$ e $m$ dados por $\epsilon_{o}=s g p_{\lambda_{1}}^{\circ}, \epsilon_{5}=s g q^{\circ}$ e $m=\frac{p_{N}^{o}}{\left|q^{\circ}\right|}$.

A prova do Teorema é consequência dos seguintes lemas: 
Lema 2.6.2. Seja $f=\left[m N+\mathcal{E}_{0} \lambda_{1}, \epsilon_{5}, 1,0\right]$ como definido acima. Então o $D_{4}$-espaço tangente unipotente é dado por

$$
\mathcal{T} \mathcal{U}^{D_{4}}(f)=\left[\mathcal{E}_{u}<N^{2}, N \lambda_{1}, \lambda_{1}^{2}, \Delta, u_{4}>, \mathcal{M}_{u}, \mathcal{M}_{u}, \mathcal{E}_{u}\right]
$$

Prova. De (2.2), um simples cálculo mostra que

$$
\mathcal{R T} \mathcal{U}^{\mathrm{D}_{4}}(f)+<\lambda_{1}, u_{4}>\overrightarrow{\mathcal{E}}_{u}=\left[\mathcal{E}_{N \Delta}<N^{2}, \Delta>, \mathcal{M}_{N \Delta}, \mathcal{M}_{N \Delta}, \mathcal{E}_{N \Delta}\right]
$$

desde que $m \neq \pm 1,0$.

Segue que

$$
\frac{\overrightarrow{\mathcal{E}}_{u}}{\mathcal{R} \mathcal{T} \mathcal{U}^{\mathrm{D}_{4}}(f)+<\lambda_{1}, u_{4}>\overrightarrow{\mathcal{E}}_{u}}
$$

é gerado por $[N, 0,0,0],[1,0,0,0],[0,1,0,0]$ e $[0,0,1,0]$ como um espaço vetorial real.

Seja $\left.R=\left[<N^{2}, N \lambda_{1}, \lambda_{1}^{2}, \Delta, u_{4}\right\rangle, \mathcal{M}_{u}, \mathcal{M}_{u}, \mathcal{E}_{u}\right]$. Mostraremos que $R=\mathcal{T} \mathcal{U}^{D_{4}}(f)$.

(i) $\mathcal{T \mathcal { U } ^ { \mathrm { D } _ { 4 } }}(f) \subset R$.

Pelo Téorema de Preparação de Malgrange, $\frac{\overrightarrow{\mathcal{E}}_{u}}{\mathcal{R} \mathcal{T} \mathcal{U}^{\mathrm{D}_{4}}(f)}$ é gerado por $[N, 0,0,0]$, $[1,0,0,0],[0,1,0,0]$ e $[0,0,1,0]$ como um $\mathcal{E}_{\left(\lambda_{1}, u_{4}\right)}$-módulo, e assim $\phi \in R$ pode ser representada como $\phi=\phi_{1}[N, 0,0,0]+\phi_{2}[1,0,0,0]+\phi_{3}[0,1,0,0]+\phi_{4}[0,0,1,0]+\tilde{\phi}$ onde $\phi_{i} \in \mathcal{E}_{\left(\lambda_{1}, u_{4}\right)}, 1 \leq i \leq 4$ e $\tilde{\phi} \in \mathcal{R} \mathcal{T} \mathcal{U}^{\mathrm{D}_{4}}(f)$.

Como $\mathcal{R} \mathcal{T} \mathcal{U}^{\mathrm{D}_{4}}(f) \subset R$, isto implica que, $\phi_{1}[N, 0,0,0]+\phi_{2}[1,0,0,0]+\phi_{3}[0,1,0,0]+$ $\phi_{4}[0,0,1,0] \in R$ e portanto $\phi_{1}, \phi_{3}, \phi_{4} \in \mathcal{M}_{\left(\lambda_{1}, u_{4}\right)}$, e $\phi_{2} \in \mathcal{E}_{\left(\lambda_{1}, u_{4}\right)}<\lambda_{1}^{2}, u_{4}>$.

Logo, $\frac{R}{\mathcal{R T \mathcal { U } ^ { \mathrm { D } _ { 4 } } ( f )}}$ é gerado por

$$
\begin{array}{llll}
{\left[N \lambda_{1}, 0,0,0\right],} & {\left[N u_{4}, 0,0,0\right],} & {\left[\lambda_{1}^{2}, 0,0,0\right],} & {\left[u_{4}, 0,0,0\right],} \\
{\left[0, \lambda_{1}, 0,0\right],} & {\left[0, u_{4}, 0,0\right],} & {\left[0,0, \lambda_{1}, 0\right],} & {\left[0,0, u_{4}, 0\right]}
\end{array}
$$

como um $\mathcal{E}_{\left(\lambda_{1}, u_{4}\right)}$-módulo.

De (2.3),

$$
\mathcal{T} \mathcal{U}^{\mathrm{D}_{4}}(f)=\mathcal{R} \mathcal{T} \mathcal{U}^{\mathrm{D}_{4}}(f)+\mathcal{E}_{\left(\lambda_{1}, u_{4}\right)}<\left[\lambda_{1}^{2}, 0,0,0\right],\left[u_{4}, 0,0,0\right],\left[0,0, \lambda_{1}, 0\right],\left[0,0, u_{4}, 0\right]>,
$$


mostrando que $\mathcal{T} \mathcal{U}^{\mathrm{D}_{4}}(f) \subset R$.

(ii) $R \subset \mathcal{T U}^{\mathrm{D}_{4}}(f)$.

Etapa 1. Considere o seguinte conjunto de geradores obtido de $\mathcal{R} \mathcal{T} \mathcal{U}^{\mathrm{D}_{4}}(f)$ módulo

$$
\begin{aligned}
& \mathcal{E}_{\left(\lambda_{1}, u_{4}\right)}<\left[\lambda_{1}^{2}, 0,0,0\right],\left[u_{4}, 0,0,0\right],\left[0,0, \lambda_{1}, 0\right],\left[0,0, u_{4}, 0\right]>\text { e com } m \neq \pm 1,0: \\
& h_{1}^{\prime}=\left[m N^{2}+\epsilon_{0} N \lambda_{1}, \epsilon_{5} N, N, 0\right], \\
& h_{2}^{\prime}=\left[m N \Delta+\epsilon_{o} \Delta \lambda_{1}, \epsilon_{5} \Delta, \Delta, 0\right] \text {, } \\
& h_{3}^{\prime}=\left[m N \lambda_{1}, \epsilon_{5} \lambda_{1}, 0,0\right] \text {, } \\
& h_{4}^{\prime}=\left[m N u_{4}, \epsilon_{5} u_{4}, 0,0\right] \text {, } \\
& h_{5}^{\prime}=\left[0,0, m N, \epsilon_{5}\right] \text {, } \\
& h_{6}^{\prime}=\left[\epsilon_{5} \Delta, m N+\epsilon_{o} \lambda_{1}, 0,1\right] \text {, } \\
& h_{7}^{\prime}=\left[0,\left(m-\epsilon_{5}\right) N+\epsilon_{0} \lambda_{1},-N, 0\right] \text {, } \\
& h_{8}^{\prime}=\left[\left(m-\epsilon_{5}\right) N \Delta+\epsilon_{o} \Delta \lambda_{1}, m N^{2}+\epsilon_{o} N \lambda_{1}-\epsilon_{5} \Delta,-\Delta,-N\right] \text {, } \\
& h_{9}^{\prime}=\left[-N u_{4},-u_{4}, m N^{2}+\epsilon_{o} N \lambda_{1}-\epsilon_{5} \Delta,\left(m-\epsilon_{5}\right) N+\epsilon_{o} \lambda_{1}\right] \text {, } \\
& h_{10}^{\prime}=\left[0, u_{4}, \epsilon_{5} \Delta, m N+\epsilon_{o} \lambda_{1}\right] \text {, } \\
& h_{11}^{\prime}=\left[-\Delta u_{4},-N u_{4},\left(m-\epsilon_{5}\right) N \Delta+\epsilon_{0} \Delta \lambda_{1}, m N^{2}+\epsilon_{0} N \lambda_{1}-\epsilon_{5} \Delta\right] \text {, } \\
& h_{12}^{\prime}=\left[\epsilon_{o} N \lambda_{1}, 0, N, 0\right] \text {, } \\
& h_{13}^{\prime}=\left[\epsilon_{o} \Delta \lambda_{1}, 0, \Delta, 0\right] \text {, } \\
& h_{14}^{\prime}=\left[m \Delta, \epsilon_{5} N, 0,0\right] \text {, } \\
& h_{15}^{\prime}=\left[0,0, \epsilon_{5} N, m\right] \text {, } \\
& h_{16}^{\prime}=\left[0,0, \epsilon_{5} \Delta, m N\right] \text {. }
\end{aligned}
$$

Etapa 2. Sejam $I$ o $\mathcal{E}_{\left(\lambda_{1}, u_{4}\right)}$-módulo gerado por $\left\{h_{1}^{\prime} \ldots h_{16}^{\prime}, \Delta h_{12}^{\prime}, \Delta h_{15}^{\prime}, N h_{6}^{\prime}, N h_{14}^{\prime}\right\}$ e $S$ o $\mathcal{E}_{\left(\lambda_{1}, u_{4}\right)}$-módulo gerado por $\left\{\left[N^{2}, 0,0,0\right],\left[N \lambda_{1}, 0,0,0\right],[N \Delta, 0,0,0],[\Delta, 0,0,0],\left[N u_{4}, 0,0,0\right],[0 ; N, 0,0]\right.$, $[0, \Delta, 0,0],\left[0, \lambda_{1}, 0,0\right],\left[0, N^{2}, 0,0\right],\left[0, u_{4}, 0,0\right],[0,0, N, 0],\left[0,0, N^{2}, 0\right],[0,0, \Delta, 0]$, $\left.[0,0, N \Delta, 0],[0,0,0,1],[0,0,0, N],\left[0,0,0, N^{2}\right],[0,0,0, \Delta]\right\}$. 
É imediato que $l \subset S$ e um longo cálculo mostra que

$$
I+\mathcal{M}_{\lambda, u_{4}} S=S
$$

Pelo Lema de Nakayama, $S \subset I$ e portanto $I=S$.

De (2.10) e (2.11),

$$
\frac{R}{\mathcal{T} \mathcal{U}^{\mathrm{D}_{4}}(f)}=\mathcal{E}_{\left(\lambda_{1}, u_{4}\right)}<\left[N \lambda_{1}, 0,0,0\right],\left[N u_{4}, 0,0,0\right],\left[0, \lambda_{1}, 0,0\right],\left[0, u_{4}, 0,0\right] .
$$

Portanto,

$$
\frac{R}{\mathcal{T} \mathcal{U}^{\mathrm{D}_{4}}(f)} \subset I \subset \mathcal{T} \mathcal{U}^{\mathrm{D}_{4}}(f), \text { isto é, } R \subset \mathcal{T} \mathcal{U}^{\mathrm{D}_{4}}(f)
$$

e, de (i) e (ii),

$$
\mathcal{T} \mathcal{U}^{\mathrm{D}_{4}}(f)=R=\left[\mathcal{E}_{u}<N^{2}, N \lambda_{1}, \lambda_{1}^{2}, \Delta, u_{4}>, \mathcal{M}_{u}, \mathcal{M}_{u}, \mathcal{E}_{u}\right]
$$

Lema 2.6.3. $\mathcal{T} \mathcal{U}^{\mathrm{D}_{4}}(f)=\left[\mathcal{E}_{u}<N^{2}, N \lambda_{1}, \lambda_{1}{ }^{2}, \Delta, u_{4}>, \mathcal{M}_{u}, \mathcal{M}_{u}, \mathcal{E}_{u}\right]$ é um submódulo intrínsico de $\overrightarrow{\mathcal{E}}_{(z, \lambda)}^{\mathrm{D}_{4}}$.

Prova. Segue direto da Proposição 2.5.1.

Prova do Teorema 2.6.1.

Pelo Lema 2.6.2.,

$$
\mathcal{T} \mathcal{U}^{\mathrm{D}_{4}}(f)=\left[\mathcal{E}_{u}<N^{2}, N \lambda_{1}, \lambda_{1}^{2}, \Delta, u_{4}>, \mathcal{M}_{u}, \mathcal{M}_{u}, \mathcal{E}_{u}\right]
$$

e, pela Proposição 2.3.3.,

$$
\text { - } \mathcal{T}_{e}^{\mathrm{D}_{4}}(f)=\mathcal{T} \mathcal{U}^{\mathrm{D}_{4}}(f)+\mathbb{R}\left\{g_{1}, g_{9}, g_{13}, \lambda_{1} g_{13}, g_{14}\right\}
$$

onde

$$
\begin{aligned}
& g_{1}=\left[m N+\epsilon_{o} \lambda_{1}, \epsilon_{5}, 1,0\right], \\
& g_{9}=\left[3 m N+\epsilon_{o} \lambda_{1}, 3 \epsilon_{5}, 1,0\right], \\
& g_{13}=\left[\epsilon_{o}, 0,0,0\right], \\
& g_{14}=[0,0,1,0] .
\end{aligned}
$$


Logo,

$$
\mathcal{T}_{e}^{\mathrm{D}_{4}}(f)=\mathcal{T} \mathcal{U}^{\mathrm{D}_{4}}(f)+\mathbb{R}\left\{m[N, 0,0,0]+\epsilon_{5}[0,1,0,0],[1,0,0,0],\left[\lambda_{1}, 0,0,0\right],[0,0,1,0]\right\},
$$

e assim, $\overrightarrow{\mathcal{E}}_{(z, \lambda)}^{\mathrm{D}_{4}}=\mathcal{T}_{\epsilon}^{\mathrm{D}_{4}}(f)+\mathbb{R}\{[N, 0,0,0]\}$.

Portanto, $f$ tem $\mathrm{D}_{4}$-codimensâo 1 e codimensão topológica 0 , pois $m$ é um parâmetro modal.

Do Lema 2.6.3 e Proposição 1.10.3.1,

$$
\mathcal{T} \mathcal{U}^{\mathrm{D}_{4}}(f)=\operatorname{Intr} \mathcal{T} \mathcal{U}^{\mathrm{D}_{4}}(f) \subset \mathbf{P}(f) .
$$

Seguindo o método clássico descrito no Capítulo 1 (pág. 42), fazemos mudanças de coordenadas usando as fórmulas $(2.8)$ e (2.9) módulo $\mathcal{T} \mathcal{U}^{\mathrm{D}_{4}}(f)$ o qual está contido no conjunto dos termos de ordem alta de $f$. Para $h=[p, q, r, s] \in \overrightarrow{\mathcal{E}}_{(z, \lambda)}^{D_{4}}$, seja $\hat{h}=h$ módulo termos em $\mathcal{T U}^{\mathrm{D}_{4}}(f)$. Então,

$$
\hat{h}=\left[p_{N}^{o} N+p_{\lambda_{1}}^{o} \lambda_{1}, q^{o}, r^{o}, 0\right] .
$$

Sejam $h^{\prime}=(I, X, \Lambda) \cdot \hat{h}=\left[p^{\prime}, q^{\prime}, r^{\prime}, s^{\prime}\right]$ e $h^{\prime \prime}=(T, I, I) \cdot h^{\prime}=\left[p^{\prime \prime}, q^{\prime \prime}, r^{\prime \prime}, s^{\prime \prime}\right]$.

Usando (2.8) e trabalhando módulo termos em $\mathcal{T U}^{\mathrm{D}_{4}}(f)$ ), obtemos

$$
\begin{aligned}
& p_{N}^{\prime \prime}=A_{10} p_{N}^{\prime}, \\
& p_{\lambda_{3}}^{\prime \prime}=A_{10} p_{\lambda_{1}}^{\prime}, \\
& q_{o}^{\prime \prime}=A_{10} q_{o}^{\prime}, \\
& r_{o}^{\prime \prime}=A_{10} r_{o}^{\prime} .
\end{aligned}
$$

Usando (2.9) e novamente trabalhando módulo termos em $\mathcal{T} \mathcal{U}^{\mathrm{D}_{4}}(f)$, segue que

$$
\begin{aligned}
& p_{N}^{\prime \prime}=A_{10} a_{o}^{3} p_{N}^{o}, \\
& p_{\lambda_{1}}^{\prime \prime}=A_{10} a_{o} \Lambda_{11} p_{\lambda_{1}}^{o}, \\
& q_{o}^{\prime \prime}=A_{10} a_{o}^{3} q^{o}, \\
& r_{o}^{\prime \prime}=A_{10} \Lambda_{20} a_{o} r^{o} .
\end{aligned}
$$

Fazendo

$$
A_{10}=\frac{1}{a_{o}^{3}\left|q^{\circ}\right|}, \Lambda_{11}=\frac{1}{A_{10} a_{o}\left|p_{\lambda_{1}}^{o}\right|} \text { e } \Lambda_{20}=\frac{1}{A_{10} a_{o} r^{\circ}}, \text { obtemos }
$$




$$
p_{N}^{\prime \prime}=m=\frac{p_{N}^{\circ}}{\left|q^{\circ}\right|}, p_{\lambda_{1}}^{\prime \prime}=\epsilon_{o}, \epsilon_{o}=s g p_{\lambda_{1}}^{o}, q_{o}^{\prime \prime}=\epsilon_{5}, \epsilon_{5}=s g q^{\circ} \text { e } \quad r_{o}^{\prime \prime}=1 \text {. }
$$

Logo,

$$
(T, X, \Lambda) \cdot h \text { módulo } \mathcal{T} \mathcal{U}^{\mathrm{D}_{4}}(f) \text { é equivalente à }\left[m N+c_{0} \lambda_{1}, \epsilon_{5}, 1,0\right] .
$$

Portanto, todos os problemas de bifurcação $\mathrm{D}_{4}, h=[p, q, r, s]$, satisfazendo $p^{o}=0, p_{N}^{o} \neq 0$, $p_{N}^{o} \neq \pm q^{\circ}, p_{\lambda_{1}}^{o} \neq 0$ e $r^{\circ} \neq 0$ são equivalentes à $f=\left[m N+\epsilon_{o} \lambda_{1}, \epsilon_{5}, 1,0\right]$ com

$$
m=\frac{p_{N}^{o}}{\left|q^{\circ}\right|}, \epsilon_{\circ}=s g p_{\lambda_{1}}^{o} \mathrm{e} \epsilon_{5}=s g q^{\circ}
$$

\subsection{Pré-forma normal}

Nesta secção apresentamos alguns resultados preliminares para obter uma pré-forma normal para problemas de bifurcação $\mathrm{D}_{4}, f=[p, q, r, s]$, quando $q^{o} \neq 0$.

Denotamos por $\mathcal{C}_{\lambda}^{\mathrm{D}_{4}}$ o subgrupo de $\mathcal{K}_{\lambda}^{\mathrm{D}_{4}}$ dado por

$$
\mathcal{C}_{\lambda}^{\mathrm{D}_{4}}=\left\{(T, \mathbf{I}, \mathbf{I}) \mid(T, \mathbf{I}, \mathbf{I}) \in \mathcal{K}_{\lambda}^{\mathrm{D}_{4}}\right\}
$$

$O \mathcal{C}_{\lambda}^{\mathrm{D}_{4}}$-espaço tangente restrito e o $\mathcal{C}_{\lambda}^{\mathrm{D}_{4}}$-espaço tangente restrito unipotente à $f$, denotado, respectivamente, por $\mathcal{R} \mathcal{T}^{\mathcal{C}}(f)$ e $\mathcal{R} \mathcal{T} \mathcal{U}^{\mathcal{C}}(f)$ são

$$
\mathcal{R} \mathcal{T}^{\mathcal{C}}(f)=\left\{T f \mid T \in \mathbf{M}_{(z, \lambda)}^{\mathrm{D}_{4}}\right\}=\mathcal{E}_{u}<f, T_{2} f \ldots T_{8} f>
$$

e

$$
\mathcal{R} \mathcal{U}^{\mathcal{C}}(f)=\left\{T f \mid T \in \mathbf{M}_{(z, \lambda)}^{\mathrm{D}_{4}} \operatorname{com} T^{o}=0\right\}=\mathcal{E}_{u}<\mathcal{M}_{(z, \lambda)}^{\mathrm{D}_{4}} f, T_{2} f \ldots T_{8} f>
$$

onde $T_{1}=\mathbf{I}_{2}, T_{2} \ldots T_{8}$ são as matrizes reais associadas às aplicações lineares $\hat{S}_{i}, 1 \leq i \leq 8$, definidas na Proposição 2.1.3.

Especificamente,

$$
\mathcal{R} \mathcal{T}^{\mathcal{C}}(f)=\mathcal{E}_{u}<g_{1}, g_{2} \ldots g_{8}>
$$

e

$$
\mathcal{R} \mathcal{T} \mathcal{U}^{\mathcal{C}}(f)=\mathcal{E}_{u}<N g_{1}, \Delta g_{1}, \lambda_{1} g_{1}, u_{4} g_{1}, g_{2} \ldots g_{8}>
$$


onde

$$
\begin{aligned}
& g_{1}=[p, q, r, s], \\
& g_{2}=\left[u_{4} r, u_{4} s, p, q\right], \\
& g_{3}=[\Delta q, p, \Delta s, r], \\
& g_{4}=[N p-\Delta q, p-N q,-N r+\Delta s,-r+N s], \\
& g_{5}=[\Delta p-N \Delta q, N p-\Delta q, N \Delta s-\Delta r, \Delta s-N r], \\
& g_{6}=\left[-N u_{4} r+\Delta u_{4} s,-u_{4} r+N u_{4} s, N p-\Delta q, p-N q\right], \\
& g_{7}=\left[\Delta u_{4} s, u_{4} r, \Delta q, p\right], \\
& g_{8}=\left[-\Delta u_{4} r+N \Delta u_{4} s,-N u_{4} r+\Delta u_{4} s, \Delta p-N \Delta q, N p-\Delta q\right] .
\end{aligned}
$$

A Proposição seguinte é a versão para a $\mathcal{C}_{\lambda}^{\mathrm{D}_{4}}$-equivalência do Teorema 1.3 ([16], pág. 168).

Proposição 2.7.1. Seja $f \in \overrightarrow{\mathcal{E}}_{(z, \lambda)}^{\mathrm{D}_{4}}$ um problema de bifurcação e $p$ um germe qualquer em $\overrightarrow{\mathcal{E}}_{(z, \lambda)}^{\mathrm{D}_{4}}$. Suponha que

$$
\mathcal{R} \mathcal{T}^{\mathcal{C}}(f+t p)=\mathcal{R} \mathcal{T}^{\mathcal{C}}(f)
$$

para todo $t \in[0,1]$. Então $f+t p$ é $\mathcal{K}_{\lambda}^{\mathrm{D}_{4}}$-equivalente à $f$ para todo $t \in[0,1]$ :

Prova. A prova é semelhante à prova do Teorema 1.3 mencionado acima e está feita com detalhes em ([16], $\S 2^{*}$, pág. 172). A idéia básica é provar que o argumento da Proposição 2.7.1. é verdadeiro numa vizinhança de $t=\bar{t}$, para todo $\bar{t} \in[0,1]$. O resultado segue de um argumento de compacidade e conexidade.

Proposição 2.7.2. Sejam $f$ um problema de bifurcação $D_{4}$ e $p \in \overrightarrow{\mathcal{E}}_{(z, \lambda)}^{\mathrm{D}_{4}}$.

Se $p \in \mathcal{R} \mathcal{T} \mathcal{U}^{\mathcal{C}}(f)$ então $\mathcal{R} \mathcal{T}^{\mathcal{C}}(f+t p)=\mathcal{R} \mathcal{T}^{\mathcal{C}}(f)$, para todo $t \in[0,1]$.

Prova. Se $p \in \mathcal{R} \mathcal{T} \mathcal{U}^{\mathcal{C}}(f)$ então $p(z, \lambda)=\sum_{i=1}^{4} \alpha_{i}(u) u_{i} f+\sum_{j=2}^{8} \beta_{j}(u) T_{j} f$ com $\alpha_{i}, \beta_{j} \in \mathcal{E}_{u}$. Recorde que $u=\left(u_{1}, u_{2}, u_{3}, u_{4}\right)$ onde $u_{1}=N, u_{2}=\Delta, \quad u_{3}=\lambda_{1}, \quad$ e $u_{4}=\lambda_{2}^{2}$.

Os geradores de $\mathcal{R} \mathcal{T}^{\mathcal{C}}(f+t p)$ são somas dos geradores de $\mathcal{R} \mathcal{T}^{\mathcal{C}}(f)$ com os geradores 
$\operatorname{det} \mathcal{R} \mathcal{T}^{\mathcal{C}}(p)$. As inclusões $\mathcal{R} \mathcal{T}^{\mathcal{C}}(p) \subset \mathcal{R} \mathcal{T}^{\mathcal{C}}(f)+\mathcal{M}_{(z, \lambda)}^{\mathrm{D}_{4}} \mathcal{R} \mathcal{T}^{\mathcal{C}}(f)$ e $\mathcal{R} \mathcal{T}^{\mathcal{C}}(f+t p) \subset \mathcal{R} \mathcal{T}^{\mathcal{C}}(f)$ são imediatas.

Pelo Lema de Nakayama, $\mathcal{R} \mathcal{T}^{\mathcal{C}}(f) \subset \mathcal{R} \mathcal{T}^{\mathcal{C}}(f+t p)$ pois

$$
\mathcal{R} \mathcal{T}^{\mathcal{C}}(f) \subset \mathcal{R} \mathcal{T}^{\mathcal{C}}(f+t p)+\mathcal{M}_{(z, \lambda)}^{\mathrm{D}_{4}} \mathcal{R} \mathcal{T}^{\mathcal{C}}(f)
$$

Portanto, $\mathcal{R T}^{\mathcal{C}}(f+t p)=\mathcal{R} \mathcal{T}^{\mathcal{C}}(f)$, para todo $t \in[0,1]$.

Corolário 2.7.3. Sejam $f$ um problema de bifurcação $\mathrm{D}_{4}$ e $p$ qualquer germe em $\overrightarrow{\mathcal{E}}_{(z, \lambda)}^{\mathrm{D}_{4}}$. Se $p \in \mathcal{R} \mathcal{T} \mathcal{U}^{\mathcal{C}}(f)$ então $f+p$ é $\mathcal{K}_{\lambda}^{\mathrm{D}_{4}}$-equivalente à $f$.

Prova. Segue das Proposições 2.7.1. e 2.7.2. fazendo $t=1$.

Observação. 2.7.4. Em particular segue que $\mathcal{R} \mathcal{T} \mathcal{U}^{\mathcal{C}}(f) \subset \mathbf{P}(f)$ que é o conjunto de termos de ordem alta de $f$ com relação à $\mathcal{C}_{\lambda}^{\mathrm{D}_{4}}$-equivalência.

Além disso, denotando por $\mathcal{U C}_{\lambda}^{\mathrm{D}_{4}}$ o grupo $\mathcal{C}_{\lambda}^{\mathrm{D}_{4}}$-equivalências unipotentes, isto é,

$$
\mathcal{U C} \mathcal{C}_{\lambda}^{\mathrm{D}_{4}}=\left\{\left(S, \mathbf{I}_{2}, \mathbf{I}_{2}\right) \in \mathcal{C}_{\lambda}^{\mathrm{D}_{4}} \mid S^{\circ}=\mathbf{I}_{2}\right\}
$$

verificamos que $\mathcal{R} \mathcal{T} \mathcal{U}^{\mathcal{C}}(f)$ é $\mathcal{U} \mathcal{C}_{\lambda}^{\mathrm{D}_{4}}$-intrínsico, ou seja, se $p \in \mathcal{R} \mathcal{T} \mathcal{U}^{\mathcal{C}}(f)$ e $\left(S, \mathbf{I}_{2}, \mathbf{I}_{2}\right)$ for uma $\mathcal{U C}_{\lambda}^{\mathrm{D}_{4}}$-equivalência então $\left(S, \mathbf{I}_{2}, \mathbf{I}_{2}\right) \cdot p \in \mathcal{R} \mathcal{T} \mathcal{U}^{\mathcal{C}}(f)$.

Lema 2.7.5. Sejam $\phi, \psi: \mathbb{R}^{n} \rightarrow \mathbb{R}$ funçôes $C^{\infty}$ definidas numa vizinhança de zero e que se anulam na origem. Suponha que

(a) $\psi(y)=0$ implica $\phi(y)=0$

(b) $\nabla_{\phi^{\circ}} \neq 0, \nabla_{\psi^{\circ}} \neq 0$

onde $\nabla$ indica o gradiente. Então $a(y)=\frac{\phi(y)}{\psi(y)}$ é $C^{\infty}$ e não nula em alguma vizinhança da origem. Além disso, $s g a^{o}=s g\left\langle\nabla_{\phi^{\circ}}, \nabla_{\psi^{\circ}}\right\rangle$.

Prova. ([15], pág. 39).

\section{Proposição 2.7.6. (Pré-Forma Normal)}

Seja $f=[p, q, r, s]$ um problema de bifurcação $\mathrm{D}_{4}$. Se $q^{o} \neq 0$ então $f$ é $\mathcal{K}_{\lambda}^{\mathrm{D}_{4}}$-equivalente à pré-forma normal $h=[\hat{p}, \epsilon, \hat{r}, 0] \operatorname{com} \epsilon=s g q^{\circ}$ e $\hat{p}, \hat{r}$ dependendo de $N, \lambda_{1}$ e $u_{4}$ somente. Prova. $f=[p, q, r, s]$ é trivialmente $\mathcal{K}_{\lambda}^{\mathrm{D}_{4}}$-equivalente à $\bar{h}=[\bar{p}, \epsilon, \bar{r}, \bar{s}] \operatorname{com} \epsilon=s g q^{\circ}$, pois $q^{\circ} \neq 0$. 
De (2.12), considere o gerador de $\mathcal{R} \mathcal{T} \mathcal{U}^{\mathcal{C}}(\bar{h})$ dado por

$$
\bar{g}_{1}=\frac{1}{\epsilon\left(1-u_{4} \bar{s}^{2}\right)}\left(g_{2}-\epsilon u_{4} \bar{s} g_{1}\right)=\left[\frac{u_{4}(\bar{r}-\epsilon \bar{p} \bar{s})}{\epsilon\left(1-u_{4} \bar{s}^{2}\right)}, 0, \frac{\bar{p}-\epsilon u_{4} \bar{s}}{\epsilon\left(1-u_{4} \bar{s}^{2}\right)}, 1\right] \text {. }
$$

Usando a Observação $2.7 .4, \bar{g}_{1} \in \mathbf{P}(f)$ e assim $\bar{h}$ módulo $\bar{s} \bar{g}_{1}$ é $\mathcal{K}_{\lambda}^{\mathrm{D}_{4}}$-equivalente à $\tilde{h}=[\tilde{p}, \epsilon, \tilde{r}, 0]$, isto é, podemos fazer $\bar{s}=0$.

Agora, considere os seguintes geradores simplificados de $\mathcal{R} \mathcal{T} \mathcal{U}^{\mathcal{C}}(\tilde{h})$ :

$$
\begin{gathered}
\tilde{g}_{2}=(\tilde{p}-\epsilon N) g_{1}-\epsilon g_{4}-\tilde{r} g_{2}=\left[\Delta+\tilde{p}^{2}-2 \epsilon N \tilde{p}-u_{4} \tilde{r}^{2}, 0,0,0\right], \\
\tilde{g}_{3}=\epsilon(\tilde{p}-\epsilon N) g_{2}-g_{6}-\epsilon u_{4} \tilde{r} g_{1}=\left[0,0, \Delta+\hat{p}^{2}-2 \epsilon N \tilde{p}-u_{4} \tilde{r}^{2}, 0\right] .
\end{gathered}
$$

Seja $Y=\left(N, \lambda_{1}, u_{4}\right)$ e defina

$$
h(Y, \Delta)=\tilde{p}^{2}-2 \epsilon N \tilde{p}-u_{4} \tilde{r}^{2}
$$

e

$$
F(Y, \Delta)=\Delta+h(Y, \Delta)
$$

$F$ satisfaz $F^{\circ}=0$ e $F_{\Delta}^{\circ} \neq 0$.

Pelo Teorema da Funçâo Implícita existem vizinhanças $I$ de 0 em $\mathbb{R}$ e $V$ de $Y$ em $\mathbb{R}^{3}$ e a função $g: V \rightarrow I$ tais que, para todo $Y \in V, \Delta=g(Y)$ é a única solução de $F(Y, \Delta)=0$ e $g^{\circ}=0$.

Defina o seguinte homomorfismo

$$
\begin{aligned}
\phi: \mathcal{E}_{(Y, \Delta)} & \rightarrow \mathcal{E}_{Y} \\
Y & \mapsto Y \\
\Delta & \mapsto g(Y) .
\end{aligned}
$$

Obviamente, $\operatorname{Ker} \phi=<\Delta-g(Y)>$. Fazendo $G(Y, \Delta)=\Delta-g(Y)$, vemos que $F$ e $G$ satisfazem as condições do Lema 2.7.5. Logo, podemos escrever

$$
G(Y, \Delta)=a(Y, \Delta) F(Y, \Delta)
$$

isto é,

$$
<\Delta-g(Y)>C<\Delta+h(Y, \Delta)>
$$

Como $<\Delta+h(Y, \Delta)>\subset \operatorname{Ker} \phi$, segue que

$$
\operatorname{Ker} \phi=\langle\Delta+h(Y, \Delta)>.
$$


Pelo Teorema do Isomorfismo,

$$
\frac{\mathcal{E}_{Y, \Delta}}{\operatorname{Ker} \phi}=\frac{\mathcal{E}_{Y, \Delta}}{<\Delta+h(Y, \Delta)\rangle} \simeq I_{\mathrm{m}} \varphi \subset \mathcal{E}_{Y} .
$$

Usando esse isomorfismo e o Corolário 2.7.3.,

$\tilde{h}=[\tilde{p}, \epsilon, \tilde{r}, 0]$ módulo $\mathcal{E}_{Y, \Delta}\left\{\tilde{g}_{2}, \tilde{g}_{3}\right\}$ is $\mathcal{K}_{\lambda}^{\mathrm{D}_{4}}$-equivalente à

$$
\hat{h}=[\hat{p}, \epsilon, \hat{r}, 0] \text { com } \hat{p} \text { e } \hat{r} \text { dependendo de } N, \lambda_{1} \text { e } u_{4} \text { somente. }
$$

Portanto, a proposição se verifica.

\subsection{Classificação parcial dos problemas $D_{4}$}

Nesta subseç̧ão resolvemos o problema do reconhecimento para as primeiras degenerjcidades da forma normal genérica dada pelo Teorema 2.6.1. Para cada forma normal também achamos o desdobramento miniversal.

Mostraremos como classificar problemas de bifurcação $\mathrm{D}_{4}$ até codimensâo topológica 2, usando Formulação por Caminhos, no Capitulo 4.

Denotamos por $\Delta_{x, y}(p, q)$ a expressão $p_{x} q_{y}-p_{y} q_{x}$.

Teorema 2.8.1. (Teorema I)

$f=[p, q, r, s] \in \overrightarrow{\mathcal{E}}_{(z, \lambda)}^{\mathrm{D}_{4}}$ é de codimensão topológica 0 ou 1 se e somente se pertencer à seguinte lista. Além disso, $f$ será $\mathcal{K}_{\lambda}^{\mathrm{D}_{4}}$-equivalente à forma normal dada em cada caso se e somente se satisfizer os correspondentes conjuntos de condições de definição e condiçôes de não-degenericidade. Em todos os casos $p^{\circ}=0$.

O desdobramento miniversal é obtido adicionandi-se à forma normal o correspondente termo do desdobramento relacionado em cada caso.

\section{CASO $I_{0}$ (Teorema 2.6.1)}

Forma genérica normal: $\left[m N+\epsilon_{o} \lambda_{1}, \epsilon_{5}, 1,0\right]$.

Coeficientes: $\quad \epsilon_{o}=s g p_{\lambda_{1}}^{o}, \epsilon_{5}=s g q^{o}, m=\frac{p_{N}^{o}}{\left|q^{o}\right|}$.

Condições de não-degenericidade: $\quad p_{N}^{o} \neq 0, p_{N}^{o} \neq \pm q^{o}, p_{\lambda_{1}}^{o} \neq 0, r^{o} \neq 0$.

Termo do desdobramento: $\quad m[N, 0,0,0]$. 
Codim topológjca / $\mathrm{D}_{4}$-codim: $0 / 1$.

\section{CASO $\mathrm{I}_{1}$}

Condição de definição: $p_{\lambda_{1}}^{o}=0$.

Forma normal: $\quad\left[m N+\epsilon_{3} N \lambda_{1}+\epsilon_{4} \lambda_{1}^{2}, \epsilon_{5}, 1,0\right]$.

Coeficientes: $\epsilon_{4}=s g p_{\lambda_{1} \lambda_{1}}^{o}, \epsilon_{5}=s g q^{o}, \epsilon_{3}=\epsilon_{5} s g\left(p_{N \lambda_{1}}^{o} q^{o}-q_{\lambda_{1}}^{o} p_{N}^{o}\right), m=\frac{p_{N}^{o}}{\left|q^{o}\right|}$.

Condições de não-degenericidade: $q^{o} \neq 0, p_{N}^{o} \neq \pm q^{o}, p_{\lambda_{1} \lambda_{1}}^{o} \neq 0, r^{o} \neq 0$,

$$
p_{N_{1} \lambda_{1}}^{o} q^{o}-q_{\lambda_{1}}^{o} p_{N}^{o} \neq 0
$$

Termos do desdobramento: $m[N, 0,0,0]+\alpha[1,0,0,0]$.

Codim topológica / $\mathrm{D}_{4}$-codim: $1 / 2$.

\section{CASO $\mathrm{I}_{2}$}

Condição de definição: $r^{\circ}=0$.

Forma normal: $\left[m N+\epsilon_{o} \lambda_{1}+n N u_{4}, \epsilon_{5}, \lambda_{1}+\epsilon_{8} u_{4}, 0\right]$.

Coeficientes: $m=\frac{p_{N}^{o}}{\left|q^{\circ}\right|}, \epsilon_{o}=s g p_{\lambda_{1}}^{o}, \epsilon_{5}=s g q^{o}$,

$$
\begin{gathered}
\epsilon_{8}=\epsilon_{o} s g \frac{\left(p_{N}^{o^{2}}-q^{o^{2}}\right) \Delta_{\lambda_{1}, u_{4}}(p, r)}{p_{\lambda_{1}}^{o}\left(q^{o} s^{o}-r_{N}^{o} p_{N}^{o}\right)+r_{\lambda_{1}}^{o}\left(p_{N}^{o}-q^{o^{2}}\right)} \\
n=\frac{\epsilon_{5} p_{\lambda_{1}}^{o}\left(q^{o^{2}}-p_{N}^{o^{2}}\right)}{q^{o^{2}}\left[p_{\lambda_{1}}^{o}\left(s^{o} q^{o}-p_{N}^{o} r_{N}^{o}\right)+r_{\lambda_{1}}^{o}\left(p_{N}^{o^{2}}-q^{o^{2}}\right)\right]^{2}} \xi_{3} \text {, onde } \\
\xi_{3}=\left(p_{N}^{o}\left(p_{N}^{o^{2}}-q^{o^{2}}\right) \Delta_{\lambda_{1}, u_{4}}(p, q)+q^{o}\left(p_{N}^{o^{2}}-q^{o^{2}}\right)\left(p_{N \lambda_{1}}^{o} p_{u_{4}}^{o}-p_{N u_{4}}^{o} p_{\lambda_{1}}^{o}\right)\right. \\
\left.+p_{\lambda_{1}}^{o} q^{o} p_{N}^{o}\left(r_{N}^{o}+s^{o^{2}}\right)-r_{N}^{o} p_{\lambda_{1}}^{o} s^{o}\left(p_{N}^{o}+q^{o^{2}}\right)\right)
\end{gathered}
$$

Condições de não-degenericidade: $q^{\circ} \neq 0, p_{N}^{o} \neq \pm q^{\circ}, p_{\lambda_{1}}^{o} \neq 0, \xi_{3} \neq 0$,

$$
\begin{array}{r}
\Delta_{\lambda_{1}, u_{4}}(p, r) \neq 0, \\
\xi_{4}=p_{\lambda_{1}}^{o}\left(q^{o} s^{o}-p_{N}^{o} r_{N}^{o}\right)+r_{\lambda_{1}}^{o}\left(p_{N}^{o^{2}}-q^{o^{2}}\right) \neq 0 .
\end{array}
$$

Termos do desdobramento: $m[N, 0,0,0]+n\left[N u_{4}, 0,0,0\right]+\alpha[0,0,1,0]$.

Codim topológica / $\mathrm{D}_{4}$-codim: $1 / 3$. 


\section{CASO II}

Condição de definição: $p_{N}^{o}=0$.

Forma normal: $\quad\left[\epsilon_{o} \lambda_{1}+\epsilon_{2} N^{2}, \epsilon_{5}, 1,0\right]$.

Coeficientes: $\quad \epsilon_{o}=s g p_{\lambda_{1}}^{o}, \epsilon_{2}=s g p_{N N}^{o}, \epsilon_{5}=s g q^{o}$.

Condições de não-degenericidade: $\quad p_{\lambda_{1}}^{o} \neq 0, q^{o} \neq 0, p_{N N}^{o} \neq 0, r^{o} \neq 0$.

Termos do desdobramento: $\alpha[N, 0,0,0]$.

Codim topológica / $\mathrm{D}_{4}$-codim: $1 / 1$.

\section{CASO III}

Condição de definição: $p_{N}^{\circ}=-q^{\circ}$.

Forma normal: $\quad\left[\epsilon_{o} \lambda_{1}-\epsilon_{5} N, \epsilon_{5}, 1+\epsilon_{12} N, 0\right]$.

Coeficientes: $\epsilon_{0}=s g p_{\lambda_{1}}^{\circ}, \epsilon_{5}=s g q^{\circ}, \epsilon_{12}=s g \xi_{1}$, onde

$$
\xi_{1}=-\frac{1}{2 q^{o}}\left(p_{N N}^{o}+2 p_{\Delta}^{o}+2 q_{N}^{o}\right)-\frac{1}{p_{\lambda_{1}}^{o}}\left(p_{N \lambda_{1}}^{o}+q_{\lambda_{1}}^{o}\right)+\frac{1}{r^{o}}\left(s^{o}+r_{N}^{o}\right)
$$

Condiçôes de nâo-degenericidade: $q^{\circ} \neq 0, p_{\lambda_{3}}^{\circ} \neq 0, r^{\circ} \neq 0, \xi_{1} \neq 0$.

Termos do desdobramento: $\alpha[N, 0,0,0]$.

Codim topológica / $\mathrm{D}_{4}$-codim: $1 / 1$.

\section{CASO IV}

Condição de definição: $\quad p_{N}^{o}=q^{\circ}$.

Forma normal: $\left[\epsilon_{1} N+\epsilon_{o} \lambda_{1}+\epsilon_{2} N^{2}, \epsilon_{1}+m_{2} \lambda_{1}, 1,0\right]$.

Coeficientes: $\epsilon_{1}=s g p_{N}^{\circ}, \epsilon_{o}=s g p_{\lambda_{1}}^{o}, \epsilon_{2}=s g\left(p_{N N}^{o}+2 p_{\Delta}^{o}-2 q_{N}^{o}\right)$,

$$
\begin{array}{r}
m_{2}=\frac{2\left|q^{o}\right|}{r^{o}\left|p_{\lambda_{1}}^{o}\right|\left|p_{N N}^{o}+2 p_{\Delta}^{o}-2 q_{N}^{o}\right|} \xi_{2}, \text { onde } \\
\xi_{2}=p_{\lambda_{1}}^{o}\left(r_{N}^{o}-s^{o}\right)+r^{o}\left(q_{\lambda_{1}}^{o}-p_{N \lambda_{1}}^{o}\right) .
\end{array}
$$


Condiçôes de nâo-degenericidade: $p_{N}^{o} \neq 0, r^{o} \neq 0, p_{\lambda_{1}}^{o} \neq 0, \xi_{2} \neq 0$

$$
p_{N N}^{o}+2 p_{\Delta}^{o}-2 q_{N}^{o} \neq 0
$$

Termos do desdobramento: $m_{2}\left[0, \lambda_{1}, 0,0\right]+\alpha[N, 0,0,0]$.

Codim topológica / $\mathrm{D}_{4}$-codim: $1 / 2$.

\section{CASO V}

Condição de definição: $\quad q^{\circ}=0$.

Forma normal: $\quad\left[\epsilon_{1} N+\epsilon_{0} \lambda_{1}, \epsilon_{6} \Delta+m_{1} \lambda_{1}, 1,0\right]$.

Coeficientes: $\epsilon_{o}=s g p_{\lambda_{1}}^{o}, \epsilon_{1}=s g p_{N}^{o}, \epsilon_{6}=\epsilon_{1} s g \Delta_{N, \Delta}(p, q)$,

$$
m_{1}=\frac{\epsilon_{1} \Delta_{N, \lambda_{3}}(p, q)}{\left|p_{\lambda_{1}}^{o}\right|\left|\Delta_{N, \Delta}(p, q)\right|^{\frac{1}{2}}} .
$$

Condições de não-degenericidade: $\quad p_{N}^{o} \neq 0, p_{\lambda_{1}}^{o} \neq 0, r^{o} \neq 0$,

$$
\Delta_{N, \Delta}(p, q) \neq 0, \Delta_{N, \lambda_{1}}(p, q) \neq 0 .
$$

Termos do desdobramento: $m_{1}\left[0, \lambda_{1}, 0,0\right]+\alpha[0,1,0,0]$.

Codim topológica / $\mathrm{D}_{4}$-codim: $1 / 2$.

Prova. Seguimos o método clássico descrito na prova do Teorema 2.6.1. Omitiremos os cálculos.

Para cada fórmula normal $g$, encontramos um submódulo intrínsico $R^{\prime}$ contido na parte intrínsica do $\mathrm{D}_{4}$-espaço tangente unipotente de $g$ e, pela Proposição 1.10.3.1, $R^{\prime}$ está contido no conjunto de termos de ordem alta de $g$. A seguir fazemos mudanças de coordenadas módulo $R^{\prime}$ para um problema de bifurcação $\mathrm{D}_{4}, f=[p, q, r, s]$, estabelecendo que $f$ será $\mathcal{K}_{\lambda}^{D_{4}}$-equivalente a $g$ se e somente se $f$ satisfizer as condições de definição e não-degenericidade escritas em cada caso.

Aplicamos o Corolário 1.7.4 para obter informação sobre os desdobramentos miniversais.

Para cada caso, escrevemos os dados básicos. Para simplificar, denotamos $\mathcal{M}_{u}$ por $\mathcal{M}$ e $\mathcal{E}_{u}$ por $\mathcal{E}$. Recordamos, novamente, que $u=\left(u_{1}, u_{2}, u_{3}, u_{4}\right)$ onde $u_{1}=N, u_{2}=\Delta$, $u_{3}=\lambda_{1}$ e $u_{4}=\lambda_{2}^{2}$. 
Caso $\mathbf{I}_{0}$ foi provado no Teorema 2.6.1.

Para o Caso $\mathbf{I}_{1}$, seja $g=\left[m N+\epsilon_{3} N \lambda_{1}+\epsilon_{4} \lambda_{1}^{2}, \epsilon_{5}, 1,0\right]$ onde $\epsilon_{3}^{2}=\epsilon_{4}^{2}=\epsilon_{5}^{2}=1 \mathrm{e}$ $m \neq \pm 1,0$. E assim,

$$
\begin{aligned}
\mathcal{T} \mathcal{U}^{\mathrm{D}_{4}}(g)=\left[\mathcal{M}^{3}+<N^{2}, \Delta, u_{4}>, \mathcal{M}^{2}+<N, \Delta, u_{4}>, \mathcal{M}, \mathcal{E}\right] & \\
& +<\left[m N \lambda_{1}, \epsilon_{5} \lambda_{1}, 0,0\right]>.
\end{aligned}
$$

$\mathcal{T}_{e}^{\mathrm{D}_{4}}(g)=\mathcal{T} \mathcal{U}^{\mathrm{D}_{4}}(g)+\mathbb{R}\left\{\left[m N, \epsilon_{5}, 0,0\right],\left[\lambda_{1}^{2}, 0,0,0\right],\left[\epsilon_{3} N+2 \epsilon_{4} \lambda_{1}, 0,0,0\right]\right.$,

$$
\left.\left[N \lambda_{1}, 0,0,0\right],[0,0,1,0]\right\} \text {. }
$$

Como consequência, $g$ tem $\mathrm{D}_{4}$-codimensão 2 e codimensão topológica 1 pois $m$ é um parâmetro modal e $[1,0,0,0]$ é, também, um termo de desdobramento.

$$
\text { Agora, seja } R^{\prime}=\left[\mathcal{M}^{3}+<N^{2}, \Delta, u_{4}>, \mathcal{M}^{2}+<N, \Delta, u_{4}>, \mathcal{M}, \mathcal{E}\right] \text {. }
$$

Pela Proposição 2.5.1, $R^{\prime}$ é intrínsico.

Para o Caso $\mathbf{I}_{2}$, seja $g=\left[m N+\epsilon_{o} \lambda_{1}+n N u_{4}, \epsilon_{5}, \lambda_{1}+\epsilon_{8} u_{4}, 0\right]$ onde $m \neq \pm 1,0$, $\epsilon_{o}^{2}=\epsilon_{5}^{2}=\epsilon_{8}^{2}=1$ e $n \neq 0$.

$$
\begin{gathered}
\mathcal{T} \mathcal{U}^{\mathrm{D}_{4}}(g)=\left[\mathcal{M}^{3}+<N^{2}, N \lambda_{1}, \Delta>+\mathcal{M}_{\left(\lambda_{1}, u_{4}\right)}^{2}, \mathcal{M}^{2}+<N, \Delta, \lambda_{1}>, \mathcal{M}^{2}+<\Delta>, \mathcal{M}\right] \\
+<\left[m N u_{4}, \epsilon_{5} u_{4}, 0,0\right],\left[0,0, N, \epsilon_{5} m\right],\left[0,0, \epsilon_{0} \lambda_{1}, \epsilon_{5}\left(1-m^{2}\right)\right],\left[\epsilon_{o} u_{4}, 0, u_{4}, 0\right]>. \\
\mathcal{T}_{e}^{\mathrm{D}_{4}}(g)=\mathcal{T} \mathcal{U}^{\mathrm{D}_{4}}(g)+\mathbb{R}\left\{\left[m N+n N u_{4}, \epsilon_{5}, 0,0\right],\left[\epsilon_{o}, 0,1,0\right],\left[\epsilon_{o} \lambda_{1}, 0, \lambda_{1}, 0\right],\right. \\
\left.\left[2 n N u_{4}, 0, \lambda_{1}, 0\right],\left[0,0, u_{4}, 0\right]\right\} .
\end{gathered}
$$

Os termos de desdobramento são $[N, 0,0,0],\left[N u_{4}, 0,0,0\right]$ e $[0,0,1,0]$. Segue que $g$ tem $\mathrm{D}_{4}$-codimensão 3 e codimensão topológica 1 , pois $m, n$ são parâmetros modais.

$$
\text { Seja } R^{\prime}=\left[\mathcal{M}^{3}+\mathcal{M}<\Delta>+\mathcal{M}_{\left(\lambda_{1}, u_{4}\right)}^{2}, \mathcal{M}^{2}, \mathcal{M}^{2}, \mathcal{M}\right]
$$

A Proposição 2.5.1 não se aplica aqui. Para mostrar que $R^{\prime}$ é intrínsico temos que usar a definição, conforme o que foi descrito na Observação 2.5.2. 
Para isso, sejam

$$
J_{1}=\mathcal{M}^{3}+\mathcal{M}<\Delta>+\mathcal{M}_{\left(\lambda_{1}, u_{4}\right)}^{2}, J_{2}=\mathcal{M}^{2}, J_{3}=\mathcal{M}, h=[p, q, r, s] \in R^{\prime}
$$

e $\epsilon=(T, X, \Lambda)$ uma $\mathcal{K}_{\lambda}^{D_{4}}$-equivalência.

Primeiro mostramos que $h^{\prime}=(I, X, \Lambda) \cdot h \in R^{\prime}$ e, para isso, decompomos

$$
h=h_{1}+h_{2}+h_{3}+h_{4} \operatorname{com} h_{1} \in J_{1}, h_{2}, h_{3} \in J_{2} \text { e } h_{4} \in J_{3}
$$

(i) Do Lema 2.4.1,

$$
\begin{aligned}
h_{1} \circ(X, \Lambda)(u) & =\tilde{p}(u)[a, b, c, d] \\
& =p\left(\tilde{N}_{1}, \tilde{\Delta}_{1}, \tilde{\lambda}_{1}, \tilde{u}_{4}\right)[a, b, c, d]+\delta \lambda_{2} p_{1}(u)[a, b, c, d]
\end{aligned}
$$

$$
+\Delta u_{4} p_{2}(u)[a, b, c, d]
$$

Por (2.5),

$$
\mathcal{M}_{\left(\tilde{N}_{1}, \tilde{\Delta}_{1}, \tilde{\lambda}_{1}, \tilde{u}_{4}\right)}^{3}+\mathcal{M}_{\left(\tilde{N}_{1}, \tilde{\Delta}_{1}, \tilde{\lambda}_{1}, \tilde{u}_{4}\right)}<\hat{\Delta}_{1}>+\mathcal{M}_{\left(\tilde{\lambda}_{1}, \tilde{u}_{4}\right)} \in J_{1}
$$

e como $p \in J_{1}$, segue que $p\left(\tilde{N}_{1}, \tilde{\Delta}_{1}, \tilde{\lambda}_{1}, \tilde{u}_{4}\right)[a, b, c, d] \in R^{\prime}$.

É imediato que $\Delta u_{4} p_{2}(u)[a, b, c, d] \in R^{\prime}$. Para verificar que

$$
\delta \lambda_{2} p_{1}(u)[a, b, c, d]=p_{1}(u)\left[\Delta u_{4} d, u_{4} c, \Delta b, a\right] \in R^{\prime}
$$

é suficiente mostrar que $p_{1} a \in \mathcal{M}$, o que é verdadeiro olhando para a ordem dos monômios de $p \in J_{1}$.

Logo, $h_{1} \circ(X, \Lambda) \in R^{\prime}$.

(ii) $h_{2} \circ(X, \Lambda)(u)=\tilde{q}(u)\left[b \Delta D_{1}+c u_{4} D_{2}, a D_{1}+d u_{4} D_{2}, d \Delta D_{1}+a D_{2}, c D_{1}+b D_{2}\right]$.

Usando o Lema 2.4.1 e (2.5) temos que $h_{2} \circ(X, \Lambda)(u) \in R^{\prime}$ se $\Delta a D_{1} q_{1} \in \mathcal{M}^{2}$. Uma inspeção dos monômios de $\tilde{q}(u)$ mostra que $q_{1} \in \mathcal{M}$ e portanto $h_{2} \circ(X, \Lambda) \in R^{\prime}$.

(iii) $h_{3} \circ(X, \Lambda)(u)=\tilde{r}(u)\left[u_{4} c \Lambda_{2}, u_{4} d \Lambda_{2}, a \Lambda_{2}, b \Lambda_{2}\right] \in R^{\prime}$ se $u_{4} a \Lambda_{2} r_{1} \in \mathcal{M}^{2}$ o que é verdadeiro, pois $r_{1} \in \mathcal{M}$. 
(iv) $h_{4} \circ(X, \Lambda)(u)=\hat{s}(u)\left[D_{1} \Lambda_{2} \Delta u_{4} d+D_{2} \Lambda_{2} u_{4} a, D_{1} \Lambda_{2} u_{4} c+D_{2} \Lambda_{2} u_{4} b\right.$

$$
\left.D_{1} \Lambda_{2} \Delta b+D_{2} \Lambda_{2} u_{4} c, D_{1} \Lambda_{2} a+D_{2} \Lambda_{2} u_{4} d\right] \text {. }
$$

É direto que $h_{4} \circ(X, \Lambda) \in J_{3}$. Segue que $h^{\prime}=h \circ(X, \Lambda) \in R^{\prime}$ e para verificar que $h^{\prime \prime}=(T, l, l) \circ h^{\prime} \in R^{\prime}$, usamos as relaçôes $(2.8)$.

Portanto, $R^{\prime}$ é intrínsico.

Para o Caso II, seja $g=\left[\epsilon_{o} \lambda_{1}+\epsilon_{2} N^{2}, \epsilon_{5}, 1,0\right]$ onde $\epsilon_{\mathrm{o}}^{2}=\epsilon_{2}^{2}=\epsilon_{5}^{2}=1$.

$$
\begin{gathered}
\mathcal{T \mathcal { U } ^ { \mathrm { D } _ { 4 } }}(g)=\left[\mathcal{M}^{3}+<N \lambda_{1}, \lambda_{1}^{2}, \Delta, u_{4}>, \mathcal{M}, \mathcal{M}, \mathcal{E}\right] . \\
\mathcal{T}_{e}^{\mathrm{D}_{4}}(g)=\mathcal{T} \mathcal{U}^{\mathrm{D}_{4}}(g)+\mathbb{R}\left\{\left[N^{2}, 0,0,0\right],[1,0,0,0],\left[\lambda_{1}, 0,0,0\right],[0,1,0,0],[0,0,1,0]\right\}
\end{gathered}
$$

O termo de desdobramento é $[N, 0,0,0]$ e por definição $g$ tem $\mathrm{D}_{4}$-codimensão e codimensão topológica iguais a 1 .

Note que neste caso, $\mathcal{T} \mathcal{U}^{\mathrm{D}_{4}}(g)$ é intrínsico pela Proposição 2.5.1.

Para Caso III, seja $g=\left[\epsilon_{o} \lambda_{1}-\epsilon_{5} N, \epsilon_{5}, 1+\epsilon_{12} N, 0\right]$ onde $\epsilon_{o}^{2}=\epsilon_{5}^{2}=\epsilon_{12}^{2}=1$.

$$
\begin{array}{r}
\mathcal{T} \mathcal{U}^{\mathrm{D}_{4}}(g)=\left[\mathcal{M}^{3}+<\Delta^{2}, u_{4}, \lambda_{1}^{2}, N \Delta>, \mathcal{M}^{2}+<\Delta, u_{4}>, \mathcal{M}^{2}+<\Delta, \lambda_{1}, u_{4}>, \mathcal{M}\right] \\
+<\left[N^{2},-N, 0,0\right],\left[\epsilon_{0} N \lambda_{1}, 0, N, 0\right],[\Delta,-N, 0,0],\left[-N \lambda_{1}, \lambda_{1}, 0,0\right] \\
{\left[\epsilon_{0} N \lambda_{1}-\epsilon_{5} N^{2}, 0,0,0\right],[0,0,-N, 1]>.} \\
\mathcal{T}_{e}^{\mathrm{D}_{4}}(g)=\mathcal{T} \mathcal{U}^{\mathrm{D}_{4}}(g)+\mathbb{R}\left\{[-N, 1,0,0],[1,0,0,0],\left[\lambda_{1}, 0,0,0\right],[0,0, N, 0],[0,0,1,0]\right\} .
\end{array}
$$

Consideramos $[N, 0,0,0]$ como termo de desdobramento e $g$ têm ambas as codimensões iguais a 1 . Seja

$$
R^{\prime}=\left[\mathcal{M}^{3}+\mathcal{M}<\Delta>+<\lambda_{1}^{2}, u_{4}>, \mathcal{M}^{2}+<\Delta, u_{4}>, \mathcal{M}^{2}+<\Delta, \lambda_{1}, u_{4}>, \mathcal{M}\right]
$$

Para verificar que $R^{\prime}$ é intrínsico, usamos a Observação 2.5.2. Todas as relações são imediatas exceto a de que $p_{1} \in \mathcal{M}$, o que se verifica analisando a ordem dos monômios de

$$
p \in \mathcal{M}^{3}+\mathcal{M}<\Delta>+<\lambda_{1}^{2}, u_{4}>
$$


Para o Caso IV, seja $g=\left[\epsilon_{1} N+\epsilon_{o} \lambda_{1}+\epsilon_{2} N^{2}, \epsilon_{1}+m_{2} \lambda_{1}, 1,0\right]$ onde $\epsilon_{o}^{2}=\epsilon_{1}^{2}=\epsilon_{2}^{2}=1 \mathrm{e}$ $m_{2} \neq 0$.

$$
\begin{aligned}
& \mathcal{T} \mathcal{U}^{D_{4}}(g)=\left[\mathcal{M}^{3}+<u_{4}, \lambda_{1}^{2}, \Delta^{2}, \Delta \lambda_{1}, \Delta N>, \mathcal{M}^{2}+<\Delta, u_{4}>, \mathcal{M}^{2}+<\Delta, \lambda_{3}, u_{4}>, \mathcal{M}\right] \\
& +<\left[N \lambda_{1}, \lambda_{1}, 0,0\right],\left[N^{2}, N, 0,0,\right],\left[N^{2}-\Delta, 0,0,0\right],\left[0, \epsilon_{0} \lambda_{1}, 0,1\right],[0,0, N, 1]>. \\
& \mathcal{T}_{e}^{\mathrm{D}_{4}}(g)=\mathcal{T} \mathcal{U}^{\mathrm{D}_{4}}(g)+\mathbb{R}\left\{\left[\epsilon_{1} N+\epsilon_{2} N^{2}, \epsilon_{1}, 0,0\right],\left[\epsilon_{2} N^{2}-\epsilon_{0} \lambda_{1}, 0,0,0\right],\right. \\
& \left.\left[\epsilon_{o}, m_{2}, 0,0\right],\left[\epsilon_{o} \lambda_{1}, m_{2} \lambda_{1}, 0,0\right],[0,0,1,0]\right\} \text {. }
\end{aligned}
$$

Escolhemos $[N, 0,0,0]$ e $\left[0, \lambda_{1}, 0,0\right]$ como termos do desdobramento e assim $g$ tem $\mathrm{D}_{4}$-codimensão 2 e codimensão topológica 1 pois $m_{2}$ é parâmetro modal.

Seja $R^{\prime}=\left[\mathcal{M}^{3}+\mathcal{M}<\Delta>+<\lambda_{1}^{2}, u_{4}>, \mathcal{M}^{2}+<\Delta, u_{4}>, \mathcal{M}^{2}+<\Delta, \lambda_{1}, u_{4}>, \mathcal{M}\right]$.

$R^{\prime}$ é intrínsico. (Veja Caso III).

Para o Caso V, seja $g=\left[\epsilon_{1} N+\epsilon_{0} \lambda_{1}, \epsilon_{6} \Delta+m_{1} \lambda_{1}, 1,0\right]$ onde $\epsilon_{o}^{2}=\epsilon_{1}^{2}=\epsilon_{6}^{2}=1$ e $m_{1} \neq 0$.

$$
\begin{array}{r}
\mathcal{T} \mathcal{U}^{\mathrm{D}_{4}}(g)=\left[\mathcal{M}^{2}+<\Delta, u_{4}>, \mathcal{M}^{2}+<\left[0, \epsilon_{1} N+\epsilon_{o} \lambda_{1}, 0,0\right], u_{4}>, \mathcal{M}, \mathcal{E}\right] . \\
\mathcal{T}_{\epsilon}^{\mathrm{D}_{4}}(g)=\mathcal{T} \mathcal{U}^{\mathrm{D}_{4}}(g)+\mathbb{R}\left\{\left[\epsilon_{1} N, \epsilon_{6} \Delta, 0,0\right],\left[0,2 \epsilon_{6} \Delta+m_{1} \lambda_{1}, 0,0\right],\left[\epsilon_{o}, m_{1}, 0,0\right],\right. \\
\left.\left[\epsilon_{o} \lambda_{1}, m_{1} \lambda_{1} 0,0\right],[0,0,1,0]\right\} .
\end{array}
$$

Sejam $\left[0, \lambda_{1}, 0,0\right]$ e $[0,1,0,0]$ os termos de desdobramento. Segue que $g$ tem $\mathrm{D}_{4^{-}}$ codimensão 2 e codimensão topológica 1 pois $m_{1}$ é parâmetro modal.

$$
\text { Seja } R^{\prime}=\left[\mathcal{M}^{2}+<u_{4}>, \mathcal{M}^{2}+<u_{4}>, \mathcal{M}, \mathcal{E}\right]
$$

$R^{\prime} \subset \mathcal{T} \mathcal{U}^{\mathrm{D}_{4}}(g)$ e para mostrar que ele é intrínsico sejam

$$
J_{1}=\mathcal{M}^{2}+\left\langle u_{4}>, J_{2}=\mathcal{M}, J_{3}=\mathcal{E}, e=(T, X, \Lambda) \text { uma } \mathcal{K}_{\lambda}^{\mathrm{D}_{4}}\right. \text {-equivalência e }
$$
$h=[p, q, r, s] \in R^{\prime}$.

Primeiro mostramos que $h^{\prime}=(I, X, \Lambda) \cdot h \in R^{\prime}$. Escrevemos

$$
\begin{aligned}
& h=h_{1}+h_{2}+h_{3}+h_{4} \operatorname{com} h_{1}=[p, 0,0,0] \in J_{1}, h_{2}=[0, q, 0,0] \in J_{1} \\
& h_{3}=[0,0, r, 0] \in J_{2}, \text { e } h_{4}=[0,0,0, s] \in J_{3} .
\end{aligned}
$$


(i) Do Lema 2.4.1,

$$
\begin{aligned}
h_{1} \circ(X, \Lambda)(u)=\tilde{p}(u)[a, b, c, d]=p\left(\hat{N}_{1}, \hat{\Delta}_{1}, \hat{\lambda}_{1}, \hat{u}_{4}\right)[a, b, c, d]+ & \delta \lambda_{2} p_{1}(u)[a, b, c, d] \\
& +\Delta u_{4} p_{2}(u)[a, b, c, d] .
\end{aligned}
$$

Usando $(2.5)$ e que $p \in J_{1}$, verificamos que $p\left(\tilde{N}_{1}, \tilde{\Delta}_{1}, \tilde{\lambda}_{1}, \tilde{u}_{4}\right)[a, b, c, d] \in R^{\prime}$ pois $\mathcal{M}^{2}{ }_{\left(\tilde{N}_{1}, \bar{\Delta}_{3}, \tilde{\lambda}_{1}, \tilde{u}_{4}\right)}+<\tilde{u}_{4}>\subset J_{1}$.

É direto que $\Delta u_{4} p_{2}(u)[a, b, c, d]$ e $\delta \lambda_{2} p_{1}(u)[a, b, c, d]$ pertencem à $R^{\prime}$.

Consequentemente, $h_{1} \circ(X, \Lambda) \in R^{\prime}$.

(ii) $h_{2} \circ(X, \Lambda)(u)=\tilde{q}(u)\left[b \Delta D_{1}+c u_{4} D_{2}, a D_{1}+d u_{4} D_{2}, d \Delta D_{1}+a D_{2}, c D_{1}+b D_{2}\right]$.

Pelo Lema 2.4.1, é suficiente mostrar que $q\left(\tilde{N}_{1}, \tilde{\Delta}_{1}, \tilde{\lambda}_{1}, \tilde{u}_{4}\right) a D_{1} \in J_{1}$. Usando que $q \in J_{1}$ $\mathrm{e}(2.5), \operatorname{temos} h_{2} \circ(X, \Lambda) \in R^{\prime}$.

(iii) $h_{3} \circ(X, \Lambda)(u)=\tilde{r}(u)\left[u_{4} c \Lambda_{2}, u_{4} d \Lambda_{2}, a \Lambda_{2}, b \Lambda_{2}\right]$.

Analogamente a (ii), ' $h_{3} \circ(X, \Lambda) \in R^{\prime}$ se e somente se $r\left(\tilde{N}_{1}, \tilde{\Delta}_{1}, \tilde{\lambda}_{1}, \tilde{u}_{4}\right)$ a $\Lambda_{2} \in \mathcal{M}$ o que se verifica por (2.5) e usando o fato de que $r \in \mathcal{M}$.

(iv) $h_{4} \circ(X, \Lambda)(u)=\tilde{s}(u)\left[D_{1} \Lambda_{2} \Delta u_{4} d+D_{2} \Lambda_{2} u_{4} a, D_{1} \Lambda_{2} u_{4} c+D_{2} \Lambda_{2} u_{4} b\right.$

$$
\left.D_{1} \Lambda_{2} \Delta b+D_{2} \Lambda_{2} u_{4} c, D_{1} \Lambda_{2} a+D_{2} \Lambda_{2} u_{4} d\right]
$$

É imediato que $h_{4} \circ(X, \Lambda) \in R^{\prime}$.

Logo, $h^{\prime}=h \circ(X, \Lambda) \in R^{\prime}$ pois $h_{i} \circ(X, \Lambda) \in J_{i}, 1 \leq i \leq 3$.

Seja $h^{\prime \prime}=(T, I, I) \cdot h^{\prime}=\left[p^{\prime \prime}, q^{\prime \prime}, r^{\prime \prime}, s^{\prime \prime}\right]$ onde $h^{\prime} \in R$. Usando $(2.8)$, segue que $h^{\prime \prime} \in R^{\prime} \mathrm{e}$ portanto, $e=(T, X, \Lambda) \cdot h \in R^{\prime}$, estabelecendo que $R^{\prime}$ é intrínsico. 


\section{Capítulo 3}

\section{Formulação por caminhos e o problema $\mathrm{D}_{4}$}

Neste capítulo, usamos a formulação por caminhos no espaço dos parâmetros para classificar problemas de bifurcaçao equivariantes, em particular, os problemas $\mathrm{D}_{4}$-equivariantes apresentados no capítulo 2 .

A idéia básica foi sugerida por Golubitsky e Schaeffer em [15] para problemas de bifurcação sem simetria nos parâmetros. Esta idéia, já presente nos primeiros artigos de Mather e Martinet para Teoria de Singularidades, tem sido estendida e aplicada (cf. $[23],[11],[4])$. A principal referência é [23].

Na secção (3.1) desenvolvemos a teoria de formulação por caminhos para problemas de bifurcação $\Gamma$ - equivariantes segundo a ação de $\Gamma$ descrita no capítulo 1 . A idéia básica é considerar problemas $\Sigma$ - equivariantes onde $\Sigma$ é o subgrupo de isotropia dos parâmetros de bifurcação e aplicar a teoria usual inicialmente proposta por [15] para essa classe de problemas. A seguir definimos uma ação no espaço dos caminhos de tal forma que o pull-back do $\Sigma$-desdobramento do centro organizador por um caminho desse espaço se torne um problema $\Gamma$-equivariante.

Na secção (3.2) definimos o espaço tangente e o espaço tangente unipotente a um caminho $\Gamma$-equivariante. $O$ resultado central estabelece um isomorfismo entre o espaço normal a um caminho $\Gamma$-equivariante e o espaço normal ao pull-back do $\Sigma$-desdobramento do centro organizador por esse caminho. A teoria aqui desenvolvida trata de uma caracterização algébrica dos espaços tangentes aos caminhos $\Gamma$-equivariantes. Fica em aberto 
uma caracterização geométrica usando a $\mathcal{K}_{V}$-equivalência definida por Damon em [11] e também apresentada em [23] e [4].

$\mathrm{Na}$ seção (3.3) mostramos que dado um problema de bifurcação $\mathrm{D}_{4}$ - equivariante, digamos $f$, existe um caminho $\mathrm{D}_{4^{-}}$equivariante que torna o pull-back do $\Sigma$ - desdobramento do centro organizador de $f$ por esse caminho um problema de bifurcação $\mathcal{K}_{\lambda}^{\mathrm{D}_{4}}$-equivalente a $f$. Provamos, também, o teorema equivalente para os respectivos desdobramentos.

\section{1 $\Sigma$-teoria para a formulação por caminhos}

Recorde do capítulo 1 que $\Gamma$ é um grupo de Lie compacto agindo no $\mathbb{R}^{m}$ e diagonalmente no $\mathbb{R}^{n+\ell}$ via as representações diagonais:

$$
\rho_{n}: \Gamma \rightarrow O(n), \quad \rho_{\ell}: \Gamma \rightarrow O(\ell) \quad \text { e } \quad \rho_{m}: \Gamma \rightarrow O(m)
$$

Definimos $\Sigma$ como o subgrupo de $\Gamma$ que deixa os parâmetros de bifurcação $\lambda$ fixados, ou seja, $\Sigma=\operatorname{Ker} \rho_{\ell}$ e, portanto, é um subgrupo normal de $\Gamma$.

Seguindo nossa notação, sejam

$$
\mathcal{E}_{x}^{\Sigma}=\left\{g:\left(\mathbb{R}^{n}, 0\right) \rightarrow \mathbb{R} \mid g\left(\sigma_{n} x\right)=g(x), \forall \sigma \in \Sigma\right\}
$$

o conjunto dos germes $\Sigma$-invariantes,

$$
\overrightarrow{\mathcal{E}}_{x}^{\Sigma}=\left\{h:\left(\mathbb{R}^{n}, 0\right) \rightarrow \mathbb{R}^{m} \mid h\left(\sigma_{n} x\right)=\sigma_{m} h(x), \forall \sigma \in \Sigma\right\}
$$

o conjunto dos germes $\Sigma$-equivariantes,

$$
\vec{\Theta}_{x}^{\Sigma}=\left\{X:\left(\mathbb{R}^{n}, 0\right) \rightarrow \mathbb{R}^{n} \mid X\left(\sigma_{n} x\right)=\sigma_{n} X(x), \forall \sigma \in \Sigma\right\}
$$

o conjunto dos campos de vetores $\Sigma$-equivariantes e

$$
\mathbf{M}_{x}^{\Sigma}=\left\{T:\left(\mathbb{R}^{n}, 0\right) \rightarrow M_{m}(\mathbb{R}) \mid T\left(\sigma_{n} x\right) \sigma_{m}=\sigma_{m} T(x), \forall \sigma \in \Sigma\right\}
$$

o conjunto das matrizes que comutam com $\Sigma$.

$$
\text { Para } h \in \overrightarrow{\mathcal{E}}_{x}^{\Sigma},
$$

- $\mathcal{T}_{e}^{\Sigma}(h)=\left\{T h+\left(D_{x} h\right) X \mid T \in \mathbf{M}_{x}^{\Sigma}, X \in \vec{\Theta}_{x}^{\Sigma}\right\}$ é o $\Sigma$-espaço tangente estendido de $h$.

- $\mathcal{N}_{e}^{\Sigma}(h)=\frac{\overrightarrow{\mathcal{E}}_{x}^{\Sigma}}{\mathcal{T}_{e}^{\Sigma}\left(f_{0}\right)}$ é o $\Sigma$-espaço normal de $h$. 
- $\operatorname{cod}^{\Sigma}(h)=\operatorname{dim}_{\mathbb{R}} \mathcal{N}_{\varepsilon}^{\Sigma}(h)$.

- Se $\operatorname{dim}_{\mathbf{R}} \mathcal{N}_{e}^{\Sigma}(h)=r \in \mathbb{N}$, e $\left\{h_{i}\right\}_{i=1}^{r} \subset \overrightarrow{\mathcal{E}}_{x}^{\Sigma}$ for uma base para $\mathcal{N}_{e}^{\Sigma}(h)$ então $F=h+\sum_{i=1}^{r} \alpha_{i} h_{i}$ será o $\Sigma$-desdobramento miniversal de $h$.

Para as provas, veja ([15]).

Seja $f \in \overrightarrow{\mathcal{E}}_{(x, \lambda)}^{\Gamma}$. O centro organizador de $f$ é definido por

$$
f_{0}(x)=f(x, 0)
$$

Obviamente, $f_{0}$ é não somente $\Sigma$-equivariante, como também, é $\Gamma$-equivariante.

Supomos $f_{0}$ um germe de codimenção finita,ou seja, $f_{0}$ satisfaz a seguinte hipótese:

$$
\operatorname{cod}^{\Sigma}\left(f_{0}\right)=r
$$

Uma observação importante é que a $\sum$-teoria para a formulação de caminhos é válida se (H0) for satisfeita. Em geral, não é verdade que se $\operatorname{cod}^{\Gamma}(f)$ for finita então $\operatorname{cod}^{\Sigma}\left(f_{0}\right)$ será finita (cf. Observação 3.2.20).

Seja $F_{0}:\left(\mathbb{R}^{n+r}, 0\right) \rightarrow \mathbb{R}^{m}$ o $\Sigma$-desdobramento miniversal de $f_{0}$ com $r$ parâmetros, $\alpha=\left(\alpha_{1} \ldots \alpha_{r}\right)$, obtido considerando-se a base $\left\{h_{i}\right\}_{i=1}^{r} \operatorname{de} \mathcal{N}_{e}^{\Sigma}\left(f_{0}\right)$, ou seja,

$$
F_{0}(x, \alpha)=f_{0}(x)+\sum_{i=1}^{r} \alpha_{i} h_{i}(x)
$$

Primeiro, construímos uma família parametrizada de caminhos $\Gamma$-equivariantes através do espaço dos parâmetros de $F_{0}$. Para isso, considere o isomofismo:

$$
\begin{gathered}
\theta: \frac{\overrightarrow{\mathcal{E}}_{x}^{\Sigma}}{\mathcal{T}_{e}^{\Sigma}\left(f_{0}\right)} \rightarrow \mathbb{R}^{r} \\
\theta([g])=\theta\left(\sum_{i=1}^{r} \alpha_{i} h_{i}\right)=\left(\alpha_{1} \ldots \alpha_{r}\right) .
\end{gathered}
$$

Seja $\varphi$ a ação of $\Gamma$ em $\overrightarrow{\mathcal{E}}_{x}^{\Sigma}$ definida por

$$
\begin{gathered}
\varphi: \Gamma \times \overrightarrow{\mathcal{E}}_{x}^{\Sigma} \rightarrow \overrightarrow{\mathcal{E}}_{x}^{\Sigma} \\
\varphi(\gamma, g)=\gamma_{m}^{t}\left(g \circ \gamma_{n}\right) .
\end{gathered}
$$


Lema 3.1.1. $\varphi$ é bem definjda.

Prova. Temos que verificar que

$$
\varphi(\gamma, g)\left(\sigma_{n} x\right)=\sigma_{m} \varphi(\gamma, g)(x), \forall x \in \mathbb{R}^{n}, \forall \sigma \in \Sigma, \forall \gamma \in \Gamma .
$$

Como $\Sigma$ é um subgroupo normal de $\Gamma$ e $g \in \overrightarrow{\mathcal{E}}_{x}^{\Sigma}$,

$$
\begin{aligned}
\varphi(\gamma, g)\left(\sigma_{n} x\right) & =\gamma_{m}^{t} g\left(\gamma_{n}\left(\sigma_{n} x\right)\right)=\gamma_{m}^{t} g\left(\sigma_{n}^{\prime}\left(\gamma_{n} x\right)\right) \\
& =\gamma_{m}^{t}\left(\sigma_{m}^{\prime} g\left(\gamma_{n} x\right)\right)=\sigma_{m}\left(\gamma_{m}^{t} g\left(\gamma_{n} x\right)\right) \\
& =\sigma_{m} \varphi(\gamma, g)(x)
\end{aligned}
$$

Proposição 3.1.2. Como $f_{0}$ é $\Gamma$-equivariante, $\mathcal{T}_{e}^{\Sigma}\left(f_{0}\right)$ é um $\varphi$-submódulo invariante de $\overrightarrow{\mathcal{E}}_{x}^{\Sigma}$

Prova. Seja $h \in \mathcal{T}_{e}^{\Sigma}\left(f_{0}\right)$, ou seja, existem $T \in \mathbf{M}_{x}^{\Sigma} e X \in \vec{\Theta}_{x}^{\Sigma}$ tais que

$$
h(x)=T(x) f_{0}(x)+\left(D_{x} f_{0}\right)(x) X(x) .
$$

Temos que mostrar que

$$
\varphi(\gamma, h) \in \mathcal{T}_{e}^{\Sigma}\left(f_{0}\right), \forall \gamma \in \Gamma
$$

Para isso, computamos

$$
\begin{aligned}
\varphi(\gamma, h)(x) & =\gamma_{m}^{t} h\left(\gamma_{n} x\right)=\gamma_{m}^{t}\left[T\left(\gamma_{n} x\right) f_{0}\left(\gamma_{n} x\right)+\left(D_{x} f_{0}\right)\left(\gamma_{n} x\right) X\left(\gamma_{n} x\right)\right] \\
& =\gamma_{m}^{t}\left[T\left(\gamma_{n} x\right) \gamma_{m} f_{0}(x)+\gamma_{m}\left(D_{x} f_{0}\right)(x) \gamma_{n}^{t} X\left(\gamma_{n} x\right)\right] \\
& =\left[\gamma_{m}^{t} T\left(\gamma_{n} x\right) \gamma_{m}\right] f_{0}(x)+\left(D_{x} f_{0}\right)(x)\left[\gamma_{n}^{t} X\left(\gamma_{n} x\right)\right] \in \mathcal{T}_{e}^{\Sigma}\left(f_{0}\right)
\end{aligned}
$$

pois

(i) $X \in \vec{\Theta}_{x}^{\Sigma}$ e refazendo os cálculos como na prova do Lema 3.1.1 mostramos que $\gamma_{n}^{t}\left(X \circ \gamma_{n}\right) \in \vec{\Theta}_{x}^{\Sigma}$

(ii) Seja, $T^{\prime}(x)=\gamma_{m}^{t} T\left(\gamma_{n} x\right) \gamma_{m}$. Como $T \in \mathbf{M}_{x}^{\Sigma}$ e $\Sigma$ é um subgroupo normal de $\Gamma$, isto é, para todo $\gamma$ em $\Gamma$ e para todo $\sigma$ em $\Sigma$,existe $\sigma^{\prime}$ em $\Sigma$ tal que $\gamma \sigma=\sigma^{\prime} \gamma$, segue que

$$
\begin{aligned}
T^{\prime}\left(\sigma_{n} x\right) & =\gamma_{m}^{t} T\left(\gamma_{n}\left(\sigma_{n} x\right)\right) \gamma_{m}=\gamma_{m}^{t} T\left(\sigma_{n}^{\prime}\left(\gamma_{n} x\right)\right) \gamma_{m} \\
& =\gamma_{m}^{t} \sigma_{m}^{\prime} T\left(\gamma_{n} x\right) \sigma_{m}^{\prime}{ }^{t} \gamma_{m}=\sigma_{m} \gamma_{m}^{t} T\left(\gamma_{n} x\right) \gamma_{m} \sigma_{m}^{t} \\
& =\sigma_{m} T^{\prime}(x) \sigma_{m}^{t}, \quad \text { ou seja, } \quad T^{\prime} \in \mathbf{M}_{x}^{\Sigma}
\end{aligned}
$$


Portanto, $\varphi(\gamma, h) \in \mathcal{T}_{e}^{\Sigma}\left(f_{0}\right)$.

Seja $\bar{\varphi}$ a açào of $\Gamma$ em $\frac{\overrightarrow{\mathcal{E}}_{x}^{\Sigma}}{\mathcal{T}_{e}^{\Sigma}\left(f_{0}\right)}$ definida por

$$
\begin{aligned}
\bar{\varphi}: \Gamma \times \frac{\overrightarrow{\mathcal{E}}_{x}^{\Sigma}}{\mathcal{T}_{\epsilon}^{\Sigma}\left(f_{0}\right)} & \rightarrow \frac{\overrightarrow{\mathcal{E}}_{x}^{\Sigma}}{\mathcal{T}_{e}^{\Sigma}\left(f_{0}\right)} \\
(\gamma,[g]) & \mapsto[\varphi(\gamma, g)] .
\end{aligned}
$$

$\bar{\varphi}$ é bem definida pois $\mathcal{T}_{e}^{\Sigma}\left(f_{0}\right)$ é um $\varphi$-submódulo invariante de $\overrightarrow{\mathcal{E}}_{x}^{\Sigma}$ pela Proposição 3.1.2. Também, para todo $1 \leq i \leq r$ e $\gamma \in \Gamma$ existem números reais bem definidos $\left(\gamma_{\tau}\right)_{i j}$, $1 \leq j \leq r$, tais que

$$
\left[\gamma_{m}^{t} h_{i} \circ \gamma_{n}\right]=\sum_{j=1}^{r}\left(\gamma_{r}\right)_{i j}\left[h_{j}\right]
$$

Pela Proposição 1.3 ([16], pág. 31), isto define uma representação

$$
\begin{aligned}
\rho_{r}: \Gamma & \rightarrow G L(r) \\
\gamma & \mapsto \gamma_{r}^{t}
\end{aligned}
$$

$\operatorname{com}\left(\gamma_{r}^{t}\right)_{i j}=\left(\gamma_{r}\right)_{j i}, 1 \leq i, j \leq r$. Como anteriormente, identificamos a ação com sua imagem.

A partir de agora, fazemos uma escolha particular da base $\left\{h_{i}\right\}$ of $\mathcal{N}_{e}^{\Sigma}\left(f_{0}\right)$ para a qual a equivariância anterior é "exata" e $\gamma_{r} \subset O(r)$, isto é, queremos que as relações

$$
\gamma_{m}^{t} h_{i}\left(\gamma_{n} x\right)=\sum_{j=1}^{\tau}\left(\gamma_{r}\right)_{i j} h_{j}(x), \quad \forall \gamma \in \Gamma, \forall 1 \leq i \leq r,
$$

sejam verdadeiras para os germes e, nao somente, para as classes e que $\rho_{r}$ seja uma representação ortogonal. Quando tal base existe, denominamos boa base.

Lema 3.1.3. Existe uma boa base.

Prova. Supondo (H0), ou seja, $\operatorname{cod}^{\Sigma}\left(f_{0}\right)=r$, existe um núméro inteiro positivo $k$ tal que

$$
\mathcal{M}_{x}^{k} \overrightarrow{\mathcal{E}}_{x}^{\Sigma} \subset \mathcal{T}_{e}^{\Sigma}\left(f_{0}\right)
$$

Denotando por $\mathrm{P}_{k-1}^{\Sigma}$ o espaço vetorial dos polinômios $\Sigma$-equivariantes de grau menor ou igual a $k-1$, segue que $\frac{\overrightarrow{\mathcal{E}}_{x}^{\Sigma}}{\mathcal{M}_{x}^{k} \overrightarrow{\mathcal{E}}_{x}^{\Sigma}}=\mathbf{P}_{k-1}^{\Sigma}$ é um subespaço $\varphi$-invariante de $\overrightarrow{\mathcal{E}}_{x}^{\Sigma}$ e $\frac{\overrightarrow{\mathcal{E}}_{x}^{\Sigma}}{\mathcal{T}_{e}^{\Sigma}\left(f_{0}\right)} \subset \mathrm{P}_{k-1}^{\Sigma}$ 
Podemos escolher a base $\left\{h_{i}\right\}_{i=1}^{r}$ para $\frac{\overrightarrow{\mathcal{E}}_{x}^{\Sigma}}{T_{e}^{\Sigma}\left(f_{0}\right)}$ em $\mathrm{P}_{k-1}^{\Sigma}$ e dessa forma,

$$
\bar{\varphi}\left(\gamma, h_{i}\right)=\gamma_{m}^{t} h_{i} \circ \gamma_{n}=\sum_{j=1}^{r}\left(\gamma_{r}\right)_{i j} h_{j}
$$

Nós poderíamos novamente fazer uma mudança de coordenadas de forma a tornar $\rho_{r}$ uma representação ortogonal.

Para uma boa base $\left\{h_{i}\right\}_{i=1}^{r}$, seja $[g]=\sum_{i=1}^{r} \alpha_{i} h_{i}$ e $\bar{\varphi}$ é explicitamente dada por:

$$
\begin{aligned}
\bar{\varphi}(\gamma,[g]) & =\sum_{i=1}^{r} \alpha_{i}\left(\gamma_{m}^{t} h_{i} \circ \gamma_{n}\right)=\sum_{i=1}^{r} \alpha_{i}\left(\sum_{j=1}^{r}\left(\gamma_{\tau}\right)_{i j} h_{j}\right)= \\
& =\sum_{j=1}^{r}\left(\sum_{i=1}^{r}\left(\gamma_{\tau}\right)_{i j} \alpha_{i}\right) h_{j}=\sum_{i=1}^{r}\left(\gamma_{r}^{t} \alpha\right)_{i} h_{i}
\end{aligned}
$$

$\operatorname{com}\left(\gamma_{r}^{i}\right)_{i j}=\left(\gamma_{r}\right)_{j i}, 1 \leq i, j \leq r$

Considerando o isomorfismo $\theta: \frac{\overrightarrow{\mathcal{E}}_{x}^{\Sigma}}{\mathcal{T}_{e}^{\Sigma}\left(f_{0}\right)} \rightarrow \mathbb{R}^{r}$, temos o diagrama comutativo:

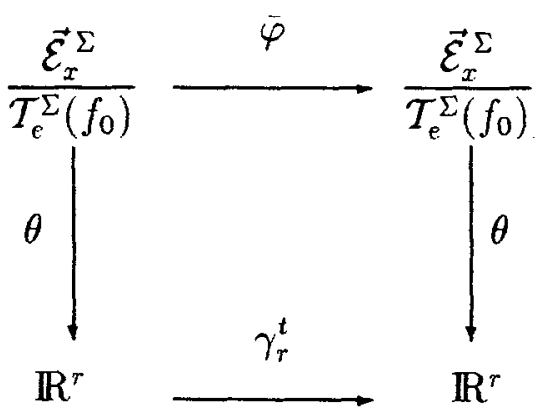

e assim, via $\theta$, para $\alpha \in \mathbb{R}^{r}, \rho_{r}(\gamma)(\alpha)=\gamma_{r}^{t} \alpha$.

Dizemos que

$$
\begin{aligned}
\alpha:\left(\mathbb{R}^{\ell}, 0\right) & \rightarrow\left(\mathbb{R}^{r}, 0\right) \\
\lambda & \mapsto \alpha(\lambda)=\left(\alpha_{1}(\lambda) \ldots \alpha_{r}(\lambda)\right)
\end{aligned}
$$

é um caminho no espaço $r$-dimensional dos parâmetros do desdobramento miniversal de $f_{0}$. 
O pull-back $\left(\bar{\alpha}^{*} F_{0}\right):\left(\mathbb{R}^{n+\ell}, 0\right) \rightarrow \mathbb{R}^{m} \quad$ é dado por

$$
\left(\bar{\alpha}^{*} F_{0}\right)(x, \lambda)=F_{0}(x, \bar{\alpha}(\lambda))
$$

Seja $\mathcal{P}_{\ell, r}=\left\{\alpha:\left(\mathbb{R}^{\ell}, 0\right) \rightarrow\left(\mathbb{R}^{r}, 0\right)\right\}$ o conjunto de caminhos no espaço dos parâmetros de desdobramento.

Definimos uma ação $\varphi_{p}$ em $\mathcal{P}_{\ell, r}$ por

$$
\begin{aligned}
\varphi_{p}: \Gamma \times \mathcal{P}_{\ell, r} & \rightarrow \mathcal{P}_{\ell, r} \\
(\gamma, \alpha) & \mapsto \gamma_{r}^{t}\left(\alpha \circ \gamma_{\ell}\right)
\end{aligned}
$$

O espaço de caminhos fundamental para o nosso trabalho é o subespaço $\mathcal{P}$ de $\mathcal{P}_{\ell, r}$ definido por

$$
\mathcal{P}=\operatorname{Fix} \varphi_{p}=\left\{\alpha \in \mathcal{P}_{\ell, r} \mid \alpha \text { é } \Gamma-\text { equivariante }\right\}
$$

ou seja, $\alpha \in \mathcal{P}$ se e somente se $\alpha\left(\gamma_{\ell} \lambda\right)=\gamma_{r} \alpha(\lambda), \forall \gamma \in \Gamma$.

Proposição 3.1.4. Para $\alpha \in \mathcal{P}$,

$$
\left(\alpha^{*} F_{0}\right)(x, \lambda)=f_{0}(x)+\sum_{i=1}^{r} \alpha_{i}(\lambda) h_{i}(x) \text { é } \Gamma \text { - equivariante }
$$

Prova. Os cálculos a seguir são imediatos.

$$
\begin{aligned}
\left(\alpha^{*} F_{0}\right)\left(\gamma_{n} x, \gamma_{\ell} \lambda\right) & =F_{0}\left(\gamma_{n} x, \alpha\left(\gamma_{\ell} \lambda\right)\right) \\
& =f_{0}\left(\gamma_{n} x\right)+\sum_{i=1}^{r} \alpha_{i}\left(\gamma_{\ell} \lambda\right) h_{i}\left(\gamma_{n} x\right) \\
& =\gamma_{m} f_{0}(x)+\sum_{i=1}^{r}\left(\sum_{j=1}^{r}\left(\gamma_{r}\right)_{i j} \alpha_{j}(\lambda)\right) h_{i}\left(\gamma_{n} x\right) \\
& =\gamma_{m}\left(f_{0}(x)+\sum_{i=1}^{r}\left(\gamma_{r}\right)_{i j} \alpha_{j}(\lambda) \gamma_{m}^{t} h_{i}\left(\gamma_{n} x\right)\right) \\
& =\gamma_{m}\left(f_{0}(x)+\sum_{j=1}^{r} \alpha_{j}(\lambda)\left(\sum_{i=1}^{r}\left(\gamma_{r}\right)_{i j} \sum_{k=1}^{r}\left(\gamma_{r}\right)_{i k} h_{k}\left(\gamma_{n} x\right)\right)\right) \\
& =\gamma_{m}\left(f_{0}(x)+\sum_{j=1}^{r} \alpha_{j}(\lambda)\left(\sum_{k=1}^{r}\left(\sum_{i=1}^{r}\left(\gamma_{r}\right)_{i j}\left(\gamma_{r}\right)_{i k} h_{k}(x)\right)\right)\right) \\
& =\gamma_{m}\left(f_{0}(x)+\sum_{j=1}^{r} \alpha_{j}(\lambda)\left(\sum_{k=1}^{r}\left(\sum_{i=1}^{r}\left(\gamma_{r}^{t}\right)_{j i}\left(\gamma_{r}\right)_{i k} h_{k}(x)\right)\right)\right) \\
& =\gamma_{m}\left(f_{0}(x)+\sum_{j=1}^{r} \alpha_{j}(\lambda)\left(\sum_{k=1}^{r} \delta_{j k} h_{k}(x)\right)\right) \\
& =\gamma_{m}\left(f_{0}(x)+\sum_{j=1}^{r} \alpha_{j}(\lambda) h_{j}(x)\right)=\gamma_{m} F_{0}(x, \alpha(\lambda)) \\
& =\gamma_{m}\left(\alpha^{*} F_{0}\right)(x, \lambda), \forall \gamma \in \Gamma .
\end{aligned}
$$

Portanto, $\alpha^{*} F_{0}$ é $\Gamma$-equivariante. 


\subsection{Espaços tangentes a um caminho}

Supondo que (H0) seja verdadeira e que $\mathcal{N}_{e}^{\Sigma}\left(f_{0}\right)$ seja gerado por uma boa base $\left\{h_{1} \ldots h_{r}\right\}$, seja

$$
\begin{aligned}
\rho_{\tau}: \Gamma & \rightarrow O(r) \\
\gamma & \mapsto \gamma_{r}^{t}
\end{aligned}
$$

a representação ortogonal definida na secção 3.1 .

Considere $\Gamma$ agindo em $\alpha \in \mathbb{R}^{r}$ por $(\gamma, \alpha) \mapsto \gamma_{r} \alpha$. O $\Sigma$-desdobramento miniversal de $f_{0}$, denotado, como anteriromente, por $F_{0}$, é $\Gamma$-equivariante.

Precisamente,

$$
\begin{aligned}
F_{0}\left(\gamma_{n} x, \gamma_{r} \alpha\right) & \left.=f_{0}\left(\gamma_{n} x\right)+\sum_{i=1}^{r}\left(\sum_{j=1}^{r}\left(\gamma_{r}\right)_{i j} \alpha_{j}\right) h_{i}\left(\gamma_{n} x\right)\right) \\
& =\gamma_{m}\left(f_{0}(x)+\sum_{j=1}^{r} \alpha_{j} h_{j}(x)\right) \\
& =\gamma_{m} F_{0}(x, \alpha)
\end{aligned}
$$

como na prova da Proposição 3.1.4.

Portanto, $F_{0}\left(\gamma_{n} x, \gamma_{r} \alpha\right)=\gamma_{m} F_{0}(x, \alpha), \forall \gamma \in \Gamma$.

No que segue escreveremos os resultados preliminares e necessários para definir o espaço tangente e o espaço tangente unipotente a um caminho $\Gamma$-equjvariante. Devido à simetria existente nos parâmetros de bifurcação, temos que ampliar o espaço de caminhos $\mathcal{P}$.

Definição 3.2.1. Seja $\hat{\mathcal{P}}=\left\{\tilde{\beta}:\left(\mathbb{R}^{\ell}, 0\right) \rightarrow\left(\mathbb{R}^{r+\ell}, 0\right) \mid \tilde{\beta}\left(\gamma_{\ell} \lambda\right)=\left(\gamma_{r} \times \gamma_{\ell}\right) \tilde{\beta}(\lambda)\right\}$ o conjunto dos caminhos $\Gamma$-equivariantes definidos por $\tilde{\beta}(\lambda)=(\tilde{\alpha}(\lambda), \lambda)$ para $\tilde{\alpha} \in \mathcal{P}$.

Seja $\pi_{r}: \mathbb{R}^{r+\ell} \rightarrow \mathbb{R}^{r}$ a projeção canônica.

Definição 3.2.2. Para $\beta=(\alpha, \lambda) \in \mathbb{R}^{r+\ell}$, seja $\hat{F}_{o}:\left(\mathbb{R}^{n+r+\ell}, 0\right) \rightarrow \mathbb{R}^{m}$ definida por

$$
\hat{F}_{o}(x, \beta)=F_{o}\left(x, \pi_{r}(\beta)\right)=\left(\mathbf{I}_{n} \times \pi_{r}\right)^{*} F_{o}(x, \beta) .
$$

Definição 3.2.3. Sejam

$$
\begin{aligned}
& \overrightarrow{\mathcal{E}}_{(x, \beta)}^{\Gamma}=\left\{\hat{f}:\left(\mathbb{R}^{n+\tau+\ell}, 0\right) \rightarrow \mathbb{R}^{m} \mid \hat{f}\left(\gamma_{n} x,\left(\gamma_{r} \times \gamma_{\ell}\right) \beta\right)=\gamma_{m} \hat{f}(x, \beta), \forall \gamma \in \Gamma\right\} . \\
& \mathbf{M}_{(x, \beta)}^{\Gamma}=\left\{\hat{S}:\left(\mathbb{R}^{n+\tau+\ell}, 0\right) \rightarrow M_{m}(\mathbb{R}) \mid \hat{S}\left(\gamma_{n} x,\left(\gamma_{r} \times \gamma_{\ell}\right) \beta\right) \gamma_{m}=\gamma_{m} \hat{S}(x, \beta), \forall \gamma \in \Gamma\right\} .
\end{aligned}
$$




$$
\begin{aligned}
& \vec{\Theta}_{(x, \beta)}^{\Gamma}=\left\{\hat{\xi}:\left(\mathbb{R}^{n+r+\ell}, 0\right) \rightarrow \mathbb{R}^{n} \mid \hat{\xi}\left(\gamma_{n} x,\left(\gamma_{r} \times \gamma_{\ell}\right) \beta\right)=\gamma_{n} \hat{\xi}(x, \beta), \forall \gamma \in \Gamma\right\} . \\
& \overrightarrow{\mathcal{E}}_{\beta}^{\Gamma}=\left\{\hat{\mu}:\left(\mathbb{R}^{r+\ell}, 0\right) \rightarrow \mathbb{R}^{r+\ell} \mid \hat{\mu}\left(\left(\gamma_{r} \times \gamma_{\ell}\right) \beta\right)=\left(\gamma_{r} \times \gamma_{\ell}\right) \hat{\mu}(\beta), \forall \gamma \in \Gamma\right\} . \\
& \mathcal{E}_{\beta}^{\Gamma}=\left\{\mu:\left(\mathbb{R}^{r+\ell}, 0\right) \rightarrow \mathbb{R} \mid \hat{\eta}\left(\left(\gamma_{r} \times \gamma_{\ell}\right) \beta\right)=\hat{\eta}(\beta), \forall \gamma \in \Gamma\right\} . \\
& \vec{\Theta}_{(x, \beta)}^{\Gamma, o}=\left\{\hat{\xi} \in \vec{\Theta}_{(x, \beta)}^{\Gamma} \mid \hat{\xi}^{o}=0\right\} .
\end{aligned}
$$

Segue que

(i) $\hat{F}_{o} \in \overrightarrow{\mathcal{E}}_{(x, \beta)}^{\Gamma}$ pois

$$
\begin{aligned}
\hat{F}_{o}\left(\gamma_{n} x,\left(\gamma_{r} \times \gamma_{\ell}\right) \beta\right) & =F_{o}\left(\gamma_{n} x, \pi_{r}\left(\gamma_{r+l} \beta\right)\right)=F_{o}\left(\gamma_{n} x, \gamma_{r} \pi_{r}(\beta)\right) \\
& =F_{o}\left(\gamma_{n} x, \gamma_{r} \alpha\right)=\gamma_{m} F_{o}(x, \alpha)=\gamma_{m} F_{o}\left(x, \pi_{r}(\beta)\right) \\
& =\gamma_{m} \hat{F}_{o}(x, \beta), \forall \gamma \in \Gamma .
\end{aligned}
$$

(ii) $\tilde{\beta}^{*} \hat{F}_{o}=\tilde{\alpha}^{*} F_{o}$ e, portanto, $\tilde{\beta}^{*} \hat{F}_{o} \in \overrightarrow{\mathcal{E}}_{(x, \lambda)}^{\Gamma}$.

De fato,

$$
\left(\tilde{\beta}^{*} \hat{F}_{o}\right)(\lambda)=\hat{F}_{o}(x, \tilde{\beta}(\lambda))=F_{o}\left(x, \pi_{r}(\tilde{\beta}(\lambda))\right)=F_{o}(x, \tilde{\alpha}(\lambda))=\left(\tilde{\alpha}^{*} F_{o}\right)(\lambda)
$$

e nós já mostramos que $\left(\tilde{\alpha}^{*} F_{o}\right) \in \overrightarrow{\mathcal{E}}_{(x, \lambda)}^{\Gamma}$ se $\tilde{\alpha} \in \mathcal{P}$ (cf. Proposição 3.1.4).

$\mathrm{O}$ espaço tangente à $\hat{f} \in \overrightarrow{\mathcal{E}}_{(x, \beta)}^{\Gamma}$ é definido por

$$
\mathbf{T}^{\Gamma}(\hat{f})=\left\{\hat{S} \hat{f}+\left(D_{x} \hat{f}\right) \hat{\xi} \mid \hat{S} \in \mathbf{M}_{(x, \beta)}^{\Gamma} \text { e } \hat{\xi} \in \vec{\Theta}_{(x, \beta)}^{\Gamma}\right\}
$$

e o espaço tangente unipotente à $\hat{f} \in \overrightarrow{\mathcal{E}}_{(x, \beta)}^{\Gamma}$ é

$\mathbf{T U}^{\Gamma}(\hat{f})=\left\{\hat{S} \hat{f}+\left(D_{x} \hat{f}\right) \hat{\xi} \mid \hat{S} \in \mathbf{M}_{(x, \beta)}^{\Gamma}, \hat{\xi} \in \vec{\Theta}_{(x, \beta)}^{\Gamma, o}\right.$ satisfazendo $\hat{S}^{o}=0$ e $\left.\left(D_{x} \hat{\xi}\right)^{\circ}=0\right\}$.

Considere a seguinte intersecção de $\mathcal{E}_{\beta}^{\Gamma}$-módulos:

$$
\mathbf{T}^{\Gamma}\left(\hat{F}_{o}\right) \cap\left(\mathcal{E}_{\beta}<h_{1} \ldots h_{r}>\right)^{\Gamma}
$$

1. Suponha que exista um número finito de geradores $h_{1}^{\prime} \ldots h_{s}^{\prime}$ tais que

$$
\mathbf{T}^{\Gamma}\left(\hat{F}_{o}\right) \cap\left(\mathcal{E}_{\beta}<h_{1} \ldots h_{r}>\right)^{\Gamma}=\mathcal{E}_{\beta}^{\Gamma}<h_{1}^{\prime} \ldots h_{s}^{\prime}>\text {. }
$$

Se (H1) for satisfeita então para cada, $1 \leq j \leq s, h_{j}^{\prime}$ poderá ser decomposta como

$$
h_{j}^{\prime}(x, \beta)=\sum_{i=1}^{r}\left(\eta_{j}\right)_{i}(\beta) h_{i}(x)
$$


onde $\eta_{j}:\left(\mathbb{R}^{r+\ell}, 0\right) \rightarrow \mathbb{R}^{r}, \eta_{3}=\left(\eta_{j 1} \ldots \eta_{j_{r}}\right)$ é $\Gamma$-equivariante, ou seja,

$$
\eta_{j}\left(\left(\gamma_{r} \times \gamma_{\ell}\right) \beta\right)=\gamma_{r} \eta_{j}(\beta), \forall \gamma \in \Gamma
$$

Definimos

$$
N=\mathcal{E}_{\beta}^{\Gamma}<\eta_{1} \ldots \eta_{s}>
$$

e para $1 \leq j \leq s$

$$
\begin{aligned}
\hat{\eta}_{j}:\left(\mathbb{R}^{r+\ell}, 0\right) & \rightarrow \mathbb{R}^{r+\ell} \\
\beta & \mapsto\left(\eta_{j}(\beta), \lambda\right) .
\end{aligned}
$$

Note que $\hat{\eta}_{j} \in \overrightarrow{\mathcal{E}}_{\beta}^{\Gamma}, 1 \leq j \leq s$, pois $\hat{\eta}_{j}\left(\left(\gamma_{r} \times \gamma_{\ell}\right) \beta\right)=\left(\eta_{j}\left(\left(\gamma_{r} \times \gamma_{\ell}\right) \beta\right), \gamma_{\ell} \lambda\right)=$ $\left(\gamma_{r} \eta_{j}(\beta), \gamma_{\ell} \lambda\right)=\left(\gamma_{r} \times \gamma_{\ell}\right)\left(\eta_{j}(\beta), \lambda\right)=\left(\gamma_{r} \times \gamma_{\ell}\right) \hat{\eta}_{j}(\beta), \forall \gamma \in \Gamma$.

Seja

$$
\hat{N}=\mathcal{E}_{\beta}^{\Gamma}<\hat{\eta}_{1} \ldots \hat{\eta}_{s}>
$$

2. Analogamente, supomos que exista um número finito de geradores $h_{1}^{\prime \prime} \ldots h_{t}^{\prime \prime}$ tais que

$$
\mathbf{T U}^{\Gamma}\left(\hat{F}_{o}\right) \cap\left(\mathcal{E}_{\beta}<h_{1} \ldots h_{r}>\right)^{\Gamma}=\mathcal{E}_{\beta}<h_{1}^{\prime \prime} \ldots h_{t}^{\prime \prime}>
$$

Note que $\mathcal{E}_{\beta}^{\Gamma}<h_{1}^{\prime \prime} \ldots h_{t}^{\prime \prime}>\subset \mathcal{E}_{\beta}<h_{1}^{\prime} \ldots h_{s}^{\prime}>$.

Se (H2) for satisfeita, para cada, $1 \leq j \leq t, h_{j}^{\prime \prime}$ poderá ser decomposta como

$$
h_{j}^{\prime \prime}(x, \beta)=\sum_{i=1}^{r}\left(\hat{\eta}_{j}\right)_{i}(\beta) h_{i}(x)
$$

onde $\tilde{\eta}_{j}:\left(\mathbb{R}^{r+\ell}, 0\right) \rightarrow \mathbb{R}^{r}, \tilde{\eta}_{j}=\left(\tilde{\eta}_{j_{1}} \ldots \tilde{\eta}_{j_{r}}\right)$ é $\Gamma$-equivariante.

Definimos

$$
\tilde{N}=\mathcal{E}_{\beta}^{\Gamma}<\tilde{\eta}_{1} \ldots \tilde{\eta}_{t}>
$$

e para, $1 \leq j \leq t$,

$$
\begin{aligned}
\hat{\tilde{\eta}}_{j}:\left(\mathbb{R}^{r+\ell}, 0\right) & \rightarrow \mathbb{R}^{r+\ell} \\
\beta & \mapsto\left(\tilde{\eta}_{j}(\beta), \lambda\right) .
\end{aligned}
$$

Note que, $\hat{\tilde{\eta}}(\beta) \in \overrightarrow{\mathcal{E}}_{\beta}^{\Gamma}, 1 \leq j \leq t$.

Seja

$$
\hat{\tilde{N}}=\mathcal{E}_{\beta}^{\Gamma}<\hat{\tilde{\eta}}_{1} \ldots \hat{\tilde{\eta}}_{t}>
$$


Definição 3.2.4. Seja $\omega_{\beta}: \overrightarrow{\mathcal{E}}_{\beta}^{\Gamma} \rightarrow \overrightarrow{\mathcal{E}}_{(x, \beta)}^{\Gamma}$ definida por

$$
\omega_{\beta}(\hat{\mu})=\left.\frac{\partial}{\partial \tau}\right|_{\tau=0}\left(\mathbf{I}_{\beta}+\tau \hat{\mu}\right)^{*} \hat{F}_{o}=\left.\frac{\partial}{\partial \tau}\right|_{\tau=0}\left(\pi_{\tau} \circ\left(\mathbf{I}_{\beta}+\tau \hat{\mu}\right)\right)^{*} F_{o}=\sum_{i=1}^{r}\left(\pi_{\tau} \circ \hat{\mu}\right)_{i} h_{i} .
$$

É imediato que $\omega_{\beta}(\hat{\mu}) \in \overrightarrow{\mathcal{E}}_{(x, \beta)}^{\Gamma}$ pois

$$
\begin{aligned}
\omega_{\beta}(\hat{\mu})\left(\gamma_{n} x,\left(\gamma_{r} \times \gamma_{\ell}\right) \beta\right) & =\sum_{i=1}^{r}\left(\pi_{r} \circ \hat{\mu}\right)_{i}\left(\left(\gamma_{r} \times \gamma_{\ell}\right) \beta\right) h_{i}\left(\gamma_{n} x\right) \\
& =\sum_{i=1}^{r}\left(\sum_{j=1}^{r}\left(\gamma_{r}\right)_{i j}\left(\pi_{r} \circ \hat{\mu}\right)_{j}(\beta)\right) h_{i}\left(\gamma_{n} x\right) \\
& =\gamma_{m} \omega_{\beta}(\hat{\mu})(x, \beta) .
\end{aligned}
$$

A última igualdade se verifica através de cálculos semelhantes aos da pág. 77.

\section{Proposição 3.2.5.}

1. $\omega_{\beta}^{-1}\left(\mathbf{T}^{\Gamma}\left(\hat{F}_{o}\right)\right)=\hat{N}, \hat{N}$ definido em $(3.2)$.

2. $\omega_{\beta}^{-1}\left(\mathbf{T U}^{\Gamma}\left(\hat{F}_{o}\right)\right)=\hat{\hat{N}}, \hat{\hat{N}}$ definido em $(3.4)$.

Prova. Mostramos 1. em duas etapas.

(i) $\hat{N} \subset \omega_{\beta}^{-1}\left(\mathbf{T}^{\Gamma}\left(\hat{F}_{o}\right)\right)$.

Seja $\hat{\eta} \in \hat{N}$, ou seja, $\hat{\eta}=\sum_{j=1}^{s} \mu_{j} \hat{\eta}_{j}$.

$$
\begin{aligned}
\omega_{\beta}(\hat{\eta}) & =\sum_{i=1}^{r}\left(\pi_{r} \circ \hat{\eta}\right)_{i} h_{i}=\sum_{i=1}^{r}\left(\sum_{j=1}^{s} \mu_{j}\left(\pi_{r} \circ \hat{\eta}_{j}\right)\right)_{i} h_{i} \\
& =\sum_{i=1}^{r}\left(\sum_{j=1}^{s} \mu_{j}\left(\pi_{r} \circ \hat{\eta}_{j}\right)_{i}\right) h_{i}=\sum_{i=1}^{r}\left(\sum_{j=1}^{s} \mu_{j}\left(\eta_{j}\right)_{i}\right) h_{i} \\
& =\sum_{j=1}^{s} \mu_{j}\left(\sum_{i=1}^{r}\left(\eta_{j}\right)_{i} h_{i}\right)=\sum_{j=1}^{s} \mu_{j} h_{j}^{\prime} .
\end{aligned}
$$

Consequentemente, $\omega_{\beta}(\hat{\eta}) \in \mathcal{E}_{\beta}<h_{1}^{\prime} \ldots h_{s}^{\prime}>\subset \mathbf{T}^{\Gamma}\left(\hat{F}_{o}\right)$, por $(\mathrm{H} 1)$.

(ii) $\omega_{\beta}^{-1}\left(\mathbf{T}^{\Gamma}\left(\hat{F}_{o}\right)\right) \subset \hat{N}$.

Seja $\hat{\mu} \in \overrightarrow{\mathcal{E}}_{\beta}^{\Gamma}$ tal que $\omega_{\beta}(\hat{\mu}) \in \mathbf{T}^{\Gamma}\left(\hat{F}_{o}\right)$. Devemos mostrar que $\hat{\mu} \in \hat{N}$.

$\omega_{\beta}(\hat{\mu})=\sum_{i=1}^{r}\left(\pi_{r} \circ \hat{\mu}\right)_{i} h_{i} \in \mathbf{T}^{\Gamma}\left(\hat{F}_{o}\right)$ implica, por $(\mathrm{H} 1)$, que $\omega_{\beta}(\hat{\mu}) \in \mathcal{E}_{\beta}^{\Gamma}<h_{1}^{\prime} \ldots h_{s}^{\prime}>$

Consequentemente,

$$
\begin{aligned}
\omega_{\beta}(\hat{\mu}) & =\sum_{j=1}^{r} \mu_{j} h_{j}^{\prime}=\sum_{j=1}^{s} \mu_{j}\left(\sum_{i=1}^{r}\left(\eta_{j}\right)_{i} h_{i}\right)=\sum_{i=1}^{r}\left(\sum_{j=1}^{s} \mu_{j}\left(\eta_{j}\right)_{i}\right) h_{i} \\
& =\sum_{i=1}^{r}\left(\sum_{j=1}^{s} \mu_{j}\left(\pi_{r} \circ \hat{\eta}_{j}\right)_{i}\right) h_{i}=\sum_{i=1}^{r}\left(\sum_{j=1}^{s}\left(\mu_{j}\left(\pi_{r} \circ \hat{\eta}_{j}\right)\right)_{i}\right) h_{i} \\
& =\omega_{\beta}\left(\sum_{j=1}^{s} \mu_{j} \hat{\eta}_{j}\right) .
\end{aligned}
$$


Por unicidade, $\hat{\mu}=\sum_{j=1}^{s} \mu_{j} \hat{\eta}_{j}$ e, portanto, $\hat{\mu} \in \hat{N}$.

A proposição segue de (i) e (ii).

Analogamente, mostramos que 2. é verdadeira.

Definição 3.2.6. Para $\tilde{\alpha} \in \mathcal{P}$, seja $\quad \omega_{\tilde{\alpha}}: \mathcal{P}_{\ell, r} \rightarrow \overrightarrow{\mathcal{E}}_{(x, \lambda)}^{\Sigma} \quad$ dada por

$$
\omega_{\hat{\alpha}}(\xi)=\left.\frac{\partial}{\partial \tau}\right|_{\tau=0}(\hat{\alpha}+\tau \xi)^{*} F_{0}=\sum_{i=1}^{r} \xi_{i} h_{i} .
$$

Proposição 3.2.7. $\omega_{\dot{\alpha}}$ é um $\mathbb{R}$-isomorfismo entre $\mathcal{P}$ e $\left(\mathcal{E}_{\lambda}<h_{1} \ldots h_{r}>\right)^{\Gamma}$.

Prova. (i) É imediato que $\omega_{\tilde{\alpha}}$ é uma aplicação $\mathbb{R}$-linear.

(ii) $\omega_{\tilde{\alpha}}$ é injetora pois $\left\{h_{i}\right\}_{i=1}^{\tau}$ é uma base de $\mathcal{N}_{e}^{\Sigma}\left(f_{0}\right)$.

(iii) $\omega_{\tilde{\alpha}}(\mathcal{P})=\left(\mathcal{E}_{\lambda}<h_{1} \ldots h_{r}>\right)^{\Gamma}$.

De fato, nós já mostramos que para $\xi \in \mathcal{P}, \omega_{\tilde{\alpha}}(\xi)$ é $\Gamma$-equivariante $\mathrm{e}$, portanto,

$$
\omega_{\bar{\alpha}}\left(\mathcal{P}^{\prime}\right) \subset\left(\mathcal{E}_{\lambda}<h_{1} \ldots h_{r}>\right)^{\Gamma}
$$

Resta verificar que $\left(\mathcal{E}_{\lambda}<h_{1} \ldots h_{r}>\right)^{\Gamma} \subset \omega_{\tilde{\alpha}}(\mathcal{P})$. Para isso, seja $\zeta \in\left(\mathcal{E}_{\lambda}<h_{1} \ldots h_{r}>\right)^{\Gamma}$, isto é, $\zeta(x, \lambda)=\sum_{i=1}^{r} \nu_{i}(\lambda) h_{i}(x)$.

Seja $\nu(\lambda)=\left(\nu_{1}(\lambda) \ldots \nu_{r}(\lambda)\right)$. Afirmamos que $\nu \in \mathcal{P}$.

Definindo $\xi=\int_{\Gamma} \gamma_{r}^{t} \nu \circ \gamma_{\ell}$ onde $\int_{\Gamma}$ é a integral de Haar e escrevendo $h(x)=$ $\left(h_{1}(x) \ldots h_{r}(x)\right)$, temos que $\xi \in \mathcal{P}$ e

$$
\begin{aligned}
\left(\omega_{\tilde{\alpha}}(\xi)\right)(x, \lambda) & =<\xi(\lambda), h(x)>=<\left(\int_{\Gamma} \gamma_{r}^{t} \nu \circ \gamma_{\ell}\right)(\lambda), h(x)> \\
& =\int_{\Gamma}<\nu\left(\gamma_{\ell} \lambda\right), \gamma_{r}^{t} h(x)>=\int_{\Gamma}<\nu\left(\gamma_{\ell} \lambda\right),\left(\mathbf{I}_{r} \otimes \gamma_{m}^{-1}\right) h\left(\gamma_{n} x\right)> \\
& =\int_{\Gamma} \gamma_{m}^{-1}<\nu\left(\gamma_{\ell} \lambda\right), h\left(\gamma_{n} x\right)>=\int_{\Gamma} \gamma_{m}^{-1} \zeta\left(\gamma_{\ell} \lambda, \gamma_{n} x\right) \\
& =\int_{\Gamma} \gamma_{m}^{-1} \gamma_{m} \zeta(x, \lambda)=\zeta(x, \lambda)=\left(\omega_{\tilde{\alpha}}(\nu)\right)(x, \lambda) .
\end{aligned}
$$

Segue, por injetividade, que $\nu=\xi$ e, portanto, $\nu \in \mathcal{P}$.

Definição 3.2.8. Para $\tilde{\beta} \in \hat{\mathcal{P}}$, definimos $\omega_{\tilde{\beta}}: \hat{\mathcal{P}} \rightarrow \overrightarrow{\mathcal{E}}_{(x, \lambda)}^{\Gamma}$ por $\omega_{\tilde{\beta}}(\tilde{\xi})=\omega_{\tilde{\alpha}}\left(\pi_{r} \circ \tilde{\xi}\right)$.

Pela Definição 3.2.6., segue que

$$
\omega_{\tilde{\beta}}(\tilde{\xi})=\sum_{i=1}^{r} \xi_{i} h_{i} \operatorname{com} \xi=\pi_{r} \circ \tilde{\xi}=\left(\xi_{1} \ldots \xi_{r}\right)
$$


e $\omega_{\tilde{\beta}}$ é um $\mathbb{R}$-isomorfismo entre $\hat{\mathcal{P}}$ e $\left(\mathcal{E}_{\lambda}<h_{1} \ldots h_{\tau}>\right)^{\Gamma}$.

Recorde que

- Para $\tilde{\beta} \in \hat{\mathcal{P}},\left(\hat{\beta}^{*} \hat{F}_{o}\right) \in \overrightarrow{\mathcal{E}}_{(x, \lambda)}^{\Gamma}$,

$\hat{\mathcal{T}}_{e}^{\Gamma}\left(\tilde{\beta}^{*} \hat{F}_{o}\right)=\left\{T\left(\tilde{\beta}^{*} \hat{F}_{o}\right)+D_{x}\left(\tilde{\beta}^{*} \hat{F}_{o}\right) X \mid T \in \mathbf{M}_{(x, \lambda)}^{\Gamma}\right.$ e $\left.X \in \vec{\Theta}_{(x, \lambda)}^{\Gamma}\right\}$.

$\hat{\mathcal{T}}^{\Gamma}\left(\tilde{\beta}^{*} \hat{F}_{o}\right)=\left\{T\left(\tilde{\beta}^{*} \hat{F}_{o}\right)+D_{x}\left(\tilde{\beta}^{*} \hat{F}_{o}\right) X \mid T \in \mathbf{M}_{(x, \lambda)}^{\Gamma}, X \in \vec{\Theta}_{(x, \lambda)}^{\Gamma, o}\right.$ satisfazendo

$$
\left.T^{\circ}=0 \text { e }\left(D_{x} X\right)^{\circ}=0\right\} \text {. }
$$

- Seguem de (3.1) e (3.3),

$N=\mathcal{E}_{\beta}^{\Gamma}<\eta_{1} \ldots \eta_{s}>, \eta_{j}:\left(\mathbb{R}^{r+\ell}, 0\right) \rightarrow \mathbb{R}^{r}$ é $\Gamma$-equivariante, $1 \leq j \leq s$,

$\tilde{N}=\mathcal{E}_{\beta}^{\Gamma}<\tilde{\eta}_{1} \ldots \tilde{\eta}_{t}>, \tilde{\eta}_{j}:\left(\mathbb{R}^{r+\ell}, 0\right) \rightarrow \mathbb{R}^{r}$ é $\Gamma$-equivariante, $1 \leq j \leq t$.

Sejam $\left(\tilde{\beta}^{*} \eta_{j}\right):\left(\mathbb{R}^{\ell}, 0\right) \rightarrow \mathbb{R}^{r}$ dada por $\left(\tilde{\beta}^{*} \eta_{j}\right)(\lambda)=\eta_{j}(\tilde{\alpha}(\lambda), \lambda), 1 \leq j \leq s, \mathrm{e}$ $\left(\tilde{\beta}^{*} \tilde{\eta}_{j}\right):\left(\mathbb{R}^{\ell}, 0\right) \rightarrow \mathbb{R}^{r}$ dada por $\left(\tilde{\beta}^{*} \tilde{\eta}_{j}\right)(\lambda)=\eta_{j}(\tilde{\alpha}(\lambda), \lambda), 1 \leq j \leq t$.

Vemos que $\hat{\beta}^{*} \eta_{j}$ e $\tilde{\beta}^{*} \tilde{\eta}_{j}$ pertencem à $\mathcal{P}$ e podemos escrever $\quad \tilde{\beta}^{*} \eta_{j}=\tilde{\alpha}^{*} \eta_{j}$ $1 \leq j \leq s$, e $\quad \hat{\beta}^{*} \tilde{\eta}_{j}=\tilde{\alpha}^{*} \tilde{\eta}_{j}, \quad 1 \leq j \leq t . \quad$ Definimos

$$
\begin{aligned}
\hat{\alpha}^{*} N & =\mathcal{E}_{\lambda}^{\Gamma}<\tilde{\alpha}^{*} \eta_{1} \ldots \tilde{\alpha}^{*} \eta_{s}> \\
\mathrm{e} & \\
\tilde{\alpha}^{*} \tilde{N} & =\mathcal{E}_{\lambda}^{\Gamma}<\tilde{\alpha}^{*} \hat{\eta}_{1} \ldots \tilde{\alpha}^{*} \hat{\eta}_{t}>.
\end{aligned}
$$

- Seguem de (3.2) e (3.4),

$\hat{N}=\mathcal{E}_{\beta}^{\Gamma}<\hat{\eta}_{1} \ldots \hat{\eta}_{s}>, \hat{\eta}_{j}:\left(\mathbb{R}^{r+\ell}, 0\right) \rightarrow \mathbb{R}^{r}$ é $\Gamma$-equivariante, $1 \leq j \leq s$,

$\hat{\tilde{N}}=\mathcal{E}_{\beta}^{\Gamma}<\hat{\tilde{\eta}}_{1} \ldots \hat{\tilde{\eta}}_{t}>, \hat{\tilde{\eta}}_{j}:\left(\mathbb{R}^{r+\ell}, 0\right) \rightarrow \mathbb{R}^{r}$ é $\Gamma$-equivariante, $1 \leq j \leq t$.

Por definição, $\left(\tilde{\beta}^{*} \hat{\eta}_{j}\right)(\lambda)=\hat{\eta}_{j}(\tilde{\alpha}(\lambda), \lambda)=\left(\eta_{j}(\tilde{\alpha}(\lambda), \lambda), \lambda\right), 1 \leq j \leq s$, e $\left(\tilde{\beta}^{*} \hat{\tilde{\eta}}_{j}\right)(\lambda)$ $=\hat{\tilde{\eta}}_{j}(\tilde{\alpha}(\lambda), \lambda)=\left(\tilde{\eta}_{j}(\tilde{\alpha}(\lambda), \lambda), \lambda\right), 1 \leq j \leq t$.

Consequentemente, $\tilde{\beta}^{*} \hat{\eta}_{j}$ e $\tilde{\beta}^{*} \hat{\tilde{\eta}}_{j}$ pertencem à $\hat{\mathcal{P}}, 1 \leq j \leq s, 1 \leq j \leq t$, respectivamente.

Definimos

$$
\begin{aligned}
\tilde{\beta}^{*} \hat{N} & =\mathcal{E}_{\beta}^{\Gamma}<\tilde{\beta}^{*} \hat{\eta}_{1} \ldots \tilde{\beta}^{*} \hat{\eta}_{s}> \\
\mathrm{e} & \\
\tilde{\beta}^{*} \hat{\tilde{N}} & =\mathcal{E}_{\beta}^{\Gamma}<\tilde{\beta}^{*} \hat{\tilde{\eta}}_{1} \ldots \tilde{\beta}^{*} \hat{\tilde{\eta}}_{t}>
\end{aligned}
$$




\section{Proposição 3.2.9.}

1. $\omega_{\tilde{\beta}}^{-1}\left(\hat{\mathcal{T}}_{e}^{\Gamma}\left(\tilde{\beta}^{*} \hat{F}_{o}\right)\right)=\tilde{\beta}^{*} \hat{N}$.

2. $\omega_{\tilde{\beta}}^{-1}\left(\hat{\mathcal{T}}^{\Gamma}\left(\tilde{\beta}^{*} \hat{F}_{o}\right)\right)=\tilde{\beta}^{*} \hat{\tilde{N}}$

onde $\omega_{\tilde{\beta}}$ é dada pela definição 3.2.8. e $\tilde{\beta}^{*} \hat{N}, \tilde{\beta}^{*} \hat{\tilde{N}}$ estão definidos em (3.7) e (3.8).

Prova. Para provar 1., primeiro mostramos que

(i) $\omega_{\tilde{\beta}}^{-1}\left(\hat{\mathcal{T}}_{e}^{\Gamma}\left(\tilde{\beta}^{*} \hat{F}_{o}\right)\right) \subset \tilde{\beta}^{*} \hat{N}$ em duas etapas.

Etapa 1. Pela definição 3.2.1, $\tilde{\beta}$ é uma imersão e, portanto existe $\Psi:\left(\mathbb{R}^{r+\ell}, 0\right) \rightarrow\left(\mathbb{R}^{\ell}, 0\right)$ tal que $\Psi \circ \tilde{\beta}=\mathbf{L}_{\ell}$. Dessa forma, é possivel exibir uma aplicação $\Gamma$-equivariante $\tilde{\Psi}$, isto é, $\tilde{\Psi} \circ\left(\gamma_{r} \times \gamma_{\ell}\right)=\gamma_{\ell} \tilde{\Psi}$, tal que $\tilde{\Psi} \circ \tilde{\beta}=\mathbf{L}_{\ell}$.

Definimos $\tilde{\Psi}=\int_{\Gamma} \gamma_{\ell}^{t} \Psi \circ\left(\gamma_{r} \times \gamma_{\ell}\right)$ onde $\int_{\Gamma}$ é a integral de Haar.

Usando as propriedades da integral de Haar, temos que

$\tilde{\Psi}:\left(\mathbb{R}^{r+\ell}, 0\right) \rightarrow\left(\mathbb{R}^{\ell}, 0\right)$ e

$$
\begin{aligned}
\tilde{\Psi} \circ\left(\gamma_{r}^{\prime} \times \gamma_{\ell}^{\prime}\right) & =\int_{\Gamma} \gamma_{\ell}^{t} \Psi \circ\left(\gamma_{r} \times \gamma_{\ell}\right) \circ\left(\gamma_{r}^{\prime} \times \gamma_{\ell}^{\prime}\right) \\
& =\int_{\Gamma} \gamma_{\ell}^{\prime} \gamma_{\ell}^{\prime t} \gamma_{\ell}^{t} \circ \Psi \circ\left(\gamma_{r} \times \gamma_{\ell}\right) \circ\left(\gamma_{r}^{\prime} \times \gamma_{\ell}^{\prime}\right) \\
& =\int_{\Gamma} \gamma_{\ell}^{\prime} \nu^{t} \Psi \circ(\nu \times \nu), \operatorname{com} \nu=\gamma \gamma^{\prime}, \\
& =\gamma_{\ell}^{\prime} \circ \tilde{\Psi} .
\end{aligned}
$$

Além disso, $\tilde{\Psi} \circ \tilde{\beta}=\int_{\Gamma} \gamma_{\ell}^{t} \Psi \circ\left(\gamma_{r} \times \gamma_{\ell}\right) \circ \tilde{\beta}=\int_{\Gamma} \gamma_{\ell}^{t} \Psi \circ \tilde{\beta} \circ \gamma_{\ell}=\int_{\Gamma} \gamma_{\ell}^{t} \gamma_{\ell}=\mathbf{I}_{\ell}$.

Via $\tilde{\Psi}$

- $\mathbf{M}_{(x, \lambda)}^{\Gamma}=\tilde{\beta}^{*} \mathbf{M}_{(x, \beta)}^{\Gamma}$ pois $\tilde{\beta}^{*} \mathbf{M}_{(x, \beta)}^{\Gamma} \subset \mathbf{M}_{(x, \lambda)}^{\Gamma}$ e para $T \in \mathbf{M}_{(x, \lambda)}^{\Gamma}$, definimos $\hat{S}(x, \beta)=\left(\tilde{\Psi}^{*} T\right)(x, \beta)=T(x, \tilde{\Psi}(\beta)) ;$ Claramente, $\hat{S} \in \mathbf{M}_{(x, \beta)}^{\Gamma}$, e $\left(\tilde{\beta}^{*} \hat{S}\right)(x, \lambda)$

$$
=\hat{S}(x, \tilde{\beta}(\lambda))=T(x, \tilde{\Psi} \circ \tilde{\beta}(\lambda))=T(x, \lambda) \text {. }
$$

- $\vec{\Theta}_{(x, \lambda)}^{\Gamma}=\tilde{\beta}^{*} \vec{\Theta}_{(x, \beta)}^{\Gamma}$ pois para $X \in \vec{\Theta}_{(x, \lambda)}^{\Gamma}$, definimos $\hat{\xi}(x, \beta)=\left(\tilde{\Psi}^{*} X\right)(x, \beta)$ $=X(x, \tilde{\Psi}(\beta)) ;$ Obviamente, $\hat{\xi} \in \vec{\Theta}_{(x, \beta)}^{\Gamma}$, e $\tilde{\beta}^{*} \hat{\xi}(x, \lambda)=\hat{\xi}(x, \tilde{\beta}(\lambda))=X(x, \tilde{\Psi} \circ \tilde{\beta}(\lambda))$

$$
=X(x, \lambda) \text {. }
$$


Etapa 2. Seja $\tilde{\xi} \in \hat{\mathcal{P}}$ tal que $\omega_{\tilde{\beta}}(\hat{\xi}) \in \hat{T}_{e}^{\Gamma}\left(\tilde{\beta}^{*} \hat{F}_{o}\right)$. Devemos mostrar que $\tilde{\xi} \in \tilde{\beta}^{*} \hat{N}$.

Note que $\omega_{\tilde{\beta}}(\tilde{\xi})=\omega_{\hat{\alpha}}\left(\pi_{r} \circ \tilde{\xi}\right)=\omega_{\tilde{\alpha}}(\xi)=\sum_{i=1}^{r} \xi_{i} h_{i} \in \hat{\mathcal{T}}_{e}^{\Gamma}\left(\hat{\beta}^{*} \hat{F}_{o}\right)$ implica que para alguma $T \in \mathbf{M}_{(x, \lambda)}^{\Gamma}$ e algum $X \in \vec{\Theta}_{(x, \lambda)}^{\Gamma}$,

$$
\omega_{\bar{\beta}}(\tilde{\xi})(x, \lambda)=T(x, \lambda)\left(\tilde{\beta}^{*} \hat{F}_{o}\right)(x, \lambda)+\left(D_{x}\left(\tilde{\beta}^{*} \hat{F}_{o}\right)\right)(x, \lambda) X(x, \lambda) .
$$

Pela etapa 1,

$\omega_{\tilde{\beta}}(\tilde{\xi})=\tilde{\beta}^{*}\left(\hat{S} \hat{F}_{o}+\left(D_{x} \hat{F}_{o}\right) \hat{\xi}\right) \in \tilde{\beta}^{*}\left(\hat{\mathbf{T}}^{\Gamma} \hat{F}_{o}\right)$ com $\hat{S} \in \mathbf{M}_{(x, \beta)}^{\Gamma}$ e $\hat{\xi} \in \vec{\Theta}_{(x, \beta)}^{\Gamma}$.

Pela proposição 3.2.5.1, $\omega_{\beta}^{-1}\left(\mathbf{T}^{\Gamma} \hat{F}_{0}\right)=\hat{N}$ e podemos escrever $\omega_{\bar{\beta}}(\tilde{\xi}) \in \tilde{\beta}^{*} \omega_{\beta}(\hat{N})$, isto é, existe $\hat{\xi} \in \hat{N}, \hat{\xi}=\sum_{j=1}^{s} \mu_{j} \hat{\eta}_{j}$, tal que

$$
\begin{aligned}
\omega_{\tilde{\beta}}(\tilde{\xi}) & =\tilde{\beta}^{*} \omega_{\beta}(\hat{\xi})=\tilde{\beta}^{*}\left(\sum_{i=1}^{r}\left(\pi_{r} \circ \hat{\xi}\right)_{i} h_{i}\right) \\
& =\tilde{\beta}^{*}\left(\sum_{i=1}^{r}\left(\sum_{j=1}^{s} \mu_{j}\left(\pi_{r} \circ \hat{\eta}_{j}\right)\right)_{i} h_{i}\right. \\
& =\sum_{i=1}^{r} \tilde{\beta}^{*}\left(\sum_{j=1}^{s} \mu_{j}\left(\pi_{r} \circ \hat{\eta}_{j}\right)\right)_{i} h_{i} \\
& \left.=\sum_{i=1}^{r} \tilde{\beta}^{*}\left(\pi_{r} \circ \sum_{j=1}^{s} \mu_{j} \hat{\eta}_{j}\right)_{i}\right) h_{i} \\
& =\sum_{i=1}^{r}\left(\pi_{r} \circ\left(\tilde{\beta}^{*} \sum_{j=1}^{s} \mu_{j} \hat{\eta}_{j}\right)\right)_{i} h_{i} \\
& =\omega_{\hat{\beta}}\left(\tilde{\beta}^{*} \hat{\xi}\right) .
\end{aligned}
$$

Por unicidade, $\tilde{\xi}=\tilde{\beta}^{*} \hat{\xi} \operatorname{com} \hat{\xi} \in \hat{N}$ e assim $\hat{\xi} \in \tilde{\beta}^{*} \hat{N}$.

Portanto, $\omega_{\tilde{\beta}}^{-1}\left(\hat{\mathcal{T}}_{e}^{\Gamma}\left(\tilde{\beta}^{*} F_{o}\right)\right) \subset \tilde{\beta}^{*} \hat{N}$.

(ii) $\omega_{\bar{\beta}}\left(\tilde{\beta}^{*} \hat{N}\right) \subset \hat{T}_{e}^{\Gamma}\left(\tilde{\beta}^{*} \hat{F}_{o}\right)$.

Seja $\hat{\xi} \in \hat{N}$, isto é, $\hat{\xi}=\sum_{j=1}^{s} \mu_{j} \hat{\eta}_{j}$. Pela proposição 3.2.5.1, $\omega_{\beta}(\hat{\xi}) \in \mathrm{T}^{\Gamma}\left(\hat{F}_{o}\right)$ e, assim, podemos escrever $\omega_{\beta}(\hat{\xi})=\hat{S} \hat{F}_{o}+\left(D_{x} \hat{F}_{o}\right) \hat{\xi}^{\prime}$ para alguma $\hat{S} \in \mathbf{M}_{(x, \beta)}^{\Gamma}$ e para algum $\hat{\xi}^{\prime} \in \vec{\Theta}_{(x, \beta)}^{r}$. Segue que,

$$
\begin{aligned}
\omega_{\tilde{\beta}}\left(\tilde{\beta}^{*} \hat{\xi}\right) & =\sum_{i=1}^{r}\left(\pi_{r} \circ \tilde{\beta}^{*} \hat{\xi}\right)_{i} h_{i}=\sum_{i=1}^{r} \tilde{\beta}^{*}\left(\pi_{r} \circ \hat{\xi}\right)_{i} h_{i} \\
& =\tilde{\beta}^{*} \sum_{i=1}^{r}\left(\pi_{r} \circ \hat{\xi}\right)_{i} h_{i}=\tilde{\beta}^{*} \omega_{\beta}(\hat{\xi})=\tilde{\beta}^{*}\left(\hat{S} \hat{F}_{o}+\left(D_{x} \hat{F}_{o}\right) \hat{\xi}^{\prime}\right) \\
& =\left(\tilde{\beta}^{*} \hat{S}\right)\left(\tilde{\beta}^{*} \hat{F}_{o}\right)+\left(D_{x}\left(\tilde{\beta}^{*} F_{o}\right)\right)\left(\tilde{\beta}^{*} \hat{\xi}^{\prime}\right) \\
& =\mathrm{T}\left(\tilde{\beta}^{*} \hat{F}_{o}\right)+\left(D_{x}\left(\tilde{\beta}^{*} F_{0}\right)\right) X
\end{aligned}
$$


para alguma $T \in \mathbf{M}_{(x, \lambda)}^{\Gamma}$ e para algum $X \in \vec{\Theta}_{(x, \lambda)}^{\Gamma}$, pela etapa 1 .

Consequentemente, $\omega_{\tilde{\beta}}\left(\hat{\beta}^{*} \hat{\xi}\right) \in \hat{\mathcal{T}}_{e}^{\Gamma}\left(\tilde{\beta}^{*} \hat{F}_{o}\right)$ e,portanto, $\omega_{\bar{\beta}}\left(\tilde{\beta}^{*} \hat{N}\right) \subset \hat{\mathcal{T}}_{e}^{\Gamma}\left(\tilde{\beta}^{*} \hat{F}_{o}\right)$.

De (i) e (ii), $\omega_{\tilde{\beta}}^{-1}\left(\hat{\mathcal{T}}_{\epsilon}^{\Gamma}\left(\tilde{\beta}^{*} \hat{F}_{o}\right)\right)=\tilde{\beta}^{*} \hat{N}$.

Resta mostrar 2. . Como a demonstração é análoga à de 1., omitiremos os cálculos.

Para mostrar que $\omega_{\tilde{\beta}}^{-1}\left(\hat{\mathcal{T}}^{\Gamma}\left(\tilde{\beta}^{*} \hat{F}_{o}\right)\right)=\tilde{\beta}^{*} \hat{\tilde{N}}$, usamos Proposiçâo 3.2.5.2., a definição de $\hat{\mathcal{T}}^{\Gamma}\left(\hat{F}_{o}\right)$ e escrevemos, via $\tilde{\Psi}$

$$
\begin{aligned}
& \left(\tilde{\beta}^{*} \hat{S}\right)(x, \lambda)=T(x, \lambda) \operatorname{com} \hat{S} \in \mathbf{M}_{(x, \beta)}^{\Gamma} \text { e } T \in \mathbf{M}_{(x, \lambda)}^{\Gamma} \text { e } \\
& \left(\tilde{\beta}^{*} \hat{\xi}\right)(x, \lambda)=X(x, \lambda) \operatorname{com} \hat{\xi} \in \vec{\Theta}_{(x, \beta)}^{\Gamma, o} \text { e } X \in \vec{\Theta}_{(x, \lambda)}^{\Gamma, o} .
\end{aligned}
$$

Note que

$$
\begin{gathered}
\left(\tilde{\beta}^{*} \hat{S}\right)^{o}=0 \quad \Leftrightarrow \quad T^{\circ}=0 \\
\text { e } \\
\left(D_{x} \tilde{\beta}^{*} \hat{\xi}\right)^{o}=0 \Leftrightarrow\left(D_{x} X\right)^{o}=0 .
\end{gathered}
$$

Portanto, a proposição se verifica.

Definição 3.2.10. Para $\tilde{\alpha} \in \mathcal{P}$, definimos oespaço tangente ao caminho $\tilde{\alpha}$ por

$$
\mathcal{T}(\tilde{\alpha})=\tilde{\alpha}^{*} N+\left(D_{\lambda} \tilde{\alpha}\right) \overrightarrow{\mathcal{E}}_{\lambda}^{\Gamma}
$$

e o espaço tangente unipotente à $\tilde{\alpha}$ por

$$
\mathcal{T} \mathcal{U}(\tilde{\alpha})=\tilde{\alpha}^{*} \tilde{N}+\left(D_{\lambda} \tilde{\alpha}\right) \mathcal{M}_{\lambda}^{2} \overrightarrow{\mathcal{E}}_{\lambda}^{\Gamma}
$$

onde $N$ e $\tilde{N}$ foram definidos em (3.1) e (3.3), respectivamente.

Proposição 3.2.11. Seja $\tilde{\alpha} \in \mathcal{P}$ e $\omega_{\tilde{\alpha}}$ como na Definição 3.2.6. Então,

1. $\omega_{\tilde{\alpha}}^{-1}\left(\hat{T}_{e}^{\Gamma}\left(\tilde{\alpha}^{*} F_{o}\right)\right)=\tilde{\alpha}^{*} N$.

2. $\omega_{\tilde{\alpha}}^{-1}\left(\hat{\mathcal{U}}^{\Gamma}\left(\tilde{\alpha}^{*} F_{o}\right)\right)=\tilde{\alpha}^{*} \tilde{N}$

Prova. Segue da Proposição 3.2.9. pois

(i) $\tilde{\alpha}^{*} F_{o}=\tilde{\beta}^{*} \hat{F}_{o}^{\prime} \quad$ (veja pág. 79).

(ii) $\omega_{\tilde{\beta}}(\tilde{\xi})=\omega_{\tilde{\alpha}}\left(\pi_{r} \circ \tilde{\xi}\right)$, pela definição 3.2 .8 . 
(iii) $\hat{\alpha}^{*} N=\tilde{\beta}^{*}\left(\pi_{r} \circ \hat{N}\right)$ e $\hat{\alpha}^{*} \tilde{N}=\hat{\beta}^{*}\left(\pi_{r} \circ \hat{\hat{N}}\right)$ com $\hat{N^{\prime}}, \hat{\hat{N}}$ dados por (3.2), (3.4), respectivamente, e $\pi_{r} \circ \hat{N}, \pi_{r} \circ \hat{\hat{N}}$ denotam os seguintes $\mathcal{E}_{\beta}^{\Gamma}$-módulos:

$$
\begin{aligned}
& \pi_{r} \circ \hat{N}=\mathcal{E}_{\beta}^{\Gamma}<\pi_{r} \circ \hat{\eta}_{1} \ldots \pi_{r} \circ \hat{\eta}_{s}> \\
& \pi_{\tau} \circ \hat{\tilde{N}}=\mathcal{E}_{\beta}^{\Gamma}<\pi_{r} \circ \hat{\tilde{\eta}}_{1} \ldots \pi_{r} \circ \hat{\tilde{\eta}}_{s}>
\end{aligned}
$$

\section{Proposição 3.2.12.}

1. $\omega_{\tilde{\alpha}}^{-1}\left(\mathcal{T}_{e}^{\Gamma}\left(\tilde{\alpha}^{*} F_{o}\right)\right)=\mathcal{T}(\tilde{\alpha})$.

2. $\omega_{\tilde{\alpha}}^{-1}\left(\mathcal{T} \mathcal{U}^{\Gamma}\left(\tilde{\alpha}^{*} F_{o}\right)\right)=\mathcal{T U}(\tilde{\alpha})$

Prova. Mostramos 1. em duas estapas:

(i) $\mathcal{T}(\tilde{\alpha}) \subset \omega_{\tilde{\alpha}}^{-1}\left(\mathcal{T}_{e}^{\Gamma}\left(\tilde{\alpha}^{*} F_{o}\right)\right)$.

Podemos decompor $\tilde{\xi} \in \mathcal{T}(\tilde{\alpha})$ como $\tilde{\xi}=\xi_{1}+\xi_{2} \operatorname{com} \xi_{1} \in \hat{\alpha}^{*} \dot{N}^{\prime}$ e $\xi_{2} \in\left(D_{\lambda} \tilde{\alpha}\right) \overrightarrow{\mathcal{E}}_{\lambda}^{\Gamma}$. Usando a Proposição 3.2.11.1, $\omega_{\tilde{\alpha}}\left(\xi_{1}\right) \in \hat{\mathcal{T}}_{e}^{\Gamma}\left(\tilde{\alpha}^{*} F_{o}\right)$.

Seja $\xi_{2}=\left(L_{1} \ldots L_{r}\right)$ onde $L_{i}=\sum_{j=1}^{\ell} \frac{\partial \hat{\alpha}_{i}}{\partial \lambda_{j}} \Lambda_{j}$. Entã.o,

$$
\begin{aligned}
\omega_{\bar{\alpha}}\left(\xi_{2}\right)(x, \lambda) & =\sum_{i=1}^{r} L_{i}(\lambda) h_{i}(x)=D_{\alpha} F_{o}(x, \tilde{\alpha}(\lambda)) D_{\lambda} \hat{\alpha}(\lambda) \Lambda(\lambda) \\
& =D_{\lambda}\left(\tilde{\alpha}^{*} F_{o}\right)(x, \lambda) \Lambda(\lambda)
\end{aligned}
$$

Consequentemente, $\omega_{\tilde{\alpha}}\left(\xi_{2}\right)=\left(D_{\lambda}\left(\tilde{\alpha}^{*} F_{o}\right)\right) \circ \Lambda$ para algum $\Lambda \in \overrightarrow{\mathcal{E}}_{\lambda}^{\Gamma}$. Segue da linearidade de $\omega_{\bar{\alpha}}$ que $\omega_{\bar{\alpha}}(\tilde{\xi}) \in \mathcal{T}_{e}^{\Gamma}\left(\tilde{\alpha}^{*} F_{0}\right)$. Portanto, (i) se verifica.

(ii) Seja $\tilde{\xi} \in \mathcal{P}$ e suponhamos que $\omega_{\tilde{\alpha}}(\tilde{\xi})=\zeta \in \mathcal{T}_{e}^{\Gamma}\left(\tilde{\alpha}^{*} F_{o}\right)$. Temos que mostrar que $\tilde{\xi} \in \mathcal{T}(\tilde{\alpha})$. Podemos decompor $\mathrm{T}_{e}^{\Gamma}\left(\tilde{\alpha}^{*} F_{o}\right)=\hat{\mathrm{T}}_{e}^{\Gamma}\left(\tilde{\alpha}^{*} \dot{F}_{o}\right)+\left\{\left(D_{\lambda}\left(\tilde{\alpha}^{*} F_{o}\right)\right) \circ \Lambda \mid \Lambda \in \overrightarrow{\mathcal{E}}_{\lambda}^{\Gamma}\right\} \mathrm{e}$ $\zeta=\zeta_{1}+\zeta_{2}$. Afirmamos que existe $\xi_{2} \in\left(D_{\lambda} \tilde{\alpha}\right) \overrightarrow{\mathcal{E}}_{\lambda}^{\Gamma}$ tal que $\omega_{\tilde{\alpha}}\left(\xi_{2}\right)=\zeta_{2}$. De fato,

$$
\begin{aligned}
& \zeta_{2}=\left(D_{\lambda}\left(\tilde{\alpha}^{*} F_{o}\right)\right) \circ \Lambda \text { para algum } \Lambda \in \overrightarrow{\mathcal{E}}_{\lambda}^{\Gamma} \quad \text { e, portanto, } \\
& \zeta_{2}=\left(D_{\lambda}\left(\tilde{\alpha}^{*} F_{o}\right)\right) \circ \Lambda=\sum_{i=1}^{r}\left(\sum_{j=1}^{\ell} \frac{\partial \tilde{\alpha}_{i}}{\partial \lambda_{j}} \Lambda_{j}\right) h_{i}=\omega_{\tilde{\alpha}}\left(\left(D_{\lambda} \tilde{\alpha}\right) \circ \Lambda\right) .
\end{aligned}
$$

Logo, existe $\xi_{2}$ satisfazendo a afirmação. 
Da linearidade de $\omega_{\bar{\alpha}}, \zeta_{1}=\omega_{\hat{\alpha}}\left(\dot{\xi}-\xi_{2}\right)$ e pela Proposição $3.2 .11, \hat{\xi}-\xi_{2} \in \hat{\alpha}^{*} N$ e, como consequência, $\omega_{\tilde{\alpha}}^{-1}\left(\mathcal{T}_{e}^{\Gamma}\left(\tilde{\alpha}^{*} F_{o}\right)\right) \subset \mathcal{T}(\tilde{\alpha})$.

De (i) e (ii), $\omega_{\tilde{\alpha}}^{-1}\left(\mathcal{T}_{\epsilon}^{\Gamma}\left(\tilde{\alpha}^{*} F_{o}\right)\right)=\mathcal{T}(\tilde{\alpha})$.

A prova de 2. é análoga à de 1. e basta usar a Proposição 3.2.11.2. e as definiçôes dos espaços tangentes unipotentes à $\tilde{\alpha}^{*} F_{0}$ e $\tilde{\alpha}$.

Denotamos por $\mathbf{P}(\tilde{\alpha})$, os termos de ordem alta de $\tilde{\alpha} \in \mathcal{P}$ e definimos

Definição 3.2.13. $\xi \in \mathbf{P}(\tilde{\alpha})$ se e somente se $\omega_{\tilde{\alpha}}(\xi) \in \mathbf{P}\left(\tilde{\alpha}^{*} F_{o}\right)$.

Corolário 3.2.14. Seja $\xi \in \mathcal{T} \mathcal{U}(\tilde{\alpha})$. Se $\omega_{\tilde{\alpha}}(\xi) \in \operatorname{Intr} \mathcal{T} \mathcal{U}^{\Gamma}\left(\tilde{\alpha}^{*} F_{o}\right)$ então $\xi \in \mathbf{P}(\tilde{\alpha})$.

Prova. Como $\xi \in \mathcal{T U}(\hat{\alpha}), \omega_{\dot{\alpha}} \in \mathcal{T} \mathcal{U}^{\Gamma}\left(\tilde{\alpha}^{*} F_{o}\right)$ pela Proposição 3.2.12.2. Por hipótese, $\omega_{\tilde{\alpha}}(\xi) \in \operatorname{Intr} \mathcal{T} \mathcal{U}^{\Gamma}\left(\tilde{\alpha}^{*} F_{o}\right)$ e, portanto, $\omega_{\tilde{\alpha}}(\xi) \in \mathbf{P}\left(\hat{\alpha}^{*} F_{o}\right)$ pela Proposição 1.10.3.1. Da Definiçâ.o 3.2.13., $\xi \in \mathbf{P}(\tilde{\alpha})$.

Definição 3.2.15. Definimos o espaço normal estendido $\grave{a} \hat{\alpha} \in \mathcal{P}$ por

$$
\mathcal{N}(\tilde{\alpha})=\frac{\mathcal{P}}{\mathcal{T}(\tilde{\alpha})} .
$$

Teorema 3.2.16. $\omega_{\tilde{\alpha}}$ induz um isomorfismo de espaço vetorial real entre $\mathcal{N}(\tilde{\alpha})$ e $\mathcal{N}_{\epsilon}^{\Gamma}\left(\tilde{\alpha}^{*} F_{o}\right)$

Além disso, se $\left\{\phi_{i}\right\} \subset \mathcal{P}$ se projetar sobre uma base de $\mathcal{N}(\tilde{\alpha})$ então $\left\{\omega_{\tilde{\alpha}}\left\{\phi_{i}\right\}\right\}$ se projetará sobre uma base de $\mathcal{N}_{e}^{\Gamma}\left(\tilde{\alpha}^{*} F_{o}\right)$.

Prova. Considere o seguinte diagrama:

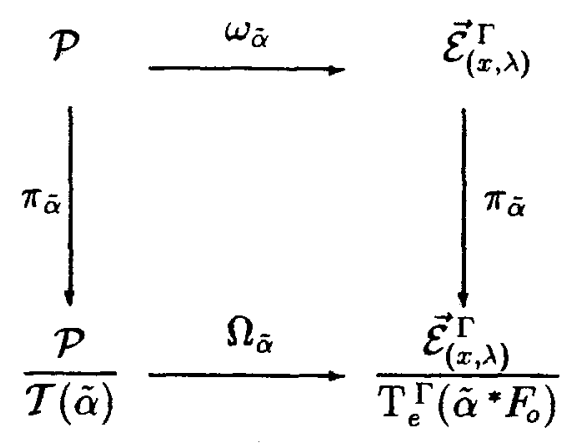


Definimos $\quad \Omega_{\tilde{\alpha}}: \mathcal{N}(\tilde{\alpha}) \rightarrow \mathcal{N}_{e}^{\Gamma}\left(\tilde{\alpha}^{*} F_{o}\right) \quad$ por $\quad \Omega_{\tilde{\alpha}}([v])=\left[\omega_{\tilde{\alpha}}(v)\right]$.

(i) $\Omega_{\tilde{\alpha}}$ é bem definida, ou seja, $\left[v_{1}\right]=\left[v_{2}\right]$ implica $\Omega_{\tilde{\alpha}}\left(\left[v_{1}\right]\right)=\Omega_{\tilde{\alpha}}\left(\left[v_{2}\right]\right)$.

Note que se $\left[v_{1}\right]=\left[v_{2}\right]$ entâo $v_{1}-v_{2} \in \mathcal{T}(\tilde{\alpha})$ e, portanto, $\omega_{\tilde{\alpha}}\left(v_{3}-v_{2}\right) \in \mathcal{T}_{e}^{\Gamma}\left(\tilde{\alpha}^{*} F_{o}\right)$ pela Proposição 3.2.12.1. Logo, $\left[\omega_{\tilde{\alpha}}\left(v_{1}\right)\right]=\left[\omega_{\tilde{\alpha}}\left(v_{2}\right)\right]$.

(ii) $\Omega_{\tilde{\alpha}}$ é linear pois $\omega_{\tilde{\alpha}}$ e as projeções são lineares.

(iii) $\Omega_{\tilde{\alpha}}$ é injetora pois se $\Omega_{\tilde{\alpha}}\left(\left[v_{1}\right]\right)=\Omega_{\tilde{\alpha}}\left(\left[v_{2}\right]\right)$ então $\omega_{\bar{\alpha}}\left(v_{1}\right)-\omega_{\tilde{\alpha}}\left(v_{2}\right) \in \mathcal{T}_{e}^{\Gamma}\left(\hat{\alpha}^{*} F_{o}\right)$. Pela Proposição 3.2.12.1, $\left(v_{1}-v_{2}\right) \in \omega_{\tilde{\alpha}}^{-1}\left(\mathcal{T}_{e}^{\Gamma}\left(\tilde{\alpha}^{*} F_{o}\right)\right)=\mathcal{T}(\tilde{\alpha})$ e,portanto, $\left[v_{1}\right]=\left[v_{2}\right]$.

(iv) $\Omega_{\bar{\alpha}}$ é sobrejetora.

$$
\mathrm{I}_{m} \Omega_{\tilde{\tilde{\alpha}^{*}}}=\left\{[f] \in \mathcal{N}_{e}^{\Gamma}\left(\tilde{\alpha}^{*} F_{o}\right) \mid[f]=\Omega_{\tilde{\alpha}}([v]) \text { para alguma }[v] \in \mathcal{N}(\tilde{\alpha})\right\}
$$

Como $\mathrm{I}_{m} \Omega_{\tilde{\alpha}} \subset \mathcal{N}_{e}^{\Gamma}\left(\tilde{\alpha}^{*} F_{o}\right)$, resta mostrar que $\mathcal{N}_{e}^{\Gamma}\left(\tilde{\alpha}^{*} F_{o}\right) \subset \mathrm{I}_{m} \Omega_{\tilde{\alpha}}$.

Seja $[f] \in \mathcal{N}_{e}\left(\tilde{\alpha}^{*} F_{o}\right)$. Usando a definição de $\Omega_{\tilde{\alpha}},[f]=\pi_{\tilde{\alpha}}\left(\omega_{\tilde{\alpha}}(\xi)\right)=\left(\Omega_{\tilde{\alpha}} \circ \pi_{\tilde{\alpha}}\right)(\xi)$ $=\Omega_{\tilde{\alpha}}([\xi])$ para algum $\xi \in \mathcal{P}$, ou seja, $[f] \in \mathrm{I}_{m} \Omega_{\tilde{\alpha}}$.

Portanto, $\Omega_{\tilde{\alpha}}$ é um isomorfismo entre $\mathcal{N}_{\mathrm{e}}(\hat{\alpha})$ e $\mathcal{N}_{e}^{\Gamma}\left(\tilde{\alpha}^{*} F_{o}\right)$. A outra afirmaçầo do teorema segue da Proposição 3.2.12.1. pois $\omega_{\tilde{\alpha}}^{-1}\left(\mathcal{T}_{e}^{\Gamma}\left(\tilde{\alpha}^{*} F_{o}\right)\right)=\mathcal{T}(\tilde{\alpha})$.

Definição 3.2.17. Para $\tilde{\alpha} \in \mathcal{P}, \operatorname{cod}^{\Gamma}(\tilde{\alpha})=\operatorname{dim}_{\mathbf{R}} \frac{\mathcal{P}}{\mathcal{T}(\tilde{\alpha})}$.

Corolário 3.2.18. $\operatorname{cod}^{\Gamma}\left(\tilde{\alpha}^{*} F_{o}\right)$ é finita se e somente se $\operatorname{cod}^{\Gamma}(\tilde{\alpha})$ é finita.

Prova. É uma consequência imediata do Teorema 3.2.16.

Observação 3.2.19. Note que se $\operatorname{cod}^{\Gamma}(f)$ e $\operatorname{cod}^{\Sigma}\left(f_{0}\right)$ forem finitas então $\operatorname{cod}^{\Gamma}\left(f_{0}\right)$ será finita.

De fato, seja $W=\frac{\overrightarrow{\mathcal{E}}_{(x, \lambda)}^{\Gamma}}{\mathcal{T}_{e}^{\Gamma}\left(f_{0}\right)}$. Como $\operatorname{cod}^{\Gamma}(f)$ é finita, $W$ é um espaço vetorial real finitamente gerado e

$$
\frac{W}{\mathcal{M}_{\lambda}^{\Gamma} \cdot W}=\mathcal{N}_{e}^{\Gamma}\left(f_{0}\right) \subset \mathcal{N}_{e}^{\Sigma}\left(f_{0}\right)
$$

Portanto, $\operatorname{cod}^{\Gamma}\left(f_{0}\right)$ é finita pois $\operatorname{cod}^{\Gamma}\left(f_{0}\right) \leq \operatorname{cod}^{\Sigma}\left(f_{0}\right)<\infty$.

Observação 3.2.20. Como mencionado na pág. 73, a $\Sigma$-teoria para a formulação de caminhos de problemas de bifurcação multiparamétricos com simetria nas variáveis -padrão e também nos parâmetros é válida se a hipótese $(\mathrm{H} 0): \operatorname{cod}^{\Sigma}\left(\mathrm{f}_{0}\right)<\infty$ for verdadeira. 
O exemplo a seguir mostra que

$$
\operatorname{cod}^{\Gamma}(f)<\infty \text { não implica que } \operatorname{cod}^{\Sigma}\left(f_{0}\right)<\infty
$$

Exemplo. Seja $O(2)$ agindo em $\mathbb{C}^{2}$ por

$$
\begin{aligned}
& \theta \cdot(z, \lambda)=\left(e^{i \theta} z, \epsilon^{i \theta} \lambda\right) \\
& \kappa \cdot(z, \lambda)=(\bar{z}, \bar{\lambda})
\end{aligned}
$$

- $\mathcal{E}_{(z, \lambda)}^{O(2)}=\left\{g:\left(\mathbb{C}^{2}, 0\right) \rightarrow \mathbb{R} \mid g(\theta \cdot(z, \lambda))=g(z, \lambda)\right.$ e $\left.g(\kappa \cdot(z, \lambda))=g(z, \lambda)\right\}$ é o anel dos germes $O(2)$-invariantes. Para cada $g \in \mathcal{E}_{(z, \lambda)}^{O(2)}$ existe $p:\left(\mathbb{R}^{3}, 0\right) \rightarrow \mathbb{R}$ tal que $g(z, \lambda)=p(u, v, \omega)$ onde $u=z \bar{z} ; v=\lambda \bar{\lambda}$ e $\omega=z \bar{\lambda}+\bar{z} \lambda$. (cf. [16], pág. 339).

- $\overrightarrow{\mathcal{E}}_{(z, \lambda)}^{O(2)}=\left\{f:\left(\mathbb{C}^{2}, 0\right) \rightarrow \mathbf{C} \mid f(\theta \cdot(z, \lambda))=\theta \cdot f(z, \lambda)\right.$ e $\left.f(\kappa \cdot(z, \lambda))=\kappa \cdot f(z, \lambda)\right\}$ é o $\mathcal{E}_{(z, \lambda)}^{O(2)}$-módulo dos germes de aplicação $O(2)$-equivariantes.

De ([16], Ex. 5.1, pág. 53). toda $f:\left(\mathbb{C}^{2}, 0\right) \rightarrow \mathbb{C}$ satisfazendo $f\left(e^{i \theta} z, e^{i \theta} \lambda\right)=e^{i \theta} f(z, \lambda)$ pode ser escrita como

$$
f(z, \lambda)=p_{1}(u, v, \omega) z+p_{2}(u, v, \omega) i z+p_{3}(u, v, \omega) \lambda+p_{4}(u, v, \omega) i \lambda
$$

Usando que $f(\bar{z}, \bar{\lambda})=\kappa \cdot f(z, \lambda)$, obtemos $p_{2} \equiv p_{4} \equiv 0$. Consequentemente, $\overrightarrow{\mathcal{E}}_{(z, \lambda)}^{O(2)}$ é o $\mathcal{E}_{(z, \lambda)}^{O(2)}$-módulo gerado por $z$ e $\lambda$, isto é,

$$
f(z, \lambda)=p(u, v, \omega) z+q(u, v, \omega) \lambda
$$

Denotamos, também, $f=[p, q]$.

- Considerando a ação de $O(2)$ em $\lambda \in \mathbb{C}$ somente,

$$
\overrightarrow{\mathcal{E}}_{\lambda}^{O(2)}=\left\{\Lambda:(\mathbf{C}, 0) \rightarrow \mathbb{C} \mid \Lambda\left(e^{i \theta} \lambda\right)=e^{i \theta} \Lambda(\lambda), \Lambda(\kappa \lambda)=\kappa \Lambda(\lambda)\right\}
$$

Toda aplicação $O(2)$-equivariante $\Lambda \in \overrightarrow{\mathcal{E}}_{\lambda}^{O(2)}$ é da forma

$$
\Lambda(\lambda)=\xi(\lambda \bar{\lambda}) \lambda=\xi(v) \lambda
$$

para algum $\xi \in \mathcal{E}_{v}$.

Para a prova veja ([16], pág. 52). 
- $\mathbf{M}_{(z, \lambda)}^{O(2)}=\left\{S:\left(\mathbb{C}^{2}, 0\right) \rightarrow G L(2) \mid S(\theta \cdot(z, \lambda)) \cdot \theta=\theta \cdot S(z, \lambda) \mathrm{e}\right.$

$$
S(\kappa \cdot(z, \lambda)) \cdot \kappa=\kappa \cdot S(z, \lambda)\}
$$

é o $\mathcal{E}_{(z, \lambda)}^{O(2)}$-módulo gerado pelas seguintes aplicações lineares em $\mathbf{C}$ :

$$
\begin{aligned}
& S_{1}(z, \lambda) w=w, \quad S_{4}(z, \lambda) w=z \lambda \bar{w} \\
& S_{2}(z, \lambda) w=\omega_{1} w, \quad S_{5}(z, \lambda) w=\lambda^{2} \bar{w} . \\
& S_{3}(z, \lambda) w=z^{2} \bar{w}
\end{aligned}
$$

onde $\omega_{1}=z \bar{\lambda}-\bar{z} \lambda$.

Para provar essa afirmação, escrevemos a aplicação linear $S(z, \lambda)$ na forma

$$
S(z, \lambda) w=f_{1}(z, \lambda) w+f_{2}(z, \lambda) \bar{w} .
$$

Usando as condições de equivariância: $S(\theta \cdot(z, \lambda)) \cdot \theta=\theta \cdot S(z, \lambda)$ e $S(\kappa \cdot(z, \lambda)) \cdot \kappa$ $=\kappa \cdot S(z, \lambda)$, obtemos as relações:

$$
\begin{aligned}
f_{1}(\theta \cdot(z, \lambda)) & =f_{1}(z, \lambda), \\
f_{2}(\theta \cdot(z, \lambda)) & =2 \theta \cdot f_{2}(z, \lambda), \\
f_{i}(\bar{z}, \bar{\lambda}) & =\kappa \cdot f_{i}(z, \lambda), i=1,2 .
\end{aligned}
$$

$f_{1}$ é $\mathbf{S}^{1}$-invariante e, portanto, podemos escrever $f_{1}(z, \lambda)=f_{1}\left(u, v, \omega, \omega_{1}\right)$.

Como $\omega_{1}^{2}-\omega^{2}=4 u v$, decompomos $f_{1}$ da seguinte maneira:

$$
f_{1}\left(u, v, \omega, \omega_{1}\right)=f_{11}(u, v, \omega)+\omega_{1} f_{12}(u, v, \omega) .
$$

Em particular, $f_{1 i}, i=1,2$, são $\kappa$-invariantes e, portanto, $f_{1 i}, i=1,2$, têm valores reais. Logo,

$$
f_{1}(z, \lambda) w=f_{11}(u, v, \omega) w+f_{12}(u, v, \omega) \omega_{1} w .
$$

Resta analisar as condições de equivariância de $f_{2}$, isto é,

$$
\begin{aligned}
f_{2}(\theta \cdot(z, \lambda)) & =2 \theta \cdot f_{2}(z, \lambda) \\
f_{2}(\bar{z}, \bar{\lambda}) & =\kappa \cdot f_{2}(z, \lambda) .
\end{aligned}
$$

Os invariantes para esta ação são $u, v, \omega$ e $\omega_{1}$. 
É suficiente achar os geradores para o módulo dos polinômios equivariantes, os quais são somas finitas de elementos da forma $a_{i j k \ell} z^{i} \bar{z}^{j} \lambda^{k} \bar{\lambda}^{\ell}, i, j, k, \ell \geq 0$. De (3.11), $a_{i j k \ell}$ é um número real. Usando os geradores dos invariantes e (3.10), obtemos

$$
f_{2}(z, \lambda) w=f_{21}(u, v, \omega) z^{2} \bar{w}+f_{22}(u, v, \omega) z \lambda \bar{w}+f_{23}(u, v, \omega) \lambda^{2} \bar{w}
$$

Substituindo (3.10) e (3.13) em (3.9), segue o resultado.

- O espaço tangente estendido à $f=[p, q] \in \overrightarrow{\mathcal{E}}_{(z, \lambda)}^{O(2)}$ é dado por

$$
\mathcal{T}_{e}^{O(2)}(f)=\left\{S f+\left(D_{x} f\right) X+\left(D_{\lambda} f\right) \Lambda \mid S \in \mathbf{M}_{(z, \lambda)}^{O(2)}, X \in \overrightarrow{\mathcal{E}}_{(z, \lambda)}^{O(2)}, \Lambda \in \overrightarrow{\mathcal{E}}_{\lambda}^{O(2)}\right\}
$$

Calculando a expressão acima,

$$
\mathcal{T}_{e}^{O(2)}(f)=\mathcal{E}_{(z, \lambda)}^{O(2)}<g_{1} \ldots g_{7}>+\mathcal{E}_{\lambda}^{O(2)}<g_{8}>
$$

onde

$$
\begin{array}{ll}
g_{1}=[p, q], & g_{5}=[-v p, \omega p+v q], \\
g_{2}=[\omega p+2 v q,-2 u p-\omega q], & g_{6}=\left[p+2 u p_{u}+\omega p_{\omega}, 2 u q_{u}+\omega q_{\omega}\right], \\
g_{3}=[u p+\omega q,-u q], & g_{7}=\left[\omega p_{u}+2 v p_{\omega}, p+\omega q_{u}+2 v q_{\omega}\right], \\
g_{4}=[v q, u p], & g_{8}=\left[2 v p_{v}+\omega p_{\omega}, q+2 v q_{v}+\omega q_{\omega}\right] .
\end{array}
$$

O exemplo é dado pelo diagrama de bifurcação genérico

$$
\begin{aligned}
& f:\left(\mathbb{C}^{2}, 0\right) \rightarrow \mathbb{C} \\
& f(z, \lambda)=\epsilon u z-\delta \lambda,
\end{aligned}
$$

onde $\epsilon^{2}=\delta^{2}=1$. Usando $(3.14)$,

$$
\mathcal{T}_{e}^{O(2)}(f)=[\mathcal{M}, \mathcal{E}]
$$

Portanto,

$$
\operatorname{cod}^{O(2)}(f)=\operatorname{dim}_{\mathbb{R}} \frac{\overrightarrow{\mathcal{E}}_{(z, \lambda)}^{O(2)}}{\mathcal{T}_{e}^{O(2)}(f)}=1 .
$$

O centro organizador de $f$ é dado por $f_{0}(z)=u z$. O subgrupo de isotropia de $\lambda$ é $\Sigma=1$, ou seja, $\Sigma$ é o grupo trivial. Logo, o $\Sigma$-espaço tangente estendido de $f_{0}$ é igual ao espaço tangente de contato de $f_{0}$, isto é, $\operatorname{cod}^{\Sigma}\left(f_{0}\right)=\operatorname{cod}^{x}\left(f_{0}\right)$. 
Pela Proposição 2.4 ([3], pág. 494), nas dimensões consideradas, todo germe $\mathcal{K}$-finito é $\mathcal{C}$-finito e usando o critério geométrico para que um germe seja $\mathcal{C}$-finito, temos que $f_{0}$ tem $\Sigma$-codimensão infinita pois $f_{0}^{-1}(0)=\{x= \pm y i\}$ para $z=x+y i$.

$\mathrm{O}$ fato de $f$ ser a forma genérica segue da teoria geral do capítulo 1 . Um cálculo direto mostra que $\mathcal{M}$ e $\langle v, w\rangle$ são ideajs intrínsicos e que $[I, J]$ é um submódulo intrínsico se e somente se $I$ e $J$ forem ideais intrínsicos e $\langle v, w\rangle J \subset I \subset J$. Para $[\epsilon u, \delta]$, com $\epsilon^{2}=\delta^{2}=1$, o conjunto de termos de ordem alta é dado por $\left[\mathcal{M}^{2}+<v, w>\mathcal{M}, \mathcal{M}\right]$. Assim, sendo $p_{u}^{o} \neq 0$ e $q^{o} \neq 0$, um simples reescalonamento mostra que $[p, q]$ é equivalente por contato à $f \operatorname{com} \epsilon=s g p_{u}^{o}$ e $\delta=s g q^{\circ}$.

\subsection{Formulação por caminhos e o problema $D_{4}$}

Seja $\Gamma_{1}$ um grupo de Lie compacto agindo em $\mathbb{R}^{n+r}$ e $\mathbb{R}^{m}$, vja representações ortogonais, onde a ação de $\Gamma_{1}$ em $(x, \alpha) \in \mathbb{R}^{n+r}$ pode ser separada da seguinte maneira:

$$
\begin{aligned}
& \gamma^{1} \cdot(x, \alpha)=\left(\gamma_{n}^{1} x, \gamma_{r}^{1} \alpha\right) \\
& \nu \cdot(x, \alpha)=\left(\nu_{n} x, \nu_{r} \alpha\right), \nu^{2}=1
\end{aligned}
$$

Seja $\tilde{\Gamma}=\Gamma_{1} \times \mathbf{Z}_{2}$ agindo em $\mathbb{R}^{n+\ell+1}$ por

$$
\begin{aligned}
& \left(\gamma^{1}, \mathbf{1}\right) \cdot(x, \alpha, \delta)=\left(\gamma_{n}^{1} x, \gamma_{r}^{1} \alpha, \delta\right) \\
& (\nu,-1) \cdot(x, \alpha, \delta)=\left(\nu_{n} x, \nu_{r} \alpha,-\delta\right)
\end{aligned}
$$

A ação em $y \in \mathbb{R}^{m}$ é denotada por $\gamma_{m}^{1} y$ e $\nu_{m} y$.

Definimos

$$
\begin{aligned}
& \overrightarrow{\mathcal{E}}_{(x, \alpha)}^{\tilde{\Gamma}}=\left\{F:\left(\mathbb{R}^{n+r}, 0\right) \rightarrow \mathbb{R}^{m} \mid F\left(\gamma_{n}^{1} x, \gamma_{r}^{1} \alpha\right)=\gamma_{m}^{1} F(x, \alpha),\right. \\
& \left.\quad F\left(\nu_{n} x, \nu_{r} \alpha\right)=\nu_{m} F(x, \alpha)\right\} . \\
& \overrightarrow{\mathcal{E}}_{(x, \alpha, \delta)}^{\bar{\Gamma}}=\left\{L:\left(\mathbb{R}^{n+r+1}, 0\right) \rightarrow \mathbb{R}^{m} \mid F\left(\gamma_{n}^{1} x, \gamma_{\tau}^{1} \alpha, \delta\right)=\gamma_{m}^{1} F(x, \alpha, \delta),\right. \\
& \left.F\left(\nu_{n} x, \nu_{r} \alpha,-\delta\right)=\nu_{m} F(x, \alpha, \delta)\right\} . \\
& \overrightarrow{\mathcal{E}}_{(x, \alpha)}^{\bar{\Gamma}} \text { e } \overrightarrow{\mathcal{E}}_{(x, \alpha, \delta)}^{\tilde{\Gamma}} \text { são módulos sobre os correspondentes anéis de invariantes. }
\end{aligned}
$$


Pelo Lema $5.11([1])$, as seguintes igualdades sâo equivalentes

$$
\begin{aligned}
\overrightarrow{\mathcal{E}}_{x}^{\Sigma} & =\mathbf{M}_{x}^{\Sigma} f_{0}+\left(D_{x} f_{0}\right) \overrightarrow{\mathcal{E}}_{x}^{\Sigma}+\mathbb{R}\left\{\frac{\partial L}{\partial \alpha_{1}}(x, 0) \ldots \frac{\partial L}{\partial \alpha_{r}}(x, 0)\right\} \\
\overrightarrow{\mathcal{E}}_{(x, \beta)}^{\Sigma} & =\mathbf{M}_{(x, \beta)}^{\Sigma} L+\left(D_{x} L\right) \overrightarrow{\mathcal{E}}_{(x, \beta)}^{\Sigma}+\mathcal{E}_{\beta}\left\{\frac{\partial L}{\partial \alpha_{1}} \ldots \frac{\partial L}{\partial \alpha_{r}}\right\} .
\end{aligned}
$$

Como

$$
-\frac{\partial L}{\partial \lambda_{2}}=-\frac{\partial K}{\partial \lambda_{2}}=-\frac{\partial f}{\partial \lambda_{2}} \in \overrightarrow{\mathcal{E}}_{(x, \beta)}^{\Sigma},
$$

existem $X \in \overrightarrow{\mathcal{E}}_{(x, \beta)}^{\Sigma}, S \in \mathbf{M}_{(x, \beta)}^{\Sigma}$ e $\xi \in \overrightarrow{\mathcal{E}}_{\beta, \alpha}$ such that

$$
-\frac{\partial L}{\partial \lambda_{2}}(x, \beta)=\sum_{j=1}^{2} X_{j}(x, \beta) \frac{\partial L}{\partial x_{j}}(x, \beta)+\sum_{i=1}^{r} \xi_{i}(\beta) \frac{\partial L}{\partial \alpha_{i}}(x, \beta)+S(x, \beta) L(x, \beta) .
$$

Consequentemente,

$$
-\frac{\partial L}{\partial \lambda_{2}}(x, \beta)=L_{x}(x, \beta) X(x, \beta)+L_{\alpha}(x, \beta) \xi(\beta)+S(x, \beta) L(x, \beta)
$$

onde $X=\left(X_{1}, X_{2}\right)$ e $\xi=\left(\xi_{1} \ldots \xi_{r}\right)$.

Além disso,

(i) $f_{\lambda_{2}}\left(\kappa x, \gamma_{r}^{\kappa} \alpha, \lambda_{2}\right)=\kappa f_{\lambda_{2}}\left(x, \alpha, \lambda_{2}\right)$,

$f_{\lambda_{2}}\left(\mu x, \gamma_{r}^{\mu} \alpha,-\lambda_{2}\right)=\mu f_{\lambda_{2}}\left(x, \alpha, \lambda_{2}\right)$.

(ii) $L_{x}\left(\kappa x, \gamma_{r}^{\kappa} \alpha, \lambda_{2}\right)=\kappa L_{x}\left(x, \alpha, \lambda_{2}\right) \kappa$,

$L_{x}\left(\mu x, \gamma_{r}^{\mu} \alpha,-\lambda_{2}\right)=\mu L_{x}\left(x, \alpha, \lambda_{2}\right) \mu$,

(iii) $L_{\alpha}\left(\kappa x, \gamma_{r}^{\kappa} \alpha, \lambda_{2}\right)=\kappa L_{\alpha}\left(x, \alpha, \lambda_{2}\right)\left(\gamma_{r}^{\kappa}\right)^{t}$,

$$
L_{\alpha}\left(\mu x, \gamma_{r}^{\mu} \alpha,-\lambda_{2}\right)=\mu L_{\alpha}\left(x, \alpha, \lambda_{2}\right)\left(\gamma_{r}^{\mu}\right)^{t} \text {. }
$$

Calculando a média de $(3.20)$ sobre $\mathrm{D}_{4}$, temos

a) $\int_{\mathrm{D}_{4}}-\kappa \frac{\partial L}{\partial \lambda_{2}}\left(\kappa x, \gamma_{r}^{\kappa} \alpha, \lambda_{2}\right)=\int_{\mathrm{D}_{4}} \kappa L_{x}\left(\kappa x, \gamma_{r}^{\kappa} \alpha, \lambda_{2}\right) X\left(\kappa x, \gamma_{r}^{\kappa} \alpha, \lambda_{2}\right)$

$$
+\int_{\mathrm{D}_{4}} \kappa L_{\alpha}\left(\kappa x, \gamma_{r}^{\kappa} \alpha, \lambda_{2}\right) \xi\left(\gamma_{r}^{\kappa} \alpha, \lambda_{2}\right)+\int_{\mathrm{D}_{4}} \kappa S\left(\kappa x, \gamma_{r}^{\kappa} \alpha, \lambda_{2}\right) L\left(\kappa x, \gamma_{r}^{\kappa} \alpha, \lambda_{2}\right),
$$

e assim

$$
\begin{aligned}
-\frac{\partial L}{\partial \lambda_{2}}(x, \beta)=L_{x}(x, \beta) \int_{\mathrm{D}_{4}} \kappa X\left(\kappa x, \gamma_{r}^{\kappa} \alpha, \lambda_{2}\right) & +L_{\alpha}(x, \beta) \int_{\mathrm{D}_{4}}\left(\gamma_{r}^{\kappa}\right)^{t} \xi\left(\gamma_{r}^{\kappa} \alpha, \lambda_{2}\right) \\
& +\int_{\mathrm{D}_{4}} \kappa S\left(\kappa x, \gamma_{r}^{\kappa} \alpha, \lambda_{2}\right) \kappa L(x, \beta)
\end{aligned}
$$


b) $\int_{D_{4}}-\mu \frac{\partial L}{\partial \lambda_{2}}\left(\mu x, \gamma_{r}^{\mu} \alpha,-\lambda_{2}\right)=\int_{D_{4}} \mu L_{x}\left(\mu x, \gamma_{r}^{\mu} \alpha,-\lambda_{2}\right) X\left(\mu x, \gamma_{r}^{\mu} \alpha,-\lambda_{2}\right)$

$+\int_{\mathrm{D}_{4}}-\mu L_{\alpha}\left(\mu x, \gamma_{\tau}^{\mu} \alpha,-\lambda_{2}\right) \xi\left(\gamma_{\tau}^{\mu} \alpha,-\lambda_{2}\right)+\int_{\mathrm{D}_{4}} \mu S\left(\mu x, \gamma_{\tau}^{\mu} \alpha,-\lambda_{2}\right) L\left(\mu x, \gamma_{\tau}^{\mu} \alpha,-\lambda_{2}\right)$.

Logo,

$$
\begin{aligned}
-\frac{\partial L}{\partial \lambda_{2}}(x, \beta) & =L_{x}(x, \beta) \int_{\mathrm{D}_{4}}-\mu X\left(\mu x, \gamma_{\tau}^{\mu} \alpha,-\lambda_{2}\right) \\
& +L_{\alpha}(x, \beta) \int_{D_{4}}-\left(\gamma_{r}^{\kappa}\right)^{t} \xi\left(\gamma_{\tau}^{\kappa} \alpha,-\lambda_{2}\right)+\int_{D_{4}}-\mu S\left(\mu x, \gamma_{\tau}^{\mu} \alpha,-\lambda_{2}\right) \mu L(x, \beta) .
\end{aligned}
$$

Portanto, podemos escrever

$$
-\frac{\partial L}{\partial \lambda_{2}}(x, \beta)=\sum_{j=1}^{2} \hat{X}_{j}(x, \beta) \frac{\partial L}{\partial x_{j}}(x, \beta)+\sum_{i=1}^{r} \tilde{\xi}_{i}(\beta) \frac{\partial L}{\partial \alpha_{i}}(x, \beta)+\tilde{S}(x, \beta) L(x, \beta)
$$

onde

- $\tilde{X}=\int_{D_{4}} \gamma^{t} X \circ \gamma, \tilde{X}=\left(\hat{X}_{1}, \hat{X}_{2}\right)$ satisfaz

$$
\tilde{X}\left(\kappa x, \gamma_{r}^{\kappa} \alpha, \lambda_{2}\right)=\kappa \tilde{X}\left(x, \alpha, \lambda_{2}\right) \quad \text { e } \quad \tilde{X}\left(\mu x, \gamma_{r}^{\mu} \alpha,-\lambda_{2}\right)=-\mu \tilde{X}\left(x, \alpha, \lambda_{2}\right),
$$

- $\tilde{\xi}=\int_{\mathrm{D}_{4}}\left(\gamma_{r}\right)^{t} \xi \circ \gamma_{r}, \tilde{\xi}=\left(\tilde{\xi}_{1} \ldots \hat{\xi}_{r}\right)$ satisfaz

$$
\hat{\xi}\left(\gamma_{r}^{\kappa} \alpha, \lambda_{2}\right)=\gamma_{r}^{\kappa} \tilde{\xi}\left(\alpha, \lambda_{2}\right) \text { e } \quad \tilde{\xi}\left(\gamma_{r}^{\mu} \alpha,-\lambda_{2}\right)=-\left(\gamma_{r}^{\mu}\right) \tilde{\xi}\left(\alpha, \lambda_{2}\right),
$$

- $\tilde{S}=\int_{\mathrm{D}_{4}} \gamma^{t} S \circ \gamma$ satisfaz

$$
\kappa \tilde{S}\left(\kappa x, \gamma_{\tau}^{\kappa} \alpha, \lambda_{2}\right) \kappa=\tilde{S}\left(x, \alpha, \lambda_{2}\right) \quad \text { e } \mu \tilde{S}\left(\mu x, \gamma_{\tau}^{\kappa} \alpha,-\lambda_{2}\right) \mu=-\tilde{S}\left(x, \alpha, \lambda_{2}\right) .
$$

Portanto, existeum campo de vetores da forma

$$
Y\left(x, \alpha, \lambda_{2}\right)=\frac{\partial}{\partial \lambda_{2}}+\sum_{j=1}^{2} \tilde{X}_{j}\left(x, \alpha, \lambda_{2}\right) \frac{\partial}{\partial x_{j}}+\sum_{i=1}^{r} \tilde{\xi}_{i}\left(x, \lambda_{2}\right) \frac{\partial}{\partial \alpha_{i}}
$$

com $\tilde{X}$ e $\tilde{\xi}$ satisfazendo as condições de equivariância do Lema 3.3.1.

Existe, também, $\tilde{S}:\left(\mathbb{R}^{2} \times \mathbb{R}^{r} \times \mathbb{R}, 0\right) \rightarrow M_{2}(\mathbb{R})$ tal que

$$
-D L \cdot Y=\tilde{S} L
$$


$\operatorname{com} \tilde{S}$ satisfazendo as condiçôes de equivariância requeridas. Pelo Lema 3.3.1., existe uma submersào $\mathrm{D}_{4}$-equivariante $B:\left(\mathbb{R}^{r} \times \mathbb{R}, 0\right) \rightarrow\left(\mathbb{R}^{r}, 0\right)$ satisfazendo $(3.18,3.19)$. É suficiente, agora, encontrar uma submersão $\mathrm{D}_{4}$-equivariante

$$
C:\left(\mathbb{R}^{r} \times \mathbb{R}^{2}, 0\right) \rightarrow\left(\mathbb{R}^{r} \times \mathbb{R}, 0\right),
$$

ou seja,

$$
C\left(\gamma_{r}^{\kappa} \alpha, \lambda\right)=\left(\gamma_{r}^{\kappa} \times \mathbf{1}\right) \cdot C \text { e } C\left(\gamma_{r}^{\mu} \alpha, \kappa \lambda\right)=\left(\gamma_{r}^{\mu} \times \kappa\right) \cdot C
$$

tal que $K$ se fatora através de $C^{*} L$. Com isso, existem $S_{C} \in \mathbf{M}_{(x, \alpha, \lambda)}^{\mathrm{D}_{4}}, X_{C} \in \overrightarrow{\mathcal{E}}_{(x, \alpha, \lambda)}^{\mathrm{D}_{4}}$ satisfazendo

$$
K(x, \alpha, \lambda)=S_{C}(x, \alpha, \lambda) L\left(X_{C}(x, \alpha, \lambda), C(\alpha, \lambda)\right), S_{C}(x, 0)=\mathbf{I}_{2}, X_{C}(x, 0)=x .
$$

No que segue, mostramos que isso conclui a prova da proposição. Sejam

- $S(x, \alpha, \lambda)=S_{C}(x, \alpha, \lambda) S_{B}(x, \alpha, \lambda)$,

- $X(x, \alpha, \lambda)=X_{B}\left(X_{C}(x, \alpha, \lambda), C(\alpha, \lambda)\right)$,

- $A=B \circ C$.

Por definiçâo, para $\forall \gamma \in \Gamma$,

$$
\begin{aligned}
& S(x, 0)=\mathbf{I}_{2}, \quad S\left(\gamma_{2} x, \gamma_{r} \alpha, \gamma_{\ell} \lambda\right) \gamma_{2}=\gamma_{2} S(x, \alpha, \lambda), \\
& X(x, 0)=x, \quad X\left(\gamma_{2} x, \gamma_{r} \alpha, \gamma_{\ell} \lambda\right)=\gamma_{2} X(x, \alpha, \lambda), \\
& A^{\circ}=0, \quad A\left(\gamma_{r} \alpha, \gamma_{\ell} \lambda\right)=\gamma_{r} A(\alpha, \lambda) \quad \mathrm{e} \\
& K(x, \alpha, \lambda)=S(x, \alpha, \lambda) F_{0}(X(x, \alpha, \lambda), A(\alpha, \lambda)) .
\end{aligned}
$$

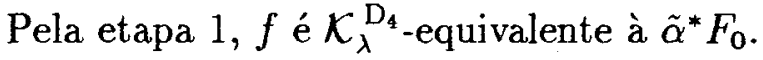

Resta provar a existência de $C$. Primeiro, na etapa 3, mostramos que exite uma submersão $C^{\prime}:\left(\mathbb{R}^{r} \times \mathbb{R}^{2}, 0\right) \rightarrow\left(\mathbb{R}^{r} \times \mathbb{R}, 0\right)$ tal' que $K$ se fatora através de $C^{\prime *} L \Sigma$ equivariantememte. Finalmente, na etapa 4 , achamos $C$ calculando a média sobre $\mathrm{D}_{4}$.

Etapa 3. Seja $\delta=\left(\alpha, \lambda_{2}, \lambda_{1}\right) . K \in \overrightarrow{\mathcal{E}}_{(x, \delta)}^{\Sigma}$ é um $\Sigma$-desdobramento de $f_{0}$ com $(r+2)$ parâmetros. Pelo Lema $5.12([1])$, as seguintes condições são equivalentes

$$
\begin{aligned}
\overrightarrow{\mathcal{E}}_{x}^{\Sigma} & =\mathbf{M}_{x}^{\Sigma} f_{0}+\left(D_{x} f_{0}\right) \overrightarrow{\mathcal{E}}_{x}^{\Sigma}+\mathbb{R}\left\{\frac{\partial K}{\partial \alpha_{1}}(x, 0) \ldots \frac{\partial K}{\partial \alpha_{r}}(x, 0)\right\} \\
\overrightarrow{\mathcal{E}}_{(x, \delta)}^{\Sigma} & =\mathbf{M}_{(x, \delta)}^{\Sigma} K+\left(D_{x} K\right) \overrightarrow{\mathcal{E}}_{(x, \delta)}^{\Sigma}+\mathcal{E}_{\delta}\left\{\frac{\partial K}{\partial \alpha_{1}} \ldots \frac{\partial K}{\partial \alpha_{r}}\right\} .
\end{aligned}
$$


Note que

$$
\begin{gathered}
\frac{\partial K^{\prime}}{\partial \delta_{r+2}}=\frac{\partial h^{\prime}}{\partial \lambda_{1}} \in \overrightarrow{\mathcal{E}}_{(x, \delta)}^{\Sigma} \text { e assim existem } X \in \overrightarrow{\mathcal{E}}_{(x, \delta)}^{\Sigma}, S \in \mathbf{M}_{(x, \delta)}^{\Sigma} \text { e } \xi \in \overrightarrow{\mathcal{E}}_{\delta, \alpha}^{\Sigma} \text { tais que } \\
-\frac{\partial K}{\partial \delta_{r+2}}=\sum_{j=1}^{2} X_{j} \frac{\partial K}{\partial x_{j}}+\sum_{i=1}^{r} \xi_{i} \frac{\partial K}{\partial \alpha_{i}}+S K .
\end{gathered}
$$

Supondo que $\xi_{r+1}(0)=0$, podemos escrever

$$
-\frac{\partial K}{\partial \delta_{r+2}}=\sum_{j=1}^{2} X_{j} \frac{\partial K}{\partial x_{j}}+\sum_{i=1}^{r+1} \xi_{i} \frac{\partial K}{\partial \alpha_{i}}+S K
$$

Portanto,

$$
Y=\frac{\partial}{\partial \delta_{r+2}}+\sum_{j=1}^{2} X_{j} \frac{\partial}{\partial x_{j}}+\sum_{i=1}^{r+1} \xi_{i} \frac{\partial}{\partial \delta_{i}}
$$

e $-D K \cdot Y=S K$

Podemos reescrever as hipóteses do Lema da Redução (Lema 3.3.2) da seguinte forma: sejam $K$ e $L$ como anteriormente e $\delta=\left(\alpha, \lambda_{2}, \lambda_{1}\right)$. Exite um germe de campo de vetores $C^{\infty} Y$ na origem do $\mathbb{R}^{2} \times \mathbb{R}^{r} \times \mathbb{R}^{2}$ dado por (3.21) e satisfazendo

(i) $X \in \overrightarrow{\mathcal{E}}_{(x, \delta)}^{\Sigma}, X=\left(X_{1}, X_{2}\right)$,

(ii) existe $S \in \mathbf{M}_{(x, \delta)}^{\Sigma}$ tal que $-D K \cdot Y=S K$.

Analogamente à prova desse Lema, encontramos uma submersâo

$$
C^{\prime}:\left(\mathbb{R}^{r} \times \mathbb{R}^{2}, 0\right) \rightarrow\left(\mathbb{R}^{r} \times \mathbb{R}, 0\right)
$$

ta] que $K$ se fatora através de $C^{\prime *} L$.

Etapa 4. Reescrevemos o Lema da Reduçâo no contexto $\mathrm{D}_{4^{-}}$equivariante.

Sejam $K$ e $L$ como antes e $\delta=\left(\alpha, \lambda_{2}, \lambda_{1}\right)$. Se existir um germe $\mathcal{X}$ de campo de vetores $C^{\infty}$ na origem do $\mathbb{R}^{2} \times \mathbb{R}^{r} \times \mathbb{R}^{2}$ da forma

$$
\mathcal{X}(x, \delta)=\frac{\partial}{\partial \delta_{r+2}}+\sum_{j=1}^{2} \tilde{X}_{j}(x, \delta) \frac{\partial}{\partial x_{j}}+\sum_{i=1}^{r+1} \tilde{\xi}_{i}(\delta) \frac{\partial}{\partial \delta_{i}}
$$

e satisfazendo

(i) $\tilde{X} \in \overrightarrow{\mathcal{E}}_{(x, \delta)}^{\mathrm{D}_{4}}, \tilde{X}=\left(\tilde{X}_{1}, \tilde{X}_{2}\right) ; \tilde{\xi} \in \overrightarrow{\mathcal{E}}_{\delta}^{\mathrm{D}_{4}}, \tilde{\xi}=\left(\tilde{\xi}_{1} \ldots \tilde{\xi}_{r+1}\right)$,

(ii) existe $\tilde{S} \in \mathbf{M}_{(x, \delta)}^{\mathrm{D}_{4}}$ tal que $-D K \cdot \mathcal{X}=\tilde{S} K$ 
então existirà uma submersão $D_{4}$-equivariante $C:\left(\mathbb{R}^{r} \times \mathbb{R}^{2}, 0\right) \rightarrow\left(\mathbb{R}^{r} \times \mathbb{R}, 0\right)$ tal que $K$ se fatora através de $C^{*} L$.

Da etapa 3 ,

$$
-\frac{\partial}{\partial \delta_{r+2}} K=\sum_{j=1}^{2} X_{j} \frac{\partial K}{\partial x_{j}}+\sum_{i=1}^{r+1} \xi_{i} \frac{\partial K}{\partial \alpha_{i}}+S K
$$

Note que

$$
\begin{gathered}
\frac{\partial}{\partial \delta_{r+2}} K=\frac{\partial}{\partial \lambda_{1}} K=\frac{\partial}{\partial \lambda_{1}} f \text { e como } \mathrm{D}_{4} \text { age trivialmente em } \lambda_{1} \text {, segue que } \\
-\frac{\partial}{\partial \delta_{r+2}} K \in \overrightarrow{\mathcal{E}}_{(x, \delta)}^{\mathrm{D}_{4}} .
\end{gathered}
$$

Portanto, calculando a média de (3.22) sobre $\mathrm{D}_{4}$,

$$
\begin{aligned}
-\frac{\partial}{\partial \delta_{r+2}} K=\sum_{j=1}^{2}\left(\int_{\mathrm{D}_{4}} \gamma_{2}{ }^{t} X_{j}\left(\gamma_{2} x, \delta\right)\right) \frac{\partial}{\partial x_{j}} K+\sum_{i=1}^{++1}\left(\int_{\mathrm{D}_{4}}{\gamma_{r}}^{t} \xi_{i}\left(\gamma_{r} \delta\right)\right) \frac{\partial}{\partial \alpha_{i}} K & \\
& +\left(\int_{\mathrm{D}_{4}} \gamma_{2}^{t} S \circ \gamma_{2}\right) K
\end{aligned}
$$

Seja

$$
\mathcal{X}^{\prime}(x, \delta)=\frac{\partial}{\partial \delta_{r+2}}+\sum_{j=1}^{2}\left(\int_{\mathrm{D}_{4}} \gamma_{2}{ }^{t} X_{j}\left(\gamma_{2} x, \lambda\right)\right) \frac{\partial}{\partial x_{j}}+\sum_{i=1}^{r+1}\left(\int_{\mathrm{D}_{4}} \gamma_{r}{ }^{t} \xi_{i}\left(\gamma_{r} \delta\right)\right) \frac{\partial}{\partial \alpha_{i}}
$$

Então, $D K \cdot \mathcal{X}=\left(\int_{\mathrm{D}_{4}} \gamma_{2}^{t} S \circ \gamma_{2}\right) K$ e as hipóteses do Lema estão satisfeitas.

Portanto, podemos reescrever a prova do Lema 3.3.2 e encontrar $C$ como desejado. Aqui também é preciso usar o argumento de unicidade das solucões das EDOs para mostrar que $C$ é $\mathrm{D}_{4}$-equivariante.

Corolário 3.3.3. Se $\operatorname{cod}^{\mathrm{D}_{4}}(\tilde{\alpha})$ for finita então $\operatorname{cod}^{\mathrm{D}_{4}}(f)$ será finita.

Prova. Pelo Corolário 3.2.18, se $\operatorname{cod}^{\mathrm{D}_{4}}(\tilde{\alpha})$ for finita então $\operatorname{cod}^{\mathrm{D}_{4}}\left(\tilde{\alpha}^{*} F_{0}\right)$ será finita. Pela Proposição 3.3.2, $\operatorname{cod}^{\mathrm{D}_{4}}(f)$ é finita.

\section{Definição 3.3.4.}

Recorde que $\mathcal{P}=\left\{\tilde{\alpha}:\left(\mathbb{R}^{2}, 0\right) \rightarrow\left(\mathbb{R}^{r}, 0\right) \mid \tilde{\alpha}\left(\gamma_{\ell} \lambda\right)=\gamma_{r} \tilde{\alpha}(\lambda)\right\}$ e consideremos $\tilde{\alpha} \in \mathcal{P}$.

Um desdobramento de $\tilde{\alpha} \operatorname{com} q$ parâmetros é um germe de aplicação

$$
\tilde{\phi}:\left(\mathbb{R}^{2+q}, 0\right) \rightarrow \mathbb{R}^{r}
$$


tal que $\dot{\phi}(\lambda, 0)=\hat{\alpha}(\lambda)$.

Seja $\mathcal{P}_{\text {un }}(q)=\left\{\dot{\phi}:\left(\mathbb{R}^{2+q}, 0\right) \rightarrow \mathbb{R}^{T} \mid \hat{\phi}(., 0) \in \mathcal{P}\right\}$ o conjunto de desdobramentos com $q$ parâmetros de um caminho em $\mathcal{P}$.

Proposição 3.3.5. Se $F \in \overrightarrow{\mathcal{E}}_{(x, \lambda, \beta)}^{\mathrm{D}_{4}}$ for um desdobramento de $f \in \overrightarrow{\mathcal{E}}_{(z, \lambda)}^{\mathrm{D}_{4}}$ com $k$ parâmetros $\beta=\left(\beta_{1} \ldots \beta_{k}\right)$ então existirá $\tilde{\phi} \in \mathcal{P}_{u n}(k)$ tal que $F$ será $\mathcal{K}_{\lambda, u n}^{D_{4}}(k)$-equivalente à $\tilde{\phi}^{*} F_{0}$.

Prova. Sejam

$$
\begin{aligned}
& \overrightarrow{\mathcal{E}}_{(x, \lambda, \alpha, \beta)}^{\mathrm{D}_{4}}=\left\{G:\left(\mathbb{R}^{2} \times \mathbb{R}^{2} \times \mathbb{R}^{r} \times \mathbb{R}^{k}, 0\right) \rightarrow \mathbb{R}^{2} \mid G\left(\gamma_{2} x, \gamma_{\ell} \lambda, \gamma_{r} \alpha, \beta\right)\right. \\
& \left.=\gamma_{2} G(x, \lambda, \alpha, \beta), \forall \gamma \in \mathrm{D}_{4}\right\}, \\
& \mathbf{M}_{(x, \lambda, \alpha, \beta)}^{D_{4}}=\left\{T:\left(\mathbb{R}^{2} \times \mathbb{R}^{2} \times \mathbb{R}^{r} \times \mathbb{R}^{k}, 0\right) \rightarrow M_{2}(\mathbb{R}) \mid T\left(\gamma_{2} x, \gamma_{\ell} \lambda, \gamma_{r} \alpha, \beta\right) \gamma_{2}\right. \\
& \left.=\gamma_{2} T(x, \lambda, \alpha, \beta), \forall \gamma \in \mathrm{D}_{4}\right\} \text {, } \\
& \vec{\Theta}_{(\lambda, \alpha, \beta)}^{D_{4}}=\left\{\Lambda:\left(\mathbb{R}^{2+r+k}, 0\right) \rightarrow \mathbb{R}^{2} \mid \Lambda\left(\gamma_{\ell} \lambda, \gamma_{r} \alpha, \beta\right)=\gamma_{\ell} \Lambda(\lambda, \alpha, \beta), \forall \gamma \in D_{4}\right\} .
\end{aligned}
$$

Considere a soma $K:\left(\mathbb{R}^{2} \times \mathbb{R}^{2} \times \mathbb{R}^{r} \times \mathbb{R}^{k}, 0\right) \rightarrow \mathbb{R}^{2}$ definjda por

$$
K(x, \lambda, \alpha, \beta)=F(x, \lambda, \beta)+F_{0}(x, \alpha)-f_{0}(x)
$$

$K \in \overrightarrow{\mathcal{E}}_{(x, \lambda, \alpha, \beta)}^{\mathrm{D}_{4}}$ e é um desdobramento de $f_{0}$ com $(2+r+k)$-parâmetros. Também, $K \in \overrightarrow{\mathcal{E}}_{(x, \lambda, \alpha, \beta)}^{\Sigma} \operatorname{com} \Sigma$ agindo trivialmente em $(\lambda, \alpha, \beta)$.

Afirmamos que existe uma submersão $D_{4}$-equivariante $A:\left(\mathbb{R}^{2+r+k}, 0\right) \rightarrow\left(\mathbb{R}^{r}, 0\right)$, isto é,

$$
A\left(\gamma_{\ell} \lambda, \gamma_{r} \alpha, \beta\right)=\gamma_{r} A(\lambda, \alpha, \beta)
$$

tal que $K$ se fatora através de $A^{*} F_{0}$.

Etapa 1. Mostraremos que essa afirmação mostra o teorema. Se ela for verdadeira então existirão $T \in \mathbf{M}_{(x, \lambda, \alpha, \beta)}^{\mathrm{D}_{4}}, X \in \overrightarrow{\mathcal{E}}_{(x, \lambda, \alpha, \beta)}^{\mathrm{D}_{4}}$ tais que

$$
K(x, \lambda, \alpha, \beta)=T(x, \lambda, \alpha, \beta) F_{0}(X(x, \lambda, \alpha, \beta), A(\lambda, \alpha, \beta))
$$

$\operatorname{com} T(x, 0,0,0)=\mathbf{I}_{2}$ e $X(x, 0,0,0)=x$.

Fazendo $\alpha=0$,

$$
\begin{aligned}
F(x, \lambda, \beta) & =K(x, \lambda, 0, \beta) \\
& =T(x, \lambda, 0, \beta) F_{0}(X(x, \lambda, 0, \beta), A(\lambda, 0, \beta)) .
\end{aligned}
$$


Sejam $S(x, \lambda, \beta)=T(x, \lambda, 0, \beta), \xi(x, \lambda, \beta)=X(x, \lambda, 0, \beta), \Lambda(\lambda, \beta)=\lambda$

e $\tilde{\phi}(\lambda, \beta)=A(\lambda, 0, \beta)$.

Como consequência, existem $S \in \mathbf{M}_{(x, \lambda, \beta)}^{\mathrm{D}_{4}}, \xi \in \overrightarrow{\mathcal{E}}_{(x, \lambda, \beta)}^{\mathrm{D}_{4}}$ e $\Lambda \in \vec{\Theta}_{\lambda, \beta}^{\mathrm{D}_{4}}$ tais que

$$
\begin{aligned}
F(x, \lambda, \beta) & =S(x, \lambda, \beta) F_{0}(\xi(x, \lambda, \beta), \tilde{\phi}(\lambda, \beta)) \\
& =S(x, \lambda, \beta)\left(\tilde{\phi}^{*} F_{0}\right)(\xi(x, \lambda, \beta), \lambda, \beta)=S(x, \lambda, \beta)\left(\hat{\phi}^{*} F_{0}\right)(\xi(x, \lambda, \beta), \Lambda(\lambda, \beta), \beta)
\end{aligned}
$$

onde $(S, \xi, \Lambda)$ é um desdobramento de um elemento de $\mathcal{K}_{\lambda}^{\mathrm{D}_{4}}$ e $\tilde{\phi}$ é um desdobramento de um caminho $\tilde{\alpha} \in \mathcal{P}$.

Em outras palavras, sejam

$$
\begin{aligned}
& S^{\prime}(x, \lambda)=S(x, \lambda, 0), S^{\prime} \in \mathbf{M}_{(x, \lambda)}^{\mathrm{D}_{4}}, S^{\prime o}=\mathbf{I}_{2}, \\
& \xi^{\prime}(x, \lambda)=\xi(x, \lambda, 0), \xi^{\prime} \in \overrightarrow{\mathcal{E}}_{(x, \lambda)}^{\mathrm{D}_{4}}, \xi^{\prime}(x, 0)=x, \\
& \Lambda^{\prime}(\lambda)=\Lambda(\lambda, 0)=\lambda, \Lambda^{\prime} \in \overrightarrow{\mathcal{E}}_{\lambda}^{\mathrm{D}_{4}},\left(D_{\lambda} \Lambda\right)^{o} \in \stackrel{\circ}{\mathcal{L}}_{\mathrm{D}_{4}}(\ell), \\
& \tilde{\alpha}(\lambda)=A(\lambda, 0,0), \hat{\phi}(\lambda, 0)=\tilde{\alpha}(\lambda) . \text { Portanto, existe } \hat{\phi} \in \mathcal{P}_{\text {un }}(k) \text { tal que } F \text { é } \mathcal{K}_{\lambda, u n}^{\mathrm{D}_{4}}(\beta)-
\end{aligned}
$$
equivalente à $\tilde{\phi}^{*} F_{0}$.

Etapa 2. Mostramos a existência de $A$ por indução em $(r+k)$.

O caso $r+k=0$ se verifica pela Proposiçào 3.3.2.

Seja $L: \mathbb{R}^{2} \times \mathbb{R}^{2} \times \mathbb{R}^{r} \times \mathbb{R}^{k-1} \rightarrow \mathbb{R}^{2}$ definida por

$$
L\left(x, \lambda, \alpha, \beta_{1} \ldots \beta_{k-1}\right)=K\left(x, \lambda, \alpha, \beta_{1} \ldots \beta_{k-1}, 0\right) .
$$

Então pela hipótese de indução, existe uma submersão $\mathrm{D}_{4}$-equivariante $B:\left(\mathbb{R}^{r+k+1}, 0\right) \rightarrow\left(\mathbb{R}^{r}, 0\right)$, isto é,

$$
B\left(\left(\gamma_{\ell} \times \gamma_{r}\right) \cdot\left(\lambda, \alpha, \beta_{1} \ldots \beta_{k-1}\right)\right)=B\left(\gamma_{\ell} \lambda, \gamma_{r} \alpha, \beta_{1} \ldots \beta_{k-1}\right)=\gamma_{r} B\left(\lambda, \alpha, \beta_{1} \ldots \beta_{k-1}\right)
$$

tal que $L$ se fatora através de $B^{*} F_{0}$.

Logo, existem $T_{B} \in \mathbf{M}_{\left(x, \lambda, \alpha, \beta^{\prime}\right)}^{\mathrm{D}_{4}}, X_{B} \in \overrightarrow{\mathcal{E}}_{\left(x, \lambda, \alpha, \beta^{\prime}\right)}^{\mathrm{D}_{4}}$ com $\beta^{\prime}=\left(\beta_{1} \ldots \beta_{k-1}\right)$ satisfazendo $L\left(x, \lambda, \alpha, \beta^{\prime}\right)=T_{B}\left(x, \lambda, \alpha, \beta^{\prime}\right) F_{0}\left(X_{B}\left(x, \lambda, \alpha, \beta^{\prime}\right), B\left(x, \lambda, \beta^{\prime}\right)\right), T_{B}(x, 0,0,0)=\mathbf{I}_{2}$

$$
\text { e } X_{B}(x, 0,0,0)=x .
$$


É suficiente encontrar

(i) Uma submersâo $C:\left(\mathbb{R}^{2+r+k}, 0\right) \rightarrow\left(\mathbb{R}^{r+k+1}, 0\right)$ tal que $C\left(\gamma_{\ell} \lambda, \gamma_{\tau} \alpha, \beta\right)=$

$$
\left(\gamma_{\ell} \times \gamma_{r}\right) \cdot C(\lambda, \alpha, \beta)
$$

(ii) $T_{C} \in \mathbf{M}_{(x, \lambda, \alpha, \beta)}^{\mathrm{D}_{4}}, X_{C} \in \overrightarrow{\mathcal{E}}_{(x, \lambda, \alpha, \beta)}^{\mathrm{D}_{4}}$ satisfazendo

$$
\begin{aligned}
K(x, \lambda, \alpha, \beta)=T_{C}(x, \lambda, \alpha, \beta) L\left(X_{C}(x, \lambda, \alpha, \beta), C(\lambda, \alpha, \beta)\right), T_{C}(x, 0,0,0)=\mathbf{I}_{2} \mathrm{e} \\
X_{C}(x, 0,0,0)=x .
\end{aligned}
$$

De fato,

$$
\begin{aligned}
K(x, \lambda, \alpha, \beta) & =T_{C}(x, \lambda, \alpha, \beta) L\left(X_{C}(x, \lambda, \alpha, \beta), C(\lambda, \alpha, \beta)\right) \\
& =T_{C}(x, \lambda, \alpha, \beta) T_{B}\left(x ; \lambda, \alpha, \beta^{\prime}\right) F_{0}\left(X_{B}\left(X_{C}(x, \lambda, \alpha, \beta), B(C(\lambda, \alpha, \beta))\right)\right) .
\end{aligned}
$$

Sejam

- $T(x, \lambda, \alpha, \beta)=T_{C}(x, \lambda, \alpha, \beta) T_{B}\left(x, \lambda, \alpha, \beta^{\prime}\right) . T(x, 0,0,0)=\mathbf{I}_{2}$,

$$
T\left(\gamma_{2} x, \gamma_{\ell} \lambda, \gamma_{\tau} \alpha, \beta\right)=\gamma_{2} T(x, \lambda, \alpha, \beta) .
$$

- $X(x, \lambda, \alpha, \beta)=X_{B}\left(X_{C}(x, \lambda, \alpha, \beta), C(x, \lambda, \alpha, \beta)\right), X(x, 0,0,0)=x$,

$$
X\left(\gamma_{2} x, \gamma_{\ell} \lambda, \eta_{r} \alpha, \beta\right)=\gamma_{2} X(x, \lambda, \alpha, \beta) .
$$

- $A=B \circ C . A^{o}=0, A\left(\gamma_{\ell} \lambda, \gamma_{r} \alpha, \beta\right)=\gamma_{r}(\lambda, \alpha, \beta)$.

Portanto, existem, $T, X, \Lambda$ satisfazendo as condiçôes de equivariância requeridas e tais que

$$
K(x, \lambda, \alpha, \beta)=T(x, \lambda, \alpha, \beta) F_{0}(X(x, \lambda, \alpha, \beta), A(\lambda . \alpha, \beta)), \text { isto é, }
$$

$K$ se fatora através de $A^{*} F_{0}$.

Resta provar a existência de $C$. A idéia é mostrar que existe uma submersão

$$
C^{\prime}:\left(\mathbb{R}^{2+r+k}, 0\right) \rightarrow\left(\mathbb{R}^{r+k+1}, 0\right)
$$

tal que $K$ se fatora, $\Sigma$-equivariantemente, através de $C^{\prime *} L$ pois $K$ e $L$ são germes de aplicação $\Sigma$-equivariantes. Para encerrar, achamos $C$ calculando a média sobre $\mathrm{D}_{4}$.

Omitiremos a prova dessa parte por ser similar às etapas 3 e 4 da Proposição 3.3.2. 


\section{Capítulo 4}

\section{Classificação dos problemas $\mathrm{D}_{4}$ usando a fomulação por caminhos}

Neste capítulo mostramos como classificar os problemas de bifurcação $\mathrm{D}_{4}$ usando a fomulação por caminhos descrita no capítulo 3 .

Recorde que $\Sigma=\mathrm{Z}_{2} \oplus \mathrm{Z}_{2}$ para o problema $\mathrm{D}_{4}$ e que $N=z \bar{z}, \delta=-\frac{1}{2}\left(z^{2}+\bar{z}^{2}\right), u_{4}=\lambda_{2}^{2}$.

Para cada $g \in \mathcal{E}_{z}^{\Sigma}$, existe $p:\left(\mathbb{R}^{2}, 0\right) \rightarrow \mathbb{R}$ tal que $g(z)=p(N, \delta) . \overrightarrow{\mathcal{E}}_{z}^{\Sigma}$ é o $\mathcal{E}_{z}^{\Sigma}$-módulo gerado por $z$ e $\bar{z}$, ou seja, $h \in \overrightarrow{\mathcal{E}}_{z}^{\Sigma}$ pode ser escrita como

$$
h(z)=p(N, \delta) z+q(N, \delta) \bar{z}
$$

e identificamos $h$ com $[p, q]$.

$\mathrm{O}$ conjunto $\mathbf{M}_{z}^{\Sigma}$ das matrizes que comutam com a ação de $\Sigma$ é o $\mathcal{E}_{z}^{\Sigma}$-módulo gerado pelas seguintes aplicações lineares em $\mathbb{C}$ :

$$
\begin{aligned}
S_{1}(z) w & =w, & S_{3}(z) w & =\frac{i}{2} \omega(w+\bar{w}), \\
S_{2}(z) w & =\bar{w}, & S_{4}(z) w & =\frac{i}{2} \omega(\bar{w}-w),
\end{aligned}
$$

onde $\omega=\frac{i}{4}\left(\bar{z}^{2}-z^{2}\right)$ 
O espaço tangente estendido é

$$
\mathcal{T}_{\varepsilon}^{\Sigma}(h)=\left\{S h+\left(D_{z} h\right) X \mid S \in \mathbf{M}_{z}^{\Sigma}, X \in \overrightarrow{\mathcal{E}}_{z}^{\Sigma}\right\}
$$

e calculando-se os geradores desse espaço temos que $\mathcal{T}_{e}^{\Sigma}(h)=\mathcal{E}_{z}^{\Sigma}<h_{1} \ldots h_{6}>$ onde

$$
\begin{aligned}
& h_{1}=[p, q], \\
& h_{2}=[q, p], \\
& h_{3}=\frac{1}{4}(p+q)[N-\delta, \delta-N] \\
& h_{4}=\frac{1}{4}(p-q)[N+\delta, N+\delta], \\
& h_{5}=\left[N p_{N}+\delta p_{\delta}, N q_{N}+\delta q_{\delta}\right], \\
& h_{6}=\left[\delta p_{N}+N p_{\delta}, \delta q_{N}+N q_{\delta}\right] .
\end{aligned}
$$

A forma normal para um problema de bifurcação $D_{4}$ é

$$
f(z, \lambda)=p(u) z+q(u) \delta \bar{z}+r(u) \lambda_{2} \bar{z}+s(u) \delta \lambda_{2} z
$$

onde $p, q, r, s \in \mathcal{E}_{u}, u=\left(N, \Delta, \lambda_{1}, u_{4}\right)$ e $p^{\circ}=0$ (cf. Capítulo 2).

O centro organizador de $f$ é

$$
f_{0}(z)=f(z, 0)=p(N, \Delta) z+q(N, \Delta) \delta \bar{z}
$$

Com base no Teorema 2.8.1., temos os seguintes centros organizadores para o problema $\mathrm{D}_{4}$ :
I. $f_{0}^{1}(z)=m N z+\epsilon_{5} \delta \bar{z} \quad$ com $\quad m=\frac{p_{N}^{0}}{\left|q^{\circ}\right|}$ e $\epsilon_{5}=s g q^{\circ}$.

As condições de não degenericidade são $m \neq \pm 1,0$ e $q^{\circ} \neq 0 . f_{0}^{1}$ é o centro organizador genérico.

II. Condição de definição: $m=0$ ou equivalentemente $p_{N}^{\circ}=0$.

Forma normal: $f_{0}^{2}(z)=\epsilon_{2} N^{2} z+\epsilon_{5} \delta \bar{z}$ com $\epsilon_{2}=s g p_{N N}^{\circ}$. 
III. Condiçâo de definiçâo: $m=-1$ ou equivalentemente $p_{N}^{o}=-q^{\circ}$.

Forma normal: $f_{0}^{3}(z)=-\epsilon_{5} N z+\epsilon_{5} \delta \bar{z}$.

IV. Condição de definição: $m=1$ ou equivalentemente $p_{N}^{o}=q^{o}$.

Forma normal: $f_{0}^{4}(z)=\epsilon_{1} N z+\epsilon_{2} N^{2} z+\epsilon_{1} \delta \bar{z}$ com $\epsilon_{1}=s g p_{N}^{o}$

$$
\text { e } \epsilon_{2}=s g\left(p_{N N}^{o}+2 p_{\Delta}^{o}-2 q_{N}^{o}\right) \text {. }
$$

V. Condição de definição: $q^{\circ}=0$.

Forma normal: $f_{0}^{5}(z)=\epsilon_{1} N z+\epsilon_{6} \Delta \delta \bar{z}$ com $\epsilon_{6}=\epsilon_{1} s g \Delta_{N, \Delta}(p, q)$.

\subsection{Classificação dos problemas $\mathrm{D}_{4}$ com centro or- ganizador $\mathrm{I}$.}

Para o caso I, $f_{0}^{1}(z)=, m N z+\epsilon_{5} \delta \bar{z}, m \neq \pm 1,0$. Usando a notação $Z_{2} \oplus Z_{2}$, escrevemos

$$
f_{0}^{1}=\left[m N, \epsilon_{5} \delta\right]
$$

Usando (4.1), um cálculo mostra que

$$
\mathcal{T}_{\epsilon}^{\Sigma}\left(f_{0}^{1}\right)=\mathcal{E}_{N, \delta}^{\Sigma}<[0, N],[\delta, 0],\left[0, \delta^{2}\right],\left[N^{2}, 0\right],\left[m N, \epsilon_{5} \delta\right]>.
$$

O $\Sigma$-espaço normal $\mathcal{N}_{e}^{\Sigma}\left(f_{0}^{1}\right)$ é gerado por $[1,0],[N, 0]$ e $[0,1]$ como um espaço vetorial real.

O $\Sigma$-desdobramento de $f_{0}^{1}$ é dado por

$$
\begin{aligned}
& F_{0}^{1}:\left(\mathbb{C} \times \mathbb{R}^{3}, 0\right) \rightarrow \mathbb{C} \\
& F_{0}^{1}(z, \alpha)=\left(m+\alpha_{2}\right) N z+\alpha_{1} z+\left(\epsilon_{5} \delta+\alpha_{3}\right) \bar{z}, \alpha=\left(\alpha_{1}, \alpha_{2}, \alpha_{3}\right) .
\end{aligned}
$$

Substituindo $\left(m+\alpha_{2}\right)$ por $\hat{m}$, obtemos

$$
F_{0}^{1}(z, \alpha)=\hat{m} N z+\alpha_{1} z+\left(\epsilon_{5} \delta+\alpha_{3}\right) \bar{z} .
$$


No que se segue, baseamo-nos na teoria descrita no Capítulo 3 (seç̧âo 3.1).

Recorde que $\mathrm{D}_{4}$ age em $(z, \lambda)$ por

$$
\begin{aligned}
& \hat{\kappa} \cdot(z, \lambda)=(\kappa \cdot z, \lambda) \text { onde } \kappa \cdot z=\bar{z} \\
& \hat{\mu} \cdot(z, \lambda)=(\mu \cdot z, \kappa \cdot \lambda) \text { onde } \mu \cdot z=i \bar{z}
\end{aligned}
$$

Considere o isomorfismo

$$
\begin{aligned}
\theta: \frac{\overrightarrow{\mathcal{E}}_{z}^{\Sigma}}{\mathcal{T}_{e}^{\Sigma}\left(f_{0}^{1}\right)} & \rightarrow \mathbb{R}^{3} \\
\left(\beta_{1} z+\beta_{2} N z+\beta_{3} \bar{z}\right) & \mapsto\left(\beta_{1}, \beta_{2}, \beta_{3}\right) .
\end{aligned}
$$

A ação $\varphi: \mathrm{D}_{4} \times \overrightarrow{\mathcal{E}}_{z}^{\Sigma} \rightarrow \overrightarrow{\mathcal{E}}_{z}^{\Sigma}$ é dada por

$$
\begin{aligned}
& \varphi(\hat{\kappa}, g)=\kappa \cdot g \circ \kappa, \\
& \varphi(\hat{\mu}, g)=\mu \cdot g \circ \mu
\end{aligned}
$$

e a ação $\bar{\varphi}: \mathrm{D}_{4} \times \frac{\overrightarrow{\mathcal{E}}_{z}^{\Sigma}}{\mathcal{T}_{e}^{\Sigma}\left(f_{0}^{1}\right)} \rightarrow \frac{\overrightarrow{\mathcal{E}}_{z}^{\Sigma}}{\mathcal{T}_{e}^{\Sigma}\left(f_{0}^{1}\right)}$ por

$$
\begin{aligned}
& \bar{\varphi}\left(\hat{\kappa},\left(\beta_{1} z+\beta_{2} N z+\beta_{3} \bar{z}\right)\right)=\beta_{1} z+\beta_{2} N z+\beta_{3} \bar{z} \\
& \bar{\varphi}\left(\hat{\mu},\left(\beta_{1} z+\beta_{2} N z+\beta_{3} \bar{z}\right)\right)=\beta_{1} z+\beta_{2} N z-\beta_{3} \bar{z}
\end{aligned}
$$

Lembrando que $\mathrm{D}_{4}$ age em $z$ como $\bar{z}$ e $i \bar{z},\{[1,0],[N, 0],[0,1]\}$ é uma boa base. As relações (4.2) definem uma representação ortogonal

$$
\begin{aligned}
\rho_{3}: \mathrm{D}_{4} & \rightarrow O(3) \\
\hat{\kappa} & \mapsto I_{3} \\
\hat{\mu} & \mapsto\left(\begin{array}{rrr}
1 & 0 & 0 \\
0 & 1 & 0 \\
0 & 0 & -1
\end{array}\right)
\end{aligned}
$$

$e$, via $\theta$, definimos uma ação em $\beta \in \mathbb{R}^{3}$ denotada por $\gamma_{3} \cdot \beta$.

Seja $\mathcal{P}_{2,3}=\left\{\tilde{\alpha}:\left(\mathbb{R}^{2}, 0\right) \rightarrow\left(\mathbb{R}^{3}, 0\right)\right\}$ o conjunto de caminhos definidos do espaço dos parâmetros de bifurcação no espaço dos parâmetros de desdobramento. 
Como antes, denotamos a açào de $\mathrm{D}_{4}$ em $\lambda=\left(\lambda_{1}, \lambda_{2}\right) \in \mathbb{R}^{2}$ por $\gamma_{\ell} \lambda$ e em $z \in \mathbb{C}$ por $\gamma_{2} z$.

A ação $\psi$ de $D_{4}$ em $\tilde{\alpha} \in \mathcal{P}_{2,3}$ é dada por $\gamma \cdot \hat{\alpha}=\gamma_{3}^{\ell} \cdot\left(\tilde{\alpha} \circ \gamma_{\ell}\right), \forall \gamma \in D_{4}$.

$\mathcal{P}$ denota o conjunto dos caminhos $\mathrm{D}_{4}$-equivariantes, isto é,

$$
\mathcal{P}=\left.\mathcal{P}_{2,3}\right|_{\text {Fix } \psi}=\left\{\tilde{\alpha} \in \mathcal{P}_{2,3} \mid \hat{\alpha}\left(\gamma_{\ell} \lambda\right)=\gamma_{3} \hat{\alpha}(\lambda)\right\}
$$

Um simples cálculo mostra que

$$
\tilde{\alpha} \in \mathcal{P} \text { se e somente } \tilde{\alpha}\left(\lambda_{1}, \lambda_{2}\right)=\left(\alpha_{1}\left(\lambda_{1}, u_{4}\right), \alpha_{2}\left(\lambda_{1}, u_{4}\right), \lambda_{2} \alpha_{3}\left(\lambda_{1}, u_{4}\right)\right) \text {. }
$$

Pela Proposição 3.3.2, $f$ é $\mathcal{K}_{\lambda}^{\mathrm{D}_{4}}$-equivalente à $\tilde{\alpha}^{*} F_{0}^{1}$ onde

$$
\begin{aligned}
\left(\tilde{\alpha}^{*} F_{0}^{1}\right)(z, \lambda) & =F_{0}^{1}(z, \tilde{\alpha}(\lambda)) \\
& =\left[\left(m+\alpha_{2}\left(\lambda_{1}, u_{4}\right)\right) N+\alpha_{1}\left(\lambda_{1}, u_{4}\right), \epsilon_{5}, \alpha_{3}\left(\lambda_{1}, u_{4}\right), 0\right] .
\end{aligned}
$$

Para encontrar o espaço tangente a um caminho $\tilde{\alpha}$, da forma (4.3), seguimos o Capítulo 3 (seç̧âo 3.2 ) e identificamos $\mathbb{R}^{2}$ com $\mathbb{C}$. Começamos calculando o espaço tangente ao germe $\hat{f} \in \overrightarrow{\mathcal{E}}_{(z, \beta)}^{\mathrm{D}_{4}}$.

Denotamos $(\alpha, \lambda)$ por $\beta$ e definimos

$\overrightarrow{\mathcal{E}}_{(z, \beta)}^{\mathrm{D}_{4}}=\left\{\hat{f}:\left(\mathbb{C} \times \mathbb{R}^{3} \times \mathbb{R}^{2}, 0\right) \rightarrow \mathbb{C} \mid \hat{f}\left(\gamma_{2} z,\left(\gamma_{3} \times \gamma_{\ell}\right) \beta\right)=\gamma_{2} \hat{f}(z, \beta), \forall \gamma \in \mathrm{D}_{4}\right\}$.

$\vec{\Theta}_{(z, \beta)}^{D_{4}, o}=\left\{\hat{\xi} \in \vec{\Theta}_{(z, \beta)}^{D_{4}} \mid \hat{\xi}^{o}=0\right\}$.

$\mathbf{M}_{(z, \beta)}^{\mathrm{D}_{4}}=\left\{\hat{S}:\left(\mathbf{C} \times \mathbb{R}^{3} \times \mathbb{R}^{2}, 0\right) \rightarrow G L(2) \mid \hat{S}\left(\gamma_{2} z,\left(\gamma_{3} \times \gamma_{\ell}\right) \beta\right) \gamma_{2}=\gamma_{2} \hat{S}(z, \beta), \forall \gamma \in \mathrm{D}_{4}\right\}$.

$A$ ação de $\mathrm{D}_{4}$ em $\mathbb{C} \times \mathbb{R}^{3} \times \mathbb{R}^{2}$ é explicitamente dada por

$$
\begin{aligned}
& \tilde{\kappa} \cdot\left(z, \alpha_{1}, \alpha_{2}, \alpha_{3}, \lambda_{1}, \lambda_{2}\right)=\left(\bar{z}, \alpha_{1}, \alpha_{2}, \alpha_{3}, \lambda_{1}, \lambda_{2}\right), \\
& \tilde{\mu} \cdot\left(z, \alpha_{1}, \alpha_{2}, \alpha_{3}, \lambda_{1}, \lambda_{2}\right)=\left(i \bar{z}, \alpha_{1}, \alpha_{2},-\alpha_{3}, \lambda_{1},-\lambda_{2}\right) .
\end{aligned}
$$


Os $\mathrm{D}_{4}$-invariantes para essa açào sào os seguintes:

$$
\begin{array}{ll}
\hat{u}_{1}=N=z \bar{z}, & \hat{u}_{6}=\alpha_{2}, \\
\hat{u}_{2}=\Delta=\delta^{2}=\frac{1}{4}\left(z^{2}+\bar{z}^{2}\right)^{2}, & \hat{u}_{7}=\alpha_{3}^{2}, \\
\hat{u}_{3}=\lambda_{1}, & \hat{u}_{8}=\delta \alpha_{3}, \\
\hat{u}_{4}=\lambda_{2}^{2}=u_{4}, & \hat{u}_{9}=\delta \lambda_{2}, \\
\hat{u}_{5}=\alpha_{1}, & \hat{u}_{10}=\alpha_{3} \lambda_{2} .
\end{array}
$$

Eles satisfazem as seguintes relações: $\hat{u}_{8}^{2}=\hat{u}_{2} \hat{u}_{7}, \hat{u}_{9}^{2}=\hat{u}_{2} \hat{u}_{4}, \hat{u}_{10}^{2}=\hat{u}_{7} \hat{u}_{4}$.

As provas das proposições 4.1.1. a 4.1.5. estão no Apêndice.

Proposição 4.1.1. $\mathcal{E}_{(z, \beta)}^{\mathrm{D}_{4}}=\hat{u}^{*} \mathcal{E}_{\bar{u}}$ onde $\hat{u}=\left(\hat{u}_{1} \ldots \hat{u}_{10}\right)$.

Proposição 4.1.2. $\overrightarrow{\mathcal{E}}_{(z, \beta)}^{\mathrm{D}_{4}}$ é gerado por $z, \delta \bar{z}, \lambda_{2} \bar{z}$ e $\alpha_{3} \bar{z}$ como um $\mathcal{E}_{(z, \beta)}^{\mathrm{D}_{4}}$-módulo.

Seja $\bar{u}=\left(\hat{u}_{1} \ldots \hat{u}_{7}\right)$ com $\hat{u}_{i}, 1 \leq i \leq 7$, dados em (4.4).

Proposição 4.1.3. $\overrightarrow{\mathcal{E}}_{(z, \beta)}^{\mathrm{D}_{4}}$ é gerado livremente por $z, \delta \bar{z}, \lambda_{2} \bar{z}, \alpha_{3} \bar{z}, \delta \lambda_{2} z, \delta \alpha_{3} z, \alpha_{3} \lambda_{2} z$ e $\delta \alpha_{3} \lambda_{2} \bar{z}$ como um $\mathcal{E}_{\bar{u}}$-módulo.

Proposição 4.1.4. $\mathbf{M}_{(z, \beta)}^{\mathrm{D}_{4}}$ é o $\mathcal{E}_{(z, \beta)}^{\mathrm{D}_{4}}$-módulo gerado pelas seguintes aplicações lineares em

$$
\begin{aligned}
& S_{1}(z, \beta) w=w, \quad S_{5}(z, \beta) w=i \omega \bar{w}, \\
& S_{2}(z, \beta) w=\lambda_{2} \bar{w}, \quad S_{6}(z, \beta) w=-i \lambda_{2} \omega w, \\
& S_{3}(z, \beta) w=\delta \bar{w}, \quad S_{7}(z, \beta) w=-i \delta \omega w \\
& S_{4}(z, \beta) w=\alpha_{3} \bar{w}, \quad S_{8}(z, \beta) w=-i \alpha_{3} w w .
\end{aligned}
$$

onde $\omega=\frac{i}{4}\left(\bar{z}^{2}-z^{2}\right)$ 
Proposição 4.1.5. $\mathbf{M}_{(2, \beta)}^{D_{4}}$ é o $\mathcal{E}_{\tilde{u}}$-módulo gerado livremente pelas seguintes aplicaçôes lineares em $\mathbb{C}$ :

$$
\begin{array}{ll}
\hat{S}_{1}(z, \beta) w=w, & \hat{S}_{9}(z, \beta) w=i \omega \bar{w}, \\
\hat{S}_{2}(z, \beta) w=\lambda_{2} \bar{w}, & \hat{S}_{10}(z, \beta) w=-i \lambda_{2} \omega w, \\
\hat{S}_{3}(z, \beta) w=\delta \bar{w}, & \hat{S}_{11}(z, \beta) w=-i \delta \omega w, \\
\hat{S}_{4}(z, \beta) w=\alpha_{3} \bar{w}, & \hat{S}_{12}(z, \beta) w=-i \alpha_{3} \omega w, \\
\hat{S}_{5}(z, \beta) w=\delta \lambda_{2} w, & \hat{S}_{13}(z, \beta) w=i \delta \lambda_{2} \omega \bar{w}, \\
\hat{S}_{6}(z, \beta) w=\delta \alpha_{3} w, & \hat{S}_{14}(z, \beta) w=i \delta \alpha_{3} \omega \bar{w}, \\
\hat{S}_{7}(z, \beta) w=\alpha_{3} \lambda_{2} w, & \hat{S}_{15}(z, \beta) w=i \alpha_{3} \lambda_{2} \omega \bar{w}, \\
\hat{S}_{8}(z, \beta) w=\delta \alpha_{3} \lambda_{2} \bar{w}, & \hat{S}_{16}(z, \beta) w=i \delta \alpha_{3} \lambda_{2} \omega w .
\end{array}
$$

Da Proposição 4.1.3., toda $\hat{f} \in \overrightarrow{\mathcal{E}}_{(z, \beta)}^{\mathrm{D}_{4}}$ pode ser escrita como

$$
\begin{aligned}
\hat{f}(z, \alpha, \lambda)=p_{1}(\bar{u}) z+p_{2}(\bar{u}) \delta \bar{z} & +p_{3}(\bar{u}) \lambda_{2} \bar{z}+p_{4}(\bar{u}) \alpha_{3} \bar{z}+p_{5}(\bar{u}) \delta \lambda_{2} z \\
& +p_{6}(\bar{u}) \delta \alpha_{3} z+p_{7}(\bar{u}) \alpha_{3} \lambda_{2} z+p_{8}(\bar{u}) \delta \alpha_{3} \lambda_{2} \bar{z}
\end{aligned}
$$

e identificamos $\hat{f} \operatorname{com}\left[p_{1} \ldots p_{8}\right]$.

O espaço tangente à $\hat{f} \in \overrightarrow{\mathcal{E}}_{(z, \beta)}^{\mathrm{D}_{4}}$ é dado por

$$
\mathbf{T}^{\mathrm{D}_{4}}(\hat{f})=\left\{\hat{S} \hat{f}+\left(D_{z} \hat{f}\right) \hat{X} \mid \hat{S} \in \mathbf{M}_{(z, \beta)}^{\mathrm{D}_{4}}, \hat{X} \in \overrightarrow{\mathcal{E}}_{(z, \beta)}^{\mathrm{D}_{4}}\right\} .
$$

Proposição 4.1.6. $\mathbf{T}^{\mathrm{D}_{4}}(\hat{f})=\mathcal{E}_{\bar{u}}<g_{1} \ldots g_{24}>$ onde

$g_{1}=\left[p_{1}, p_{2}, p_{3}, p_{4}, p_{5}, p_{6}, p_{7}, p_{8}\right]$,

$g_{2}=\left[u_{4} p_{3}, u_{4} p_{5}, p_{1}, u_{4} p_{7}, p_{2}, u_{4} p_{8}, p_{4}, p_{6}\right]$,

$g_{3}=\left[\Delta p_{2}, p_{1}, \Delta p_{5}, \Delta p_{6}, p_{3}, p_{4}, \Delta p_{8}, p_{7}\right]$,

$g_{4}=\left[\alpha_{3}^{2} p_{4}, \alpha_{3}^{2} p_{6}, \alpha_{3}^{2} p_{7}, p_{1}, \alpha_{3}^{2} p_{8}, p_{2}, p_{3}, p_{5}\right]$,

$g_{5}=\left[\Delta u_{4} p_{5}, u_{4} p_{3}, \Delta p_{2}, \Delta u_{4} p_{8}, p_{1}, u_{4} p_{7}, \Delta p_{6}, p_{4}\right]$,

$g_{6}=\left[\Delta \alpha_{3}^{2} p_{6}, \alpha_{3}^{2} p_{4}, \Delta \alpha_{3}^{2} p_{8}, \Delta p_{2}, \alpha_{3}^{2} p_{7}, p_{1}, \Delta p_{5}, p_{3}\right]$,

$g_{7}=\left[\alpha_{3}^{2} u_{4} p_{7}, \alpha_{3}^{2} u_{4} p_{8}, \alpha_{3}^{2} p_{4}, u_{4} p_{3}, \alpha_{3}^{2} p_{6}, u_{4} p_{5}, p_{1}, p_{2}\right]$, 


$$
\begin{aligned}
& g_{8}=\left[\Delta \alpha_{3}^{2} u_{4} p_{8}, \alpha_{3}^{2} u_{4} p_{7}, \Delta \alpha_{3}^{2} p_{6}, \Delta u_{4} p_{5}, \alpha_{3}^{2} p_{4}, u_{4} p_{3}, \Delta p_{2}, p_{1}\right] \\
& g_{9}=\left[N p_{1}-\Delta p_{2}, p_{1}-N p_{2}, \Delta p_{5}-N p_{3}, \Delta p_{6}-N p_{4}, N p_{5}-p_{3}, N p_{6}-p_{4},\right. \\
& \left.N p_{7}-\Delta p_{8}, p_{7}-N p_{8}\right] \\
& g_{10}=\left[\Delta u_{4} p_{5}-N u_{4} p_{3}, N u_{4} p_{5}-u_{4} p_{3}, N p_{1}-\Delta p_{2}, N u_{4} p_{7}-\Delta u_{4} p_{8},\right. \\
& \left.p_{1}-N p_{2}, u_{4} p_{7}-N u_{4} p_{8}, \Delta p_{6}-N p_{4}, N p_{6}-p_{4}\right\} \text {, } \\
& g_{11}=\left[\Delta p_{1}-N \Delta p_{2}, N p_{1}-\Delta p_{2}, N \Delta p_{5}-\Delta p_{3}, N \Delta p_{6}-\Delta p_{4}, \Delta p_{5}-N p_{3},\right. \\
& \left.\Delta p_{6}-N p_{4}, \Delta p_{7}-N \Delta p_{8}, N p_{7}-\Delta p_{8}\right], \\
& g_{12}=\left[\Delta \alpha_{3}^{2} p_{6}-N \alpha_{3}^{2} p_{4}, N \alpha_{3}^{2} p_{6}-\alpha_{3}^{2} p_{4}, N \alpha_{3}^{2} p_{7}-\Delta \alpha_{3}^{2} p_{8}, N p_{1}-\Delta p_{2}, \alpha_{3}^{2} p_{7}-N \alpha_{3}^{2} p_{8}\right. \\
& \left.p_{1}-N p_{2}, \Delta p_{5}-N p_{3}, N p_{5}-p_{3}\right] \text {, } \\
& g_{13}=\left[N \Delta u_{4} p_{5}-\Delta u_{4} p_{3}, \Delta u_{4} p_{5}-N u_{4} p_{3}, \Delta p_{1}-N \Delta p_{2}, \Delta u_{4} p_{7}-N \Delta u_{4} p_{8}\right. \\
& \left.N p_{1}-\Delta p_{2}, N u_{4} p_{7}-\Delta u_{4} p_{8}, N \Delta p_{6}-\Delta p_{4}, \Delta p_{6}-N p_{4}\right] \text {, } \\
& g_{14}=\left[N \Delta \alpha_{3}^{2} p_{6}-\Delta \alpha_{3}^{2} p_{4}, \Delta \alpha_{3}^{2} p_{6}-N \alpha_{3}^{2} p_{4}, \Delta \alpha_{3}^{2} p_{7}-N \Delta \alpha_{3}^{2} p_{8}, \Delta p_{1}-N \Delta p_{2},\right. \\
& \left.N \alpha_{3}^{2} p_{7}-\Delta \alpha_{3}^{2} p_{8}, N p_{1}-\Delta p_{2}, N \Delta p_{5}-\Delta p_{3}, \Delta p_{5}-N p_{3}\right], \\
& g_{15}=\left[N u_{4} \alpha_{3}^{2} p_{7}-u_{4} \alpha_{3}^{2} \Delta p_{8}, u_{4} \alpha_{3}^{2} p_{7}-N u_{4} \alpha_{3}^{2} p_{8}, \Delta \alpha_{3}^{2} p_{6}-N \alpha_{3}^{2} p_{4}, \Delta u_{4} p_{5}-N u_{4} p_{3},\right. \\
& \left.N \alpha_{3}^{2} p_{6}-\alpha_{3}^{2} p_{4}, N u_{4} p_{5}-u_{4} p_{3}, N p_{1}-\Delta p_{2}, p_{1}-N p_{2}\right] \\
& g_{16}=\left[\Delta \alpha_{3}^{2} u_{4} p_{7}-N \Delta \alpha_{3}^{2} u_{4} p_{8}, N \alpha_{3}^{2} u_{4} p_{7}-\Delta \alpha_{3}^{2} u_{4} p_{8}, N \Delta \alpha_{3}^{2} p_{6}-\Delta \alpha_{3}^{2} p_{4}, N \Delta u_{4} p_{5}-\Delta u_{4} p_{3}\right. \\
& \left.\alpha_{3}^{2} \Delta p_{6}-N \alpha_{3}^{2} p_{4}, \Delta u_{4} p_{5}-N u_{4} p_{3}, \Delta p_{1}-N \Delta p_{2}, N p_{1}-\Delta p_{2}\right], \\
& g_{17}=\left[2 N p_{1 N}+4 \Delta p_{1 \Delta}+p_{1}, 2 N p_{2 N}+4 \Delta p_{2 \Delta}+3 p_{2}, 2 N p_{3 N}+4 \Delta p_{3 \Delta}+p_{3}\right. \text {, } \\
& 2 N p_{4 N}+4 \Delta p_{4 \Delta}+p_{4}, 2 N p_{5 N}+4 \Delta p_{5 \Delta}+3 p_{5}, 2 N p_{6 N}+4 \Delta p_{6 \Delta}+3 p_{6} \text {, } \\
& \left.2 N p_{7 N}+4 \Delta p_{7 \Delta}+p_{7}, 2 N p_{8 N}+4 \Delta p_{8 \Delta}+3 p_{8}\right] \\
& g_{18}=\left[-2 \Delta p_{1 N}-4 N \Delta p_{1 \Delta}+\Delta p_{2},-2 \Delta p_{2 N}-4 N \Delta p_{2 \Delta}-2 N p_{2}+p_{1},\right. \\
& -2 \Delta p_{3 N}-4 N \Delta p_{3 \Delta}+\Delta p_{5},-2 \Delta p_{4 N}-4 N \Delta p_{4 \Delta}+\Delta p_{6} \\
& -2 \Delta p_{5 N}-4 N \Delta p_{5 \Delta}-2 N p_{5}+p_{3},-2 \Delta p_{6 N}-4 N \Delta p_{6 \Delta}-2 N p_{6}+p_{4}, \\
& \left.-2 \Delta p_{7 N}-4 N \Delta p_{7 \Delta}+\Delta p_{8},-2 \Delta p_{8 N}-4 N \Delta p_{8 \Delta}-2 N p_{8}+p_{7}\right] \text {, } \\
& g_{19}=\left[-2 \Delta u_{4} p_{5 N}-4 N \Delta u_{4} p_{5 \Delta}-2 N u_{4} p_{5}+u_{4} p_{3},-2 u_{4} p_{3 N}-4 N u_{4} p_{3 \Delta}+u_{4} p_{5},\right. \\
& -2 \Delta p_{2 N}-4 N \Delta p_{2 \Delta}-2 N p_{2}+p_{1},-2 \Delta u_{4} p_{8 N}-4 N \Delta u_{4} p_{8 \Delta}-2 N u_{4} p_{8}+u_{4} p_{7}, \\
& -2 p_{1 N}-4 N p_{1 \Delta}+p_{2},-2 u_{4} p_{7 N}-4 N u_{4} p_{7 \Delta}+u_{4} p_{8}, \\
& \left.-2 \Delta p_{6 N}-4 N \Delta p_{6 \Delta}-2 N p_{6}+p_{4},-2 p_{4 N}-4 N p_{4 \Delta}+p_{6}\right] \text {, }
\end{aligned}
$$




$$
\begin{aligned}
& g_{20}=\left[\alpha_{3}^{2} p_{4}-2 N \alpha_{3}^{2} p_{6}-2 \Delta \alpha_{3}^{2} p_{6 N}-4 N \Delta \alpha_{3}^{2} p_{6 \Delta}, \alpha_{3}^{2} p_{6}-2 \alpha_{3}^{2} p_{4 N}-4 N \alpha_{3}^{2} p_{4 \Delta}\right. \text {, } \\
& \alpha_{3}^{2} p_{7}-2 \Delta \alpha_{3}^{2} p_{8 N}-4 N \Delta \alpha_{3}^{2} p_{8 \Delta}-2 N \alpha_{3}^{2} p_{8}, p_{1}-2 \Delta p_{2 N}-4 N \Delta p_{2 \Delta}-2 N p_{2}, \\
& -2 \alpha_{3}^{2} p_{7 N}-4 \alpha_{3}^{2} N p_{7 \Delta}+\alpha_{3}^{2} p_{8},-2 p_{1 N}-4 N p_{1 \Delta}+p_{2}, p_{3}-2 \Delta p_{5 N}-4 N \Delta p_{5 \Delta}-2 N p_{5} \text {, } \\
& \left.p_{5}-2 p_{3 N}-4 N p_{3 \Delta}\right] \\
& g_{21}=\left[2 N \Delta u_{4} p_{5 N}+4 \Delta^{2} u_{4} p_{5 \Delta}+3 \Delta u_{4} p_{5}, 2 N u_{4} p_{3 N}+4 \Delta u_{4} p_{3 \Delta}+u_{4} p_{3},\right. \\
& 2 N \Delta p_{2 N}+4 \Delta^{2} p_{2 \Delta}+3 \Delta p_{2}, 2 N \Delta u_{4} p_{8 N}+4 \Delta^{2} u_{4} p_{8 \Delta}+3 \Delta u_{4} p_{8} \\
& 2 N p_{1 N}+4 \Delta p_{1 \Delta}+p_{1}, 2 N u_{4} p_{7 N}+4 \Delta u_{4} p_{7 \Delta}+u_{4} p_{7} \\
& \left.2 N \Delta p_{6 N}+4 \Delta^{2} p_{6 \Delta}+3 \Delta p_{6}, 2 N p_{4 N}+4 \Delta p_{4 \Delta}+p_{4}\right] \\
& g_{22}=\left[2 N \Delta \alpha_{3}^{2} p_{6 N}+4 \Delta^{2} \alpha_{3}^{2} p_{6 \Delta}+3 \Delta \alpha_{3}^{2} p_{6}, 2 N \alpha_{3}^{2} p_{4 N}+4 \Delta \alpha_{3}^{2} p_{4 \Delta}+\alpha_{3}^{2} p_{4},\right. \\
& 2 N \Delta \alpha_{3}^{2} p_{8 N}+4 \Delta^{2} \alpha_{3}^{2} p_{8 \Delta}+3 \Delta \alpha_{3}^{2} p_{8}, 2 N \Delta p_{2 N}+4 \Delta^{2} p_{2 \Delta}+3 \Delta p_{2}, \\
& 2 N \alpha_{3}^{2} p_{7 N}+4 \Delta \alpha_{3}^{2} p_{7 \Delta}+\alpha_{3}^{2} p_{7}, 2 N p_{1 N}+4 \Delta p_{1 \Delta}+p_{1}, 2 N \Delta p_{5 N}+4 \Delta^{2} p_{5 \Delta}+3 \Delta p_{5}, \\
& \left.2 N p_{3 N}+4 \Delta p_{3 \Delta}+p_{3}\right] \\
& g_{23}=\left[2 N u_{4} \alpha_{3}^{2} p_{7 N}+4 \Delta \alpha_{3}^{2} u_{4} p_{7 \Delta}+u_{4} \alpha_{3}^{2} p_{7}, 2 N u_{4} \alpha_{3}^{2} p_{8 N}+4 \Delta u_{4} \alpha_{3}^{2} p_{8 \Delta}+3 \alpha_{3}^{2} u_{4} p_{8},\right. \\
& 2 N \alpha_{3}^{2} p_{4 N}+4 \Delta \alpha_{3}^{2} p_{4 \Delta}+\alpha_{3}^{2} p_{4}, 2 N u_{4} p_{3 N}+4 \Delta u_{4} p_{3 \Delta}+u_{4} p_{3}, \\
& 2 N \alpha_{3}^{2} p_{6 N}+4 \Delta \alpha_{3}^{2} p_{6 \Delta}+3 \alpha_{3}^{2} p_{6}, 2 N u_{4} p_{5 N}+4 \Delta u_{4} p_{5 \Delta}+3 u_{4} p_{5} \\
& \left.2 N p_{1 N}+4 \Delta p_{1 \Delta}+p_{1}, 2 N p_{2 N}+4 \Delta p_{2 \Delta}+3 p_{2}\right], \\
& g_{24}=\left[-2 \Delta \alpha_{3}^{2} u_{4} p_{7 N}-4 N \Delta \alpha_{3}^{2} u_{4} p_{7 \Delta}+\Delta \alpha_{3}^{2} u_{4} p_{8}\right. \\
& -2 \Delta \alpha_{3}^{2} u_{4} p_{8 N}-4 N \Delta \alpha_{3}^{2} u_{4} p_{8 \Delta}-2 N u_{4} \alpha_{3}^{2} p_{8}+u_{4} \alpha_{3}^{2} p_{7} \text {, } \\
& -2 \Delta \alpha_{3}^{2} p_{4 N}-4 N \Delta \alpha_{3}^{2} p_{4 \Delta}+\Delta \alpha_{3}^{2} p_{6},-2 \Delta u_{4} p_{3 N}-4 N \Delta u_{4} p_{3 \Delta}+u_{4} \Delta p_{5}, \\
& -2 \Delta \alpha_{3}^{2} p_{6 N}-4 N \Delta \alpha_{3}^{2} p_{6 \Delta}-2 N \alpha_{3}^{2} p_{6}+\alpha_{3}^{2} p_{4}, \\
& -2 \Delta u_{4} p_{5 N}-4 N \Delta u_{4} p_{5 \Delta}-2 N u_{4} p_{5}+u_{4} p_{3}, \\
& \left.-2 \Delta p_{1 N}-4 N \Delta p_{1 \Delta}+\Delta p_{2},-2 \Delta p_{2 N}-4 N \Delta p_{2 \Delta}-2 N p_{2}+p_{1}\right] \text {. }
\end{aligned}
$$

Prova. Os geradores $g_{1} \ldots g_{16}$ são obtidos por

$$
g_{i}=S_{i}\left[p_{1}, p_{2} \ldots p_{8}\right], 1 \leq i \leq 16
$$

onde os $S_{i}^{\prime}$ s são os geradores livres de $\mathbf{M}_{(z, \beta)}^{\mathrm{D}_{4}}$ da Proposição 4.1.5. e os geradores $g_{17} \ldots g_{24}$ obtidos por

$$
D_{z}(\hat{f}) \hat{f}_{j}, 1 \leq j \leq 8
$$

onde os $\hat{f}_{j}^{\prime} s$ são os geradores livres de $\overrightarrow{\mathcal{E}}_{(z, \beta)}^{\mathrm{D}_{4}}$ da Proposição 4.1.3. 
Proposição 4.1.7. O espaço tangente unipotente à $\hat{f} \in \overrightarrow{\mathcal{E}}_{(z, \beta)}^{\mathrm{D}_{4}}$ é

$$
\begin{array}{r}
\operatorname{TU}^{\mathrm{D}_{4}}(\hat{f})=\mathcal{E}_{\bar{u}}<N g_{1}, \Delta g_{1}, \alpha_{1} g_{1}, \alpha_{2} g_{1}, \alpha_{3}^{2} g_{1}, \lambda_{1} g_{1}, u_{4} g_{1}, g_{2} \ldots g_{16}, N g_{17}, \\
\Delta g_{17}, \alpha_{1} g_{17}, \alpha_{2} g_{17}, \alpha_{3}^{2} g_{17}, \lambda_{1} g_{17}, u_{4} g_{17}, g_{18} \ldots g_{24}>
\end{array}
$$

onde $g_{1} \ldots g_{24}$ são dados pela Proposição 4.1.6.

Prova. $\operatorname{TU}^{\mathrm{D}_{4}}(\hat{f})=\left\{\hat{S} \hat{f}+\left(D_{z} \hat{f}\right) \hat{X} \mid \hat{S} \in \mathbf{M}_{(z, \beta)}^{\mathrm{D}_{4}}, \hat{X} \in \overrightarrow{\mathcal{M}}_{(z, \beta)}^{\mathrm{D}_{4}}\right.$ e $\left.\hat{S}^{o}=0,\left(D_{z} \hat{X}\right)^{o}=0\right\}$

Um simples cálculo mostra que

$\mathrm{TU}^{\mathrm{D}_{4}}(\hat{f})=\left\{\mathcal{M}_{(z, \beta)}^{\mathrm{D}_{4}} g_{1}, g_{2} \ldots g_{16}, \mathcal{M}_{(z, \beta)}^{\mathrm{D}_{4}} g_{17}, g_{18} \ldots g_{24}>\right.$ com $g_{i}, 1 \leq i \leq 24$, dados pela Proposição 4.1.6.

Recorde de (4.3) que um caminho $\tilde{\alpha} \in \mathcal{P}$ é dado por

$$
\tilde{\alpha}\left(\lambda_{1}, \lambda_{2}\right)=\left(\alpha_{1}\left(\lambda_{1}, u_{4}\right), \alpha_{2}\left(\lambda_{1}, u_{4}\right), \lambda_{2} \alpha_{3}\left(\lambda_{1}, u_{4}\right)\right)
$$

Calculamos o espaço tangente à $\tilde{\alpha}$ em duas etapas:

Etapa 1. $\hat{F}_{0}^{1}:\left(\mathbb{C} \times \mathbb{R}^{3} \times \mathbb{R}^{2}, 0\right) \rightarrow \mathbb{C}$ é definida por

$$
\hat{F}_{0}^{1}\left(z, \alpha_{1}, \alpha_{2}, \alpha_{3}, \lambda_{1}, \lambda_{2}\right)=F_{0}^{1}\left(z, \alpha_{1}, \alpha_{2}, \alpha_{3}\right)=\left(\hat{m} N+\alpha_{1}\right) z+\epsilon_{5} \delta \bar{z}+\alpha_{3} \bar{z}
$$

onde $\hat{m}=m+\alpha_{2}$.

Usando a Proposição 4.1.6, um longo cálculo mostra que $\mathbf{T}^{\mathrm{D}_{4}}\left(\hat{F}_{0}^{1}\right)=\mathcal{E}_{\bar{u}}\left\langle g_{1} \ldots g_{15}\right\rangle$ onde

$$
\begin{aligned}
& g_{1}=\epsilon_{5} \delta \bar{z}+\hat{m} N z \\
& g_{2}=\left(\hat{m}^{2}-1\right) N \lambda_{2} \bar{z}+\hat{m} \alpha_{1} \lambda_{2} \bar{z}+\hat{m} \alpha_{3} \lambda_{2} z \\
& g_{3}=\hat{m} \Delta z+\alpha_{1} N z \\
& g_{4}=\left(\hat{m}^{2}-1\right) \Delta \lambda_{2} \bar{z}-\alpha_{1} \alpha_{3} \lambda_{2} z-\alpha_{1}^{2} \lambda_{2} \bar{z} \\
& g_{5}=\epsilon_{5}\left(1-\hat{m}^{2}\right) \delta \lambda_{2} z+\alpha_{1} \lambda_{2} \bar{z}+\alpha_{3} \lambda_{2} z \\
& g_{6}=\epsilon_{5}\left(\hat{m}^{2}-1\right) \delta \alpha_{3} \lambda_{2} \bar{z}+\alpha_{1}{ }^{2} \lambda_{2} \bar{z}+\alpha_{1} \alpha_{3} \lambda_{2} z, \\
& g_{7}=\hat{m}^{2} N \delta \alpha_{3} z+\epsilon_{5} \alpha_{1}^{2} N z
\end{aligned}
$$




$$
\begin{aligned}
& g_{8}=\left(1-\hat{m}^{2}\right) N^{2} \lambda_{2} \bar{z}+\alpha_{1} \alpha_{3} \lambda_{2} z+\alpha_{1}^{2} \lambda_{2} \bar{z} \\
& g_{9}=\hat{m} N^{2} z+\alpha_{1} N z \\
& g_{10}=\alpha_{1} z+\alpha_{3} \bar{z} \\
& g_{11}=\alpha_{1} \alpha_{3} \lambda_{2} z+\alpha_{3}^{2} \lambda_{2} \bar{z} \\
& g_{12}=\hat{m} \alpha_{3}^{2} z+\left(1-\hat{m}^{2}\right) \alpha_{1} N z+\hat{m} \alpha_{1} \alpha_{3} \bar{z} \\
& g_{13}=\alpha_{1} \alpha_{3} \lambda_{2} z+\hat{m}\left(1-\hat{m}^{2}\right) N \alpha_{3} \lambda_{2} z+\alpha_{1}^{2} \lambda_{2} \bar{z} \\
& g_{14}=\alpha_{1} \alpha_{3}^{2} z+\hat{m}\left(1-\hat{m}^{2}\right) \alpha_{3}^{2} N z+\alpha_{1}^{2} \alpha_{3} \bar{z} \\
& g_{15}=\hat{m} \alpha_{3}^{2} \alpha_{3} \lambda_{2} z+\left(1-\hat{m}^{2}\right) \alpha_{1} N \alpha_{3} \lambda_{2} z+\hat{m} \alpha_{1} \alpha_{3}^{2} \lambda_{2} \bar{z} .
\end{aligned}
$$

Segue que

$$
\begin{aligned}
\mathbf{T}^{\mathrm{D}_{4}}\left(\hat{F}_{0}^{1}\right) \cap\left(\mathcal{E}_{\beta}<z, N z, \bar{z}>\right)^{\mathrm{D}_{4}}=\mathcal{E}_{\beta}^{\mathrm{D}_{4}}<h_{1}^{\prime}, h_{2}^{\prime}, h_{3}^{\prime}, h_{4}^{\prime}, h_{5}^{\prime}, h_{6}^{\prime}>\text { onde } \\
h_{1}^{\prime}=\hat{m} \alpha_{3}^{2} z+\left(1-\hat{m}^{2}\right) \alpha_{1} N z+\hat{m} \alpha_{1} \alpha_{3} \bar{z} \\
h_{2}^{\prime}=\alpha_{1} \alpha_{3} \lambda_{2} z+\hat{m}\left(1-\hat{m}^{2}\right) N \alpha_{3} \lambda_{2} z+\alpha_{1}^{2} \lambda_{2} \bar{z} \\
h_{3}^{\prime}=\alpha_{1} \alpha_{3}^{2} z+\hat{m}\left(1-\hat{m}^{2}\right) \alpha_{3}^{2} N z+\alpha_{1}^{2} \alpha_{3} \bar{z} \\
h_{4}^{\prime}=\hat{m} \alpha_{3}^{2} \alpha_{3} \lambda_{2} z+\left(1-\hat{m}^{2}\right) \alpha_{1} N \alpha_{3} \lambda_{2} z+\hat{m} \alpha_{1} \alpha_{3}^{2} \lambda_{2} \bar{z} \\
h_{5}^{\prime}=\alpha_{1} z+\alpha_{3} \bar{z} \\
h_{6}^{\prime}=\alpha_{1} \alpha_{3} \lambda_{2} z+\alpha_{3}^{2} \lambda_{2} \bar{z} .
\end{aligned}
$$

Sejam $\eta_{j}:\left(\mathbb{R}^{3} \times \mathbb{R}^{2}, 0\right) \rightarrow \mathbb{R}^{3}, 1 \leq j \leq 6$, dadas por

$$
\begin{aligned}
& \eta_{1}(\alpha, \lambda)=\left(\hat{m} \alpha_{3}^{2},\left(1-\hat{m}^{2}\right) \alpha_{1}, \hat{m} \alpha_{1} \alpha_{3}\right), \\
& \eta_{2}(\alpha, \lambda)=\left(\alpha_{1} \alpha_{3} \lambda_{2}, \hat{m}\left(1-\hat{m}^{2}\right) \alpha_{3} \lambda_{2}, \alpha_{1}^{2} \lambda_{2}\right), \\
& \eta_{3}(\alpha, \lambda)=\left(\alpha_{1} \alpha_{3}^{2}, \hat{m}\left(1-\hat{m}^{2}\right) \alpha_{3}^{2}, \alpha_{1}^{2} \alpha_{3}\right), \\
& \eta_{4}(\alpha, \lambda)=\left(\hat{m} \alpha_{3}^{3} \lambda_{2},\left(1-\hat{m}^{2}\right) \alpha_{1} \alpha_{3} \lambda_{2}, \hat{m} \alpha_{1} \alpha_{3}^{2} \lambda_{2}\right), \\
& \eta_{5}(\alpha, \lambda)=\left(\alpha_{1}, 0, \alpha_{3}\right), \\
& \eta_{6}(\alpha, \lambda)=\left(\alpha_{1} \alpha_{3} \lambda_{2}, 0, \alpha_{3}^{2} \lambda_{2}\right) .
\end{aligned}
$$


As aplicaşôes $\eta_{j}^{\prime} s$ são $\mathrm{D}_{4}$-equivariantes pois

$$
\eta_{j}\left(\alpha_{1}, \alpha_{2},-\alpha_{3}, \lambda_{1},-\lambda_{2}\right)=\gamma_{3} \cdot \eta_{j}\left(\alpha_{1}, \alpha_{2}, \alpha_{3}, \lambda_{1}, \lambda_{2}\right)
$$

onde

$$
\gamma_{3} \cdot \eta_{j}=\left(\eta_{j_{1}}, \eta_{j_{2}},-\eta_{j_{3}}\right), 1 \leq j \leq 6
$$

Seja. $N=\mathcal{E}_{\beta}^{\mathrm{D}_{4}}<\eta_{1} \ldots \eta_{6}>$.

Para $\tilde{\alpha} \in \mathcal{P}$, definimos

$$
\tilde{\alpha}^{*} N=\mathcal{E}_{\lambda}^{\mathrm{D}_{4}}<\tilde{\alpha}^{*} \eta_{1} \ldots \tilde{\alpha}^{*} \eta_{6}>
$$

onde

$$
\begin{aligned}
& \tilde{\alpha}^{*} \eta_{1}=\left(\hat{m} u_{4} a_{3}^{2},\left(1-\hat{m}^{2}\right) \alpha_{1}, \hat{m} \alpha_{1} \alpha_{3} \lambda_{2}\right), \\
& \tilde{\alpha}^{*} \eta_{2}=\left(u_{4} \alpha_{1} \alpha_{3}, \hat{m}\left(1-\hat{m}^{2}\right) u_{4} \alpha_{3}, \alpha_{1}^{2} \lambda_{2}\right), \\
& \tilde{\alpha}^{*} \eta_{3}=\left(u_{4} \alpha_{1} \alpha_{3}^{2}, \hat{m}\left(1-\hat{m}^{2}\right) u_{4} \alpha_{3}^{2}, \alpha_{1}^{2} \alpha_{3} \lambda_{2}\right), \\
& \tilde{\alpha}^{*} \eta_{4}=\left(\hat{m} \alpha_{3}^{3} u_{4}^{2},\left(1-\hat{m}^{2}\right) \alpha_{1} \alpha_{3} u_{4}, \hat{m} \alpha_{1} u_{4} \alpha_{3}^{2} \lambda_{2}\right), \\
& \tilde{\alpha}^{*} \eta_{5}=\left(\alpha_{1}, 0, \alpha_{3} \lambda_{2}\right), \\
& \tilde{\alpha}^{*} \eta_{6}=\left(\alpha_{1} u_{4} \alpha_{3}, 0, u_{4} \alpha_{3}^{2} \lambda_{2}\right) .
\end{aligned}
$$

Como $\alpha_{i} \in \mathcal{E}_{\lambda}^{\mathrm{D}_{4}}, \quad$ temos que $\quad \tilde{\alpha}^{*} N=\mathcal{E}_{\lambda}^{\mathrm{D}_{4}}<\tilde{h}_{1}, \tilde{h}_{2}, \tilde{h}_{3}>\quad$ com

$$
\begin{aligned}
& \tilde{h}_{1}=\left(\hat{m} u_{4} \alpha_{3}^{2},\left(1-\hat{m}^{2}\right) \alpha_{1}, \hat{m} \alpha_{1} \alpha_{3} \lambda_{2}\right), \\
& \tilde{h}_{2}=\left(u_{4} \alpha_{1} \alpha_{3}, \hat{m}\left(1-\hat{m}^{2}\right) u_{4} \alpha_{3}, \alpha_{1}^{2} \lambda_{2}\right), \\
& \tilde{h}_{3}=\left(\alpha_{1}, 0, \alpha_{3} \lambda_{2}\right) .
\end{aligned}
$$

Etapa 2.

$$
\left(D_{\lambda} \tilde{\alpha}\right)=\left(\begin{array}{ll}
\left(\alpha_{1}\right)_{\lambda_{1}} & 2 \lambda_{2}\left(\alpha_{1}\right)_{u_{4}} \\
\left(\alpha_{2}\right)_{\lambda_{1}} & 2 \lambda_{2}\left(\alpha_{2}\right)_{u_{4}} \\
\lambda_{2}\left(\alpha_{3}\right)_{\lambda_{1}} & \alpha_{3}+2 u_{4}\left(\alpha_{3}\right)_{u_{4}}
\end{array}\right)
$$


Pela Proposiçào 2.1.4., $\overrightarrow{\mathcal{E}}_{\lambda}^{\mathrm{D}_{4}}$ é gerado por $\left(\begin{array}{l}1 \\ 0\end{array}\right)$ e $\left(\begin{array}{l}0 \\ \lambda_{2}\end{array}\right)$ como um $\mathcal{E}_{\lambda}^{\mathrm{D}_{4}}$-módulo. Sejam

$$
\begin{aligned}
& \hat{h}_{4}=\left(D_{\lambda} \tilde{\alpha}\right)\left(\begin{array}{c}
1 \\
0
\end{array}\right)=\left(\left(\alpha_{1}\right)_{\lambda_{1}} \cdot\left(\alpha_{2}\right)_{\lambda_{3}},\left(\alpha_{3}\right)_{\lambda_{3}} \lambda_{2}\right), \\
& \hat{h}_{5}=\left(D_{\lambda} \tilde{\alpha}\right)\left(\begin{array}{c}
0 \\
\lambda_{2}
\end{array}\right)=\left(2 u_{4}\left(\alpha_{1}\right)_{u_{4}}, 2 u_{4}\left(\alpha_{2}\right)_{u_{4}}, \alpha_{3} \lambda_{2}+2\left(\alpha_{3}\right)_{u_{4}} u_{4} \lambda_{2}\right) .
\end{aligned}
$$

Pela Definição 3.2.10, o espaço tangente à $\tilde{\alpha} \in \mathcal{P}$ é

$$
\begin{gathered}
\mathcal{T}(\tilde{\alpha})=\tilde{\alpha}^{*} N+\left(D_{\lambda} \tilde{\alpha}\right) \overrightarrow{\mathcal{E}}_{\lambda}^{\mathrm{D}_{4}}, \text { ou seja, } \mathcal{T}(\tilde{\alpha})=\mathcal{E}_{\lambda}^{\mathrm{D}_{4}}<\tilde{h}_{1} \ldots \tilde{h}_{5}>\text { onde } \\
\tilde{h}_{1}=\left(\hat{m} u_{4} \alpha_{3}^{2},\left(1-\hat{m}^{2}\right) \alpha_{1}, \hat{m} \alpha_{1} \alpha_{3} \lambda_{2}\right) \\
\tilde{h}_{2}=\left(u_{4} \alpha_{1} \alpha_{3}, \hat{m}\left(1-\hat{m}^{2}\right) u_{4} \alpha_{3}, \alpha_{1}^{2} \lambda_{2}\right) \\
\tilde{h}_{3}=\left(\alpha_{1}, 0, \alpha_{3} \lambda_{2}\right) \\
\tilde{h}_{4}=\left(\left(\alpha_{1}\right)_{\lambda_{1}},\left(\alpha_{2}\right)_{\lambda_{1}},\left(\alpha_{3}\right)_{\lambda_{1}} \lambda_{2}\right) \\
\tilde{h}_{5}=\left(2 u_{4}\left(\alpha_{1}\right)_{u_{4}}, 2 u_{4}\left(\alpha_{2}\right)_{u_{4}}, \alpha_{3} \lambda_{2}+2\left(\alpha_{3}\right)_{u_{4}} u_{4} \lambda_{2}\right) .
\end{gathered}
$$

Usamos a Proposição 4.1.7., para achar o espaço tangente unipotente à $\tilde{\alpha}$. Um longo cálculo mostra que $\mathbf{T U}^{\mathrm{D}_{4}}\left(\hat{F}_{0}^{1}\right)=\mathcal{E}_{\bar{u}}\left\langle h_{1} \ldots h_{27}\right\rangle$ 'onde

$$
\begin{aligned}
& h_{1}=\alpha_{1} \alpha_{3} \lambda_{2} z+\alpha_{3}^{2} \lambda_{2} \bar{z} \\
& h_{2}=\alpha_{1}^{2} z+\alpha_{1} \alpha_{3} \bar{z} \\
& h_{3}=\alpha_{1} \alpha_{2} z+\alpha_{2} \alpha_{3} \bar{z} \\
& h_{4}=\alpha_{1} \lambda_{1} z+\lambda_{1} \alpha_{3} \bar{z} \\
& h_{5}=\alpha_{1} \alpha_{3}^{2} z+\alpha_{3}^{2} \alpha_{3} \bar{z} \\
& h_{6}=\alpha_{1} u_{4} z+u_{4} \alpha_{3} \bar{z} \\
& h_{7}=\hat{m} \alpha_{3}^{2} z+\left(1-\hat{m}^{2}\right) \alpha_{1} N z+\hat{m} \alpha_{1} \alpha_{3} \bar{z}, \\
& h_{8}=\alpha_{1} \alpha_{3} \lambda_{2} z+\hat{m}\left(1-\hat{m}^{2}\right) N \alpha_{3} \lambda_{2} z+\alpha_{1}^{2} \lambda_{2} \bar{z},
\end{aligned}
$$




$$
\begin{aligned}
& h_{9}=\alpha_{1} \alpha_{3}^{2} z+\hat{m}\left(1-\hat{m}^{2}\right) \alpha_{3}^{2} N z+\alpha_{1}^{2} \alpha_{3} \bar{z} \\
& h_{10}=\hat{m} \alpha_{3}^{2} \alpha_{3} \lambda_{2} z+\left(1-\hat{m}^{2}\right) a_{1} N \alpha_{3} \lambda_{2} z+\hat{m} \alpha_{1} \alpha_{3}^{2} \lambda_{2} \bar{z} \text {. } \\
& h_{11}=\alpha_{1} N z+\alpha_{3} N \bar{z} \\
& h_{12}=\hat{m} \Delta a_{3} \bar{z}-\alpha_{1}^{2} N z \text {, } \\
& h_{13}=\left(\hat{m}^{2}-1\right) N \lambda_{2} \bar{z}+\hat{m} \alpha_{1} \lambda_{2} \bar{z}+\hat{m} \alpha_{3} \lambda_{2} z \\
& h_{14}=\hat{m} \Delta z+\alpha_{1} N z \text {, } \\
& h_{15}=\left(\hat{m}^{2}-1\right) \Delta \lambda_{2} \bar{z}-\alpha_{1} \alpha_{3} \lambda_{2} z-\alpha_{1}^{2} \lambda_{2} \bar{z}, \\
& h_{16}=\epsilon_{5}\left(1-\hat{m}^{2}\right) \delta \lambda_{2} z+\alpha_{1} \lambda_{2} \bar{z}+\alpha_{3} \lambda_{2} z, \\
& h_{17}=\epsilon_{5}\left(\hat{m}^{2}-1\right) \delta \alpha_{3} \lambda_{2} \bar{z}+\alpha_{1} \alpha_{3} \lambda_{2} z+\alpha_{1}^{2} \lambda_{2} \bar{z}, \\
& h_{18}=\hat{m} \alpha_{1} N z+\epsilon_{5} \alpha_{1} \delta \bar{z}, \\
& h_{19}=\hat{m} \alpha_{2} N z+\epsilon_{5} \alpha_{2} \delta \bar{z}, \\
& h_{20}=\hat{m} \lambda_{1} N z+\epsilon_{5} \lambda_{1} \delta \bar{z} \\
& h_{21}=\hat{m} \alpha_{3}^{2} N z+\epsilon_{5} \alpha_{3}^{2} \delta \bar{z}, \\
& h_{22}=\hat{m} u_{4} N z+\epsilon_{5} u_{4} \delta \bar{z}, \\
& h_{23}=\hat{m} N^{2} z+\alpha_{1} N z \\
& h_{24}=\epsilon_{5} \hat{m}\left(\hat{m}^{2}-1\right) N \delta \lambda_{2} z+\alpha_{1}^{2} \lambda_{2} \bar{z}+\alpha_{1} \alpha_{3} \lambda_{2} z, \\
& h_{25}=\hat{m}^{2} N \delta \alpha_{3} z+\epsilon_{5} \alpha_{1}^{2} N z \\
& h_{26}=\epsilon_{5} N \delta \bar{z}-\alpha_{1} N z, \\
& h_{27}=\epsilon_{5} \hat{m} \Delta \delta \bar{z}-\alpha_{1}^{2} N z \text {. }
\end{aligned}
$$

Segue que 
$\mathbf{T U}^{\mathrm{D}_{4}}\left(\hat{F}_{0}^{1}\right) \cap\left(\mathcal{E}_{\beta}<z, N z, \bar{z}>\right)^{\mathrm{D}_{4}}=\mathcal{E}_{\bar{u}}<h_{1}^{\prime} \ldots h_{10}^{\prime}>$ onde

$$
\begin{aligned}
& h_{1}^{\prime}=\alpha_{1} \alpha_{3} \lambda_{2} \bar{z}+\alpha_{3}^{2} \lambda_{2} \bar{z}, \\
& h_{2}^{\prime}=\alpha_{1}^{2} z+\alpha_{1} \alpha_{3} z \\
& h_{3}^{\prime}=\alpha_{1} \alpha_{2} z+\alpha_{2} \alpha_{3} \bar{z} \\
& h_{4}^{\prime}=\alpha_{1} \lambda_{1} z+\lambda_{1} \alpha_{3} \bar{z} \\
& h_{5}^{\prime}=\alpha_{1} \alpha_{3}^{2} z+\alpha_{3}^{2} \alpha_{3} \bar{z} \\
& h_{6}^{\prime}=\alpha_{1} u_{4} z+u_{4} \alpha_{3} \bar{z} \\
& h_{7}^{\prime}=\hat{m} \alpha_{3}^{2} z+\left(1-\hat{m}^{2}\right) \alpha_{1} N z+\hat{m} \alpha_{1} \alpha_{3} \bar{z}, \\
& h_{8}^{\prime}=\alpha_{1} \alpha_{3} \lambda_{2} z+\hat{m}\left(1-\hat{m}^{2}\right) N \alpha_{3} \lambda_{2} z+\alpha_{1}^{2} \lambda_{2} \bar{z}, \\
& h_{9}^{\prime}=\alpha_{1} \alpha_{3}^{2} z+\hat{m}\left(1-\hat{m}^{2}\right) \alpha_{3}^{2} N z+\alpha_{1}^{2} \alpha_{3} \bar{z}, \\
& h_{10}^{\prime}=\hat{m} \alpha_{3}^{2} \alpha_{3} \lambda_{2} z+\left(1-\hat{m}^{2}\right) \alpha_{1} N \alpha_{3} \lambda_{2} z+\hat{m} \alpha_{1} \alpha_{3}^{2} \lambda_{2} \bar{z} .
\end{aligned}
$$

Sejam $\tilde{\eta}_{j}:\left(\mathbb{R}^{3} \times \mathbb{R}^{2}, 0\right) \rightarrow \mathbb{R}^{3}, 1 \leq j \leq 10$ dadas por

$$
\begin{aligned}
& \tilde{\eta}_{1}\left(\alpha_{1}, \alpha_{2}, \alpha_{3}, \lambda_{1}, \lambda_{2}\right)=\left(\alpha_{1} \alpha_{3} \lambda_{2}, 0, \alpha_{3}^{2} \lambda_{2}\right) \\
& \tilde{\eta}_{2}\left(\alpha_{1}, \alpha_{2}, \alpha_{3}, \lambda_{1}, \lambda_{2}\right)=\left(\alpha_{1}^{2}, 0, \alpha_{1} \alpha_{3}\right) \\
& \tilde{\eta}_{3}\left(\alpha_{1}, \alpha_{2}, \alpha_{3}, \lambda_{1}, \lambda_{2}\right)=\left(\alpha_{1} \alpha_{2}, 0, \alpha_{2} \alpha_{3}\right), \\
& \tilde{\eta}_{4}\left(\alpha_{1}, \alpha_{2}, \alpha_{3}, \lambda_{1}, \lambda_{2}\right)=\left(\alpha_{1} \lambda_{1}, 0, \lambda_{1} \alpha_{3}\right) \\
& \tilde{\eta}_{5}\left(\alpha_{1}, \alpha_{2}, \alpha_{3}, \lambda_{1}, \lambda_{2}\right)=\left(\alpha_{1} \alpha_{3}^{2}, 0, \alpha_{3}^{2}\right) \\
& \tilde{\eta}_{6}\left(\alpha_{1}, \alpha_{2}, \alpha_{3}, \lambda_{1}, \lambda_{2}\right)=\left(\alpha_{1} u_{4}, 0, u_{4} \alpha_{3}\right), \\
& \tilde{\eta}_{7}\left(\alpha_{1}, \alpha_{2}, \alpha_{3}, \lambda_{1}, \lambda_{2}\right)=\left(\hat{m} \alpha_{3}^{2},\left(1-\hat{m}^{2}\right) \alpha_{1}, \hat{m} \alpha_{1} \alpha_{3}\right), \\
& \tilde{\eta}_{8}\left(\alpha_{1}, \alpha_{2}, \alpha_{3}, \lambda_{1}, \lambda_{2}\right)=\left(\alpha_{1} \alpha_{3} \lambda_{2}, \hat{m}\left(1-\hat{m}^{2}\right) \alpha_{3} \lambda_{2}, \alpha_{1}^{2} \lambda_{2}\right) \\
& \tilde{\eta}_{9}\left(\alpha_{1}, \alpha_{2}, \alpha_{3}, \lambda_{1}, \lambda_{2}\right)=\left(\alpha_{1} \alpha_{3}^{2}, \hat{m}\left(1-\hat{m}^{2}\right) \alpha_{3}^{2}, \alpha_{1}^{2} \alpha_{3}\right), \\
& \tilde{\eta}_{10}\left(\alpha_{1}, \alpha_{2}, \alpha_{3}, \lambda_{1}, \lambda_{2}\right)=\left(\hat{m} \alpha_{3}^{3} \lambda_{2},\left(1-\hat{m}^{2}\right) \alpha_{1} \alpha_{3} \lambda_{2}, \hat{m} \alpha_{1} \alpha_{3}^{2} \lambda_{2}\right)
\end{aligned}
$$


É imediato ver que as $\tilde{\eta}_{j}{ }^{\prime} s, 1 \leq j \leq 10$, sâo $\mathrm{D}_{4}$-equivariantes. Recorde que $\tilde{\alpha}\left(\lambda_{1}, \lambda_{2}\right)=\left(\alpha_{1}\left(\lambda_{1}, u_{4}\right), \alpha_{2}\left(\lambda_{1}, u_{4}\right), \lambda_{2} \alpha_{3}\left(\lambda_{1}, u_{4}\right)\right)$.

Seja $\tilde{N}=\mathcal{E}_{\beta}^{D_{4}}<\tilde{\eta}_{1} \ldots \tilde{\eta}_{10}>$. Vimos que $\tilde{\alpha}^{*} \tilde{N}=\mathcal{E}_{\lambda}^{\mathrm{D}_{4}}<\tilde{\alpha}^{*} \tilde{\eta}_{1} \ldots \tilde{\alpha}^{*} \tilde{\eta}_{10}>$ onde

$$
\begin{aligned}
& \tilde{\alpha}^{*} \tilde{\eta}_{1}=\left(\alpha_{1} u_{4} \alpha_{3}, 0, u_{4} \alpha_{3}^{2} \lambda_{2}\right), \\
& \hat{\alpha}^{*} \tilde{\eta}_{2}=\left(\alpha_{1}^{2}, 0, \alpha_{1} \alpha_{3} \lambda_{2}\right), \\
& \tilde{\alpha}^{*} \tilde{\eta}_{3}=\left(\alpha_{1} \alpha_{2}, 0, \alpha_{2} \alpha_{3} \lambda_{2}\right), \\
& \tilde{\alpha}^{*} \tilde{\eta}_{4}=\left(\alpha_{1} \lambda_{1}, 0, \lambda_{1} \alpha_{3} \lambda_{2}\right), \\
& \tilde{\alpha}^{*} \tilde{\eta}_{5}=\left(\alpha_{1} u_{4} \alpha_{3}^{2}, 0, u_{4} \alpha_{3}^{3} \lambda_{2}\right), \\
& \tilde{\alpha}^{*} \tilde{\eta}_{6}=\left(\alpha_{1} u_{4}, 0, u_{4} \alpha_{3} \lambda_{2}\right), \\
& \tilde{\alpha}^{*} \tilde{\eta}_{7}=\left(\hat{m} u_{4} \alpha_{3}^{2},\left(1-\hat{m}^{2}\right) \alpha_{1}, \hat{m} \alpha_{1} \alpha_{3} \lambda_{2}\right), \\
& \tilde{\alpha}^{*} \tilde{\eta}_{8}=\left(\alpha_{1} \alpha_{3} u_{4}, \hat{m}\left(1-\hat{m}^{2}\right) u_{4} \alpha_{3}, \alpha_{1}^{2} \lambda_{2}\right), \\
& \hat{\alpha}^{*} \tilde{\eta}_{9}=\left(\alpha_{1} u_{4} \alpha_{3}^{2}, \hat{m}\left(1-\hat{m}^{2}\right) u_{4} \alpha_{3}^{2}, \alpha_{1}^{2} \alpha_{3} \lambda_{2}\right), \\
& \tilde{\alpha}^{*} \tilde{\eta}_{10}=\left(\hat{m} u_{4}^{2} \alpha_{3}^{3},\left(1-\hat{m}^{2}\right) \alpha_{1} \alpha_{3} u_{4}, \hat{m} \alpha_{1} u_{4} \alpha_{3}^{2} \lambda_{2}\right) .
\end{aligned}
$$

Como $\alpha_{i}, 1 \leq i \leq 3$, e $u_{4}$ pertencem a $\mathcal{E}_{\lambda}^{\mathrm{D}_{4}}$, temos que

$$
\begin{aligned}
& \hat{\alpha}^{*} \tilde{N}=\mathcal{E}_{\lambda}^{\mathrm{D}}<\hat{h}_{1} \ldots \hat{h}_{5}>\text { onde } \\
& \hat{h}_{1}=\left(\alpha_{1}^{2}, 0, \alpha_{1} \alpha_{3} \lambda_{2}\right), \\
& \hat{h}_{2}=\left(\alpha_{1} \alpha_{2}, 0, \alpha_{2} \alpha_{3} \lambda_{2}\right), \\
& \hat{h}_{3}=\left(\alpha_{1} \lambda_{1}, 0, \lambda_{1} \alpha_{3} \lambda_{2}\right), \\
& \hat{h}_{4}=\left(\alpha_{1} u_{4}, 0, u_{4} \alpha_{3} \lambda_{2}\right), \\
& \hat{h}_{5}=\left(\hat{m} u_{4} \alpha_{3}^{2},\left(1-\hat{m}^{2}\right) \alpha_{1}, \hat{m} \alpha_{1} \alpha_{3} \lambda_{2}\right), \\
& \hat{h}_{6}=\left(\alpha_{1} \alpha_{3} u_{4}, \hat{m}\left(1-\hat{m}^{2}\right) u_{4} \alpha_{3}, \alpha_{1}^{2} \lambda_{2}\right) .
\end{aligned}
$$

Os geradores em (4.7), quando comparados com os apresentados em (4.5), satisfazem as relações a seguir. Essas relações são, na realidade, consequência da definição do 
espaço tangente unipotente.

$$
\begin{array}{ll}
\hat{h}_{1}=\alpha_{1} \tilde{h}_{3}, & \hat{h}_{4}=u_{4} \hat{h}_{3}, \\
\hat{h}_{2}=\alpha_{2} \tilde{h}_{3}, & \hat{h}_{5}=\tilde{h}_{1}, \\
\hat{h}_{3}=\lambda_{1} \tilde{h}_{3}, & \hat{h}_{6}=\tilde{h}_{2} .
\end{array}
$$

Segue que $\tilde{\alpha}^{*} \tilde{N}=\mathcal{E}_{\lambda}^{\mathrm{D}_{4}}<\tilde{h}_{1}, \tilde{h}_{2}, \alpha_{1} \tilde{h}_{3}, \alpha_{2} \tilde{h}_{3}, \lambda_{1} \tilde{h}_{3}, u_{4} \tilde{h}_{3}>$.

Usando a Definição 3.2.10,

$$
\mathcal{T U}(\tilde{\alpha})=\tilde{\alpha}^{*} \tilde{N}+\left(D_{\lambda} \tilde{\alpha}\right) \mathcal{M}_{\lambda}^{2} \overrightarrow{\mathcal{E}}_{\lambda}^{\mathrm{D}_{4}}
$$

Portanto,

$$
\mathbf{T U}(\tilde{\alpha})=\mathcal{E}_{\lambda}^{\mathrm{D}_{4}}<\tilde{h}_{1}, \tilde{h}_{2}, \alpha_{1} \tilde{h}_{3}, \lambda_{1} \tilde{h}_{3}, u_{4} \tilde{h}_{3}, \lambda_{1}^{2} \tilde{h}_{4}, u_{4} \tilde{h}_{4}, \lambda_{1} \tilde{h}_{5}, u_{4} \tilde{h}_{5}>
$$

$\operatorname{com} \tilde{h}_{i}{ }^{\prime} s, 1 \leq i \leq 5$, dados em (4.6).

O Teorema seguinte apresenta a classificação de problemas $\mathrm{D}_{4}$ de codimensâo topológica 2 e com centro organizador dado por

$$
f_{0}^{1}(z)=m N z+\epsilon_{5} \delta \bar{z}, m \neq \pm 1,0
$$

\section{TEOREMA 4.1.8. (Teorema do Reconhecimento para $f_{0}^{1}$ )}

Seja $f=[p, q, r, s]$ um problema de bifurcaçâo $\mathrm{D}_{4}$ com centro organizador $f_{0}^{1}$. Então $f$ será $\mathcal{K}_{\lambda}^{\mathrm{D}_{4}}$-equivalente às formas normais abaixo se e somente se $f$ satisfizer as correspondentes condições de definição e não degenericidade. Em todos os casos $p^{\circ}=0$.

O desdobramento miniversal é obtido adiçionando-se à forma normal os correspondentes termos do desdobramento dados em cada caso.

I: $\left[m N+\epsilon_{o} \lambda_{1}, \epsilon_{5}, 1,0\right]$ (Forma genérica normal).

Coeficientes: $\quad m=\frac{p_{N}^{o}}{\left|q^{\circ}\right|}, \epsilon_{o}=s g p_{\lambda_{1}}^{\circ}, \epsilon_{5}=s g q^{\circ}$.

Condições de não-degenericidade: $p_{N}^{o} \neq 0, p_{N}^{o} \neq \pm q^{o}, p_{\lambda_{1}}^{o} \neq 0, r^{o} \neq 0$. 
Termos do desdobramento: $\quad m[N, 0,0,0]$.

Codim topológica / $\mathrm{D}_{4}$-codim: $0 / 1$.

$\mathrm{I}_{1}:\left[m N+\epsilon_{3} N \lambda_{1}+\epsilon_{4} \lambda_{1}^{2}, \epsilon_{5}, 1,0\right]$.

Condição de definição: $\quad p_{\lambda_{1}}^{o}=0$.

Coeficientes: $\epsilon_{4}=s g p_{\lambda_{1} \lambda_{1}}^{o}, \epsilon_{5}=s g q^{o}$,

$$
\begin{aligned}
& \epsilon_{3}=\epsilon_{5} s g\left(p_{N \lambda_{1}}^{o} q^{o}-q_{\lambda_{1}}^{o} p_{N}^{o}\right), \\
& m=\frac{p_{N}^{o}}{\left|q^{o}\right|} .
\end{aligned}
$$

Condições de não-degenericidade: $p_{N}^{o} \neq 0, p_{N}^{o} \neq \pm q^{\circ}$,

$$
\begin{aligned}
& p_{\lambda_{1} \lambda_{1}}^{o} \neq 0, r^{o} \neq 0, \\
& p_{N \lambda_{1}}^{o} q^{\circ}-q_{\lambda_{1}}^{o} p_{N}^{o} \neq 0 .
\end{aligned}
$$

Termos do desdobramento: $m[N, 0,0,0]+\alpha[1,0,0,0]$.

Codim topológica / $\mathrm{D}_{4}$-codim: $1 / 2$.

$\mathrm{I}_{2}:\left[m N+\epsilon_{7} N \lambda_{1}^{2}+\epsilon_{4} \lambda_{1}^{2}, \epsilon_{5}, 1,0\right]$.

Condições de definição: $p_{\lambda_{1}}^{o}=0, p_{N \lambda_{1}}^{o} q^{o}-q_{\lambda_{1}}^{o} p_{N}^{o}=0$.

Coeficientes: $\epsilon_{4}=s g p_{\lambda_{1} \lambda_{1}}^{o}, m=\frac{p_{N}^{o}}{\left|q^{o}\right|}, \epsilon_{5}=s g q^{\circ}$,

$$
\begin{aligned}
\epsilon_{7}= & \epsilon_{1} \epsilon_{5} s g \xi_{7} \text { com } \epsilon_{1}=s g p_{N}^{o} \mathrm{e} \\
\xi_{7}= & p_{\lambda_{1} \lambda_{1}}^{o} p_{u_{4}}^{o} q^{o}\left(p_{N}^{o}-q^{o}\right)+p_{N}^{o} r_{o}^{2}\left(p_{N \lambda_{1} \lambda_{1}}^{o} q^{o}-p_{N}^{o} q_{\lambda_{1} \lambda_{1}}^{o}\right)+ \\
& p_{\lambda_{1} \lambda_{1}}^{o} q^{o} r^{o}\left(s^{o} q^{o}-p_{N}^{o} r_{N}^{o}\right)+\frac{1}{2} p_{\lambda_{1} \lambda_{1}}^{o} r^{o 2}\left(2 p_{N}^{o} q_{N}^{o}-2 p_{\Delta}^{o} q^{o}-p_{N N}^{o} q^{o}\right) .
\end{aligned}
$$

Condições de não-degenericidade: $r^{o} \neq 0, p_{\lambda_{1} \lambda_{1}}^{o} \neq 0, \xi_{7} \neq 0, p_{N}^{o} \neq 0, p_{N}^{o} \neq \pm q^{o}$. 
Termos do desdobramento: $m[N, 0,0,0]+\alpha[1,0,0,0]+\beta\left[N \lambda_{1}, 0,0,0\right]$.

Codim topológica / $\mathrm{D}_{4}$-codim: $2 / 3$.

$\mathrm{I}_{3}:\left[m N+\epsilon_{3} N \lambda_{1}+\epsilon_{11} \lambda_{1}^{3}, \epsilon_{5}, 1,0\right]$.

Condiçôes de definição: $p_{\lambda_{1}}^{o}=0, p_{\lambda_{1} \lambda_{1}}^{o}=0$.

Coeficientes: $\epsilon_{3}=\epsilon_{5} s g\left(p_{N \lambda_{1}}^{o} q^{o}-q_{\lambda_{1}}^{o} p_{N}^{o}\right)$,

$$
\epsilon_{11}=s g p_{\lambda_{1} \lambda_{1} \lambda_{1}}^{o}, m=\frac{p_{N}^{o}}{\left|q^{o}\right|}, \epsilon_{5}=s g q^{o} .
$$

Condiçôes de não-degenericidade: $r^{o} \neq 0, p_{\lambda_{1} \lambda_{1} \lambda_{1}}^{o} \neq 0, p_{N}^{o} \neq 0, p_{N}^{o} \neq \pm q^{\circ}$,

$$
p_{N \lambda_{1}}^{o} q^{o}-q_{\lambda_{3}}^{o} p_{N}^{o} \neq 0 .
$$

Termos do desdobramento: $m[N, 0,0,0]+\alpha[1,0,0,0]+\beta\left[\lambda_{1}, 0,0,0\right]$.

Codim topológica / $\mathrm{D}_{4}$-codim: $2 / 3$.

$\mathrm{I}_{4}:\left[m N+\epsilon_{0} \lambda_{1}+n N u_{4}, \epsilon_{5}, \lambda_{1}+\epsilon_{8} u_{4}, 0\right]$.

Condição de definição: $r^{\circ}=0$.

Coeficientes: $m=\frac{p_{N}^{o}}{\left|q^{o}\right|}, \epsilon_{o}=s g p_{\lambda_{1}}^{o}, \epsilon_{5}=s g q^{o}$,

$$
\begin{aligned}
& \epsilon_{8}=\epsilon_{0} s g \frac{1}{\xi_{4}}\left(p_{N}^{o} 2-q^{o 2}\right) \Delta_{\lambda_{1}, u_{4}}(p, r) \text { onde } \\
& \xi_{4}=p_{\lambda_{1}}^{o}\left(q^{o} s^{o}-r_{N}^{o} p_{N}^{o}\right)+r_{\lambda_{1}}^{o}\left(p_{N}^{o}-q^{o 2}\right), \\
& n=\epsilon_{5} p_{\lambda_{1}}^{o}\left(q^{o 2}-p_{N}^{o}\right) \frac{\xi_{3}}{q^{o 2} \xi_{4}^{2}} \text { onde } \\
& \xi_{3}=\left[p_{N}^{o}\left(p_{N}^{o}-q^{o 2}\right) \Delta_{\lambda_{1}, u_{4}}(p, q)+q^{o}\left(p_{N}^{o}{ }^{2}-q^{o 2}\right)\left(p_{N \lambda_{1}}^{o} p_{u_{4}}^{o}-p_{N u_{4}}^{o} p_{\lambda_{1}}^{o}\right)\right. \\
& \left.\quad+p_{\lambda_{1}}^{o} q^{o} p_{N}^{o}\left(r_{N}^{o}+s^{o 2}\right)-r_{N}^{o} p_{\lambda_{1}}^{o}{ }^{o}\left(p_{N}^{o}+q^{o 2}\right)\right] .
\end{aligned}
$$

Condições de não-degenericidade: $p_{N}^{o} \neq 0, p_{N}^{o} \neq \pm q^{\circ}$,

$$
p_{\lambda_{1}}^{o} \neq 0, \Delta_{\lambda_{1}, u_{4}}(p, r) \neq 0, \xi_{3} \neq 0, \xi_{4} \neq 0 .
$$

Termos do desdobramento: $m[N, 0,0,0]+n\left[N u_{4}, 0,0,0\right]+\alpha[0,0,1,0]$. 
Codim topológica / $\mathrm{D}_{4}$-codim: $1 / 3$.

$\mathrm{I}_{5}:\left[m N+\epsilon_{3} N \lambda_{1}+\epsilon_{4} \lambda_{1}^{2}+n_{1} u_{4}, \epsilon_{5}, \lambda_{1}, 0\right]$.

Condiçôes de definição: $r^{\circ}=0, p_{\lambda_{1}}^{o}=0$.

Coeficientes: $\quad m=\frac{p_{N}^{o}}{\left|q^{o}\right|}, \epsilon_{5}=s g q^{o}, \epsilon_{3}=\epsilon_{5} s g\left(p_{N \lambda_{1}}^{o} q^{o}-q_{\lambda_{1}}^{o} p_{N}^{o}\right)$,

$$
\epsilon_{4}=s g p_{\lambda_{1} \lambda_{1}}^{o}, n_{1}=\frac{p_{u_{4}}^{o}\left|p_{\lambda_{1} \lambda_{1}}^{o}\right|}{2 r_{\lambda_{1}}^{o}}
$$

Condições de não-degenericidade: $p_{N}^{o} \neq 0, q^{\circ} \neq 0, p_{N}^{o} \neq \pm q^{o}$,

$$
\xi_{8}=p_{u_{4}}^{o} r_{\lambda_{1}}^{o} p_{\lambda_{1} \lambda_{1}}^{o}\left(p_{N \lambda_{1}}^{o} q^{o}-q_{\lambda_{1}}^{o} p_{N}^{o}\right) \neq 0
$$

Termos do desdobramento: $m[N, 0,0,0]+n_{1}\left[u_{4}, 0,0,0\right]+\alpha[1,0,0,0]+\beta[0,0,1,0]$.

Codim topológica / $\mathrm{D}_{4}$-codim: $2 / 4$.

$\mathrm{I}_{6}:\left[m N+\epsilon_{0} \lambda_{1}+\epsilon_{9} N u_{4}, \epsilon_{5}, u_{4}, 0\right]$.

Condições de definição: $r^{o}=0, \xi_{4}=0$.

Coeficientes: $\epsilon_{o}=s g p_{\lambda_{1}}^{o}, m=\frac{p_{N}^{o}}{\left|q^{\circ}\right|}, \epsilon_{5}=s g q^{\circ}$,

$$
\epsilon_{9}=\epsilon_{o} \epsilon_{5} s g\left(p_{N}^{o}-q^{o 2}\right) \xi_{3} \text {. }
$$

Condições de não-degenericidade: $p_{\lambda_{1}}^{o} \neq 0, \xi_{3} \neq 0, p_{N}^{o} \neq 0, p_{N}^{o} \neq \pm q^{o}$,

$$
\xi_{5}=p_{u_{4}}^{o}\left(s^{o} q^{o}-p_{N}^{o} r_{N}^{o}\right)+r_{u_{4}}^{o}\left(p_{N}^{o}{ }^{2}-q^{o}\right) \neq 0 .
$$

Termos do desdobramento: $m[N, 0,0,0]+\alpha\left[0,0, \lambda_{1}, 0\right]+\beta[0,0,1,0]$.

Codim topológica / $\mathrm{D}_{4}$-codim: $2 / 3$. 
$I_{7}:\left[m N+\epsilon_{0} \lambda_{1}, \epsilon_{5}, \lambda_{1}+\epsilon_{8} u_{4}, 0\right]$.

Condiçôes de definição: $r^{o}=0$ e $\xi_{3}=p_{N}^{o} \Delta_{\lambda_{1}, u_{4}}(p, q)\left(p_{N}^{o}{ }^{2}-q^{o 2}\right)$

$$
\begin{array}{r}
+q^{o}\left(p_{N \lambda_{1}}^{o} p_{u_{4}}^{o}-p_{N u_{4}}^{o} p_{\lambda_{1}}^{o}\right)\left(p_{N}^{o}{ }^{2}-q^{o 2}\right) \\
+p_{\lambda_{1}}^{o} q^{o} p_{N}^{o}\left(r_{N}^{o}{ }^{2}+s^{o 2}\right)-r_{N}^{o} p_{\lambda_{1}}^{o} s^{o}\left(p_{N}^{o}{ }^{2}+q^{o 2}\right)=0 .
\end{array}
$$

Coeficientes: $\quad \epsilon_{o}=s g p_{\lambda_{\mathrm{J}}}^{o}, m=\frac{p_{N}^{o}}{\left|q^{\circ}\right|}, \epsilon_{5}=s g q^{\circ}$,

$$
\epsilon_{8}=\epsilon_{o} s g\left[\frac{\left(p_{N}^{o} 2-q^{o 2}\right) \Delta_{\lambda_{1}, u_{4}}(p, r)}{p_{\lambda_{1}}^{o}\left(q^{o} s^{o}-r_{N}^{o} p_{N}^{o}\right)+r_{\lambda_{1}}^{o}\left(p_{N}^{o}{ }^{2}-q^{o 2}\right)}\right] \text {. }
$$

Condiçôes de não-degenericidade: $\quad p_{\lambda_{1}}^{o} \neq 0, \Delta_{\lambda_{1}, u_{4}}(p, r) \neq 0, p_{N}^{o} \neq 0, p_{N}^{o} \neq \pm q^{o}$,

$$
\xi_{4}=p_{\lambda_{1}}^{o}\left(q^{o} s^{o}-r_{N}^{o} p_{N}^{o}\right)+r_{\lambda_{1}}^{o}\left(p_{N}^{o}{ }^{2}-q^{o 2}\right) \neq 0 .
$$

Termos do desdobramento: $m[N, 0,0,0]+\alpha[1,0,0,0]+\beta\left[N u_{4}, 0,0,0\right]$.

Codim topológica / $\mathrm{D}_{4^{-}}$codim: $2 / 3$.

$\mathrm{I}_{8}:\left[m N+\epsilon_{0} \lambda_{1}+n N u_{4}, \epsilon_{5}, \lambda_{1}+\epsilon_{10} u_{4}^{2}, 0\right]$.

Condições de definição: $r^{\circ}=0$ e $\Delta_{\lambda_{1}, u_{4}}(p, r)=0$.

Coeficientes: $\epsilon_{o}=s g p_{\lambda_{1}}^{o}, m=\frac{p_{N}^{o}}{\left|q^{\circ}\right|}, \epsilon_{5}=s g q^{o}$,

$$
\begin{aligned}
& n= \frac{\epsilon_{5} p_{\lambda_{1}}^{o}\left(q_{o}^{2}-p_{N}^{o}\right) \xi_{3}}{q_{o}^{2}\left[p_{\lambda_{1}}^{o}\left(s^{o} q^{o}-p_{N}^{o} r_{N}^{o}\right)+r_{\lambda_{1}}^{o}\left(p_{N}^{o}{ }^{2}-q^{o}\right)\right]^{2}} \\
& \epsilon_{10}=s g \frac{\xi_{6}}{\xi_{4}}\left(p_{\lambda_{1}}^{o}\left(p_{N}^{o}{ }^{2}-q^{o}\right)\right) \text { onde } \\
& \xi_{6}=r_{\lambda_{1}}^{o} p_{u_{4}}^{o}\left(p_{u_{4} \lambda_{1}}^{o} p_{\lambda_{1}}^{o}-p_{\lambda_{1} \lambda_{1}}^{o} p_{u_{4}}^{o}\right)+ \\
& \quad p_{\lambda_{1}}^{o} p_{u_{4}}^{o}\left(r_{\lambda_{1} \lambda_{1}}^{o} p_{u_{4}}^{o}-r_{u_{4} \lambda_{1}}^{o} p_{\lambda_{1}}^{o}\right)-p_{\lambda_{1}}^{o} \Delta_{u_{4} u_{4}, \lambda_{1}}(p, r) .
\end{aligned}
$$

Condições de não-degenericidade: $\quad p_{\lambda_{1}}^{o} \neq 0, \xi_{3} \neq 0, \xi_{4} \neq 0, \xi_{6} \neq 0, p_{N}^{o} \neq 0$,

$$
p_{N}^{o} \neq \pm q^{o} \text {. }
$$

Termos do desdobramento: $m[N, 0,0,0]+n\left[N u_{4}, 0,0,0\right]+\alpha\left[u_{4}, 0,0,0\right]+\beta[0,0,1,0]$. Codim topológica / $\mathrm{D}_{4}$-codim: $2 / 4$. 


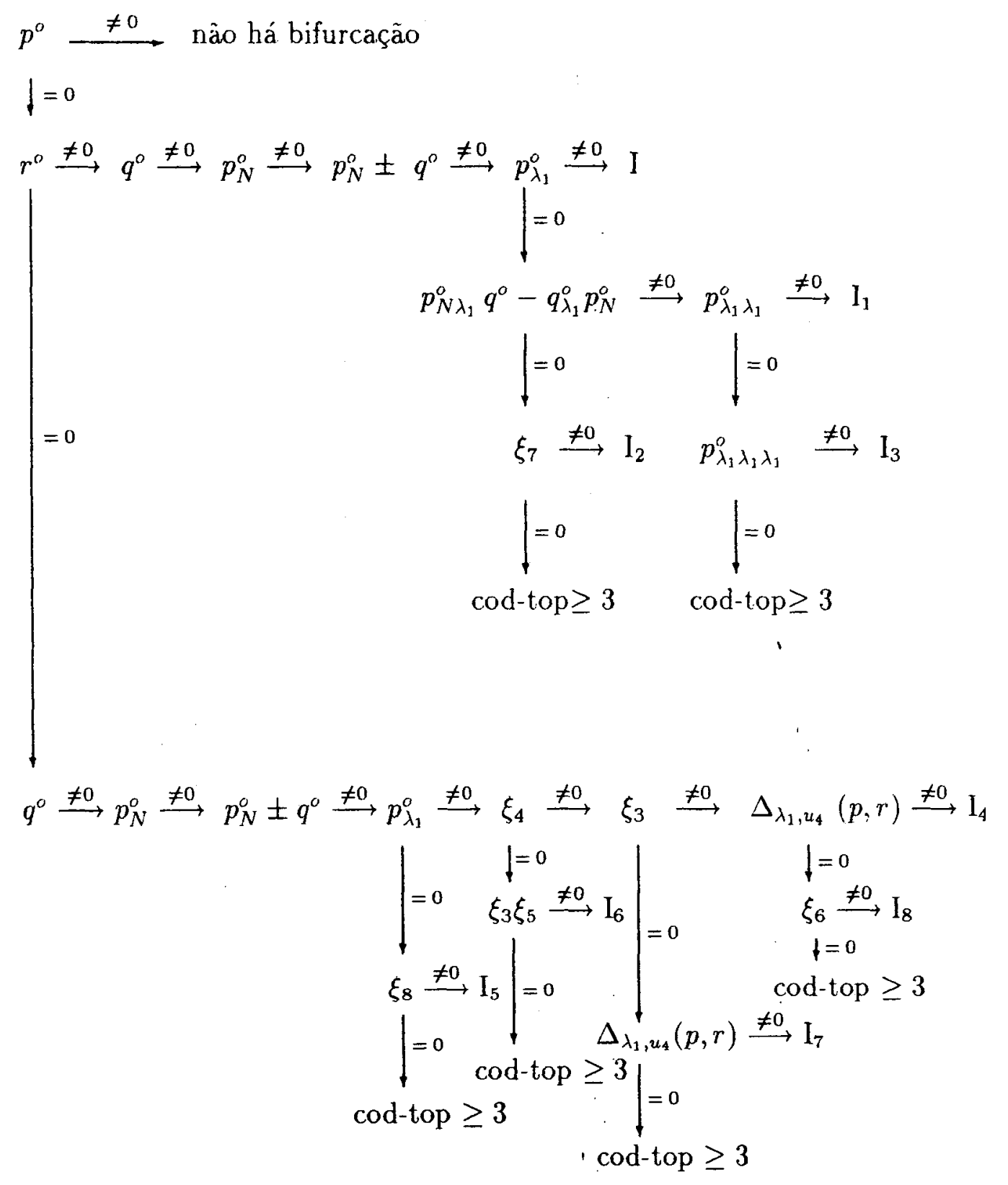

Tabela 4.1: Fluxograma para o centro organizador $f_{o}^{1}$ 


\section{Prova do Teorema 4.1.8.}

Denotamos por $g$ a forma normal em cada caso. Usamos (4.6) e (4.8) para calcular, respectivamente, o espaço tangente e espaço tangente unipotente ao caminho associado a $g$.

A primeira parte do Teorema segue da Proposição 3.2.12. e Corolário 3.2.14. Para obtermos a forma normal e as condiçôes de não-degenericidade, fazemos a mudança de coordenadas módulo um submódulo intrínsico contido na parte intrínsica de $\mathcal{T U}^{\mathrm{D}_{4}}(g)$, o qual está contido em $\mathcal{P}(g)$ pela Proposição 1.10.3.1.

O desdobramento miniversal segue do Teorema 3.2.15. e Corolário 1.7.4.

Para concluir a prova, escrevemos os dados básicos para cada caso.

CASO I. $g=\left[m N+\epsilon_{0} \lambda_{1}, \epsilon_{5}, 1,0\right]$.

Foi provado usando a método clássico no Teorema 2.6.1.

Caminho associado: $\tilde{\alpha}(\lambda)=\left(\epsilon_{0} \lambda_{1}, 0, \lambda_{2}\right)$.

$\mathcal{T}(\tilde{\alpha})=\mathcal{E}_{\lambda}^{\mathrm{D}_{4}}<(1,0,0),\left(0, \lambda_{1}, 0\right),\left(0, u_{4}, 0\right),\left(0,0, \lambda_{2}\right)>$.

$\mathcal{N}(\tilde{\alpha})=\langle(0,1,0)\rangle ; m$ é um parâmetro modal.

$\mathcal{T} \mathcal{U}(\tilde{\alpha})=\mathcal{E}_{\lambda}^{\mathrm{D}_{4}}<\left(\lambda_{1}^{2}, 0,0\right),\left(u_{4}, 0,0\right),\left(0, \lambda_{1}, 0\right)\left(0, u_{4}, 0\right),\left(0,0, \lambda_{1} \lambda_{2}\right),\left(0,0, u_{4} \lambda_{2}\right)>$.

CASO I $. g=\left[m N+\epsilon_{3} N \lambda_{1}+\epsilon_{4} \lambda_{1}^{2}, \epsilon_{5}, 1,0\right]$ (cf. Teorema 2.8.1).

Caminho associado: $\hat{\alpha}(\lambda)=\left(\epsilon_{4} \lambda_{1}^{2}, \epsilon_{3} \lambda_{1}, \lambda_{2}\right)$.

$\mathcal{T}(\tilde{\alpha})=\mathcal{E}_{\lambda}^{\mathrm{D}_{4}}<\left(u_{4}, 0,0\right),\left(0, \lambda_{1}, 0\right),\left(0, u_{4}, 0\right),\left(2 \epsilon_{4} \lambda_{1}, \epsilon_{3}, 0\right),\left(0,0, \lambda_{2}\right)>$.

$\mathcal{N}(\tilde{\alpha})=\langle(1,0,0),(0,1,0)\rangle ; m$ é um parâmetro modal.

$\mathcal{T U}(\tilde{\alpha})=\mathcal{E}_{\lambda}^{\mathrm{D}_{4}}<\left(u_{4}, 0,0\right),\left(\lambda_{1}^{3}, 0,0\right),\left(0, \lambda_{1}^{2}, 0\right),\left(0, u_{4}, 0\right),\left(0,0, \lambda_{1} \lambda_{2}\right),\left(0,0, u_{4} \lambda_{2}\right)>$. 
CASO I ${ }_{2} . g=\left[m N+\epsilon_{7} N \lambda_{1}^{2}+\epsilon_{4} \lambda_{1}^{2}, \epsilon_{5}, 1,0\right]$.

Caminho associado: $\hat{\alpha}(\lambda)=\left(\epsilon_{4} \lambda_{1}^{2}, \epsilon_{7} \lambda_{1}^{2}, \lambda_{2}\right)$.

$\mathcal{T}(\tilde{\alpha})=\mathcal{E}_{\lambda}^{\mathrm{D}_{4}}<\left(u_{4}, 0,0\right),\left(\lambda_{1}^{2}, 0,0\right),\left(\epsilon_{4} \lambda_{1}, \epsilon_{7} \lambda_{1}, 0\right),\left(0, u_{4}, 0\right),\left(0,0, \lambda_{2}\right)>$.

$\mathcal{N}(\tilde{\alpha})=\left\langle(1,0,0),(0,1,0),\left(0, \lambda_{1}, 0\right)\right\rangle ; m$ é um parâmetro modal.

$\mathcal{T U}(\hat{\alpha})=\mathcal{E}_{\lambda}^{D_{4}}<\left(m u_{4}, \epsilon_{4}\left(1-m^{2}\right) \lambda_{1}^{2} .0\right),\left(0, u_{4}, 0\right),\left(\lambda_{1}^{3}, 0,0\right),\left(0, \lambda_{1}^{3}, 0\right)$,

$\left(0,0, \lambda_{1} \lambda_{2}\right),\left(0,0, u_{4} \lambda_{2}\right)>$.

CASO I $\mathbf{I}_{3} . g=\left[m N+\epsilon_{3} N \lambda_{1}+\epsilon_{11} \lambda_{1}^{3}, \epsilon_{5}, 1,0\right]$.

Caminho associado: $\tilde{\alpha}(\lambda)=\left(\epsilon_{11} \lambda_{1}^{3}, \epsilon_{3} \lambda_{1}, \lambda_{2}\right)$.

$\mathcal{T}(\tilde{\alpha})=\mathcal{E}_{\lambda}^{\mathrm{D}_{4}}<\left(m u_{4}+\epsilon_{3} u_{4} \lambda_{1}, 0,0\right),\left(0, u_{4}, 0\right),\left(\lambda_{1}^{3}, 0,0\right),\left(3 \epsilon_{11} \lambda_{1}^{2}, \epsilon_{3}, 0\right),\left(0,0, \lambda_{2}\right)>$.

$\mathcal{N}(\tilde{\alpha})=\left\langle(0,1,0),(1,0,0),\left(\lambda_{1}, 0,0\right)\right\rangle ; m$ é um parâmetro modal.

$\mathcal{T U}(\hat{\alpha})=\mathcal{E}_{\lambda}^{\mathrm{D}_{4}}<\left(u_{4}, 0,0\right),\left(\lambda_{1}^{4}, 0,0\right),\left(0, u_{4}, 0\right),\left(0, \lambda_{1}^{2}, 0\right),\left(0,0, \lambda_{1} \lambda_{2}\right),\left(0,0, u_{4} \lambda_{2}\right)>$.

CASO $\mathrm{I}_{4} . g=\left[m N+\epsilon_{0} \lambda_{1}+n N u_{4}, \epsilon_{5}, \lambda_{1}+\epsilon_{8} u_{4}, 0\right]$. (cf. Teorema 2.8.1.).

Caminho associado: $\hat{\alpha}(\lambda)=\left(\epsilon_{o} \lambda_{1}, n u_{4},\left(\lambda_{1}+\epsilon_{8} u_{4}\right) \lambda_{2}\right)$.

$\mathcal{T}(\hat{\alpha})=\mathcal{E}_{\lambda}^{\mathrm{D}_{4}}<\left(-2 m \lambda_{1}^{2}, \epsilon_{0}\left(1-m^{2}\right) \lambda_{1}, 0\right),\left(u_{4}, 0,0\right),\left(\epsilon_{0}, 0, \lambda_{2}\right)$,

$\left(-\epsilon_{o} \lambda_{1}, 2 n u_{4}, 0\right),\left(\epsilon_{o} m\left(1-m^{2}\right) \lambda_{1}^{2}, 0,2 n \lambda_{1}^{2} \lambda_{2}\right)>$.

$\mathcal{N}(\tilde{\alpha})=\left\langle(0,1,0),\left(0, u_{4}, 0\right),\left(0,0, \lambda_{2}\right)\right\rangle ; m$ e $n$ sấo parâmetros modais.

$\mathcal{T U}(\tilde{\alpha})=\mathcal{E}_{\lambda}^{\mathrm{D}_{4}}<\left(\lambda_{1}^{2}, 0,0\right),\left(u_{4}^{2}, 0,0\right),\left(0, \lambda_{1}, 0\right),\left(0, u_{4}^{2}, 0\right),\left(0,0, u_{4} \lambda_{1} \lambda_{2}\right)$,

$\left(\epsilon_{0} u_{4}, 0, u_{4} \lambda_{2}\right),\left(0,0, \lambda_{1}^{2} \lambda_{2}\right)>$.

CASO $\mathbf{I}_{5} . g=\left[m N+\epsilon_{3} N \lambda_{1}+\epsilon_{4} \lambda_{1}^{2}+n_{1} u_{4}, \epsilon_{5}, \lambda_{1}, 0\right]$

Caminho associado: $\tilde{\alpha}(\lambda)=\left(\epsilon_{4} \lambda_{1}^{2}+n_{1} u_{4}, \epsilon_{3} \lambda_{1}, \lambda_{1} \lambda_{2}\right)$.

$\mathcal{T}(\tilde{\alpha})=\mathcal{E}_{\lambda}^{D_{4}}<\left(m u_{4} \lambda_{1}^{2},\left(1-m^{2}\right) n_{1} u_{4}, m \lambda_{1} \lambda_{2}\right),\left(4 n_{1} u_{4} \lambda_{1}^{3}, 0, \epsilon_{4} \lambda_{1}^{2} \lambda_{2}\right)$,

$\left(2 n_{1} u_{4}, 0, \lambda_{1} \lambda_{2}\right),\left(2 \epsilon_{4} \lambda_{1}, \epsilon_{3}, \lambda_{2}\right),\left(0, \lambda_{1}, 0\right)>$.

$\mathcal{N}(\tilde{\alpha})=\left\langle(1,0,0),\left(u_{4}, 0,0\right),(0,1,0),(0,0,1)\right\rangle ; m$ e $n_{1}$ são parâmetros modais.

$\mathcal{T U}(\tilde{\alpha})=\mathcal{E}_{\lambda}^{D_{4}}<\left(\epsilon_{4} m \lambda_{1}^{4},\left(1-m^{2}\right) n_{1}^{2} u_{4}, 2 \epsilon_{4} n_{1} m \lambda_{1}^{4} \lambda_{2}\right),\left(u_{4} \lambda_{1}^{3}, 0,0\right),\left(0, \lambda_{1}^{2}, 0\right)$, 


$$
\begin{aligned}
& \left(0, u_{4} \lambda_{1}, 0\right),\left(2 n_{1} u_{4} \lambda_{1}, 0, \lambda_{1}^{2} \lambda_{2}\right),\left(2 \epsilon_{4} u_{4} \lambda_{1}, c_{3} u_{4}, u_{4} \lambda_{2}\right), \\
& \left(n_{1} u_{4} \lambda_{1}-\epsilon_{4} \lambda_{1}^{3}, 0,0\right),\left(n_{1} u_{4}^{2}-\epsilon_{4} u_{4} \lambda_{1}^{2}, 0,0\right)>.
\end{aligned}
$$

CASO I $6 . \quad g=\left[m N+\epsilon_{0} \lambda_{1}+\epsilon_{9} N u_{4}, \epsilon_{5}, u_{4}, 0\right]$.

Caminho associado: $\hat{\alpha}(\lambda)=\left(\epsilon_{o} \lambda_{1}, \epsilon_{9} u_{4}, u_{4} \lambda_{2}\right)$.

$$
\begin{aligned}
& \mathcal{T}(\tilde{\alpha})=\mathcal{E}_{\lambda}^{\mathrm{D}_{4}}<(1,0,0),\left(0, \lambda_{1}, 0\right),\left(0, u_{4}, 0\right),\left(0,0, \lambda_{1}^{2} \lambda_{2}\right),\left(0,0, u_{4} \lambda_{2}\right)> \\
& \mathcal{N}(\tilde{\alpha})=\left\langle(0,1,0),\left(0,0, \lambda_{2}\right),\left(0,0, \lambda_{1} \lambda_{2}\right)>; m\right. \text { é um parâmetro modal. } \\
& \mathcal{T} \mathcal{U}(\hat{\alpha})=\mathcal{E}_{\lambda}^{\mathrm{D}_{4}}<\left(u_{4}, 0,0\right),\left(\lambda_{1}^{2}, 0,0\right),\left(0, \lambda_{1}, 0\right),\left(0, u_{4}^{2}, 0\right),\left(0,0, \lambda_{1}^{2} \lambda_{2}\right), \\
& \left(0,0, u_{4} \lambda_{1} \lambda_{2}\right),\left(0,0, u_{4}^{2} \lambda_{2}\right)>
\end{aligned}
$$

CASO I $. g=\left[m N+\epsilon_{0} \lambda_{1}, \epsilon_{5}, \lambda_{1}+\epsilon_{8} u_{4}, 0\right]$.

Caminho associado: $\tilde{\alpha}(\lambda)=\left(\epsilon_{0} \lambda_{1}, 0,\left(\lambda_{1}+\epsilon_{8} u_{4}\right) \lambda_{2}\right)$.

$$
\begin{aligned}
& \mathcal{T}(\tilde{\alpha})=\mathcal{E}_{\lambda}^{\mathrm{D}_{4}}<_{1}\left(0, \lambda_{1}, 0\right),\left(0, u_{4}^{2}, 0\right),\left(0,0, u_{4} \lambda_{2}\right),\left(\epsilon_{o}, 0, \lambda_{2}\right),\left(\lambda_{1}, 0,0\right)> \\
& \mathcal{N}(\tilde{\alpha})=\left\langle(1,0,0),(0,1,0),\left(0, u_{4}, 0\right)>; m\right. \text { é um parâmetro modal. } \\
& \mathcal{T} \mathcal{U}(\tilde{\alpha})=\mathcal{E}_{\lambda}^{\mathrm{D}_{4}}<\left(0, \lambda_{1}, 0\right),\left(0, u_{4}^{2}, 0\right),\left(0,0, u_{4} \lambda_{1} \lambda_{2}\right),\left(0,0, u_{4}^{2} \lambda_{2}\right),\left(\lambda_{1}^{2}, 0,0\right), \\
& \quad\left(c_{o} u_{4}, 0, u_{4} \lambda_{2}\right),\left(0,0, \lambda_{1}^{2} \lambda_{2}\right)>.
\end{aligned}
$$

CASO I $. g=\left[m N+\epsilon_{0} \lambda_{1}+n N u_{4}, \epsilon_{5}, \lambda_{1}+\epsilon_{10} u_{4}^{2}, 0\right]$.

Caminho associado: $\tilde{\alpha}(\lambda)=\left(\epsilon_{\circ} \lambda_{1}, n u_{4},\left(\lambda_{1}+\epsilon_{10} u_{4}^{2}\right) \lambda_{2}\right)$.

$$
\begin{aligned}
& \mathcal{T}(\tilde{\alpha})=\mathcal{E}_{\lambda}^{\mathrm{D}_{4}}<\left(\epsilon_{o}, 0, \lambda_{2}\right),\left(\epsilon_{o} \lambda_{1}, 0, u_{4}^{2} \lambda_{2}\right),\left(0,2 n u_{4}, \lambda_{1} \lambda_{2}\right),\left(0, \lambda_{1}, 0\right)>. \\
& \mathcal{N}(\tilde{\alpha})=<\left(u_{4}, 0,0\right),\left(0, u_{4}, 0\right),(0,1,0),\left(0,0, \lambda_{2}\right)>; m \text { e } n \text { são parâmetros modais. }
\end{aligned}
$$$$
\mathcal{T U}(\tilde{\alpha})=\mathcal{E}_{\lambda}^{\mathrm{D}_{4}}<\left(\lambda_{1}^{2}, 0,0\right),\left(\epsilon_{o} u_{4}, 0, u_{4} \lambda_{2}\right),\left(0, \lambda_{1}, 0\right),\left(0,2 n u_{4}^{2}, u_{4} \lambda_{1} \lambda_{2}\right),\left(0,0, u_{4}^{2} \lambda_{1} \lambda_{2}\right),
$$

$$
\left(0,0, u_{4}^{3} \lambda_{2}\right) ;\left(0,0, \lambda_{1}^{2} \lambda_{2}\right)>\text {. }
$$




\section{Capítulo 5}

\section{Diagramas de bifurcação}

A descrição geométrica das formas normais obtidas é feita através dos diagramas de bifurcação. Dado um problema de bifurcação $g$, o diagrama de bifurcaçâao associado é dado pelo conjunto de zeros de $g$. A construção de tais diagramas para os problemas $\mathrm{D}_{4^{-}}$equivariantes em estudo e também para os seus correspondentes desdobramentos é feita através da análise das regiôes do plano dos parâmetros $\left(\lambda_{1}, \lambda_{2}\right)$ onde as soluções do problema aparecem. Os diagramas são construídos através do que chamamos de representação radial pois consideramos a parametrização

$$
\begin{aligned}
& \lambda_{1}=R \cos (s) \\
& \lambda_{2}=R \operatorname{sen}(s)
\end{aligned}
$$

com $R>0$. Em alguns casos, os diagramas apresentam comportamentos diferentes quando $R$ varia.

Paralelamente, fazemos o estudo da estabilidade assintótica das soluções.

\subsection{Soluções do problema $D_{4}$}

Recorde que a forma geral de um problema de bifurcação $\mathrm{D}_{4}$-equivariante é dada por

$$
\begin{aligned}
g:\left(\mathbf{C} \times \mathbb{R}^{2}, 0\right) & \rightarrow \mathbb{C} \\
\left(z, \lambda_{1}, \lambda_{2}\right) & \mapsto p\left(N, \Delta, \lambda_{1}, u_{4}\right) z+q\left(N, \Delta, \lambda_{1}, u_{4}\right) \delta \bar{z}+r\left(N, \Delta, \lambda_{1}, u_{4}\right) \lambda_{2} \bar{z} \\
& +s\left(N, \Delta, \lambda_{1}, u_{4}\right) \delta \lambda_{2} z .
\end{aligned}
$$

Identificando $\mathbb{C}$ com $\mathbb{R}^{2} \mathrm{e} z$ com $\left(x_{1}, x_{2}\right)$, a expressão de $g$, em coordenadas reais $x_{1}, x_{2}$, é dada por

$$
g\left(x_{1}, x_{2}, \lambda_{1}, \lambda_{2}\right)=\left(g_{1}\left(x_{1}, x_{2}, \lambda_{1}, \lambda_{2}\right), g_{2}\left(x_{1}, x_{2}, \lambda_{1}, \lambda_{2}\right)\right)
$$


com

$$
\begin{aligned}
& g_{1}\left(x_{1}, x_{2}, \lambda_{1}, \lambda_{2}\right)=p x_{1}+q x_{1} x_{2}^{2}-q x_{1}^{3}+\lambda_{2}\left(r x_{1}+s x_{1} x_{2}^{2}-s x_{1}^{3}\right) \\
& g_{2}\left(x_{1}, x_{2}, \lambda_{1}, \lambda_{2}\right)=p x_{2}+q x_{1}^{2} x_{2}-q x_{2}^{3}+\lambda_{2}\left(-r x_{2}-s x_{1}^{2} x_{2}+s x_{2}^{3}\right)
\end{aligned}
$$

e $p, q, r, s$ funções de $\left(N, \Delta, \lambda_{1}, u_{4}\right)$ sendo $N=x_{1}^{2}+x_{2}^{2}, \Delta=\left(x_{2}^{2}-x_{1}^{2}\right)^{2}$ e $u_{4}=\lambda_{2}^{2}$.

O diagrama de bifurcação de $g$ é dado pelos zeros de $g$ e corresponde a achar os pontos $\left(x_{1}, x_{2}, \lambda_{1}, \lambda_{2}\right) \in \mathbb{R}^{2} \times \mathbb{R}^{2}$ que satisfazem as equações

$$
g_{1}\left(x_{1}, x_{2}, \lambda_{1}, \lambda_{2}\right)=0 \quad \text { e } g_{2}\left(x_{1}, x_{2}, \lambda_{1}, \lambda_{2}\right)=0
$$

Note que achar os zeros da equação $g(x, \lambda)=0$ corresponde a obter pontos estacionários em função dos parâmetros para a família de sistemas de equações diferenciajs ordinárias

$$
\begin{aligned}
& \frac{d}{d t} x_{1}+g_{1}\left(x_{1}, x_{2}, \lambda_{1}, \lambda_{2}\right)=0 \\
& \frac{d}{d t} x_{2}+g_{2}\left(x_{1}, x_{2}, \lambda_{1}, \lambda_{2}\right)=0
\end{aligned}
$$

Recordamos que o subgrupo de isotropia do grupo $\mathrm{D}_{4}$ de um ponto $x=\left(x_{1}, x_{2}\right) \in \mathbb{R}^{2}$ é o subgrupo

$$
\Sigma_{x}=\left\{\sigma \in D_{4} \mid \sigma \cdot x=x\right\}
$$

e o subespaço de pontos fixos de um subgrupo $\Sigma$ de $D_{4}$ é o subespaço vetorial do $\mathbb{R}^{2}$

$$
\operatorname{Fix}(\Sigma)=\left\{x \in \mathbb{R}^{2} \mid \sigma \cdot x=x, \forall \sigma \in \Sigma\right\}
$$

A órbita de um ponto $x \in \mathbb{R}^{2}$ é o conjunto

$$
\mathrm{D}_{4} \cdot x=\left\{\gamma \cdot x, \forall \gamma \in \mathrm{D}_{4}\right\}
$$

$\mathrm{O}$ conjunto solução da equação $g(x, \lambda)=0$ é preservado pela simetria $\mathrm{D}_{4}$, ou seja, se $x$ é solução então $\gamma \cdot x$ é solução para todo $\gamma$ em $D_{4}$. Existe uma relação natural entre os subgrupos de isotropia de pontos numa mesma órbita dada por:

$$
\Sigma_{\gamma \cdot x}=\gamma \cdot \Sigma_{x} \cdot \gamma^{-1}, \forall \gamma \in D_{4}
$$

Neste caso, dizemos que $\Sigma_{x}$ e $\Sigma_{\gamma \cdot x}$ são subgrupos de isotropias conjugados e vamos daqui para frente denotar $\Sigma_{\gamma \cdot x}$ por $\Sigma_{x}^{c}$. 


\begin{tabular}{|c||c|}
\hline $\begin{array}{c}\text { SUBGRUPOS DE } \\
\text { ISOTROPIA }\end{array}$ & $\begin{array}{c}\text { SUBESPACOS DE } \\
\text { PONTOS FIXOS }\end{array}$ \\
\hline $\mathrm{D}_{4}$ & $x_{1}=x_{2}=0$ \\
\hline $\mathrm{Z}_{2}(\kappa)=<\kappa>$ & $x_{2}=0$ \\
\hline $\mathrm{Z}_{2}^{c}(\kappa)$ & $x_{1}=0$ \\
\hline $\mathrm{Z}_{2}(\xi \kappa)=\left\langle\xi \kappa>, \xi=e^{\frac{i \pi}{2}}\right.$ & $x_{1}=x_{2}$ \\
\hline $\mathrm{Z}_{2}^{c}(\xi \kappa)$ & $x_{1}=-x_{2}$ \\
\hline 1 & $\forall\left(x_{1}, x_{2}\right)$ \\
\hline
\end{tabular}

TABELA 5.1

Para o grupo $\mathrm{D}_{4}$, os subgrupos de isotropia com seus respectivos conjugados e subespaços de pontos fixos são dados na tabela acima.

A justificativa da tabela 5.1 pode ser encontrada em ([16], pág. 97). Note que a solução nula é sempre solução de (5.1) por se tratar de um problema de bifurcação. Para cada $\lambda=\left(\lambda_{1}, \lambda_{2}\right)$, temos que $\left(0,0, \lambda_{1}, \lambda_{2}\right)$ é, também, sempre solução de (5.1) e chamada solução trivial. $\mathrm{O}$ subgrupo de isotropia da solução trivial é o próprio $\mathrm{D}_{4}$. Outras soluções diferentes da trivial podem ocorrer para o problema de bifurcação $g$ porém, com simetria $\Sigma_{x}$, onde $\Sigma_{x}$ denota os subgrupos de isotropia da tabela 5.1. Esse fenômeno é conhecido como quebra de simetria espontânea.

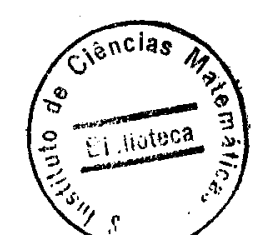




\subsection{Estabilidade orbital}

Definição 5.2.1. Seja $\left(x_{0}, \lambda_{0}\right)$ um ponto de equilíbrio do sistema de equações diferenciais ordinárias $\frac{d x}{d t}+g(x, \lambda)=0$, ou, equivalentemente um zero da equação $g(x, \lambda)=0$. Dizemos que $\left(x_{0}, \lambda_{0}\right)$ é orbitalmente linearmente estável se for estável e se os auto-valores da matriz Jacobiana $\left(D_{x} g\right)$ calculada em $\left(x_{0}, \lambda_{0}\right)$, não forçados pela ação do grupo a serem nulos, tiverem parte real positiva.

Os auto-valores nulos correspondem a auto-vetores que estâo no espaço tangente à órbita $\mathrm{D}_{4} \cdot x_{0}$ no ponto $x_{0}$.

Teorema 5.2.2. Estabilidade orbital linear implica estabilidade orbital assintótica.

Prova. ([16], Teorema 4.3, pág. 88).

Assim como os zeros de um problema de bifurcação são preservados por $\mathcal{K}_{\lambda}^{\mathrm{D}_{4}}$-equivalência, enunciaremos os resultados que estabelecem quando a estabilidade linear de uma solução de equilíbrio $g\left(x_{\circ}, \lambda_{\circ}\right)=0$, para o sistema de EDOs

$$
\frac{d x}{d t}+g(x, \lambda)=0
$$

é também preservada por $\mathcal{K}_{\lambda}^{\mathrm{D}_{4}}$-equivalência.

Proposição 5.2.3. A estabilidade orbital linear de $\left(x_{o}, \lambda_{o}\right)$ de $g$ é preservada por $\mathcal{K}_{\lambda}^{\mathrm{D}_{4}}$ equivalência se e somente se for preservada por $\mathrm{D}_{4}$-equivalências da forma $S(x, \lambda) g(x, \lambda)$ com $S$ como na definição de $\mathcal{K}_{\lambda}^{\Gamma}$-equivalência no Capítulo 1 (seç̧âo 1.3 ) e com $\Gamma$ sendo $D_{4}$. Prova. ([16], Proposição 5.1, pág. 200).

Observação 5.2.4. O sinal do determinante de $\left(D_{x} g\right)$ é também um invariante por $\mathcal{K}_{\lambda}^{\mathrm{D}_{4} \text {-equivalência. }}$

Teorema 5.2.5. Seja $\Sigma$ um subgrupo de isotropia de $D_{4}$ e $\left(x_{o}, \lambda_{o}\right)$ solução de $g=0$ com $\Sigma_{x_{0}}=\Sigma$. Se for possível decompor $V$ como $V=V_{1} \oplus \ldots \oplus V_{k}$, onde cada $V_{j}$ é uma representação absolutamente irredutível pela ação de $\Sigma$ então a estabilidade orbital linear de $\left(x_{0}, \lambda_{0}\right)$ é preservada por $\mathcal{K}_{\lambda}^{\mathrm{D}_{4}}$-equivalência.

Prova. ([16], Teorema 5.3, pág. 201).

Lembramos que a ação de $\Sigma$ em $V_{j}$ é dita absolutamente irredutível se as únicas 
aplicaçōes lineares sobre $V_{j} \Sigma$-equivariantes sâo múltiplas da identidade.

\subsection{Dados para o problema $D_{4}$}

Vimos que a forma geral de um problema $\mathrm{D}_{4}$-equivariante é dada por

$$
\begin{aligned}
g:\left(\mathbf{C} \times \mathbb{R}^{2}, 0\right) & \rightarrow \mathbb{C} \\
\left(z, \lambda_{1}, \lambda_{2}\right) & \mapsto p\left(N, \Delta, \lambda_{1}, u_{4}\right) z+q\left(N, \Delta, \lambda_{1}, u_{4}\right) \delta \bar{z}+r\left(N, \Delta, \lambda_{1}, u_{4}\right) \lambda_{2} \bar{z} \\
&
\end{aligned}
$$

Identificando $z$ com $\left(x_{1}, x_{2}\right)$, escrevemos $g=\left(g_{1}, g_{2}\right)$ sendo

$$
\begin{array}{r}
g_{1}=p x_{1}+q x_{1} x_{2}^{2}-q x_{1}^{3}+\lambda_{2}\left(r x_{1}+s x_{1} x_{2}^{2}-s x_{1}^{3}\right), \\
g_{2}=p x_{2}+q x_{1}^{2} x_{2}-q x_{2}^{3}+\lambda_{2}\left(-r x_{2}-s x_{1}^{2} x_{2}+s x_{2}^{3}\right) .
\end{array}
$$

A matriz Jacobiana $\left(D_{x} g\right)$ tem as seguintes entradas:

$$
\begin{aligned}
\frac{\partial}{\partial x_{1}} g_{1}= & \left(2 p_{N} x_{1}^{2}+p-4 p_{\Delta} \delta x_{1}^{2}+q x_{2}^{2}-3 q x_{1}^{2}+2 q_{N} \delta x_{1}^{2}-4 q_{\Delta} \Delta x_{1}^{2}\right) \\
& +\lambda_{2}\left(r+2 r_{N} x_{1}^{2}-4 r_{\Delta} \delta x_{1}^{2}+s x_{2}^{2}-3 s x_{1}^{2}+2 s_{N} \delta x_{1}^{2}-4 \Delta s_{\Delta} x_{1}^{2}\right) \\
\frac{\partial}{\partial x_{2}} g_{1}= & \left(2 p_{N} x_{1} x_{2}+4 p_{\Delta} \delta x_{1} x_{2}+2 q x_{1} x_{2}+2 q_{N} \delta x_{1} x_{2}+4 q_{\Delta} \Delta x_{1} x_{2}\right) \\
& +\lambda_{2}\left(2 r_{N} x_{1} x_{2}+4 r_{\Delta} \delta x_{1} x_{2}+2 s x_{1} x_{2}+2 s_{N} \delta x_{1} x_{2}+4 s_{\Delta} \Delta x_{1} x_{2}\right) \\
\frac{\partial}{\partial x_{1}} g_{2}= & \left(2 p_{N} x_{1} x_{2}-4 p_{\Delta} \delta x_{1} x_{2}+2 q x_{1} x_{2}-2 q_{N} \delta x_{1} x_{2}+4 q_{\Delta} \Delta x_{1} x_{2}\right) \\
& +\lambda_{2}\left(-2 r_{N} x_{1} x_{2}+4 r_{\Delta} \delta x_{1} x_{2}-2 s x_{1} x_{2}+2 s_{N} \delta x_{1} x_{2}-4 s_{\Delta} \Delta x_{1} x_{2}\right) \\
\frac{\partial}{\partial x_{2}} g_{2}= & \left(2 p_{N} x_{2}^{2}+p+4 p_{\Delta} \delta x_{2}^{2}+q x_{1}^{2}-3 q x_{2}^{2}-2 q_{N} x_{2}^{2} \delta-4 q_{\Delta} \Delta x_{2}^{2}\right) \\
& +\lambda_{2}\left(-r-2 r_{N} x_{2}^{2}-4 r_{\Delta} \delta x_{2}^{2}+3 s x_{2}^{2}-s x_{1}^{2}+2 s_{N} \delta x_{2}^{2}+4 s_{\Delta} \Delta x_{2}^{2}\right)
\end{aligned}
$$

Note que

$$
\begin{aligned}
\frac{\partial}{\partial x_{1}} g_{1}\left(x_{1}, x_{2}, \lambda_{1}, \lambda_{2}\right) & =\frac{\partial}{\partial x_{2}} g_{2}\left(x_{2}, x_{1}, \lambda_{1},-\lambda_{2}\right), \\
\frac{\partial}{\partial x_{2}} g_{1}\left(x_{1}, x_{2}, \lambda_{1}, \lambda_{2}\right) & =\frac{\partial}{\partial x_{1}} g_{2}\left(x_{2}, x_{1}, \lambda_{1},-\lambda_{2}\right) .
\end{aligned}
$$

Usaremos a notação $\left(D_{x} g\right)_{\mid}$, para indicar a restrição dos elementos da matriz Jacobiana $\left(D_{x} g\right)$ aos respectivos ramos de soluções encontrados. Além disso, $\operatorname{tr}\left(D_{x} g\right)$ denotará o traço da matriz $\left(D_{x} g\right)$ e $\operatorname{det}\left(D_{x} g\right)$ o seu determinante. 
As soluçôes do problema (5.1), correspondentes aos subespaços de pontos fixos dos subgrupos de isotropia do $\mathrm{D}_{4}$ com seus respectivos conjugados, assim como as expressôes que fornecem os sinais dos auto-valores estâo na tabela 5.2 . 


\begin{tabular}{|c|c|c|}
\hline $\begin{array}{l}\text { SUBESPACOOS } \\
\text { FIXADOS }\end{array}$ & SOLUÇÕES & $\begin{array}{c}\text { SINAIS DOS } \\
\text { AUTO - VALORES }\end{array}$ \\
\hline $\operatorname{Fix}\left(D_{4}\right)$ & $\left(0,0, \lambda_{1}, \lambda_{2}\right)$ & $p+\lambda_{2} r$ e $p-\lambda_{2} r$ \\
\hline $\operatorname{Fix}\left(Z_{2}(\kappa)\right)$ & $\begin{array}{c}x_{2}=0 \\
p=q x_{1}^{2}-\lambda_{2} r+\lambda_{2} s x_{1}^{2}\end{array}$ & $\begin{array}{r}\left(p_{N}-q+\left(2 p_{\Delta}-q_{N}\right) x_{1}^{2}-2 q_{\Delta} x_{1}^{4}\right) \\
+\lambda_{2}\left(r_{N}-s+\left(2 r_{\Delta}-s_{N}\right) x_{1}^{2}-2 s_{\Delta} x_{1}^{4}\right) \\
\text { e }\left(q x_{1}^{2}-\lambda_{2} r\right)\end{array}$ \\
\hline $\operatorname{Fix}\left(Z_{2}^{c}(\kappa)\right)$ & $\begin{array}{c}x_{1}=0 \\
p=q x_{2}^{2}+\lambda_{2} r-s \lambda_{2} x_{2}^{2}\end{array}$ & $\begin{array}{r}\left(p_{N}-q+\left(2 p_{\Delta}-q_{N}\right) x_{2}^{2}-2 q_{\Delta} x_{2}^{4}\right) \\
+\lambda_{2}\left(-r_{N}+s-\left(2 r_{\Delta}-s_{N}\right) x_{2}^{2}+2 s_{\Delta} x_{2}^{4}\right) \\
\text { e }\left(q \dot{x}_{2}^{2}+\lambda_{2} r\right)\end{array}$ \\
\hline $\begin{array}{c}\operatorname{Fix}\left(\mathrm{Z}_{2}(\xi \kappa)\right) \\
\mathrm{e} \\
\operatorname{Fix}\left(\mathrm{Z}_{2}^{c}(\xi \kappa)\right)\end{array}$ & $\begin{array}{c}x_{1}= \pm x_{2} \\
p=0 \text { e } \lambda_{2}=0\end{array}$ & $\begin{array}{c}p_{N} \\
\mathrm{e}(-q)\end{array}$ \\
\hline $\operatorname{Fix}(1)$ & $\begin{array}{c}p+\delta \lambda_{2} s=0 \\
\mathrm{e} \\
\lambda_{2} r+\delta q=0\end{array}$ & $\begin{array}{r}\operatorname{tr}\left(D_{x} g\right)_{\mid}=N p_{N}+2 \Delta p_{\Delta}-N q-\Delta q_{N} \\
-2 \Delta N q_{\Delta}+\delta \lambda_{2}\left(-r_{N}-2 N r_{\Delta}+s\right. \\
\left.+N s_{N}+2 \Delta s_{\Delta}\right) \\
\operatorname{det}\left(D_{x} g\right)_{\mid}=-4 q p_{N}+8 \Delta\left(p_{\Delta} q_{N}-p_{N} q_{\Delta}\right) \\
+\delta \lambda_{2}\left[8\left(p_{\Delta} r_{N}-p_{N} r_{\Delta}\right)+4\left(s q_{N}-q s_{N}\right)\right. \\
\left.+8 \Delta\left(s_{\Delta} q_{N}-q_{\Delta} s_{N}\right)\right] \\
+u_{4}\left[4 s r_{N}+8 \Delta\left(s_{\Delta} r_{N}-s_{N} r_{\Delta}\right)\right]\end{array}$ \\
\hline
\end{tabular}

TABELA 5.2 
A seguir construíremos os diagramas de bifurcaçào para a forma normal genérica e para a forma normal quando $p_{\lambda_{1}}^{o}=0$ e também os diagramas para os seus respectivos desdobramentos versais.

\subsection{Diagramas para a forma normal genérica}

Como vimos a forma normal genérica para os problemas $\mathrm{D}_{4}$-equivariantes é dada por

$$
\begin{aligned}
g:\left(\mathbb{C} \times \mathbb{R}^{2}, 0\right) & \rightarrow \mathbb{C} \\
\left(z, \lambda_{1}, \lambda_{2}\right) & \mapsto\left(m N+\epsilon_{0} \lambda_{1}\right) z+\epsilon_{5} \delta \bar{z}+\lambda_{2} \bar{z}
\end{aligned}
$$

$\operatorname{com} m \neq \pm 1,0$ e $\epsilon_{o}^{2}=\epsilon_{5}^{2}=1$.

Os diagramas serâo construídos para o caso $\epsilon_{o}=\epsilon_{5}=1$.

Em coordenadas $\left(x_{1}, x_{2}, \lambda_{1}, \lambda_{2}\right)$ a expressão de $g$ é

$$
g\left(x_{1}, x_{2}, \lambda_{1}, \lambda_{2}\right)=\left(\begin{array}{l}
\left(\lambda_{1}+\lambda_{2}\right) x_{1}+(m-1) x_{1}^{3}+(m+1) x_{1} x_{2}^{2} \\
\left(\lambda_{1}-\lambda_{2}\right) x_{2}+(m-1) x_{2}^{3}+(m+1) x_{1}^{2} x_{2}
\end{array}\right)
$$

e a expressão do seu desdobramento versal é

$$
G\left(x_{1}, x_{2}, \lambda_{1}, \lambda_{2}, \beta\right)=\left(\begin{array}{c}
\left(\lambda_{1}+\lambda_{2}\right) x_{1}+(m+\beta-1) x_{1}^{3}+(m+\beta+1) x_{1} x_{2}^{2} \\
\left(\lambda_{1}-\lambda_{2}\right) x_{2}+(m+\beta-1) x_{2}^{3}+(m+\beta+1) x_{1}^{2} x_{2}
\end{array}\right)
$$

A análise será feita de acordo com a variaçâo dos sinais de $m$ e $\beta$.

Caso 1. $m>1$ e $\beta=0$

Os dados para a construção do diagrama de $g$ estão na tabela 5.3 e são obtidos substituindo-se os correspondentes dados do problema na tabela 5.2 . 


\begin{tabular}{|c|c|c|c|}
\hline $\begin{array}{c}\text { SUBESPAÇOS } \\
\text { FIXADOS } \\
\end{array}$ & $\begin{array}{c}\text { REGIÕES DO } \\
\text { PLANO }\left(\lambda_{1}, \lambda_{2}\right) \\
\end{array}$ & SOLUÇÕES & $\begin{array}{c}\text { SINAIS DOS } \\
\text { AUTO - VALORES }\end{array}$ \\
\hline $\operatorname{Fix}\left(D_{4}\right)$ & $\forall\left(\lambda_{1}, \lambda_{2}\right)$ & $x_{1}=x_{2}=0$ & $++\left\{\begin{array}{l}\lambda_{1}+\lambda_{2}>0 \\
\lambda_{1}-\lambda_{2}>0\end{array}\right.$ \\
\hline $\operatorname{Fix}\left(\mathrm{Z}_{2}(\kappa)\right)$ & $\lambda_{1}+\lambda_{2} \leq 0$ & $\begin{array}{c}x_{2}=0 \\
x_{1}^{2}=\frac{1}{(1-m)}\left(\lambda_{1}+\lambda_{2}\right)\end{array}$ & $\begin{array}{l}++\left\{\begin{array}{l}\lambda_{1}+\lambda_{2}<0 \\
\lambda_{2}+\frac{1}{m} \lambda_{1}<0\end{array}\right. \\
+-\left\{\begin{array}{l}\lambda_{1}+\lambda_{2}<0 \\
\lambda_{2}+\frac{1}{m} \lambda_{1}>0\end{array}\right.\end{array}$ \\
\hline $\operatorname{Fix}\left(\mathrm{Z}_{2}^{c}(\kappa)\right)$ & $\lambda_{2}-\lambda_{1} \geq 0$ & $\begin{array}{c}x_{1}=0 \\
x_{2}^{2}=\frac{1}{(1-m)}\left(\lambda_{1}-\lambda_{2}\right)\end{array}$ & $\begin{array}{l}++\left\{\begin{array}{l}\lambda_{2}-\lambda_{1}>0 \\
\lambda_{2}-\frac{1}{m} \lambda_{1}>0\end{array}\right. \\
+-\left\{\begin{array}{l}\lambda_{2}-\lambda_{1}>0 \\
\lambda_{2}-\frac{1}{m} \lambda_{1}<0\end{array}\right.\end{array}$ \\
\hline $\begin{array}{l}\operatorname{Fix}\left(Z_{2}(\xi \kappa)\right) \\
\operatorname{Fix}\left(Z_{2}^{c}(\xi \kappa)\right)\end{array}$ & $\begin{array}{l}\lambda_{1} \leq 0 \\
\lambda_{2}=0\end{array}$ & $\begin{array}{c}x_{1}= \pm x_{2} \\
x_{1}^{2}=-\frac{1}{2 m} \lambda_{1}\end{array}$ & +- \\
\hline $\operatorname{Fix}(1)$ & $\begin{array}{c}\lambda_{1} \leq 0 \\
\left\{\begin{array}{l}\lambda_{2} \geq 0 \\
\lambda_{2}+\frac{1}{m} \lambda_{1} \leq 0\end{array}\right. \\
\left\{\begin{array}{l}\lambda_{2}<0 \\
\lambda_{2}-\frac{1}{m} \lambda_{1} \geq 0\end{array}\right.\end{array}$ & $\begin{array}{c}m x_{1}^{2}+m x_{2}^{2}=-\lambda_{1} \\
\mathrm{e} \\
x_{1}^{2}-x_{2}^{2}=\lambda_{2}\end{array}$ & +- \\
\hline
\end{tabular}

TABELA 5.3: $m>1$ 
Para a construção do diagrama, fazemos a análise do número de soluçôes do problema para cada valor fixado do par $\left(\lambda_{1}, \lambda_{2}\right)$.

As soluçôes correspondentes ao $\operatorname{Fix}\left(Z_{2}(\kappa)\right)$ tem a componente $x_{2}$ nula e a componente $x_{1}$ dada por valores simétricos, para cada par fixado $\left(\lambda_{1}, \lambda_{2}\right)$ satisfazendo $\lambda_{1}+\lambda_{2} \leq 0$. Quando $\lambda_{1}+\lambda_{2}=0$ temos a solução trivial. O mesmo vale para as soluções correspondentes ao $\operatorname{Fix}\left(Z_{2}^{c}(\kappa)\right)$ para cada par $\left(\lambda_{1}, \lambda_{2}\right)$ fixado na região $\lambda_{2}-\lambda_{1} \geq 0$ e trocando-se $x_{1}$ por $x_{2}$. Para cada par fixado $\left(\lambda_{1}, 0\right)$ com $\lambda_{1}<0$, a solução correspondente ao $\operatorname{Fix}\left(\mathrm{Z}_{2}(\xi \kappa)\right)$ e seu conjugado é dada por quatro pares de pontos $\left(x_{1}, x_{2}\right)$ com $x_{1}= \pm x_{2}$ e satisfazendo $x_{1}^{2}=-\frac{1}{2 m} \lambda_{1}$. As soluções correspondentes ao Fix(1) correspondem aos pontos de intersecção de uma circunferência com uma hipérbole para cada par $\left(\lambda_{1}, \lambda_{2}\right)$ fixado na região definida por $\lambda_{1} \leq 0, \lambda_{2} \geq 0$ e $\lambda_{2}+\frac{1}{m} \lambda_{1} \leq 0$ ou $\lambda_{1} \leq 0, \lambda_{2}<0$ e $\lambda_{2}-\frac{1}{m} \lambda_{1} \geq 0$. A representação esquemática dessas observaçôes está na figura 5.1. Observe ainda que

1. Quando $\lambda_{2}=-\frac{1}{m} \lambda_{1}$ as soluçôes correspondentes ao $\operatorname{Fix}\left(Z_{2}(\kappa)\right)$ e $\operatorname{Fix}(1)$ coincidem.

2. Quando $\lambda_{2}=\frac{1}{m} \lambda_{1}$, as soluçôes correspondentes ao $\operatorname{Fix}\left(Z_{2}^{c}(\kappa)\right)$ e $\operatorname{Fix}(\mathbf{1})$ coincidem.

3. Quando $\lambda_{2}=0$, as soluçôes correspondentes ao $\mathrm{Fix}(1)$ e Fix $\left(Z_{2}(\xi \kappa), Z_{2}^{c}(\xi \kappa)\right)$ também coincidem.

Finalmente para a construção do diagrama, consideramos a parametrização polar

$$
\begin{aligned}
& \lambda_{1}=R \text { coss } \\
& \lambda_{2}=R \text { sens }
\end{aligned}
$$

com $R>0$. Os ramos de soluções, obtidos em função dos parâmetros de bifurcação, são desenhados no espaço $\left(x_{1}, s, x_{2}\right)$. Neste caso, o comportamento qualitativo dos diagramas não depende do raio $R$.

Note, também, que quando $\frac{3 \pi}{4} \leq s \leq \frac{7 \pi}{4}$, temos duas "pitchforks" se encontrando no plano $\left(x_{1}, s\right)$. O mesmo ocorre no plano $\left(s, x_{2}\right)$, para valores $\frac{\pi}{4} \leq s \leq \frac{5 \pi}{4}$. Na região do plano dos parâmetros $\left(\lambda_{1}, \lambda_{2}\right)$ definida acima por $\lambda_{1} \leq 0, \lambda_{2} \geq 0$ e $\lambda_{2}+\frac{1}{m} \lambda_{1} \leq 0$ ou $\lambda_{1} \leq 0, \lambda_{2}<0$ e $\lambda_{2}-\frac{1}{m} \lambda_{1} \geq 0$, o ramo de solução em $\left(x_{1}, s, x_{2}\right)$ tem a propriedade de que, para cada $s$ fixado nessa região, aparecem quatro pontos como solução. Os pontos 
de contato deste ramo com os "pitchforks" correspondem à coincidência das soluçôes dos respectivos subespaços fixados, conforme comentado acima.

O diagrama está na figura 5.2 e as letras $s$ e $u$ correspondem, respectivamente, aos ramos de soluçâo estáveis e instáveis.

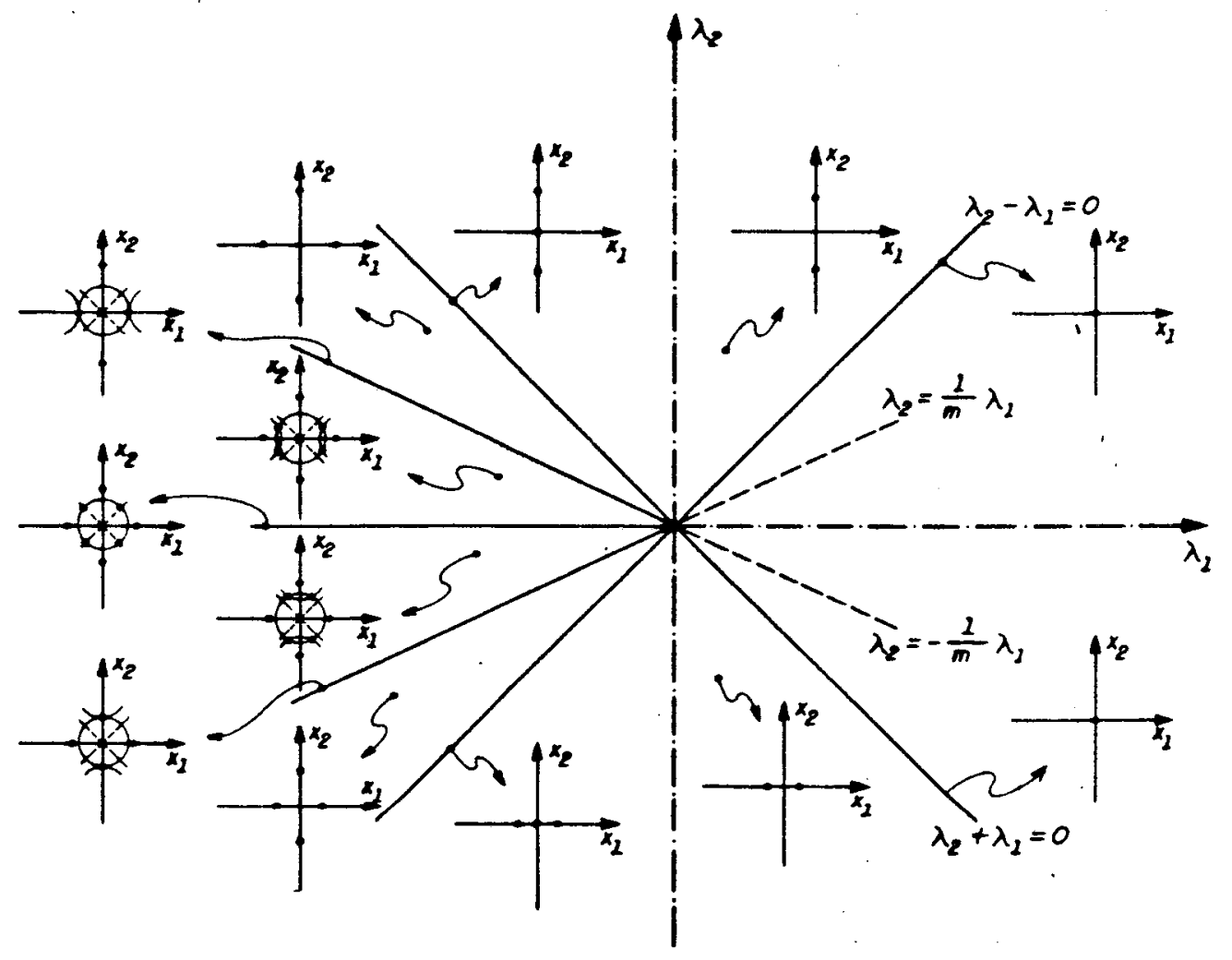

FIG. $5.2: m>1$ 

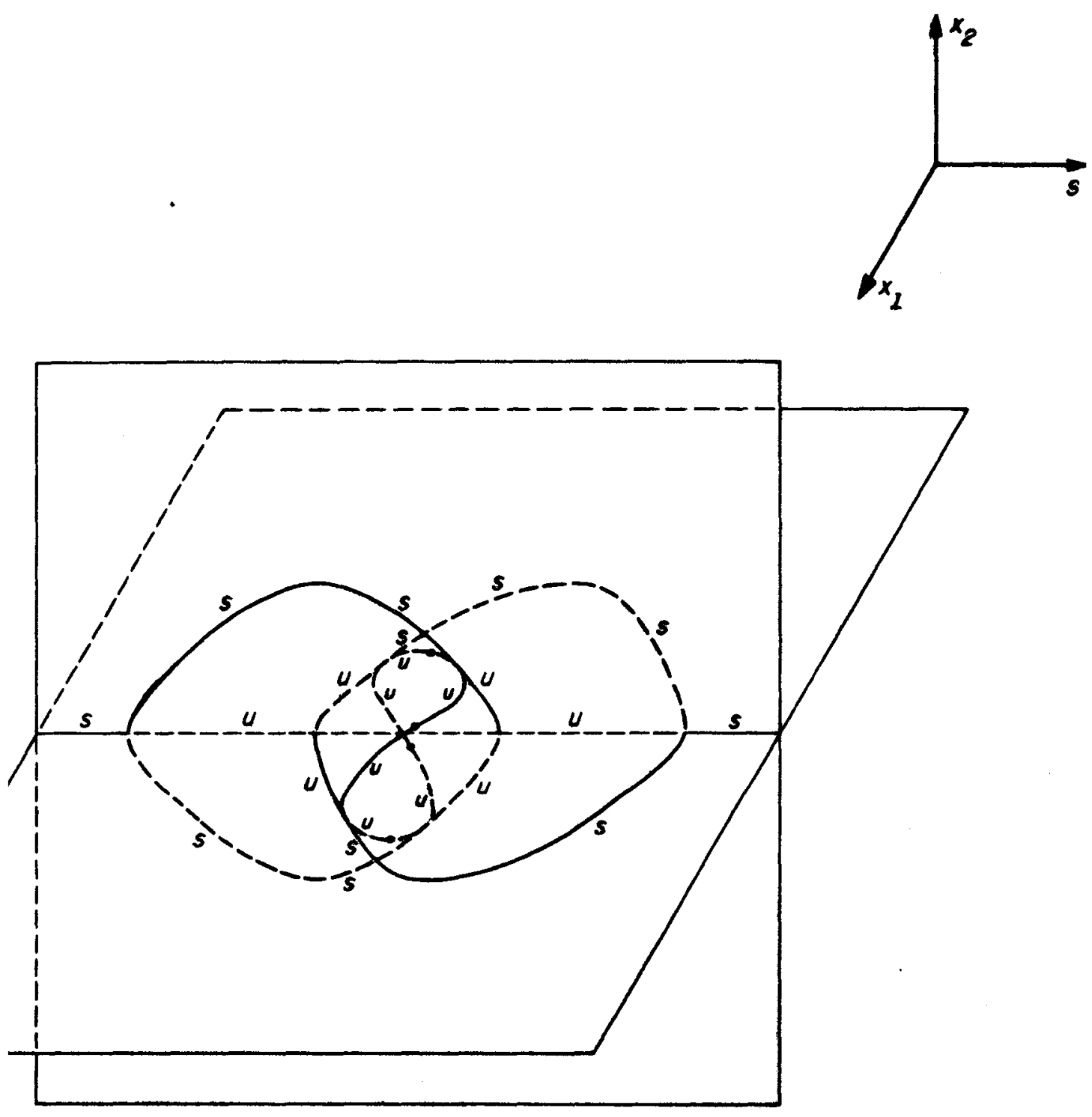

FIG. 5.2 - DIAGRAMA DE EIFUACACÃO: $m>1$ 
Comentários análogos feitos para as soluções no caso $m>1$ são válidos em todos os demais casos que se seguem e por isso apresentaremos apenas a tabela com os dados sobre as soluções, a representação esquemática do número de soluções do problema nas regiões do plano $\left(\lambda_{1}, \lambda_{2}\right)$ e o diagrama de bifurcação.

A construção dos diagramas de bifurcação do desdobramento versal $G$ de $g$ quando $m>1$ é dividido em dois casos: no caso (i) consideramos valores de $\beta$ positivos e próximos de zero e no caso (ii), valores de $\beta$ negativos e também próximos de zero. A tabela 5.4 contem os dadọs relativos a ambos casos. Esse dados variam de acordo com o sinal de $\beta$.

Neste caso não há mudanças qualitativas nos diagramas de bifurcação do desdobramento $G$ de $g$ quando comparado com o diagrama de bifurcação de $g$ pois trata-se de um germe topologicamente estável. O mesmo acorre para os casos seguintes que analisaremos. 


\begin{tabular}{|c|c|c|c|}
\hline $\begin{array}{c}\text { SUBESPAÇOS } \\
\text { FIXADOS } \\
\end{array}$ & $\begin{array}{l}\text { REGIÕES DO } \\
\text { PLANO }\left(\lambda_{1}, \lambda_{2}\right) \\
\end{array}$ & SOLUÇÕES & $\begin{array}{c}\text { SINAIS DOS } \\
\text { AUTO - VALORES } \\
\end{array}$ \\
\hline $\operatorname{Fix}\left(D_{4}\right)$ & $\forall\left(\lambda_{1}, \lambda_{2}\right)$ & $x_{1}=x_{2}=0$ & $++\left\{\begin{array}{l}\lambda_{1}+\lambda_{2}>0 \\
\lambda_{1}-\lambda_{2}>0\end{array}\right.$ \\
\hline $\operatorname{Fix}\left(Z_{2}(\kappa)\right)$ & $\lambda_{1}+\lambda_{2} \leq 0$ & $\begin{array}{c}x_{2}=0 \\
x_{1}^{2}=\frac{\left(\lambda_{1}+\lambda_{2}\right)}{(1-m-\beta)}\end{array}$ & $\begin{array}{l}++\left\{\begin{array}{l}\lambda_{1}+\lambda_{2}<0 \\
\lambda_{2}+\frac{1}{(m+\beta)} \lambda_{1}<0\end{array}\right. \\
+-\left\{\begin{array}{l}\lambda_{1}+\lambda_{2}<0 \\
\lambda_{2}+\frac{1}{(m+\beta)} \lambda_{1}>0\end{array}\right.\end{array}$ \\
\hline $\operatorname{Fix}\left(\mathrm{Z}_{2}^{c}(\kappa)\right)$ & $\lambda_{2}-\lambda_{1} \geq 0$ & $\begin{array}{c}x_{1}=0 \\
x_{2}^{2}=\frac{\left(\lambda_{1}-\lambda_{2}\right)}{(1-m-\beta)}\end{array}$ & $\begin{array}{l}++\left\{\begin{array}{l}\lambda_{2}-\lambda_{1}>0 \\
\lambda_{2}-\frac{1}{(m+\beta)} \lambda_{1}>0\end{array}\right. \\
+-\left\{\begin{array}{l}\lambda_{2}-\lambda_{1}>0 \\
\lambda_{2}-\frac{1}{(m+\beta)} \lambda_{1}<0\end{array}\right.\end{array}$ \\
\hline $\begin{array}{l}\operatorname{Fix}\left(\mathrm{Z}_{2}(\xi \kappa)\right) \\
\operatorname{Fix}\left(\mathrm{Z}_{2}^{c}(\xi \kappa)\right)\end{array}$ & $\begin{array}{l}\lambda_{1} \leq 0 \\
\lambda_{2}=0\end{array}$ & $\begin{array}{c}x_{1}= \pm x_{2} \\
x_{1}^{2}=-\frac{1}{2(m+\beta)} \lambda_{1}\end{array}$ & +- \\
\hline $\operatorname{Fix}(\mathbf{1})$ & $\begin{array}{c}\lambda_{1} \leq 0 \\
\left\{\begin{array}{l}\lambda_{2} \geq 0 \\
\lambda_{2}+\frac{1}{(m+\beta)} \lambda_{1} \leq 0 \\
\lambda_{2}<0 \\
\lambda_{2}-\frac{1}{(m+\beta)} \lambda_{1} \geq 0\end{array}\right.\end{array}$ & $\begin{array}{c}(m+\beta) x_{1}^{2}+ \\
(m+\beta) x_{2}^{2}=-\lambda_{1} \\
\text { e } \\
x_{1}^{2}-x_{2}^{2}=\lambda_{2}\end{array}$ & +- \\
\hline
\end{tabular}

TABELA 5.4: $m>1, \beta \neq 0$ 


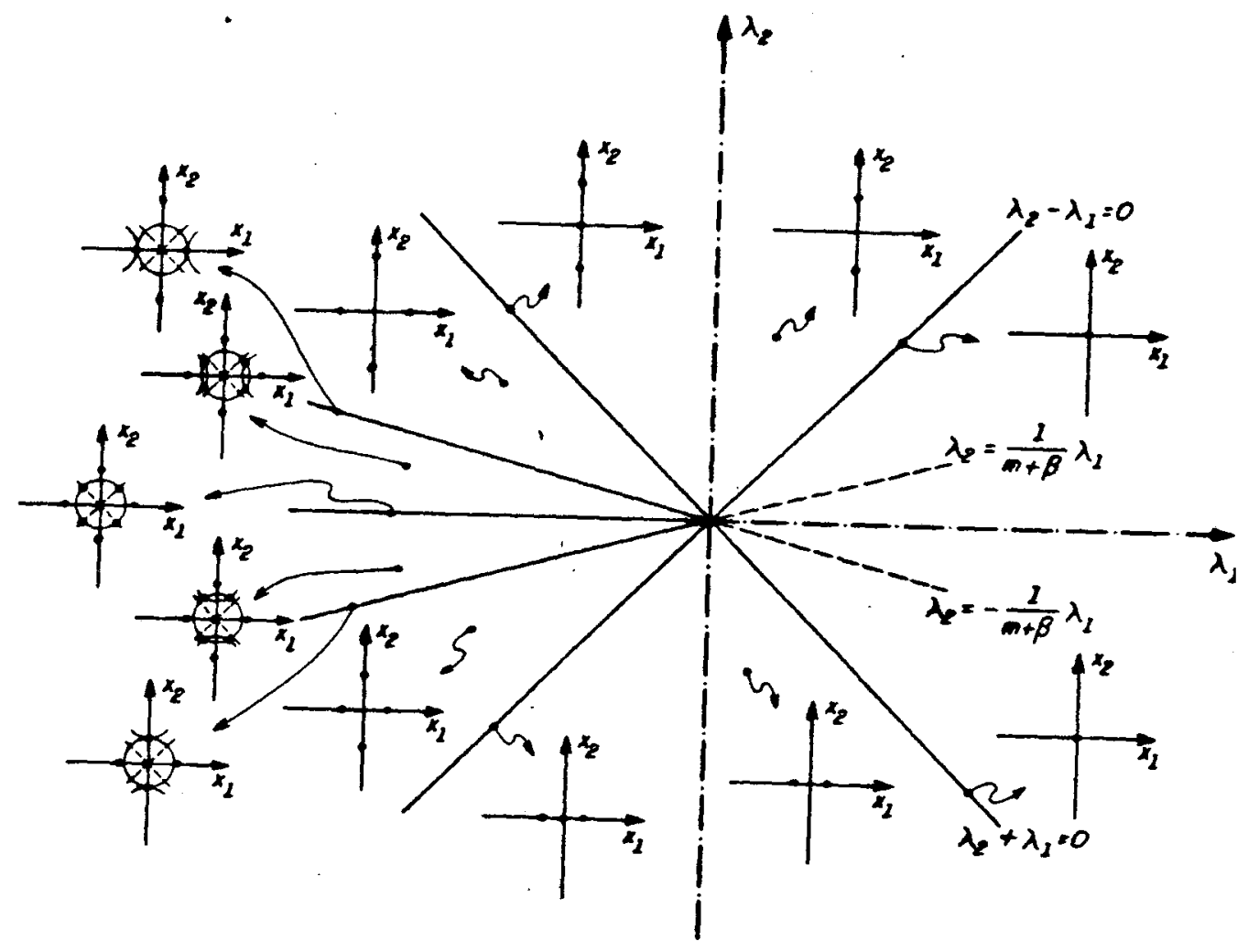

Fi6. 5.3:m>1, $\beta>0$ 

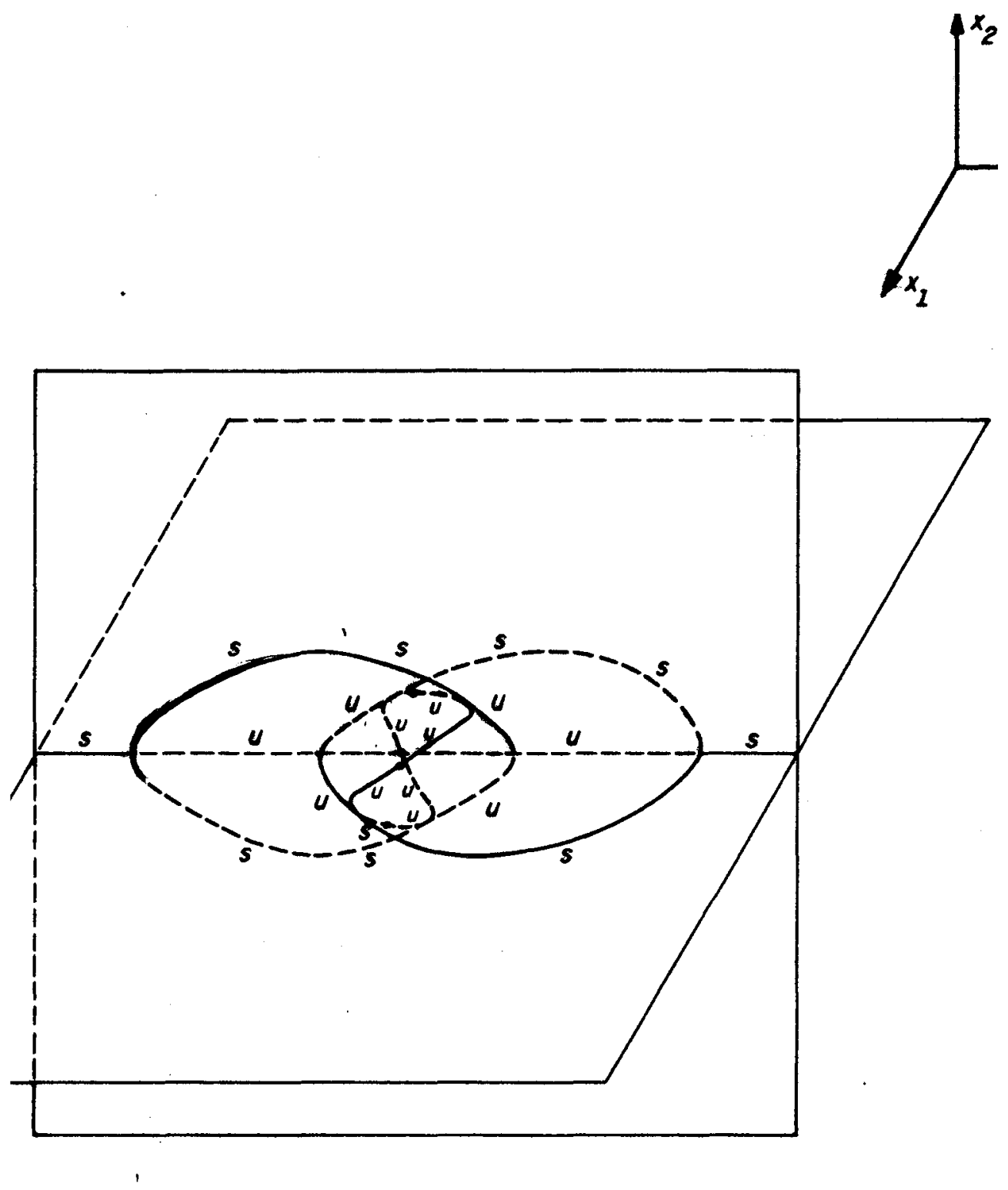

FIG.5.4-DIAGRAMA DE BIFURCACĀO: $m>1, \beta>0$ 


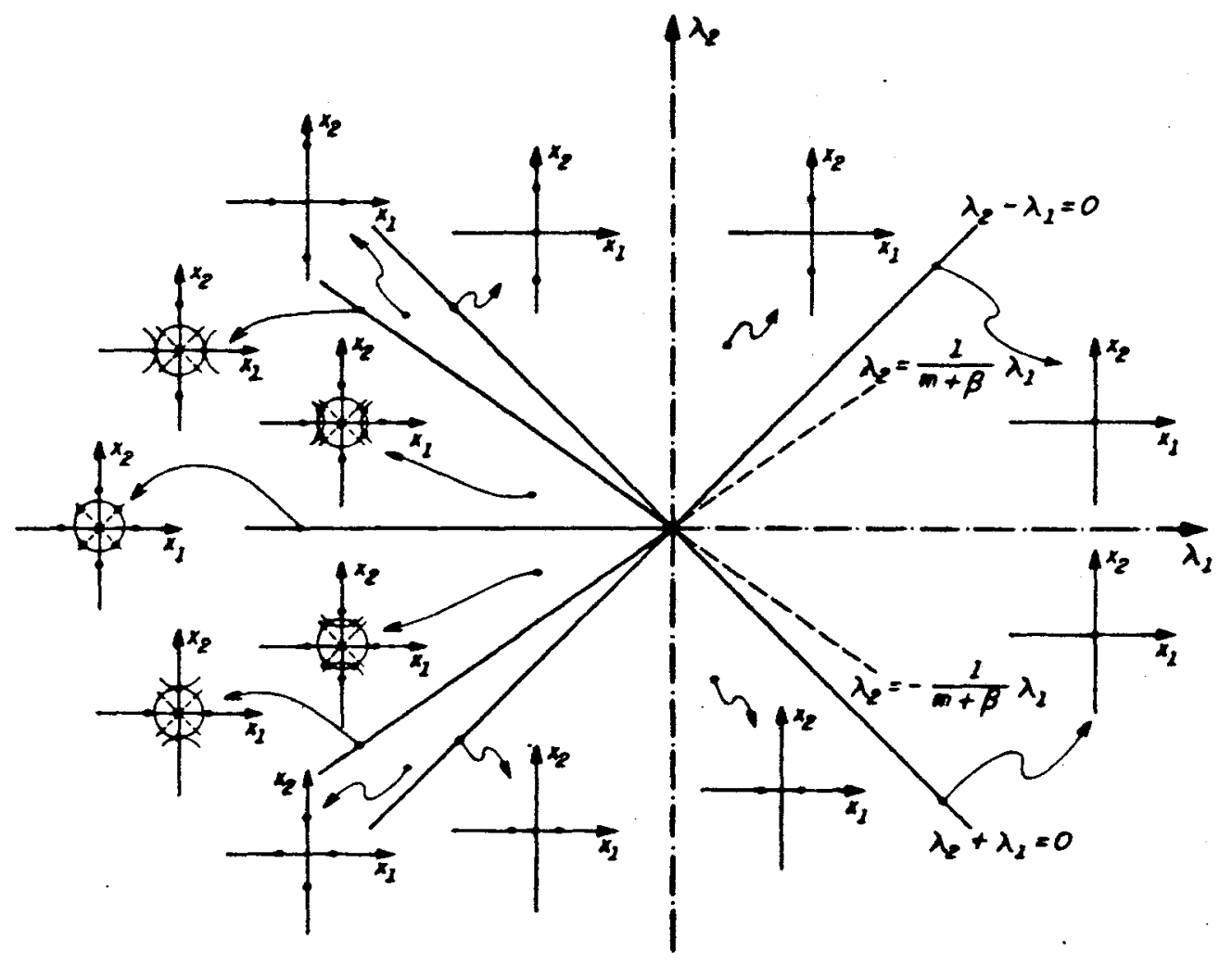

F16. $5.5: m>1, \beta<0$ 

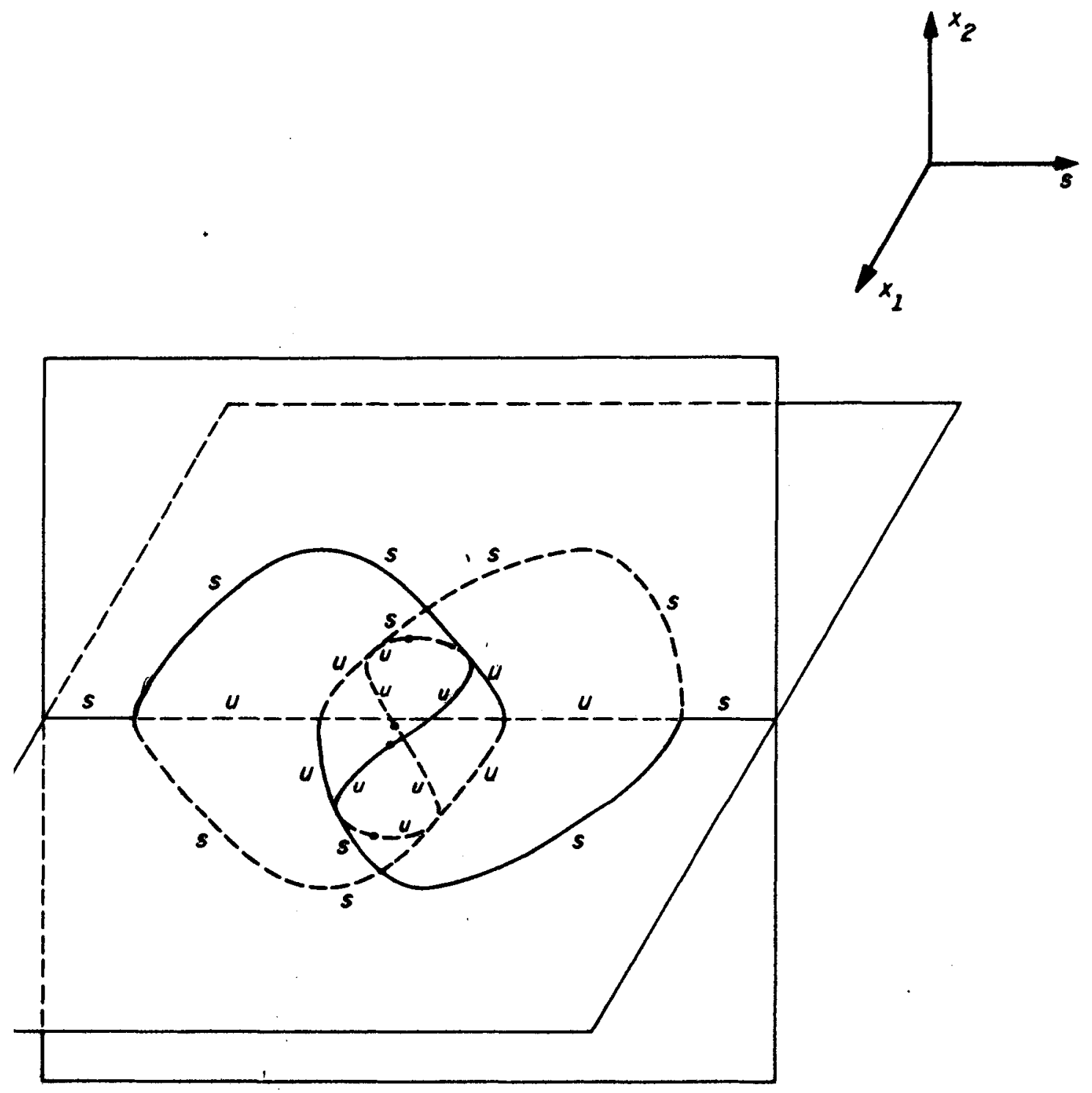

FIG.5.6-DIAGRAMA DE GIFURCACÃO: $m>1, \beta<0$ 


\begin{tabular}{|c|c|c|c|}
\hline $\begin{array}{c}\text { SUBESPACOS } \\
\text { FIXADOS } \\
\end{array}$ & $\begin{array}{c}\text { REGIÕES DO } \\
\text { PLANO }\left(\lambda_{1}, \lambda_{2}\right) \\
\end{array}$ & SOLUÇÕES & $\begin{array}{c}\text { SINAIS DOS } \\
\text { AUTO - VALORES } \\
\end{array}$ \\
\hline $\operatorname{Fix}\left(D_{4}\right)$ & $\forall\left(\lambda_{1}, \lambda_{2}\right)$ & $x_{1}=x_{2}=0$ & $++\left\{\begin{array}{l}\lambda_{1}+\lambda_{2}>0 \\
\lambda_{1}-\lambda_{2}>0\end{array}\right.$ \\
\hline $\operatorname{Fix}\left(Z_{2}(\kappa)\right)$ & $\lambda_{1}+\lambda_{2} \geq 0$ & $\begin{array}{c}x_{2}=0 \\
x_{1}^{2}=\frac{1}{(1-m)}\left(\lambda_{1}+\lambda_{2}\right)\end{array}$ & -+ \\
\hline $\operatorname{Fix}\left(\mathrm{Z}_{2}^{c}(\kappa)\right)$ & $\lambda_{2}-\lambda_{1} \leq 0$ & $\begin{array}{c}x_{1}=0 \\
x_{2}^{2}=\frac{1}{(1-m)}\left(\lambda_{1}-\lambda_{2}\right)\end{array}$ & -+ \\
\hline $\begin{array}{l}\operatorname{Fix}\left(Z_{2}(\xi \kappa)\right) \\
\operatorname{Fix}\left(Z_{2}^{c}(\xi \kappa)\right)\end{array}$ & $\begin{array}{l}\lambda_{1} \leq 0 \\
\lambda_{2}=0\end{array}$ & $\begin{array}{c}x_{1}= \pm x_{2} \\
x_{1}^{2}=-\frac{1}{2 m} \lambda_{1}\end{array}$ & +- \\
\hline $\operatorname{Fix}(1)$ & $\begin{array}{c}\lambda_{1} \leq 0 \\
\left\{\begin{array}{l}\lambda_{2} \geq 0 \\
\lambda_{2}+\frac{1}{m} \lambda_{1} \leq 0\end{array}\right. \\
\left\{\begin{array}{l}\lambda_{2}<0 \\
\lambda_{2}-\frac{1}{m} \lambda_{1} \geq 0\end{array}\right.\end{array}$ & $\begin{array}{c}m x_{1}^{2}+m x_{2}^{2}=-\lambda_{1} \\
\mathrm{e} \\
x_{1}^{2}-x_{2}^{2}=\lambda_{2}\end{array}$ & +- \\
\hline
\end{tabular}

TABELA 5.5: $0<m<1$

Neste caso todas as soluções não triviais são instáveis. 


\begin{tabular}{|c|c|c|c|}
\hline $\begin{array}{l}\text { SUBESPACYOS } \\
\text { FIXADOS } \\
\end{array}$ & $\begin{array}{c}\text { REGIÔES DO } \\
\operatorname{PLANO}\left(\lambda_{1}, \lambda_{2}\right)\end{array}$ & SOLUÇÕES & $\begin{array}{c}\text { SINAIS DOS } \\
\text { AUTO - VALORES }\end{array}$ \\
\hline $\operatorname{Fix}\left(D_{4}\right)$ & $\forall\left(\lambda_{1}, \lambda_{2}\right)$ & $x_{1}=x_{2}=0$ & $++\left\{\begin{array}{l}\lambda_{1}+\lambda_{2}>0 \\
\lambda_{1}-\lambda_{2}>0\end{array}\right.$ \\
\hline $\operatorname{Fix}\left(\mathrm{Z}_{2}(\kappa)\right)$ & $\dot{\lambda}_{1}+\lambda_{2} \geq 0$ & $\begin{array}{c}x_{2}=0 \\
x_{1}^{2}=\frac{\left(\lambda_{1}+\lambda_{2}\right)}{(1-m-\beta)}\end{array}$ & -+ \\
\hline $\operatorname{Fix}\left(Z_{2}^{c}(\kappa)\right)$ & $\lambda_{2}-\lambda_{1} \leq 0$ & $\begin{array}{c}x_{1}=0 \\
x_{2}^{2}=\frac{\left(\lambda_{1}-\lambda_{2}\right)}{(1-m-\beta)}\end{array}$ & -+ \\
\hline $\begin{array}{l}\operatorname{Fix}\left(\mathrm{Z}_{2}(\xi \kappa)\right) \\
\operatorname{Fix}\left(\mathrm{Z}_{2}^{c}(\xi \kappa)\right)\end{array}$ & $\begin{array}{l}\lambda_{1} \leq 0 \\
\lambda_{2}=0\end{array}$ & $\begin{array}{c}x_{1}= \pm x_{2} \\
x_{1}^{2}=-\frac{1}{2(m+\beta)} \lambda_{1}\end{array}$ & +- \\
\hline $\operatorname{Fix}(1)$ & $\begin{array}{c}\lambda_{1} \leq 0 \\
\left\{\begin{array}{l}\lambda_{2} \geq 0 \\
\lambda_{2}+\frac{1}{(m+\beta)} \lambda_{1} \leq 0\end{array}\right. \\
\left\{\begin{array}{l}\lambda_{2}<0 \\
\lambda_{2}-\frac{1}{(m+\beta)} \lambda_{1} \geq 0\end{array}\right.\end{array}$ & $\begin{array}{c}(m+\beta) x_{1}^{2}+ \\
(m+\beta) x_{2}^{2}=-\lambda_{1} \\
\text { e } \\
x_{1}^{2}-x_{2}^{2}=\lambda_{2}\end{array}$ & + \\
\hline
\end{tabular}

TABELA 5.6: $0<m<1, \beta \neq 0$

Todas as soluções não triviais são instáveis. 


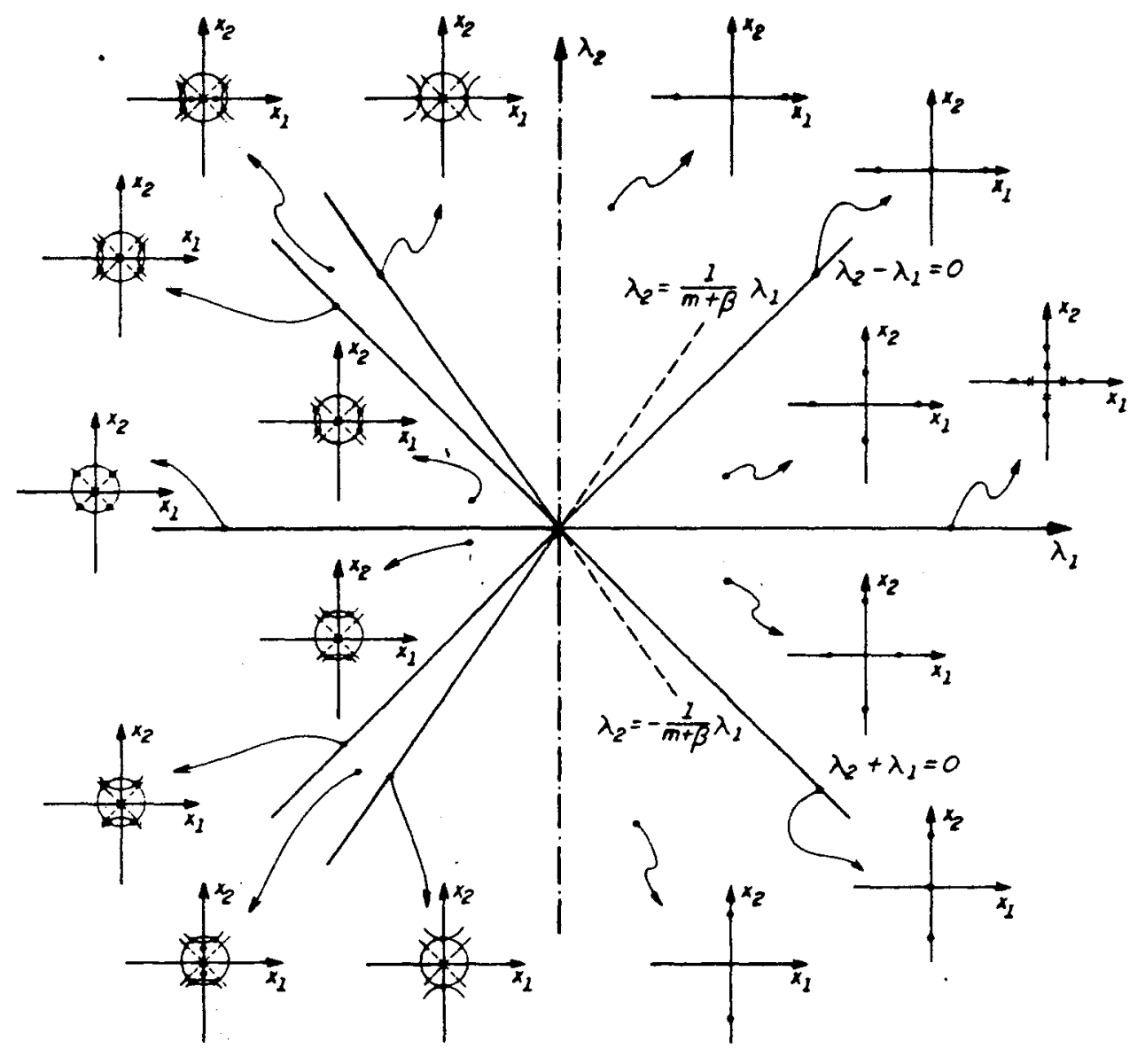

FIG. 5.9: $0<m<1, \beta>0$ 

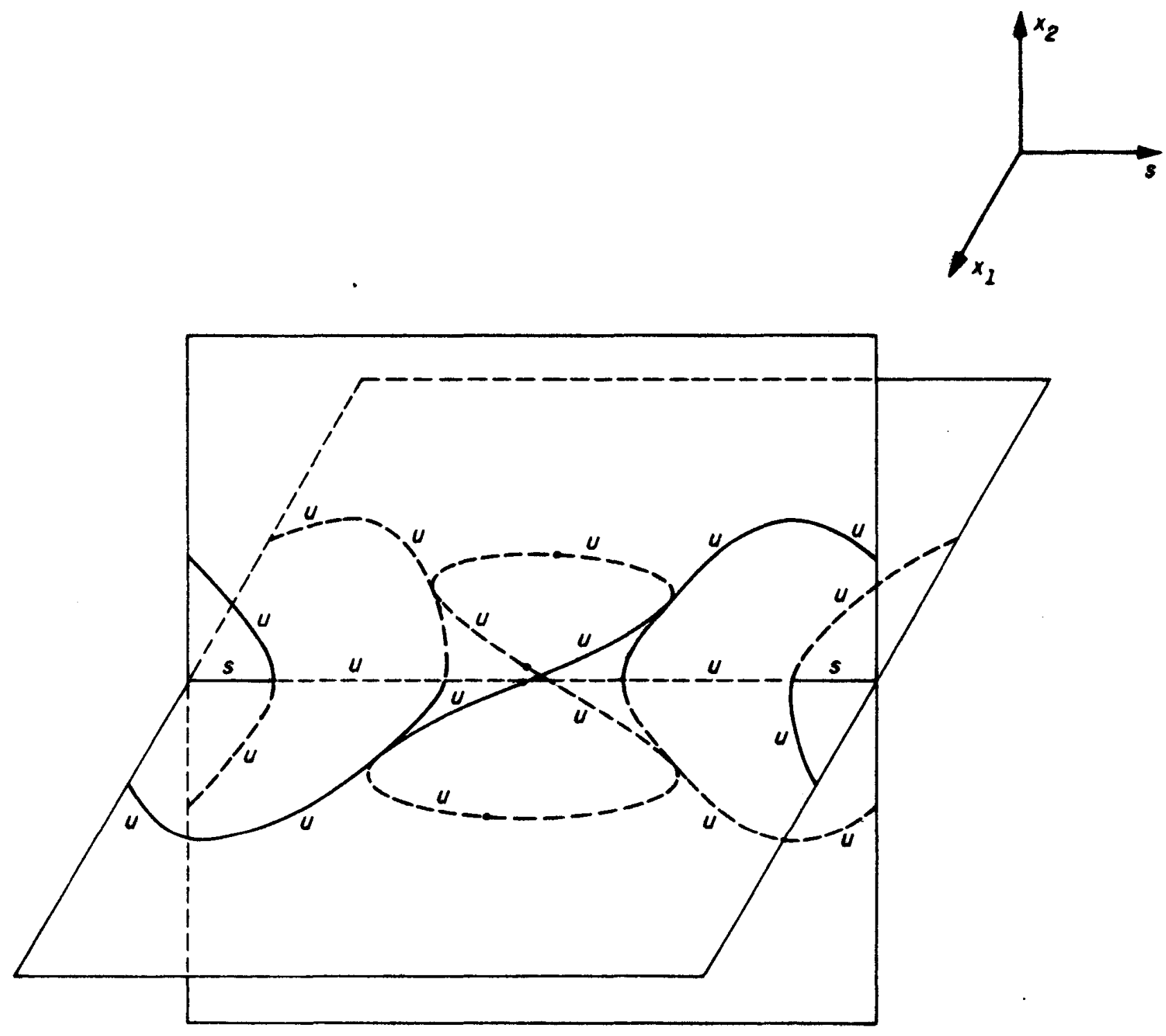

FIG.5.10- DIAGRAMA DE BIFURCACÃO: $0<m<1, \beta>0$ 


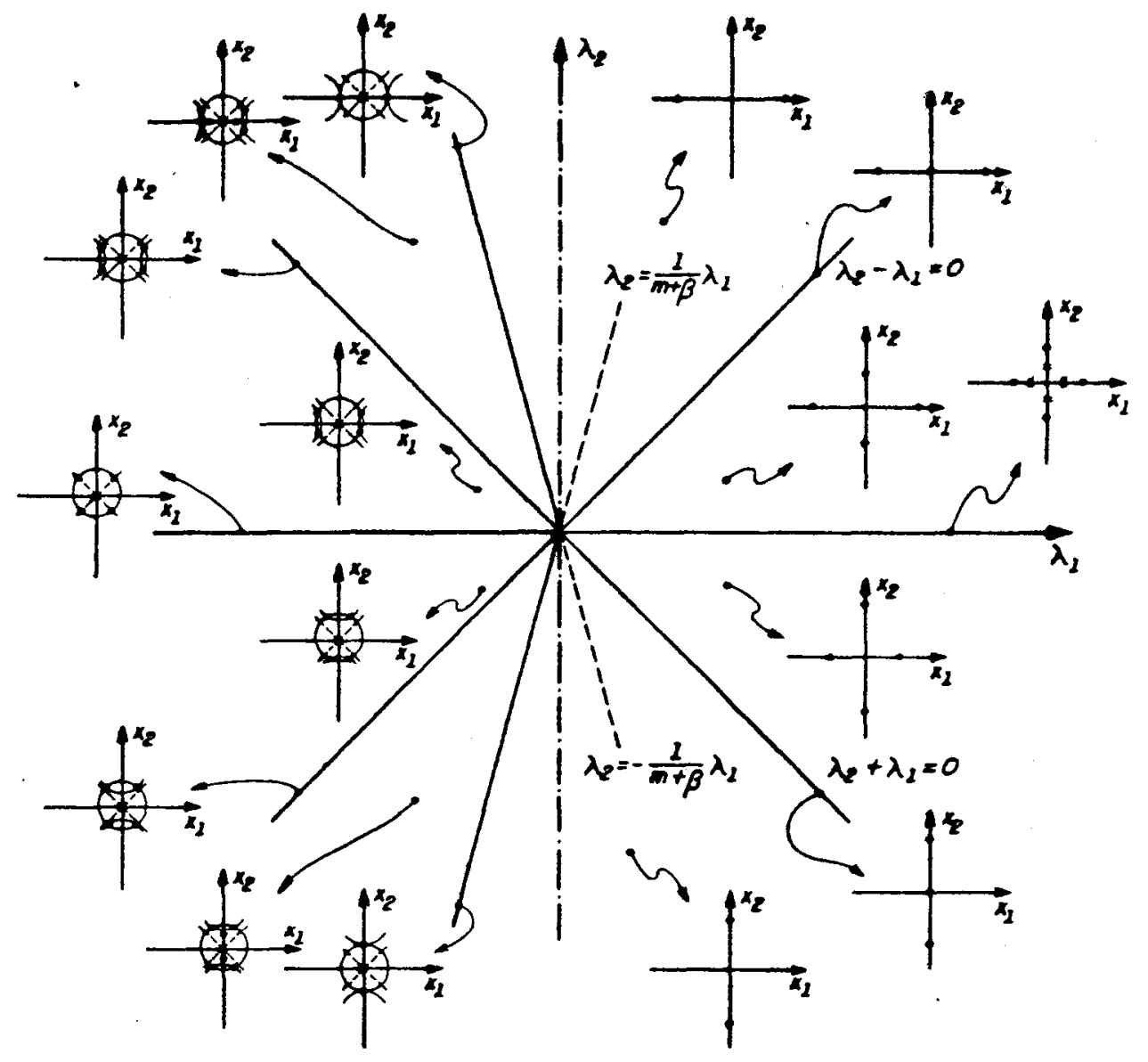

FIG. S.11: $0<m<1, \rho<0$ 

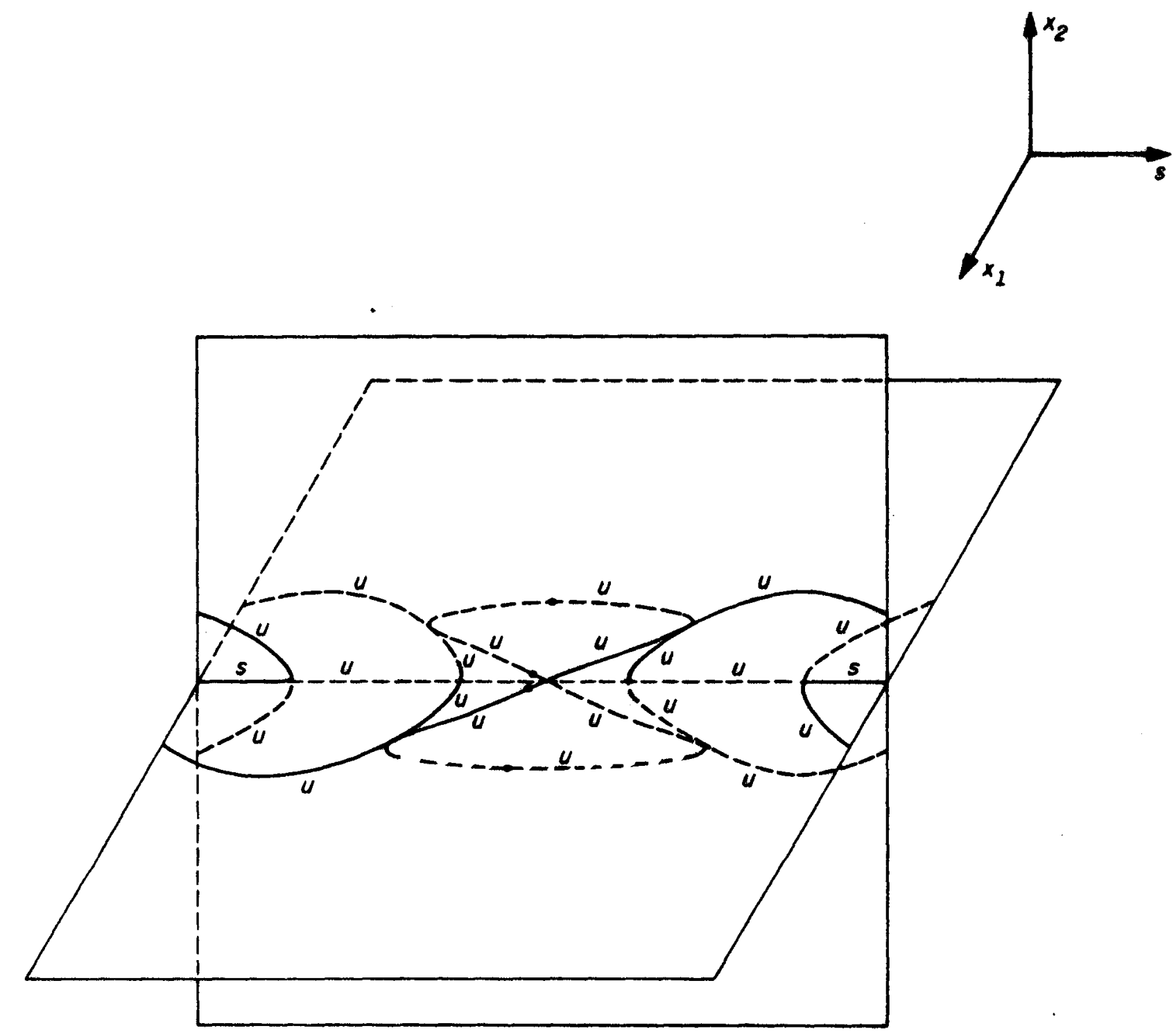

FIG. 5.12 - DIAGRAMA DE BIFURCACÃO: $0<m<1, \beta<0$ 


\begin{tabular}{|c|c|c|c|}
\hline $\begin{array}{c}\text { SUBESPAÇOS } \\
\text { FIXADOS }\end{array}$ & $\begin{array}{c}\text { REGIŌES DO } \\
\text { PLANO }\left(\lambda_{1}, \lambda_{2}\right) \\
\end{array}$ & SOLUÇÕES & $\begin{array}{c}\text { SINAIS DOS } \\
\text { AUTO - VALORES } \\
\end{array}$ \\
\hline $\operatorname{Fix}\left(D_{4}\right)$ & $\forall\left(\lambda_{1}, \lambda_{2}\right)$ & $x_{1}=x_{2}=0$ & $++\left\{\begin{array}{l}\lambda_{1}+\lambda_{2}>0 \\
\lambda_{1}-\lambda_{2}>0\end{array}\right.$ \\
\hline $\operatorname{Fix}\left(\mathrm{Z}_{2}(\kappa)\right)$ & $\lambda_{1}+\lambda_{2} \geq 0$ & $\begin{array}{c}x_{2}=0 \\
x_{1}^{2}=\frac{1}{(1-m)}\left(\lambda_{1}+\lambda_{2}\right)\end{array}$ & -+ \\
\hline $\operatorname{Fix}\left(\mathrm{Z}_{2}^{c}(\kappa)\right)$ & $\lambda_{2}-\lambda_{1} \leq 0$ & $\begin{array}{c}x_{1}=0 \\
x_{2}^{2}=\frac{1}{(1-m)}\left(\lambda_{1}-\lambda_{2}\right)\end{array}$ & -+ \\
\hline $\begin{array}{l}\operatorname{Fix}\left(\mathrm{Z}_{2}(\xi \kappa)\right) \\
\operatorname{Fix}\left(\mathrm{Z}_{2}^{c}(\xi \kappa)\right)\end{array}$ & $\begin{array}{l}\lambda_{1} \geq 0 \\
\lambda_{2}=0\end{array}$ & $\begin{array}{c}x_{1}= \pm x_{2} \\
x_{1}^{2}=-\frac{1}{2 m} \lambda_{1}\end{array}$ & +- \\
\hline Fix (1) & $\begin{array}{c}\lambda_{1} \geq 0 \\
\left\{\begin{array}{l}\lambda_{2} \geq 0 \\
\lambda_{2}+\frac{1}{m} \lambda_{1} \leq 0\end{array}\right. \\
\left\{\begin{array}{l}\lambda_{2}<0 \\
\lambda_{2}-\frac{1}{m} \lambda_{1} \geq 0\end{array}\right.\end{array}$ & $\begin{array}{c}m x_{1}^{2}+m x_{2}^{2}=-\lambda_{1} \\
\mathrm{e} \\
x_{1}^{2}-x_{2}^{2}=\lambda_{2}\end{array}$ & +- \\
\hline
\end{tabular}

TABELA 5.7: $-1<m<0$

Neste caso todas as soluções não triviais são instáveis. 


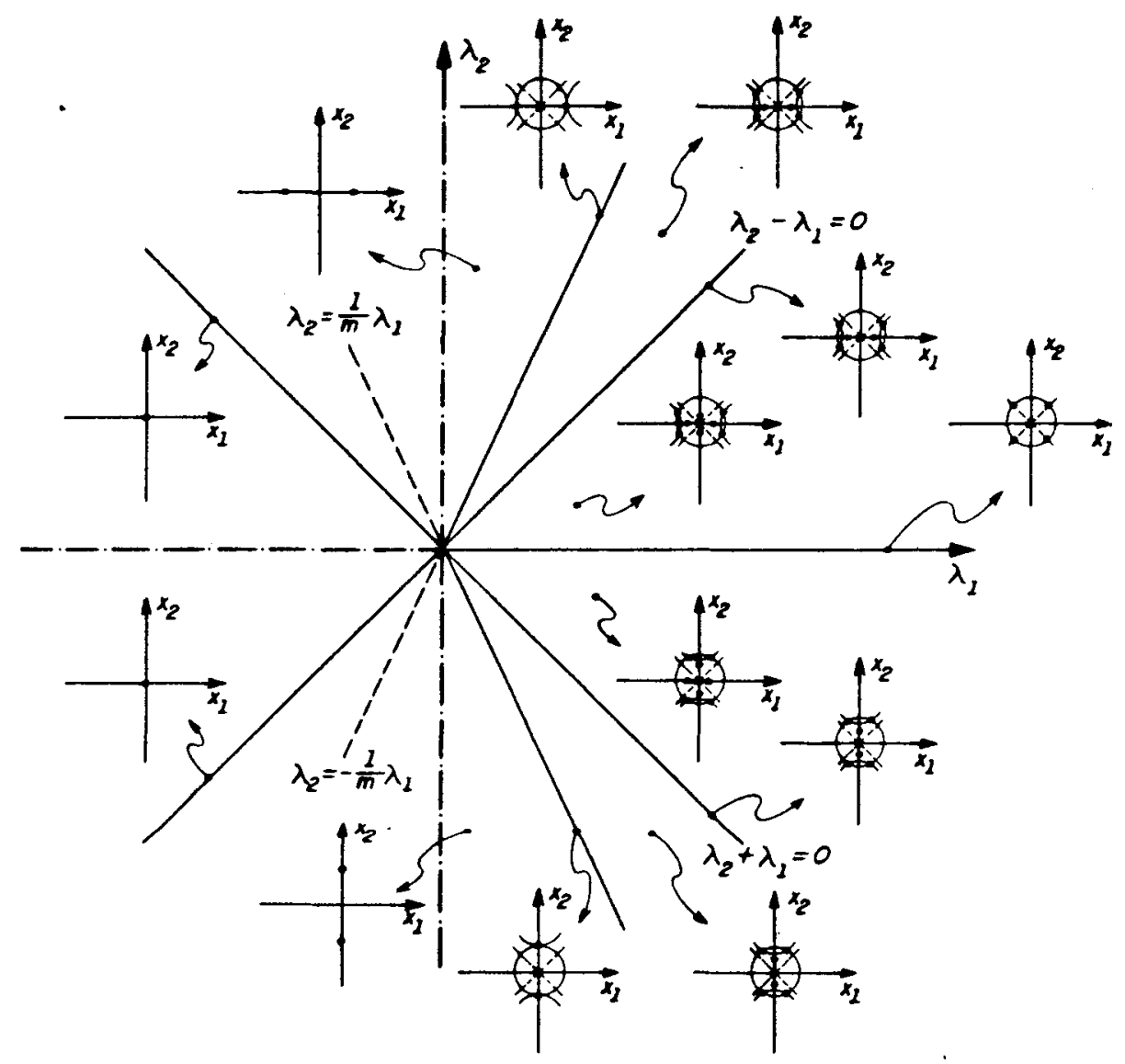

FIG. 5.13:-2<a<0 

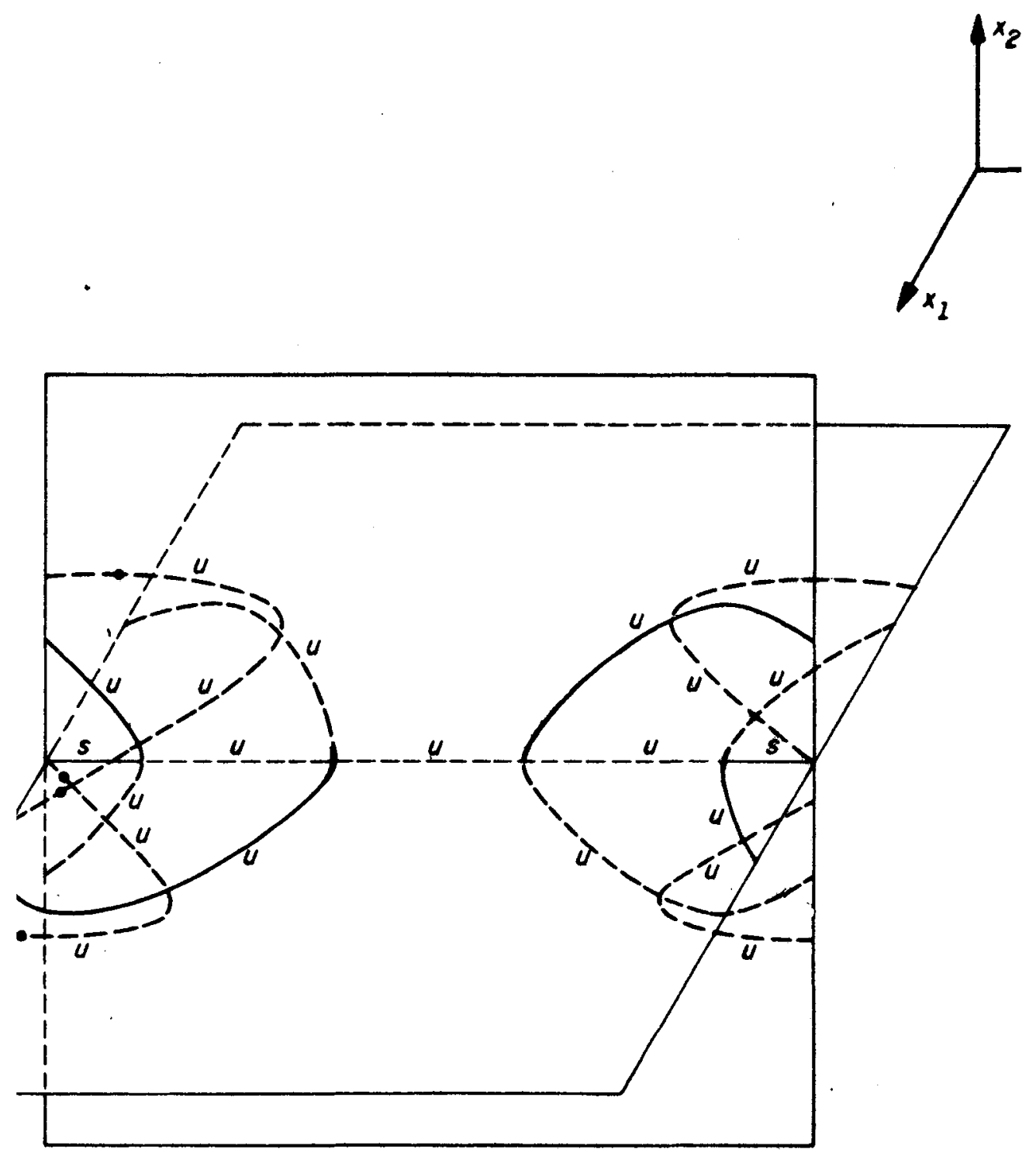

FIG.5.14 - DIAGRAMA DE BIFURCACÃO: $-1<m<0$ 


\begin{tabular}{|c|c|c|c|}
\hline $\begin{array}{c}\text { SUBESPAÇOS } \\
\text { FIXADOS } \\
\end{array}$ & $\begin{array}{c}\text { REGIÕES DO } \\
\operatorname{PLANO}\left(\lambda_{1}, \lambda_{2}\right) \\
\end{array}$ & SOLUÇÕES & $\begin{array}{c}\text { SINAIS DOS } \\
\text { AUTO - VALORES } \\
\end{array}$ \\
\hline $\operatorname{Fix}\left(D_{4}\right)$ & $\forall\left(\lambda_{1}, \lambda_{2}\right)$ & $x_{1}=x_{2}=0$ & $++\left\{\begin{array}{l}\lambda_{1}+\lambda_{2}>0 \\
\lambda_{1}-\lambda_{2}>0\end{array}\right.$ \\
\hline $\operatorname{Fix}\left(Z_{2}(\kappa)\right)$ & $\lambda_{1}+\lambda_{2} \geq 0$ & $\begin{array}{c}x_{2}=0 \\
x_{1}^{2}=\frac{\left(\lambda_{1}+\lambda_{2}\right)}{(1-m-\beta)}\end{array}$ & -+ \\
\hline $\operatorname{Fix}\left(Z_{2}^{c}(\kappa)\right)$ & $\lambda_{2}-\lambda_{1} \leq 0$ & $\begin{array}{c}x_{1}=0 \\
x_{2}^{2}=\frac{\left(\lambda_{1}-\lambda_{2}\right)}{(1-m-\beta)}\end{array}$ & -+ \\
\hline $\begin{array}{l}\operatorname{Fix}\left(\mathrm{Z}_{2}(\xi \kappa)\right) \\
\operatorname{Fix}\left(\mathrm{Z}_{2}^{c}(\xi \kappa)\right)\end{array}$ & $\begin{array}{l}\lambda_{1} \geq 0 \\
\lambda_{2}=0\end{array}$ & $\begin{array}{c}x_{1}= \pm x_{2} \\
x_{1}^{2}=-\frac{1}{2(m+\beta)} \lambda_{1}\end{array}$ & +- \\
\hline $\operatorname{Fix}(1)$ & $\begin{array}{l}\lambda_{1} \geq 0 \\
\lambda_{2} \geq 0 \\
\lambda_{2}+\frac{1}{(m+\beta)} \lambda_{1} \leq 0 \\
\lambda_{2}<0 \\
\lambda_{2}-\frac{1}{(m+\beta)} \lambda_{1} \geq 0\end{array}$ & $\begin{array}{l}(m+\beta) x_{1}^{2}+ \\
(m+\beta) x_{2}^{2}=-\lambda_{1} \\
\mathbf{e} \\
x_{1}^{2}-x_{2}^{2}=\lambda_{2}\end{array}$ & +- \\
\hline
\end{tabular}

TABELA 5.8: $-1<m<0, \beta \neq 0$

Todas as soluções não triviais são instáveis. 


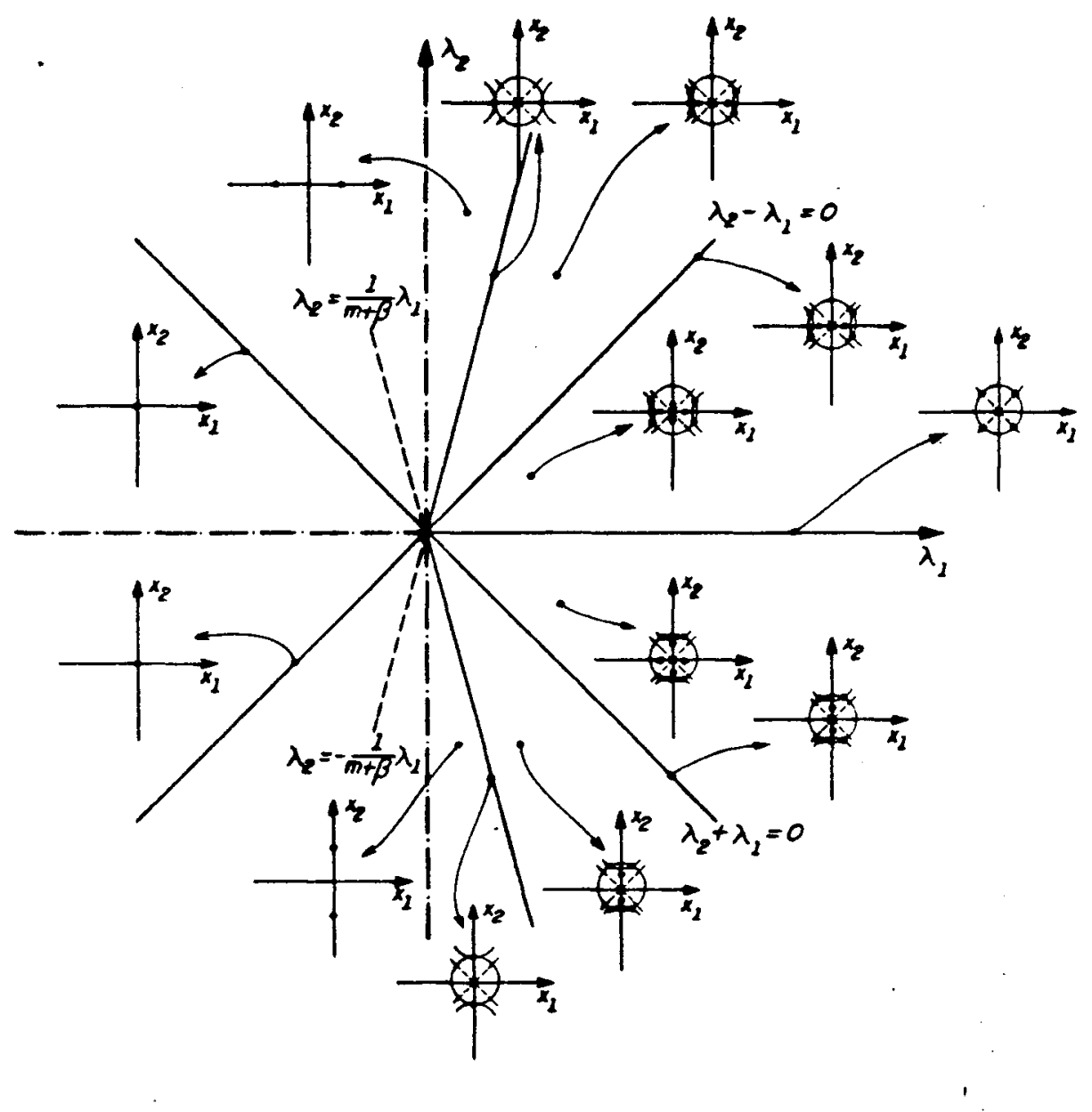

FIG. $5.15:-1<m<0, \beta>0$ 

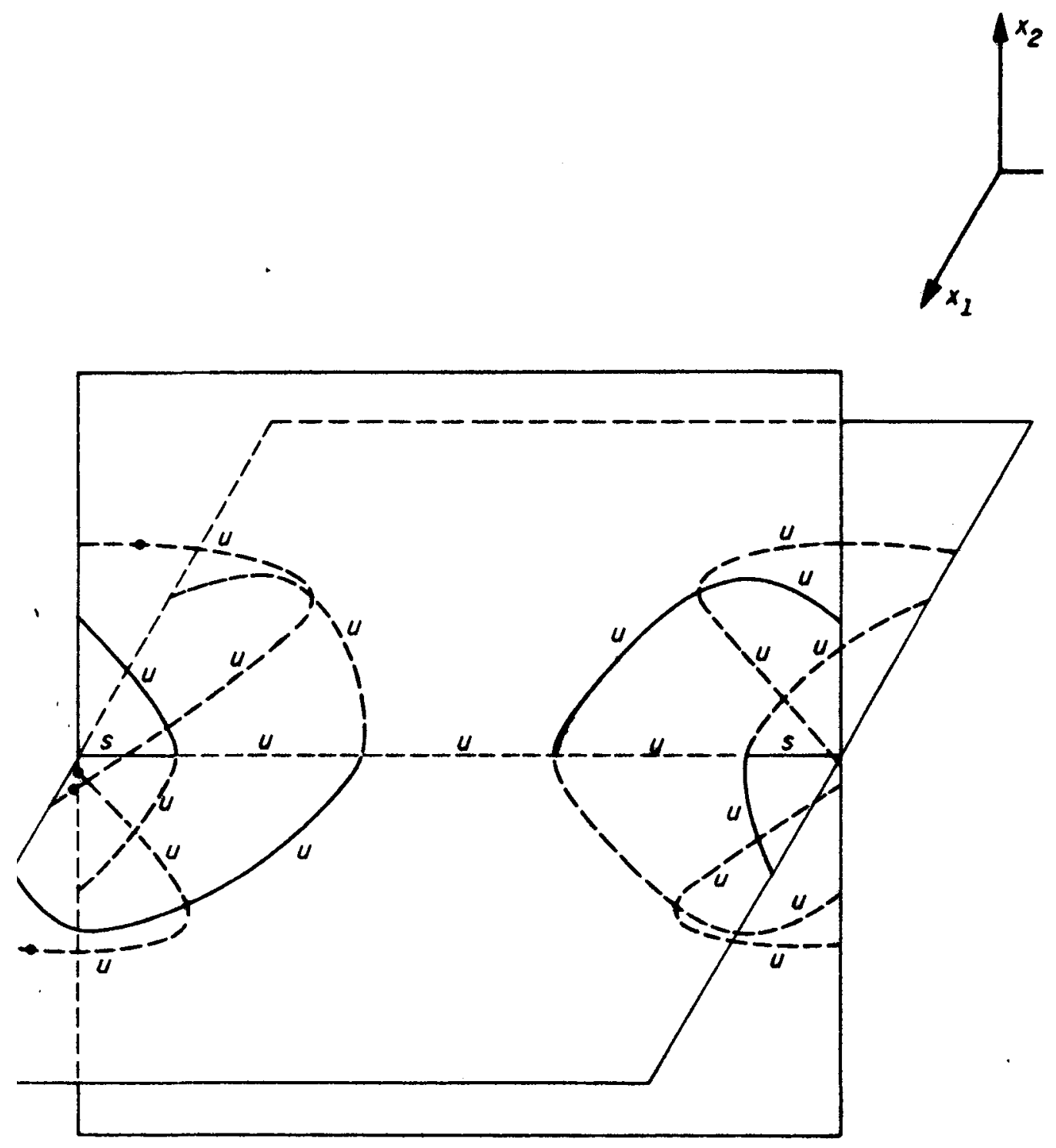

FIG.5.16 - DIAGRAMA DE BIFURCACÃO: $-1<m<0, \beta>0$ 


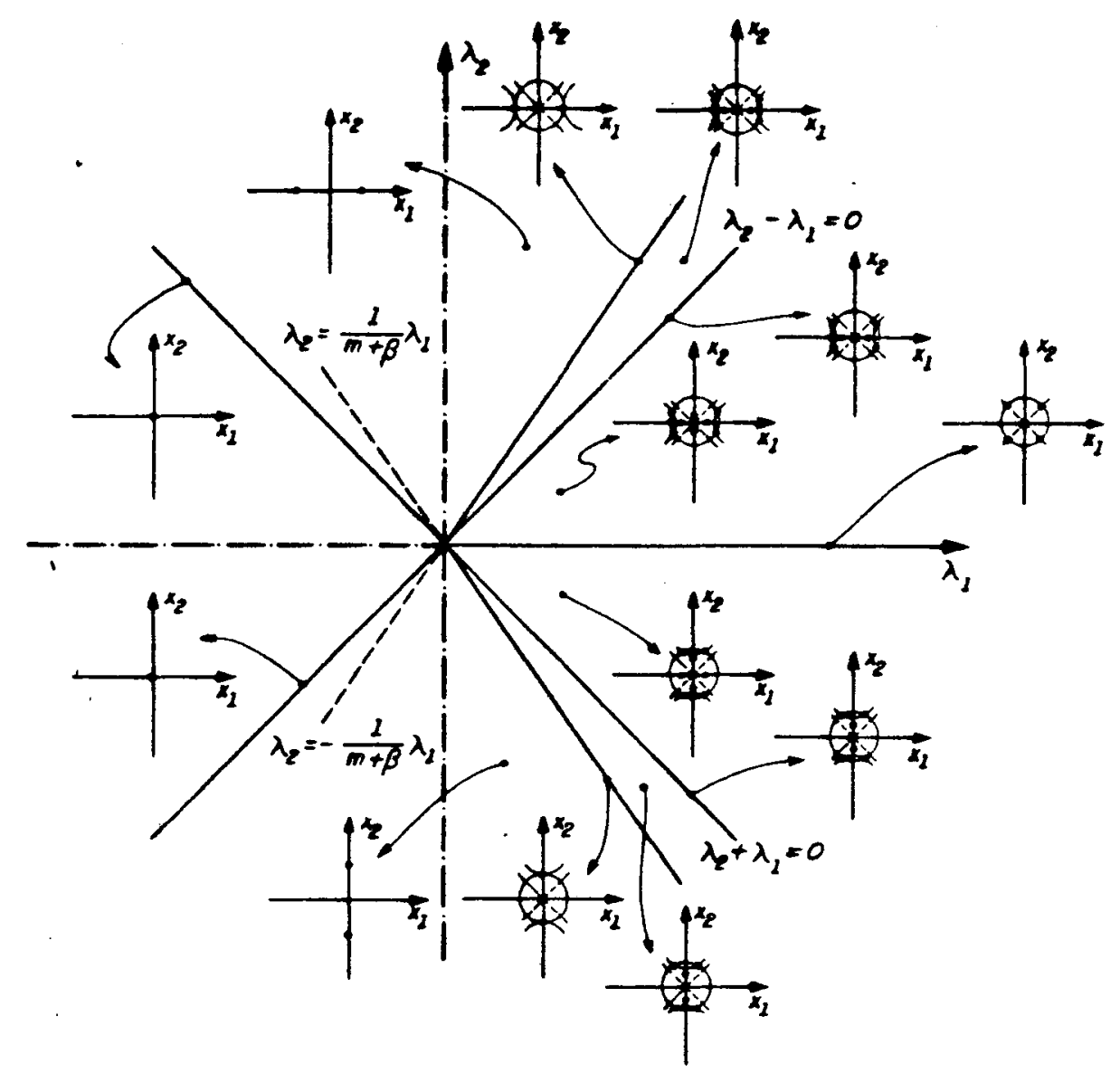

FIG. $5.17:-2<m<0, \beta<0$ 


\begin{tabular}{|c|c|c|c|}
\hline $\begin{array}{c}\text { SUBESPAÇOS } \\
\text { FIXADOS } \\
\end{array}$ & $\begin{array}{c}\text { REGIÕES DO } \\
\operatorname{PLANO}\left(\lambda_{1}, \lambda_{2}\right) \\
\end{array}$ & SOLUÇÔES & $\begin{array}{c}\text { SINAIS DOS } \\
\text { AUTO - VALORES }\end{array}$ \\
\hline $\operatorname{Fix}\left(D_{4}\right)$ & $\forall\left(\lambda_{1}, \lambda_{2}\right)$ & $x_{1}=x_{2}=0$ & $++\left\{\begin{array}{l}\lambda_{1}+\lambda_{2}>0 \\
\lambda_{1}-\lambda_{2}>0\end{array}\right.$ \\
\hline $\operatorname{Fix}\left(\mathrm{Z}_{2}(\kappa)\right)$ & $\lambda_{1}+\lambda_{2} \geq 0$ & $\begin{array}{c}x_{2}=0 \\
x_{1}^{2}=\frac{1}{(1-m)}\left(\lambda_{1}+\lambda_{2}\right)\end{array}$ & -+ \\
\hline $\operatorname{Fix}\left(\mathrm{Z}_{2}^{c}(\kappa)\right)$ & $\lambda_{2}-\lambda_{1} \leq 0$ & $\begin{array}{c}x_{1}=0 \\
x_{2}^{2}=\frac{1}{(1-m)}\left(\lambda_{1}-\lambda_{2}\right)\end{array}$ & $\begin{array}{l}-+ \\
\text {. }\end{array}$ \\
\hline $\begin{array}{l}\operatorname{Fix}\left(Z_{2}(\xi \kappa)\right) \\
\operatorname{Fix}\left(Z_{2}^{c}(\xi \kappa)\right)\end{array}$ & $\begin{array}{l}\lambda_{1} \geq 0 \\
\lambda_{2}=0\end{array}$ & $\begin{array}{c}x_{1}= \pm x_{2} \\
x_{1}^{2}=-\frac{1}{2 m} \lambda_{1}\end{array}$ & + \\
\hline $\operatorname{Fix}(1)$ & $\begin{array}{c}\lambda_{1} \geq 0 \\
\left\{\begin{array}{l}\lambda_{2} \geq 0 \\
\lambda_{2}+\frac{1}{m} \lambda_{1} \leq 0\end{array}\right. \\
\left\{\begin{array}{l}\lambda_{2}<0 \\
\lambda_{2}-\frac{1}{m} \lambda_{1} \geq 0\end{array}\right.\end{array}$ & $\begin{array}{c}m x_{1}^{2}+m x_{2}^{2}=-\lambda_{1} \\
\mathrm{e} \\
x_{1}^{2}-x_{2}^{2}=\lambda_{2}\end{array}$ & +- \\
\hline
\end{tabular}

TABELA 5.9: $m<-1$

Neste caso todas as soluções não triviais são instáveis.

Para esse caso $m<-1$, construiremos apenas o diagrama de bifurcação de $g$ devido a semelhança dos diagramas do seu desdobramento com o caso anterior. 


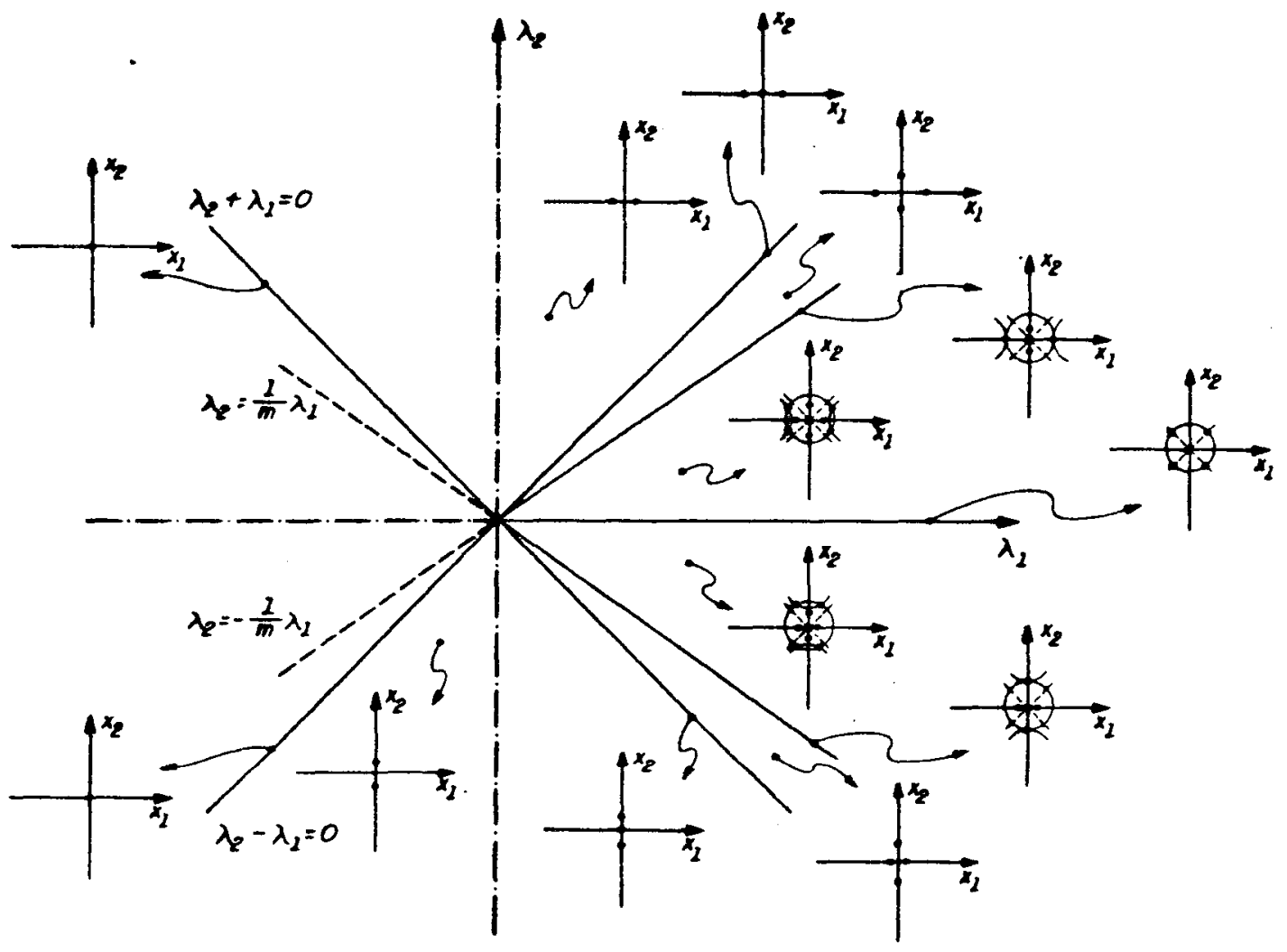

F16. 5.19: $n<-2$ 


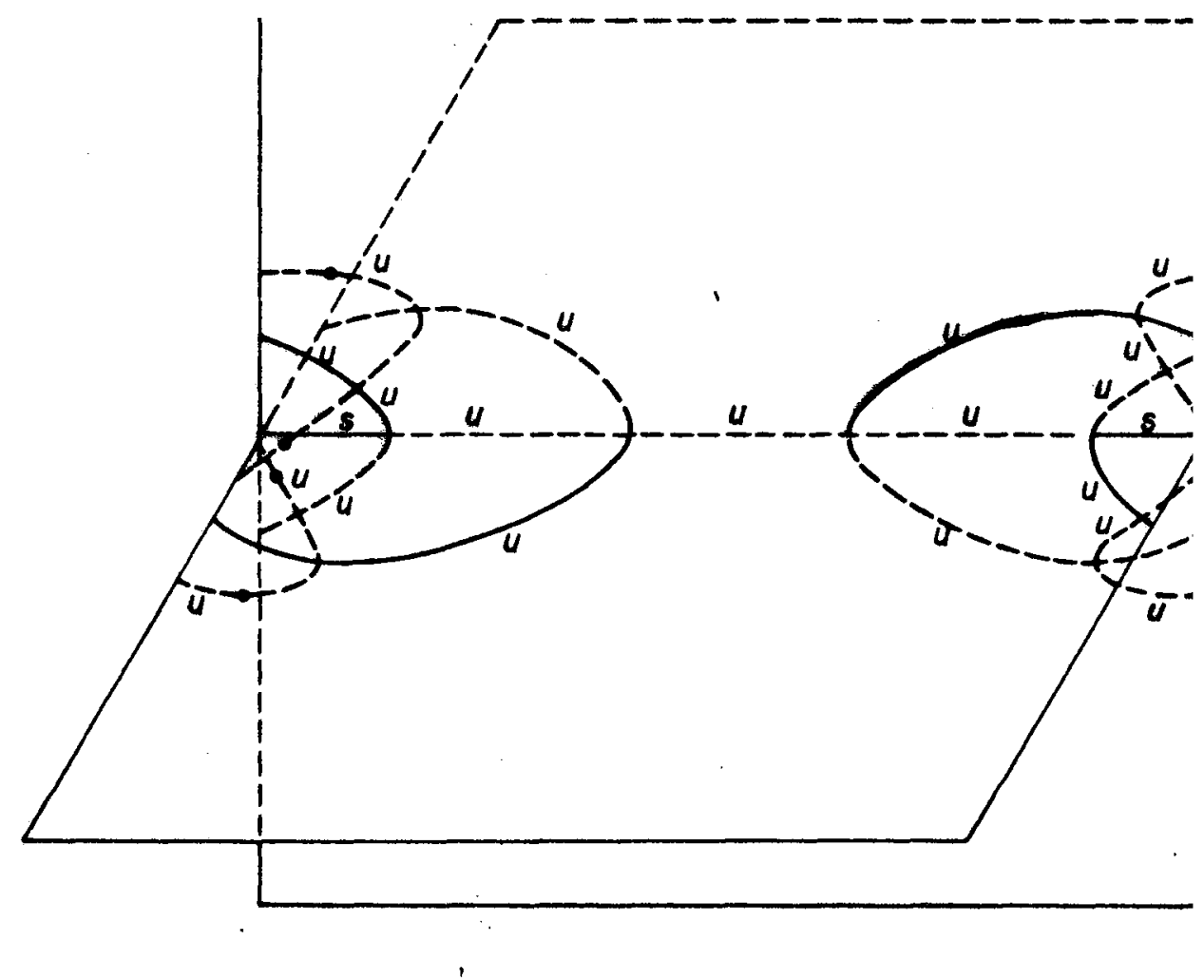

FIG.5.20 - DIAGRAMA OE BIFURCACĀO: $m<-1$ 
Observação. Para os diagramas de bifurcação da forma normal genérica que apresentamos, fizemos a escolha $\epsilon_{o}=\epsilon_{5}=1$. Há, na realidade, 16 diagramas qualitativamente distintos correspondendo às 4 possíveis escolhas para $\epsilon_{0}$ e $\epsilon_{5}$ e aos 4 intervalos de variaçâo de $m$ pois $m \neq \pm 1,0$. Entretanto, usando a mudança de coordenadas $g \mapsto-g$ que inverte a estabilidade e a mudança $\left(\lambda_{1}, \lambda_{2}\right) \mapsto\left(\lambda_{1},-\lambda_{2}\right)$ que inverte as regiões do plano, vemos que basta analisar os casos $\epsilon_{o}=\epsilon_{5}=1$ e $\epsilon_{o}=1$ e $\epsilon_{5}=-1$. Optamos pela construção dos diagramas para $\epsilon_{0}=\epsilon_{5}=1$.

\subsection{Diagramas de bifurcação para a forma normal quando $p_{\lambda_{1}}^{o}=0$}

A forma normal quando $p_{\lambda_{1}}^{o}=0$ é o CASO $\mathrm{I}_{1}$ do Teorema 2.8.1. e é dada por

$$
\begin{aligned}
g:\left(\mathbb{R}^{2} \times \mathbb{R}^{2}, 0\right) & \rightarrow \mathbb{R}^{2} \\
\left(x_{1}, x_{2}, \lambda_{1}, \lambda_{2}\right) & \mapsto\left(m N+\epsilon_{3} N \lambda_{1}+\epsilon_{4} \lambda_{1}^{2}\right)\left(\begin{array}{l}
x_{1} \\
x_{2}
\end{array}\right)+\epsilon_{5} \delta\left(\begin{array}{r}
x_{1} \\
-x_{2}
\end{array}\right)+\lambda_{2}\left(\begin{array}{r}
x_{1} \\
-x_{2}
\end{array}\right)
\end{aligned}
$$

e seu desdobramento, para cada valor isolado de $m$, é

$$
\begin{aligned}
& G:\left(\mathbb{R}^{2} \times \mathbb{R}^{2} \times \mathbb{R}, 0\right) \rightarrow \mathbb{R}^{2} \\
&\left(x_{1}, x_{2}, \lambda_{1}, \lambda_{2}, \alpha\right) \mapsto\left(m N+\epsilon_{3} N \lambda_{1}+\epsilon_{4} \lambda_{1}^{2}+\alpha\right)\left(\begin{array}{c}
x_{1} \\
x_{2}
\end{array}\right)+\epsilon_{5} \delta\left(\begin{array}{r}
x_{1} \\
-x_{2} \\
x_{1} \\
-x_{2}
\end{array}\right) . \\
&+\lambda_{2}
\end{aligned}
$$

Analisaremos o caso quando $\epsilon_{3}=\epsilon_{4}=\epsilon_{5}=1$. Os dados para a construção dos diagramas de bifurcação são obtidos substituindo-se os dados do problema na tabela 5.2. Seguindo o mesmo esquema anterior apresentamos a tabela com os principais dados sobre as soluções em cada caso analisado, as regiôes do plano $\left(\lambda_{1}, \lambda_{2}\right)$ onde as soluções existem e o diagrama de bifurcação desenhado no espaço $\left(x_{1}, s, x_{2}\right)$ devido a parametrização

$$
\begin{aligned}
& \lambda_{1}=R \text { coss } \\
& \lambda_{2}=R \text { sens }
\end{aligned}
$$

$\operatorname{com} R>0$.

Para esse problema de bifurcação observamos que, para valores isolados de $m$, ocorrem variações do diagrama de bifurcação quando $R$ varia. 


\begin{tabular}{|c|c|c|c|}
\hline $\begin{array}{l}\text { SUBESPAÇOS } \\
\text { FIXADOS }\end{array}$ & $\begin{array}{l}\text { REGIÕES DO } \\
\text { PLANO }\left(\lambda_{1}, \lambda_{2}\right) \\
\end{array}$ & SOLUÇÕES & $\begin{array}{c}\text { SINAIS DOS } \\
\text { AUTO - VALORES }\end{array}$ \\
\hline $\operatorname{Fix}\left(D_{4}\right)$ & $\forall\left(\lambda_{1}, \lambda_{2}\right)$ & $x_{1}=x_{2}=0$ & $\begin{array}{l}++\left\{\begin{array}{l}\lambda_{1}^{2}-\lambda_{2}>0 \\
\lambda_{1}^{2}+\lambda_{2}>0\end{array}\right. \\
+-\left\{\begin{array}{l}\lambda_{1}^{2}-\lambda_{2}<0 \\
\lambda_{1}^{2}+\lambda_{2}>0\end{array}\right.\end{array}$ \\
\hline $\operatorname{Fix}\left(\mathrm{Z}_{2}(\kappa)\right)$ & $\lambda_{1}^{2}+\lambda_{2} \leq 0$ & $\begin{array}{c}x_{2}=0 \\
x_{1}^{2}=\frac{\lambda_{1}^{2}+\lambda_{2}}{1-m-\lambda_{1}}\end{array}$ & ++ \\
\hline $\operatorname{Fix}\left(Z_{2}^{c}(\kappa)\right)$ & $\lambda_{1}^{2}-\lambda_{2} \leq 0$ & $\begin{array}{c}x_{1}=0 \\
x_{2}^{2}=\frac{\lambda_{1}^{2}-\lambda_{2}}{1-m-\lambda_{1}}\end{array}$ & ++ \\
\hline $\begin{array}{l}\operatorname{Fix}\left(\mathrm{Z}_{2}(\xi \kappa)\right) \\
\operatorname{Fix}\left(\mathrm{Z}_{2}^{\mathrm{c}}(\xi \kappa)\right)\end{array}$ & & $\emptyset$ & \\
\hline $\operatorname{Fix}(\mathbf{1})$ & & 0 & \\
\hline
\end{tabular}

TABELA 5.10: $m>1$ 


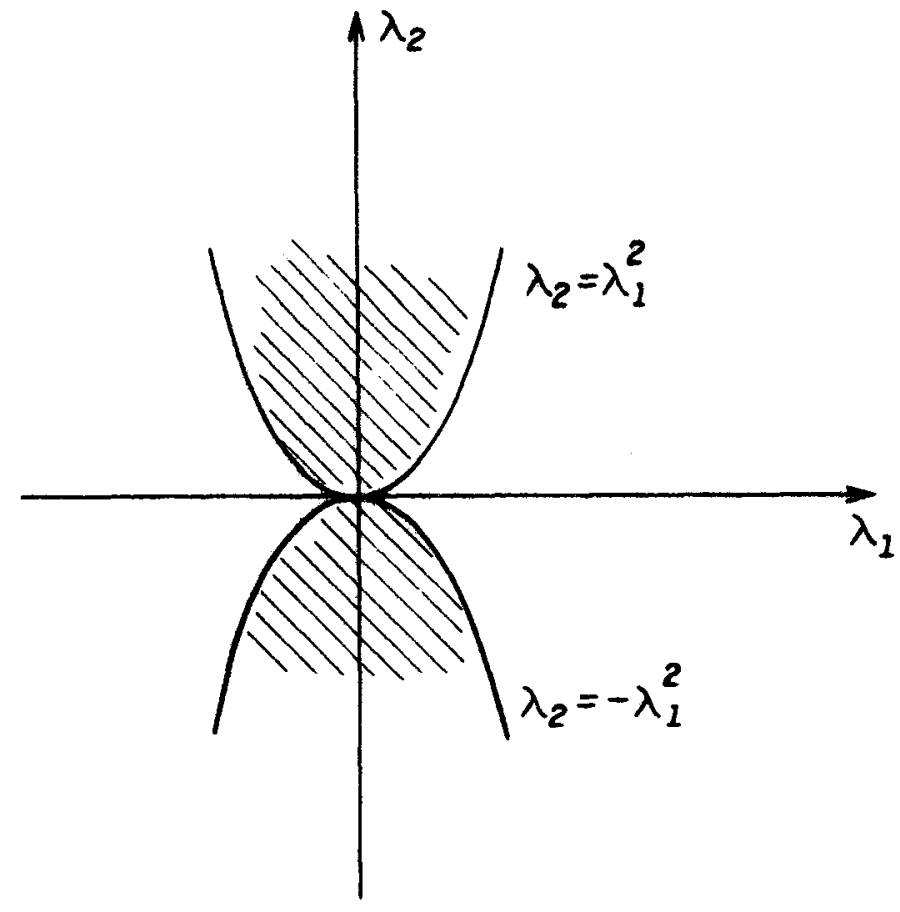

FIG.5.21: $m>1$

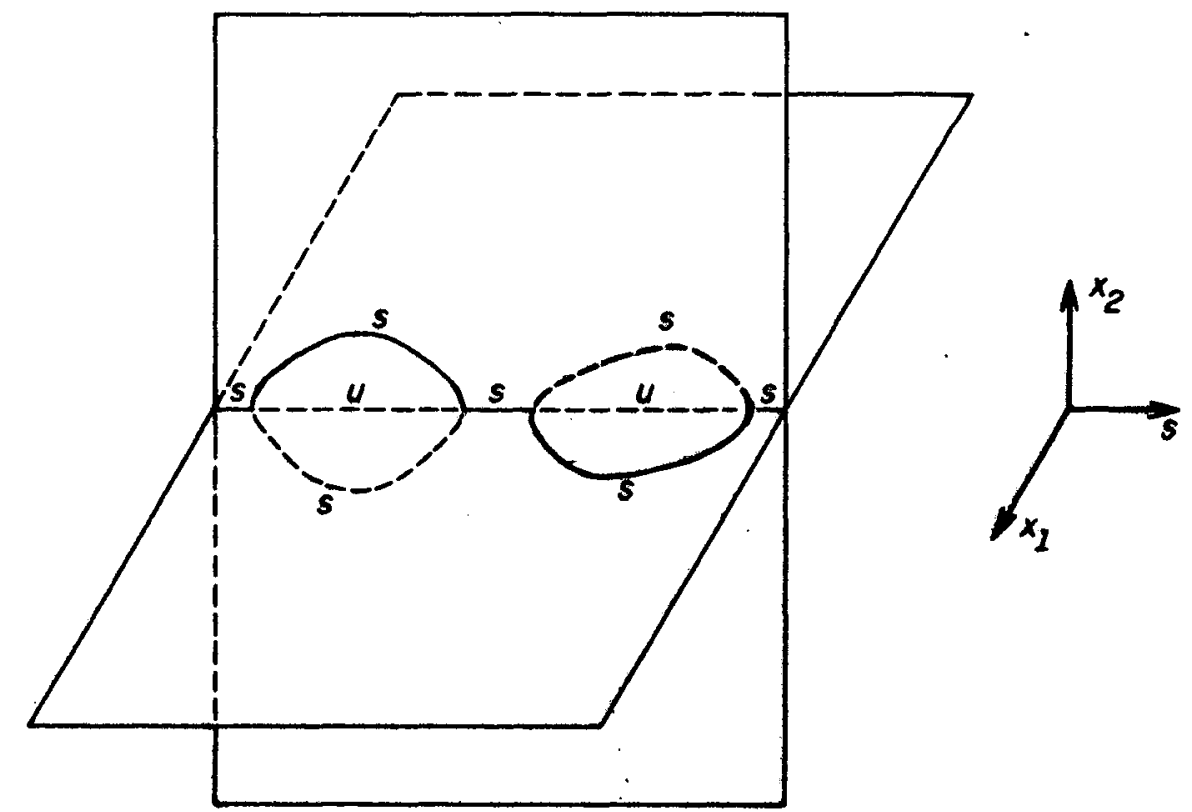

FIG.5.22 - DIAGRAMA DE BIFURCACÃO: $m>1$ 


\begin{tabular}{|c|c|c|c|}
\hline $\begin{array}{c}\text { SUBESPAÇOS } \\
\text { FIXADOS } \\
\end{array}$ & $\begin{array}{c}\text { REGIÕES DO } \\
\operatorname{PLANO}\left(\lambda_{1}, \lambda_{2}\right) \\
\end{array}$ & SOLUÇÕES & $\begin{array}{c}\text { SINAIS DOS } \\
\text { AUTO - VALORES } \\
\end{array}$ \\
\hline $\operatorname{Fix}\left(D_{4}\right)$ & $\forall\left(\lambda_{1}, \lambda_{2}\right)$ & $x_{1}=x_{2}=0$ & $\begin{array}{c}+t\left\{\begin{array}{c}\lambda_{1}^{2}+\lambda_{2}+\alpha>0 \\
\lambda_{1}^{2}-\lambda_{2}+\alpha>0\end{array}\right. \\
+-\left\{\begin{array}{l}\lambda_{1}^{2}+\lambda_{2}+\alpha>0(<0) \\
\lambda_{1}^{2}-\lambda_{2}+\alpha<0(>0)\end{array}\right.\end{array}$ \\
\hline $\operatorname{Fix}\left(\mathrm{Z}_{2}(\kappa)\right)$ & $\lambda_{1}^{2}+\lambda_{2}+\alpha \leq 0$ & $\begin{array}{c}x_{2}=0 \\
x_{1}^{2}=\frac{\lambda_{1}^{2}+\lambda_{2}+\alpha}{1-m-\lambda_{1}}\end{array}$ & ++ \\
\hline $\operatorname{Fix}\left(Z_{2}^{c}(\kappa)\right)$ & $\lambda_{1}^{2}-\lambda_{2}+\alpha \leq 0$ & $\begin{array}{c}x_{1}=0 \\
x_{2}^{2}=\frac{\lambda_{1}^{2}-\lambda_{2}+\alpha}{1-m-\lambda_{1}}\end{array}$ & ++ \\
\hline $\begin{array}{l}\operatorname{Fix}\left(Z_{2}(\xi \kappa)\right) \\
\operatorname{Fix}\left(Z_{2}^{c}(\xi \kappa)\right)\end{array}$ & & $\emptyset$ & \\
\hline $\operatorname{Fix}(\mathbf{1})$ & & $\theta$ & \\
\hline
\end{tabular}

TABELA 5.11: $m>1, \alpha>0$ 


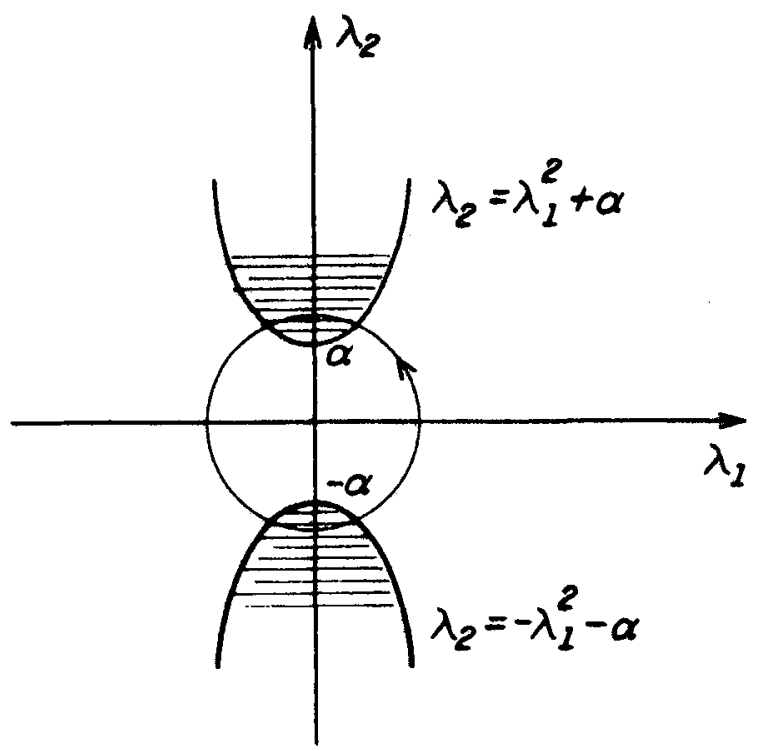

FIG. $5.23: m>1, \alpha>0$

DIAGRAMAS DE BIFURCACĀO: $m>1, \alpha>0$

1. $\lambda_{1}=R_{0} \cos s \quad R_{0}=\alpha$

$\lambda_{2}=R_{0} \operatorname{sen} s$

2. $\lambda_{1}=R_{1} \cos s \quad R_{1}>\alpha$ $\lambda_{2}=R_{1} \operatorname{sen} s$

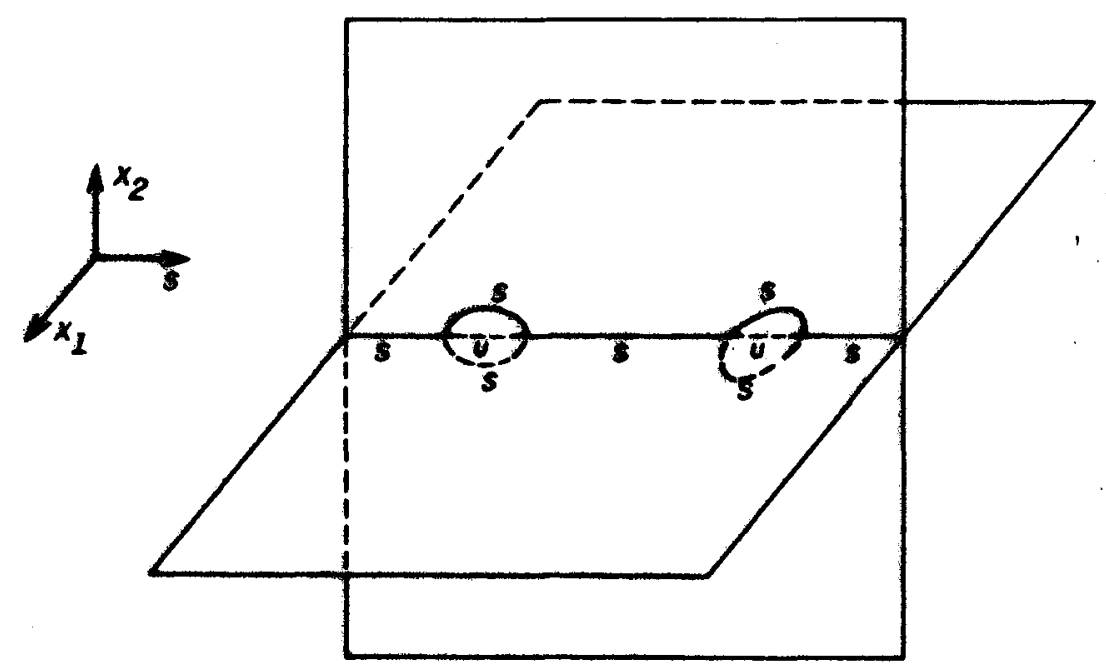

F/G. 5.24 


\begin{tabular}{|c|c|c|c|}
\hline $\begin{array}{c}\text { SUBESPAÇOS } \\
\text { FIXADOS } \\
\end{array}$ & $\begin{array}{c}\text { REGIÕES DO } \\
\text { PLANO }\left(\lambda_{1}, \lambda_{2}\right) \\
\end{array}$ & SOLUÇÕES & $\begin{array}{c}\text { SINAIS DOS } \\
\text { AUTO - VALORES } \\
\end{array}$ \\
\hline $\operatorname{Fix}\left(D_{4}\right)$ & $\forall\left(\lambda_{1}, \lambda_{2}\right)$ & $x_{1}=x_{2}=0$ & $\begin{array}{c}++\left\{\begin{array}{l}\lambda_{1}^{2}+\lambda_{2}+\alpha>0 \\
\lambda_{1}^{2}-\lambda_{2}+\alpha>0\end{array}\right. \\
+-\left\{\begin{array}{l}\lambda_{1}^{2}+\lambda_{2}+\alpha>0(<0) \\
\lambda_{1}^{2}-\lambda_{2}+\alpha<0(>0)\end{array}\right. \\
--\left\{\begin{array}{l}\lambda_{1}^{2}+\lambda_{2}+\alpha<0 \\
\lambda_{1}^{2}-\lambda_{2}+\alpha<0\end{array}\right.\end{array}$ \\
\hline $\operatorname{Fix}\left(\mathrm{Z}_{2}(\kappa)\right)$ & $\lambda_{1}^{2}+\lambda_{2}+\alpha \leq 0$ & $\begin{array}{c}x_{2}=0 \\
x_{1}^{2}=\frac{\lambda_{1}^{2}+\lambda_{2}+\alpha}{1-m-\lambda_{1}}\end{array}$ & $\begin{array}{l}++\left\{\begin{array}{l}\lambda_{2} \leq 0 \text { ou } \lambda_{2}>0 \mathrm{e} \\
\lambda_{1}^{2}+m \lambda_{2}+\lambda_{1} \lambda_{2}+\alpha<0\end{array}\right. \\
+-\left\{\begin{array}{l}\lambda_{2}>0 \mathrm{e} \\
\lambda_{1}^{2}+m \lambda_{2}+\lambda_{1} \lambda_{2}+\alpha>0\end{array}\right.\end{array}$ \\
\hline $\operatorname{Fix}\left(\mathrm{Z}_{2}^{c}(\kappa)\right)$ & $\lambda_{1}^{2}-\lambda_{2}+\alpha \leq 0$ & $\begin{array}{c}x_{1}=0 \\
x_{2}^{2}=\frac{\lambda_{1}^{2}-\lambda_{2}+\alpha}{1-m-\lambda_{1}}\end{array}$ & $\begin{array}{l}++\left\{\begin{array}{l}\lambda_{2} \geq 0 \text { ou } \lambda_{2}<0 \mathrm{e} \\
\lambda_{1}^{2}-m \lambda_{2}-\lambda_{1} \lambda_{2}+\alpha<0\end{array}\right. \\
+-\left\{\begin{array}{l}\lambda_{2}<0 \mathrm{e} \\
\lambda_{1}^{2}-m \lambda_{2}-\lambda_{1} \lambda_{2}+\alpha>0\end{array}\right.\end{array}$ \\
\hline
\end{tabular}

TABELA 5.12: $m>1, \alpha<0$ 


\begin{tabular}{|c|c|c|c|}
\hline $\begin{array}{c}\text { SUBESPACOS } \\
\text { FIXADOS } \\
\end{array}$ & $\begin{array}{c}\text { REGIÕES DO } \\
\text { PLANO }\left(\lambda_{1}, \lambda_{2}\right)\end{array}$ & SOLUÇÕES & $\begin{array}{c}\text { SINAIS DOS } \\
\text { AUTO - VALORES }\end{array}$ \\
\hline $\begin{array}{l}\operatorname{Fix}\left(\mathrm{Z}_{2}(\xi \kappa)\right) \\
\operatorname{Fix}\left(\mathrm{Z}_{2}^{c}(\xi \kappa)\right)\end{array}$ & $\begin{array}{c}\lambda_{2}=0 \\
\lambda_{1}^{2}+\alpha \leq 0\end{array}$ & $\begin{array}{c}x_{1}= \pm x_{2} \\
x_{1}^{2}=-\frac{\lambda_{1}^{2}+\alpha}{2\left(m+\lambda_{1}\right)}\end{array}$ & +- \\
\hline $\operatorname{Fix}(1)$ & $\begin{array}{c}\lambda_{2} \geq 0 \\
\lambda_{1}^{2}+m \lambda_{2} \\
+\lambda_{1} \lambda_{2}+\alpha \leq 0 \\
\\
\lambda_{2}<0 \\
\lambda_{1}^{2}-m \lambda_{2} \\
-\lambda_{1} \lambda_{2}+\alpha \leq 0\end{array}$ & $\begin{array}{c}x_{1}^{2}=x_{2}^{2}+\lambda_{2} \\
x_{2}^{2}=-\frac{\left(\lambda_{1}^{2}+m \lambda_{2}+\lambda_{1} \lambda_{2}+\alpha\right)}{2\left(m+\lambda_{1}\right)} \\
x_{2}^{2}=-\lambda_{2}+x_{1}^{2} \\
x_{1}^{2}=-\frac{\left(\lambda_{1}^{2}-m \lambda_{2}-\lambda_{1} \lambda_{2}+\alpha\right)}{2\left(m+\lambda_{1}\right)}\end{array}$ & +- \\
\hline
\end{tabular}

Continuação da TABELA 5.12: $m>1, \alpha<0$ 
Observamos que quando $\lambda_{2}=0$, as soluçôes correspondentes a $\operatorname{Fix}(1), \operatorname{Fix}\left(Z_{2}(\xi \kappa)\right)$ e $\operatorname{Fix}\left(Z_{2}^{c}(\xi \kappa)\right)$ coincidem. A coincidência entre as soluções correspondentes a Fix $(1)$ com $\operatorname{Fix}\left(\mathrm{Z}_{2}(\kappa)\right.$ e Fix $(\mathbf{1}) \operatorname{com} \mathrm{Fix}\left(\mathrm{Z}_{2}^{c}(\kappa)\right)$ ocorrem, respectivamente, quando $\lambda_{1}^{2}+m \lambda_{2}+\lambda_{1} \lambda_{2}+$ $\alpha=0$ e $\lambda_{2}>0$ ou $\lambda_{1}^{2}-m \lambda_{2}-\lambda_{1} \lambda_{2}+\alpha=0$ e $\lambda_{2}<0$. Esse comentário se estende a todos os demais casos a seguir, onde ocorrem as intersecções acima mencionadas.

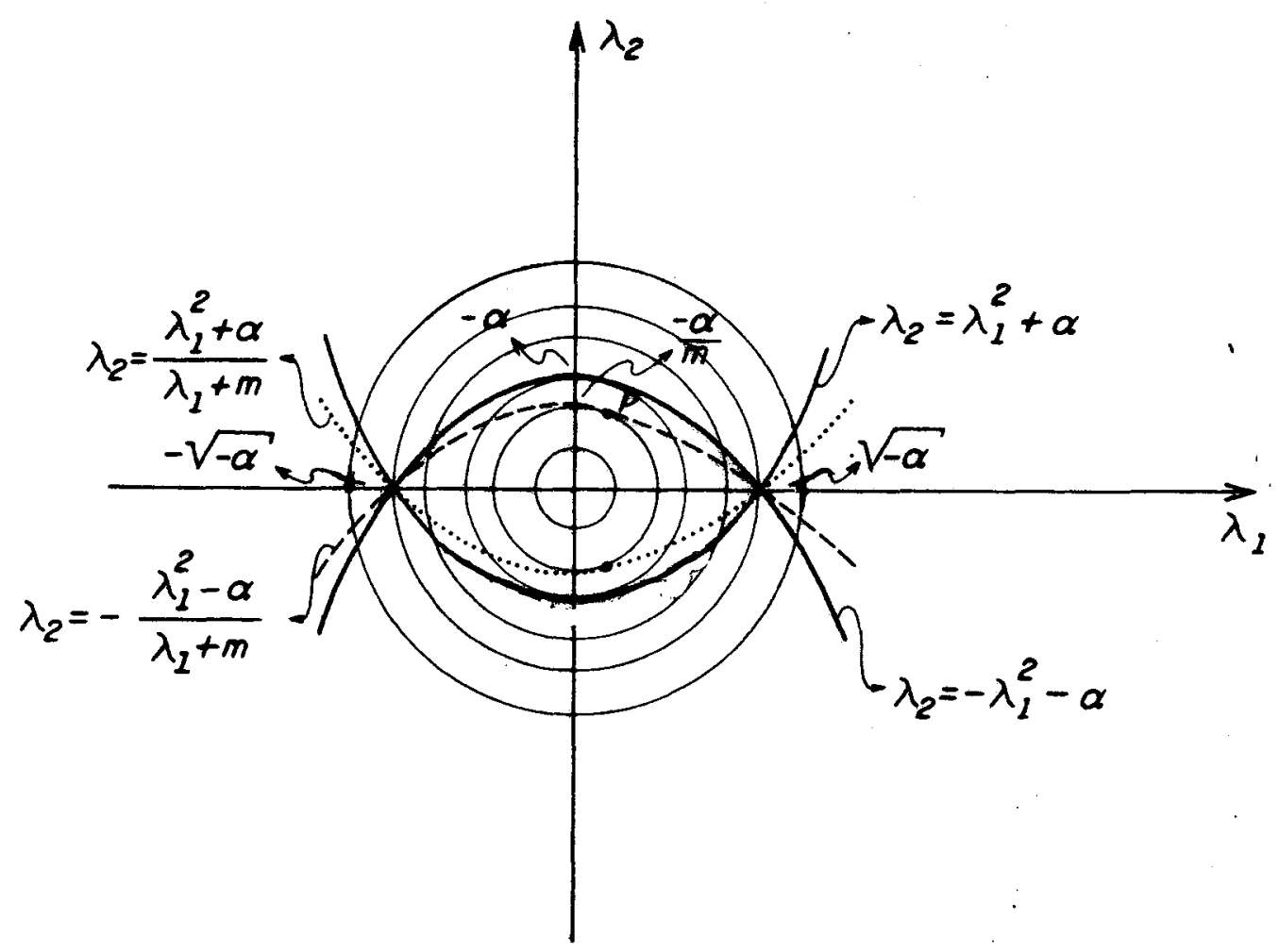

FIG. $5.25: m>1, \alpha<0$ 
DIAGRAMAS DE BIFURCACĀO: $m>1, \alpha<0$

\section{1. $R<|\overrightarrow{O P}|$}
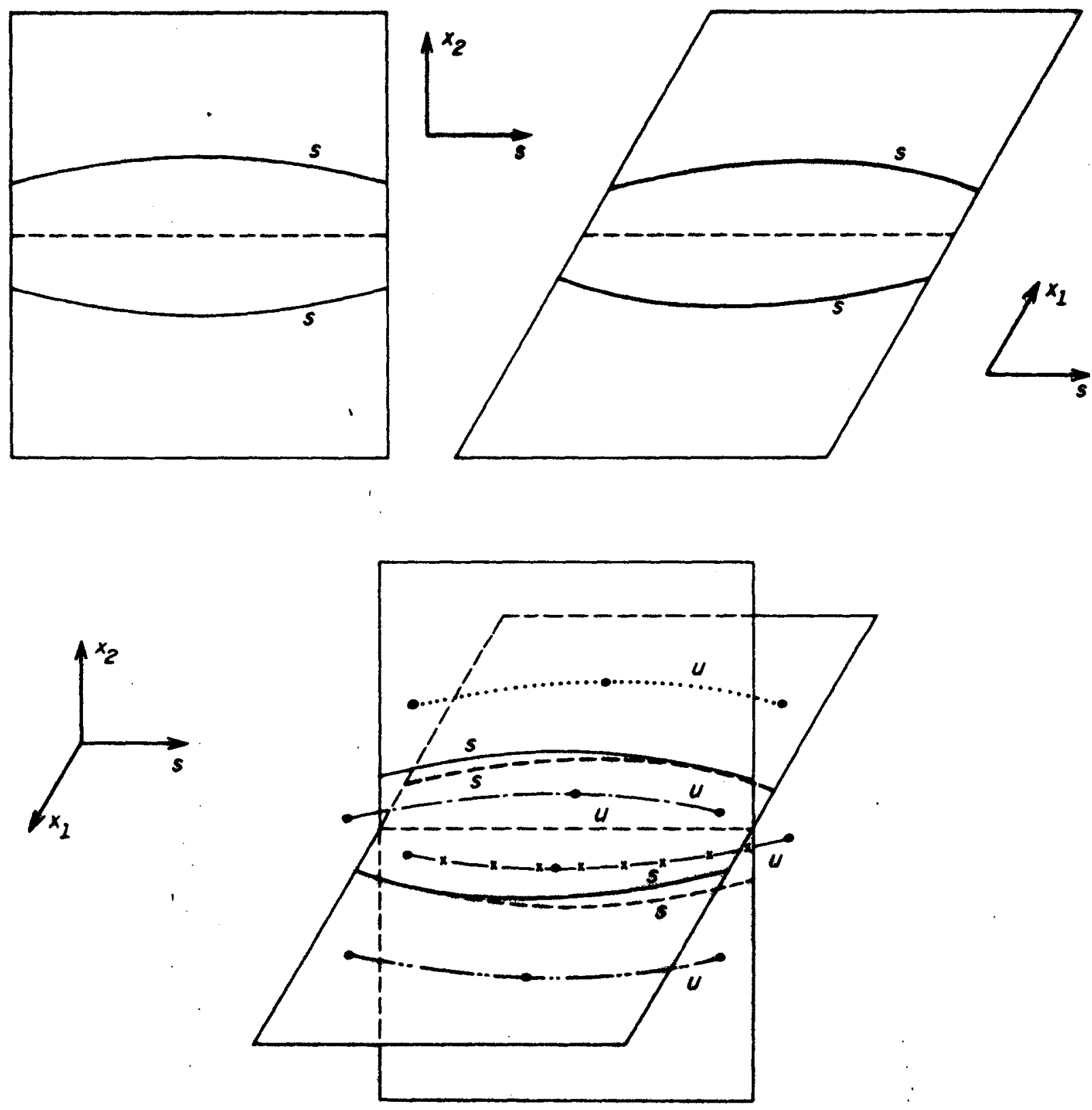

F/G. 5.26 
2. $R=|\overrightarrow{O P}|$
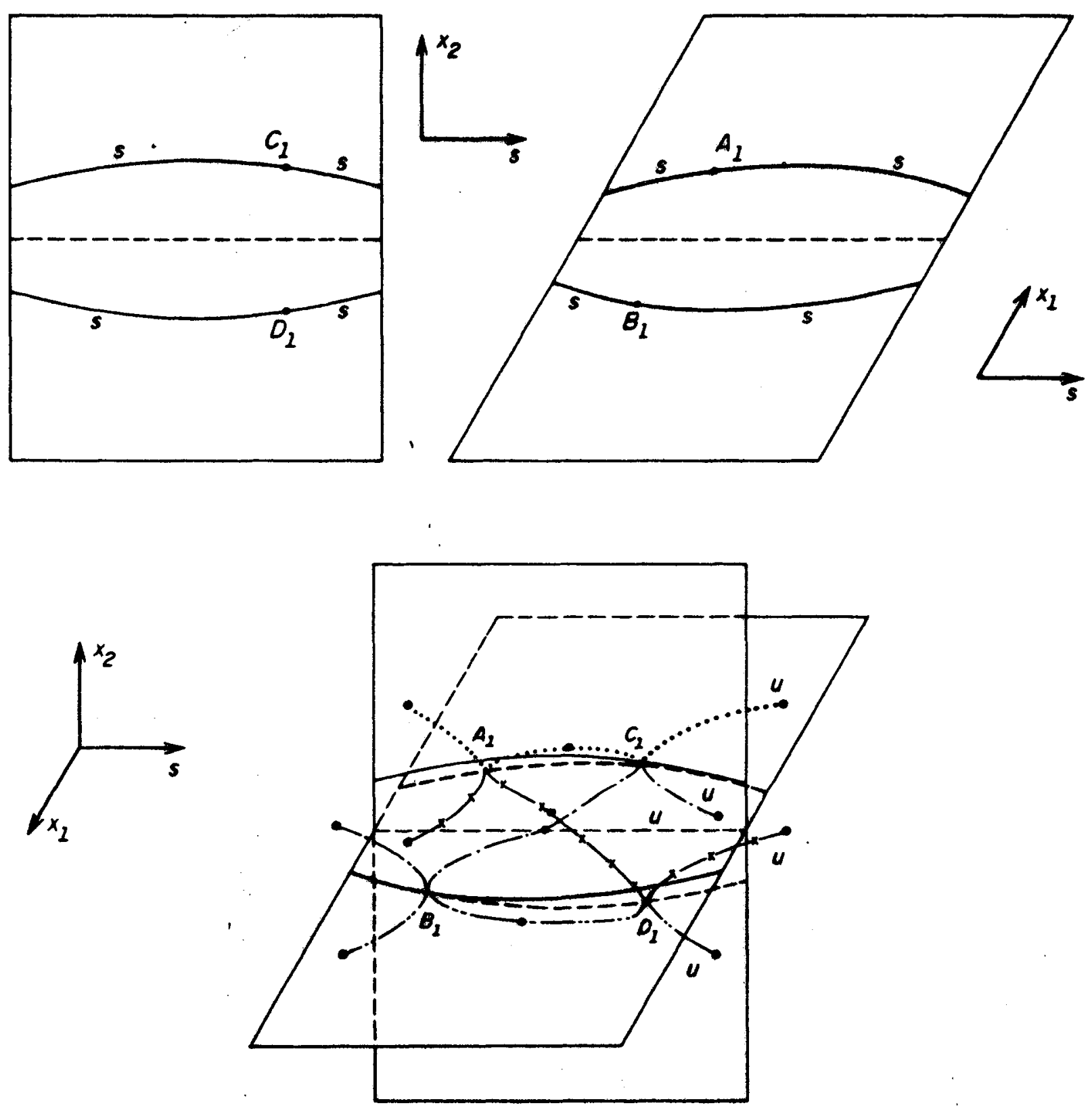

F/G. 5.26 
3. $|\overrightarrow{O P}|<R<-\alpha$
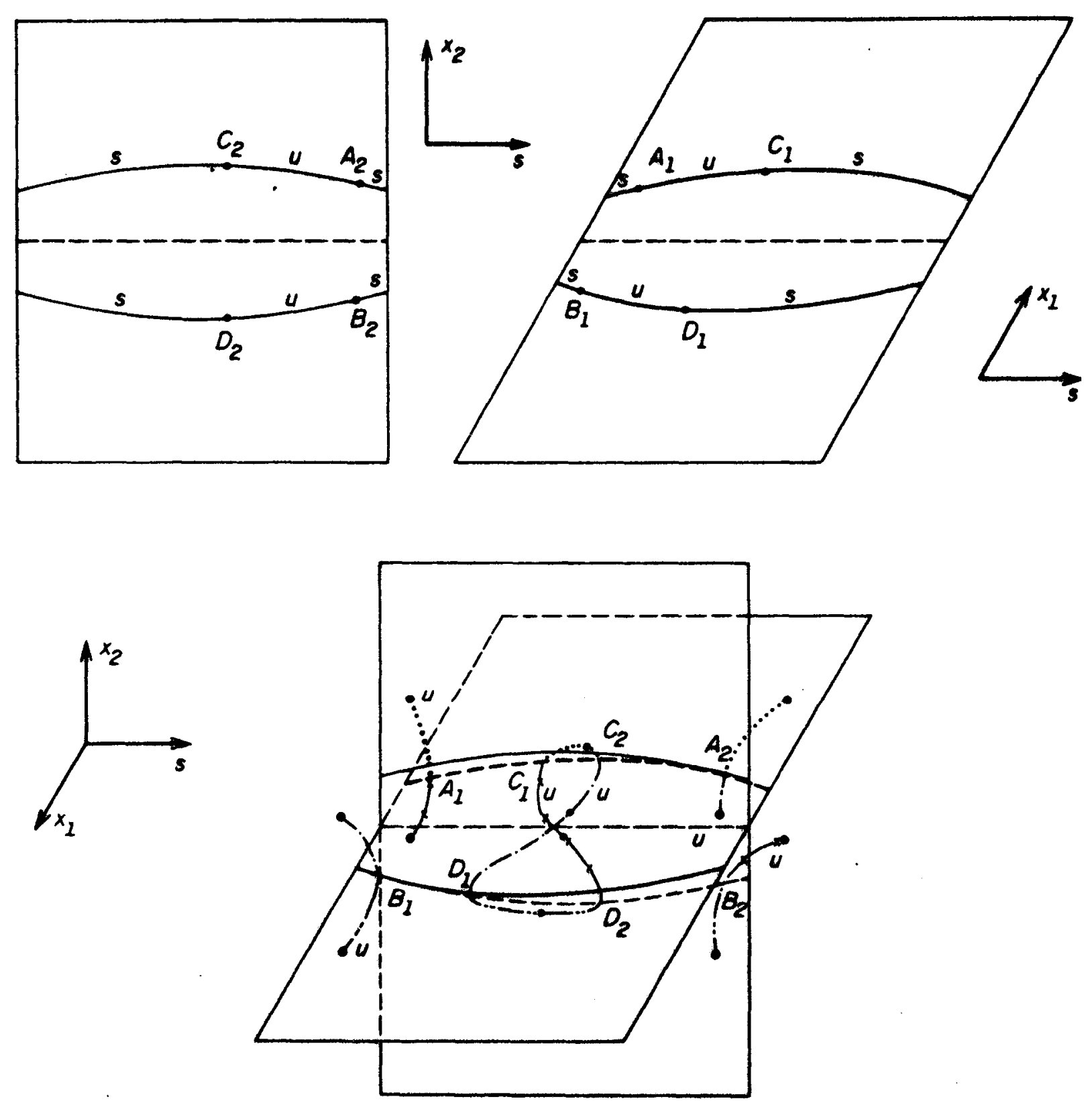

F/G. 5.26 
4. $R=-\alpha$
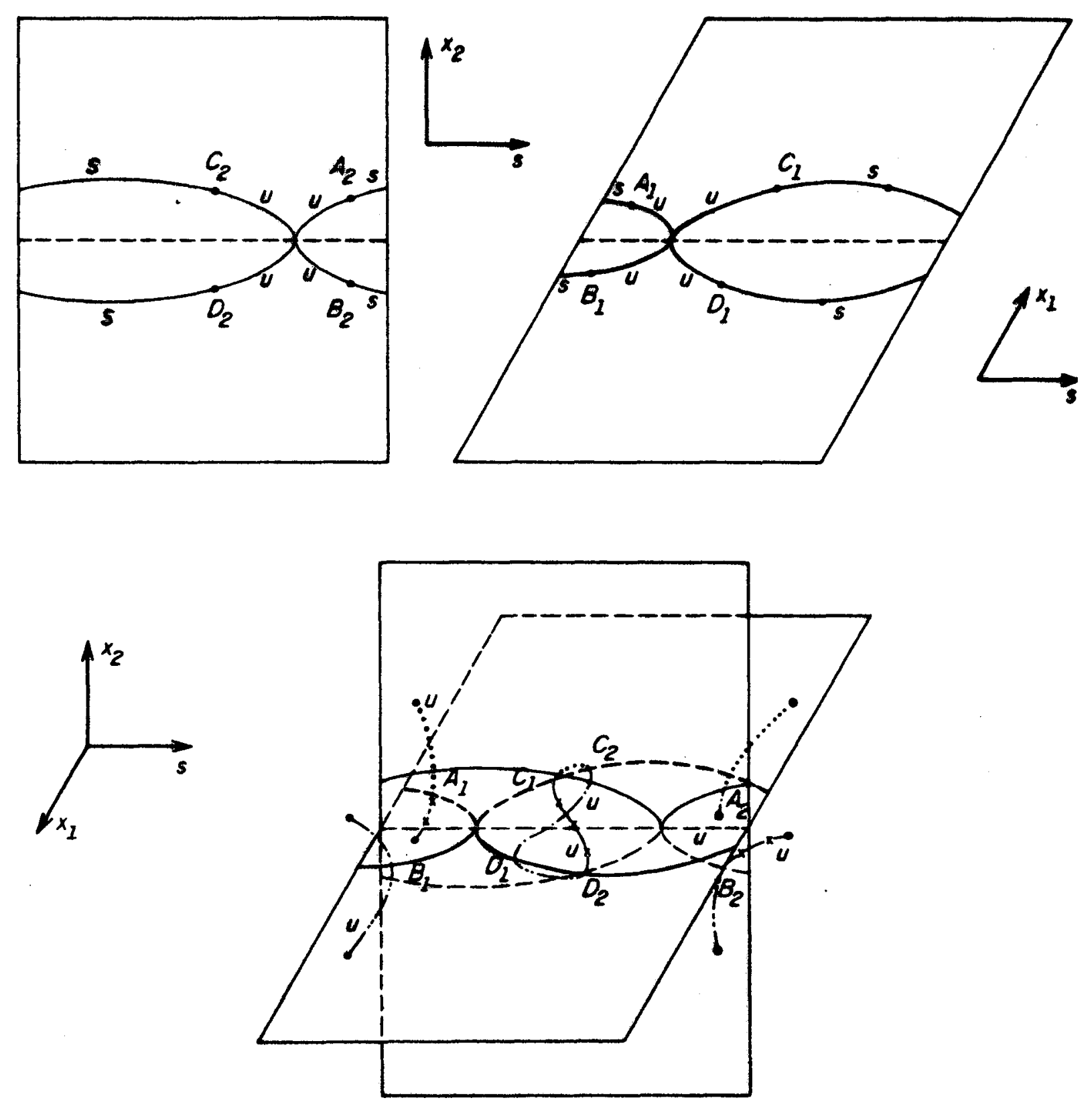

FIG. 5.26 
5. $-\alpha<R<\sqrt{-\alpha}$
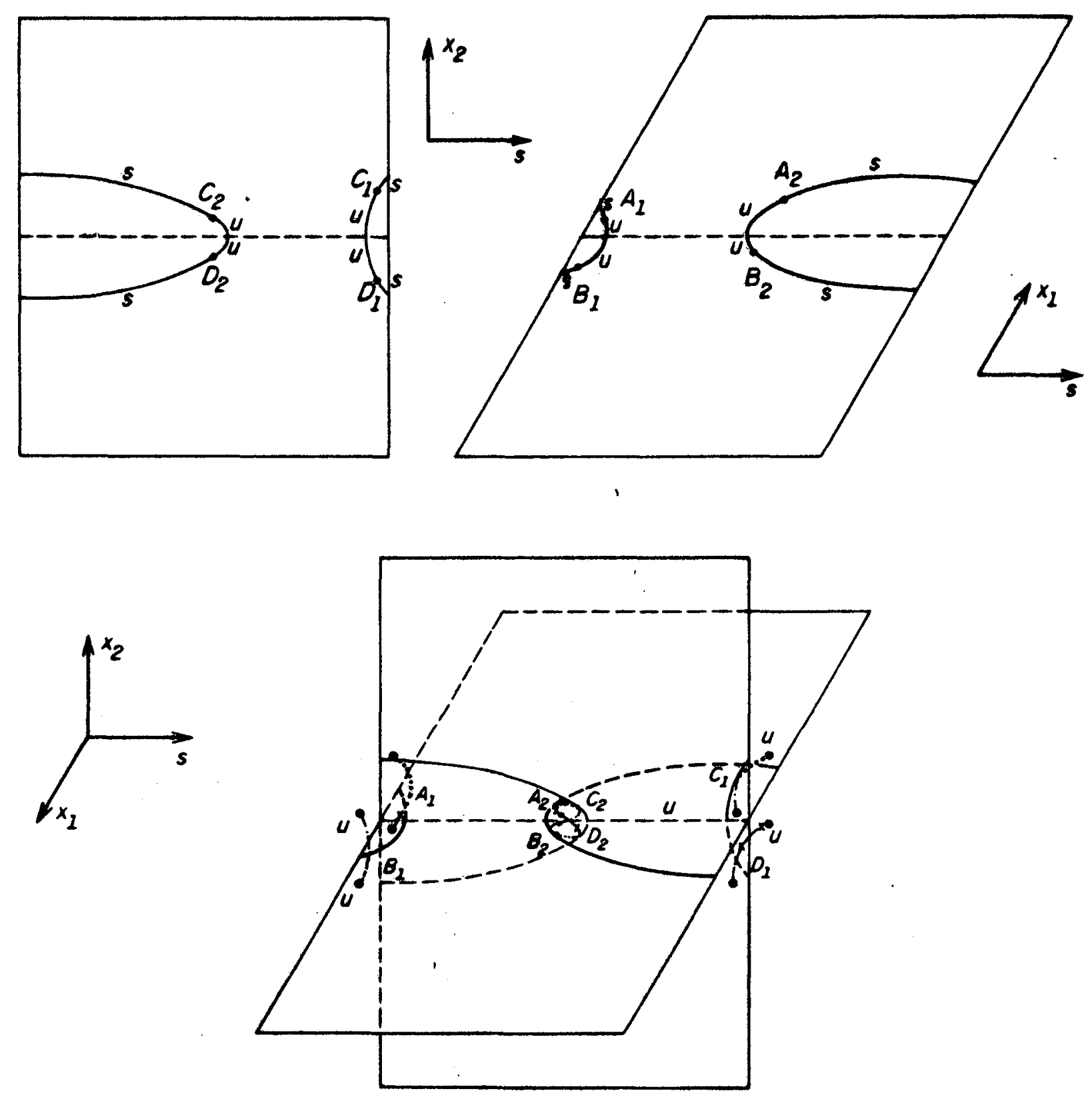

F/G. 5.26 

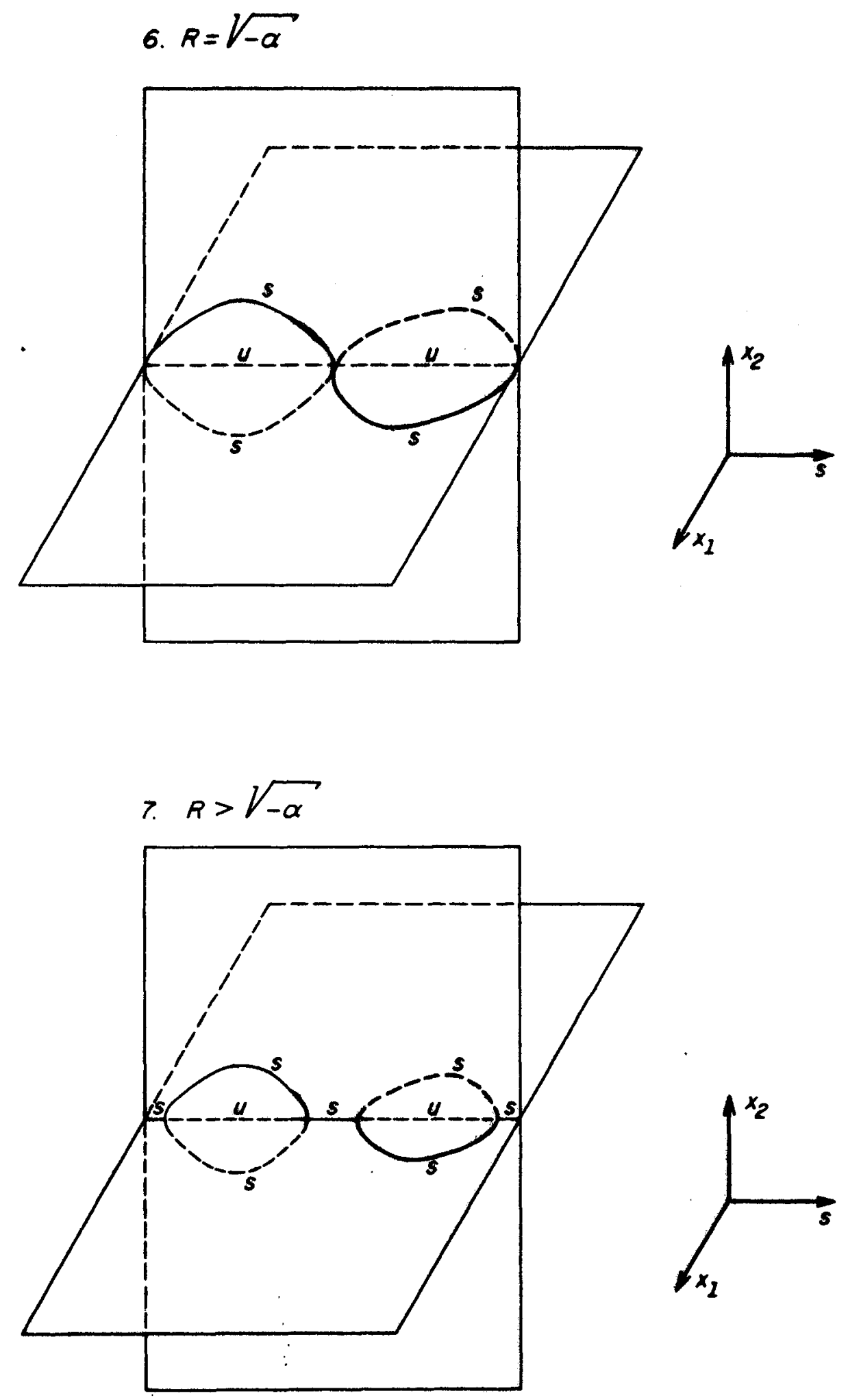

F/G. 5.26 


\begin{tabular}{|c|c|c|c|}
\hline $\begin{array}{l}\text { SUBESPAÇOS } \\
\text { FIXADOS }\end{array}$ & $\begin{array}{l}\text { REGIÕES DO } \\
\text { PLANO }\left(\lambda_{1}, \lambda_{2}\right)\end{array}$ & SOLUÇÕES & $\begin{array}{c}\text { SINAIS DOS } \\
\text { AUTO - VALORES } \\
\end{array}$ \\
\hline $\operatorname{Fix}\left(D_{4}\right)$ & $\forall\left(\lambda_{1}, \lambda_{2}\right)$ & $x_{1}=x_{2}=0$ & $\begin{array}{l}++\left\{\begin{array}{l}\lambda_{1}^{2}-\lambda_{2}>0 \\
\lambda_{1}^{2}+\lambda_{2}>0\end{array}\right. \\
+-\left\{\begin{array}{l}\lambda_{1}^{2}-\lambda_{2}<0 \\
\lambda_{1}^{2}+\lambda_{2}>0\end{array}\right.\end{array}$ \\
\hline $\operatorname{Fix}\left(\mathrm{Z}_{2}(\kappa)\right)$ & $\lambda_{1}^{2}+\lambda_{2} \geq 0$ & $\begin{array}{c}x_{2}=0 \\
x_{1}^{2}=\frac{\lambda_{1}^{2}+\lambda_{2}}{1-m-\lambda_{1}}\end{array}$ & +- \\
\hline $\operatorname{Fix}\left(\mathrm{Z}_{2}^{c}(\kappa)\right)$ & $\lambda_{1}^{2}-\lambda_{2} \geq 0$ & $\begin{array}{c}x_{1}=0 \\
x_{2}^{2}=\frac{\lambda_{1}^{2}-\lambda_{2}}{1-m-\lambda_{1}}\end{array}$ & +- \\
\hline $\begin{array}{l}\operatorname{Fix}\left(\mathrm{Z}_{2}(\xi \kappa)\right) \\
\operatorname{Fix}\left(\mathrm{Z}_{2}^{c}(\xi \kappa)\right)\end{array}$ & & 0 & \\
\hline $\operatorname{Fix}(1)$ & & 0 & \\
\hline
\end{tabular}

TABELA 5.13: $0<m<1$ 


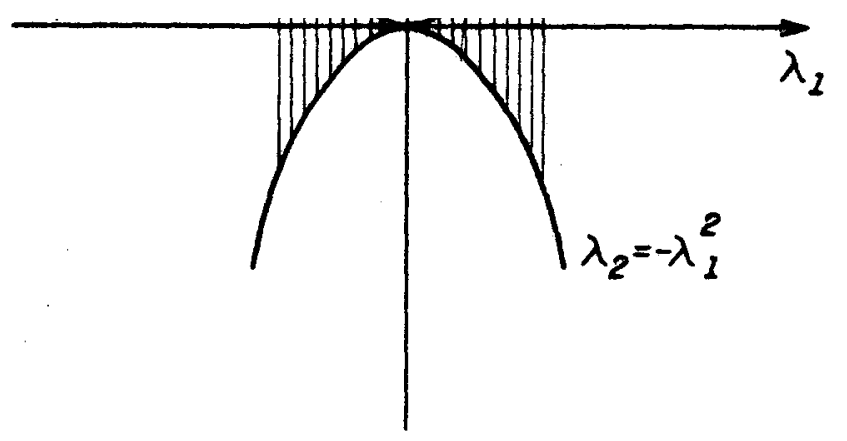

FIG. 5.27: $0<m<1$

DIAGRAMA DE BIFURCACĀO : $0<m<1$

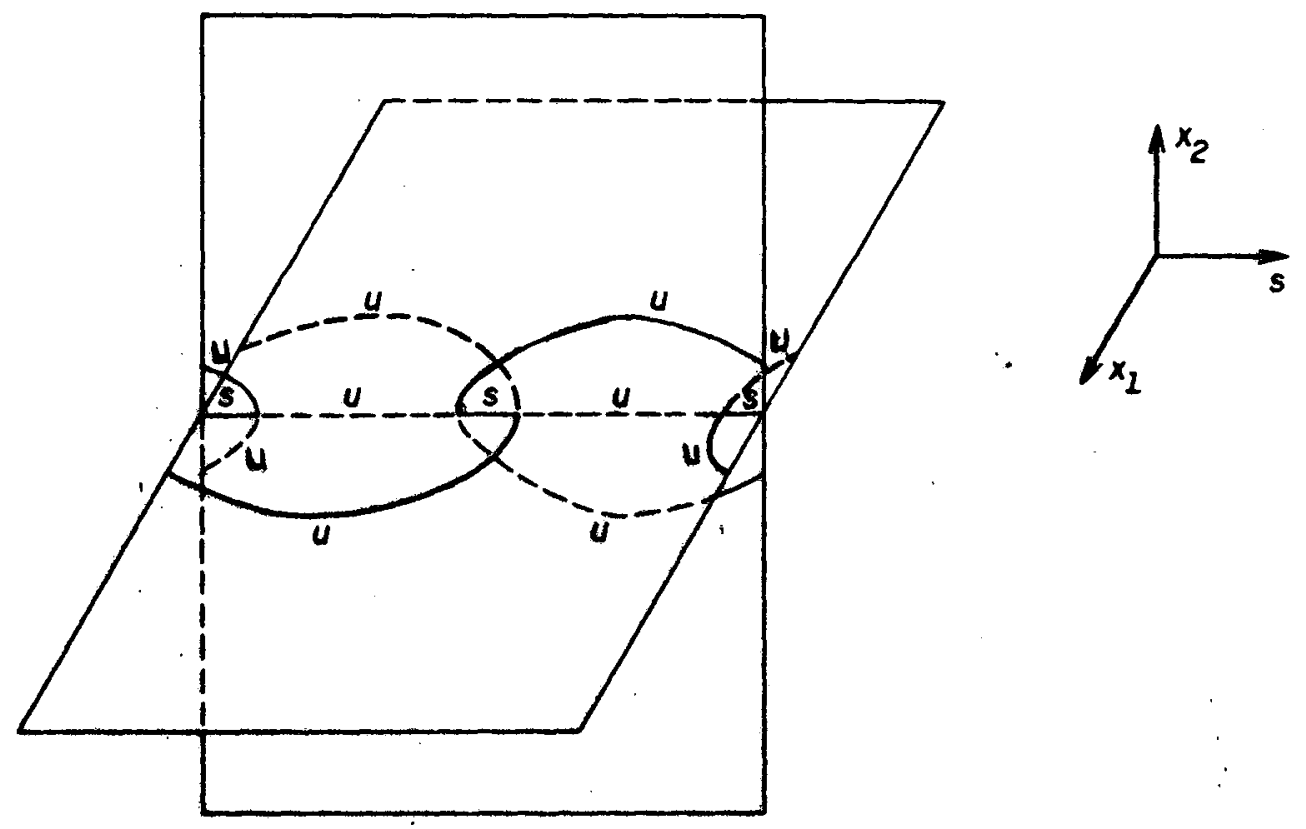

FIG. 5.28 


\begin{tabular}{|c|c|c|c|}
\hline $\begin{array}{c}\text { SUBESPAÇOS } \\
\text { FIXADOS } \\
\end{array}$ & $\begin{array}{l}\text { REGIÕES DO } \\
\text { PLANO }\left(\lambda_{1}, \lambda_{2}\right) \\
\end{array}$ & SOLUÇÕES & $\begin{array}{c}\text { SINAIS DOS } \\
\text { AUTO - VALORES } \\
\end{array}$ \\
\hline $\operatorname{Fix}\left(D_{4}\right)$ & $\forall\left(\lambda_{1}, \lambda_{2}\right)$ & $x_{1}=x_{2}=0$ & $\begin{array}{l}++\left\{\begin{array}{l}\lambda_{1}^{2}+\lambda_{2}+\alpha>0 \\
\lambda_{1}^{2}-\lambda_{2}+\alpha>0\end{array}\right. \\
--\left\{\begin{array}{l}\lambda_{1}^{2}+\lambda_{2}+\alpha<0 \\
\lambda_{1}^{2}-\lambda_{2}+\alpha<0\end{array}\right.\end{array}$ \\
\hline $\operatorname{Fix}\left(\mathrm{Z}_{2}(\kappa)\right)$ & $\lambda_{1}^{2}+\lambda_{2}+\alpha \geq 0$ & $\begin{array}{c}x_{2}=0 \\
x_{1}^{2}=\frac{\lambda_{1}^{2}+\lambda_{2}+\alpha}{1-m-\lambda_{1}}\end{array}$ & +- \\
\hline $\operatorname{Fix}\left(Z_{2}^{c}(\kappa)\right)$ & $\lambda_{1}^{2}-\lambda_{2}+\alpha \geq 0$ & $\begin{array}{c}x_{1}=0 \\
x_{2}^{2}=\frac{\lambda_{1}^{2}-\lambda_{2}+\alpha}{1-m-\lambda_{1}}\end{array}$ & +- \\
\hline $\begin{array}{l}\operatorname{Fix}\left(Z_{2}(\xi \kappa)\right) \\
\operatorname{Fix}\left(Z_{2}^{c}(\xi \kappa)\right)\end{array}$ & & $\emptyset$ & \\
\hline $\operatorname{Fix}(1)$ & & $\emptyset$ & \\
\hline
\end{tabular}

TABELA 5.14: $0<m<1, \alpha>0$ 


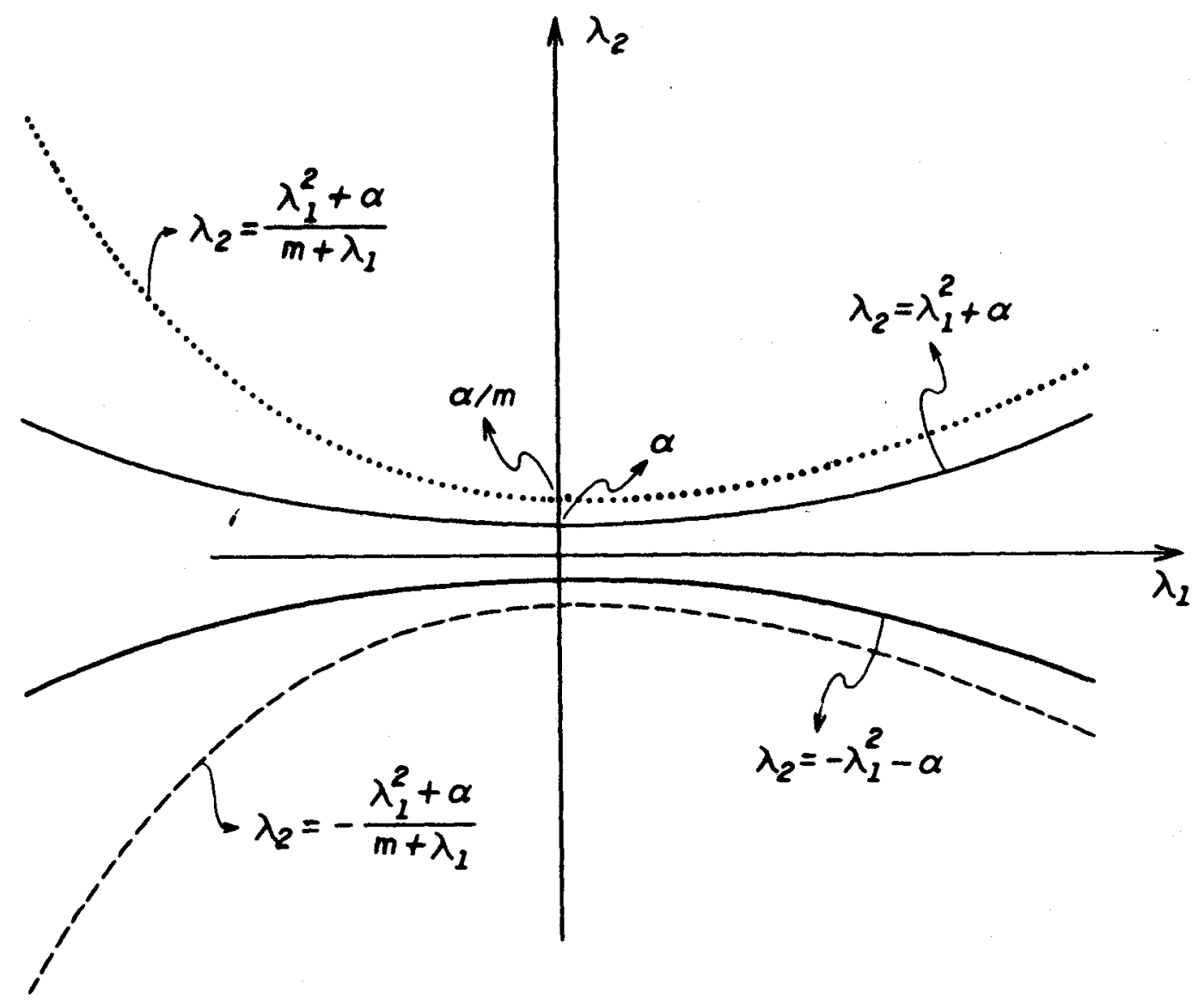

FIG. $5.29: 0<m<1, \alpha>0$ 
DIAGRAMAS DE BIFURCACATO: $0<m<1, \alpha>0$

1. $R<\alpha$

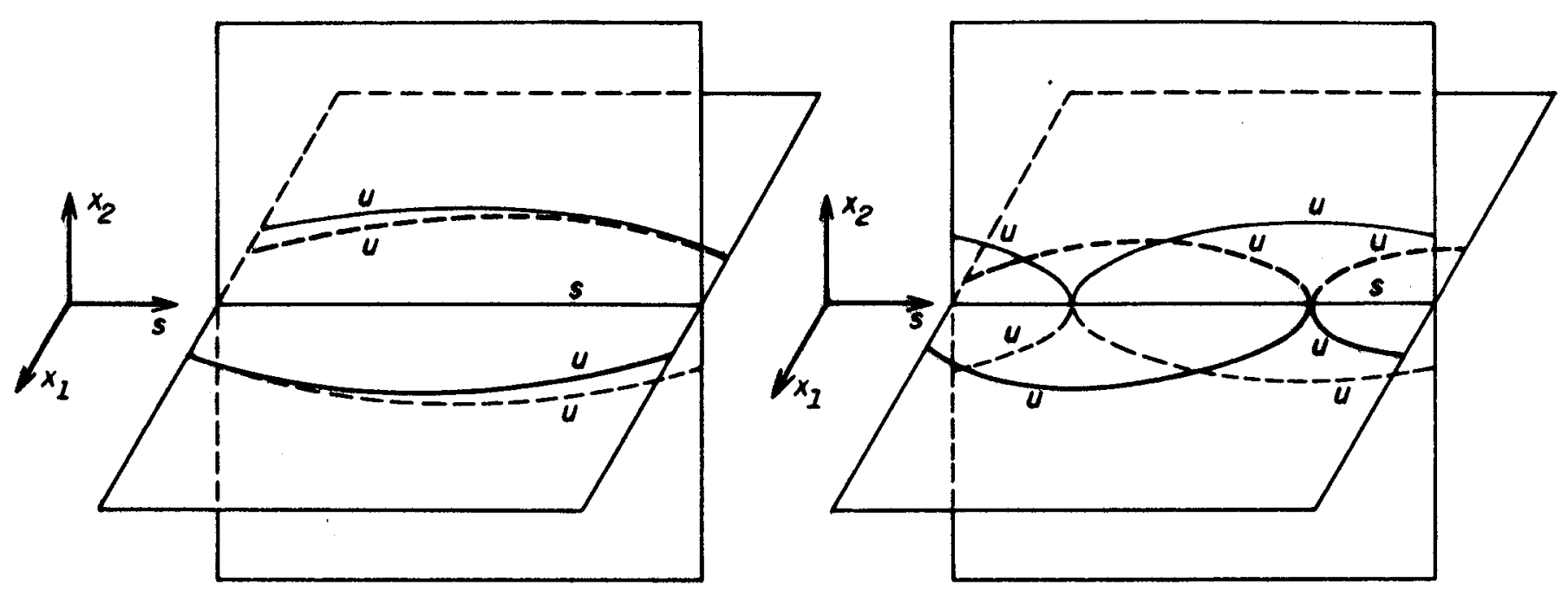

3. $R>\alpha$

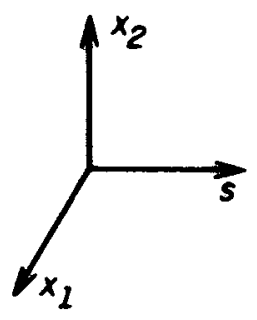

2. $R=\alpha$

1

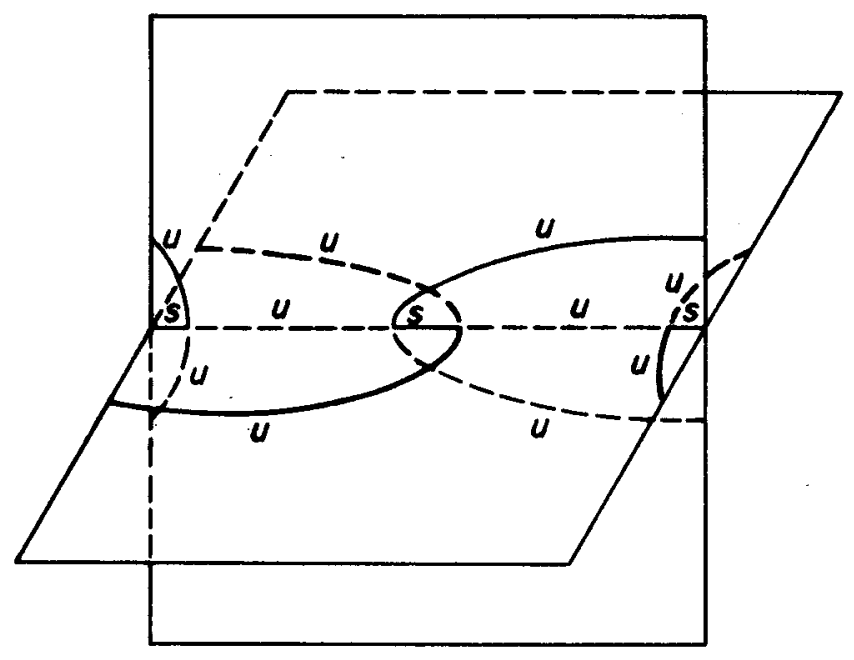

F/G. 5.30 


\begin{tabular}{|c|c|c|c|}
\hline $\begin{array}{c}\text { SUBESPAÇOS } \\
\text { FIXADOS } \\
\end{array}$ & $\begin{array}{c}\text { REGIÕES DO } \\
\operatorname{PLANO}\left(\lambda_{1}, \lambda_{2}\right) \\
\end{array}$ & SOLUÇÕES & $\begin{array}{c}\text { SINAIS DOS } \\
\text { AUTO - VALORES } \\
\end{array}$ \\
\hline $\operatorname{Fix}\left(D_{4}\right)$ & $\forall\left(\lambda_{1}, \lambda_{2}\right)$ & $x_{1}=x_{2}=0$ & $\begin{array}{c}+\left\{\begin{array}{l}\lambda_{1}^{2}-\lambda_{2}+\alpha>0 \\
\lambda_{1}^{2}+\lambda_{2}+\alpha>0\end{array}\right. \\
+-\left\{\begin{array}{l}\lambda_{1}^{2}-\lambda_{2}+\alpha>0(<0) \\
\lambda_{1}^{2}+\lambda_{2}+\alpha<0(>0)\end{array}\right. \\
--\left\{\begin{array}{l}\lambda_{1}^{2}-\lambda_{2}+\alpha<0 \\
\lambda_{1}^{2}+\lambda_{2}+\alpha<0\end{array}\right.\end{array}$ \\
\hline $\operatorname{Fix}\left(\mathrm{Z}_{2}(\kappa)\right)$ & $\lambda_{1}^{2}+\lambda_{2}+\alpha \geq 0$ & $\begin{array}{c}x_{2}=0 \\
x_{1}^{2}=\frac{\lambda_{1}^{2}+\lambda_{2}+\alpha}{1-m-\lambda_{1}}\end{array}$ & $\begin{array}{l}+-\left\{\lambda_{1}^{2}+m \lambda_{2}+\lambda_{1} \lambda_{2}+\alpha>0\right. \\
--\left\{\lambda_{1}^{2}+m \lambda_{2}+\lambda_{1} \lambda_{2}+\alpha<0\right.\end{array}$ \\
\hline $\operatorname{Fix}\left(Z_{2}^{c}(\kappa)\right)$ & $\lambda_{1}^{2}-\lambda_{2}+\alpha \geq 0$ & $\begin{array}{c}x_{1}=0 \\
x_{2}^{2}=\frac{\lambda_{1}^{2}-\lambda_{2}+\alpha}{1-m-\lambda_{1}}\end{array}$ & $\begin{array}{l}+-\left\{\lambda_{1}^{2}-m \lambda_{2}-\lambda_{1} \lambda_{2}+\alpha>0\right. \\
--\left\{\lambda_{1}^{2}-m \lambda_{2}-\lambda_{1} \lambda_{2}+\alpha<0\right.\end{array}$ \\
\hline
\end{tabular}

TABELA 5.15: $0<m<1, \alpha<0$ 


\begin{tabular}{|c|c|c|c|}
\hline $\begin{array}{c}\text { SUBESPACOS } \\
\text { FIXADOS } \\
\end{array}$ & $\begin{array}{l}\text { REGIÕES DO } \\
\operatorname{PLANO}\left(\lambda_{1}, \lambda_{2}\right)\end{array}$ & SOLUÇÕES & $\begin{array}{c}\text { SINAIS DOS } \\
\text { AUTO - VALORES } \\
\end{array}$ \\
\hline $\begin{array}{l}\operatorname{Fix}\left(\mathrm{Z}_{2}(\xi \kappa)\right) \\
\operatorname{Fix}\left(\mathrm{Z}_{2}^{c}(\xi \kappa)\right)\end{array}$ & $\begin{array}{c}\lambda_{2}=0 \\
\lambda_{1}^{2}+\alpha \leq 0\end{array}$ & $\begin{array}{c}x_{1}= \pm x_{2} \\
x_{1}^{2}=-\frac{\lambda_{1}^{2}+\alpha}{2\left(m+\lambda_{1}\right)}\end{array}$ & +- \\
\hline $\operatorname{Fix}(1)$ & $\begin{array}{c}\quad \lambda_{2} \geq 0 \\
\lambda_{1}^{2}+m \lambda_{2} \\
+\lambda_{1} \lambda_{2}+\alpha \leq 0 \\
\\
\lambda_{2}<0 \\
\lambda_{1}^{2}-m \lambda_{2} \\
-\lambda_{1} \lambda_{2}+\alpha \leq 0\end{array}$ & $\begin{array}{c}x_{1}^{2}=x_{2}^{2}+\lambda_{2} \\
x_{2}^{2}=-\frac{\left(\lambda_{1}^{2}+m \lambda_{2}+\lambda_{1} \lambda_{2}+\alpha\right)}{2\left(m+\lambda_{1}\right)} \\
x_{2}^{2}=-\lambda_{2}+x_{1}^{2} \\
x_{1}^{2}=-\frac{\left(\lambda_{1}^{2}-m \lambda_{2}-\lambda_{1} \lambda_{2}+\alpha\right)}{2\left(m+\lambda_{1}\right)}\end{array}$ & +- \\
\hline
\end{tabular}

Continuação da TABELA 5.15: $0<m<1, \alpha<0$ 


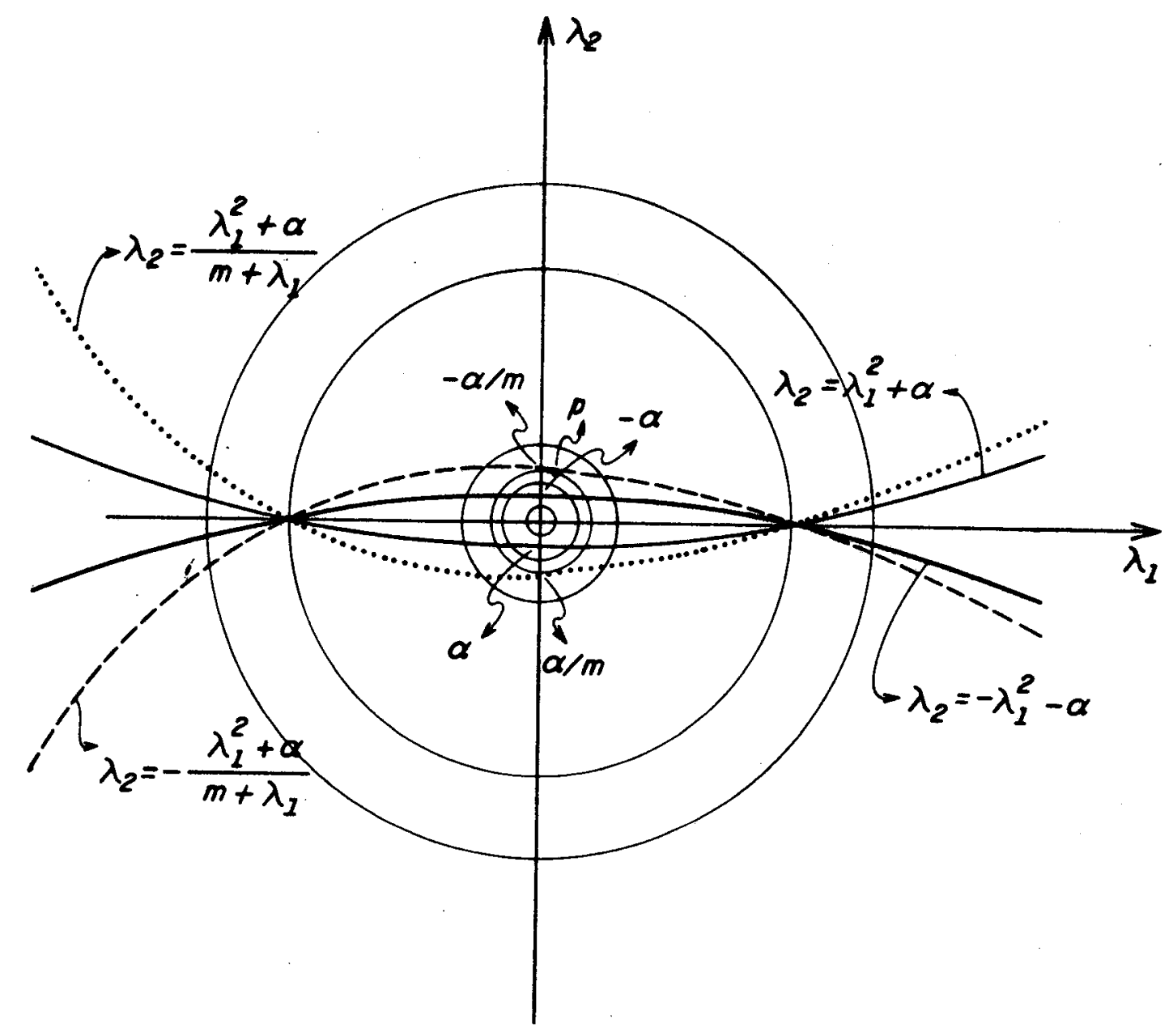

$F / G .5 .31: 0<m<1, \alpha<0$ 
DIAGRAMAS DE BIFURCAFATO: $0<m<1, \alpha<0$
1. $R<-\alpha$
2. $R=-\alpha$

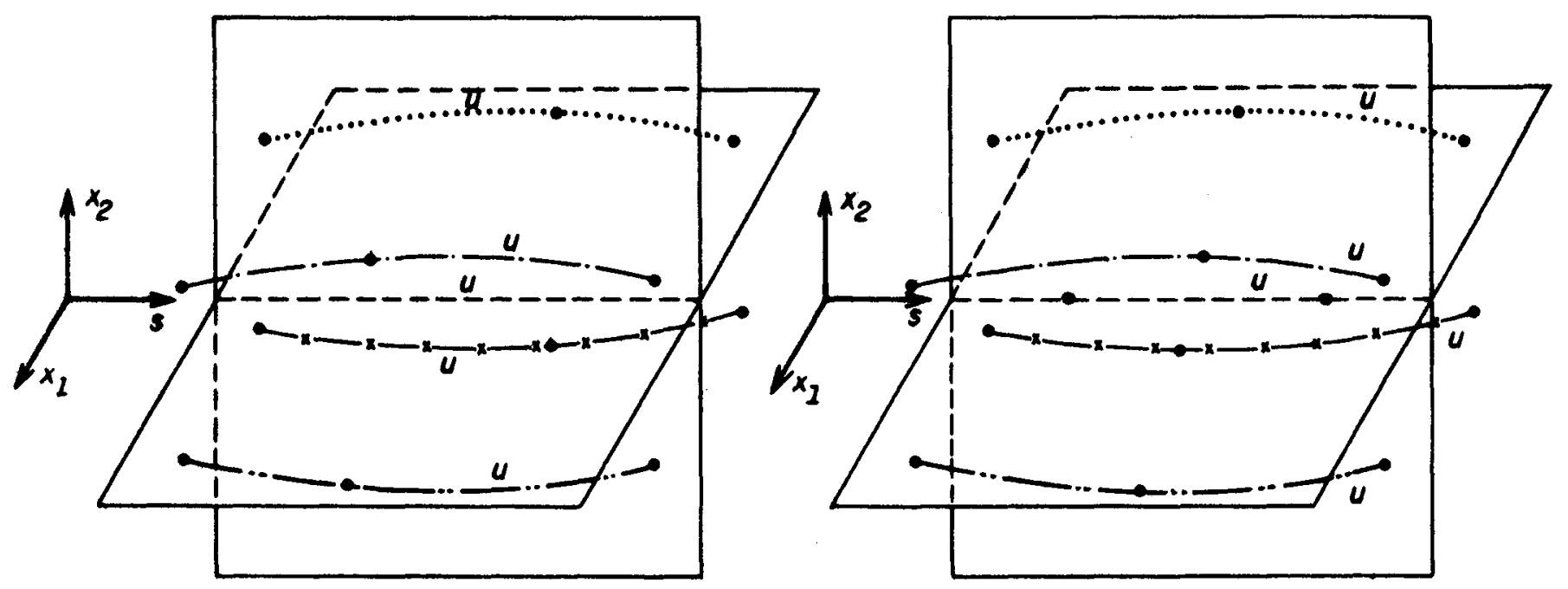

3. $-\alpha<R<|\overrightarrow{O P}|$
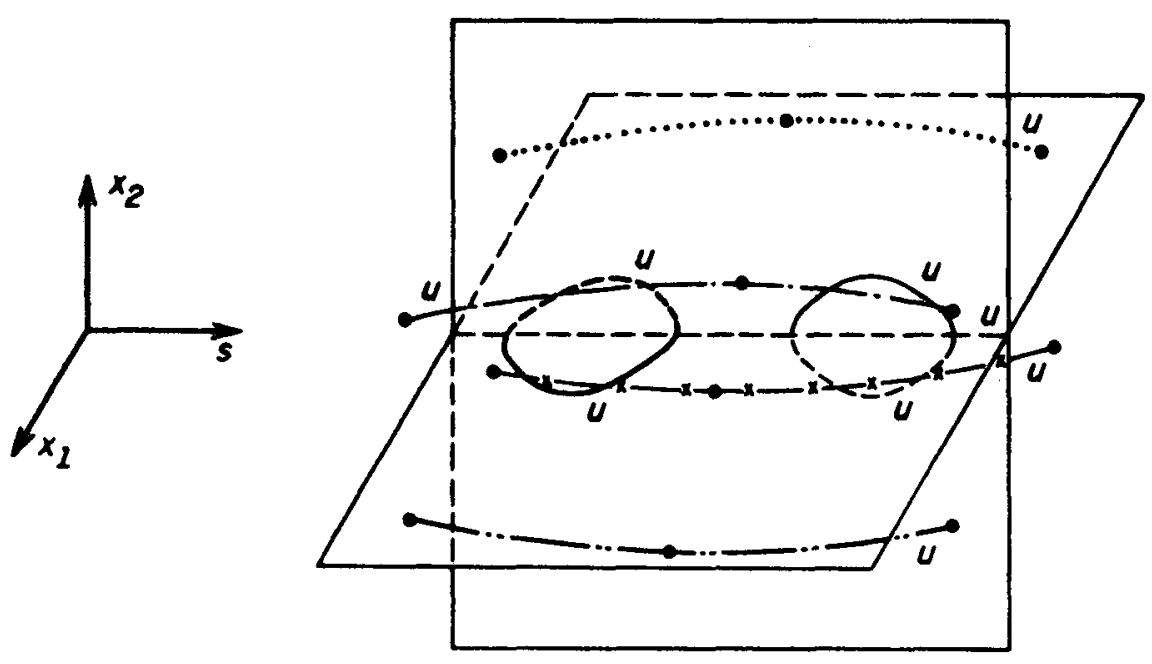

F/G. 5.32 
4. $R=|\overrightarrow{O P}|$
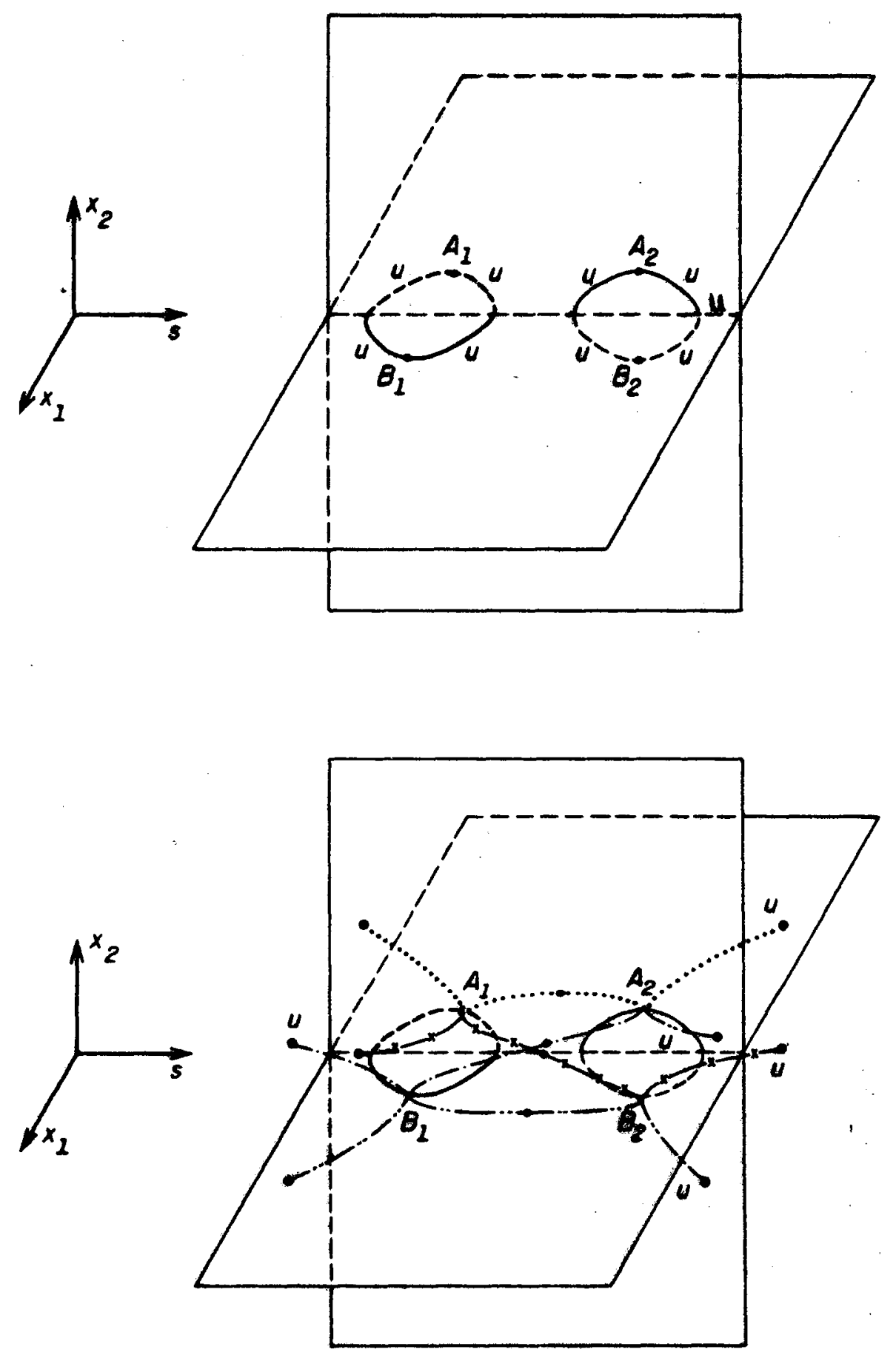

F/G. 5.32 
5. $|\overrightarrow{O P}|<R<\sqrt{-\alpha}$
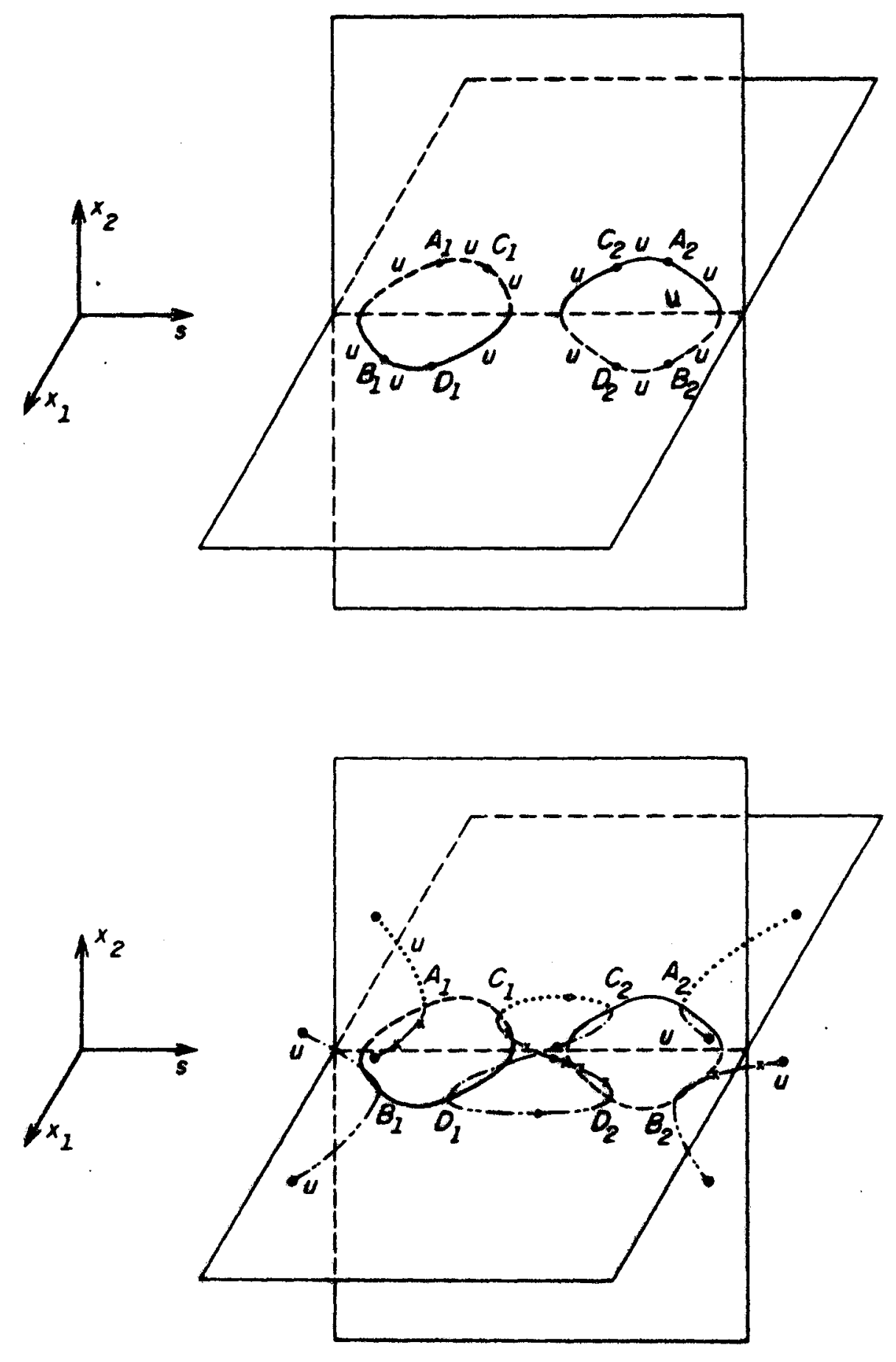

F/G. 5.32 

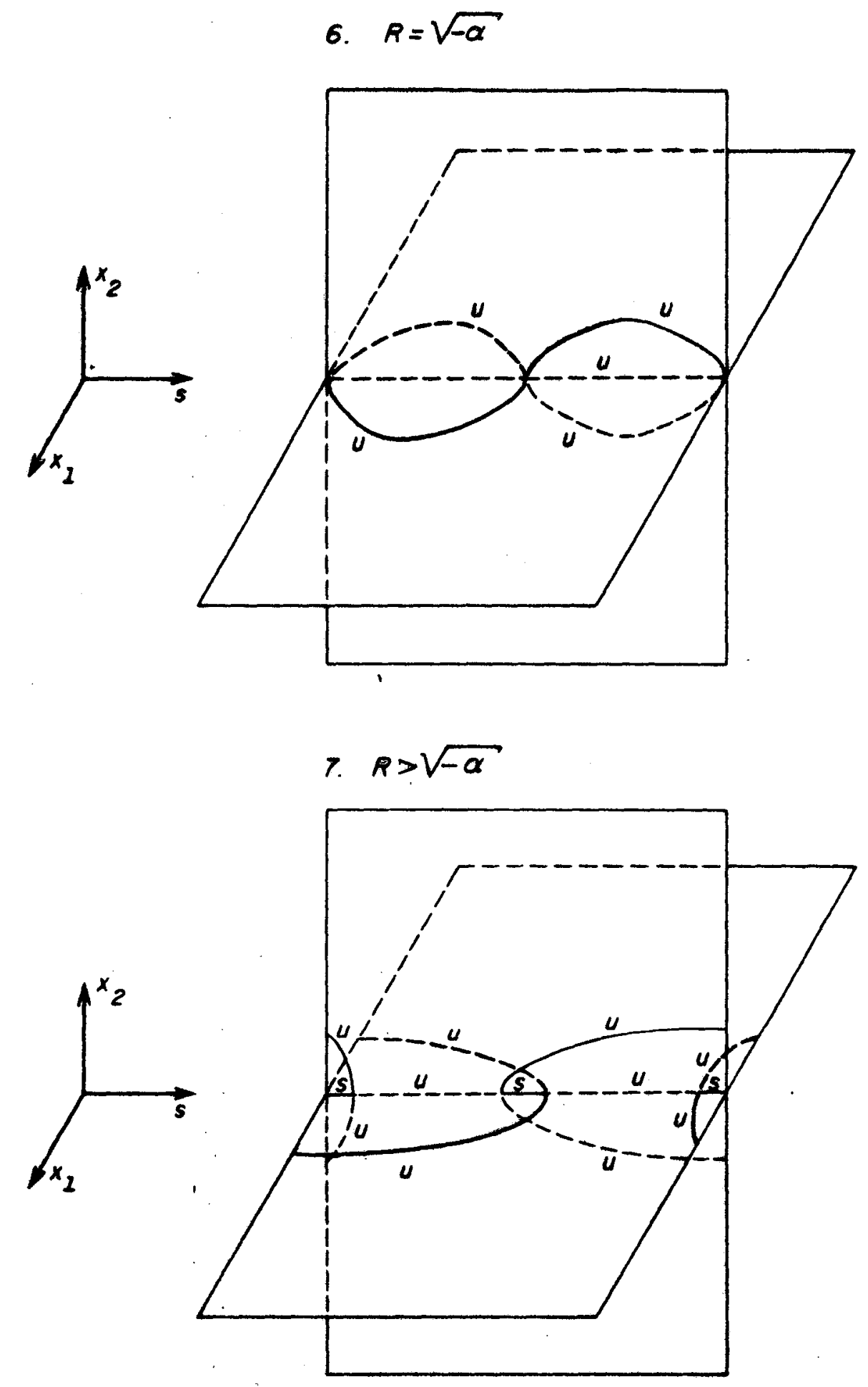

F/G. 5.32 


\begin{tabular}{|c|c|c|c|}
\hline $\begin{array}{c}\text { SUBESPAÇOS } \\
\text { FIXADOS } \\
\end{array}$ & $\begin{array}{l}\text { REGIÕES DO } \\
\text { PLANO }\left(\lambda_{1}, \lambda_{2}\right) \\
\end{array}$ & SOLUÇÕES & $\begin{array}{c}\text { SINAIS DOS } \\
\text { AUTO - VALORES } \\
\end{array}$ \\
\hline $\operatorname{Fix}\left(D_{4}\right)$ & $\forall\left(\lambda_{1}, \lambda_{2}\right)$ & $x_{1}=x_{2}=0$ & $\begin{array}{l}+\left\{\begin{array}{l}\lambda_{1}^{2}-\lambda_{2}>0 \\
\lambda_{1}^{2}+\lambda_{2}>0\end{array}\right. \\
+-\left\{\begin{array}{l}\lambda_{1}^{2}-\lambda_{2}<0 \\
\lambda_{1}^{2}+\lambda_{2}>0\end{array}\right.\end{array}$ \\
\hline $\operatorname{Fix}\left(\mathrm{Z}_{2}(\kappa)\right)$ & $\lambda_{1}^{2}+\lambda_{2} \geq 0$ & $\begin{array}{c}x_{2}=0 \\
x_{1}^{2}=\frac{\lambda_{1}^{2}+\lambda_{2}}{1-m-\lambda_{1}}\end{array}$ & $\begin{array}{l}-+\left\{\begin{array}{l}\lambda_{2} \leq 0 \text { ou } \lambda_{2}>0 \mathrm{e} \\
\lambda_{1}^{2}+m \lambda_{2}+\lambda_{1} \lambda_{2}>0\end{array}\right. \\
--\left\{\begin{array}{l}\lambda_{2}>0 \mathrm{e} \\
\lambda_{1}^{2}+m \lambda_{2}+\lambda_{1} \lambda_{2}<0\end{array}\right.\end{array}$ \\
\hline $\operatorname{Fix}\left(Z_{2}^{c}(\kappa)\right)$ & $\lambda_{1}^{2}-\lambda_{2} \geq 0$ & $\begin{array}{c}x_{1}=0 \\
x_{2}^{2}=\frac{\lambda_{1}^{2}-\lambda_{2}}{1-m-\lambda_{1}}\end{array}$ & $\begin{array}{l}-+\left\{\begin{array}{l}\lambda_{2} \geq 0 \text { ou } \lambda_{2}<0 \mathrm{e} \\
\lambda_{1}^{2}-m \lambda_{2}-\lambda_{1} \lambda_{2}>0\end{array}\right. \\
--\left\{\begin{array}{l}\lambda_{2}<0 \mathrm{e} \\
\lambda_{1}^{2}-m \lambda_{2}-\lambda_{1} \lambda_{2}<0\end{array}\right.\end{array}$ \\
\hline
\end{tabular}

TABELA 5.16: $-1<m<0$ 


\begin{tabular}{|c|c|c|c|}
\hline $\begin{array}{c}\text { SUBESPACOS } \\
\text { FIXADOS } \\
\end{array}$ & $\begin{array}{c}\text { REGIÕES DO } \\
\text { PLANO }\left(\lambda_{1}, \lambda_{2}\right) \\
\end{array}$ & SOLUÇÕES & $\begin{array}{c}\text { SINAIS DOS } \\
\text { AUTO - VALORES } \\
\end{array}$ \\
\hline $\begin{array}{l}\operatorname{Fix}\left(\mathrm{Z}_{2}(\xi \kappa)\right) \\
\operatorname{Fix}\left(\mathrm{Z}_{2}^{c}(\xi \kappa)\right)\end{array}$ & $\lambda_{2}=0$ & $\begin{array}{c}x_{1}= \pm x_{2} \\
x_{1}^{2}=-\frac{\lambda_{1}^{2}}{2\left(m+\lambda_{1}\right)}\end{array}$ & -- \\
\hline $\operatorname{Fix}(\mathbf{1})$ & $\begin{array}{c}\lambda_{2} \geq 0 \\
\lambda_{1}^{2}+m \lambda_{2}+\lambda_{1} \lambda_{2} \geq 0 \\
\lambda_{2}<0 \\
\lambda_{1}^{2}-m \lambda_{2}-\lambda_{1} \lambda_{2} \geq 0\end{array}$ & $\begin{array}{c}x_{1}^{2}=x_{2}^{2}+\lambda_{2} \\
x_{2}^{2}=-\frac{\left(\lambda_{1}^{2}+m \lambda_{2}+\lambda_{1} \lambda_{2}\right)}{2\left(m+\lambda_{1}\right)} \\
x_{2}^{2}=-\lambda_{2}+x_{1}^{2} \\
x_{1}^{2}=-\frac{\left(\lambda_{1}^{2}-m \lambda_{2}-\lambda_{1} \lambda_{2}\right)}{2\left(m+\lambda_{1}\right)}\end{array}$ & -- \\
\hline
\end{tabular}

Continuação da TABELA 5.16: $-1<m<0$ 


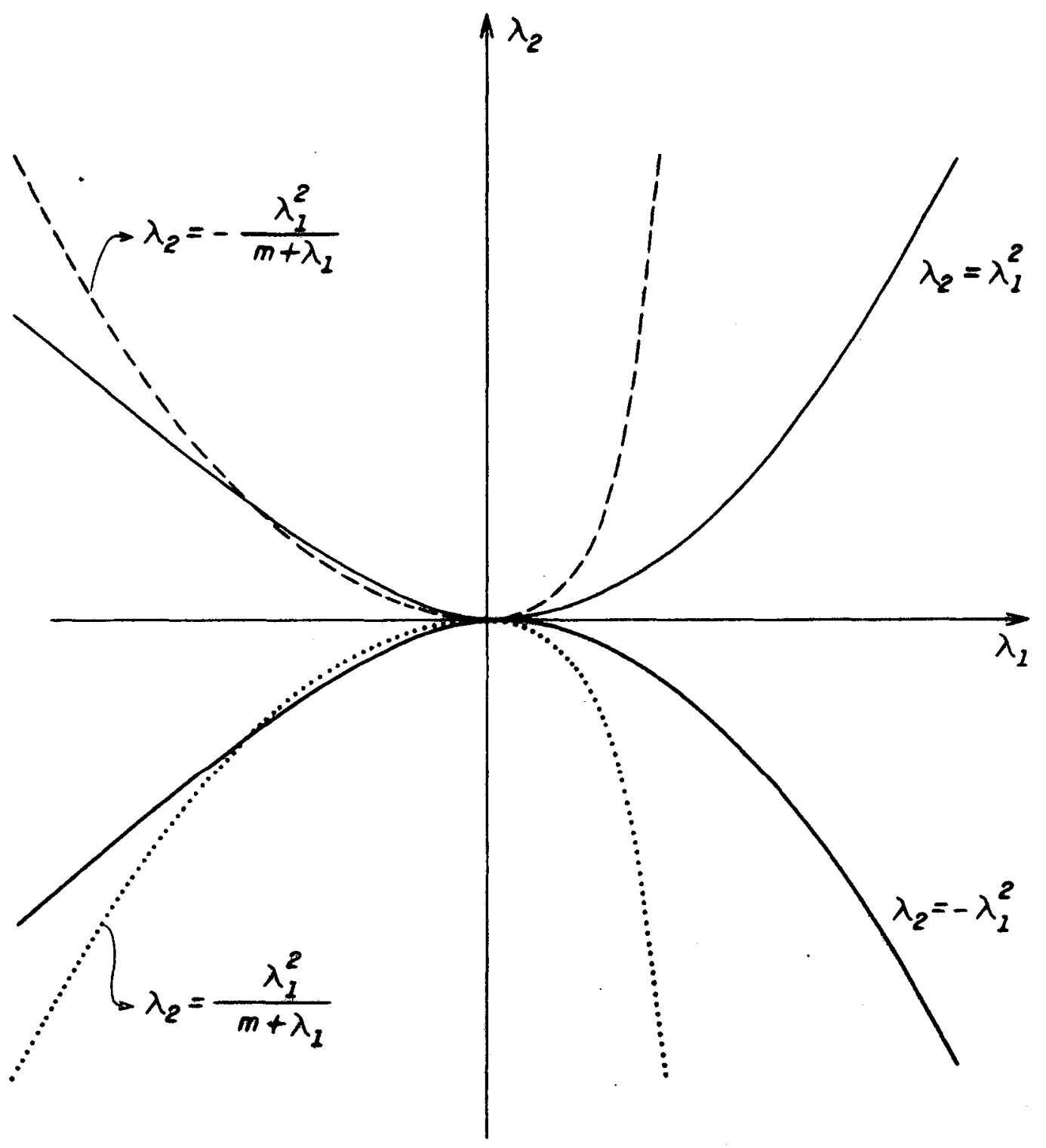

FIG. $5.33:-1<m<0$ 
DIAGRAMAS DE BIFURCACĀO: $-1<m<0$
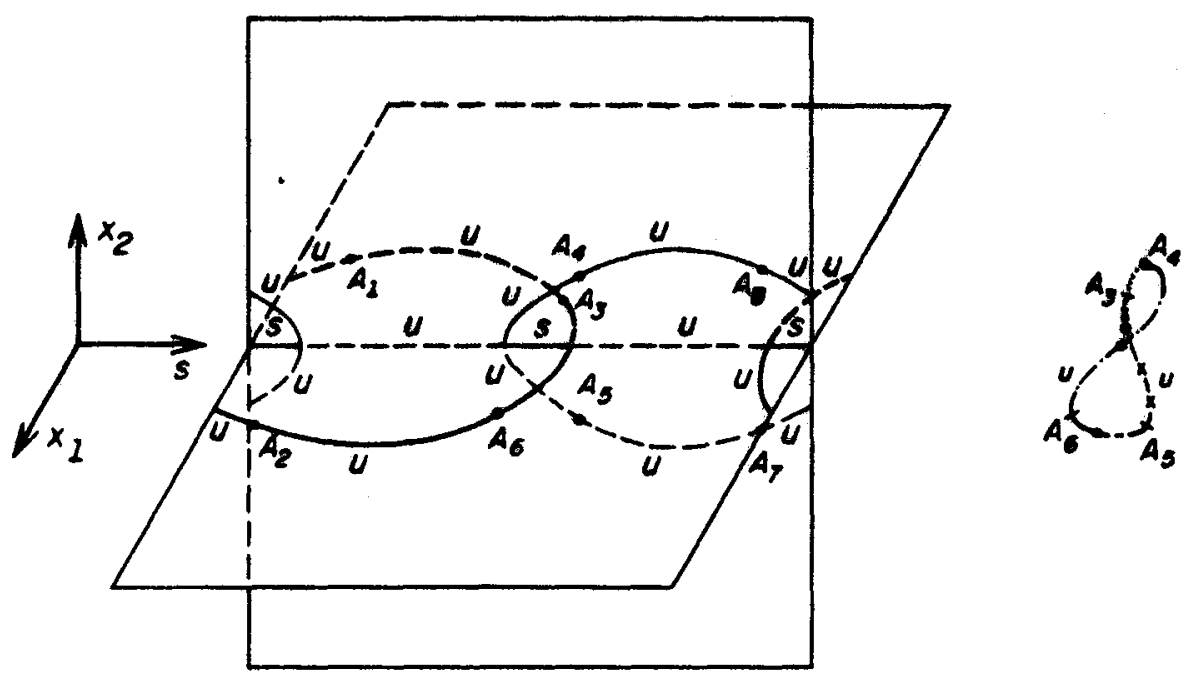

"LOOP"

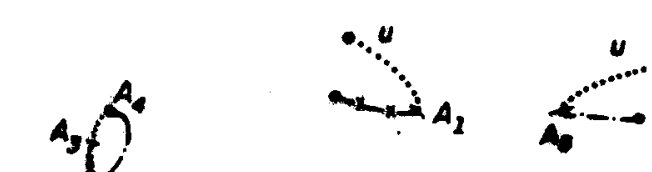

$\sigma^{A_{2}}$

in
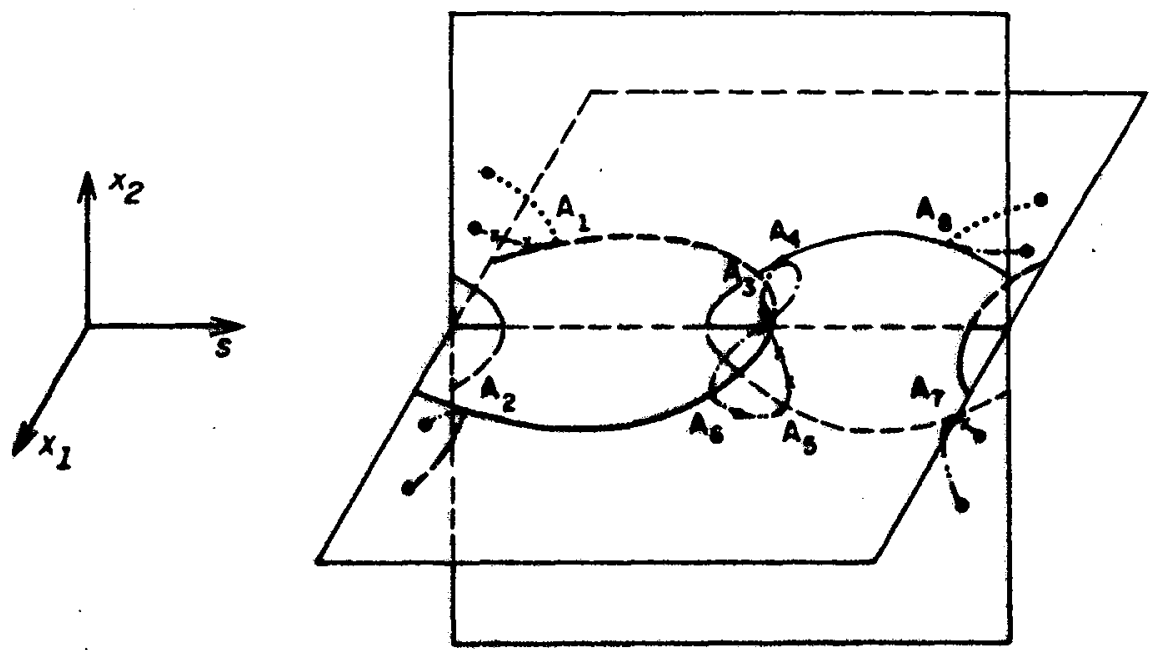

FIG. 5.34 


\begin{tabular}{|c|c|c|c|}
\hline $\begin{array}{c}\text { SUBESPAÇOS } \\
\text { FIXADOS } \\
\end{array}$ & $\begin{array}{c}\text { REGIÕES DO } \\
\text { PLANO }\left(\lambda_{1}, \lambda_{2}\right) \\
\end{array}$ & SOLUÇÕES & $\begin{array}{c}\text { SINAIS DOS } \\
\text { AUTO - VALORES } \\
\end{array}$ \\
\hline $\operatorname{Fix}\left(D_{4}\right)$ & $\forall\left(\lambda_{1}, \lambda_{2}\right)$ & $x_{1}=x_{2}=0$ & $\begin{array}{c}++\left\{\begin{array}{l}\lambda_{1}^{2}+\lambda_{2}+\alpha>0 \\
\lambda_{1}^{2}-\lambda_{2}+\alpha>0\end{array}\right. \\
+-\left\{\begin{array}{l}\lambda_{1}^{2}+\lambda_{2}+\alpha>0(<0) \\
\lambda_{1}^{2}-\lambda_{2}+\alpha<0(>0)\end{array}\right.\end{array}$ \\
\hline $\operatorname{Fix}\left(\mathrm{Z}_{2}(\kappa)\right)$ & $\lambda_{1}^{2}+\lambda_{2}+\alpha \geq 0$ & $\begin{array}{c}x_{2}=0 \\
x_{1}^{2}=\frac{\lambda_{1}^{2}+\lambda_{2}+\alpha}{1-m-\lambda_{1}}\end{array}$ & $\begin{array}{l}+-\left\{\begin{array}{l}\lambda_{2} \leq 0 \text { ou } \lambda_{2}>0 \mathrm{e} \\
\lambda_{1^{\prime}}^{2}+m \lambda_{2}+\lambda_{1} \lambda_{2}+\alpha>0\end{array}\right. \\
--\left\{\begin{array}{l}\lambda_{2}>0 \mathrm{e} \\
\lambda_{1}^{2}+m \lambda_{2}+\lambda_{1} \lambda_{2}+\alpha<0\end{array}\right.\end{array}$ \\
\hline $\operatorname{Fix}\left(\mathrm{Z}_{2}^{c}(\kappa)\right)$ & $\lambda_{1}^{2}-\lambda_{2}+\alpha \geq 0$ & $\begin{array}{c}x_{1}=0 \\
x_{2}^{2}=\frac{\lambda_{1}^{2}-\lambda_{2}+\alpha}{1-m-\lambda_{1}}\end{array}$ & $\begin{array}{l}+-\left\{\begin{array}{l}\lambda_{2} \geq 0 \text { ou } \lambda_{2}<0 \mathrm{e} \\
\lambda_{1}^{2}-m \lambda_{2}-\lambda_{1} \lambda_{2}+\alpha>0\end{array}\right. \\
--\left\{\begin{array}{l}\lambda_{2}<0 \mathrm{e} . \\
\lambda_{1}^{2}-m \lambda_{2}-\lambda_{1} \lambda_{2}+\alpha<0\end{array}\right.\end{array}$ \\
\hline
\end{tabular}

TABELA 5.17: $-1<m<0, \alpha>0$ 


\begin{tabular}{|c|c|c|c|}
\hline $\begin{array}{c}\text { SUBESPAÇOS } \\
\text { FIXADOS } \\
\end{array}$ & $\begin{array}{l}\text { REGIÕES DO } \\
\operatorname{PLANO}\left(\lambda_{1}, \lambda_{2}\right)\end{array}$ & SOLUÇÕES & $\begin{array}{c}\text { SINAIS DOS } \\
\text { AUTO - VALORES } \\
\end{array}$ \\
\hline $\begin{array}{l}\operatorname{Fix}\left(\mathrm{Z}_{2}(\xi \kappa)\right) \\
\operatorname{Fix}\left(\mathrm{Z}_{2}^{c}(\xi \kappa)\right)\end{array}$ & $\lambda_{2}=0$ & $\begin{array}{c}x_{1}= \pm x_{2} \\
x_{1}^{2}=-\frac{\lambda_{1}^{2}+\alpha}{2\left(m+\lambda_{1}\right)}\end{array}$ & -- \\
\hline $\operatorname{Fix}(1)$ & $\begin{array}{c}\lambda_{2} \geq 0 \\
\lambda_{1}^{2}+m \lambda_{2} \\
+\lambda_{1} \lambda_{2}+\alpha \geq 0 \\
\\
\lambda_{2}<0 \\
\lambda_{1}^{2}-m \lambda_{2} \\
-\lambda_{1} \lambda_{2}+\alpha \geq 0\end{array}$ & $\begin{array}{c}x_{1}^{2}=x_{2}^{2}+\lambda_{2} \\
x_{2}^{2}=-\frac{\left(\lambda_{1}^{2}+m \lambda_{2}+\lambda_{1} \lambda_{2}+\alpha\right)}{2\left(m+\lambda_{1}\right)} \\
x_{2}^{2}=-\lambda_{2}+x_{1}^{2} \\
x_{1}^{2}=-\frac{\left(\lambda_{1}^{2}-m \lambda_{2}-\lambda_{1} \lambda_{2}+\alpha\right)}{2\left(m+\lambda_{1}\right)}\end{array}$ & $-\cdots$ \\
\hline
\end{tabular}

Continuação da TABELA 5.17: $-1<m<0, \alpha>0$ 


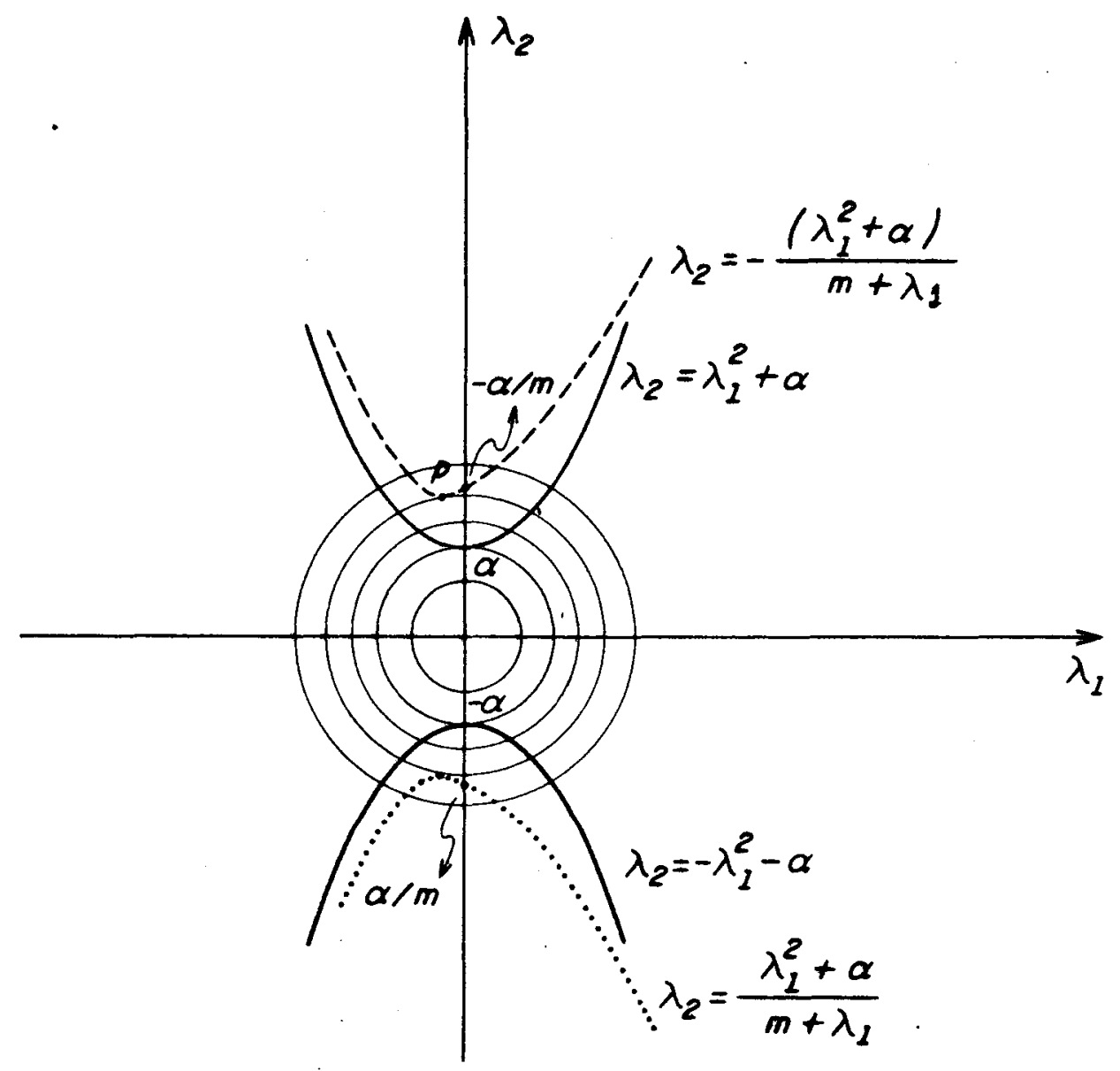

FIG. $5.35:-1<m<0, \alpha>0$ 
DIAGRAMAS DE BIFURCACĀO: $-1<m<0, \alpha>0$

1. $R<\alpha$

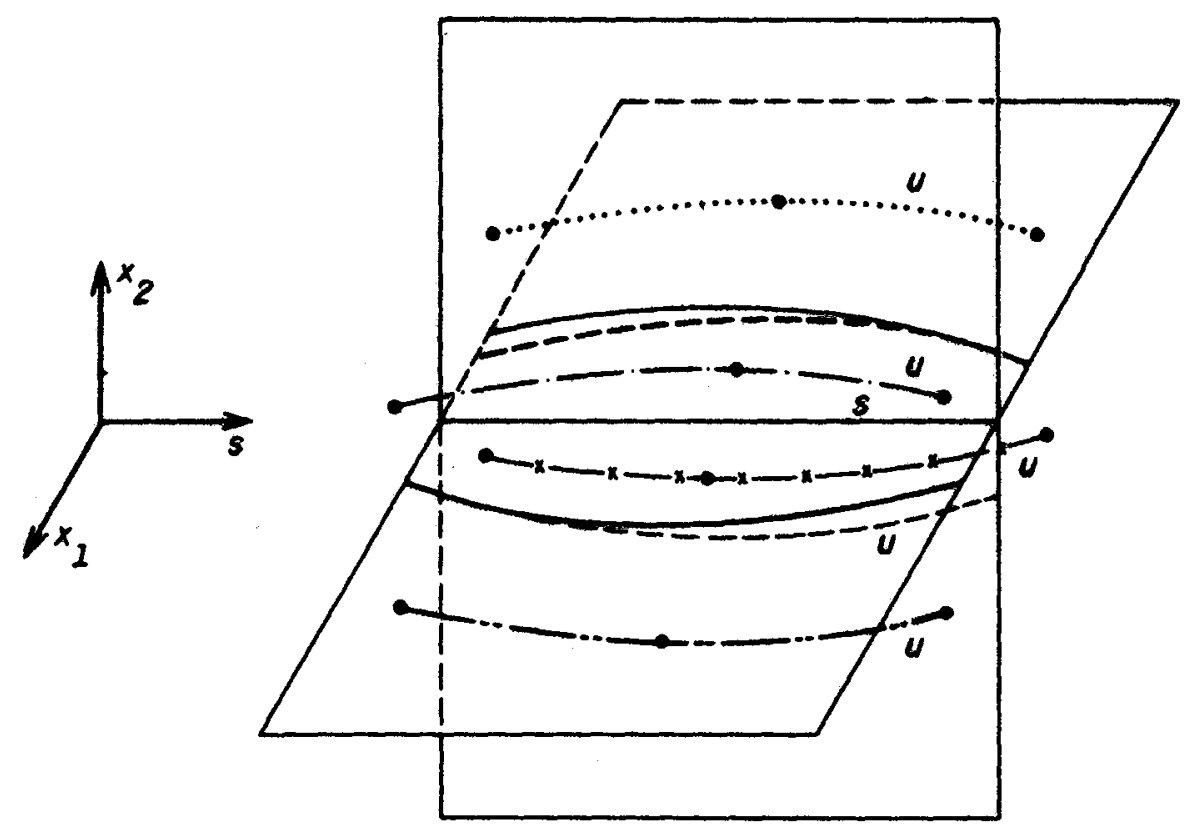

2. $R=\alpha$

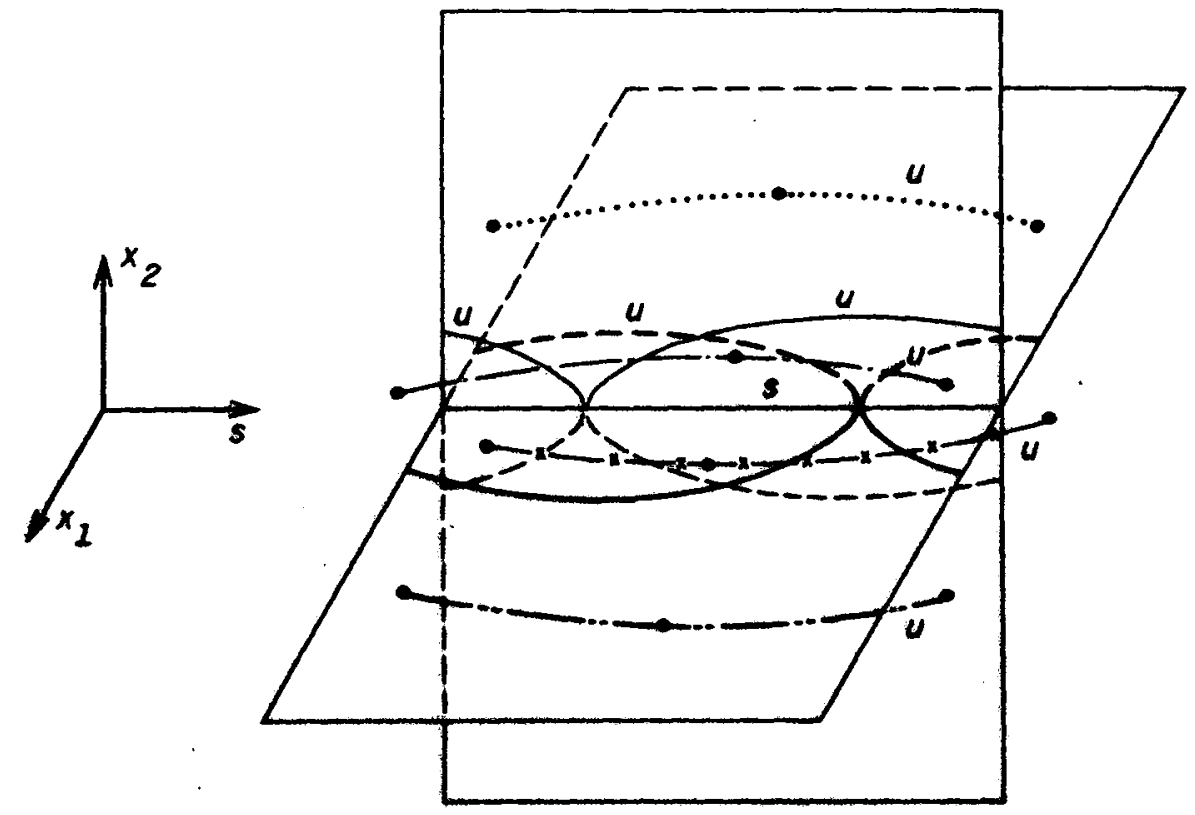

FIG. 5.36 
3. $\quad \alpha<R<|\overrightarrow{O P}|$
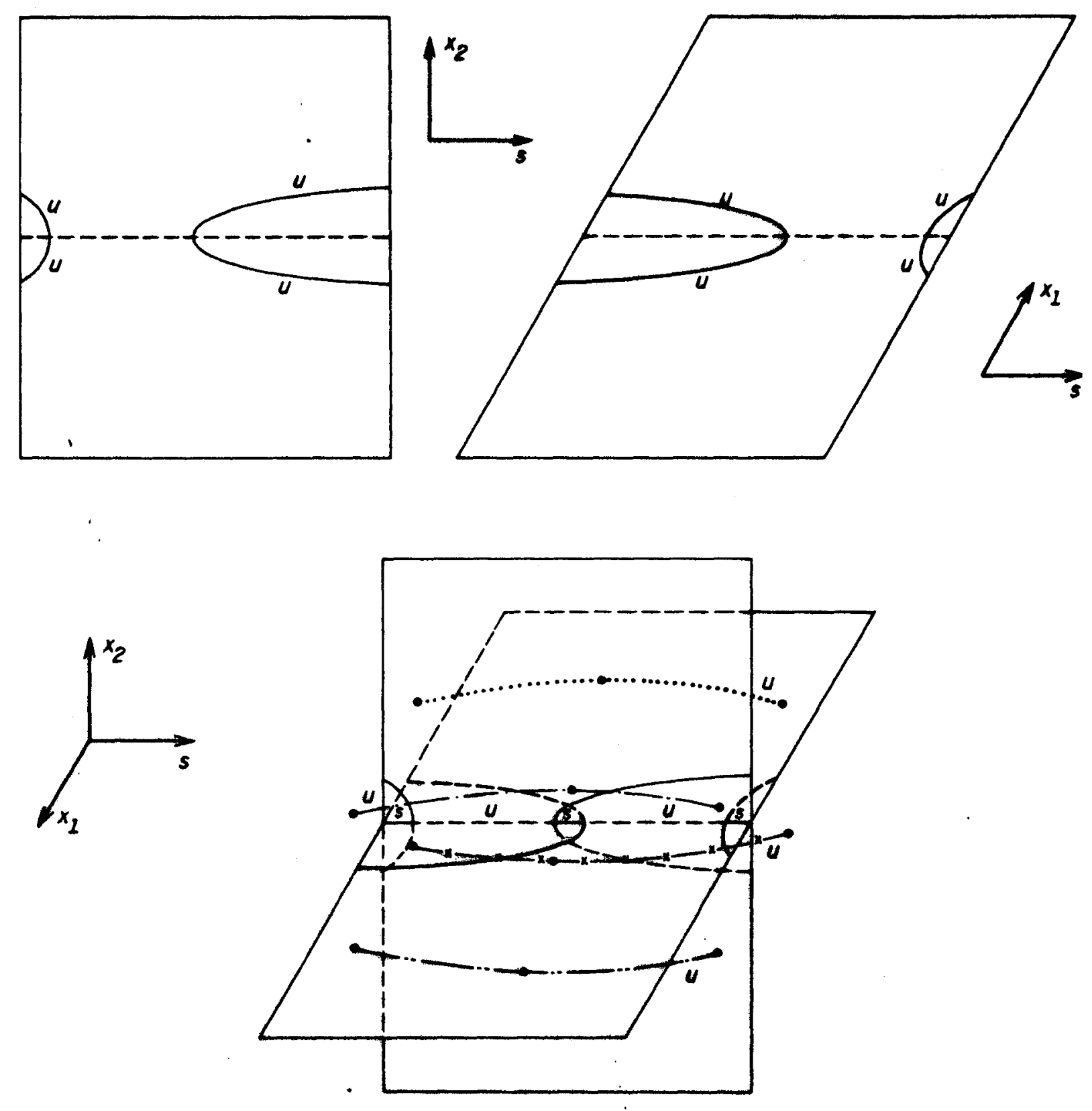

FIG. 5.36 
4. $R=|\overrightarrow{O P}|$
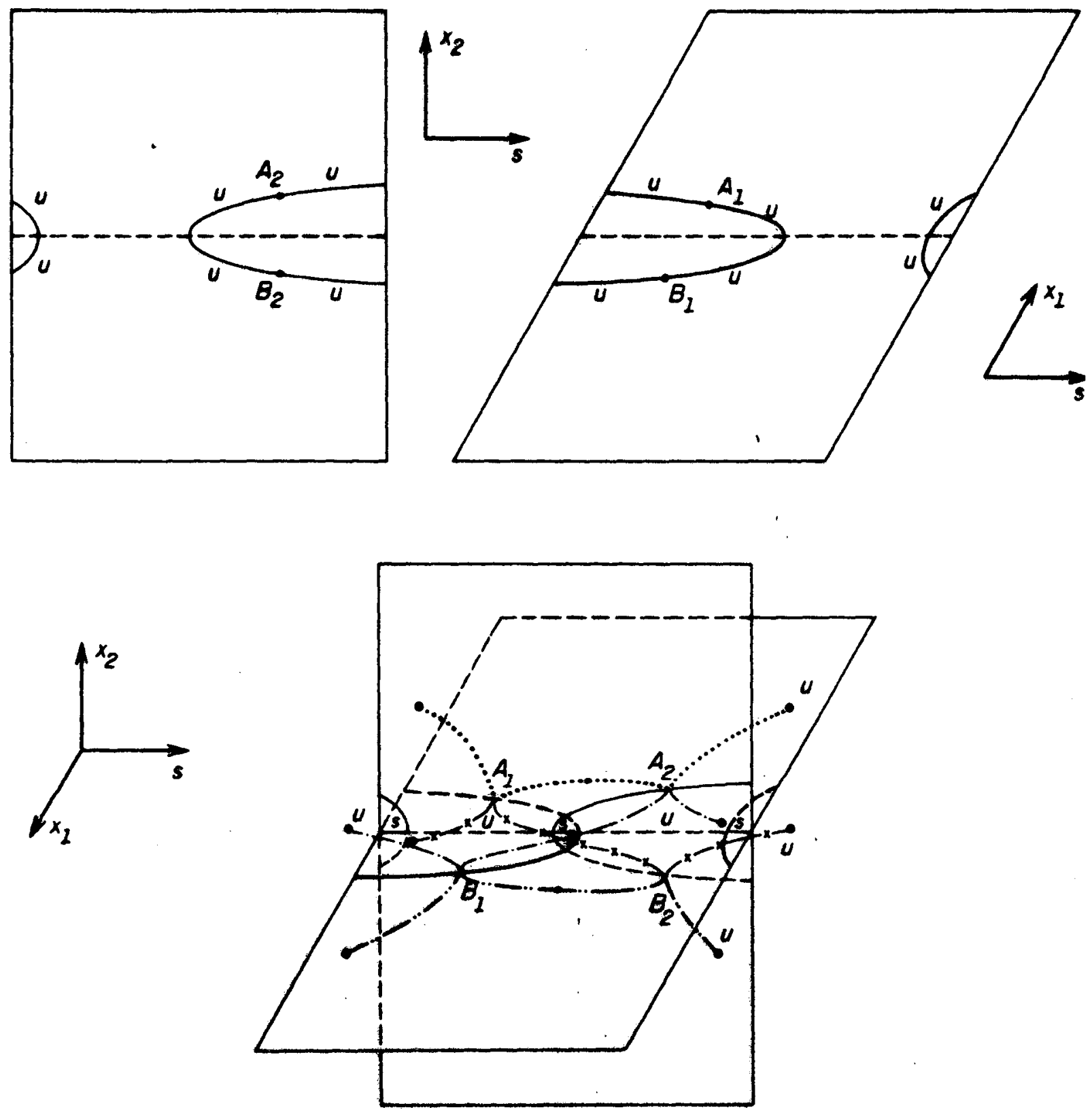

F/G. 5.36 
5. $R>|\overrightarrow{O P}|$
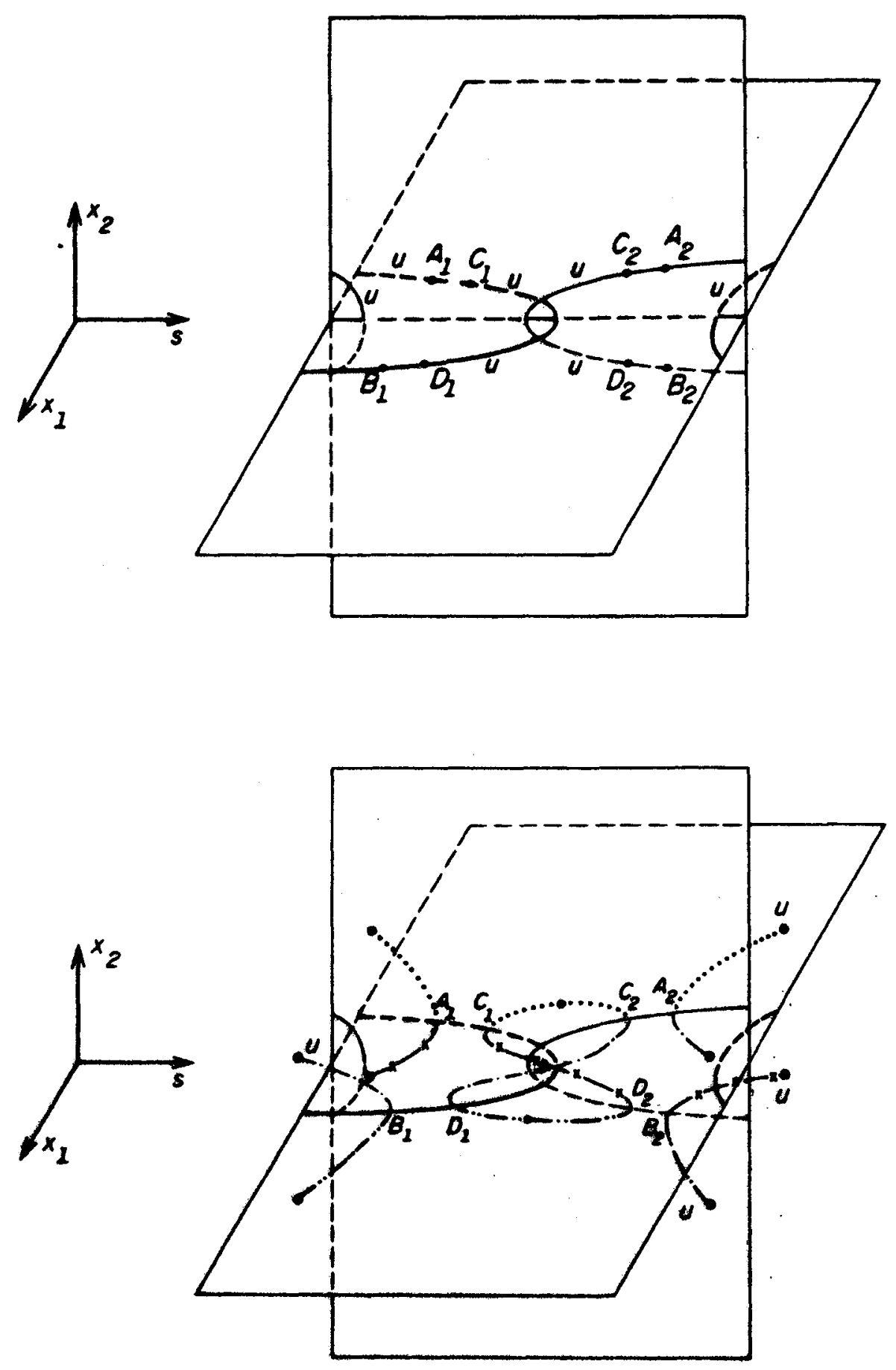

F/G. 5.36 


\begin{tabular}{|c|c|c|c|}
\hline $\begin{array}{c}\text { SUBESPAÇOS } \\
\text { FIXADOS }\end{array}$ & $\begin{array}{c}\text { REGIÕES DO } \\
\operatorname{PLANO}\left(\lambda_{1}, \lambda_{2}\right)\end{array}$ & SOLUÇÕES & $\begin{array}{c}\text { SINAIS DOS } \\
\text { AUTO - VALORES }\end{array}$ \\
\hline $\operatorname{Fix}\left(D_{4}\right)$ & $\forall\left(\lambda_{1}, \lambda_{2}\right)$ & $x_{1}=x_{2}=0$ & $\begin{array}{c}+\left\{\begin{array}{l}\lambda_{1}^{2}-\lambda_{2}+\alpha>0 \\
\lambda_{1}^{2}+\lambda_{2}+\alpha>0\end{array}\right. \\
+-\left\{\begin{array}{l}\lambda_{1}^{2}-\lambda_{2}+\alpha>0(<0) \\
\lambda_{1}^{2}+\lambda_{2}+\alpha<0(>0)\end{array}\right. \\
--\left\{\begin{array}{l}\lambda_{1}^{2}-\lambda_{2}+\alpha<0 \\
\lambda_{1}^{2}+\lambda_{2}+\alpha<0\end{array}\right.\end{array}$ \\
\hline $\operatorname{Fix}\left(\mathrm{Z}_{2}(\kappa)\right)$ & $\lambda_{1}^{2}+\lambda_{2}+\alpha \geq 0$ & $\begin{array}{c}x_{2}=0 \\
x_{1}^{2}=\frac{\lambda_{1}^{2}+\lambda_{2}+\alpha}{1-m-\lambda_{1}}\end{array}$ & $\begin{array}{l}+-\left\{\lambda_{1}^{2}+m \lambda_{2}+\lambda_{1} \lambda_{2}+\alpha>0\right. \\
--\left\{\lambda_{1}^{2}+m \lambda_{2}+\lambda_{1} \lambda_{2}+\alpha<0\right.\end{array}$ \\
\hline $\operatorname{Fix}\left(Z_{2}^{c}(\kappa)\right)$ & $\lambda_{1}^{2}-\lambda_{2}+\alpha \geq 0$ & $\begin{array}{c}x_{1}=0 \\
x_{2}^{2}=\frac{\lambda_{1}^{2}-\lambda_{2}+\alpha}{1-m-\lambda_{1}}\end{array}$ & $\begin{array}{l}+-\left\{\lambda_{1}^{2}-m \lambda_{2}-\lambda_{1} \lambda_{2}+\alpha>0\right. \\
--\left\{\lambda_{1}^{2}-m \lambda_{2}-\lambda_{1} \lambda_{2}+\alpha<0\right.\end{array}$ \\
\hline
\end{tabular}

TABELA 5.18: $-1<m<0, \alpha<0$ 


\begin{tabular}{|c|c|c|c|}
\hline $\begin{array}{l}\text { SUBESPAÇOS } \\
\text { FIXADOS } \\
\end{array}$ & $\begin{array}{l}\text { REGIÕES DO } \\
\text { PLANO }\left(\lambda_{1}, \lambda_{2}\right) \\
\end{array}$ & SOLUÇÕES & $\begin{array}{c}\text { SINAIS DOS } \\
\text { AUTO - VALORES } \\
\end{array}$ \\
\hline $\begin{array}{l}\operatorname{Fix}\left(\mathrm{Z}_{2}(\xi \kappa)\right) \\
\operatorname{Fix}\left(\mathrm{Z}_{2}^{c}(\xi \kappa)\right)\end{array}$ & $\begin{array}{c}\lambda_{2}=0 \\
\lambda_{1}^{2}+\alpha \geq 0\end{array}$ & $\begin{array}{c}x_{1}= \pm x_{2} \\
x_{1}^{2}=-\frac{\lambda_{1}^{2}+\alpha}{2\left(m+\lambda_{1}\right)}\end{array}$ & -- \\
\hline $\operatorname{Fix}(1)$ & $\begin{array}{c}\quad \lambda_{2} \geq 0 \\
\lambda_{1}^{2}+m \lambda_{2} \\
+\lambda_{1} \lambda_{2}+\alpha \geq 0 \\
\lambda_{2}<0 \\
\lambda_{1}^{2}-m \lambda_{2} \\
-\lambda_{1} \lambda_{2}+\alpha \geq 0\end{array}$ & $\begin{array}{c}x_{1}^{2}=x_{2}^{2}+\lambda_{2} \\
x_{2}^{2}=-\frac{\left(\lambda_{1}^{2}+m \lambda_{2}+\lambda_{1} \lambda_{2}+\alpha\right)}{2\left(m+\lambda_{1}\right)} \\
x_{2}^{2}=-\lambda_{2}+x_{1}^{2} \\
x_{1}^{2}=-\frac{\left(\lambda_{1}^{2}-m \lambda_{2}-\lambda_{1} \lambda_{2}+\alpha\right)}{2\left(m+\lambda_{1}\right)}\end{array}$ & -- \\
\hline
\end{tabular}

Continuação da TABELA 5.18: $-1<m<0, \alpha<0$ 


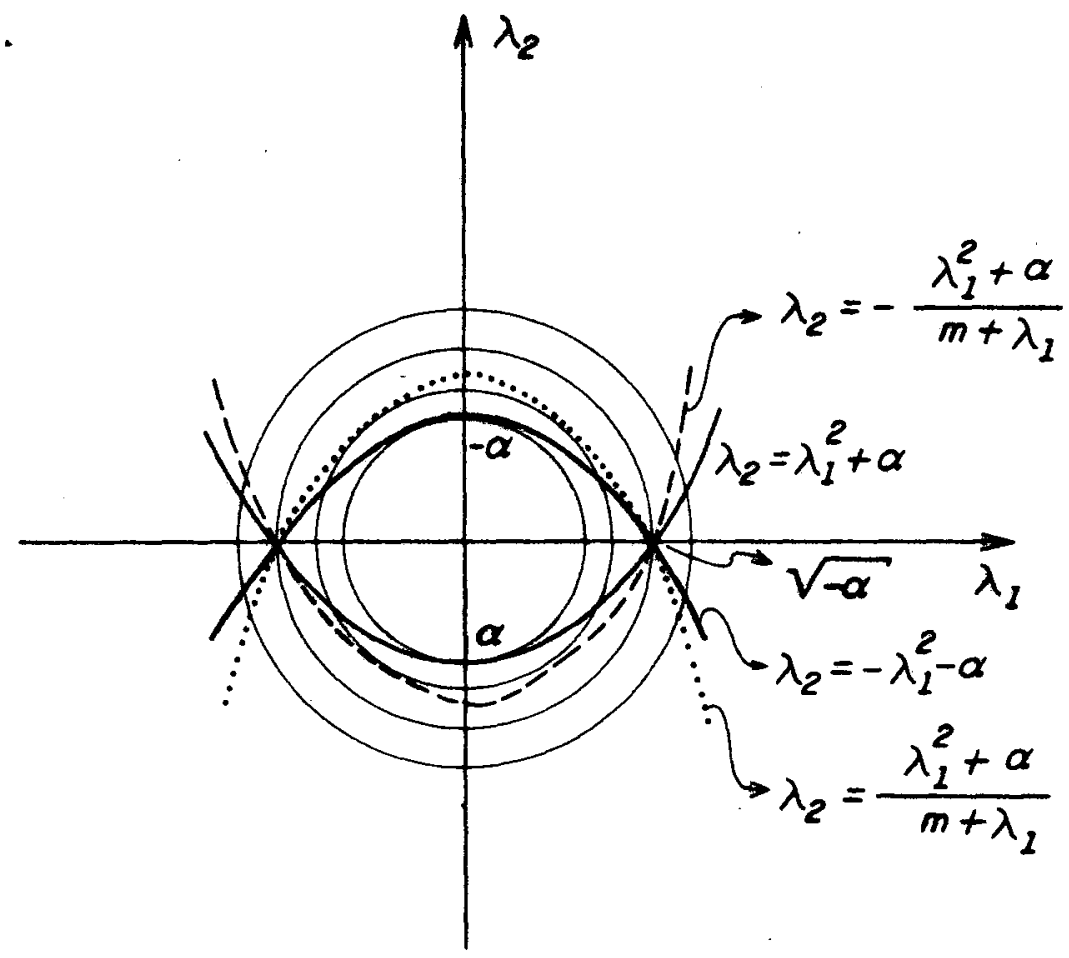

FIG. 5. 37: $-1<m<0, a<0$ 
DIAGRAMAS DE BIFURCASATO: $-1<m<0, \alpha<0$

1. $R=-\alpha \quad--\frac{u}{\pi / 2}-\frac{u}{3 \pi / 2}-\frac{u}{s}$

2. $-\alpha<R<\sqrt{-\alpha}$

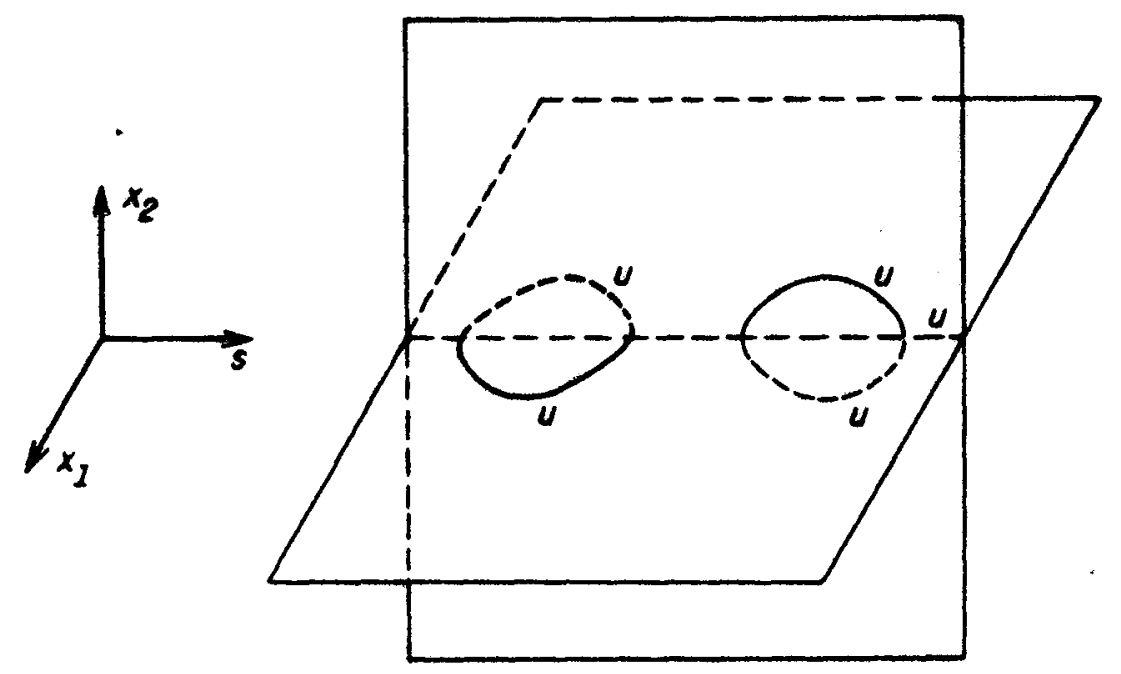

3. $R=\sqrt{-\alpha}$

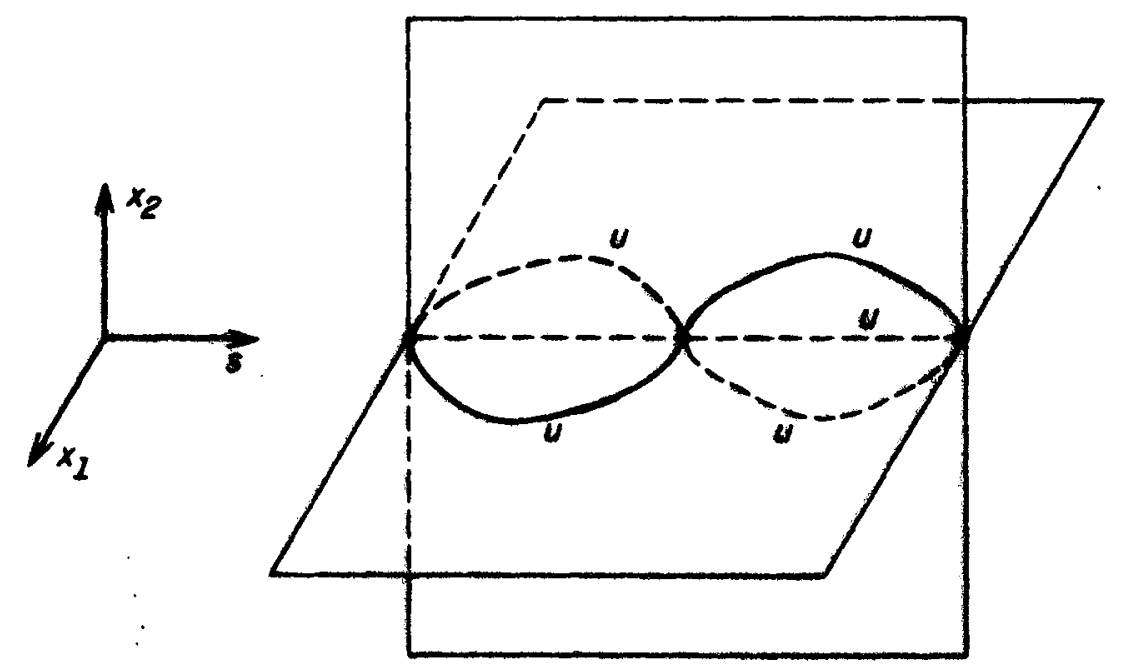

F/G. 5.38 

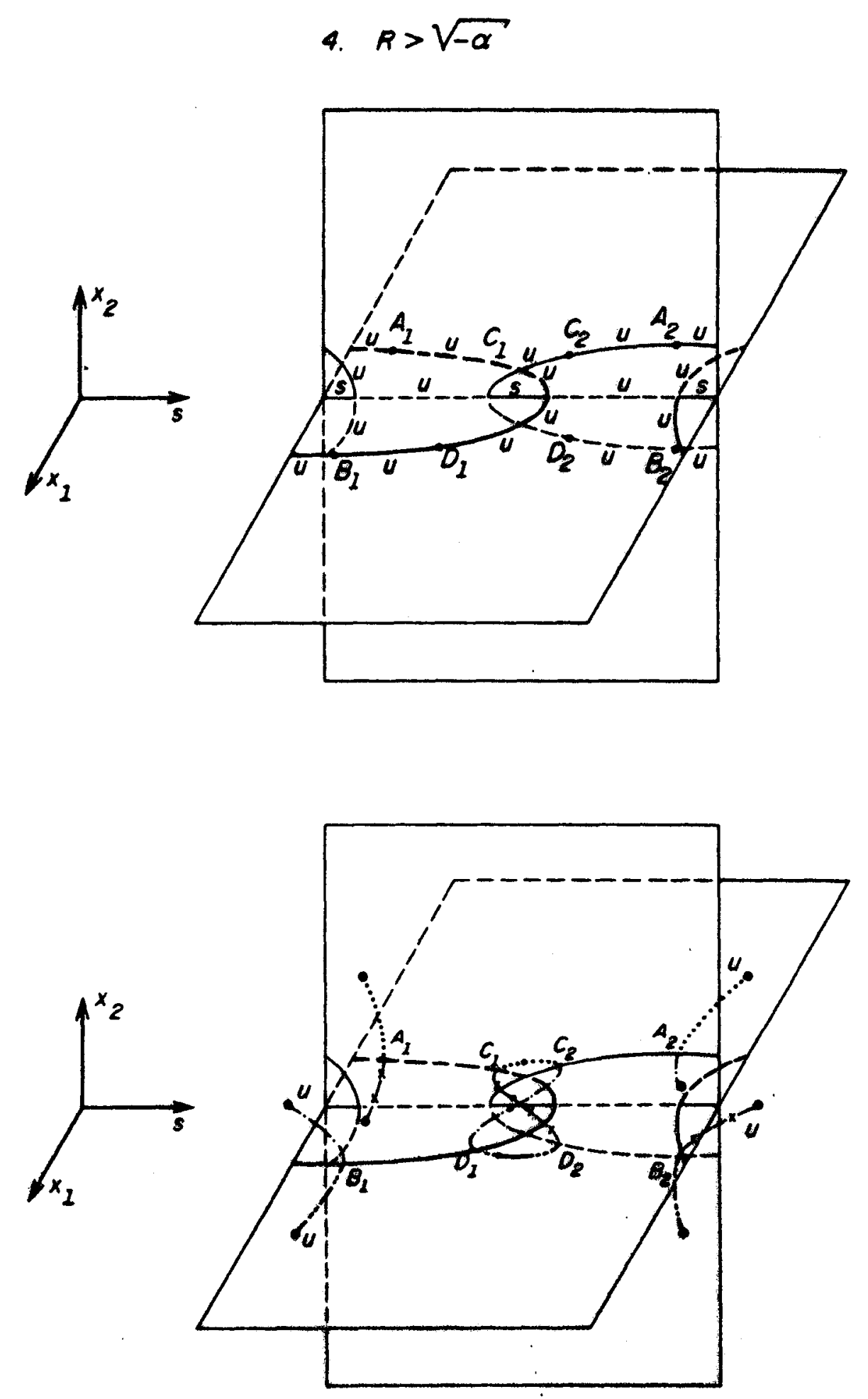

F/G. 5.38 
A tabela com os dados para o caso $m<-1$ é igual a tabela 5.16 com a ressalva da variação de $m$ nesse caso. Por isso, faremos apenas a representação no plano $\left(\lambda_{1}, \lambda_{2}\right)$ e os diagramas de bifurcação.

A diferença entre o diagrama de bifurcação desse caso e o diagrama do caso anterior, $-1<m<0$, está na posição do "laço".

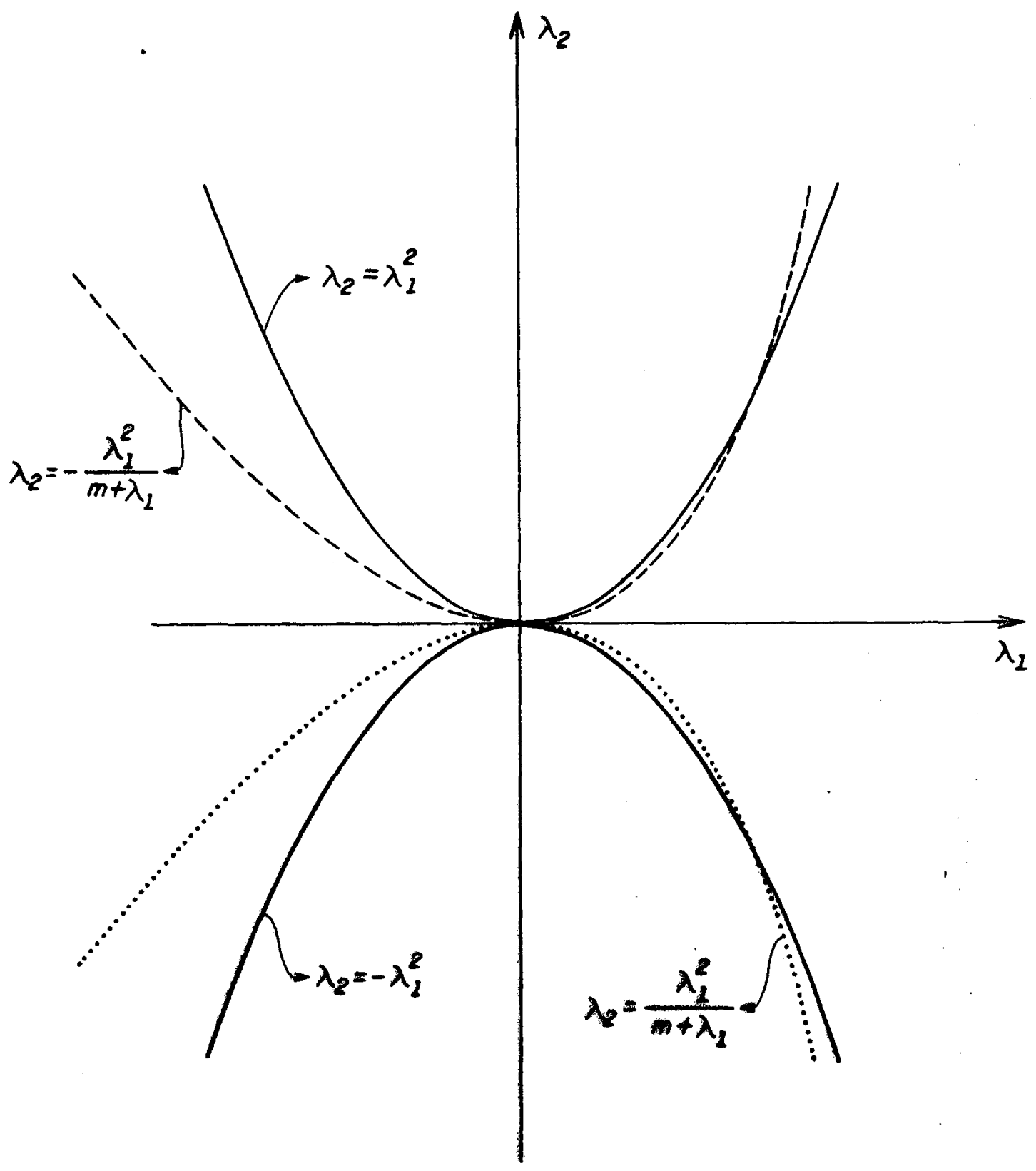

F16. 5.39: $m<-1$ 
DIAGRAMA DE BIFURCACÃO: $m<-1$

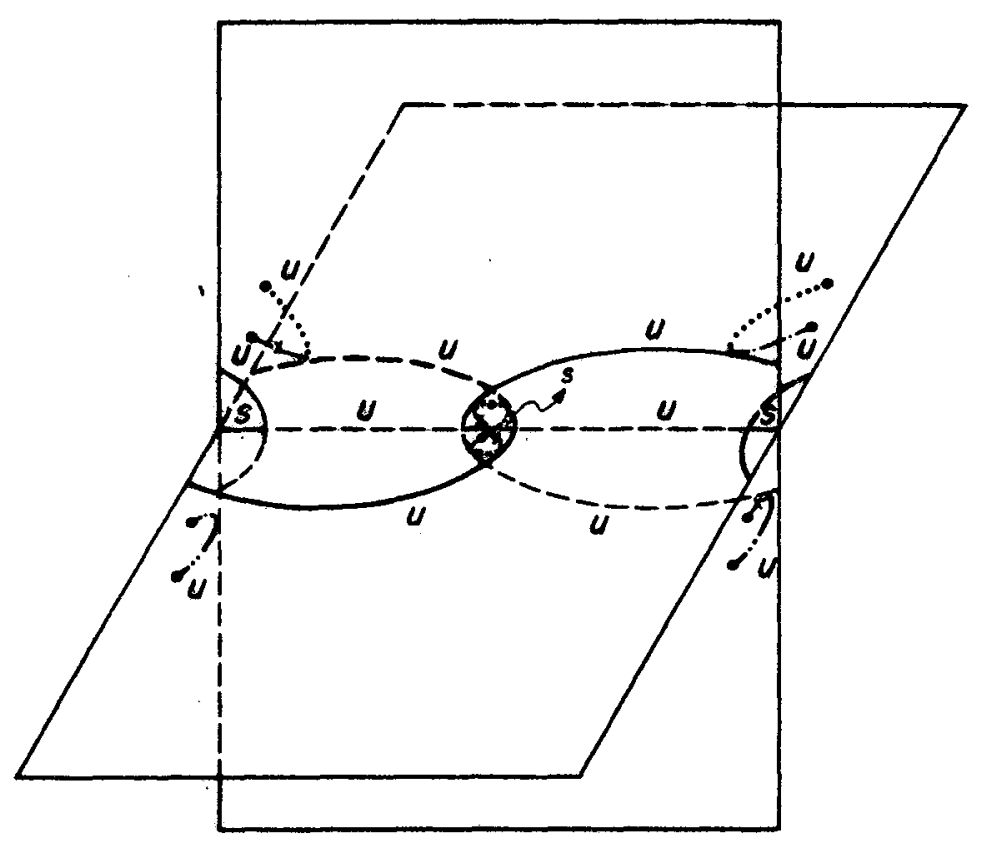

FIG. 5.40 
Para valores do parâmetro $\alpha$ positivos e próximos do zero, os dados para a construção do diagrama do desdobramento dessa forma são iguais aos da tabela 5.17. Para o caso onde $\alpha$ é negativo, os dados podem ser vistos na tabela 5.18. Por isso, faremos apenas a representação no plano $\left(\lambda_{1}, \lambda_{2}\right)$ e os correspondentes diagramas.

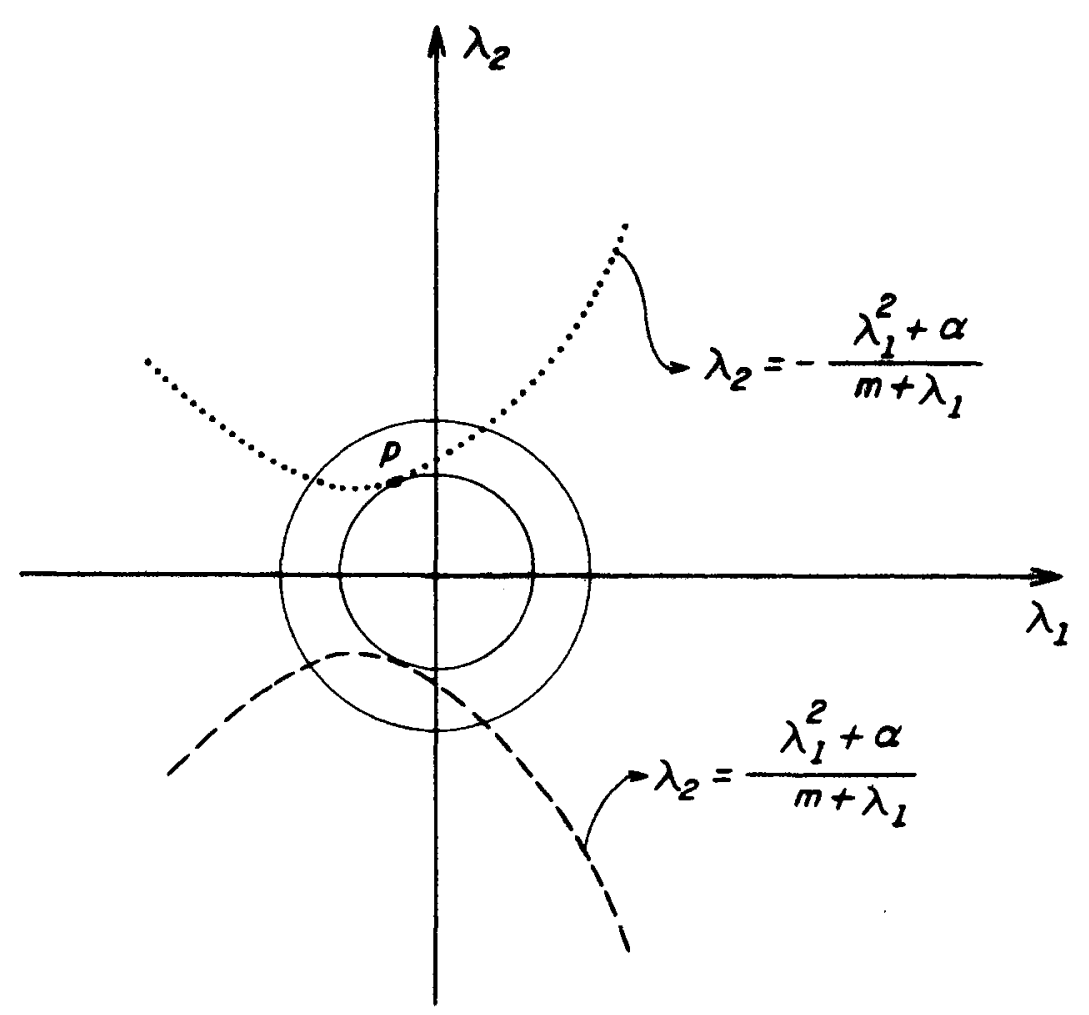

FI6. $5.41: m<-1, \alpha>0$ 
DIAGRAMAS DE BIFURCACATO: $m<-1, \alpha>0$

1. $R<|\overrightarrow{O P}|$
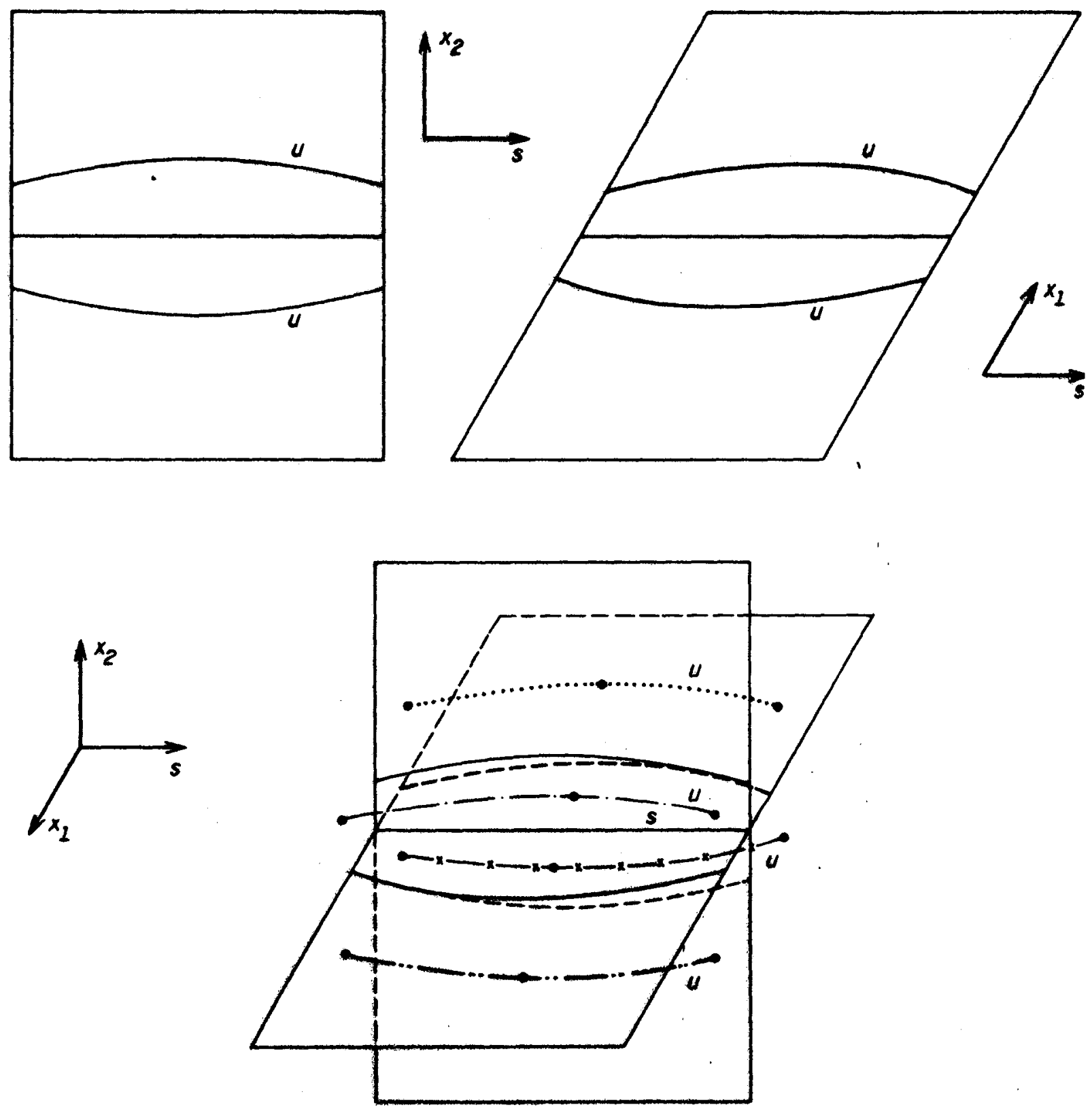

FIG. 5.42 
2. $R=|\overrightarrow{O P}|$
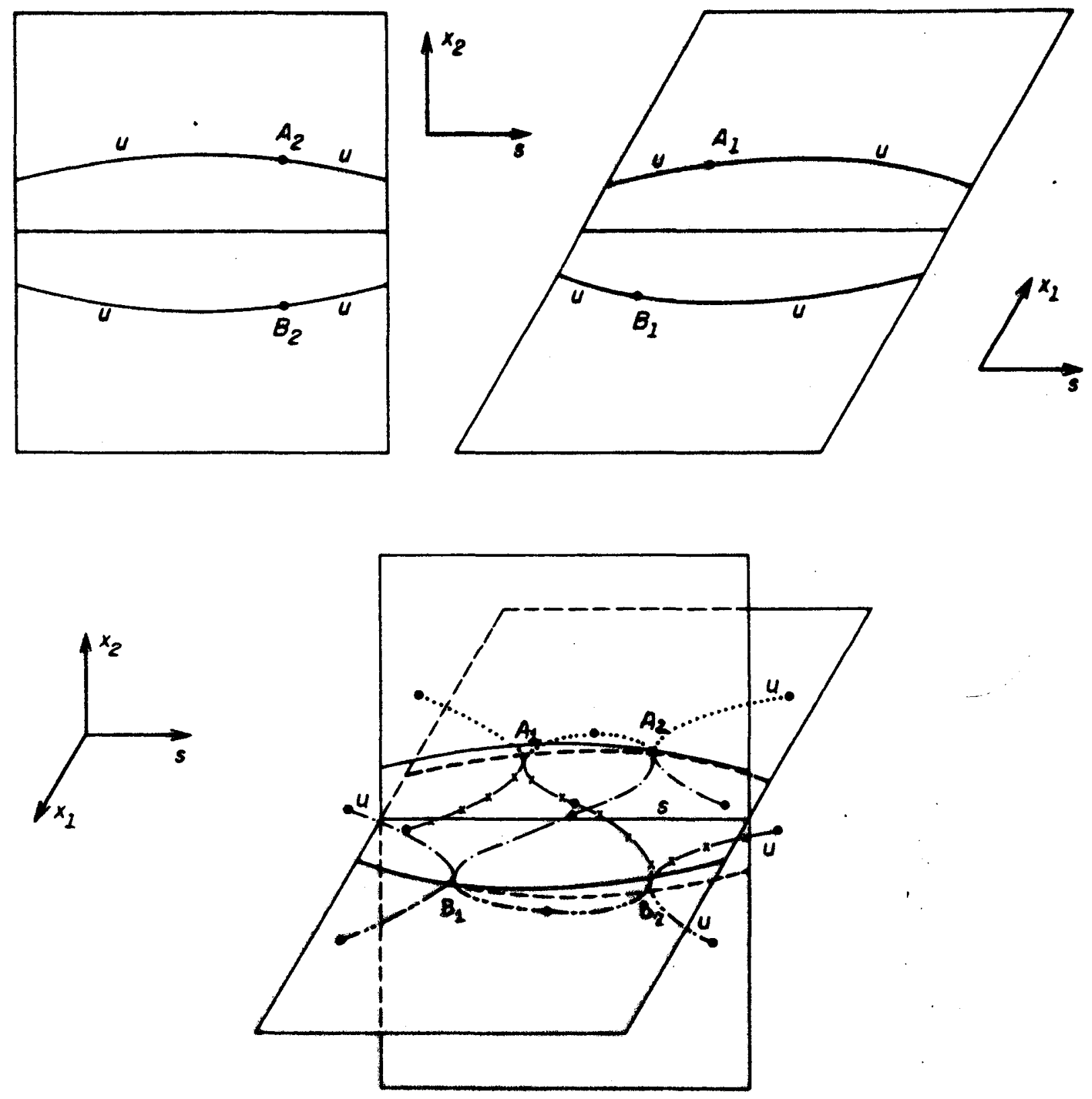

FIG. 5.42 
3. $|\overrightarrow{O P}|<R<\alpha$
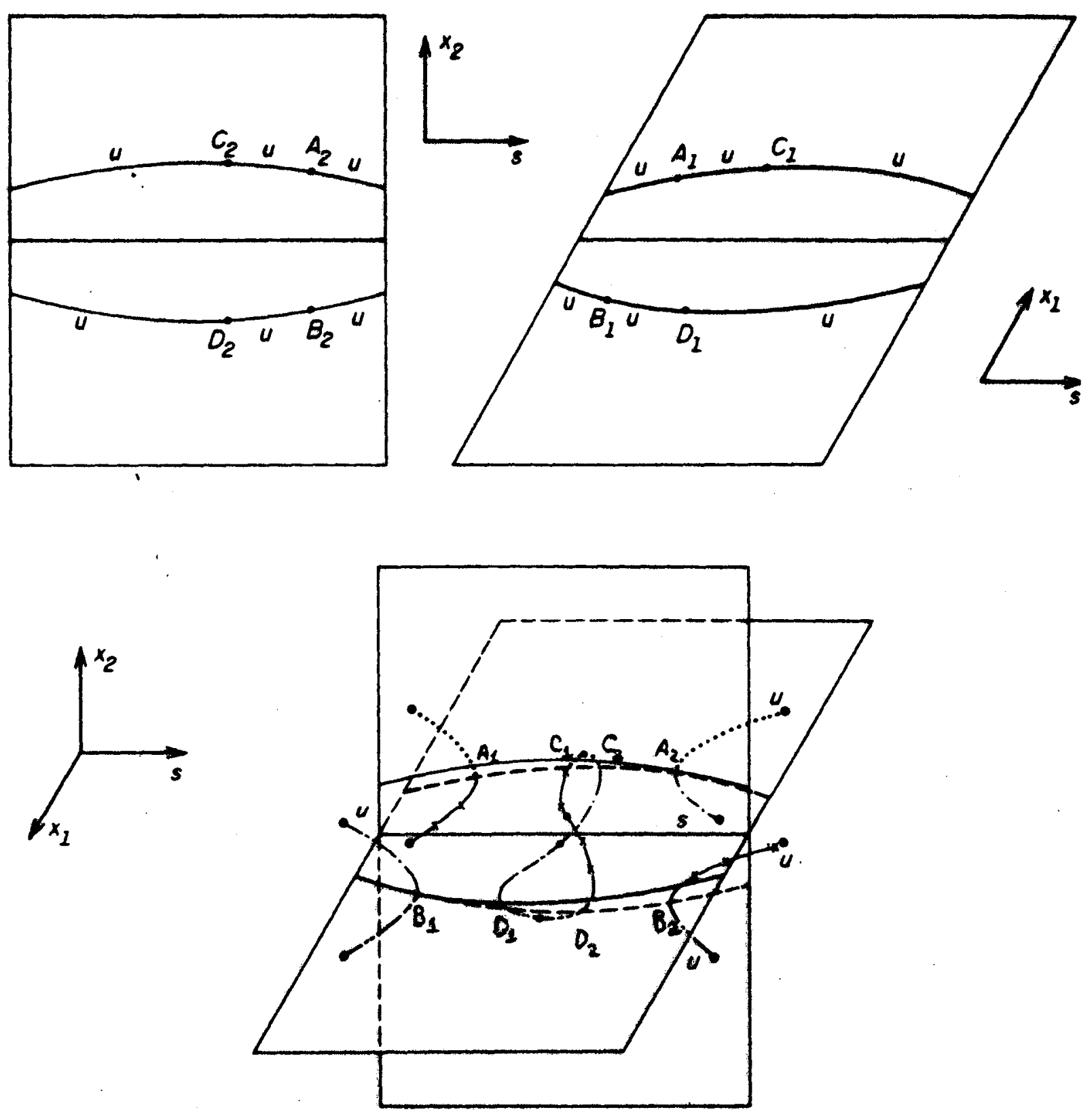

F/G. 5.42 
4. $R=\alpha$
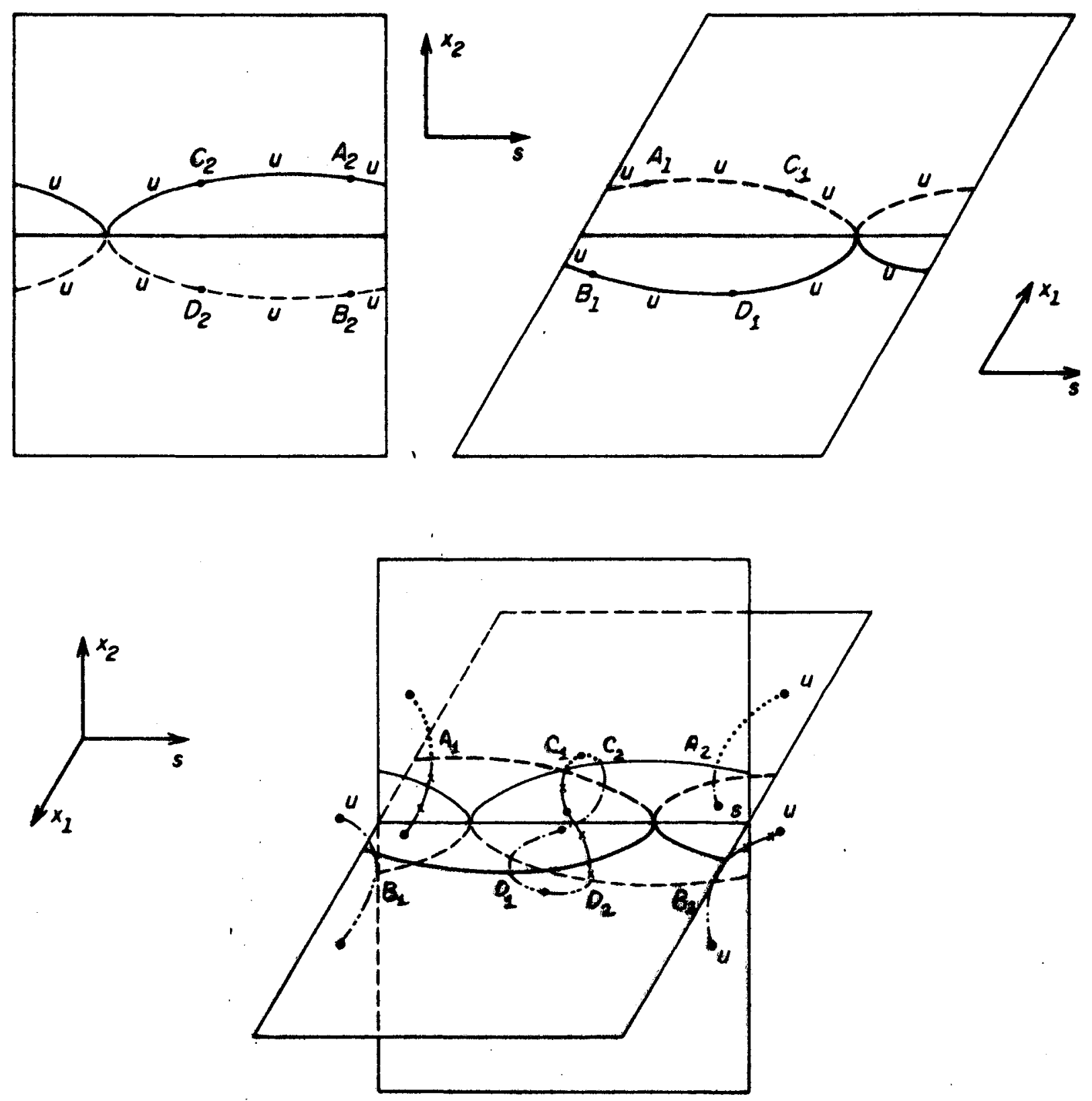

FIG. 5.42 
5. $R>\alpha$
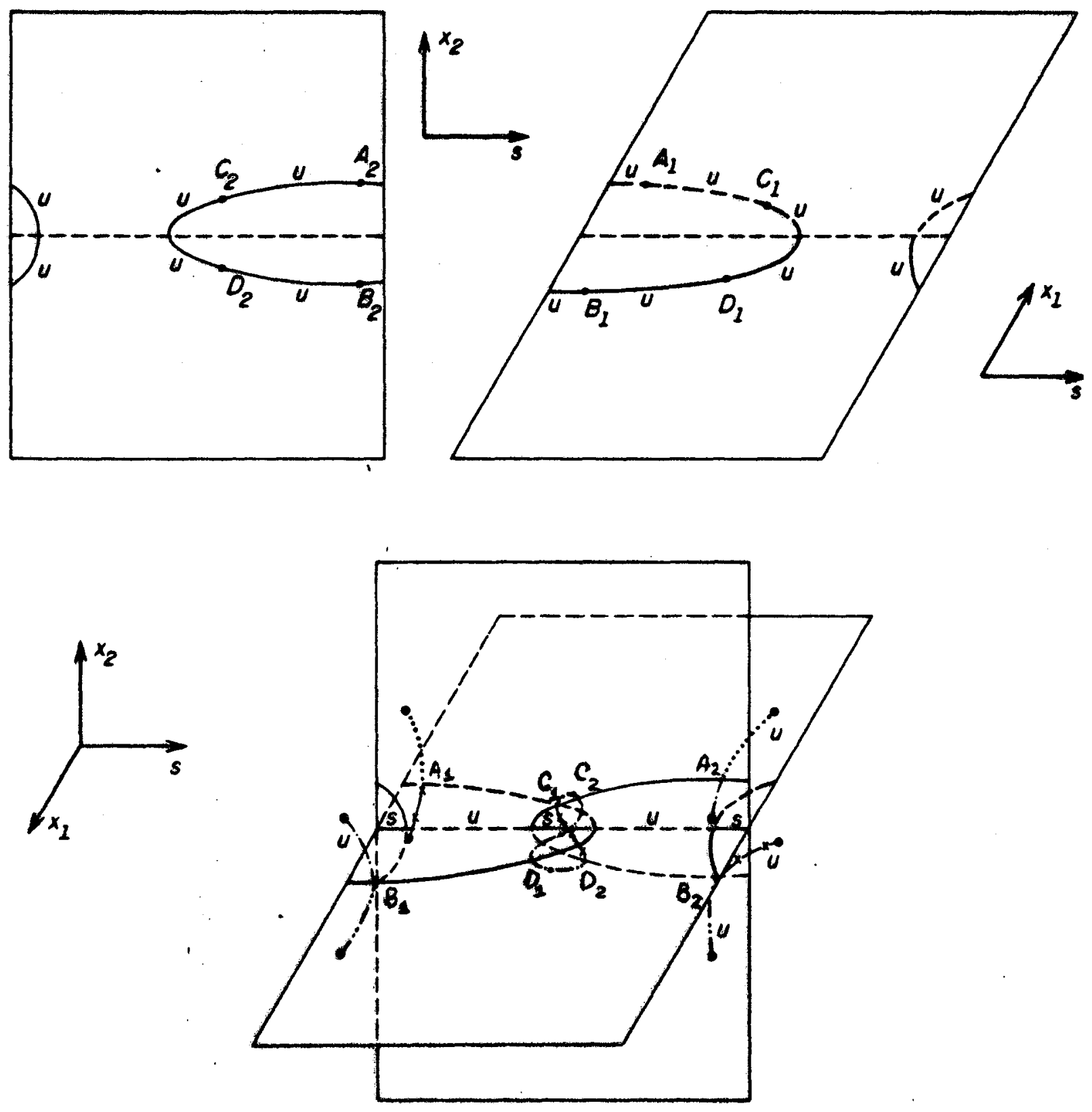

F/G. 5.42 


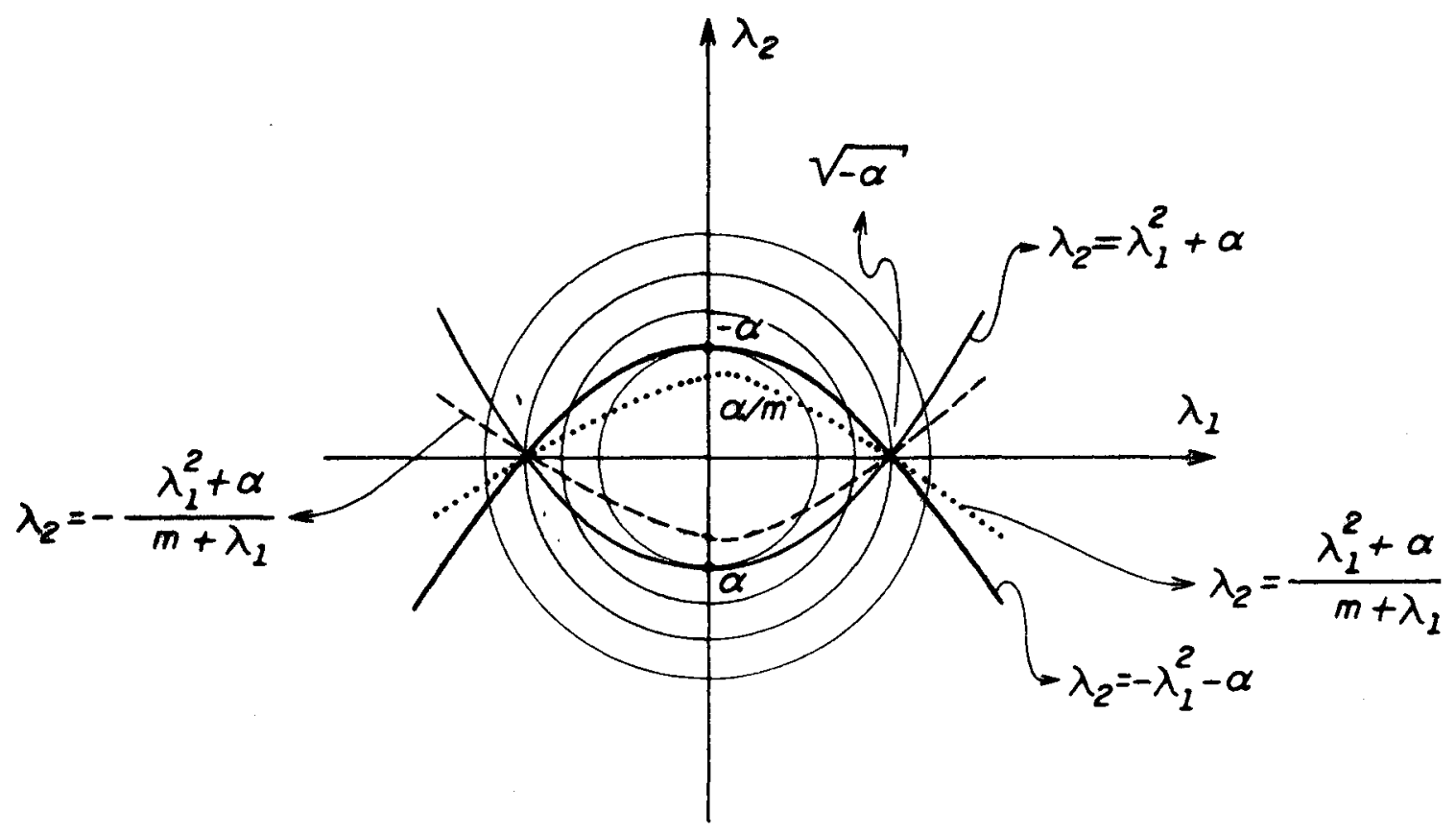

F/6. $5.43: m<-1, \alpha<0$ 
DIAGRAMAS DE GIFURCAGÃO: $m<1, \alpha<0$

1. $R=-\alpha$

$-\stackrel{u}{\pi / 2}-\frac{u}{3 \pi / 2}-\frac{u}{3}--\rightarrow$

2. $-\alpha<R<\sqrt{-\alpha}$
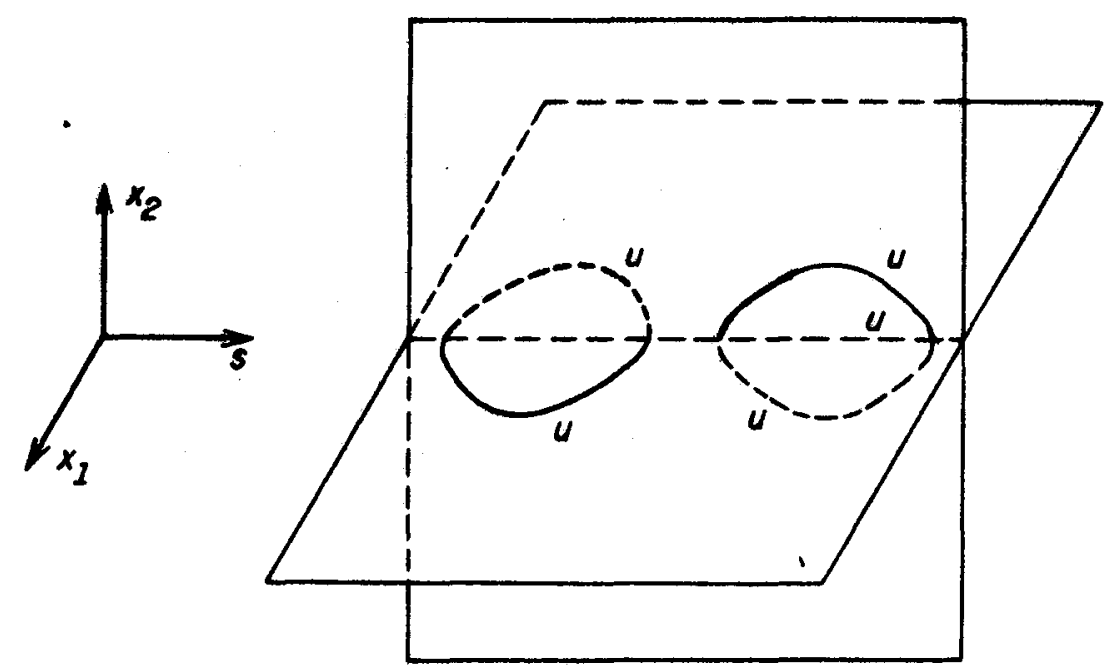

3. $R=\sqrt{-\alpha}$

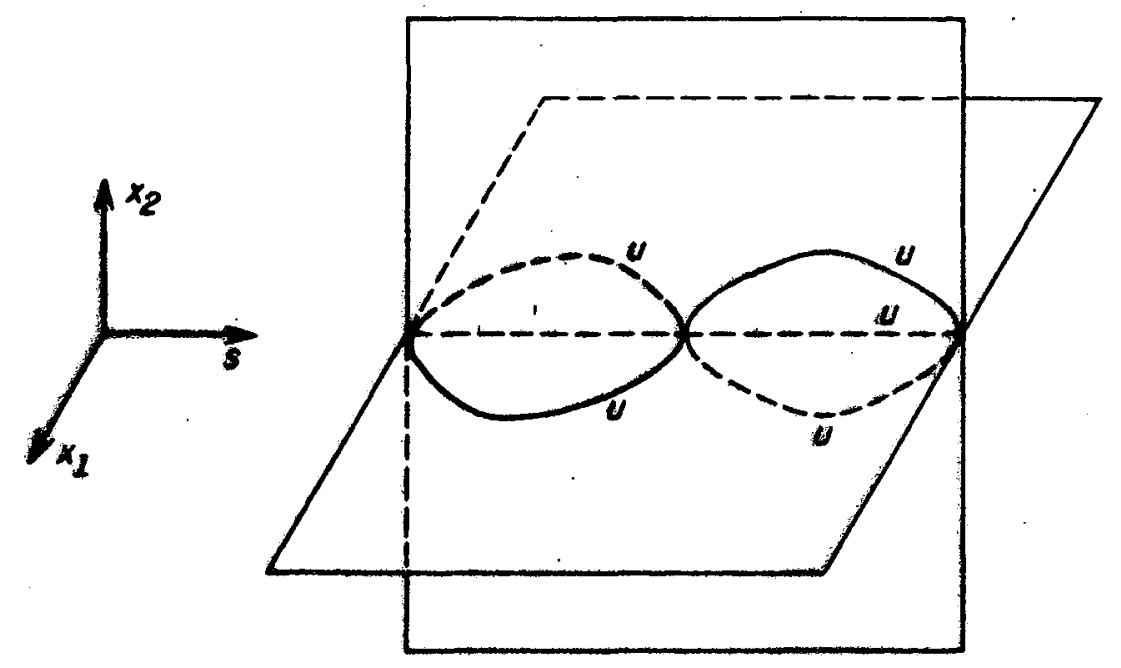

F/G. 5.44 
4. $R>\sqrt{-\alpha}$
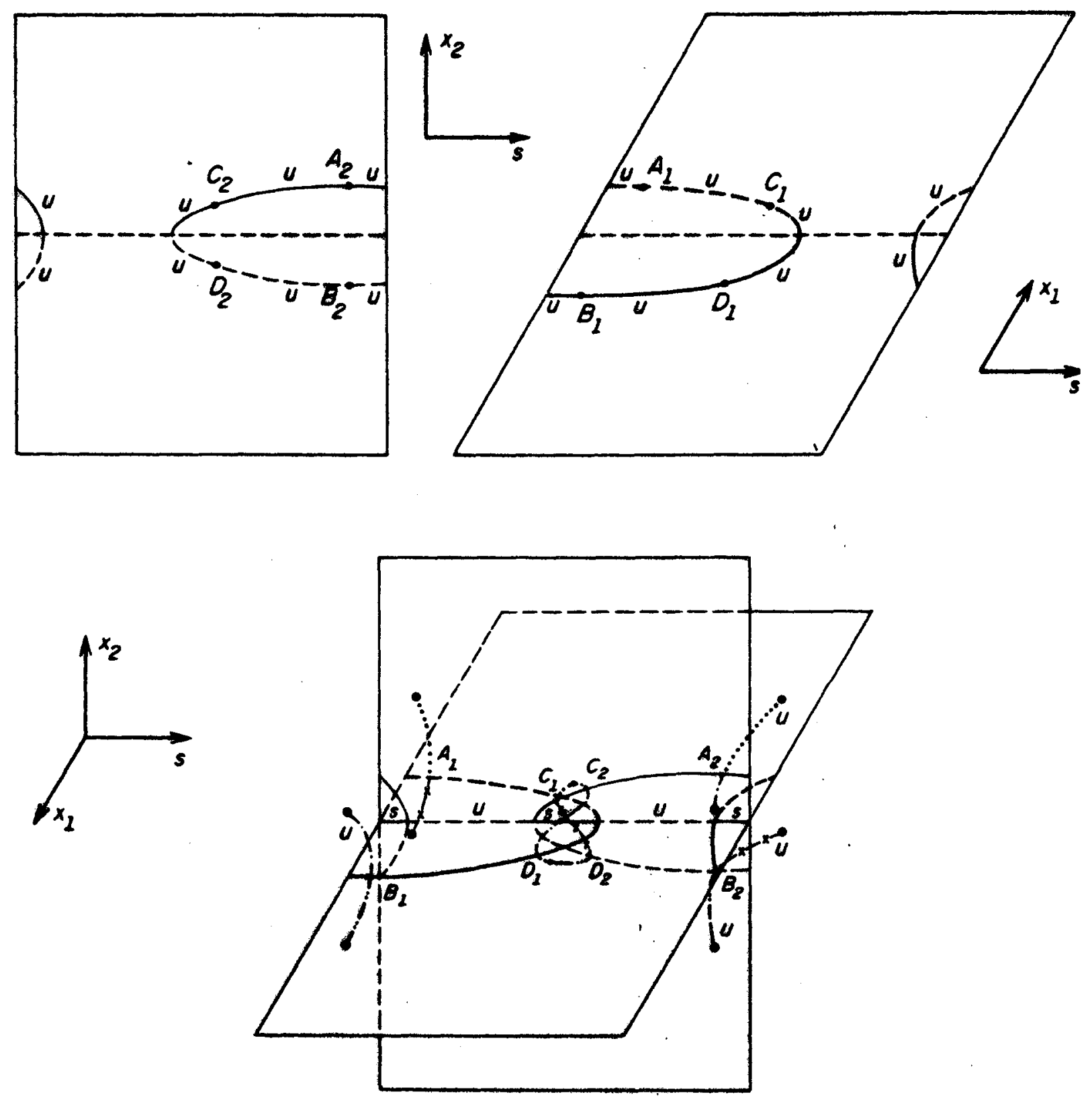

FIG. 5.44 
Observação: Para essa forma normal, como $m \neq \pm 1,0$, temos quatro intervalos reais de variação de $m$ e para cada intervalo, oito possibilidades de variação dos sinais de $\epsilon_{3}, \epsilon_{4}, \epsilon_{5}$. Usando a mudança de coordenadas $g \mapsto-g$ que inverte a estabilidade e a. mudança $\left(\lambda_{1}, \lambda_{2}\right) \mapsto\left(\lambda_{1},-\lambda_{2}\right)$ que inverte as regiôes do plano $\left(\lambda_{1}, \lambda_{2}\right)$, vemos que basta analisar os seguintes casos:

\begin{tabular}{|l|ccc|}
\hline & $\epsilon_{3}$ & $\epsilon_{4}$ & $\epsilon_{5}$ \\
\hline \hline \multirow{3}{*}{$\begin{array}{l}m>1 \quad \mathrm{e} \\
m<-1\end{array}$} & 1 & 1 & 1 \\
\cline { 2 - 4 } & 1 & 1 & -1 \\
\cline { 2 - 4 } & 1 & -1 & 1 \\
\cline { 2 - 4 } & 1 & -1 & -1 \\
\hline \hline \multirow{3}{*}{$0<m<1 \quad \mathrm{e}$} \\
$-1<m<0$ & 1 & 1 & 1 \\
\cline { 2 - 4 } & 1 & 1 & -1 \\
\cline { 2 - 4 } & 1 & -1 & 1 \\
\cline { 2 - 4 } & 1 & -1 & -1 \\
\hline
\end{tabular}

Fizemos a opção $\epsilon_{3}=\epsilon_{4}=\epsilon_{5}=1$ e analisamos todas as variações de $m$. 


\section{Capítulo 6}

\section{Aplicações}

\subsection{Motivação física}

Este capítulo trata da descrição de problemas onde a teoria apresentada neste trabaIho poderá ser aplicada. Alguns dos problemas estâo sendo por nós considerados e outros poderão ser estudados futuramente. $O$ uso de técnicas da teoria de singularidades aplicadas à teoria de bifurcação tem sido motivo de estudo de problemas em Física, Química, Mecânica, Teoria de Elasticidade, etc. Como vimos no capítulo 2, o nosso problema foi motivado por um problema em Mecânica.

Em [15], (Capítulo X, Caso 3, pág. 439), Golubitsky and Schaeffer descrevem a envergadura de uma placa elástica não espessa e isotrópica sujeita a uma força compressivel, e uniformemente distribuída sobre as extremidades da placa conforme a figura 6.1.

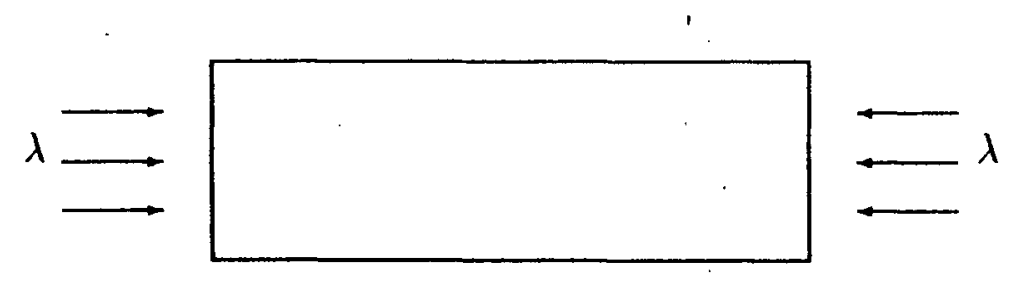

Fig. 6.1: Placa sujeita a uma força compressível 
A formulação de um modelo matemático para essa situação é feita através das equações de von Kármán e o problema se reduz a um problema de bifurcação $Z_{2} \oplus Z_{2}$-equivariante com um parâmetro de bifurcação sem simetria dado por $\lambda$. No caso clássico do modelo de von Kármán para uma placa não espessa, temos que, no caso da placa quadrada, o núcleo é 1-dimensional no ponto de bifurcação principal, ou seja, não há "competing modes". A quebra de simetria é descrita através de um problema com dois parâmetros, porém com simetria $Z_{2}$. Se considerarmos porém que a placa é feita de um material não isotrópico, então teremos um núcleo 2-dimensional no auto-valor principal. Neste caso teremos um problema representando dois "competing modes" para a envergadura. Dependendo do material, é possível obter uma bifurcação secundária com dois "competing modes" ao longo do ramo obtido após a primeira envergadura.

$O$ que pretendemos é descrever a envergadura de uma placa quadrada não espessa e não isotrópica quando um par de forças $F_{1}$ e $F_{2}$ atua, horizontalmente e verticalmente, sobre a placa, como na figura 6.2 .

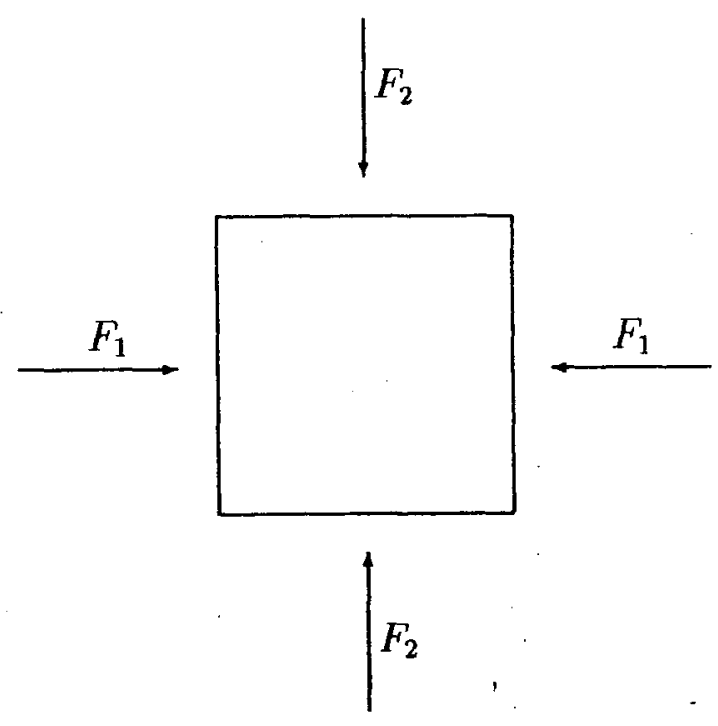

Fig. 6.2

A idéia é escrever as equações de von Kármán nesse contexto e reduzir o problema a um problema $\mathrm{D}_{4}$-equivariante com simetria nos dois parâmetros de bifurcação, e assim aplicar a teoria apresentada nesse trabalho. 
res, acoplados com a simetria do quadrado, podem ser estudados através de uma versão simétrica da bifurcação de Hopf padrão. Ele estudou as dinâmicas de todas as possíveis Bifurcações de Hopf com a simetria do quadrado.

Para sistemas de equaçôes de reação-difusão, o modelo a ser estudado seria o Brusselator. O Brusselator é o mais simples modelo para reações químicas que exibem comportamento oscilatório. (cf. [15], capítulo 1, pág. 312). O Brusselator exibe uma grande variedade de fenômenos de bifurcaçâo. Em [20], Ashwin estudou a Redução de Liapunov Schmidt para bifurcação de Hopf de equações do Brusselator no quadrado.

Em Teoria de Elasticidade, poderá ser estudada a deformação de uma placa quadrada formada de material elástico levemente compressível. Se a energia armazenada do material for do tipo Ogden com termos pares, como no caso dos materiais de Mooney-Rivlin, o problema pode ser reduzido ao nosso contexto de simetria. Como referência bibliográfica principal citamos [21]. 


\section{Apêndice}

\section{Provas das Proposições 4.1.1 a 4.1.5 do Capítulo 4}

Recorde que $\beta=(\alpha, \lambda), \alpha=\left(\alpha_{1}, \alpha_{2}, \alpha_{3}\right), \lambda=\left(\lambda_{1}, \lambda_{2}\right)$ e $D_{4}$ age em $\mathbb{C} \times \mathbb{R}^{3} \times \mathbb{R}^{2}$ da seguinte forma:

$$
\begin{aligned}
& \tilde{\kappa} \cdot(z, \alpha, \lambda)=\left(\bar{z}, \alpha_{1}, \alpha_{2}, \alpha_{3}, \lambda_{1}, \lambda_{2}\right), \\
& \tilde{\mu} \cdot(z, \alpha, \lambda)=\left(i \bar{z}, \alpha_{1}, \alpha_{2},-\alpha_{3}, \lambda_{1},-\lambda_{2}\right) .
\end{aligned}
$$

\section{Invariantes}

Definimos $N=z \bar{z}, \quad \delta=-\frac{1}{2}\left(z^{2}+\bar{z}^{2}\right), \quad \Delta=\delta^{2}, \quad u_{4}=\lambda_{2}^{2} \quad \mathrm{e}$

$$
\begin{array}{lll}
\hat{u}_{1}=N, \hat{u}_{2}=\Delta, & \hat{u}_{5}=\alpha_{1}, & \hat{u}_{8}=\delta \alpha_{3}, \\
\hat{u}_{3}=\lambda_{1}, & \hat{u}_{6}=\alpha_{2}, & \hat{u}_{9}=\delta \lambda_{2}, \\
\hat{u}_{4}=u_{4}, & \hat{u}_{7}=\alpha_{3}^{2}, & \hat{u}_{10}=\alpha_{3} \lambda_{2} .
\end{array}
$$

Proposição 4.1.1. $\mathcal{E}_{z, \beta}^{\mathrm{D}_{4}}=\hat{u}^{*} \mathcal{E}_{\tilde{u}}, \operatorname{com} \hat{u}=\left(\hat{u}_{1} \ldots \hat{u}_{10}\right)$.

Prova. Por um teorema de Schwarz [7], é suficiente mostrar que todo polinômio $\mathrm{D}_{\mathbf{4}^{-}}$ invariante pode der escrito como um polinômio em $\hat{u}$.

Seja $\bar{h}$ um polinômio $D_{4}$-invariante em $\mathbb{R}[z, \beta]$. Isso é equivalente à duas condições:

$$
\begin{aligned}
& \bar{h}\left(z, \alpha_{1}, \alpha_{2}, \alpha_{3}, \lambda_{1}, \lambda_{2}\right)=\bar{h}\left(\bar{z}, \alpha_{1}, \alpha_{2}, \alpha_{3}, \lambda_{1}, \lambda_{2}\right) \\
& \bar{h}\left(z, \alpha_{1}, \alpha_{2}, \alpha_{3}, \lambda_{1}, \lambda_{2}\right)=\bar{h}\left(i \bar{z}, \alpha_{1}, \alpha_{2},-\alpha_{3}, \lambda_{1},-\lambda_{2}\right)
\end{aligned}
$$

Do Capítulo 3, o subgrupo de isotropia de $\lambda$ é $\Sigma=\mathrm{Z}_{2} \oplus \mathrm{Z}_{2}$ e os invariantes para essa ação são $N$ e $\delta$. Logo, podemos escrever

$$
\bar{h}\left(z, \alpha_{1}, \alpha_{2}, \alpha_{3}, \lambda_{1}, \lambda_{2}\right)=\tilde{h}\left(N, \delta, \alpha_{1}, \alpha_{2}, \alpha_{3}, \lambda_{1}, \lambda_{2}\right) .
$$

Segue de (3) que

$$
\tilde{h}\left(N, \delta, \alpha_{1}, \alpha_{2}, \alpha_{3}, \lambda_{1}, \lambda_{2}\right)=\tilde{h}\left(N,-\delta, \alpha_{1}, \alpha_{2},-\alpha_{3}, \lambda_{1},-\lambda_{2}\right)
$$

e assim

$$
\tilde{h}\left(N, \delta, \alpha_{1}, \alpha_{2}, \alpha_{3}, \lambda_{1}, \lambda_{2}\right)=\hat{h}\left(\hat{u}_{1} \ldots \hat{u}_{10}\right)
$$


com $\hat{u}_{i}, 1 \leq i \leq 10$, dados em (1). O resultado segue de (4).

\section{Equivariantes}

Proposição 4.1.2 $\overrightarrow{\mathcal{E}}_{(z, \beta)}^{\mathrm{D}_{4}}$ é gerado por $z, \delta \bar{z}, \lambda_{2} \bar{z}, \alpha_{3} \bar{z}$ como um $\mathcal{E}_{(z, \beta)}^{\mathrm{D}_{4}}$-módulo.

Prova. Pelo teorema de Poénaru [24], seja $f:\left(\mathbb{C} \times \mathbb{R}^{3} \times \mathbb{R}^{2}, 0\right) \rightarrow \mathbb{W}$ um polinômio $\mathrm{D}_{4}$-equivariante, ou seja,

$$
\begin{aligned}
f\left(\bar{z}, \alpha_{1}, \alpha_{2}, \alpha_{3}, \lambda_{1}, \lambda_{2}\right) & =\kappa \cdot f\left(z, \alpha_{1}, \alpha_{2}, \alpha_{3}, \lambda_{1}, \lambda_{2}\right) \\
f\left(i \bar{z}, \alpha_{1}, \alpha_{2},-\alpha_{3}, \lambda_{1},-\lambda_{2}\right) & =i \kappa \cdot f\left(z, \alpha_{1}, \alpha_{2}, \alpha_{3}, \lambda_{1}, \lambda_{2}\right) .
\end{aligned}
$$

Considerando primeiro a ação de $\Sigma=Z_{2} \oplus Z_{2}$, podemos escrever

$$
f\left(z, \alpha_{1}, \alpha_{2}, \alpha_{3}, \lambda_{1}, \lambda_{2}\right)=p\left(N, \delta, \alpha_{1}, \alpha_{2}, \alpha_{3}, \lambda_{1}, \lambda_{2}\right) z+q\left(N, \delta, \alpha_{1}, \alpha_{2}, \alpha_{3}, \lambda_{1}, \lambda_{2}\right) \bar{z}
$$

Usando (6),

$$
p\left(N,-\delta, \alpha_{1}, \alpha_{2},-\alpha_{3}, \lambda_{1},-\lambda_{2}\right)=p\left(N, \delta, \alpha_{1}, \alpha_{2}, \alpha_{3}, \lambda_{1}, \lambda_{2}\right)
$$

e

$$
q\left(N,-\delta, \alpha_{1}^{\prime}, \alpha_{2},-\alpha_{3}, \lambda_{1},-\lambda_{2}\right)=-q\left(N, \delta, \alpha_{1}, \alpha_{2}, \alpha_{3}, \lambda_{1}, \lambda_{2}\right),
$$

o que é equivalente à

$$
p\left(N, \delta, \alpha_{1}, \alpha_{2}, \alpha_{3}, \lambda_{1}, \lambda_{2}\right)=p_{1}(\hat{u})
$$

e

$$
q\left(N, \delta, \alpha_{1}, \alpha_{2}, \alpha_{3}, \lambda_{1}, \lambda_{2}\right)=\delta q_{1}(\hat{u})+\alpha_{3} q_{2}(\hat{u})+\lambda_{2} q_{3}(\hat{u})
$$

De (7),

$$
f\left(z, \alpha_{1}, \alpha_{2}, \alpha_{3}, \lambda_{1}, \lambda_{2}\right)=p_{1}(\hat{u}) z+q_{1}(\hat{u}) \delta \bar{z}+q_{2}(\hat{u}) \alpha_{3} \bar{z}+q_{3}(\hat{u}) \lambda_{2} \bar{z}
$$

e a proposição está verificada.

Observação. Seja $\bar{u}=\left(\hat{u}_{1} \ldots \hat{u}_{7}\right)$ e $p \in \mathcal{E}_{\hat{u}}$. Então, existem germes $p_{i} \in \mathcal{E}_{\hat{u}}, 1 \leq i \leq 4$, tais que

$$
p(\hat{u})=p_{1}(\bar{u})+\delta \lambda_{2} p_{2}(\bar{u})+\delta \alpha_{3} p_{3}(\bar{u})+\alpha_{3} \lambda_{2} p_{4}(\bar{u}) .
$$

Esta decomposição é facilmente verificada usando-se o Teorema de Taylor com parâmetros ([15], pág. 77). 
Proposição 4.1.3. $\overrightarrow{\mathcal{E}}_{(z, \beta)}^{\mathrm{D}_{4}}$ é gerado livremente por $z, \delta \bar{z}, \lambda_{2} \bar{z}, \alpha_{3} \bar{z}, \delta \lambda_{2} z, \delta \alpha_{3} z, \alpha_{3} \lambda_{2} z$ e $\delta \alpha_{3} \lambda_{2} \bar{z}$ como um $\mathcal{E}_{\bar{u}}$-módulo.

Prova. Pela Proposição $4.1 .2, \hat{f} \in \overrightarrow{\mathcal{E}}_{(z, \beta)}^{\mathrm{D}_{4}}$ pode ser escrito como

$$
\hat{f}(z, \beta)=p_{1}(\hat{u}) z+p_{2}(\hat{u}) \delta \bar{z}+p_{3}(\hat{u}) \lambda_{2} \bar{z}+p_{4}(\hat{u}) \alpha_{3} \bar{z}
$$

$\operatorname{com} p \in \mathcal{E}_{\hat{u}}, 1 \leq i \leq 5$.

Usando (8), para cada $1 \leq i \leq 4$, existem germes $p_{i j} \in \mathcal{E}_{\bar{u}}, 1 \leq j \leq 4$, tais que

$$
\begin{aligned}
& p_{1}(\hat{u}) z=p_{11}(\bar{u}) z+p_{12}(\bar{u}) \delta \lambda_{2} z+p_{13}(\bar{u}) \delta \alpha_{3} z+p_{14}(\bar{u}) \alpha_{3} \lambda_{2} z \\
& p_{2}(\hat{u}) \delta \bar{z}=p_{21}(\bar{u}) \delta \bar{z}+p_{22}(\bar{u}) \Delta \lambda_{2} \bar{z}+p_{23}(\bar{u}) \Delta \alpha_{3} \bar{z}+p_{24}(\bar{u}) \delta \alpha_{3} \lambda_{2} \bar{z} \\
& p_{3}(\hat{u}) \lambda_{2} \bar{z}=p_{31}(\bar{u}) \lambda_{2} \bar{z}+p_{32}(\bar{u}) \delta u_{4} \bar{z}+p_{33}(\bar{u}) \delta \alpha_{3} \lambda_{2} \bar{z}+p_{34}(\bar{u}) \alpha_{3} u_{4} \bar{z} \\
& p_{4}(\hat{u}) \alpha_{3} \bar{z}=p_{41}(\bar{u}) \alpha_{3} \bar{z}+p_{42}(\bar{u}) \delta \alpha_{3} \lambda_{2} \bar{z}+p_{43}(\bar{u}) \delta \alpha_{3}^{2} \bar{z}+p_{44}(\bar{u}) \alpha_{3}^{2} \lambda_{2} \bar{z}
\end{aligned}
$$

Substituindo (10) em (9), segue que $z, \delta \bar{z}, \lambda_{2} \bar{z}, \alpha_{3} \bar{z}, \delta \lambda_{2} z, \delta \alpha_{3} z, \alpha_{3} \lambda_{2} z$ e $\delta \alpha_{3} \lambda_{2} \bar{z}$ geram $\overrightarrow{\mathcal{E}}_{(z, \beta)}^{\mathrm{D}_{4}}$ como um $\mathcal{E}_{\bar{u}}$-módulo.

Resolvendo o sistema,

$$
\begin{array}{r}
p_{1}(\bar{u}) z+p_{2}(\bar{u}) \delta \bar{z}+p_{3}(\bar{u}) \lambda_{2} \bar{z}+p_{4}(\bar{u}) \alpha_{3} \bar{z}+p_{5}(\bar{u}) \delta \lambda_{2} z+ \\
p_{6}(\bar{u}) \delta \alpha_{3} z+p_{7}(\bar{u}) \alpha_{3} \lambda_{2} z++p_{8}(\bar{u}) \delta \alpha_{3} \lambda_{2} \bar{z} \equiv 0
\end{array}
$$

obtemos $p_{i} \equiv 0,1 \leq i \leq 8$, o que prova o teorema.

\section{Matrizes Comutantes}

Proposição 4.1.4. $\mathbf{M}_{(z, \beta)}^{\mathrm{D}_{4}}$ é o $\mathcal{E}_{(z, \beta)}^{\mathrm{D}_{4}}$-módulo gerado pelas seguintes aplicações em $\mathbb{C}$ :

$$
\begin{aligned}
S_{1}(z, \beta) w & =w, & S_{5}(z, \beta) w & =i \omega \bar{w} \\
S_{2}(z, \beta) w & =\lambda_{2} \bar{w}, & S_{6}(z, \beta) w & =-i \lambda_{2} \omega w \\
S_{3}(z, \beta) w & =\delta \bar{w}, & S_{7}(z, \beta) w & =-i \delta \omega w \\
S_{4}(z, \beta) w & =\alpha_{3} \bar{w}, & S_{8}(z, \beta) w & =-i \alpha_{3} \omega w .
\end{aligned}
$$

onde $\omega=\frac{i}{4}\left(\bar{z}^{2}-z^{2}\right)$ 
Prova. $\hat{S} \in \mathbf{M}_{(z, \beta)}^{\mathrm{D}_{4}}$ pode ser escrita como $\hat{S}(z, \beta) w=a(z, \beta) w+b(z, \beta) \bar{w}$ satisfazendo

$$
\begin{aligned}
\hat{S}\left(\bar{z}, \alpha_{1}, \alpha_{2}, \alpha_{3}, \lambda_{1}, \lambda_{2}\right) w & =\kappa \cdot \hat{S}\left(z, \alpha_{1}, \alpha_{2}, \alpha_{3}, \lambda_{1}, \lambda_{2}\right) \bar{w} \\
\hat{S}\left(i \bar{z}, \alpha_{1}, \alpha_{2},-\alpha_{3}, \lambda_{1},-\lambda_{2}\right) w & =i \kappa \cdot \hat{S}\left(z, \alpha_{1}, \alpha_{2}, \alpha_{3}, \lambda_{1}, \lambda_{2}\right) i \bar{w}
\end{aligned}
$$

Do Capítulo 4, um conjunto equivalente de aplicações lineares que geram $\mathbf{M}_{(z, \beta)}^{\Sigma}$, $\Sigma=\mathrm{Z}_{2} \oplus \mathrm{Z}_{2}$, como um $\mathcal{E}_{(N, \delta, \beta)}$-módulo é

$$
\begin{aligned}
\tilde{S}_{1}(z, \beta) w & =w, & \tilde{S}_{3}(z, \beta) w & =i w \bar{w} \\
\hat{S}_{2}(z, \beta) w & =\bar{w}, & S_{4}(z, \beta) w & =-i \omega w .
\end{aligned}
$$

Como $\hat{S} \in \mathbf{M}_{(z, \beta)}^{\Sigma}$,

$$
\hat{S}(z, \beta) w=p_{1}(N, \delta, \beta) w+p_{2}(N, \delta, \beta) \bar{w}+p_{3}(N, \delta, \beta) i \omega \bar{w}-p_{4}(N, \delta, \beta) i \omega w
$$

Usando a condição de equivariância (12) para $\hat{S}$, as seguintes igualdades devem ser satisfeitas:

$$
\begin{aligned}
& p_{1}\left(N,-\delta, \alpha_{1}, \alpha_{2},-\alpha_{3}, \lambda_{1},-\lambda_{2}\right)=p_{1}\left(N, \delta, \alpha_{1}, \alpha_{2}, \alpha_{3}, \lambda_{1}, \lambda_{2}\right), \\
& p_{2}\left(N,-\delta, \alpha_{1}, \alpha_{2},-\alpha_{3}, \lambda_{1},-\lambda_{2}\right)=-p_{2}\left(N, \delta, \alpha_{1}, \alpha_{2}, \alpha_{3}, \lambda_{1}, \lambda_{2}\right), \\
& p_{3}\left(N,-\delta, \alpha_{1}, \alpha_{2},-\alpha_{3}, \lambda_{1},-\lambda_{2}\right)=p_{3}\left(N, \delta, \alpha_{1}, \alpha_{2}, \alpha_{3}, \lambda_{1}, \lambda_{2}\right), \\
& p_{4}\left(N,-\delta, \alpha_{1}, \alpha_{2},-\alpha_{3}, \lambda_{1},-\lambda_{2}\right)=-p_{4}\left(N, \delta, \alpha_{1}, \alpha_{2}, \alpha_{3}, \lambda_{1}, \lambda_{2}\right) .
\end{aligned}
$$

Consequentemente,

$$
\begin{aligned}
& p_{1}\left(N, \delta, \alpha_{1}, \alpha_{2}, \alpha_{3}, \lambda_{1}, \lambda_{2}\right)=q_{1}(\hat{u}) \\
& p_{2}\left(N, \delta, \alpha_{1}, \alpha_{2}, \alpha_{3}, \lambda_{1}, \lambda_{2}\right)=\delta q_{2}(\hat{u})+\alpha_{3} q_{3}(\hat{u})+\lambda_{2} q_{4}(\hat{u}) \\
& p_{3}\left(N, \delta, \alpha_{1}, \alpha_{2}, \alpha_{3}, \lambda_{1}, \lambda_{2}\right)=q_{5}(\hat{u}) \\
& p_{4}\left(N, \delta, \alpha_{1}, \alpha_{2}, \alpha_{3}, \lambda_{1}, \lambda_{2}\right)=\delta q_{6}(\hat{u})+\alpha_{3} q_{7}(\hat{u})+\lambda_{2} q_{8}(\hat{u})
\end{aligned}
$$

A proposição segue da substituição de (14) em (13). 
Proposição 4.1.5. $\mathrm{M}_{(z, \beta)}^{\mathrm{D}_{4}}$ é o $\mathcal{E}_{\bar{u}}$-módulo livremente gerado pelas seguintes aplicações lineares em $\mathbb{C}$ :

$$
\begin{array}{ll}
\hat{S}_{1}(z, \beta) w=w, & \hat{S}_{9}(z, \beta) w=i \omega \bar{w} \\
\hat{S}_{2}(z, \beta) w=\lambda_{2} \bar{w}, & \hat{S}_{10}(z, \beta) w=-i \lambda_{2} \omega w \\
\hat{S}_{3}(z, \beta) w=\delta \bar{w}, & \hat{S}_{11}(z, \beta) w=-i \delta \omega w \\
\hat{S}_{4}(z, \beta) w=\alpha_{3} \bar{w}, & \hat{S}_{12}(z, \beta) w=-i \alpha_{3} \omega w \\
\hat{S}_{5}(z, \beta) w=\delta \lambda_{2} w, & \hat{S}_{13}(z, \beta) w=i \delta \lambda_{2} \omega \bar{w} \\
\hat{S}_{6}(z, \beta) w=\delta \alpha_{3} w, & \hat{S}_{14}(z, \beta) w=i \delta \alpha_{3} \omega \bar{w} \\
\hat{S}_{7}(z, \beta) w=\alpha_{3} \lambda_{2} w, & \hat{S}_{15}(z, \beta) w=i \alpha_{3} \lambda_{2} \omega \bar{w} \\
\hat{S}_{8}(z, \beta) w=\delta \alpha_{3} \lambda_{2} \bar{w}, & \hat{S}_{16}(z, \beta) w=-i \delta \alpha_{3} \lambda_{2} \omega w .
\end{array}
$$

Prova. Pela proposição 4.1.4., $\hat{S} \in \mathbf{M}_{(z, \beta)}^{\mathrm{D}_{4}}$ pode ser escrita como

$$
\begin{array}{r}
\hat{S}(z, \beta) w=p_{1}(\hat{u}) w+p_{2}(\hat{u}) \lambda_{2} \bar{w}+p_{3}(\hat{u}) \delta \bar{w}+p_{4}(\hat{u}) \alpha_{3} \bar{w}+p_{5}(\hat{u}) i \omega \bar{w} \\
-p_{6}(\hat{u}) i \omega \lambda_{2} w-p_{7}(\hat{u}) i \omega \delta w-p_{8}(\hat{u}) i \omega \alpha_{3} w
\end{array}
$$

Usando (8), para cada $1 \leq i \leq 8$, existem germes $p_{i j} \in \mathcal{E}_{\bar{u}}, \quad i \leq j \leq 4$, tais que

$$
\begin{aligned}
& p_{1}(\hat{u}) w=p_{11}(\bar{u}) w+p_{12}(\bar{u}) \delta \lambda_{2} w+p_{13}(\bar{u}) \delta \alpha_{3} w+p_{14}(\bar{u}) \alpha_{3} \lambda_{2} w \\
& p_{2}(\hat{u}) \lambda_{2} \bar{w}=p_{21}(\bar{u}) \lambda_{2} \bar{w}+p_{22}(\bar{u}) \delta u_{4} \bar{w}+p_{23}(\bar{u}) \delta \alpha_{3} \lambda_{2} \bar{w}+p_{24}(\bar{u}) \alpha_{3} u_{4} \bar{w} \\
& p_{3}(\hat{u}) \delta \bar{w}=p_{31}(\bar{u}) \delta \bar{w}+p_{32}(\bar{u}) \Delta \lambda_{2} \bar{w}+p_{33}(\bar{u}) \Delta \alpha_{3} \bar{w}+p_{34}(\bar{u}) \delta \alpha_{3} \lambda_{2} \bar{w} \\
& p_{4}(\hat{u}) \alpha_{3} \bar{w}=p_{41}(\bar{u}) \alpha_{3} \bar{w}+p_{42}(\bar{u}) \delta \alpha_{3} \lambda_{2} \bar{w}+p_{43}(\bar{u}) \alpha_{3}^{2} \delta \bar{w}+p_{44}(\bar{u}) \alpha_{3}^{2} \lambda_{2} \bar{w} \\
& p_{5}(\hat{u}) i \omega \bar{w}=p_{51}(\bar{u}) i \omega \bar{w}+p_{52}(\bar{u}) i \omega \delta \lambda_{2} \bar{w}+p_{53}(\bar{u}) i \omega \delta \alpha_{3} \bar{w}+p_{54}(\bar{u}) i \omega \alpha_{3} \lambda_{2} \bar{w} \\
& p_{6}(\hat{u}) i \omega \lambda_{2} w=p_{61}(\bar{u}) i \omega \lambda_{2} w+p_{62}(\bar{u}) u_{4} i \omega \delta w+p_{63}(\bar{u}) i \omega \delta \alpha_{3} \lambda_{2} w+p_{64}(\bar{u}) u_{4} i \omega \alpha_{3} w \\
& p_{7}(\hat{u}) i \omega \delta w=p_{71}(\bar{u}) i \omega \delta w+p_{72}(\bar{u}) \Delta i \omega \lambda_{2} w+p_{73}(\bar{u}) \Delta i \omega \alpha_{3} w+p_{74}(\bar{u}) i \omega \delta \alpha_{3} \lambda_{2} w \\
& p_{8}(\hat{u}) i \omega \alpha_{3} w=p_{81}(\bar{u}) i \omega \alpha_{3} w+p_{82}(\bar{u}) i \omega \delta \alpha_{3} \lambda_{2} w+p_{83}(\bar{u}) i \alpha_{3}^{2} \omega \delta w+p_{84}(\bar{u}) i \alpha_{3}^{2} \omega \lambda_{2} w .
\end{aligned}
$$


Substituindo-se (16) em (15), segue que $\mathbf{M}_{(z, \beta)}^{\mathrm{D}_{4}}$ é gerado por $\hat{S}_{i}(z, \beta), 1 \leq i \leq 16$, como um $\mathcal{E}_{\bar{u}}$-módulo.

Então, resolvendo o sistema $\sum_{i=1}^{16} q_{i}(\bar{u}) \hat{S}_{i}(z, \beta) \equiv 0$, obtemos $q_{i} \equiv 0,1 \leq i \leq 16$. Portanto, as aplicações lineares $\hat{S}_{i}(z, \beta), 1 \leq i \leq 16$, geram livremente $\mathbf{M}_{(z, \beta)}^{\mathrm{D}_{4}}$ sobre $\mathcal{E}_{\bar{u}}$. 


\section{Bibliografia}

[1] A. Lari-Lavassani, Multiparameter bifurcation with symmetry via singularity theory. Ph.D. Thesis, Ohio State University, 1990.

[2] A. Vanderbauwhede, Local bifurcation and symmetry. Research Notes in Math. 75. Pitman, Boston, 1982.

[3] C.T.C. Wall, Finite determinacy of smooth map-germs. Bull. London Math. Soc. $13,481-539,1981$.

[4] D. Mond and J. Montaldi, Deformations of maps on complete intersections, Da-

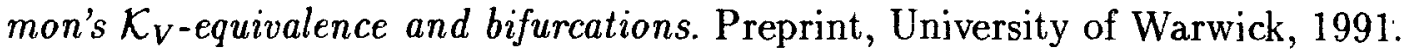

[5] E. Bierstone, The structure of orbit spaces and the singularities of equivariant maps. IMPA, Rio de Janeiro, 1980.

[6] E.J. Holder and D.G. Schaeffer, Boundary conditions and mode jumping in the von Kármán equations. SIAM J. Math. Anal. 15, 446-458, 1984.

[7] G. Schwarz, Smooth functions invariant under the action of a compact Lie group. Topology 14, 63-68, 1975.

[8] I. Melbourne, The recognition problem for equivariant singularities. Nonlinearity 1, 215-240, 1987.

[9] J.C. Alexander and G. Auchmuty, Global bifurcations of phase-locked oscilators. Arch. Rational Mech. Anal. 93, 253-270, 1986.

[10] J. Damon, The unfolding and determinacy theorems for subgroups of $\mathcal{A}$ and $\mathcal{K}$. Memoirs of the American Mathematical Society 50, no 306, 1984. 
[11] J. Damon, Deformations of sections of singularities and Gorenstein surface singularities. Am. J. Math. 109, 695-722, 1987.

[12] J. Martinet, Déploiements versels des applications différentiables et classification des applications stables. Singularités d'applications differéntiables, Plans-Sur-Bex, Springer Lecture Notes 535, 1-44, 1975.

[13] J. Martinet, Singularities of smooth functions and maps. London Mathematical Society Lecture Notes Ser. 58. Cambridge University Press, Cambridge, 1982.

[14] J.W. Swift, Hopf bifurcation with the symmetry of the square. Nonlinearity 1, 333$377,1988$.

[15] M. Golubitsky, D.G. Schaeffer, Singularities and groups in bifurcation theory. Vol. I, Applied Math. Sciences 51. Springer Verlag, New York, 1985.

[16] M. Golubitsky, I. Stewart and D.G. Schạeffer, Singularities and groups in bifurcation theory. Vol II, Applied Math. Sciences 69. Springer Verlag, New York, 1988.

[17] M. Golubitsky and M. Roberts, A classification of degenerate Hopf bifurcations with $O(2)$ symmetry. J. Diff. Eqns. 69, 216-264, 1987.

[18] M. Peters, Classification of two-parameter bifurcations. Ph.D. Thesis, University of Warwick, 1991.

[19] J.N. Mather, Stability of $\mathrm{C}^{\infty}$ mappings, III. Finitely determined germs. Publ. Math. I.H.E.S. 35, 127-156, 1968.

[20] P. Ashwin, Liapunov-Schmidt reduction at Hopf bifurcation of the Brusselator equations on a square. Preprint, University of Warwick, 1992.

[21] R.W. Ogden, Large deformation isotropic elasticity: on the correlation of theory and experiment for compressible rubber like solids. Proc. R. Soc. Lond. A. 328; $567-583,1972$.

[22] T. Gaffney, Some new results in the classification theory of bifurcation problems. In: Multiparameter Bifurcation Theory. Contemporary Mathematics 56, A.M.S., Providence, 1986. 
[23] T.J. Bridges and J.E. Furter, Singularity theory and equivariant symplectic maps. Springer Lectures Notes 1558, Heidelberg, 1993.

[24] V. Poénaru, Singularités $\mathrm{C}^{\infty}$ en présenc de symétrie. Lecture Notes in Mathematics 510, Springer Verlag, Berlin, 1976.

[25] V.I. Arnold, Ordinary Differential Equations. The MIT Press, Cambridge, seventh edition, 1990.

[26] D.R.J. Chillingworth, Bifurcation from an orbit of symmetry. In: Singularities and dynamical systems, North Holland, 285-294, 1986. 
